\title{
AN EXPERIMENTAL STUDY OF
}

THE NEUTRONICS OF THE FIRST

GAS COOLED FAST REACTOR BENCHMARK ASSEMBLY (GCFR PHASE I ASSEMBLY)

by

S. K. Bhattacharyya

ARGONNE NATIONAL LABORATORY, ARGONNE, ILLINOIS

Prepared for the U.S. ENERGY RESEARCH AND DEVELOPMENT ADMINISTRATION under Contract W-31-109-Eng-38 
The facilities of Argonne National Laboratory are owned by the United States Government. Under the terms of a contract (W-31-109-Eng-38) between the U. S. Energy Research and Development Administration, Argonne Universities Association and The University of Chicago, the University employs the staff and operates the Laboratory in accordance with policies and programs formulated, approved and reviewed by the Association

\section{MEMBERS OF ARGONNE UNIVERSITIES ASSOCIATION}

The University of Arizona

Carnegie-Mellon University

Case Western Reserve University

The University of Chicago

University of Cincinnat

Illinois Institute of Technology

University of Illinois

Indiana University

Iowa State University

The University of Iowa
Kansas State University

The University of Kansas

Loyola University

Marquette University

Michigan State University

The University of Michigan

University of Minnesota

University of Missouri

Northwestern University

University of Notre Dame
The Ohio State University

Ohio University

The Pennsylvania State University

Purdue University

Saint Louis University

Southern Illinois University

The University of Texas at Austin

Washington University

Wayne State University

The University of Wisconsin

This report was prepared as an account of work sponsored by the United States Government. Neither the United States nor the United States Energy Research and Development Administration, nor any of their employees, nor any of their contractors, subcontractors, or their employees, makes any warranty, express or implied, or assumes any legal liability or responsibility for the accuracy, completeness or usefulness of any information, apparatus, product or process disclosed, or represents that its use would not infringe privately-owned rights. Mention of commercial products, their manufacturers, or their suppliers in this publication does not imply or connote approval or disapproval of the product by Argonne National Laboratory or the U. S. Energy Research and Development Administration.

Printed in the United States of America

Available from

National Technical Information Service

U. S. Department of Commerce 5285 Port Royal Road

Springfield, Virginia 22161

Price: Printed Copy $\$ 7.75 ;$ Microfiche $\$ 3.00$ 


\section{DISCLAIMER}

This report was prepared as an account of work sponsored by an agency of the United States Government. Neither the United States Government nor any agency Thereof, nor any of their employees, makes any warranty, express or implied, or assumes any legal liability or responsibility for the accuracy, completeness, or usefulness of any information, apparatus, product, or process disclosed, or represents that its use would not infringe privately owned rights. Reference herein to any specific commercial product, process, or service by trade name, trademark, manufacturer, or otherwise does not necessarily constitute or imply its endorsement, recommendation, or favoring by the United States Government or any agency thereof. The views and opinions of authors expressed herein do not necessarily state or reflect those of the United States Government or any agency thereof. 


\section{DISCLAIMER}

Portions of this document may be illegible in electronic image products. Images are produced from the best available original document. 
Distribution Category:

Gas Cooled Reactor Technology (UC-77)

ANL $-76-36$

\title{
ARGONNE NATIONAL LABORATORY
}

9700 South Cass Avenue

Argonne, Illinois 60439

AN EXPERIMENTAL STUDY OF

THE NEUTRONICS OF THE FIRST

GAS COOLED FAST REACTOR BENCHMARK ASSEMBLY

(GCFR PHASE I ASSEMBLY)

by

S. K. Bhattacharyya

Applied Physics Division

\begin{abstract}
This report was prepared as an account of work sponsored by the United States Covernment Nerthe the United States nor the Unitad States Energy ther employes, nor eny of thes contractors, subcontractors, or their employees, makes any warranty, express or impled, or assumes any legal uabuluty of responsobulity for the aceuracy, completenes or usefulness of any information, apparatus, product or process disclosed, of represents that its use would no unfinge privately owned rights
\end{abstract}

December 1976 
The work described in this report has involved the efforts of a number of people associated with various stages of the GCFR Phase-I Project. The material in the report was compiled Argonne National Laboratory Internal Memoranda authored by the responsible experimenters and analysts. In addition to these memoranda, the data have been published in several open literature articles.

As Project Coordinator, S. K. Bhattacharyya was associated with all stages of the project. W. R. Robinson served as the Experiment Coordinator and was directly involved in several experiments. R. D. McKnight provided the primary analytical support. In addition, the following other individuals were involved with the various stages of the project.

1. Program P1anning:

2. Experiments:

3. Analysis:

4. Reactor Operations:
E. M. Bohn and L. G. LeSage.

J. A. Morman, D. M. Smith, K. E. Freese,

T. J. Yule and E. F. Bennett.

D. C. Wade.

G. K. Rusch, F. H. Martens and the Reactor Operations Crew. 
ABSTRACT . . . . . . . . . . . . . . . . . . . .

I. INTRODUCTION AND OUTLINE OF PROGRAM . . . . . . . . . 1

II. DESCRIPTION OF ASSEMBLY AND ANALYTICAL METHODS. . • . . • • 4

A. Introduction. . . . . . . . . . . . . . 4

B. Description of the As sembly ............. 4

C. The Approach-To-Critical Experiment . . . . . . . 5

D. Corrections to the As-Built Critical Mass . . . . . . 7

1. Excess Reactivity Correction. . . . . . . . 7

2. In terface Gap Worth . . . . . . . . . . 7

3. Temperature Correction. . . . . . . . . 7

4. Source Subcriticality . . . . . . . . . 8

5. Edge Smoothing Correction . . . . . . . . 8

E. Generation of Atom Concentrations . . . . . . . . . 9

F. Operational Measurements. . . . . . . . . . . 10

1. Measurement of Temperature "Coefficient" of the As sembly................. . . 10

2. Core Drawer Worth Measurements. . . . . . . 11

3. Measurements of Control Rod Worths. . . . . . . 11

4. Configuration Reproducibility . . . . . . . . 11

5. Reactivity Worth of Decay of ${ }^{241} \mathrm{Pu}$........ 11

G. Analysis.................. 12

1. Cross Section Generation. . . . . . . . . 12

2. Evaluation of Neutron Streaming Effects ....... 13

3. Reactor Model ................. 14

4. Results of Eigenvalue Calculations. . . . . . . 15

H. Discussion of Results . . . . . . . . . . . 16 
III。 NEUTRON SPECTRUM MEASUREMENT. . . . . . . . . . . 18

A. Introduction. . . . . . . . . ...... 18

B. Experimental Technique. . . . . . . . . . 19

C. As sembly Configuration. . . . . . . . . . . 19

D. Results ................... 19

E. Systematic Measurement Errors . . . . . . . . . 20

F. Discussion. . . . . . . . . . . . . 21

IV. CENTRAL REACTION RATE MEASUREMENTS. . . . . . . . . . . . 21

A. Introduction................... 21

B. Experimental Procedure............. 21

1. Fission Rate Measurements .......... 21

2. Capture Rate Measurements . . . . . . . . 22

C. Data Reduction................ 22

1. Fission Rates............. . 22

2. Capture Rates . . . . . . . . . . . 23

D. Calculational Procedure . . . . . . . . . . 23

E. Results and Discussion. . . . . . . . . 24

V. SMALL SAMPLE REACTTVITY WORTH MEASUREMENTS. . • • • • • • . 25

A. Introduction. . . . . . . . . . . . . 25

B. Reactor Configuration . . . . . . . . . . 26

C. Experimental Me thod and Equipment . . . . . . . . 26

D. Calibration of the Fine Autorod (FAR) . . . . . . . 27

1. FAR Calibration for Central Worth Measurement . . . 27

2. FAR Calibration for the Radial Traverse Measurements. - 28

E. Calculational Methods . . . . . . . . . . . 28 
1. Central Worth . . . . . . . . . . . 28

2. Radial Worth Traverse . . . . . . . . . 28

F. Reactivity Worth Results. . . . . . . . . . . 29

1. Small Sample Central Worths . . . . . . . . 29

2. Elemental and Isotopic Central Worths . . . . . . 29

3. Small Sample Radial Worth Travers ........ 30

G. Determination of the Perturbation Denominator . . . . 30

1. The Central Fission Rate $\mathrm{R}_{\mathrm{f}}$. . . . . . . . . 31

2. The Worth of ${ }^{252} \mathrm{Cf}$ sample $[\rho(C f)] . . . . . . .32$

3. Results and Comparison with Calculations. . . . . 33

H. Discussion of Reactivity Worth Results. . . . . . . 33

1. Central Reactivity Worths ........... 33

2. Small Sample Radial Worth Traverses . . . . . . 35

I. Conclusions . . . . . . . . . . . . 35

VI. VILIDATION OF KINETICS PARAMETERS . . . . . . . . . . 36

A. Introduction. ................ 36

B. Experimental Procedure and Results. . . . . . . . . 36

1. Measurement of Relative Yields and Decay Constants. . . 36

2. Measurement of Beta Effective . . . . . . . . 37

c. Discussion................ 39

VIT. REACTION RATE MAPPINGS AND UNIT CELI MEASUREMENTS . • • • • . 39

A. Introduction. . . . . . . . . . . 39

B. Experimental Procedure.................. 40

1. Unit Cell Measurements in Normal Core . . . . . . 40

2. Radial Traverses in Normal and Checkerboard Octant. . 40 
3. Axial Traverses in Normal Core. . . . . . . . 41

C. Data Reduction. . . . . . . . . . . . 42

1. Fission Rates . . . . . . . . . . 4 42

2. Capture Rates . . . . . . . . . . . 42

3. Unit Cell and Stainless Steel Corrections . . . . . 42

4. Normalization . . . . . . . . . . . 43

D. Reactivity Worth Measurements . . . . . . . . . 43

E. Analysis. . . . . . . . . . . . . 43

F. Experimental Results. . . . . . . . . . . 45

1. Norma1 Core Traverses . . . . . . . . . . 45

2. Checkerboard Core Traverses . . . . . . . . 46

G. Discussion of Results . . . . . . . . . . . 46

1. Effect of Knees and Bed of the Reactor. . . . . . 46

2. Comparison of the Calculated and Experimental Radial
Traverses . . . . . . . . . . . 46

3. Comparison of the Calculated and Experimenta1 Axia1 Reaction Rate Traverses in the Normal Core. . . . .

4. Comparison Between the Reaction Rate Distributions in the Normal and Checkerboard Cases . . . . . . .

H. Conclusions . . . . . . . . . . . . . 48

VIII. THE SIMULATED S TEAM ENTRY EXPERIMENT. . . . . . . . . 49

A. Introduction. . . . . . . . . . . . 49

B. Experiment Description. . . . . . . . . . 50

C. Results of Measurements . . . . . . . . . . 51

D. Analysis. . . . . . . . . . . . 51

1. Broad Group Cross Section Generation. . . . . . .

2. Neutron Streaming Effects . . . . . . . . . 


\section{TABLE OF CONTENTS}

$\underline{\text { Page }}$

3. Eigenvalue Calculations . . . . . . . . 52

4. Exact Perturbation Theory Calculations. . . . . 53

5. X-Y Exact Perturbation Theory Mode1 . . . . . . 54

E. Discussion. . . . . . . . . . . 54

IX. SUMMARY AND CONCLUSIONS . . . . . . . . . . 55

APPENDIXES

A. CORRECTION FOR DECAY OF CAPTURE PRODUCT IN CENTRAL REACTION RATE

MEASUREMENT. . . . . . . . . . . . . . . . . 58

B. DETERMINATION OF ISOTOPIC FISSION RATES FROM FOIL DATA . . . . 62

REFERENCES . . . . . . . . . . . . . . . . . . 64 


\section{LIST OF FIGURES}

No.

Title

1. Unit Ce11 Structures (actual and mode1) for the Core, Radial Blanket and Axia1 Blanket in the ZPR-GCFR Phase I Critical Assembly.......................... 68

2. Drawer Master for Drawers in Core Region 1.......... 68

3. Drawer Master for Drawers in the Radial Blanket Region. . . . . 69

4. Sketch of the Stationary Half of the Assembly Showing the As-Built Configuration ....................... 70

5. Sketch of the Movable Half of the Assembly Showing the As-Built Configuration. . . . . . . . . . . . . . . . . 70

6. Approach-to-Critical P1ots from ${ }^{235} \mathrm{U}$ Fission Chamber Located at the Core Center ................... 70

7. Approach-to-Critical Plots from ${ }^{238} \mathrm{U}$ Fission Chamber Located at Core Center .................... 71

8. Approach-to-Critical Plots from $235 \mathrm{U}$ Fission Chamber Located at Core/Axial Blanket Interface. . . . . . . . . . . . 71

9. Approach-to-Critical Plots from $\mathrm{BF}_{3}$ Ionization Chamber Located at Outside Boundary of Radial Blanket . . . . . . . . . 72

10. Approach-to-Critical P1ots from $235 \mathrm{U}$ Fission Chamber Located at Core Center, using the 0lson-Palmer Method. . . . . . . . 72

11. Approach-to-Critical Plots from ${ }^{235} \mathrm{U}$ Fission Chamber Located at Core Center, with All Control Rods in their Positions of Least Reactivity。................. 73

12. Outline of Quadrant of the Core of the Assembly showing the Smoothed Core-Blanket Interface . . . . . . . . . . 73

13. Experimental Data and Least Squares Fitted Straight Lines used in the Determination of the Temperature "Coefficient" of the Assembly. 74

14. Experimental Data and Least Squares Fitted Line used in the Determination of the Reactivity Worth of ${ }^{24 l} \mathrm{Pu}$ Decay. . . . . . 75

15. Flowchart of the Calculation Method . . . . . . . . . 76

16. $\mathrm{MC}^{2}$ U1trafine Group Spectrum at the Core Center . . . . . . . . 77

17. The R-Z Mode1 of the GCFR Phase-I Assemb1y. . . . . . . . . 78

18. SCX Fine-Group (USS226) Spectra for the Core Center of the GCFR Phase-I Assembly. . . . . . . . . . . . . . . . . . 


\section{LIST OF FIGURES}

No.

19. SDX Fine-Group (USS226) Spectra for the Axial Blanket of the GCFR Phase-I Assembly. . . . . . . . . . . . . . . . . 79

20. Comparison of 11- and 29-Broad Group Real F1ux for the GCFR Phase-I Assembly. . . . . . . . . . . . . . . . 80

21. Comparison of 11- and 29-Broad Group Adjoint Flux for the GCFR Phase-I Assembly. . . . . . . . . . . . . . . . . .

22. Comparison of Real Group Flux for Streaming and Nonstreaming Ca1culations . . . . . . . . . . . . . . . . . . .

23. Comparison of Adjoint Group Flux for Streaming and Nonstreaming Calculations........................ 83

24. The XY Full Plane Model Used in the 3D Calculation. . . . . . 83

25. Schematic Showing In-Core Detector, Preamplifier, and Reactor Material Placement. . . . . . . . . . . . . 84

26. Measured and Calculated Spectra at the Center of the GCFR Phase-I Reference Core. . . . . . . . . . . . . . 84

27. Effect of an Uncertainty in $\mathrm{W}$ on a Typica1 LMFBR Spectrum . . . . 85

28. Correction to Account for Wall and End Effects. . . . . . . . 85

29. Measured and Calculated Spectra at the Center of the FTP-EMC Core - 85

30. ZPR-9 Core Configuration for the GCFR Phase-I Assembly . . . . . 86

31. Position of Fission Chamber Within Stationary Half Drawers. . . . 86

32. Example of Computer Fit to the $227.6 \mathrm{-keV}$ Photopeak in the Decay

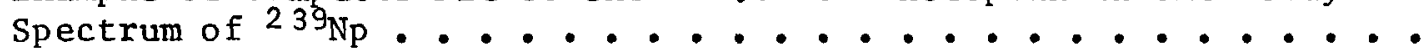

33. Example of Computer Fit to the 311.9-keV Photopeak in the Decay Spectrum of ${ }^{23} \mathrm{~Pa}$....................

34. Comparison of Undistorted ARC and Distorted PIT Broad Group FIux Spectra . . . . . . . . . . . . . . . . . . . •

35. Reactor Configuration for the Small Sample Central Reactivity Worth Measurements in the GCFR Phase-I Assembly . . . . . . . . 88

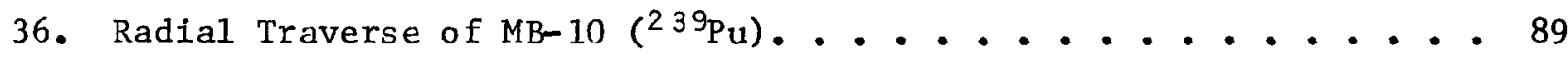

7. Radial Traverse of $\mathrm{MB}-35\left(238_{U}\right)$. . . . . . . . . . . 89

38. Radial Traverse of $\mathrm{CH}_{2}(\mathrm{~F})-4$ (Polyethylene Foam). . . . . . 89 


\section{LIST OF FIGURES}

No.

39. Radial Traverse of 304 SST. . . . . . . . . . . . . 90

40. Variation of the Reactivity Worth of ${ }^{252} \mathrm{Cf}$ With Inverse Power

Level . . . . . . . . . . . . . . . . . . . 91

41. Central Reactivity Worth vs Sample Mass of $\mathrm{H}_{2} \mathrm{O}, \mathrm{CH}_{2}$, and $\mathrm{CH}_{2}$

Foam Samples...... . . . . . . . . . . . . . 92

42. ZPR-9 Core Configuration for the GCFR Phase-I Assemb1y. . . . . 93

43. Illustration of the Checkerboard Loading Concept. . . . . . . . 93

44. Unit Cel1 Structures for the Checkerboard Streaming Experiment in the GCFR Phase-I As sembly as Viewed From Between the As semb1y Halves, Facing the Stationary Half. . . . . . . . . . . . 94

45. Foil and Horizontal1y Loaded Drawer Locations for the Checkerboard Streaming Experiment in the GCFR Phase-I Assembly . . . . . . . 94

46. Aluminum (0.001 in. thick) Foil Holders for Foil Irradiations Showing Positions of Plutonium (P) Foils and Enriched (E) and Depleted (D) Uranium Foils. . . . . . . . . . . . . .

47. Depleted (D) and Enriched (E) Uranium and Plutonium (P) Foil Locations for Unit Cell Measurements in the Core Region . . . . .

48. Depleted (D) and Enriched (E) Uranium Foil Locations for Unit Cell Measurements in the Radial Blanket Region . . . . . . . . .

49. Depleted (D) and Enriched (E) Uranium Foil Locations for Unit Ce11 Measurements in the Axial Blanket Region. . . . . . . . . .

50. Axial Traverse Foil Holders (0.001 in. aluminum) Showing Positions of Enriched (E) and Depleted (D) Uranium Foils, and Plutonium (F) Foils and Spacers $(S)$. . . . . . . . . . . . .

51. Pin Zone Location in the Movable Half of ZPR-9. . . . . . .

52. Location of Pins Containing Enriched (E) and Depleted (D) Uranium Foils in the Pin Zone, and Position of Foils on top of Pins (Depleted on top of M23/22, Enriched on top of M23/24). . . . .

53. Approximate Location of Enriched and Depleted Uranium Foils Within Selected Rods of the Pin Zone . . . . . . . . . . . . .

54. Typical ${ }^{238} \mathrm{U}$ Reaction Rate Distributions in a Core Region Unit Ce11.........................

55. Typical ${ }^{238} \mathrm{U}$ Reaction Rate Distributions in a Radial Blanket

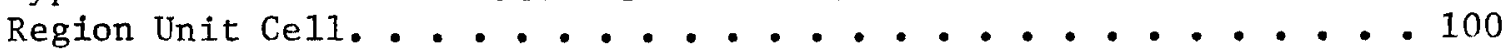




\section{LIST OF FIGURES}

No.

$\underline{\text { Title }}$

Page

56. Typical ${ }^{238} \mathrm{U}$ Reaction Rate Distributions in an Axial Blanket

Region Unit Ce11. . . . . . . . . . . . . . . . 101

57. Radial ${ }^{235}$ Fission Rate Distribution, Diagonal . . . . . . 102

58. Radial 235 Fission Rate Distribution, Diagona1 . . . . . . 103

59. Radia1 235U Fission Rate Distribution, Column 23. . . . . . . 104

60. Radia1 238U Fission Rate Distribution, Row 23........ 105

61. Radia1 $238_{\mathrm{U}}$ Fission Rate Distribution, Diagonal . . . . . . 106

62. Radial ${ }^{238} \mathrm{U}$ Fission Rate Distribution, Column 23. . . . . 107

63. Radial Pu Foil Fission Rate Distribution, Row 23. . . . . . 108

64. Radial Pu Foil Fission Rate Distribution, Diagona1. . . . . . 109

65. Radial Pu Foil Fissinn Rate Distribution, Column 23 . . . . . . 110

66. Radial ${ }^{238}$ U Capture Rate Distribution, Row $23 . . . . . . . .111$

67. Radial ${ }^{238}$ U Capture Rate Distribution, Diagonal . . . . . . . 112

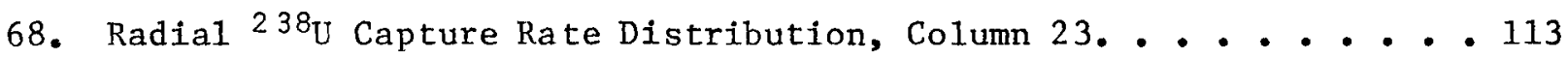

69. Axial $235 \mathrm{U}$ Fission Rate Distribution, Experimental (Symbols) and Calculated (Line) . . . . . . . . . . . . . . 114

70. Axial ${ }^{238} \mathrm{U}$ Fission Rate Distribution, Experimental (Symbols) and Calculated (Line) . . . . . . . . . . . . . . . 115

71. Axial ${ }^{238}$ U Capture Rate Distribution, Experimental (Symbols) and Calculated (Line) . . . . . . . . . . . . . . 116

72. Axial Pu Foil Fission Rate Distribution, Stationary Half of ZPR-9, Experimental (Symbols) and Calculated (Line). . . . . . . . 117

73. Radial ${ }^{238} \mathrm{U}$ Fission Rate Distribution (Row 23) in the Checkerboard Octant. . . . . . . . . . . . . . . . . 118

74. Radial $238_{U}$ Fission Rate Distribution (Diagonal) in the Checker-

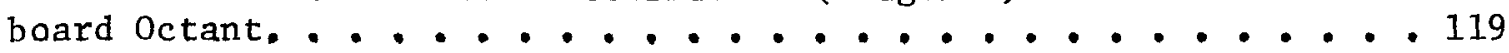

75. Radial ${ }^{238_{U}}$ Fission Rate Distribution (Column 23) in the

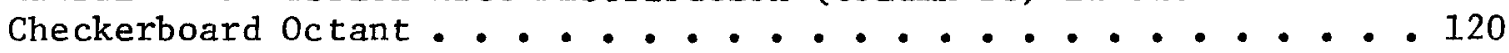

6. Radial ${ }^{239} \mathrm{Pu}$ Fission Rate Distribution (Row 23) in the Checkerboard Octant. . . . . . . . . . . . . . . . . 121 
77. Radial ${ }^{239} \mathrm{Pu}$ Fission Rate Distribution (Diagona1) in the Checkerboard Octant. . . . . . . . . . . . . . . . 122

78. Radial ${ }^{239} \mathrm{Pu}$ Fission Rate Distribution (Column 23) in the Checkerboard Octant . . . . . . . . . . . . . . . 123

79. Radial ${ }^{238} \mathrm{U}$ Capture Rate Distribution (Row 23) in the Checkerboard Octant. . . . . . . . . . . . . . . . 124

80. Radial ${ }^{238} \mathrm{U}$ Capture Rate Distribution (Diagona1) in the Checkerboard Octant. . . . . . . . . . . . . . . . . 125

81. Radial ${ }^{238} \mathrm{U}$ Capture Rate Distribution (Column 23) in the Checkerboard Octant. . . . . . . . . . . . . . . 126

82. Radial $235 \mathrm{U}$ Fission Rate Distribution (Row 23) in the Checkerboard 0ctant...................... 127

83. Radial $235 \mathrm{U}$ Fission Rate Distribution (Diagona1) in the Checkerboard Octant. . . . . . . . . . . . . . . . 128

84. Radial ${ }^{235_{U}}$ Fission Rate Distribution (Column 23) in the Checkerboard 0ctant. . . . . . . . . . . . . . . . . 129

85. Comparison of the ${ }^{239} \mathrm{Pu}$ Fission Rate Distribution in the Three Radial Directions . . . . . . . . . . . . 130

86. Comparison of the ${ }^{238} \mathrm{U}$ Fission Rate in the Three Radial Directions. 131

87. Comparison of the Experimental ${ }^{239} \mathrm{Pu}$ Fission Rate in the $90^{\circ}$ Direction (Column 23) for the Norma1 and Checkerboard Cases . . 132

88. Comparison of the Experimental ${ }^{238} \mathrm{U}$ Fission Rate in the $90^{\circ}$ Direction (Column 23) for the Normal and Checkerboard Cases . . . 133

89. Comparison of the Calculated ${ }^{2}{ }^{38} \mathrm{U}$ Fission Rate Distribution in the $0^{\circ}$ Direction (Row 23) for the Normal and Checkerboard Cases . . 134

90. Comparison of the Calculated ${ }^{2}{ }^{38} \mathrm{U}$ Fission Rate Distribution in the $90^{\circ}$ Direction (Column 23) for the Normal and Checkerboard Cases . . 135

91. Comparison of the Calculated ${ }^{2}{ }^{39} \mathrm{Pu}$ Fission Rate Distribution in the $0^{\circ}$ Direction (Row 23) for the Normal and Checkerboard Cases . . . 136

92. Comparison of the Calculated $2{ }^{39} \mathrm{Pu}$ Fission Rate Distribution in the $90^{\circ}$ (Column 23) for the Normal and Checkerboard Cases . . . . 137

93. Location of the Experimental Zone for the Steam Entry Measurements. 138

94. RZ Model Used in the Analysis of the Steam Zone Measurements. . . 138 


\section{LIST OF FIGURES}

No.

Title

$\underline{\text { Page }}$

95. Intermediate Group Spectra at Core Center for "Norma1" and

"Steam-Filled" Cases. . . . . . . . . . . . . . . 139

96. Intermediate Group Spectra at Center of Test Zone for Normal

and Steam-Filled Cases (Steam Density No. 1). . . . . . . . 140

97. Intermediate Group Spectra at Center of Test Zone for Normal

and Steam-Filled Cases (Steam Density No. 2). . . . . . . . 141

98. Intermediate Group Spectra at Center of Test Zone for Norma1

and Steam-Filled Case (Steam Density No. 3). . . . . . . . . 142

99. Broad Group Adjoint Spectrum at Core Center for Reference Configuration . . . . . . . . . . . . . . . . 143

100. Comparative Broad (29) Group Spectra at Center of Test Zone for the Reference and Steam Density No. 1 Filled Zone Cases . . . . . 144

101. Comparative Broad (29) Group Spectra at Center of Test Zone for the Reference and Steam Density No. 2 Filled Zone Cases . . . . . . 145

102. Comparative Broad (29) Group Spectra at Center of Test Zone for the Reference and Steam Density No. 2b Filled Zone Cases. . . . . 146

103. Comparative Broad (29) Group Spectra at Center of Test Zone for the Reference and Steam Density No. 3 Filled Zone Cases . . . . 147

104. Results of Small Zone Steam-Entry Experiments . . . . . . 148 
No.

I. Geometric Specifications of Core Unit Cel1. . . . . . . . . . . 149

II. Geometric Specifications of Blanket Unit Cell . . . . . . . . . 149

III. GCFR-Phase I Thermocouple Locations . . . . . . . . . . . . 150

IV. Location of Detectors for Use in Approach-to-Critical

Measurement .. . . . . . . . . . . . . 150

V. Fuel Loading Steps in the Approach-to-Critical. . . . . . . . 151

VI. Predicted Critical Masses from the Responses of All

Detectors during the Approach-to-Critical Measurements

Using a $1 / \mathrm{C}$ vs. $\mathrm{M}_{\mathrm{f}}$. . . . . . . . . . . . . . . . . . . . 151

VII. Predicted Critical Masses from the Responses of A11

Detectors during the Approach-to-Critical Measurements

Using a $M_{f} / C$ vs. M Formulation . . . . . . . . . . . . 151

VIII. Summary of Reactivity and Mass Correction for the GCFR

Phase I Assembly. . . . . . . . . . . . . . . . 152

IX. Heterogeneous Atom Concentrations in Core Unit Ce11

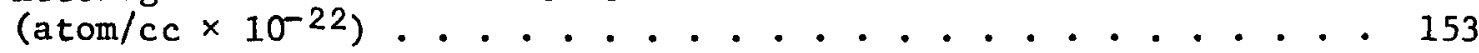

X. Heterogeneous Atom Concentrations in Radial Blanket

Unit Cel1 (atoms $/ c c \times 1 \sigma^{22}$ ). . . . . . . . . . . . 153

XI. Heterogeneous Atom Concentrations in Axial Blanket

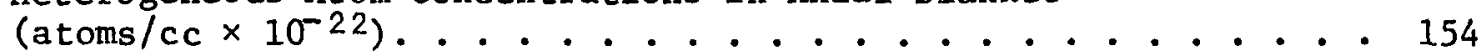

XII. As-Built ZPR-GCFR Phase I Composition Specification-

Homogeneous Unit Cell Atom Densities (atoms/cc $\times 10^{-21}$ ) . . . . 154

XIII. Atom Concentrations of Isotopes in the Core Unit Cell

Computed Using the Wade-Gelbard Prescription

(atoms/cc $\times 10^{-21}$ ).................. 155

XIV. Atom Concentrations of Isotopes in the Axial Blanket

Unit Cell Computed Using the Wade-Gelbard Prescription

(atoms $/ \mathrm{cc} \times 10^{-21}$ )................. 155

XV. ZPR-GCFR Phase I - Radial Blanket Composition Specifications

Atom Densities; Atoms $/ c c \times 10^{-21}$ ) . . . . . . . . . . 155

XVI. Results of Operational Measurements . . . . . . . . . . . 156

XVII. Experimentally Determined Control Rod Worths in

GCFR Phase I Assembly 


\section{LIST OF TABLES}

No.

XVIII. Energy Group Structure for the 11-Group Cross

Section Set. . . . . . . . . . . . . . . . . . 156

XIX. Energy Group Structure for the 29-Group Cross

Section Set. . . . . . . . . . . . . . . . . . .

$\mathrm{XX}$. Homogeneous Atom Densities $\left(x 10^{-21}\right)$ Used in $\mathrm{R}-\mathrm{Z}$

Model of GCFR Phase I Assembly . . . . . . . . . . 157

XXI. Bi-directional Diffusion Coefficients for Core

Composition in GCFR Phase I Assembly . . . . . . . . . . . . 158

XXII. Bi-directional Diffusion Coefficients for Axial

Blanket Composition in GCFR Phase I Assembly . . . . . . . . . 158

XXIII. Bi-directional Diffusion Coefficients for Radial

Blanket Composition in GCFR Phase I Assembly . . . . . . . . . 159

XXIV. Bi-directional Diffusion Coefficient Modifiers

for GCFR Phase I Assembly. . . . . . . . . . . . . . . 159

XXV. Results of Criticality Calculations of As-Built

Phase I Assembly . . . . . . . . . . . . . . . . 160

XXVI. Sensitivity of Eigenvalue to Calculated Parameters . . . . . 160

XXVII. Final Results of Criticality Calculations Performed
in Two-Dimensions. . . . . . . . . . . . 160

XXVIII. Comparison of Real and Adjoint Spectra for 11- and
29-Group Nonstreaming Calculations . . . . . . . . . . 161

XXIX. Comparison of 29-Group Real and Adjoint Spectra for

the With Streaming and Without Streaming Calculations. . . . . 162

XXX. Basic Physics Parameters of the ZPR-GCFR Phase I Core. . . . 163

XXXI. Kinetics Parameters of the ZPR-GCFR Phase I Core . . . . . 163

XXXII. Delayed Neutron Kinetics Parameters for the GCFR Phase I Assembly (29 Energy Group R-Z Diffusion Theory Calculation, With Streaming). . . . . . . . . . . . . . 164

XXXIII. Condensed Kinetics Parameters Used in the Analyses of the Phase I Experiments. . . . . . . . . . . . . . . . 164

XXXIV. Breakdown of Reaction Rates by Region ${ }^{a}$ in the GCFR Phase I Assembly. . . . . . . . . . . . . . 165 


\section{LIST OF TABLES}

No.

$\underline{\text { Title }}$

Page

XXXV. Isotopic Breakdown of Reaction Rate in Central

XXXVI. Summary of Calculated Eigenvalues ${ }^{a}$ for Several

Fast Critical Assemblies . . . . . . . . . . . 166

XXXVII. Summary of Eigenvalue Calculations of GCFR

Phase I Assembly . . . . . . . . . . . . . . . . 166

XXXVIII. Fission Rates Measured in Three Centra1 Drawers ${ }^{a}$. . . . 166

XXXIX. Mass and Composition of Fission Counter Sources. . . . . . 167

XL. Reaction Rate Ratios at the Center of the GCFR

Phase I Assembly . . . . . . . . . . . . . . . . .

XLI. Calculated Reaction Rates at the Center of the

GCFR Phase I Assembly. . . . . . . . . . . . . . .

XIII. Calculated Reaction Rate Ratios (Relative to

${ }^{239} \mathrm{Pu}$ ) at the Center of GCFR Phase I Assembly. . . . . .

XLIII. Comparison of ARC and PIT Group Fluxes and

${ }^{238}$ U Fission Rate by Group ............... 168

XLIV. Comparison of Reaction Rate Ratios in GCFR Phase I

Assemb1y and ZPR-6 Assemb1y 7. . . . . . . . . . 169

XLV. Sample Description . . . . . . . . . . . . . 169

XLVI. Sample Composition . . . . . . . . . . . . . 170

XLVII. Experimental Small Sample Central Reactivity Worths

in the GCFR Phase I Assembly . . . . . . . . . . . . .

XLVIII. Calculated Small Sample Central Reactivity Worths in the GCFR Phase I Assembly with Comparison to

Experimenta1 Worths.

XLIX. Isotopic or Elemental Central Reactivity Worths in

the GCFR Phase I Assembly: Experimental and

L. Experimental Sma11 Sample Radial Reactivity Worth

Traverses in the GCFR Phase I Assembly . . . . . . . . . . .

LI. Parameters Used in the Determination of the

Perturbation Denominator 
LII. Isotopic Fission Rates at the Center of the GCFR Phase I Assembly. . . . . . . . . . . . . 176

LIII. Parameters Used in the Determination of the Perturbation Denominator . . . . . . . . . . . . . .

LIV. A Comparison of the $\mathrm{C} / \mathrm{E}$ Values for Isotopic Central Worths . . . . . . . . . . . . . . . . .

LV. A Comparison of Reactivity Worth Results For GCFR Phase I Assembly and ZPR-6 Assembly 7 . . . . . . . . 178

LVI. VMM-Fitted Amplitudes and Decay Constants $\left(a_{i}, \lambda_{i}\right) . . . . . . .178$

LVII. Comparison of Calculated and Collapsed Set of Measured Values for Relative Yields and Decay Constants. . . . . . . . . . . . . . . . . .

LVIII. Variation of $\tau \sigma^{2}$ Versus $\tau$ as Multiples of the Basic Sampling Interval, DT $=0.1 \mathrm{Sec} . . . . . . . . . . .$.

LIX. Description of Activation Folls. . . . . . . . . . . . 179

LX. Unit Ce11 Measurement Locations. . . . . . . . . . . . . 180

LXI. Cell-Averaged Reaction Rate to Mapping Foil

Reaction Rate Ratios . . . . . . . . . . . . . . . 180

LXII. Average Unit Ce11 Correction Factors . . . . . . . . . . . 180

LXIII. Reactivity Worths of Steps Toward the Establishment of the Stationary Half of Checkerboard Quadrant. . . . . . . . 180

LXIV. Comparison of Experimental and Calculated Uranium Fission Rates, $0^{\circ}$ Radial Traverse. . . . . . . . . . . . 181

LXV. Comparison of Experimental and Calculated Uranium Fission Rates, $45^{\circ}$ Radial Traverse . . . . . . . . . . . 181

LXVI. Comparison of Experimental and Calculated Uranium Fission Rates, $90^{\circ}$ Radial Traverse . . . . . . . . . . 182

LXVII. Comparison of Experimental and Calculated Plutonium Fission and $23{ }^{8} \mathrm{U}$ Capture Rates, $0^{\circ}$ Radial Traverse . . . . . . 182

LXVIII. Comparison of Experimental and Calculated Plutonium Fission and $238 \mathrm{U}$ Capture Rates, $45^{\circ}$ Radial Traverse. . . . . 
LXX. Comparison of Experimental and Calculated

Uranium Fission Rates, Stationary Half

Axial Traverse. . . . . . . . . . . . . . . . .

LXXI. Comparison of Experimental and Calculated

Plutonium Fission and $238 \mathrm{U}$ Capture Rates,

Stationary Half Axial Traverse. . . . . . . . . . . .

LXXII. Experimental Uranium Fission Rates, and $238 \mathrm{U}$

Capture Rate, Moveable Half Axial Traverse. . . . . . . .

LXXIII. Normalized Relative Calculated and Measured

Uranium Fission Rates for the Row 23, $0^{\circ}$

Traverse, in the GCFR Phase I Normal and

Checkerboard Core Configurations. . . . . . . . . . . .

LXXIV. Normalized Relative Calculated and Measured

Plutonium Fission Rates and ${ }^{238} \mathrm{U}$ Capture Rates

for the Row 23, $0^{\circ}$ Traverse in the GCFR Phase I

Normal and Checkerboard Core Configurations . . . . . . .

LXXV. Normalized Relative Calculated and Measured Uranium

Fission Rates for the Diagonal, $45^{\circ}$ Traverse, in

the GCFR Phase I Normal and Checkerboard Core

Configuration .....................

LXXVI. Normalized Relative Calculated and Measured

Plutonium Fission Rates and $238_{\mathrm{U}}$ Capture Rates

for the Diagona1, $45^{\circ}$ Traverse, in the GCFR

Phase I Normal and Checkerboard Core Configurations . . . . .

LXXVII. Normalized Relative Calculated and Measured Uranium

Fission Rates for the Column 23, $90^{\circ}$ Traverse, in

the GCFR Phase I Normal and Checkerboard Core

Configurations. . . . . . . . . . . . . . . . . .

LXXVIII. Normalized Relative Ca1culated and Measured

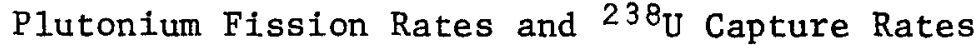

for the Column $23,90^{\circ}$ Traverse, in the GCFR

Phase I Normal and Checkerboard Core Configurations . . . .

LXXIX. Ratio of Reaction Rates at Monitor Foil Location

(S16/12) and at Diagonally Opposite Location in

Checkerboard Octant . . . . . . . . . . . . . . . .

LXXX. Summary of the $\mathrm{CH}_{2}$ Mass and Atom Concentrations

Used in the Simulated Steam Entry Experiment. . . . . . . . .

LXXXI. Inverse Kinetics Data . . . . . . . . . . . . . .

LXXXII. Analysis of Simulated Steam Entry Experiment. . . . . . 


\section{LIST OF TABLES}

No.

IXXXIII. Comparison of the Benoist Coefficents for

LXXXIV. Summary of Experimental and Calculated Results

for Steam Zone Experiments . . . . . . . . . . . . . 191

LXXXV. Comparison of the Integral Parameters of the

Dry Reference Assembly and the Experimental

Assembly with $\mathrm{CH}_{2}$ in Test Zone . . . . . . . . . . . 192

LXXXVI. Isotopic Breakdown of Reaction Rates in the

Central Test Zone - With Steam in Test Zone. . . . . . . 192

LXXXVII. Isotopic Breakdown of Reaction Rates in the

Central Test Zone - Dry Reference Case . . . . . . . . 193

LXXXVIII. Analysis of Steam Worth Components . . . . . . . . . . 193

B-I. Calculated ${ }^{238_{U} / 235}$ U (F8/F5) Fission Rate Ratios . . . . . 194 


\title{
AN EXPERIMENTAL STUDY OF THE NEUTRONICS OF THE FIRST GAS COOLED FAST REACTOR BENCHMARK ASSEMBLY \\ (GCFR PHASE I ASSEMBLY)
}

by

S. K. Bhattacharyya

\begin{abstract}
The Gas Cooled Fast Reactor (GCFR) Phase I Assembly is the first in a series of ZPR-9 critical assemblies designed to provide a reference set of reactor physics measurements in support of the 300 MWe GCFR Demonstration Plant designed by General Atomic Company. The Phase I Assembly was the first complete mockup of a GCFR core ever built. This assembly was a uniform, single composition core with loading that matched the average enrichment (17.3\%) and coolant volume fraction (53\%) of the GCFR Demonstration plant. The loading was especially simple; a single drawer unit cell that facilitated calculational comparisons. The harder neutron spectrum within a GCFR represents a different neutron environment than the situations for which integral experimental data are presently available. The anisotropic effects of preferential neutron streaming along coolant channels in a GCFR presents an additional degree of complexity in the analysis of GCFR neutronics. A set of basic reactor physics measurements were performed in the assembly to characterize the neutronics of the assembly and assess the impact of the neutron streaming on the various integral parameters. The analysis of the experiments was carried out using ENDF/B-IV based data and two-dimensional diffusion theory methods. The Benoist method of using directional diffusion coefficients was used to treat the anisotropic effects of neutron streaming within the framework of diffusion theory. An unequivocal validation of the Benoist method was not possible from the results of the present measurements because reflection effects from the reactor support structures obscured the analysis of some of the streaming effects. However, it was concluded that the method was not substantially in error. While the eigenvalue was sensitive to the values of the Benoist bi-directional diffusion coefficients, the integral parameters were relatively insensitive to them. Calculated predictions of most integral parameters in the GCFR showed the same kinds of agreements with experiment as in earlier LMFBR assemblies.
\end{abstract}

\section{INTRODUCTION AND OUTLINE OF PROGRAM}

The GCFR Phase I assembly is the initial GCFR benchmark critical assembly. forms the first step in a systematic sequence of critical assemblies anned for the study of the physics of the GCFR Demonstration Plant designed by General Atomic Company. The assembly was built on the Zero Power Reactor-9 
(ZPR-9) at Argonne National Laboratory. All earlier experimental studies of integral reactor parameters have focussed on LMFBR systems. The neutronics design of the GCFR differs from the LMFBR due primarily to certain characteristic features associated with the use of helium gas as a coolant in the GCFR. Helium gas, even at the high pressures inside the core $(\sim 85 \mathrm{~atm})$, is relatively transparent to neutrons; the mean free paths are large and the coolant contributes little to neutron moderation. Relative to the LMFBR, the neutron spectrum is harder and this fact has important implications for the physics of a GCFR. The capture-to-fission ratio, $\alpha$, in the fuel isotopes decreases with the hardened spectrum and the potential breeding gain of a GCFR system is enhanced. At the same time, the hardened spectrum represents a slightly different neutron environment, one for which relatively few integral studies of basic nuclear data are available. Anisotropic neutron streaming through coolant channels within and between subassemblies presents an added degree of difficulty in GCFR physics analysis for core and blanket design and for nearcore shielding structures. In order to avoid high differential back-pressures on the coolant pumping system, power flattening through the use of multi-core enrichment regions becomes an important consideration in the GCFR design.

While many of the safety aspects of the GCFR are similar to those of LMFBR systems, significant differences do exist between them. The Doppler coefficient, somewhat reduced by the hardened neutron spectrum, remains an important reactivity feedback mechanism. Core expansion effects are different in nature due to the unique single-grid-plate, fuel subassembly suspension design in the GCFR. The loss of helium coolant is estimated to add only a small positive reactivity to a GCFR system in contrast to the possible significant positive feedback associated with loss of sodium coolant in the LMFBR. An important additional safety consideration in a GCFR is the possible entry of steam from the higher pressure, primary heat transfer loop into the circulating helium coolant.

Thus, there are several features unique to the GCFR design or sufficiently different from the much-studied LMFBR systems that warrant study. During 1974, the physics design of the GCFR-300 MW(e) Demonstration Plant was assessed, meetings were held with the industrial designers (GAC) and the USAEC, and a critical experiments program for the study of the GCFR was developed. The program is aimed at the study of the important features of the GCFR concept relating to economic performance and safety operation. As originally conceived, the GCFR critical experiments program was composed of several phases or assemblies. In each assembly, the unit-cell loading patterns are made up of standard ZPR plutonium fuel (plates or pins), iron and uranium oxide and stainless steel void devices. The void devices, containing air at atmospheric pressure, simulate the helium coolant volume of the GCFR. Calculations have shown that the lack of helium in the ZPR simulation has 1ittle effect upon the physics parameters of the reactor and, hence, void volume is a good coolant simulation.

The GCFR Program began with a clean benchmark assembly (Phase I) designed to facilitate tests of analytical methods and nuclear data. The Phase II Assembly was intended to investigate specific features of the GCFR concept primarily safety coefficients and neutron streaming effects. In Phase III effects of a multi-zoned core were studied particularly with regard to power distributions. 
The above discussion provides a background of the GCFR criticals experiments program and indicates where the GCFR Phase I Assemb1y experiments fits into the larger picture. In this report we will present the results of the experiments performed on the GCFR Phase I Assembly. The assembly went critical on April 3, 1975 with an evaluated fissile mass of $1128.8 \pm 6.8 \mathrm{~kg}$, and the experimental program was completed on June 25, 1975.

The experiments that were performed in the assembly fall into two broad categories. The first encompasses the experiments designed to characterize the general neutronics of the assembly while in the second are classed the special purpose experiments to study effects of steam entry in a GCFR and an experiment to study the neutron streaming effects. A complete list of the experiments performed is given below.

1. Approach-to-Critical measurements and evaluation of critical mass.

2. Measurement of neutron spectra at core center using the proton-recoil technique.

3. Measurement of spectral indices $\left(f^{22} / f^{49}, f^{23 / f^{49}}, f^{25 / f^{49}}, f^{28} / f^{49}\right.$, $\left.\mathrm{f}^{40} / \mathrm{f}^{49}, \mathrm{c}^{22} / \mathrm{f}^{49}, \mathrm{c}^{28 / \mathrm{f}^{49}}\right)$ at core center using fission flow counters for fission rates and foil irradiations for the capture-rate.

4. Measurement of reactivity worths of a large number of samples of interest in the GCFR program using the sample oscillation reactivity difference technique. In addition to the usual samples, the worths of ${ }^{232} \mathrm{Th}, 233 \mathrm{U}, \mathrm{H}_{2} \mathrm{O}$ and $\mathrm{CH}_{2}$ samples were measured. The reactivity worth of a ${ }^{252} \mathrm{Cf}$ sample was used to determine the perturbation denominator of the assembly. The radial variation of the worths of some of the materials was also measured.

5. Measurement of the radial and axial profiles of the fission rates in ${ }^{235} \mathrm{U},{ }^{238} \mathrm{U}$ and ${ }^{239} \mathrm{Pu}$ and the capture rate in ${ }^{238} \mathrm{U}$. The measurements were made using foil irradiation techniques. The radial traverses were made in three different azimuthal directions to obtain data on neutron streaming effects.

6. Measurement of the kinetics parameters of the assembly (delayed neutron relative yields, on decay constants) using the rod-drop die-away flux profile technique. In addition, the detector-variance method was used to determine $\beta_{\text {eff }}{ }^{\circ}$

7. Measurement of radial reaction rate traverses within an octant of the assembly in which every alternate drawer was loaded with plates positioned horizontally instead of vertically. This loading cut off the direct streaming paths in one direction and the experiment was designed to study the effect of this reduction in streaming on the eigenvalue and the reaction rate profiles.

8. Measurement of the reactivity worth of a simulated steam entry into a central core zone. The entry of steam into a GCFR is a hypothetical accident condition and the experiment was designed to study the reactivity consequences of such an entry. Polyethylene foam strips were used to simulate steam for purposes of this experiment. 


\section{DESCRIPTION OF ASSEMBLY AND ANALYTICAL METHODS}

\section{A. Introduction}

The GCFR Phase I assembly is the initial GCFR benchmark critical assembly. The assembly has been designed and built as a clean, homogeneous system to provide information of the general features of the GCFR, viz. critical mass, spectral and reactivity coefficients and neutron streaming effects. In this chapter the experimental determination of the critical mass is presented along with an evaluation of the mass.

The GCFR Phase I assembly provides a clean, homogeneous, benchmark reference for development and testing of the methods to be used in the design and safety analysis of the GCFR. The Benoist formalism ${ }^{1}$ has been used to treat the anisotropic neutron streaming phenomenon in the leaky assembly. Criticality calculations have been performed for the assembly using conventional twodimensional diffusion theory methods (with isotropic diffusion coefficients) and also with anisotropic diffusion coefficients to study the effect of neutron streaming. A discussion of the analytical methods and results of calculations based on ENDF/B-IV data are presented.

\section{B. Description of the Assemb1y}

The basic features of the GCFR Phase I assembly were designed to match general GCFR design characteristics. The core is a homogeneous single-zone region with a single-drawer unit cell. The unit cell has one $1 / 4$ in. wide column of $\mathrm{Pu}-\mathrm{U}-\mathrm{Mo}$ fuel plate sandwiched between two $1 / 8$ in. $\mathrm{Fe}_{2} \mathrm{O}_{3}$ plates, a $1 / 4$ in. $U_{3} O_{8}$ plate and $1-1 / 4 \mathrm{in}$. of void space provided by 3 void devices. Air at atmospheric pressure, contained in these void devices, simulates the helium coolant in the GCFR. The unit cell loading results in a core that matches the average GCFR Demonstration Plant fissile enrichment (17.3\%) and void fraction. In the GCFR Demonstration Plant, the void fraction, composed of coolant channel plus gaps, is 55\% while the Phase I assemb1y core has a $53 \%$ void fraction. The radial and axial blanket unit cells are composed of $\mathrm{U}_{3} \mathrm{O}_{8}$, depleted uranium and void devices. The void fraction in the axial blanket matches that of the core, while the void fraction of the radial blanket is $33 \%$. Figure 1 shows the three unit cells. Table I and II provide a detailed geometric description of the unit cells.

The actual layout of material in the core, axial blanket and radial blanket are depicted diagrammatically in drawer masters. Figures 2 and 3 show some typical drawer masters for various compositions used in the assembly. The core and axial blanket compositions were loaded in single $36 \mathrm{in}$. (91.54 cm) drawers. The first 24 in. of these drawers contained core composition; the last 12 in. contained axial blanket composition. The radial blanket composition was contained in a 24 in. drawer plus a 12 in. drawer loading. Because of fuel platelet size (length) limitations in the fuel inventory, the whole core could not be assembled with identical fuel platelet-length loadings. Five core drawer loading patterns of slightly different fuel platelet-length combinations were used and these loadings have been identified in Figs. 4 and 5. The homogeneous atom concentrations for the various isotopes for each the fuel platelet loading patterns do not differ significantly from each other. The void devices used in the assembly were made of $0.015 \mathrm{in}$. $(0.038 \mathrm{~mm})$ thick 304 stainless steel. The cans, specially fabricated for the assembly, 
are open at both ends to provide the appropriate coolant channel simulation. The rest of the materials come from the existing inventory of reactor materials.

The final (just-critical) evaluated core radius was determined to be 90.60 $\mathrm{cm}$ with an outer radial blanket radius of $115.61 \mathrm{~cm}$. The core half height was $61.04 \mathrm{~cm}$ and the axial blanket thickness was $30.52 \mathrm{~cm}$. Figures 4 and 5 show the two halves of the as-built reactor with the locations of the twelve ${ }^{10} \mathrm{~B}$ control rods, ten dual purpose fueled control rods and the in-core thermocouples. Table III lists the positions of the iron-constantan thermocouples used for monitoring the core temperatures for operational and experimental purposes. The thermocouples were positioned so as to provide a representative core temperature sampling. The average of the sixteen experimental thermocouple readings gave the average core temperature at a given configuration.

\section{The Approach-To-Critical Experiment}

The approach-to-critical measurement provides the first indications of the correctness of design calculations with regard to the prediction of the critical mass. In addition, the start-up process or approach-to-critical in a large fast reactor is of importance in itself and, thus the experiment will be discussed in some detail.

The approach-to-critical began from the fully preloaded configuration with all the materials other than $\mathrm{Pu}$ fuel fully loaded in the matrix. Aluminum plates were positioned at the $\mathrm{Pu}$ fuel plate locations and the core was preloaded to the dimensions computed from preanalysis. The first batch of fuel loaded in the core was in the ten fuel bearing dual-purpose control rods. Following that, fuel was loaded in discrete steps in concentric regions beginning in the center of the core and going radially outward. At each step, count rates were recorded for a series of detectors. Table IV 1ists the kinds and locations of the detectors used.

From the point reactor kinetics equations under equilibrium conditions, one can show that

$$
\frac{\mathrm{n}_{0}}{\ell} \equiv \frac{\mathrm{s}}{1-\mathrm{k}}
$$

where $\mathrm{n}_{0}$ is the equilibrium neutron density and $S$ the source strength. By definition $1 /(1-k)$ is the subcritical multiplication $M_{0}$ of the assembly and

$$
\frac{\mathrm{n}_{0}}{\ell}=\mathrm{M}_{0} \mathrm{~S}
$$

This expression gives the neutron production rate per unit volume in the reactor. The count rates recorded in a neutron detector placed in or adjacent to the assembly can be assumed to be proportional to $\mathrm{M}_{0} \mathrm{~S}$.

$$
\mathrm{C} \propto \mathrm{M}_{0} \mathrm{~S} \text {. }
$$

With the assumption that reactivity increase is proportional to incremental fissile mass loaded in the core, the inverse count rate (1/C) varies linearly with fissile mass loaded, $M_{f}$. 
This is the principle used in conventional approach-to-critical measurements. From extrapolations of a plot of $1 / \mathrm{C}$ vs $\mathrm{M}_{\mathrm{f}}$ an estimate of the critical mass can be made at each loading stage. The presence of $240 \mathrm{Pu}$ in the p1utonium fuel introduces an additional neutron source in the subcritical assembly thus altering the multiplication and the detector response. Assuming this source to be proportional to the amount of fissile mass added the function $M_{f} / C$ varies approximately linearly with the fissile mass loaded $M_{f}$. In the analysis of the present data, both methods were used to plot the subcriticality data and to predict the critical mass at each loading step.

Six loading steps were needed to bring the assembly to a critical configuration. Table $\mathrm{V}$ shows the amount of fissile mass added in each step and the corresponding core radius at each step. The core radius was calculated from the relation

\section{$R=[$ Number of fuel drawers per reactor half $\times$ cross sectional area of a drawer $/ \pi]^{\frac{2}{2}}$}

At each loading step the count rates on all the detectors (C) were measured and a prediction of the critical mass was made based on a $1 / \mathrm{C}$ vs $M_{f}$ plot and a $\mathrm{M}_{\mathrm{f}} / \mathrm{C}$ vs $\mathrm{M}_{\mathrm{f}}$ plot for each detector. The results of these predictions at each of the loading steps for the various detectors are shown in Table VI for the $1 / C$ case and in Table VII for the $M_{f} / C$ case. The $M_{f} / C$ vs $M_{f}$ plot is seen to give a much better representation of events and, particularly for the central detectors, the predictions are constant, implying a linearity in the plot.

Figures 6-9 show the $1 / C$ vs $M_{f}$ and $M_{f} / C$ vs $M_{f}$ plots for ${ }^{235} U$ fission chamber No. 3 located at core center, ${ }^{238} \mathrm{U}$ fission chamber No. 1 located at core center, ${ }^{235} \mathrm{U}$ fission chamber No. 4 located at core axial blanket boundary and $\mathrm{BF}_{3}$ ion chamber No. 4 located at the outer boundary of the radial blanket. For the in-core central fission chambers, the $M_{f} / C$ vs $M_{f}$ curve is linear throughout the range of $M_{f}$. The $1 / C$ vs $M_{f}$ curve shows a conservative "concave upward" shape in all these cases. For the out-of-core $\mathrm{BF}_{3}$ detector both the curves are of the concave shape. The initial multiplication sensed by this detector is larger than at subsequent stages because of the building up of fuel toward it. For central detectors the $M_{f} / C$ formulation does seem to provide a good representation of the $240 \mathrm{Pu}$ spontaneous fission source. Results of approach-to-critical measurements performed on ZPR-6 Assembly 7 led to the same conclusion. ${ }^{2}$

Another method of plotting subcriticality data for prediction of critical mass at a relatively early stage in the loading was proposed by 01son and Palmer. ${ }^{3}$ They suggested plotting $R^{5 / 2} / C$ vs core radius, $R$, and observed good linearity for sample Pu-fueled cores from low multiplications $(k>0.6)$. The $\mathrm{R}^{5 / 2} / \mathrm{C}$ vs $\mathrm{R}$ plot for the present approach to critical measurements is shown in Fig. 10 for the ${ }^{235} \mathrm{U}$ fission chamber No. 3. The curve shows a nonconservative convex trend initially followed by a linear stretch to the critical radius. From the present data it appears that this method is not as good as the $M_{f} / C$ vs Mf plotting method in $\mathrm{Pu}$ fueled fast critical systems.

During each stage of the loading to critical the subcriticality measurements were also made with all the control rods in their least reactive position. 
The difference in predicted critical masses from the two measurements for each detector gave the amount of control available (in equivalent $\mathrm{kg}$ of fuel). Figure 11 shows a typical plot of the subcriticality data with control rods in their least reactive position. The prediction of the control available was quite erratic until the loading was close to critical.

As indicated in Tables VI and VII, the final critical mass prediction (total fissile mass) from all the detectors converged to a value of $\sim 1136 \mathrm{~kg}$. This value had to be adjusted to correct for the several small effects discussed in the next section to provide a final "evaluated" critical mass value.

\section{Corrections to the As-Built Critical Mass}

Several corrections have to be made to the as-constructed critical mass to define an equivalent critical configuration for a smooth, uniform cylindrical core for use in simple RZ calculational modeling. Among the important corrections are the accounting of the excess reactivity in the as-built core, source-subcriticality effects, smoothing effects of the outer radius of the core outline, and temperature effects. In this section, a detailed discussion of these corrections is presented. The final reactivity worths associated with these corrections and the corresponding change in critical mass are shown in Table VIII.

\section{Excess Reactivity Correction}

The as-built Phase I assembly had a built-in reactivity excess of $+75.3 \pm 2.0$ Ih for operational purposes. Based upon an experimentally determined core edge drawer worth of $+6.67 \pm 0.25 \mathrm{Ih} / \mathrm{drawer}$, the $\Delta \rho / \Delta \mathrm{M}$ at the core edge is $9.90 \pm 0.38 \mathrm{Ih} / \mathrm{kg}$. The excess reactivity thus translates to a reduction of critical mass by $7.61 \pm 0.35 \mathrm{~kg}$.

\section{Interface Gap Worth}

The reactivity worth of the possible void between the reactor halves when they are brought together has been evaluated in some earlier determinations of critical mass. The reported gap widths vary from 0 to $10 \mathrm{mils}$ and the measured gap reactivity worth is of the order of $0.8 \mathrm{Ih} / \mathrm{mil} .^{4}$ The precise value of the gap during the reference measurement could not be measured with a great degree of accuracy and the correction for interface gap was taken to be zero. An uncertainty of 2 Ih was associated with this correction - this value being the product of an average estimated gap of $2.5 \mathrm{mils}$ in this assembly and the $0.8 \mathrm{Ih} / \mathrm{mil}$ gap worth determined from earlier assemblies. No additional correction was made of the effect of homogenizing the stainless steel drawer fronts at the interface between the reactor halves; this correction has been investigated and found to be negligible for a large critical assembly (ZPR6-7) of nearly the same dimensions as the GCFR Phase I assembly.

\section{Temperature Correction}

The excess reactivity values were corrected to a standard temperaure of $25^{\circ} \mathrm{C}$. An experimental temperature index of reactivity was determined as described in section II F to be $-2.42 \pm 0.04 \mathrm{Ih} /{ }^{\circ} \mathrm{C}$. The average correction applied to the data was +3.4 Ih. 


\section{Source Subcriticality}

The spontaneous fission in ${ }^{240} \mathrm{Pu}$ constitutes a built-in source in a11 Pu fueled cores. The effect of this source is to make an apparently critical assembly, actually subcritical by an amount

$$
\Delta \mathrm{k}=-\frac{\mathrm{S} \ell}{\mathrm{n}}
$$

where

$$
\begin{aligned}
\mathrm{S} \equiv & \text { effective neutron volumetric source for }{ }^{240} \mathrm{Pu} \text { spontaneous } \\
& \text { fission }\left(4.30 \times 10^{2} \mathrm{n} / \mathrm{sec} \text { per gm of } 240 \mathrm{Pu} \text { present }\right) \\
\ell= & \text { neutron lifetime } \\
\mathrm{n}= & \text { neutron density. }
\end{aligned}
$$

Clearly the effect is inversely proportional to the power level (neutron density and the correction is necessary because assemblies are operated at different power levels. Usually this correction is lumped with the excess reactivity correction. At the power level of measurement, $\Delta \mathrm{k}$ was $-1.95 \mathrm{Ih}$. This translates to an increase of $0.198 \pm 0.008 \mathrm{~kg}$ of fuel inventory in the core for a zero excess core.

\section{Edge Smoothing Correction}

The actual core-radial blanket interface boundary is not a smooth circle; it is defined by a step-like boundary line (see Fig. 12). An "equalarea" circular section is obtained for the core by defining an equivalent core radius as

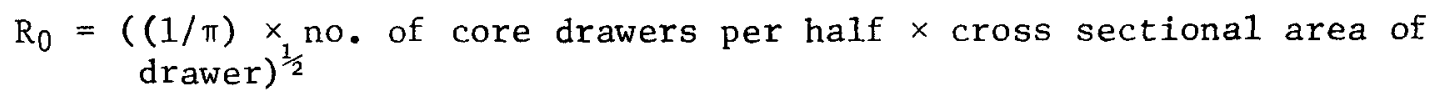

Figure 12 shows the outline of a quadrant of one half of the core with the corresponding part of the equivalent circle. (The Phase I assembly is completely symmetrical about the major axial and radial axes.) The edge smoothing correction consists of making a determination of the reactivity-worth of moving the core drawers outside of the circle to the area inside the circle and, moving the blanket drawers inside the circle to the area outside the circle. Since this involves fuel movement radially inward, a positive reactivity effect is anticipated. From past experience it is known that this correction for large cores such as the GCFR-Phase I assembly is small. The loading plans were designed to minimize asymmetry and thus the edge smoothing correction. Thus a simple semi-empirical method was used to evaluate the correction.

At the as-determined core blanket interface $\left(R_{0}=90.60 \mathrm{~cm}\right)$ the worth of exchanging a fueled core drawer for a radial blanket drawer was experimenta11y determined to be $6.68 \pm 0.25 \mathrm{Ih} / \mathrm{drawer}$. This value was used to normalize the radial $\mathrm{Pu}$ worth profile (which was assumed to be the same as the drawer wor profile) calculated for the preanalysis of the Phase I experiments. From Fig. 12 the area of fueled region to be moved inward corresponded to 16.4 drawers per half. The worth profile at radius $R=R_{0}$ was linear for small radial separations with a slope of 


$$
\left.\frac{\Delta\left(\rho / \rho_{0}\right)}{\Delta R}\right)_{R=R_{0}}=0.007 \mathrm{~cm}^{-1} \text {. }
$$

The fuel movement necessary for the edge smoothing correction was reduced to the motion of a radial strip corresponding to the 16.4 drawer area outside $R_{0}$ to the radial strip corresponding to the same area inside $R_{0}$. Assuming the worth of fuel within the strips to have average values $\left(\rho_{2} \rho_{1}\right)$ at the median radii of the strips $\left(R_{2}, R_{1}\right)$ the edge smoothing reactivity offect can be written as

$$
\begin{aligned}
\left(\rho_{2}-\rho_{1}\right) & \left.=\rho_{0} \frac{\Delta\left(\rho / \rho_{0}\right)}{\Delta R}\right)_{R=R_{0}} *\left(R_{2}-R_{1}\right) \\
& =(16.4 \times 6.67) * 0.007 *(0.88) \\
& =0.674 \text { Ih/half }
\end{aligned}
$$

For the whole reactor (both halves) the effect is doubled:

$$
\therefore \Delta \mathrm{p})_{\text {edge smoothing }}=1.35 \mathrm{Ih} \text {. }
$$

The uncertainty in this value is taken to be $50 \%$ of the estimated value of $\Delta \rho$. This effect causes a reduction in critical mass by $0.136 \pm 0.068 \mathrm{~kg}$.

Table VIII presents a summary of all of the corrections made to the critical mass. For the $k_{\text {eff }}$ unity (zero-excess reactivity) core, the evaluated critical mass is $1128.8 \pm 6.8 \mathrm{~kg}$, and the evaluated core radius is $90.60 \mathrm{~cm}$. The other dimensions of the assembly are also listed.

\section{E. Generation of Atom Concentrations}

Three sets of atom concentrations were needed for the analysis of the assembly. The first is a set of heterogeneous or plate-homogenized number densities needed for the calculation of self-shielding factors and crosssection generation. These number densities are characteristic of a ray taken through the center of the plates perpendicular to the direction of the plate columns and the densities obtained preserve the optical thickness through the plates. Homogeneous unit cell-averaged atom concentrations are required in two-dimensional diffusion theory calculations. In addition, for the calculation of Benoist diffusion coefficient modifiers, "stretched heterogeneous" atom concentrations are needed in accordance with the Wade-Gelbard prescription. 6 The heterogeneous number densities for the core, radial blanket and axial blanket compositions are shown in Tables IX-XI. The method of generation of the other two atom concentrations are discussed below.

Since the assembly was composed of the single drawer unit cells the calculation of cell averaged number densities was reasonably straightforward. The total weight of an isotope within the unit cell was computed from the nown loading scheme and the isotopic composition and weights of the plates obtained from relevant ANL internal memoranda. The structural material from the drawer ends and matrix tube top and bottom were transferred to the 
non-fissile regions in proportion to the volume fractions of the regions to maintain the resonance absorber to scattering atom ratios within the fuel plates. The atom density of the isotope was then given by the relation

$$
C_{i}=\frac{\text { Isotope Weight in Unit Cells }}{\text { Unit-Ce11 Volume }} * \frac{\text { Avagadro's Number }}{\text { Isotope Atomic Weight }}
$$

In computing the unit-cell volumes the cross section of drawer init cel1 was taken as a square of side $2.175 \mathrm{in}$. $(5.5245 \mathrm{~cm})$. This includes the thickness of the matrix tubes which form part of the unit cell. The axial heights of the three unit cells were defined so as to include the fronts and back of drawers and end springs where appropriate. The axial heights used in the computation are listed below:

$$
\begin{array}{ll}
\text { 1. Core: } & 24.032 \mathrm{in.}(61.041 \mathrm{~cm}) \\
\text { 2. Axial Blanket: } & 12.015 \mathrm{in} .(30.518 \mathrm{~cm}) \\
\text { 3. Radial Blanket: } & 36.596 \mathrm{in.}(92.954 \mathrm{~cm})
\end{array}
$$

The value of Avogadro's number used in these computations was $6.023 \times 10^{23}$ atoms/mole. The atomic weights were normalized to ${ }^{12} \mathrm{C}$ as usual. Table XII lists the cell averaged atom concentrations in the various regions of the assembly. The differences in plate loading schemes in the various core regions resulted in very small differences in atom concentrations in these regions. For the generation of the "stretched heterogeneous" number densities the Wade-Gelbard prescription was used. Tables XIII-XV give the stretched heterogeneous number densities for the core, axial blanket and radial blanket number densities.

\section{F. Operational Measurements}

A series of measurements were performed in the critical Phase I configuration to define the operational characteristics of the assembly. The measurements are described briefly in this section and the results of the measurements are summarized in Tables XVI and XVII.

\section{Measurement of Temperature "Coefficient" of the Assembly}

Reactivity measurements are typically performed on different days under different temperature conditions. An experimentally determined temperature coefficient is used to correct these measurements to the standard reference temperature. The temperature coefficient was determined by observing the reactivity change associated with heating up the reactor from 24 to $31.5^{\circ} \mathrm{C}$ and, subsequently, in cooling it back down to $24^{\circ} \mathrm{C}$. The air conditioning and core cooling fans were kept on all night prior to the measurement to create low initial temperature conditions. After startup, the cooling fans were turned off and the core was allowed to heat up. A calibrated dual purpose control rod was used to maintain the power level, thus providing a measurement of reactivity variation with temperature. The temperature range covered (24 to $31.5^{\circ} \mathrm{C}$ ) spans the normal operating core temperature range. The cooling were then turned on and the procedure was repeated for cooling down of the core. The reactivity excess data for the "heating" and "cooling" stages were fitted to linear functions in temperature and the average of the two slopes 
so determined was taken to be the temperature coefficient of reactivity of the core. Figure 13 shows the experimental data points and the fitted curves. The temperature coefficient was $-2.42 \pm 0.04 \mathrm{Ih} /{ }^{\circ} \mathrm{C}$.

\section{Core Drawer Worth Measurements}

The worth of the core crawers at the center of the matrix (1ocations S/M 23/23) and at the core-blanket interface are needed for operational purposes. A number of later experiments involved substitution of experimental drawers in place of the central core drawer whereas the blanket-core interface provides for adjustments to criticality for different configurations. The central drawer worth was measured relative to void in that location while the edge core drawer worth was measured relative to radial blanket drawers. Ten radial blanket drawers were replaced by core drawers for the edge drawer measurements, the replacements being made symmetrically around the circumference of the core to average out any azimuthal asymetry effects. The measurements were performed by a determination of the reactivity excess of the various configurations by means of a calibrated dual purpose rod. The experimental value for the central drawer worth was $62.99 \pm 1.09$ Ih while the edge drawer worth was $6.68 \pm 0.25$ Ih.

\section{Measurements of Control Rod Worths}

The worths of the $10_{\mathrm{B}}$ and fueled dual purpose control rods were measured using the rod-drop inverse-kinetics method. The experimentally determined rod worths are shown in Table XVII.

\section{Configuration Reproducibility}

The achievement of a given configuration on the reactor involves the moving of the tables together and the moving of the rods to the desired position. There is a small uncertainty in the final positions of the table and the rods. The reactivity uncertainty associated with this configuration uncertainty was determined by measuring the reactivity excess of the same configuration several times in a short space of time with complete scram between measurements. The measurements were performed in a short time span to minimize effects of ${ }^{24} \mathrm{Pu}$ decay. The experimental configuration reproducibility uncertainty was \pm 1.4 Ih. However, based on earlier experience, a slightly higher value $( \pm 2.0 \mathrm{Ih})$ was taken as the configuration reproducibility uncertainty used to compute the final uncertainties of experimental reactivity worth values.

\section{Reactivity Worth of Decay of $241 \mathrm{Pu}$}

${ }^{24}{ }^{1} \mathrm{Pu}$ has a half 1 ife of $14.5 \pm 0.4$ years $^{5}$ and hence there is a discernable depletion of $24 \mathrm{l} \mathrm{Pu}$ inventory in the core during the course of the experiments. There is a measurable reduction in the reactivity excess of configurations caused by this depletion of ${ }^{24 l} \mathrm{Pu}$ decay. The reactivity worth of ${ }^{24} \mathrm{P}$ u decay was experimentally determined by measuring the reactivity excess of the reference GCFR Phase I configuration at different times over a period of 62 days during the experimental program. The reactivity data were fit to linear function in time. The slope of this fitted line gave the reactivity brth of ${ }^{241} \mathrm{Pu}$ decay in the assembly. Figure 14 shows the experimental data and the fitted line. The decay worth is $-0.15 \pm 0.02 \mathrm{Ih} / \mathrm{day}$. The approximate 
decay worth of ${ }^{24 l} \mathrm{Pu}$ can be deduced from the experimentally determined central worth of ${ }^{241} \mathrm{Pu}$. The value so obtained was in agreement with the value reported here.

\section{G. Analysis}

The analysis of the GCFR Phase I Assembly Experiments was performed using standard methods of design computations (ENDF/B Version IV data and multigroup diffusion theory). Composition and spatially dependent broad group cross section sets appropriate for the heterogeneous plate environment were generated using the ETOE- $2 / \mathrm{MC}^{2}-2 / \mathrm{SDX}\left({ }^{7}\right)$ cross section processing codes. These multigroup cross-sections were used in diffusion theory calculations for an appropriate model of the zero-excess reactivity critical assembly. The calculations included the use of directional diffusion coefficients to treat anisotropic neutron diffusion effects within the framework of diffusion theory. Figure 15 shows a layout of the calculational steps.

\section{Cross Section Generation}

The ENDF/B Version IV library processed by ETOE-2(8) was used for the generation of all cross sections. This 1ibrary, with an ultra-fine group structure consisting of 2082 groups with $\Delta u=1 / 120$ extending from $14.190675 \mathrm{MeV}$ to $0.414 \mathrm{eV}$, was used with $\mathrm{MC}^{2}-2^{(9)}$ to generate an intermediate fine group cross section library. This homogeneous $\mathrm{MC}^{2}-2$ spectrum calculation utilized the homogeneous atom densities for the GCFR Phase I core composition. Sixteen principalconstituent isotopes were included at their actual homogeneous number densities and twenty-four additional isotopes were included at infinite dilution. An inconsistent $P_{1}$ fundamental mode option was used for the $M^{2}-2$ calculation and produced a critica1 $(k=0.99983)$ buckling $\mathrm{B}_{\text {crit }}^{2}=7.6928 \times 10^{-4}$. The resultant 2082 group spectrum used to collapse to the intermediate group structure is shown in Fig. 16.

The selection of the intermediate broad group structure was based upon ANSI Standard N411, the American National Standard for Nuclear Data Sets for Reactor Design Calculations. The energy group structure used for the intermediate base 1ibrary was the Universal Supergroup, 226 group structure (USS 226). As recommended by the ANS-19 Standards Subcommittee, this group structure has a variable lethargy width in the energy range from $14.190675 \mathrm{MeV}$ to $0.414 \mathrm{eV}$. The 226 group structure represents a more detailed energy description of the cross-sections at the intermediate base 1ibrary level than earlier sets used for LMFBR assembly studies (156 groups).10

The collapse of the cross section set to the final broad group structure was performed with the space-dependent multigroup cross section generation code SDX. ${ }^{1}$ This code provides a one-dimensional treatment of unit-cell heterogeneity and of spatial flux effects throughout the assembly. Two different broad group cross section sets (11 broad groups and 29 broad groups) were generated for the analysis of the Phase I assembly. The energy group structures for these cross section sets are given in Tables XVIII and XIX. Eigenvalue calculations were performed for the evaluated zero-excess reactivity configura tion of GCFR Phase I using both the 11- and 29-group cross sections. For ead of the two broad group energy structures, two separate calculations were performed to generate broad group cross sections in the radial and the axial directions. In order to partially account for the spatial effect upon the 
collapsing spectrum, the core and the blanket regions were each divided into two sub-regions (core, core-near-blanket, blanket-near-core, blanket) for both the radial and axial SDX calculations. The sub-regions used for the generation of space- and composition-dependent cross sections are defined in Fig. 17. The calculated 226 fine group spectra used by SDX to collapse to the broad group level are shown for the core, and axial blanket in Figs. 18 and 19. Successive SDX calculations were made to iterate upon the critical transverse bucklings. For the radial calculation the critical transverse buckling (taken to be region independent $B_{z}^{2}=4.059 \times 10^{-4}$; for the axial calculation the critical transverse buckling $\mathrm{B}_{\mathrm{r}}^{2}=4.634 \times 10^{-4}$. These buckling values yielded $k=1.000$ for the respective SDX calculations when collapsing to both of the broad group energy structures. The 29 group cross-section set was used for all the two-dimensional calculations of eigenvalue and analysis of the experiments. The 11 group set was used for scoping and sensitivity analyses and also for the three-dimensional diffusion theory calculation.

In addition to the unit-cell averaged, heterogeneity corrected cross sections used in the diffusion calculation, homogeneous "detector" cross-sections were generated for use in the analysis of small sample central worths and reaction rates. These cross-sections are designed to preserve reaction rates in an infinitely dilute foil spanning a unit cell, and have incorporated in them effects of energy self-shielding, but exclude spatial self shielding effects.

\section{Evaluation of Neutron Streaming Effects}

It has been demonstrated that conventional diffusion theory calculations using homogeneous isotropic diffusion coefficients substantially underpredict the leakage in the axial direction in the leaky GCFR cores. The method of Benoist ${ }^{1}$ provides a means of calculating directional diffusion coefficients which enables a treatment of the anisotropy within the framework of diffusion theory. In the Benoist formulation, the group diffusion coefficient in the $k$ th direction is given by the expression

$$
D_{k}=1 / 3 \frac{\sum_{j} v_{i} \phi_{i} \lambda_{j} P_{i j, k}}{\sum_{i} v_{i} \phi_{i}}
$$

where $V_{i}$ is the volume of the region $i, \phi_{i}$ is the average flux in region $i$, $\lambda_{j}$ is the transport mean free path in region $j$ and $P_{i j, k}$ is the directional probability that neutrons appearing in region $i$ have their first collision in region $j$. A free standing computer code ${ }^{12}$ developed by Kier was used to evaluate $P_{i j, k}$ and $D_{k}$ from input value of $V_{i}, \phi_{i}$ and $\lambda_{i}$.

The $\mathrm{D}_{k}$ terms were computed for all different unit cells within the assembly (the core, radial blanket and axial blanket regions). Wade and Gelbard ${ }^{6}$ showed that the magnitudes of the directional diffusion coefficients computed by this method depended crucially upon the modeling procedure adopted produce a one dimensional representation of the three-dimensional unit cells. ed upon comparison with Monte Carlo calculations, Wade and Gelbard also proposed a prescription for the modeling that gave Benoist coefficients that appeared to adequately described the streaming process in a zero-leakage ZPR 
unit-cell. 6 This modeling prescription was used in the present calculation. The details of the calculational method have been described in an earlier publication. 13

The calculations gave anisotropic diffusion coefficients in directions paralle1 (D $\mid$ ) and perpendicular (D ) to the void channels in each unit cell. For each drawer unit cell D|| acts along the length of the drawer (axially in the reactor) and D/ cuts across the plates. For a given reactor model the bi-directional diffusion coefficients were constructed from these two parameters. For the diffusion theory calculations the appropriate directional diffusion coefficients were input to the code as multipliers to the isotropic diffusion coefficients. One of the aims of the GCFR Phase I assembly criticals program was to check the Benoist method of treating anisotropic diffusion and also to validate the various prescriptions used to evaluate the directional diffusion coefficients.

\section{Reactor Mode1}

The initial calculations for the assembly were performed using a two-dimensional cylindrical (R-Z) model of the reactor. Figure 17 shows a layout of the model with solid lines indicating region and composition boundaries. The axial dimensions represent the actual axial dimensions of the assembly. The radial dimensions were calculated such that the cylindrical regions contain the same volume as the matrix locations comprising these regions. The dashed lines in Fig. 17 indicate the sub-regions used in the radial and axial SDX calculations from which space-dependent cross sections were obtained. The homogeneous atom densities for each composition have been described in Section $\mathrm{E}$ of this chapter. For the calculations, only three distinct core regions (with individually computed atom concentrations) were used in place of the five regions actually loaded. The atom densities used in the calculations are shown in Table XX. The concentrations indicated for core region 3 represent a volume-weighted average of the actual number densities listed in Table XII for fuel platelet loadings 3, 4 and 5. The mesh spacing for the multigroup diffusion calculations performed with this $\mathrm{R}-\mathrm{Z}$ model are indicated in Fig. 17. In order to assess the effect of anisotropic neutron diffusion upon the basic physics parameters for the Phase I assembly, the diffusion calculations were performed in two ways: (a) using a homogeneous diffusion coefficient for each composition, and (b) using bi-directional diffusion coefficients for each composition.

The bi-directional Benoist diffusion coefficients were calculated in the manner described in Section G-2. For the $R-Z$ calculation the $D / \mid$ represents exactly the situation in the (axial) $Z$ direction and $D_{z}$ was taken equal to $D / \mid$. In the $R$ direction, however, the streaming pattern is more complicated because of the drawer loading pattern* and the characteristic diffusion coefficient varies uniformly from $D$ | in the horizontal direction to almost $D||$ in the vertical direction. Hence $D_{R}$ was taken to be the arithmetic mean of $D \perp$ and $\mathrm{D} \mid$. Values of the $\mathrm{R}-\mathrm{Z}$ bi-directional diffusion coefficients are listed in Tables XXI-XXIII. The diffusion coefficient modifiers used in the calculations are shown in Table XXIV.

* Since the void devices line up vertically, there are clear streaming paths vertically blocked only by the tops and bottoms of the void devices, drawer bottoms and matrix tubes. 
For an analysis of the experiments involving radial traverses, an $\mathrm{X}-\mathrm{Y}$

model of the assembly was developed. The model used a quarter core symmetry condition at the inner boundaries for both axes. The transverse buckling for the calculation was determined by a buckling search to obtain the calculated eigenvalue from the $R-Z$ model. This group and region independent axial buckling $\left(\mathrm{B}_{Z}^{2}=4.123247 \times 10^{-4}\right)$ corresponds to an extrapolated half height of $77.3571 \mathrm{~cm}$. To provide a detailed spatial description, a uniform spacing of two mesh intervals per drawer was used in both directions. The bi-directional Benoist coefficients used in this model were $D_{y}=D \mid f$ and $D_{x}=D \perp$ which appropriately described the streaming characteristics.

The initial models used the condition of zero flux at physical boundaries. The models were later refined to use a logarithmic external boundary condition. The initial model also neglected the effects of the reactor support bed and knees - data accumulated during the program made it clear that the reflection effects were important and these were included in the refined models.

\section{Results of Eigenvalue Calculations}

In the calculations performed for the assembly the neutron streaming correction was defined as the difference in eigenvalue obtained by introducing the Benoist bi-directional diffusion coefficients in place of the isotropic diffusion coefficients $\left(D=1 / 3 \Sigma_{t r}\right)$. Table XXV summarizes the results of initial eigenvalue calculations obtained with both the 11 and 29 group crosssection sets. The agreement between the 11- and 29-broad group calculated eigenvalues is very good $(20.0004 \mathrm{\delta})$ for both the with-streaming and withoutstreaming cases. The 29 broad group calculation including the Benoist treatment of streaming produces an eigenvalue of 0.9718 . This includes a $\delta \mathrm{k}_{\mathrm{streaming}}$ of -0.0230 . This large underprediction of the calculated eigenvalue prompted a closer look at the calculational methods and the models. The experimental evidence from the reaction rate traverse measurements indicated a significant reflection effect from the knees and bed of the reactor and a more refined $X-Y$ model was constructed that included these structures. A logarithmic boundary condition was used at the external boundaries and the results of the $R-Z$ and $\mathrm{X}-\mathrm{Y}$ calculations were combined to reconstruct the "three-dimensional" eigenvalue (the knees and bed could not be conveniently modeled in R-Z geometry and the reflection effect $(\Delta k)$ thus was calculated in $X-Y$ geometry and added on to the basic R-Z eigenvalue). Table XXVI lists the sensitivity of the eigenvalue to the various calculational parameters. Table XXVII shows the composite result of the refined two-dimensional calculations. The sensitivity of the eigenvalue to the various calculational parameters are also shown. The final eigenvalue of 0.9763 included a $\delta k_{\text {streaming of }}-0.0229$ and showed a large bias that needed further scrutiny. This is discussed in the next section in greater detail.

Even though the 11- and 29-group calculations predict the same eigenvalue, there are non-negligible differences in the calculated real and adjoint group fluxes. These group fluxes from the non-streaming calculations are given in Table XXVII. They are compared in Figs. 20 and 21. Some of the differences - Wue to the greater energy detail of the 29-group calculation would appear to be gnificant for the prediction of spectrum-sensitive reactor parameters, such as material worths and threshold reaction rates. 
Some of the effects which neutron streaming has upon reactor parameters can be understood by considering the effect which streaming has upon the neutron spectra. The calculated 29-group group real and adjoint fluxes for the with-streaming and without-streaming calculations are given in Table XXIX. These spectra are further compared in Figs. 22 and 23 . There is a small ( $~ 1$ to $2 \%$ ) increase in the group fluxes above $\sim 200 \mathrm{keV}$ and a corresponding decrease in the lower energy group fluxes, for the with-streaming case. This slight spectral hardening effect has been observed in earlier studies (see Ref. 6) and is caused by the reduction of the downscatter source term to lower group as a result of preferential leakage of high energy neutrons. The group adjoint fluxes are smaller for the with streaming case for all groups above $\sim 10 \mathrm{keV}$, indicating the decreased importance of the higher energy neutrons which have a higher probability of leakage. The basic physics parameters including kinetics parameters for the Phase $I$ assembly are given in Tables XXX and XXXI. Of the calculated parameters included in these tables, only the eigenvalue is significantly affected by streaming. The calculated $\beta_{\text {eff }}$ is increased $<0.5 \%$ and the reactivity conversion factor $\mathrm{Ih} / \% \delta \mathrm{k} / \mathrm{k}$ is decreased $<0.1 \%$. The general conclusion from the analysis was that while the leakage and eigenvalue were very sensitive to the streaming effects, the value of central integral physics parameters was not significantly affected by these effects.

For the analysis of experiments, the kinetics parameters obtained from 29 energy group R-Z calculations were used. The parameters so obtained are listed in Table XXXII. It has been shown in earlier work that a single set of kinetics parameters (condensed over isotopes) provides for reduced computation time without sacrificing accuracy. The collapsing is performed by defining effective isotope averaged parameters as the following:

$$
\begin{aligned}
& \beta_{j}= \sum_{i} \beta_{i j} \\
& \lambda_{j}= \sum_{i} \beta_{i j} \\
& \sum_{i} \beta_{i j} \lambda_{i j}
\end{aligned}
$$

Where $i$ is the isotope index and $j$ the delayed family index. Table XXXIII gives the condensed kinetics data set actually used in the analysis of the experiment along with the other kinetics data.

Table XXXIV gives a neutron balance by region for the assembly. In Table XXXV the isotope reaction rates for the core region are summarized.

The results of the analyses of individual experiments have been reported in the subsequent chapters along with the experimental results. Additional analytical work beyond the basics described here were needed for the analysis of some of the experiments and these have been discussed in the appropriate chapters.

\section{H. Discussion of Results}

The predicted elgenvalue (which has been obtained using standard methods of design analysis) for the GCFR Phase I assembly is 0.9763 . This value includes a $2.29 \% \delta k$ due to streaming. This discrepancy of $2.37 \%$ in the 
calculated eigenvalue is larger than that obtained for other fast critical assemblies. A review of the current status for predicting the eigenvalue for several fast benchmark critical assemblies is given in Table XXXVI. It can be noted that the eigenvalue is consistently underpredicted. There is a negative bias of $1-1.5 \% \delta \mathrm{k}$ for the LMFBR critical assemblies listed in the Table when analyzed by standard calculational methods (ENDF/B Version IV data, 27 broad groups, two-dimensional diffusion theory). This bias in predicting the eigenvalue for these assemblies is larger than the desired accuracy $(<0.5-1.0 \% \delta \mathrm{k})$ in fast reactor design. The bias in predicting the eigenvalue for GCFR Phase $I$ is more than $1 \% \delta k$ larger.

The LMFBR calculations quoted herein have not included any effects due to neutron streaming, whereas the GCFR Phase analysis includes a $\delta \mathrm{k}_{\text {streaming }}$ of $2.29 \%$. Recent studies show that the LMFBR assemblies have much smaller)

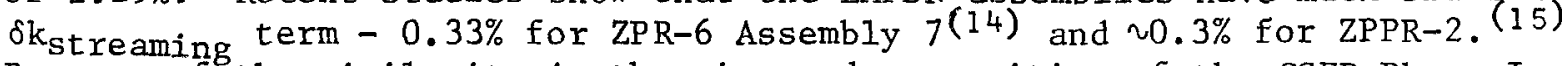
Because of the similarity in the size and composition of the GCFR Phase I assembly and ZPR-6 Assembly 7 a comparison of the results for the two assemblies suggested a possible inconsistency in the $\delta k_{\text {streaming calculations for }}$ the two assemblies. It was necessary to investigate the validity of the Benoist method in the light of these experimental results.

The value of the diffusion coefficient, D, determines the neutron leakage in diffusion theory calculations. Wade and Gelbard ${ }^{6}$ compared calculated values of D for several cases, including (a) homogeneous D, (b) Benoist D, and (c) anisotropic D corrected for higher powers of $B^{2}$ obtained from the numerical solution of a transport equation. They found the uncorrected Benoist $D$ to be slightly $(22 \%)$ too large relative to the more exact results. In order to assess the sensitivity of the calculated eigenvalue for GCFR Phase I to errors in the diffusion coefficient, eigenvalue calculations were repeated using modified values for $D$. The calculations were identical (same cross sections, reactor model and calculational methods) except for a uniform change (over the entire energy range) in the values for D. As shown in Table XXVI a $5 \%$ change in $\mathrm{D}$ produces a $1.5 \%$ change in $k_{e}$ ff. These calculations suggest that the uncertainty in the values of $\mathrm{D}$ can produce an uncertainty in the calculated eigenvalue for the GCFR Phase I assembly of the order of $1 \% \delta \mathrm{k}$. Thus, a plausible explanation of the discrepancy in eigenvalue was an overprediction of the Benoist $D$ values.

Analysis of the reaction rate traverse measurements (described in Chapter VII) in the normal and checkerboard loadings revealed that the calculated shapes of the reaction rate profiles were mch steeper than the experimental profile, i.e., the calculation underpredicted the reaction rate values in the blanket. It became increasingly apparent that there were larger reflection effects than was being accounted for in the model. Additionally it was clear that the basic $\mathrm{R}-\mathrm{Z}$ calculation did not adequately model the complete assembly. The presence of the massive bed at the bottom of the matrix (with no corresponding structure on top) and the much thinner knees on the vertical side made the assembly azimuthally asymmetric and not representable in the $R-Z$ plane. A set of three-dimensional 16 diffusion theory calculations were performed to study the effects of the modeling difficulties in two-dimensions. An addition1 use of the three-dimensional calculation was to provide a check of the alidity of using the prescription $D_{R}=1 / 2(D|+D| \mid)$ in the $R-Z$ calculation. 
The three dimensional calculations were performed using the flux synthesis code, SYN3D. 17 This variational code was used in preference to the existing three dimensional finite difference code $\mathrm{DIF} 3 \mathrm{D}^{18}$ because at the time of the calculations the latter code was limited to a small number of mesh points. A series of test problems were run to check the validity of SYN3D against DIF3D. In addition, the handling of the Benoist diffusion coefficients by the three dimensional codes were checked by comparison with DIF2D for a degenerate 3D problem. The checks showed that SYN3D did provide a valid eigenvalue and flux solution in the assembly for the with-streaming case.

The bulk of the calculations were performed with a $1 / 8$ reactor model (quarter core symmetry assumed in the radial mid-plane and symmetry assumed above the axial mid-plane). This model suffered from one of the problems with the earlier two-dimensional representations in that the reactor bed was, in effect, introduced on the top of the reactor because of the symmetry conditions. The vertical knees existed on both sides of the reactor and the modeling procedure accounted for these accurately. One-half core model calculation was performed to study the effect of this modeling error. Figure 24 shows the model of the assembly used for the calculations. The calculations were performed in 11 energy groups with a fairly coarse ( 1 mesh point per drawer) spatial representation. Earlier studies with the DIF2D code had shown that this coarseness of the spatial mesh and energy group structure did not affect the eigenvalue significantly.

For the calculations $30 \mathrm{~cm}$ of axial reflector was introduced to account for axial reflection of neutrons from outside the blanket. This reflector region consisted of homogenized matrix tube material and was assigned an isotropic diffusion coefficient. Table XXXVII gives the best results obtained from the three dimensional and two dimensional diffusion theory calculations.

The "final" eigenvalue of 0.99088 has a bias of $-0.912 \%$. This is very comparable to the earlier LMFBR assemblies. There is still a $0.65 \%$ discrepancy between this eigenvalue and the eigenvalue of ZPR-6 Assembly 7. That might reflect a general problem of calculating leakage terms by $2-D$ models or errors

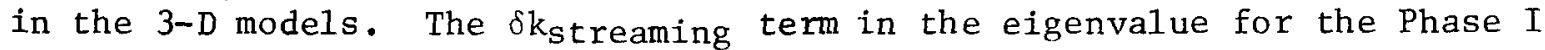
assembly determined from the 3-D calculations is $1.91 \%$ which is not very much different from what was obtained from 2-D mode1s.

\section{NEUTRON SPECTRUM MEASUREMENT}

\section{A. Introduction}

The central neutron spectrum was measured in the GCFR Phase I critical assembly using in-core proton-recoil proportional counters. This measurement represents the first neutron spectrum measurement in a full-scale mock-up of a GCFR. The only earlier GCFR spectrum measurements reported were performed in test lattices constructed in the central region of the thermally driven PROTEUS reactor.19,20 The measured central spectrum is compared with a calculated spectrum based on ENDF/B Version IV data. The measured GCFR spectrum is also compared with a typical LMFBR spectrum. 


\section{Experimental Technique}

The proton-recoil measurement technique has been described in Ref. 21. For the measurement reported here counting tubes of a special design were used. These are cylindrical tubes with a modified end structure made to reduce the response distortion from non-uniform internal electric fields. The counters are sufficiently free from electric-field-response effects that no corrections for this effect were required to the measured pulse-height distributions. The counting tubes have a 10 -mil-thick wall and are filled with hygrogen or methane gas. The hydrogen-filled tube has a $3 / 8 \mathrm{in.}$ inside diameter and a $3 / 4$ in. effective length, while the methane-filled tube is $5 / 8$ in. in diameter and 1-1/4 in. long. The active counters are enclosed in a sleeve (stee1, 10-mi1thick) having an outer diameter of 1 in. The overall length of the container is 7 in., including a pumpout tube and external ceramic insulator. Electrical insulation is of minimal mass and is well removed from the effective counting volume. During the measurement the counter center was placed 4-1/2 in. from the front face of the drawer. A schematic of the detector layout is shown in Fig. 25. The counter assembly rested on the bottom of the drawer. That part of the drawer not containing the detector, preamplifier, and lead-in cables was filled with nominal central-core material composition. The plate-material pattern is also indicated in Fig. 25. Evidence from past experiments ${ }^{21}$ indicates that effects of permuting the platelet arrangement in the detector drawer (and thus changing the local environment of the detector) are insignificant compared to measurement uncertainties. Such effects as do occur are either above or below the measurement range ( $1 \mathrm{keV}$ to $2 \mathrm{MeV}$ ).

Measurements were made sequentially with the methane-filled and hydrogenfilled detectors. The methane-filled detector is used to measure proton-recoil events with energies greater than $100 \mathrm{keV}$; the hydrogen-filled detector is used for lower energies.

\section{Assembly Configuration}

The configuration was that of the initial Phase I reference core in a state of maximum shutdown with all DP rods removed and all boron blades inserted. The reactor was approximately $2 \%$ subcritical. Ordinarily, it is desirable to achieve the necessary degree of subcriticality (required to limit detector count rates) by a core-reload in order to minimize any spectral effects due to the insertion of the boron safety blades. In this measurement no boron blades were closer to the core center than $30 \mathrm{~cm}$ and calculations indicate that there are no observable perturbations on the central spectrum over the measurement range, which is above $1 \mathrm{keV}$.

\section{Results}

The measured central spectrum is shown in Fig. 26. (A calculated spectrum is also shown; the calculation and comparison are discussed below.) The measurement extends from $1 \mathrm{keV}$ to $2 \mathrm{MeV}$. The error bars on the measured points reflect only the statistical uncertainty. The experimental resolution, i.e., the full width at half maximum, is $8 \%$ over most of the energy range. At low nergies the statistics in the ionization process broaden the resolution so hat at $1 \mathrm{keV}$ the resolution is about 20\%. The oxygen resonances at $400 \mathrm{keV}$ and $1 \mathrm{MeV}$ and the iron resonance at $30 \mathrm{keV}$ are clearly seen. 


\section{E. Systematic Measurement Errors}

Several sources of systematic error have been identified using the protonrecoil measurement technique. One of the more serious, relating to the quality of detector internal field structure is not expected to be significant for this measurement series due to the use of the spectally designed counting tubes.

The use of methane gas for the higher-energy measurement requires that a correction be made for the energy-ionization relationship for protons stopping in methane and also the effect of carbon recoils resulting from neutron scattering from carbon in methane. These corrections are small (1ess than 5\%) and based upon fairly good data. They tend to compensate each other to some extent and we do not consider them to be especially unreliable to make. Results reported here are corrected for these effects.

Energy-scale calibration errors are present in the reported data above about $1 \mathrm{MeV}$ and may bias the calibration by about $10 \%$. These are traceable to the inherent resolution of the detector and to the effects of increasing track lengths as energy increases. The above-mentioned errors related to the use of methane gas and could all be eliminated by using a mixture of hydrogen and xenon at high pressures with good resolution. The upper-energy limit for measurements would be extended by 1 or $2 \mathrm{MeV}$, there would be no scattering correction, and good energy versus ionization proportionality for such a mixture is expected. There is a clear need for technique improvement along these lines.

The low-ionization data is affected by how well the background from fastelectron events that extend under the proton-peak can be represented. Simply using the electron energy-loss distributions from a ${ }^{60} \mathrm{Co}$ source to represent the distributions from the GCFR gamma-ray environment introduces negligible effects in the background subtraction.

The two largest sources of systematic error in proton-recoil measurements occur at the low- and high-energy extremities. Variations in $W$, the energy loss by a proton per ion pair formed, will affect results below about $10 \mathrm{keV}$ and wall-and-end effects, wherein protons leave the active volume of the detector before stopping, will distort results at high energies. Experimental data are inadequate for use in treating the low-energy $W$ variation. Fortunately, $\mathrm{W}$ for protons stopping in hydrogen is remarkably constant even to very low energies and the assumption of a constant value everywhere for $W$ is as well justified by existing experimental evidence as is any other prescription for W variation. More complicated prescriptions have been used in the past; to indicate the extent of possible systematic errors from this source, Fig. 27 shows a plot of a typical LMFBR central spectrum from 1 to $20 \mathrm{keV}$ as analyzed by assuming $W$ to be constant and by assuming $W$ to vary with the ionization dependence 1isted in Ref. 22. Although the effect upon absolute flux is relatively modest, the uncertainty in relative flux near the depleted-flux region at $3 \mathrm{keV}$ induced by large sodium scattering resonance at this energy could make a ratio comparison of measurement and theory totally erroneous. Since the GCFR spectrum does not contain this feature, the effect of the uncertainty in $\mathrm{W}$ is considerably smaller.

Wall-and-end effects set in strongly at about $1 \mathrm{MeV}$ and effective1y terminate the useful range of measurements at between 2 and $3 \mathrm{MeV}$ for the counters 
used for measurements reported here. A correction procedure for cylindrical counters has been described previously. ${ }^{22}$ When the procedure is applied to the GCFR spectrum, the resulting correction as a function of energy is as shown in Fig. 28. Only those data are reported for which the wall-and-end correction do not exceed $20 \%$ - this corresponds to a high-energy limit of approximately $2 \mathrm{MeV}$.

\section{F. Discussion}

The measured and calculated spectra are compared in Fig. 26. The measurement extends from $1 \mathrm{keV}$ to $2 \mathrm{MeV}$. As indicated previously, the error bars on the measured points reflect only the statistical uncertainty, and do not take into account uncertainties associated with the various corrections. The corrections (see Section $\mathrm{E}$ ) over most of the energy range introduce less than $5 \%$ changes. The histogram is the result of an SDX spectrum calculation with USS226 group structure. The calculation was smoothed with a Gaussian response function, whose width as a function of energy was determined by the experimental resolution. Smoothing of spectra in the vicinity of strong resonance-related structure depends sensitively upon the response shape. Since the Gaussian prescription is only approximate, it is not surprising to find the greatest apparent disagreement between theory and experiment at or just below large resonances. The calculation was normalized to the measurement to preserve the flux over the measurement interval.

In general, the agreement between measurement and calculation is good. The calculation overpredicts the flux near $600 \mathrm{keV}$; the measured low-energy flux is consistently higher than the prediction.

Figure 29 shows a typical LMFBR spectrum measured with the same counters. The spectrum is the central core spectrum in the Engineering Mockup Critical Assembly of the Fast Test Reactor. ${ }^{23}$ The GCFR spectrum is significantly harder at high energies, especially around $600 \mathrm{keV}$. Note that in this region the calculations overpredict the flux for both assemblies. At low energies the flux is underpredicted. The absence of the sodium resonance in the GCFR spectrum is clearly seen; in fact, the GCFR spectrum is quite similar to that found in a sodium-voided LMFBR core.

\section{CENTRAL REACTION RATE MEASUREMENTS}

\section{A. Introduction}

Fission rates were measured at the center of the Phase I assembly using Kirn-type fission counters. In addition, the capture rates in $232 \mathrm{Th}$ and ${ }^{238} \mathrm{U}$ were measured using a foil irradiation technique. The results are compared to calculations and the effect of the fission counter material on the reaction rates is discussed.

\section{B. Experimental Procedure}

\section{Fission Rate Measurements}

Absolute fission rates were measured for ${ }^{232} \mathrm{Th},{ }^{233} \mathrm{U},{ }^{235} \mathrm{U},{ }^{238} \mathrm{U}$, ${ }^{239} \mathrm{Pu}$ and ${ }^{240} \mathrm{Pu}$ samples using $5.1-\mathrm{cm}$ diameter Kirn-type fission counters. 
Three counters were located in drawers S21/25, S23/23 and S25/21 of the matrix as shown in Fig. 30. The center of the assembly is matrix location 23/23. Figure 31 shows the position of the detector within the drawer, with the fission source on the front plate of the detector located $1.91 \mathrm{~cm}$ from the front of the drawer. Fission rates for three of the sources were measured at more than one location in order to assess the magnitude of the flux change between positions. A fourth fission counter with a ${ }^{239} \mathrm{Pu}$ source 10 cated in drawer S23/12 served as a monitor for normalization between runs.

Scaler data for all four counters were accumulated simultaneously, giving a monitor count rate for each set of scaler counts. In addition, fission fragment energy spectra were recorded and used to calculate tailing correction factors which were applied to the scaler data.

\section{Capture Rate Measurements}

Capture rates in ${ }^{232} \mathrm{Th}$ and ${ }^{238} \mathrm{U}$ were measured by placing four foils (1.27- cm diameter, $0.0127-\mathrm{cm}$ thickness) of each material in a square array centered in front of two of the fission counters. This positioning put the capture-rate foils in almost exactly the same location as the fission counter sources. These foils were counted off-line with a high-resolution Ge(Li) detector system to determine the absolute capture rate in the foils.

\section{Data Reduction}

\section{Fission Rates}

Scaler data from the fission counters was corrected for the low-energy tailing or alpha-particle contributions by using a factor calculated from the multichannel analyzer spectra. Table XXXVIII shows the results of the fission rate measurements at the three detector positions. The numbers listed are fissions per second per microgram of source material normalized to 150 monitor counts. No isotopic corrections have been made to these results. Also listed

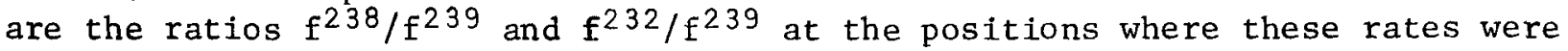
measured in the same drawers. In those cases where a particular fission rate was not measured in the central detector, the rates were normalized to location $23 / 23$ by assuming a constant ratio with the ${ }^{239} \mathrm{Pu}$ fission rate at all three detector positions. For example, the ${ }^{233} \mathrm{U}$ fission rate was measured only S21/25. To calculate the ${ }^{233} \mathrm{U}$ fission rate in the center, it was assumed that $\left(f^{233} / f^{239}\right)$ was the same in $S 21 / 25$ and $S 23 / 23$. Since the off-center detectors were located only two drawers from the center position in a very large, homogeneous core, this assumption introduces negligible error, as verified by the results of Table XXXVIII.

Isotopic corrections were also applied to the uranium and plutonium fission rates. Table XXXIX lists the isotopic composition and the mass of the fission sources. Since the fission rates of ${ }^{234} \mathrm{U},{ }^{236} \mathrm{U},{ }^{241} \mathrm{Pu}$ and ${ }^{242} \mathrm{Pu}$ were not measured, calculated values were used for the corrections due to these isotopes.

Data were taken at a reactor power level of approximately 250 watt and all results were normalized to an arbitrary value of 150 counts/sec in the monitor counter. This monitor count rate corresponds to a reactor power level of approximately 60 watts. 


\section{Capture Rates}

The eight foils irradiated to determine the capture rates were counted with high-resolution $\mathrm{Ge}(\mathrm{Li})$ detectors in an automatic foil counting system. 23 Gamma-ray spectra from each of the foils were accumulated with a computer-based multichannel analyzer system. Each foil was counted many times in each of the two sample changer-detector systems.

The determination of the absolute capture rate is based on the detection of gamma rays from one isotope in the capture-product decay chain. For $238 \mathrm{U}$ capture, the $277.6-\mathrm{keV}$ gamma ray following the decay of $23{ }^{9} \mathrm{~Np}$ is counted, and for ${ }^{232} \mathrm{Th}$ capture, the $311.9-\mathrm{keV}$ gamma ray from the decay of $233 \mathrm{~Pa}$ is counted. The number of gamma rays detected is taken to be the area of the photopeak above background in the spectrum. This area is determined by fitting a multiparameter function to the data points using a modified version of the computer code SKEWGAUS. ${ }^{25}$ Figure 32 shows a typical fit to the $277.6 \mathrm{-keV}$ photopeak in the $23{ }^{9} \mathrm{~Np}$ spectrum and Fig. 33 shows a fit to the $311.9-\mathrm{keV}$ peak in the ${ }^{23} 3 \mathrm{~Pa}$ spectrum.

The detector efficiency is determined by fitting the peaks in the spectrum of a calibrated source with the same computer code. Using the efficiency of the detector, the absolute number of gamma rays emitted can be calculated. Then using the branching ratios for the transitions under consideration, the number of decays of either ${ }^{233} \mathrm{~Pa}$ or ${ }^{239} \mathrm{~Np}$ can be calculated. For $233 \mathrm{~Pa}, 311.9 \mathrm{keV}$, a gamma intensity of 0.3771 is used, and for ${ }^{239} \mathrm{~Np}$, $277.6 \mathrm{keV}$, a ratio of 0.1412 is used.

Since each recorded spectrum from the foils is accumulated at a different time after the irradiation, the disintegration rate must be corrected for the decay half-life of the capture product. Also, the power history of the reactor must be known and accounted for in order to determine the absolute number of captures in the foils. For these measurements, the power history data was taken from the monitor fission counter. The equations involved in these corrections are derived and their use explained in Appendix A. Halflives of 2.355 days and 27.0 days were used for the decays of ${ }^{239} \mathrm{~Np}$ and ${ }^{233} \mathrm{~Pa}$ respectively.

The final capture rate in each foil is then taken to be a statistically weighted average of all the corrected peak areas for that foil. Since the four foils covered the center section of the counter, an average of the rates in the four foils was taken to be the central capture rate. The data for ${ }^{232} \mathrm{Th}$ and ${ }^{238} \mathrm{U}$ capture were evaluated on each of the two Ge (Li) systems. The results differed by less than $0.5 \%$ between the two systems, and were averaged to give the final results. The final experimental reaction rate ratios are given in Table $\mathrm{XL}$.

\section{Calculational Procedure}

The fluxes used to calculate the reaction rates are from the 29-group, twodimensional, $X-Y$ diffusion calculation described in Cahpter III. A uniform acing of two mesh intervals per drawer was used in both directions. Since ne material in the fission counter foils is very thin (the deposited fission foils are $6-100 \mu \mathrm{gm} / \mathrm{cm}^{2}$ or much less than $1 \mathrm{mil}$ thick and the capture foils are $\sim 5$ mils thick), there is no spatial self-shielding in the sample. For 
this reason "detector" or homogeneous cross sections are appropriate when calculating the fission counter reaction rates, and these were used in the analysis.

Because of the unique environment of the fission (or capture) foil material, the flux spectra seen by the sample is not the same as the calculated flux spectra at the core center. The foils are encased in the stainless-steel Kirn-type fission chamber which is placed in a cavity that is "shielded" from the core loading by the stainless steel drawer and matrix. The thicknesses of the drawer and matrix are $32 \mathrm{mils}$ and $40 \mathrm{mils}$, respectively. To account for the shift in spectra produced by the stainless steel detector environment, the distorted flux in the vicinity of the foil was calculated using the PIT code. 26 This computer program provides a one-dimensional integral transport formulation for the computation of the distorted fluxes. The PIT calculations were performed for a spherical model of the two-inch cubical cavity in which the detector was positioned. The stainless steel of the matrix, drawer, and fission chamber was homogenized throughout the spherical model of the cavity. Two sets of foil reaction rates were then computed using the homogeneous cross sections, first with the undistorted (ARC-DIF2D) fluxes and then with the distorted (PIT) fluxes.

\section{E. Results and Discussion}

Table XII lists the reaction rate ratios at matrix position $523 / 23$, the center of the core. Also listed are the calculated values obtained using the homogeneous cross sections and the distorted (PIT) fluxes. All the C/E values are within $1 \%$ of unity except for the threshold ratios of ${ }^{238} \mathrm{U}$ and ${ }^{23} \mathrm{Th}$ (relative to $249 \mathrm{Pu})$. The calculated values for these ratios have been decreased slightly over $10 \%$ due to the softening of the flux spectra by the stainless stee1. These effects are summarized in Tables XLI and XLII. Table XLI 1ists the calculated reaction rates obtained using both homogeneous (detector) and heterogeneous (cell-averaged) cross sections with both undistorted and distorted fluxes. Also shown are ratios of the heterogeneous/homogeneous and distorted flux/undistorted flux reaction rates. These quantities are given in Table XLII as fission ratios relative to ${ }^{239} \mathrm{Pu}$. These calculations indicate that the differences between homogeneous and cell-average reaction rates are sma11 $(<1 \%)$ except for capture $(-3 \%)$ and fission $(+6 \%)$ in ${ }^{238} U$ and fission (+6\%) in ${ }^{240} \mathrm{Pu}$ (relative to homogeneous). Further, the effect of the stainless steel surroundings upon the flux spectra seen by the foil is relatively small ( $~ 11$ to $2 \%$ ), except for the threshold fission rates in ${ }^{2}{ }^{32} \mathrm{Th}(-10 \%),{ }^{238} \mathrm{U}(-10 \%)$, and ${ }^{240} \mathrm{Pu}(-3 \%)$. The calculated undistorted (ARC) and distorted (PIT) flux spectra are plotted in Fig. 34 Table XLIII shows a comparison of these group fluxes and the corresponding calculated ${ }^{238} \mathrm{U}$ fission rates by group. As indicated by these calculations, $75 \%$ of the fissions in ${ }^{238} \mathrm{U}$ occur in groups 4 and 5 (90\% in groups $\left.3-5\right)$. The presence of the stainless steel reduces the flux in these higher energy groups, sharply affecting the calculated ${ }^{238} \mathrm{U}$ fission rate.

This calculated effect on the threshold fission rate is comparable to the differences reported for the ${ }^{238} \mathrm{U}:{ }^{239} \mathrm{Pu}$ fission ratio measured with foils and fission chambers. The cell-averaged ${ }^{238} \mathrm{U}(\mathrm{n}, \mathrm{f}):{ }^{239} \mathrm{Pu}(\mathrm{n}, \mathrm{f})$ reaction rate rat measured with foils in ZPPR-2 was $0.0243 \pm 0.0002$, while that measured with chambers was $0.0219 \pm 0.0005 .(27)$ In SNEAK $3 \mathrm{~A}-2$ the central ${ }^{238} \mathrm{U}:{ }^{239} \mathrm{Pu}$ fission ratio in the normal cel1 was 0.0338 measured by foils compared to 0.0313 
measured with fission chambers. ${ }^{28}$ The simplified calculational model used in the PIT calculations of this study (i.e., homogenizing the stainless steel in a spherical region) produce a comparable effect $(-10 \%)$ upon the calculated ${ }^{238} \mathrm{U}: 239 \mathrm{Pu}$ fission ratio.

This modeling procedure should be overpredicting these effects somewhat for the following reasons. The region surrounding the stainless steel zone has been taken to be homogenized core composition, when in fact it is, for the width of a drawer, core composition with reduced stainless steel. That is, although the calculation accounts for the spectral softening of the stainless steel surrounding the fission chamber, it neglects the spectral change (hardening) in the region adjacent to the stainless steel zone. Furthermore, the foils are not exactly at the center of the cavity, but in fact are on the face of the detector. The flux seen by the foil is comprised of neutrons which have passed through perhaps 2 or 3 detector walls or perhaps none at all. This has almost no effect on the non-threshold reaction rate ratios, but a slight effect $(\sim 0.1-0.3 \%)$ on the threshold ratios.

In the interpretation of these calculated and experimental results some fundamental problems should be kept in mind. The PIT calculations provide reasonably accurate corrections for the flux distortion effects of the surrounding stainless steel on the fission rates. The capture rates in ${ }^{238} \mathrm{U}$ and 232 Th are dominated by resonance capture events which are affected by the a1terations in the microstructure of the energy spectrum caused by the stainless steel. Since the PIT calculations are performed at the broad group level, these ultra fine group effects are not computable and the distortion effects of the stainless steel or the capture rates are miscalculated. Monte Carlo calculations are needed to compute these effects of flux distortion for capture rates. The experimental results do not directly correspond to the reaction rates within the GCFR Phase I assembly composition, because of the configurarion of the measurements. They provide a test of spectrum and capture rate calculations.

Table XIIV shows a comparison of the experimental values and C/E ratios of the central reaction ratios in the GCFR Phase I Assembly and ZPR-6 Assembly 7 - a benchmark LMFBR assembly. No flux perturbation effects have been considered for this comparison. The harder GCFR spectrum results in increased fission rates in $238 \mathrm{U}$ and $240 \mathrm{Pu}$. The calculated predictions are generally slightly better in the GCFR assembly than in the LMFBR assembly.

\section{SMALL SAMPLE REACTIVITY WORTH MEASUREMENTS}

\section{A. Int roduction}

This chapter describes the small-sample reactivity worth measurements that were made in the GCFR Phase I Assembly. The measurements included small-sample central worth determinations of a number of materials of interest to the GCFR program, viz., ${ }^{239} \mathrm{Pu},{ }^{240} \mathrm{Pu},{ }^{241} \mathrm{Pu},{ }^{23} \mathrm{U}^{3},{ }^{235} \mathrm{U},{ }^{238} \mathrm{U}$, for ${ }^{239} \mathrm{Pu}$, $238 \mathrm{U}, \mathrm{CH}_{2}$-foam, and SST samples. In addition central worth measurements of a ${ }^{52} \mathrm{Cf}$ spontaneous fission source at several reactor power levels have provided determination of the perturbation denominator for the Assembly. 
The small-sample worth measurements were conducted in two phases in two slightly differing configurations. The first phase included the central worth and perturbation denominator measurements while the second phase consisted of the radial traverses. The differences in the two configurations are described in the next section. The experimental results are compared to results of First order Perturbation Theory calculations. The discrepancies between calculated and experimental values obtained in the present work are comparable to past experience with LMFBR assemblies.

The central worth and radial worth traverse measurements are described in Sections B - F. In Section $G$ the technique and results of the perturbation denomination determination are discussed. Finally, all the results are discussed in Section $\mathrm{H}$.

\section{B. Reactor Configuration}

The basic GCFR Phase I core configuration was modified slightly for these measurements to include (1) the insertion hole for the radial sample changer traverse tube, (2) the installation of the fine autorod (FAR) and (3) adjustments at the core - radial blanket boundary and adjacent to the FAR to compensate for the reactivity losses due to ( 1 ) and (2), above.

The insertion hole was approximately $2.86 \mathrm{~cm}$ in diameter located in the stationary half, $4.45 \mathrm{~cm}$ axially from the core midplane, and extended radially along matrix row 23 from colum 21, near the center of the core, to the outer edge of the radial blanket (column 44).

The autorod was installed in matrix position S/M-23/14. Matrix position $\mathrm{S} / \mathrm{M}-23 / 13$ adjacent to the autorod position, was loaded with a double column of fuel to compensate for the fuel removed from S/M-23/14.

For the central reactivity worth and perturbation denominator measurements, the core-radial blanket boundary adjustments were made by replacing blanket composition with core composition at matrix positions S/M-19/7, $-11 / 12,-39 / 19$, $-7 / 27$, and $-27 / 39$. This configuration, which had a measured excess reactivity of $\sim 89 \mathrm{Ih}$, is shown in Fig. 35 .

For the radial worth traverses, core material was substituted for blanket material only at matrix positions $\mathrm{S} / \mathrm{M}-19 / 7,-39 / 19$, and $-27 / 39$. In addition a dual ${ }^{10} B$ safety blade in matrix positions $M-19 / 15$ and $-19 / 17$ was substituted for a single $10_{B}$ safety blade in matrix position $M-19 / 16$ as part of a reactor operations test. This configuration, had a measured excess reactivity of $\sim 56 \mathrm{Ir}$. Since the changes were made far away from the radial traverse measurement location and the $10 \mathrm{~B}$ blades were withdrawn during the measurement, the influence of the changed configuration on the results is negligibly small.

\section{Experimental Method and Equipment}

The reactivity worths were determined by measuring the reactivity change (given by the change in the critical position of a calibrated autorod) caused by pneumatically oscillating an encapsulated sample radially in and out of assembly with the sample changer. The autorod consisted of an autorod drawel, a 5/8 inch thick tapered polyethylene blade that extended axially through the core and axial blankets, a servo-control system, and a rod position 
potentiometer. The sample changer consisted of a turret containing eight sample positions with remote position selection capability and a double walled stainless steel traverse tube. The radial position of the traverse tube was also remotely adjustable from the control room allowing a sample capsule to be pneumatically injected into a prescribed radial position in the assembly.

Three compensated ion chambers (SP-10) connected in parallel and located above the movable half of the reactor continuously monitored the neutron flux at that location as the servo-control system positioned the autorod to maintain a constant current from these chambers. The output signal of the rod position potentiometer along with the sample capsule position signal (sample-in or -out) from the sample changer were digitized and transmitted to digital scalers interfaced with an on-line computer where the autorod position versus sample capsule position (sample-in or -out) information was accumulated. The reactivity worth of the sample capsule was determined from the change in position of the calibrated autorod for each sample-in and -out cycle.

Data were accumulated for several sample-in and -out cycles for each sample, and the method of Bennett and Long ${ }^{29}$ was used to analyze the data so as to eliminate the effect of linear drift in the reactor power level. The on-1ine acquisition and analysis of reactivity measurement data is described in greater detail in Ref. 30 .

The samples used in these measurements are described in detail in Tables XLV and XLVI. Reactivity worth measurements of sample capsules and empty capsules, both types contained in stainless steel sample-changer capsule holders, were performed. The reactivity worth of the empty capsule was subtracted from the worth of the sample capsule to yield the worth of the sample.

\section{Calibration of the Fine Autorod (FAR)}

The FAR was calibrated separately for each of the two phases of the smal1sample worth measurements. Details of these calibrations are described below.

\section{FAR Calibration for Central Worth Measurement}

A calibration of the position of the FAR and the reactivity of the reactor configuration was performed prior to the actual measurement. The computer-aided calibration followed the method developed by Cohn et a1. 31

The FAR was moved repeatedly at steady speed back and forth between the limits of its travel (at the desired power level) and its position was recorded at $0.5 \mathrm{sec}$ intervals. At each of these intervals the reactor flux data were also recorded. Data were taken for two calibration runs of approximately half an hour duration each. The flux data were processed by inversekinetics methods to yield reactivities at each time point. The reactivity data reflected a superposition of the effect of the rod position and slow drift effect caused by temperature changes. A least-squares fitting method was used to fit the position and time data to the reactivities. Orthogonal polynomials were used to represent the position dependence of the FAR worth hile Gram polynomials were used to account for the time variation of the eactivity. For the determination of the calibration parameters ENDF/B-IV based kinetics data collapsed over isotopes were used (Table XXXIII). 
The total worth of the FAR was determined for each calibration by using the calibration parameters. As in earlier studies a linear fit to reactivity vs rod position was seen to provide an adequate representation of the data. The total worth was also determined by the conventional inverse kinetics technique (used for dual purpose control rod worth determination). Neutron flux and rod position information were accumulated while the autorod made one pass from its full-in to full-out position. The value selected for the total worth of the FAR for the central worth measurements was $5.224 \pm 0.052$ inhours the weighted average of the means of the values obtained from the two calibration techniques. The uncertainty of \pm 0.052 Ih was obtained by applying a conservative estimate of $1 \%$ uncertainty to the total worth of the FAR. This value includes both the uncertainty in the weighted average and an estimate of the uncertainty in the linearity along the length of the autorod. Linearity tests of the same autorod made during the central worth measurements in the FTR-EMC environment $^{10}$ demonstrated that the non-linearity in the FAR was quite small.

\section{FAR Calibration for the Radial Traverse Measurements}

One calibration of the FAR by the oscillation technique was conducted for the radial traverse measurements. The worth was determined to be 5.181 Ih which agreed to within $1 \%$ of the worth used in the central worth measurements. Again, the uncertainty in the FAR total worth was estimated to be $1 \%$ of the total worth or $\pm 0.052 \mathrm{Ih}$.

\section{E. Calculational Methods}

\section{Central Worth}

The reactivity worths were calculated by first order perturbation theory methods from the 29 energy group, two-dimensional diffusion theory mode1 of the GCFR Phase I Assembly. Homogeneous isotopic cross sections were used for the test samples. The fluxes were calculated by two-dimensional diffusion theory methods using the Benoist bi-directional diffusion coefficients to account for the anisotropic effects of neutron streaming. The central worth calculations were performed in $\mathrm{X}-\mathrm{Y}$ geometry. The sample insertion tube was offset from the axial midplane of the core by $4.45 \mathrm{~cm}$ and hence the "central" sample was removed from the midplane by that amount during the experiment. This effect was explicitly taken into account in the X-Y calculations by the specification of the appropriate transverse integration height in the perturbation calculations.

\section{Radial Worth Traverse}

For the calculation of the radial worth traverses the methods used were identical to those described for the central sample. It may be noted that this model did not include the reflection effects of the knees and bed of the reactor. This will only have an effect on the worth results deep in the radial

blanket. Perturbation regions were defined in drawers corresponding to the experimental sample location. The reactivity worths of the samples were determined by averaging the perturbation results at the four mesh points within the drawer location. The effect of the anisotropic diffusion coefficients in $t$ leakage component of the perturbation numerator was taken into account in thcalculations. As expected, for the scattering materials this proved to be a significant effect. 
For the evaluation of the perturbation denominator the isotopic fission rates at core center were calculated. The central $R-Z$ flux was used along with unit cell averaged isotopic cross-sections to determine the reaction rate. The calculations used fluxes that were normalized to a power of 1 watt in the reactor and then were renormalized to the power level of the measurement for the evaluation.

F. Reactivity Worth Results

\section{Small Sample Central Worths}

Table XLVII contains the results of the small sample central worth measurements in the GCFR Phase I Assembly. The reported sample worths are those obtained for the entire composite sample - the reactivity worths of individual isotopes within the sample have not been evaluated here. For each sample the gross worth of the corresponding dummy capsule and capsule holder are tabulated. The gross worths were determined by off-line analysis of the reactivity worth data using first order autorod calibration parameters corresponding to the previously determined average autorod worth of $5.224 \pm 0.052 \mathrm{Ih}$. The uncertainty, $E_{G}$ including the estimated uncertainty in the autorod calibration was given by the following expression:

$$
E_{G}=\rho_{G}\left(\frac{E_{B}}{\rho_{G}}\right)^{2}+(0.01)^{2} \frac{1}{2}
$$

where $E_{B}$ is the statistical uncertainty, determined by the Bennett technique in the gross worth $P_{G}$, of each sample, and 0.01 is the FAR worth uncertainty of $1 \%$ applied to the total worth of the autorod. The ratio of the sum of the sample capsule and capsule holder masses to the sum of the dummy capsule and capsule holder masses is tabulated for each sample. This ratio multiplied by the dummy capsule plus capsule holder worth is then subtracted from the sample plus sample capsule plus sample holder worth to yield the net sample worth. The uncertainty in the net sample worth, $E_{N}$, was determined from the uncertainties in the gross worths of the sample plus hardware, $E_{G S}$, and the dummy plus hardware, $E_{G D}$, as follows:

$$
E_{N}=\left(E_{G S}^{2}+E_{G D}^{2}\right)^{\frac{1}{2}}
$$

The specific worth of the sample was then obtained by dividing the net sample worth by the sample mass.

Table XIVIII lists the calculated central worths and the $\mathrm{C} / \mathrm{E}$ values for the composite small samples. For cases where more than one sample size worth was measured the worth of the smallest mass sample was used for the $\mathrm{C} / \mathrm{E}$ determination. No sample size effect corrections were made.

\section{Elemental and Isotopic Central Worths}

Table XLIX lists the experimental and calculated central worths of dividual isotopes and elements. The worths of ${ }^{23} \mathrm{U}, \mathrm{Th}, \mathrm{C}$, and Fe were measured directly from almost pure samples while the isotopic worths of ${ }^{235} \mathrm{U}$, ${ }^{238} \mathrm{U},{ }^{10} \mathrm{~B}$, and the $\mathrm{Pu}$ isotopes were determined from the experimental worths of 
$\mathrm{U}, \mathrm{B}$, and $\mathrm{Pu}$ samples by solving the following simultaneous equations relating isotopic worths to sample worths:

$$
\sum m_{i j} \rho_{i}=\bar{\rho}_{j}
$$

where $m_{i j}=$ mass fraction of isotope $i$ in sample $j$

$$
\begin{aligned}
& \rho_{i}=\text { worth of isotope } i \\
& \bar{\rho}_{j}=\text { worth of sample } j
\end{aligned}
$$

The C/E values are also listed in Table XLIX. As in Table XLVIII all experimental results reported in Table XLIX are for, or were determined from samples of smallest mass for cases where more than one sample size was measured. Again, no sample size effect corrections have been made.

\section{Small Sample Radia1 Worth Traverse}

Table $L$ lists the results of the experimental radial worth traverses. These results were determined similarly to the results in Table XlVII. First order autorod calibration parameters corresponding to an autorod worth of $5.181 \pm 0.052$ Ih were used. Small cylindrical samples of plutonium $(96.9 \%$ $239 \mathrm{Pu})$, depleted uranium $(99.77 \% 238 \mathrm{U})$, and type 304 stainless steel in addition to polyethylene foam used to simulate steam were included in the measurements. Table LI and Figures 36 through 39 provide a comparison of the experimental and calculated radial traverses. The figures graphically compare the normalized experimental and calculated reactivity worths, each normalized to the worth at the center (radial distance $=0.0 \mathrm{~cm}$ ) as a function of radius at the assembly midplane. On the figures, radial distance $=0.0 \mathrm{~cm}$ is the center of matrix location 23/23. No sample size effect corrections were applied.

\section{G. Determination of the Perturbation Denominator}

The perturbation denominator is defined as the importance-weighted neutron production rate in a reactor. In first order perturbation theory the reactivity worth of a sample $i$ in a core $j$ is expressed in terms of the perturbation denominator $\left(D_{j}\right)$ as follows:

$$
\rho_{i j}=N_{i j} / D_{j}
$$

where $N_{i j}$ is the local effect of the sample on the neutron population and $D_{j}$ is a global quantity characteristic of a given core configuration. The general expression for $D_{j}$ is $D_{j}=\left(\phi_{j}^{*}, S \phi_{j}\right)$.

In the GCFR Phase I assembly the perturbation denominator was determined from a measurement of the apparent reactivity worth of a ${ }^{252} \mathrm{Cf}$ sample. The technique was developed and used in earlier assemblies.32-34 At a given power level, the worth of a ${ }^{252} \mathrm{Cf}$ sample inserted at core center is given by the expression:

$$
\rho(C f)=\frac{\left.S(C f) \iint E_{X^{X f}}(E) \phi *(E) d E\right)}{D}
$$


where $S(C f)$ is the strength of the ${ }^{252} \mathrm{Cf}$ source and $x^{C f}$ (E) is the fission neutron spectrum of the source, Denoting the term in brackets by the expression $\phi^{*}(\mathrm{Cf})$ the perturbation denominator can be expressed as follows:

$$
D=\frac{S(C f) \bar{\phi}_{f}^{*}(C f)}{\rho(C f)}
$$

The worth of the ${ }^{252} \mathrm{Cf}$ source is dependent on the power level of the measurement and the absolute values of $\phi_{f}^{*}$ are dependent on the normalizations used. Thus the results are reported relative to a normalization term, $n$. The normalization term is chosen to be the product of the real and adjoint source terms at the center of the core.

$$
\mathrm{n}=\int \nu \Sigma_{f}(E) \phi_{0}(E) d E \int \chi\left(E^{\prime}\right) \phi \gamma\left(E^{\prime}\right) d E^{\prime} \equiv S_{0} \cdot S \gamma \equiv \bar{v} \cdot R_{f} \cdot \bar{\phi} \frac{S_{f}}{*}
$$

Then, the normalized perturbation denominator is given by the expression:

$$
D / n=\frac{S(C f)}{\rho(C f) \cdot \bar{v} \cdot R_{f}} \frac{\phi \stackrel{*}{f}(C f)}{\bar{\phi} \frac{*}{f}}
$$

where the quantities not refered to ${ }^{252} \mathrm{Cf}$ are values for the core material at the experimental position, $\bar{v}$ is the average number of neutrons emitted per fission and $R_{f}$ is the measured fission rate for the core material at the experimental position. The ratio $\bar{\phi} *(\mathrm{Cf}) / \overline{\phi_{\mathrm{f}}^{*}}$ has been shown to be close to 1 for a large number of assemblies 35 and has been taken to be unity for the present case. The source strength was determined from a calibrated strength of $(2.038 \pm 0.014) \times 10^{7} \mathrm{n} / \mathrm{sec}$ on February $4,1974(36)$ and a ${ }^{252} \mathrm{Cf}$ half life of $2.640 \pm 0.008$ years. The value of $\bar{v}$ was determined* from ENDF/B-IV based data to be $2.931 \pm 1 \%$ neutrons/fission. The $R_{f}$ and $p(C f)$ terms were determined experimentally in the manner described below.

1. The Central Fission Rate $R_{f}$

The central isotope fission rate $\bar{R}_{f i}$ was determined experimentally using fission flow counters for ${ }^{235} \mathrm{U},{ }^{238} \mathrm{U},{ }^{239} \mathrm{Pu}$ and ${ }^{240} \mathrm{Pu}$. These experimental values were used in the computation of the core material fission rate

$$
\bar{v}=\frac{\sum_{i j} v_{i j} \Sigma_{f i j}{ }^{\phi_{0}}{ }}{\sum_{i j} \sum_{f i j}{ }^{\phi_{0}}{ }_{j}}
$$

where $i$ is an isotope index and $j$ is a group index. $\phi_{0}$ is the $f 1$ ux at core enter. 
using calculated values for ${ }^{24 l} \mathrm{Pu}$, and ${ }^{242} \mathrm{Pu}$. The measured fission rates from the flow counters were assumed to be a close representation of the fission rate in the unit cell. Table LII shows the experimental values and the evaluated $R_{f i}$ in fissions/sec-cc of core materials due to each isotope. $R_{f i}$ is given by the expression:

$$
\mathrm{R}_{f i}=\overline{\mathrm{R}}_{f i} \cdot \frac{\text { Monitor count at power level of interest }}{\text { Monitor count at power level of measurement }} \times \frac{\mathrm{N}_{i} \cdot \mathrm{A}_{i}}{\mathrm{~N}_{0}}
$$

The total fission rate, $R_{f}$, is given by the expression,

$$
R_{f}=\sum_{i} R_{f i} \cdot
$$

where

$$
\begin{aligned}
& \mathrm{N}_{i} \text { = atom concentration of isotope } i \text { at core center } \\
& \mathrm{A}_{i} \text { - atomic weight of isotope } i \\
& \mathrm{~N}_{0}=\text { Avogadro's number. }
\end{aligned}
$$

The corresponding $R_{f i}$ for ${ }^{241} \mathrm{Pu}$ and ${ }^{242} \mathrm{Pu}$ were obtained from calculated fluxes using an appropriate normalization factor determined from the measured and calculated values for ${ }^{235} \mathrm{U}$. The calculated values are also shown in Table LI. of $\pm 2 \%$.

The total $\mathrm{R}_{\mathrm{f}}$ was $5.175 \times 10^{6}$ fission/cc sec with an estimated uncertainty

\section{The Worth of ${ }^{252} \mathrm{Cf}$ sample [o(Cf)]}

The apparent worth of the ${ }^{252}$ Cf sample was determined by the sampleoscillation technique at different power levels. The worth at a given power level $i$ is given by

$$
\mathrm{W}_{i}=\mathrm{A}+\mathrm{B} / \mathrm{P}_{\mathrm{i}}
$$

where the first term is the worth of the sample holder, etc, and the second term is the acutal worth of the ${ }^{252} \mathrm{Cf}$ sample. The count rate on a $\mathrm{BF}_{3}$ detector, $\mathrm{SP}-11$, was used as a power level indicator. The measured worths, $W_{i}$, were fitted to a linear function in $1 / P_{i}$ (see $F i g .40$ ) and from the fit a value of $B$ was determined. The worth of the ${ }^{252} \mathrm{Cf}$ sample at the required power level $\mathrm{P}_{\mathrm{j}}$ was given by the expression:

$$
\rho^{\prime}(\mathrm{Cf})=\mathrm{B} / \mathrm{P}_{j}
$$

Table LIII lists the value of $p^{\prime}(\mathrm{Cf})$. 


\section{Results and Comparison with Calculations}

The perturbation denominator was calculated using the same $\mathrm{R}-\mathrm{Z}$ model used to obtain the fluxes for calculating the ${ }^{241} \mathrm{Pu}$ and $242 \mathrm{Pu}$ fission rates. The calculated value was normalized to $\mathrm{S}_{0}$ - So as was the experimental value and the two were directly comparable when the $I h / \Delta k / k$ conversion factor was taken into account. Table LIII presents numerical values for all the parameters used in the determination of the perturbation denominator (see Eq. V.8). The normalized experimental perturbation denominator had an uncertainty of about $3 \%$. The $C / E$ value for the perturbation denominator was 0.75 .

It is of interest to note that the $\mathrm{C} / \mathrm{E}$ value is in close agreement with the $\mathrm{C} / \mathrm{E}$ value reported earlier ${ }^{4}$ for $\mathrm{ZPR}-6$ Assembly 7 using fission flow counters for the determination of $R_{f}$. A subsequent measurement ${ }^{37}$ in that assembly using radiochemical methods gave a different $R_{f}$ and better agreement between calculations and experiment $(\mathrm{C} / \mathrm{E}=0.85)$. Since the same ${ }^{252} \mathrm{Cf}$ source was used in both the measurements, the increased discrepancy between the calculated and measured perturbation denominator can be attributed wholly to the $\mathrm{R}_{\mathrm{f}}$ measurement.

\section{H. Discussion of Reactivity Worth Results}

In the course of the present experiments, a very large number of sma11 sample reactivity worths were determined. The measured composite sample and isotopic worths reported herein have not been corrected for sample size effects. For several materials, the worths were measured using samples of varying sizes. These measurements can be extrapolated to estimate the "zero-size" sample worth. For the heavy metal samples these effects are small ( \pm 1 to $2 \%$ ), however, for the heavy absorbers and light scattering materials these effects can be large $( \pm 50 \%)$. The measured central worths for $\mathrm{H}_{2} \mathrm{O}, \mathrm{CH}_{2}$ and $\mathrm{CH}_{2}$ foam samples are plotted in Fig. 41 as a function of sample mass. These measured values indicate the sample size correction factors range up to 1.5 for the larger water and polyethylene samples. Inclusion of these effects should greatly improve the C/E ratios for these materials. A complete evaluation of sample size effects would include a comparison of calculated and measured worth shapes and an extrapolation of the calculated shapes to "zero" sample size. However, since a complete set of calculated sample size correction factors for all samples were not available, these effects have not been included in the present results. From the available data it appears that the correction factors would be in general agreement with results of earlier experiments. 38,39 for ${ }^{10} \mathrm{~B}$ and the heavy isotopes. The reactivity worth of the smallest available sample was used in the determination of isotopic worths. Since the reactivity signal of the smallest sample was usually small, there was a large uncertainty associated with it, which was propagated through to the evaluated isotopic worths. In all cases where multiple sample worths were measured, the uncertainty in the data is lower than the isotopic worths indicate. All the basic data has been presented in this chapter so that the larger sample worths could be used in the future when usable sample size correction factors become available.

\section{Central Reactivity Worths}

The results of isotopic worths evaluated from the experimental data (Table XIIX) provide a clearer insight into the behavior of the real and adjoint spectra (a check of isotopic cross sections) than the corresponding 
composite sample worth results (Table XLVII). The C/E values for all the heavy isotopes are slightly greater in general, than the corresponding values for earlier LMFBR assemblies. The light scattering material worths are poorly predicted as usual. The polyethylene worth was calculated to be negative whereas the measured worth was positive.

The worth of light scatterers in general and hydrogeneous materials in particular are extremely sensitive to the calculations of the real and adjoint energy spectrum. The worth of polyethylene has been measured to be positive in all assemblies - for both LMFBR and GCFR mockups. In sodium-filled LMFBR assemblies, a positive worth of polyethylene has been calculated (e.g., in ZPPR-2 and ZEBRA assemblies). However, in sodium-voided LMFBR and GCFR assemblies, the calculations have given negative results, mis-predicting the sign of the measured worths. A review of the analytical procedures is being undertaken to pinpoint the reasons for the discrepancy. Both errors in the cross-sections used to compute the real and adjoint spectra and approximations in the processing codes are being considered.

Recent work has focused on the use of bilinear weighted cross-sections and fine group spectrum calculations in the computations of the central worth of scatters. 40 Preliminary results show very large differences compared to usual broad group calculations using flux-weighted cross-sections. Further work on this is in progress.

The high values of the $\mathrm{C} / \mathrm{E}$ for the reactivity worths of most of the materials are consistent with the low $\mathrm{C} / \mathrm{E}$ for the perturbation denominator. In Table LIV the normal $\mathrm{C} / \mathrm{E}$ values have been shown for the various isotopes along with "adjusted" values of the C/E. The first adjustment was made by multiplying all the normal $\mathrm{C} / \mathrm{E}$ values by the $\mathrm{C} / \mathrm{E}$ for the perturbation denominator. The adjusted values so obtained give an indication of the $\mathrm{C} / \mathrm{E}$ for the perturbation numerator which is of greater interest. A second adjustment method used was to divide the normal $\mathrm{C} / \mathrm{E}$ values by the $\mathrm{C} / \mathrm{E}$ value of ${ }^{239} \mathrm{Pu}$. If it is assumed that the discrepancy between experiment and calculation for the ${ }^{2}{ }^{39} \mathrm{Pu}$ worth is caused entirely by the perturbation denominator evaluation, the two adjustments should be equivalent. From Table LIV it appears that the two adjustments differ by about $5 \%$.

The most significant deviation from unity in the adjusted C/E values occurs for the ${ }^{10} \mathrm{~B}$ central worth. To compare the magnitude of this deviation, with results for a corresponding LMFBR assembly, the reactivity worth measurements made in ZPR-6 Assembly $7(4,38,41)$ were analyzed. The adjusted values of the central worths of several isotopes are shown in Table LV. The adjusted $\mathrm{C} / \mathrm{E}$ value for ${ }^{10} \mathrm{~B}$ is 0.93 for the LMFBR assembly as constrasted to 0.88 for the GCFR assembly. While this is not a large difference, it appears that the low energy part of the GCFR spectrum is underpredicted by calculation. Similar effects have been noted in sodium voided LMFBRs (e.g., low prediction of the ${ }^{238} \mathrm{U}$ Doppler effect in these assemblies). It appears that the underprediction of the low energy part of the spectrum is common to hard spectrum system.

Table LV also shows the ratios of the experimental central worths of ${ }^{239} \mathrm{Pu}, 235 \mathrm{U}, 238 \mathrm{U}$ and Fe measured in the two assemblies, along with norm ized ratios. The normalized ratios were obtained by dividing all the unnormar ized ratios by the ratio for ${ }^{239} \mathrm{Pu}$. The normalization of the ratios essentially eliminates the effects of the difference between the perturbation denominators 
of the two assemblies, and directly compares the local effects caused by the perturbation samples.

The results of the table clearly indicate the effect of the considerably harder spectrum in the GCFR assembly relative to the LMFBR assembly (greatly decreased ${ }^{10} \mathrm{~B}$ worth and ${ }^{238} \mathrm{U}$ worth in the GCFR assembly).

\section{Sma11 Sample Radial Worth Traverses}

The results of the radial worth traverses (see Table LI and Figures 37-40) show good agreement between the normalized experimental worths and the shape of the normalized calculated curves for the ${ }^{239} \mathrm{Pu}$ and ${ }^{238} \mathrm{U}$ samples. The comparison is not as good for the $304 \mathrm{SST}$ and quite poor for the hydrogen bearing steam simulation material, polyethylene foam. For the polyethylene sample, the mis-prediction of the sign of the central worth makes a comparison of the worth profile meaningless. However, the experimental profile does reproduce the calculated trends at the core-blanket boundary and in the radial blanket. Sample self shielding factors have not been included in any of the calculated results. Though self shielding corrections are usually small in the center of the core for most materials, they are usually of greater significance away from the center for all materials, particularly for scattering materials like stainless steel and polyethylene foam. Similar results for stainless steel were reported earlier for $2 P R-6$ assembly 7. (39)

\section{Conclusions}

The following general conclusions are drawn from the large mass of data accumulated during the reactivity worth measurements.

1. The normalized central worths of isotopes of interest are generally predicted with the same degree of certainty in the GCFR Phase I assembly as in earlier LMFBR assemblies.

2. A comparison of the normalized central worths show clear evidence of the harder GCFR spectrum relative to the LMFBR spectrum (e.g., 30\% reduction in ${ }^{10} \mathrm{~B}$ worth).

3. The prediction of the worth of light scatters is bad in general and very poor for hydrogeneous material. The problem with light scatters has been seen in the past and is generally attributed to a miscalculation of the shape of the adjoint spectrum. However, the misprediction of the sign of the $\mathrm{CH}_{2}$ worth points to more fundamental problems - the inadequacy of First Order Perturbation theory methods, even for a small hydrogeneous sample.

4. The low calculated prediction of the $10^{0} \mathrm{~B}$ worth relative to earlier experience with LMFBRs is probably indicative of an underprediction of the low energy spectrum. Because of the lack of other suitable experimental results, there is no corroborating evidence for this conclusion, at this time. 


\section{VALIDATION OF KINETICS PARAMETERS}

\section{A. Introduction}

The delayed-neutron relative yields, $a_{i}$, and decay constants, $\lambda_{i}$, were measured for the ZPR-GCFR Phase I core by the rod-drop die-away flux profile analysis technique. The primary interest in these parameters lies in their use in the interpretation of rod drop inverse kinetics measurements of subcriticality and in the calculation of reactivity conversion factors. Errors in the $a_{i}, \lambda_{i}$ sets are transmitted directly to the results of subcriticality measurements.

A measurement of the effective delayed neutron fraction was made using the detector variance method. Earlier work ${ }^{42}$ with beta-effective determination using the pile noise fluctuation measurements has shown that the effects of reactor drift can be kept to negligible levels by the use of a joint-localvariance definition of fluctuations. The effective delayed neutron fraction is defined in terms of the total fission rate in the assembly and a spatially dependent Diven factor $D$, by the expression

$$
D / \beta^{2} F=2 / 3(1+\$)^{2}\left(\tau \sigma^{2}\right)
$$

where $\tau$ is the flux sampling interval

$\sigma^{2}$ is the joint-local-variance.

The value of $\tau$ was kept short ( 0.2 secs) to avoid effects of delayed neutrons on the term $\tau \sigma^{2}$. The major uncertainty in the beta measurement is in the determination of $F$, the total fission rate for all species in the core and blanket at the subcriticality level of the measurement. In addition to the uncertainties in the basic determination of the fission rates, errors in $F$ occur due to inaccuracies of monitor conversions from the irradiation power level. The Diven factor is calculated from available codes and has associated with it the usual uncertainties in spectrum calculations.

B. Experimental Procedure and Results

1. Measurement of Relative Yields and Decay Constants

The procedure and analysis used for the present measurements was essentially similar to that used in earlier studies described in Ref. 43. With the reactor close to critical and steady, various combinations of moveable control rods were scrammed, and the flux profile was recorded. The final subcriticality, ranging from 6 to 7 dollars, was sufficiently large so that the shape of the neutron flux decay profile was not greatly different from $\sum a_{i} e^{-\lambda}{ }_{i}^{t}$. A Variable Metric Minimization (VMM) 44 method was used to infer an equivalent six-group set of amplitudes. The $a_{i}, \lambda_{i}$ values so derived are only weakly dependent upon subcriticality level when the subcriticality value is larger than about 5 dollars.

Table LVI summarizes the reuslts of the five measurements that were made. A comparison of the mean delayed neutron lifetime, 


$$
l_{0}=\sum_{i}{ }_{i} / \lambda_{i}
$$

between the various sets of data is important since this term is almost the inhour-to-dollar conversion factor (apart from the constant $3600 \mathrm{sec} / \mathrm{hr}$ ),

$$
\Sigma \frac{a_{i}}{1+3600 \lambda_{i}}
$$

For the experiments reported here, the values for $\ell$ varied from 10.83 to $11.35 \mathrm{sec}$. This scatter is consistent with experimental uncertainty. The drop point can be defined to no better than about $\pm D T / 2$ where $D T$ is the basic data sampling interval ( 0.2 secs). The finite scram time of the ZPR rods ( 0.2 secs) and the overall uncertainty in definition of scram time leads to an uncertainty in $\ell_{0}$ of about $\pm 3 \%$, which is essentially the same as the observed scatter in $\ell_{0}$ from the various drops.

A single "best" set of $a_{i}, \lambda_{i}$ as derived from these measurements was formed by collapsing the individual sets derived from each of the five drops together in a manner identical to the method used for collapsing sets of delayed neutron data for different fissionable isotopes (Chapter I). The collapsing procedure preserves the mean value for $\sum a_{i} / \lambda_{i}$. The result is shown in Table LVII together with the calculated $a_{i}, \lambda_{i}$ from basic ENDF/B Version 4 and Keepin data for this GCFR core.

\section{Measurement of Beta Effective}

The experiment consisted of a determination of $\tau \sigma^{2}$ (Eq. 1) at known subcriticality. The total fission rate $F$ was determined from available experimental and calculational information and the spatial Diven factor $D$ was calculated, allowing one to solve for the unknown $\beta^{2}$ froin Eq. VI.1. Since $\beta^{2}$ occurs in an expression involving $D, \tau \sigma^{2}$ and $F$ linearly, the uncertainty in $\beta$ is only one-half of that from accumulated uncertainties in these other data.

In the course of measurement, 47 consecutive 2100-point arrays of flux data were recorded from two independent $\mathrm{BF}_{3}$ detector units (designated as SP-10 and SP-11) with a sampling time of 0.1 sec per point. Rod drops were made at the beginning and at the end of the 25 hour run. Subcriticality determined from these drops was found to be 0.8 dollars $\pm 2 \%$. The $2 \%$ represents agreement between the rod drops at the beginning and at the end of the run and is consistent with purely statistical errors in rod drop measurements of subcriticality at the 0.8 dollar level. From pairs of records, mean-square joint averages as defined in Ref. 42 were extracted.

Table LVIII shows the variation of $\tau \sigma^{2}$ with $\tau$ as multiples of the basic sampling interval of $0.1 \mathrm{sec}$. A small correction in $\tau \sigma^{2}$ was made for delayed neutron dependence. An average of the $\tau \sigma^{2}$ values for $\tau=0.4,0.8$ and $1.6 \mathrm{sec}\left(\tau \sigma^{2}=0.207 \times 10^{-5} \pm 2 \%\right)$ was used in Eq. VI.1. The corresnding value of $D / \beta^{2} \mathrm{~F}$ from $\mathrm{Eq}$. VI. I was

$$
D / B^{2} \mathrm{~F}=4.47 \times 10^{-6} \pm 2.7 \% \text {, }
$$


where the uncertainty was obtained by propagating the statistical uncertainties in $\$$ and $\tau \sigma^{2}$.

The spatial Diven factor, D, is defined ${ }^{4}$ as

$$
D=\frac{\int F(\vec{r}) d \vec{r} \int \nu(\nu-1) F(\vec{r}) I^{2}(\vec{r}) d \vec{R}}{\left.\iint V F(\vec{r}) I(\vec{r}) d \vec{r}\right]^{2}}
$$

where

$$
\begin{aligned}
& \nu=\text { neutron emission per fission } \\
& F(\vec{r})=\int \sum_{f}(\vec{r}, E) \phi(\vec{r}, E) d E \\
& I(\vec{r})=\int X(\vec{r}, E) \phi^{+}(\vec{r}, E) d E \\
& X(\vec{r}, E)=\text { energy distribution of neutrons from fission and where } \phi \text { and } \\
& \phi+\text { are the real and adjoint fluxes, respectively. }
\end{aligned}
$$

For a11 practical purposes, the dispersion in neutron emission $v(\nu-1) / \nu^{2}$ has the constant value $0.805 \pm 21.5 \%$ and $c$ an be extracted from within the integral operations in Eq. (VI.2), which then becomes

$$
\frac{v(\nu-1)}{\nu^{2}} \cdot \frac{\int F(\vec{r}) d \vec{r} \int F(\vec{r}) I^{2}(\vec{r}) d \vec{r}}{\left.\iint F(\vec{r}) I(\vec{r}) d \vec{r}\right]^{2}}
$$

Central 235 absolute fission rates from a pulse detector with known $235 \mathrm{U}$ mass were measured and related to ion chamber current levels. Twodimensional diffusion-theory calculations were made and used to construct the total core fission rate relative to the central 235 fission rate per unit mass of $235 \mathrm{U}$. From these data it was inferred that the total (all species included) core fission rate at the variance-measurement flux level was

$$
F=1.60 \times 10^{10} \pm 4 \% \text { fissions } / \mathrm{sec}
$$

The $\pm 4 \%$ uncertainty is the same as was estimated earlier for fission rate determination on earlier assemblies. From Eq. VI.1

$$
\beta^{2} / D=1.40 \times 10^{-5} \pm 4.8 \%
$$

The point-model value of $D$ is 20.80 and the corresponding value of Beff is $0.00335 \pm 2.4 \%$. The $E N D F / B$ based value obtained from an $R-Z$ model calcu lation neglected the reflection effects of the reactor bed and knees but th effect of that on Beff is expected to be small. Recently, the SYN3D code has been used to compute the core total fission rate and Diven factor using a 
full core three-dimensional mode1.46 The value of $F$ so obtained was $1.494 \times 10^{10}$ fissions/sec and $D$ was evaluated to be 0.929 . The resulting value of $\beta$ eff is $0.00360 \pm 2.4 \%$.

\section{Discussion}

The $a_{i}, \lambda_{i}$ results reported in this chapter are consistent with either Keepin or ENDF/B data. The $3.5 \%$ discrepancy between the present results and ENDF/B-4 calculated value and the $1.7 \%$ discrepancy with the Keepin data are probably associated with the uncertainty in drop point as a consequence of the $0.2 \mathrm{sec}$ averaging interval. Choice of a smaller $(0.1 \mathrm{sec})$ sampling interval for future experiments might permit a selection between the two data sets.

The $\mathrm{C} / \mathrm{E}$ value for the Beff determination using the best calculated results is 0.90 . While one might be tempted to relate this underprediction of Beff to the central worth discrepancy (the results are consistent) it has to be recognized that the uncertainties in $F$ and $D$ are larger than the purely statistical uncertainties reported here. In view of those uncertainties, one can only conclude that the present experiments are in general agreement with $\mathrm{ENDF} / \mathrm{B}-4$ values.

VII. REACTION RATE MAPPINGS AND UNIT CELL MEASUREMENTS

\section{A. Introduction}

Foil irraditaions were performed in the GCFR Phase I Assembly in order to determine the spatial distribution of the ${ }^{235} \mathrm{U}, 2{ }^{38} \mathrm{U}$ and $\mathrm{Pu}$ fission rates and the ${ }^{238} \mathrm{U}$ capture rate. In addition, several detailed unit cell measurements were performed at selected locations in the core and blanket to study the local reaction rate distributions within the single drawer unit cells. The reaction rate distributions were measured in three radial directions in one octant of the assembly in order to assess the effects of neutron streaming paths in the assembly (Fig. 42). Axial reaction rates were measured in both directions from the midplane. A small pin zone was loaded in the center of the axial blanket region in one half of the reactor and axial reaction rate traverses were measured in the pin zone for comparison with corresponding measurements in the plate environment in the other half of the reactor.

To provide an experimental evaluation of neutron streaming effects in the Phase I core, an additional experiment was performed in which a checkerboard loading pattern was formed in one quadrant of the stationary half of the reactor. Alternate drawers within the checkerboard quadrant were loaded with the plates positioned horizontally. In the normal drawer loading with all plates positioned vertically, direct streaming channels exist in the $y$ and $z$ direction (see Fig. 43). In the checkerbaord loading, with alternate drawers having a horizontal plate structure, direct streaming channels exist only in the $\mathrm{z}$ direction. Radial reaction rate traverses were made in the same three directions in the checkerboard octant as the normal octant to determine the change aused by the checkerboard loading. In addition, a measurement was made of he change in eigenvalue of the assembly caused by the checkerboard loading. No axial reaction rates were measured in the checkerboard case. Figure 44 shows the unit cell plate loadings in the drawers and Fig. 45 shows the 
checkerboard matrix loading pattern and the directions of the radial reaction rate traverses.

Table LIX describes the foils used in the experiments. In each case two separate irradiations were used to provide the desired reaction rate mappings and unit cell measurements. One irradiation was for the plutonium radial and axial traverses and the unit cell measurements, and the second was for the enriched and depleted uranium traverses. No unit ce11 measurements were per formed for the checkerboard octant.

B. Experimental Procedure

\section{Unit Cel1 Measurements in Normal Core}

Several two-inch cubical regions of drawers in both the core and blanket of the assembly were selected for detailed unit-cell analysis. Table LX lists the locations of the unit cell positions.

Foils were held in $0.001-i n$. thick aluminum folders as shown in Fig. 46. Folders $46(\mathrm{~b})$ and $46(\mathrm{c})$ were used in the unit cells. In the core locations, foils were positioned as shown in Fig. 47. Note that the normal 0.25-in. thick fuel plate has been replaced with two 0.125-in. thick fuel plates. The fuel in these two thin plates is equivalent to that in the normal plate. Figure 48 shows the foil positions for the unit cells in the radial blanket and Fig. 49 for the axial blanket. Wherever possible, the uranium oxide plates were replaced by $0.125-i n$. thick plates for a more detailed analysis.

The fuel plates are clad in 0.015-in. thick stainless stee1. Thus the thin fuel plates introduce $0.030 \mathrm{in}$. of extra stainless steel into the unit cell. In an attempt to determine the effect of the stainless steel clad on the reaction rates measured between the fuel plates an additional measurement was performed. A pair of thin fuel plates was put into drawer S22/24 (replacing the 0.25-in. thick plate). A foil packet was placed between 0.010-in. thick pieces of stainless steel and then positioned between these plates, with normal foil packets on either side of the pair of fuel plates. These extra 0.010-in. shims bring the total stainless steel between the thin fuel plates to 0.050 in. Thus, with two different thicknesses of clad at the foil location an extrapolation to "zero" clad thickness is possible and the true reaction rate at the center of a $0.25-i n$. fuel plate may be determined.

Also in drawer $\mathrm{S} 22 / 24$ the $0.25-i n$. uranium oxide plate was replaced by a pair of 1-in. high, 0.25-in. thick plates. A slot was machined in the lower plate to hold a small rectangular integrating foil, 0.05-in. thick, at the vertical center of the drawer. The ratio of the reaction rates in the foils on either side of the plate to the rectangular foil was compared to the ratio using a circular foil between two $0.125-i n . \mathrm{U}_{3} \mathrm{O}_{8}$ plates.

2. Radia1 Traverses in Normal and Checkerboard Octant

Radial distributions of the reaction rates were measured in thre directions from the core center. Figures 42 and 45 show the locations of the drawers used for the traverses. The $0^{\circ}$ traverse (row 23) is perpendicular to the axial and vertical streaming paths in the assembly. The $90^{\circ}$ traverse 
(column 23) is parallel to the vertical paths, and the $45^{\circ}$ traverse (diagonal drawers) is intermediate. Thus, any effect that the platelet streaming paths may have on reaction rate distributions can be evaluated by comparing traverses in the different directions.

Foils were placed in the front portion of the stationary-half drawers for the traverses. Each foil was placed at the mid-height of its drawer with the plutonium foils positioned 1.0-in. from the midplane, the enriched uranium foils at 1.625-in. and the depleted uranium foils at $0.357-i n$. In the core region the foils were placed against the left side of the fuel plate in each drawer. In the radial blanket drawers, the foils were placed between the right-hand pair of uranium oxide plates (see Fig. 44). The plutonium foils were held inside folded lengths of 1-mil thick aluminum as shown in Fig. 46(a). A strip of stianless steel, 15-mil thick, 0.125-in. wide was used on each side of the folder to prevent compression of the clad foil. The depleted and enriched foils were held in the folders shown in Fig. 46(b). In the checkerboard octant, all foils were loaded into drawers having the vertical plate structure. Monitor foils were positioned in matrix 1ocations S16/12, S30/12 and $M 22 / 22$ during the irradiations in both configurations to provide a measure of any gross flux tilts caused by the checkerboard loading.

\section{Axial Traverses in Normal Core}

Axial distributions of the plutonium and uranium fission rates and the $238_{U}$ capture rate were measured in the stationary half drawer $23 / 23$ at the radial center of the core. The traverses covered 24-in. of core region and the 12-in. axial blanket. The plutonium foils were placed in a 1 -mil thick aluminum folder as shown in Fig. 50(a). Foils were alternately positioned with 15-mil thick stainless steel spacers as shown. The depleted and enriched uranium foils weere positioned in a folder as shown in Fig. 50(b). In both irradiations the axial traverse foil holder was positioned against the left side of the fuel plates in drawer S23/23. The 0.125-in. and 0.250-in. thick uranium oxide plates in the axial blanket were interchanged to provide a continuous path for the folder.

The axial distributions of the uranium fission rates and the ${ }^{238} \mathrm{U}$ capture rate were also measured in the moveable half of the reactor. Within the axial blanket of the moveable half, a 49-drawer pin zone was formed as shown in Fig. 51. The back 12-in. (i.e., the axial blanket) of these drawers were loaded with a square array of 16 pins as shown in Fig. 52.

Through the core region of the moveable-half drawer 23/23, a foil holder similar to that in Fig. 50(b) was placed in a position symmetric with the stationary half folder (i.e., against the right side of the fuel plates). In the pin zone, one drawer was selected to hold enriched uranium foils (M23/24) and one to hold depleted uranium foils (M23/22). Several foils were placed above the pins of these drawers as shown in Fig. 52.

Each pin is loaded with depleted uranium oxide pellets approximately 0.625-in. long. Within three pins of each of the two drawers, as marked in g. 52,0.346-in. diameter foils were placed between the pellets. Their istribution was approximately that shown in Fig. 53. Monitor foils were positioned in locations S/M 22-24 in. from the axial midplane to assess any gross flux tilts caused by the loading of the pin zone in the axial blanket. 
C. Data Reduction

\section{Fission Rates}

For the fission rate analysis, the gamma activity of the irradiated foils was counted with $\mathrm{NaI}$ (T1) scintillator detectors in an automatic foil counting system. ${ }^{47}$ Fission rates were determined by couting fission-product gamma rays with energies greater than $550 \mathrm{keV}$. The counting data were then processed using the computer codes NURF and COMBO. ${ }^{48}$ With these codes, the data are corrected for decay rates, counting system dead-time and background radiation. From the normalized data, the relative reaction rates per unit mass of foil material were calculated for the enriched uranium, depleted uranium and plutonium foils. The fission rates in the individual isotopes of $\mathrm{Pu}$ and $U$ were determined from the foil data using the method outlined in Appendix $B$. Table LIX gives the isotopic compositions of the $\mathrm{Pu}$ isotopes in the $\mathrm{Pu}$ foils.

\section{Capture Rates}

After the fission-product counting of the depleted uranium foils, the same foils were counted for the relative capture rates. Differential counting was used, the window being set on the composite gamma-ray/X-ray peak at $106 \mathrm{keV}$ in the decay spectrum of $2{ }^{39} \mathrm{~Np}$. Coincidences between two NAI (Tl) crystals were recorded and used to determine the relative capture rate for each foil. 50

\section{Unit Ce 11 and Stainless Steel Corrections}

Since the foils were located between plates within the unit-cell, the reaction rates measured from the foil irradiations represented local ince11 values and not unit-cell averaged reaction rates. The calculated reaction rates were unit-cell averaged values, and for comparison, the experimental in-plate reaction rate values were converted to unit-cell values using experimentally determined correction factors. These factors were obtained from the foil data of the detailed unit-cell measurements as the ratio of the cellaveraged reaction rate to the rate at the traverse foil position. Appropriate factors were evaluated for the core and blanket regions. For the case of plutonium foils within the blankets no unit cell correction factors were applied because there was no $\mathrm{Pu}$ in the blankets.

Figure 54 shows the distribution of the depleted uranium fission and $238 \mathrm{U}$ capture rates in a core-region unit cell. The reaction rates have been normalized to absolute fission counter results as discussed below. Figure 55 shows the depleted uranium fission and ${ }^{2{ }^{38}} \mathrm{U}$ capture rate distributions in a radial blanket unit cell and Fig. 56 in an axial blanket unit cell. The data were corrected for radial and axial distance from core center to eliminate the effects of gross flux differences at the foil positions. A zero-order Bessel function $\mathrm{fit}$ to the $0^{\circ}$ radial traverse data for each reaction was used for radial corrections and a cosine fit to the axial traverse data was used for axial corrections. In addition, the effect of the stainless steel cladding on the fuel plate was accounted for as discussed below.

A smooth curve drawn between the data points across the plates was numerically integrated to give the reaction rates in the plates. These plate reaction rates, weighted by the appropriate atom densities, were averaged to give the unit cell reaction rate. 
Table LXI lists the ratios of the unit cell-averaged reaction rate to the traverse foil reaction rate for the six unit cell positions. The unit-cell correction factors for the traverse data were calculated by averaging the unit cell positions which cover a particular traverse. For example, the average of the results from S22/22 at 0-22 in. and 22-24 in. was used for correcting axial traverse data in the core region. Results from S22/22 at 24-26 in. and 34-36 in. were averaged to correct the axial traverse data in the axial blanket region. Table LXII lists the unit cell correction factors used in the different regions and for the two types of traverses.*

In order to check the effect of the fuel plate cladding on the measured reaction rates, three drawers (S22/23, S21/23 and S20/23) had extra stainless steel placed between the foils and the fuel plate. After correcting the data for the different radial positions of the foils (using experimental radial traverse results) a linear least-squares fit was made to the reaction rates vs. stainless steel thickness. Extrapolating to zero thickness, a correction factor of $0.9924 \pm 0.0036$ was calculated for the ${ }^{238} \mathrm{U}$ capture rates. Corrections to the other reaction rates were essentfally unity within limits of experimental accuracy.

\section{Normalization}

A11 foil data were normalized to absolute fission counter results at the center of the core. The relative reaction rates output by the analysis code COMBO were fit to a zero-order Bessel function over the first $40 \mathrm{~cm}$ of the radius in each direction for each reaction rate. For each reaction rate, the average of the fit results extrapolated to the center of the core in the three directions was taken to be the reaction rate at the core center and was equated to the absolute measured rate.

\section{Reactivity Worth Measurements}

Reactivity measurements for the reference state and for the two steps toward establishing the checkerboard pattern were made with the assembly in a subcritical state $\left[10_{B}\right.$ rod numbers $3(M 27 / 30)$ and 8 (S19/30) fully inserted]. The subcriticality of each state was measured using the rod-drop inversekinetics technique. The two banks of $\mathrm{BF}_{3}$ chambers used for the measurements (1abelled SP-10 and SP-11) were located at the core midplane above the moveable and stationary halves respectively. The data were all adjusted to a reference temperature of $25^{\circ} \mathrm{C}$ using a $-2.42 \mathrm{Th} /{ }^{\circ} \mathrm{C}$ reactivity-temperature coefficient. A ${ }^{241} \mathrm{Pu}$ reactivity decay effect of $-0.15 \mathrm{Ih} /$ day was used to account for the time difference between measurements. The uncertainties in the worths were determined by the usual method of error propagation and include a table-closure uncertainty of \pm 2.0 Ih added in quadrature to each statistical measurement uncertainty. The reactivity worth measurement data is shown in Table LXIII.

\section{E. Analysis}

For the analysis of the radial reaction rate traverses the fluxes used were obtained from the appropriate 29-group two dimensional $X-Y$ diffusion alculations with zero flux boundary conditions at the extrapolated dimensions.

* Since normalized relative reaction rates are being considered in all cases, these correction factors are not necessary, once the consistency of the factors throughout the core are recognized. 
The knees and bed of the reactor were included in the model to account for reflection effects at the edges of the matrix. A uniform spacing of two mesh intervals per drawer was used in both directions. The cell averaged isotopic cross sections were used in a11 cases where appropriate and homogeneous (detector) cross-sections were used where the cell average cross sections did not apply (e.g., for Pu isotope reaction rates in the blanket). The reaction rates were calculated at the center of each mesh interval and the value at each foil location was determined by interpolation between two adjacent mesh values. No interpolation was performed across composition boundaries. The axial reaction rate distributions for the normal core were calculated with the $\mathrm{R} \sim \mathrm{Z}$ model $\mathrm{flux}$ and appropriate cross sections. A mesh spacing of $\sim 1.9 \mathrm{~cm}$ was used in the axial direction. The calculated results were normalized by assuming that the calculated value at core center was equal to the central reaction rate measured with absolute fission counters. For the R-Z geometry calculation for the normal core configuration the Benoist coefficients used were $D_{Z}=D_{\|}$, and $D_{R}=1 / 2\left(D_{\|}+D_{L}\right)$.

The calculation was repeated for the checkerboard configuration using identical cross sections and geometry but the diffusion coefficients were

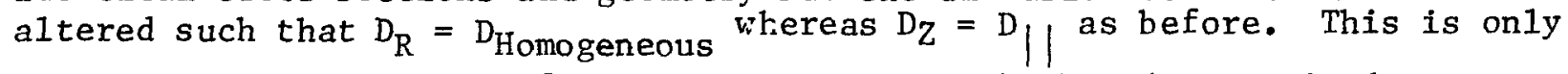
an approximate treatment of streaming in the checkerboard zone; it does represent the fact that the streaming in the axial directions (parallel to the void slots) is unchanged due to checkerboarding while in the radial direction the direct streaming paths have been blocked. The difference in eigenvalue obtained from the two calculations gave the effect of a checkerboard loading of the whole core. Since the checkerboard was loaded only in one octant of the core, the eigenvalue difference was divided by eight to give the calculated change in reactivity for the experiment. The calculated value was 111 Ih, much larger than the experimental value of $23.3 \pm 4.0 \mathrm{Ih}$.

All calculations were repeated in X-Y geometry. For the normal-core assembly the group and region independent buckling was generated by matching the $k_{\text {eff }}$ to that obtained from the $R-Z$ calculation. The mesh size was selected such that there were 2 mesh points per drawer in each direction. For the normal core calculation $D_{y}$ was taken to be $D_{\|}$and $D_{x}$ was set equal to $\mathrm{D}_{\mathcal{L}}$. The model used a quarter core symmetry condition. In the checkerboard case the same model was used with $D_{x}$ and $D_{y}$ now set equal to $\mathrm{D}_{\text {Homogeneous }}$. The same transverse buckling was used in this case as in the normal-core calculation. The eigenvalue difference obtained from the two calculations was divided by eight to give the reactivity change for the checkerboard octant corresponding to the experiment. The calculated value was 111 Ih from this model. The effects of the reactor bed and knees were explicitly taken into account in the $X-Y$ model. Both models (X-Y and $R-Z$ ) were admittedly crude because of the symmetry conditions assumed (which did not reproduce experimental conditions). Under the calculational conditions the calculated predictions based on the Benoist method did not agree with the experimental results.

As an additional check, the fluxes computed by the three-dimensional culations using the SYN3D code were also used to calculate the radial and axial reaction rate profiles. 16 The calculational models have been described in some detail in Chapter II. The bed and knees of the reactor were modeled 
explicitly as were the empty-matrix tubes surrounding the core. The slight uncertainty of the bed thickness (caused by its ribbed construction) was not significant.

\section{F. Experimental Results}

\section{Normal Core Traverses}

Table LXIV - LXVI compare the experimental and calculated values of the ${ }^{235} \mathrm{U}$ and ${ }^{238} \mathrm{U}$ fission rates, and also list the experimental values of the enriched and depleted uranium foil fission rates, at $0^{\circ}, 45^{\circ}$, and $90^{\circ}$, respectively. The numbers in the table are unit cell-averaged values, normalized to absolute fission rates at a reactor power of approximately 100 watts.

Figures 57 - 59 are graphical presentations of the $235 \mathrm{U}$ fission rates, both experimental and calculated (the two-dimensional mode1), at $0^{\circ}$, $45^{\circ}$, and $90^{\circ}$. Figures $60-62$ compare the experimental and calculated ${ }^{238} \mathrm{U}$ fission rates in the three directions.

Tables LXVII - LXIX compare the experimental and calculated values of the plutonium foil fission rate and the ${ }^{238} \mathrm{U}$ capture rate at $0^{\circ}, 45^{\circ}$ and $90^{\circ}$, respectively. Rather than use calculated reaction rate data to correct the plutonium foil data for isotopic impurities $\left(94.98 \%{ }^{239} \mathrm{Pu}\right.$, see Table LI), the calculated values of the isotopic rates were combined to match the foil composition.

Figures 63 - 65 graphically compare the plutonium foil fission rates with the experimental results at $0^{\circ}, 45^{\circ}$ and $90^{\circ}$. Figures $66-68$ are graphs comparing the experimental and calculated ${ }^{238} \mathrm{U}$ capture rates at $0^{\circ}, 45^{\circ}$ and $90^{\circ}$.

Table LXX 1 ists the experimental and calculated results for the axial distribution of the ${ }^{235} \mathrm{U}$ and ${ }^{238} \mathrm{U}$ fission rates in the stationary half of the reactor, and also the experimetnal depleted and enriched uranium foil rates. Table LXXI lists similar results for the plutonium foil fission and ${ }^{238} \mathrm{U}$ capture rates.

Table LXXII 1ists the experimental results for the ${ }^{235} \mathrm{U}$ and ${ }^{238} \mathrm{U}$ fission and ${ }^{238} \mathrm{U}$ capture rate distributions in the moveable half of the reactor, including the pin zone.

In a11 the tabulated results, the statistical uncertainty of the data ranges from approximately $0.3 \%$ for foils near the core center to $2.7 \%$ for foils in the blankets.

Figures 69 - 71 show the comparison between experimental and calculated axial distributions of the ${ }^{235} \mathrm{U}$ and ${ }^{23}{ }^{8} \mathrm{U}$ fission rate and ${ }^{23}{ }^{8} \mathrm{U}$ capture rate, respectively. Since a full core calculation including the pin zone has not been performed, calculated results are present only for the stationary half. Figure 72 compares the experimental and calculated lutonium foil axial distribution. 


\section{Checkerboard Core Traverses}

Tables LXXIII - LXXVIII Iist the calculated and measured reaction rate data for the normal and checkerboard core configuration for each matrix location in the three radial traverses: row $23,0^{\circ}$ traverse, column $23,90^{\circ}$ traverse and the diagonal, $45^{\circ}$ traverse.

Columns 2 and 8 of Tables LXXIII, LXXV and LXXVII show the unit cell corrected fission rate values for the depleted and enriched uranium foils for the normal Phase I Assembly. These values do not include the isotopic corrections and are identical to the values used for the normal assembly. However, the normalized ${ }^{238} \mathrm{U}$ and $235 \mathrm{U}$ fission rates for the normal core configuration shown in the table differ slightly from the corresponding fission rates used for the normal assembly. For fission rates in the normal assembly isotopic rates were calculated directly from enriched and depleted uranium rates normalized to absolute fission counter results. For the checkerboard irradiations, absolute fission counter measurements were not performed and the isotopic corrections were determined using calculated values of the ratio F8/F5. For consistency in the comparisons, the fission rates for the normal core have been evaluated using calculated fission rates.

Figure 73 - 84 show the comparison between the calculated and measured reaction rates in the checkerboard core configuration for the three radial traverses. In the figures, the experimental numbers are plotted at the actual radial foil locations while the calculated values are located at the center of each matrix position.

\section{G. Discussion of Results}

The following subsections present a systematic discussion of the results and conclusions of the foil irradiation measurements.

1. Effect of Knees and Bed of the Reactor

Initial calculations of the traverses used a reactor model in which the meehanite bed and knees were neglected. When the knees and bed were taken into account the $\mathrm{C} / \mathrm{E}$ values near the blanket edge improved dramatically. The reflection effects from the knees and bed were much larger than original1y anticipated. The calculated ${ }^{2{ }^{3}} \mathrm{U}$ fission rate traverse showed no change when the model was altered since the reflection of slower energy neutrons has no impact on the threshold fission rate. It was also observed that a1tering the boundary conditions from zero flux at the physical assembly boundaries to a logarithmic boundary conditions improved the agreement between calculated and experimental distributions, particularly in the blankets.

\section{Comparison of the Calculated and Experimenta1 Radial Traverses}

For the $238 \mathrm{U}$ fission rate the calculated and experimental rates were in good agreement for the entire traverse for all three directions $\left(0^{\circ}, 45^{\circ}\right.$, $\left.90^{\circ}\right)$. The other three reaction rates showed the same trends, viz.

a. good agreement in core region but lower calculated values (as compared to experiment) in the blanket 
b. progressively increased $\mathrm{C} / \mathrm{E}$ discrepancy between the $0^{\circ}, 45^{\circ}$ and $90^{\circ}$ traverse.

A comparison of the experimental traverses in the three directions (see Fig. 85) for ${ }^{239} \mathrm{Pu}$ fission shows that the $0^{\circ}$ traverse falls off most rapidly near and in the radial blanket while the measured reaction rates are larger at the blanket edge at $45^{\circ}$ and largest at $90^{\circ}$. This trend is seen for the ${ }^{238} \mathrm{U}$ capture rate and ${ }^{235} \mathrm{U}$ fission rate also. The effect is expected, since the $90^{\circ}$ direction was parallel to the streaming path. However, only a small effect was observed for the ${ }^{238}$ U fission rate traverse (Fig 86 ). While these small effects may be attributed to streaming effects, it appears that the bulk of the effect seen in the non-threshold reaction arises from neutron reflection effects. A larger reflection occurs from the thicker reactor bed than from the knees, leading to a larger measured reaction rate at the blanket edge in the $90^{\circ}$ direction than in the $0^{\circ}$ direction. This tends to mask the effects of neutron streaming.

The results for the irradiations in the checkerboard octant followed the same trends in all cases.

3. Comparison of the Calculated and Experimental Axial Reaction Rate Traverses in the Normal Core

The calculated and experimental axial reaction rate distributions show considerable disagreement for the three non threshold reactions and fair agreement for the $238_{U}$ fission rate. Even though the axial direction is also parallel to a streaming direction, the $\mathrm{C} / \mathrm{E}$ values are poorer in the blanket than in the $90^{\circ}$ radial traverse. The evidence points to substantial neutron reflection occurring from the empty matrix tubes beyond the axial blankets. This reflection was neglected in the analysis.

There is 1ittle discernible difference between the axial distributions in the stationary and movable halves of the reactor. There is a slight increase in the reaction rates in the movable half core, just in front of the pin-zone but the shapes of the distributions in pin-zone axial blanket and plate-loaded axial blanket are nearly identical. Although the streaming effects in the blanket pin-zone are expected to differ from the plate-loaded blanket, the composition difference between the two and the possible reflection effects in the axial direction mask any effect that can be attributed solely to streaming changes.

4. Comparison Between the Reaction Rate Distributions in the Normal and Checkerboard Cases

In order to compare the experimental reaction rates determined from the two separate sets of irradiations, it is necessary to factor out any gross flux tilt effects caused by the checkerboard loading. Monitor foils (depleted and enriched uranium and plutonium) were located in matrix location S16/12 during both sets of irradiations. The ratio of the corresponding reaction rates in the monitor location and a location diagonally away in the checkerpard octant for the two cases provided an indicator of the flux tilt effects aused by the checkerboard. The data, shown in Table LXXIX indicates that there was no measurable flux tilt introduced by the checkerboard loading and that the experimental traverses could be compared directly if they were 
similarly normalized. For all the cases investigated the reaction rate profiles for the checkerboard core were identical (to within experimental error) to the pro files for the normal core as exemplified in Figs. 87 and 88 . Similar results were obtained for the other reaction rates. This conclusion is in contradiction to calculated predictions of a more rapid drop off of the distributions in the checkerboard case than in the normal case (particularly in the $90^{\circ}$ direction). Figures 89 - 92 provide examples of the calculated predictions for the reaction rate.

\section{H. Conclusions}

It is apparent from the experimental results that the reflection of neutrons from the meehanite bed and knees is larger than was anticipated and is more pronounced from the bed than the knees. This explains the increased reaction rate values ( ${ }^{2}{ }^{38} \mathrm{U}$ capture, $235_{\mathrm{U}}$ fission and $\mathrm{Pu}$ fission) at the blanket edge (relative to central values) for the $90^{\circ}$ traverse as compared to the $0^{\circ}$ and $45^{\circ}$ traverses. The ${ }^{23} 8_{\mathrm{U}}$ fission rate profile, however, is identical in all the three traverses. Since ${ }^{238} \mathrm{U}$ fission is a threshold event, it is insensitive to reflection effects (neutrons that are scattered back are of low energy). Thus the indications are that the differences in profiles of the other reaction rates are consequences of enhanced reflection from the bed and not streaming effects (which might have been expected to show a similar trend).

The presence of the bed in only the bottom of the reactor and the difference in thickness between the bed and knees caused the assembly to be azimuthally asymmetric. The basic two dimensional R-Z model was, therefore, inaccurate. Results of the three dimensional diffusion theory analyses gave excellent agreement with experiment, generally better than $5 \%$ even in the radial blanket. 51 The axial traverses were the least accurately calculated with as much as $15 \%$ underpredictions deep in the blanket. The greatest uncertainty resulted from difficulties in modelling the reflection effect from the empty matrix tube. Physically, the axial reflector consisted of $\sim 30 \mathrm{~cm}$ of empty matrix tubes backed up by a steel end plate. This was modeled by $30 \mathrm{~cm}$ of homogenized matrix tube composition and isotopic diffusion coefficients. Since the empty matrix tubes were 2 in. $\times 2$ in. square streaming slots, the use of isotopic diffusion coefficients is open to question. The $30 \mathrm{~cm}$ thickness was selected because it gave the best match with the experimental reaction rate distribution with the choice of the isotopic diffusion coefficients. Clearly, however, the choice of the thickness and the diffusion coefficients were far from unamgibious. The $\mathrm{C} / \mathrm{E}$ discrepancy in the reaction rate traverse appears to be caused primarily by modelling difficulties in two dimensions.

The two-dimensional calculational models grossly overpredicted the change in the eigenvalue of the assembly caused by the checkerboard loading of the octant. The calculational models ( $R-Z$ and $X-Y$ ) both used quarter-core symmetry conditions and thus did not reproduce the experimental conditions closely. No difference was observed in the experimental reaction rate profiles in the two configurations, to within experimental error. The calculations, however, predict a difference in the profiles, particularly for the $90^{\circ}$ (column 23) traverses. The Benoist diffusion coefficient modifier in that direction $\left(D_{y} / D_{\text {Homogeneous }}\right)$ for the normal core is much larger than unity and a 
larger leakage would be expected for the normal core as compared to the checkerboard core. Consequently the reaction rates in this direction should be larger for the normal core than the checkerboard core. The fact that this effect was not seen experimentally appears to be caused more by experiment design than experimental method. The checkerboard was loaded only in one octant of the core and the foils were located within the octant only two inches from the boundary between the checkerboard and normal regions. Apparently the folls did not sense the effect of the checkerboard because of the proximity of the normal core loading. The small difference between the reaction rate profiles in the normal and checkerboard cases might have been measureable if the foils had been located deep inside the checkerboard octant.

In summary, the presence of the reactor bed and knees close to the edge of the large Phase I assembly masked the effects of neutron streaming by introducing large reflection effects. The positions of the irradiation foils was also not ideally suited for the measurement of the small streaming effects. An unambigious validation of the Benoist method of treating neutron streaming was not possible from these reaction rate experiments alone because of the nature of the results. Finally, the checkerboard octant experiment was not analyzable by standard methods.

\section{THE S IMULATED STEAM ENTRY EXPERIMENT}

\section{A. Introduction}

Since the steam pressure in the steam generator in a typical GCFR is higher than the core coolant pressure, there is a possiblity of steam leaks into the GCFR in the event of a failure of a steam generator tube. The effects of such a steam entry have been investigated calculationally by several authors.52-54 The general conclusion of all these analytical studies was that steam entry would result in a negative reactivity effect for a wide range of steam densities and a broad range of reactor conditions. Only one prior experimental study of the effect has been attempted, ${ }^{55}$ but that study confined itself to a measurement of reaction rates and relying on analytical arguments about the reactivity effects. The present experiments provided a clean, homogeneous simulation of the steam within a small central zone and a direct measurement of the reactivity change which could be analyzed by available methods.

The typical steam densities that can be expected under accident conditions for the GCFR Demonstration plant have been discussed by Broido and Rothstein. 52 In summary, a steam density of $0.003 \mathrm{~g} / \mathrm{cm}^{3}$ can be expected under accident conditions before an overpressure scram occurred and the control rods are inserted to shut down the reactor. If all the water in one of the steam generators were dumped in the core, a steam density of $0.006 \mathrm{~g} / \mathrm{cm}^{3}$, would be reached. This forms the maximum steam density that $c$ an be hypothesized realistically. The hypothetical limit for steam density in the core is $0.052 \mathrm{~g} / \mathrm{cm}^{3}$, obtained by assuming a complete replacement of helium by saturated steam at the PCRV relief valve pressure (1375 psia). It should be emphasized

at the inventory of all three steam generators would be insufficient to prode the amount of steam needed for this. 
For the present experiment, polyethylene $\left(\mathrm{CH}_{2}\right)$ foam strips were used to simulate steam. The foam density was $0.035 \mathrm{~g} / \mathrm{cm}^{3}$. The reactivity effects of inserting steam of several densities into a central core zone were investigated. Four different steam densities were simulated by suitable arrangements of perforating foam strips and alternate drawer loadings. The "steam" entry was seen to introduce a positive reactivity insertion in all cases studied. The reactivity changes were analyzed with ENDF/B-IV based data, using both direct eigenvalue difference and exact perturbation theory methods.

\section{B. Experiment Description}

The experimental zone consisted of the central $5 \times 5$ matrix tube bundle. Steam entry was simulated in this zone to an axial half height of 12 inches. Four densities of steam were simulated within the zone by the following methods.

1. Polyethylene foam strips were inserted into all the void cans within the zone. This constituted a nominal $\mathrm{CH}_{2}$ density of $20.035 \mathrm{~g} / \mathrm{cm}^{3}$ in the void channels or $20.0169 \mathrm{~g} / \mathrm{cm}^{3}$ homogeneously in the experimental zone. The equivalent homogeneous steam $\left(\mathrm{H}_{2} \mathrm{O}\right)$ density was $00.0217 \mathrm{~g} / \mathrm{cm}^{3}$. This was referred to as the full density steam loading.

2. The above drawer loading pattern was used in a "checkerboard" fashion, such that every alternate drawer had a normal core loading, resulting in approximately a half density steam loading in the zone. The $\mathrm{CH}_{2}$ density here was about $0.00878 \mathrm{~g} / \mathrm{cm}^{3}$ and the corresponding $\mathrm{H}_{2} \mathrm{O}$ density $20.0113 \mathrm{~g} / \mathrm{cm}^{3}$.

3. A half density steam loading was simulated by loading perforated poly ethylene strips in all the void cans in the zone. The performations were made such that approximately $52 \%$ of the material was removed. The nominal density attained by this method was almost the same as that in Step 2, and the purpose of this simulation was to check the effect of the more heterogeneous steam simulation in Step 2 .

4. A quarter density steam loading was simulated by a checkerboard loading of the perforated polyethylene foam strips. The nominal $\mathrm{CH}_{2}$ density in the zone was $0.00418 \mathrm{~g} / \mathrm{cm}^{3}$ and the equivalent $\mathrm{H}_{2} \mathrm{O}$ density was $0.00537 \mathrm{~g} / \mathrm{cm}^{3}$.

Table LxxX lists the exact weights and densities of the $\mathrm{CH}_{2}$ in the zone and the equivalent $\mathrm{H}_{2} \mathrm{O}$ densities. Figure 93 shows the location of the steam zone in the assembly.

A reference subcritical configuration was established by inserting the operational ${ }^{10} \mathrm{~B}$ control blades No. 2 and No. 11 in the normal GCFR Phase I assembly. The subcriticality was determined by the rod-drop inverse kinetics method. The four steam densities were then individually loaded and the subcriticality for each configuration was measured by the rod-drop method. The reactivity worths of each configuration were determined from these subcriticality data. A set of eight rod drop measurements were performed for each case and the subcriticality was determined to better than $1.5 \%$ in all cases. Table LXXXI presents the rod-drop measurement data for the two $\mathrm{BF}_{3}$ detector bank (SP-10 and SP-11) used for the experiment. The SP-11 data were used for $t$ analysis of the experi ment and the SP-10 data were used as a check. Both detectors are located on top of the reactor matrix at about the axial midplane of the core. 
The kinetics parameters used for the analysis of the data were obtained from ENDF/B-IV based isotopic values (Table XXXV). The reactivity worth of ${ }^{2}{ }^{1} \mathrm{Pu}$ decay was determined specifically for the period of the experiment, and the value used here $(-0.183 \pm 0.003 \mathrm{Ih} /$ day $)$ differs slightly from the number reported earlier $(-0.15 \pm 0.02 \mathrm{Ih} /$ day $)$ which was obtained by fitting the data for the entire Phase I experimental period. All the data were adiusted to a reference temperature of $25^{\circ}$ using the previously measured temperature coefficient of $-2.42 \pm 0.04 \mathrm{Ih} /{ }^{\circ} \mathrm{C}$.

\section{Results of Measurements}

Table LXXXII presents a summary of the results of the measurements. The reactivity worth of each steam simulation was obtained from the measured subcriticalities for each reactor configuration. For a configuration $i$ the reactivity is given by the expressions:

$$
\rho_{i}=B_{i}-S_{i}
$$

where

$$
\begin{aligned}
& \mathrm{B}_{i}=\text { worth of } 10_{\mathrm{B}} \text { control rods inserted in configuration } i, \\
& \mathrm{~S}_{i}=\text { subcriticality of configuration. }
\end{aligned}
$$

The reactivity worth of a change relative to a reference configuration is then given by:

$$
\begin{aligned}
& \rho_{i}-\rho_{\text {ref }}=\left(B_{i}-s_{i}\right)-\left(B_{\text {ref }}-s_{\text {ref }}\right) \\
& \rho_{i}-\rho_{\text {ref }}=\left(s_{\text {ref }}-s_{i}\right)-\left(B_{\text {ref }}-B_{i}\right)
\end{aligned}
$$

The two ${ }^{10} \mathrm{~B}$ control rods inserted to create the subcritical configuration were farthest from the steam zone and experimental results showed that the worths of the operational control rods did not change with the addition of $\mathrm{CH}_{2}$ in the zone. Thus the term ( $B_{\text {ref }}-\mathrm{B}_{i}$ ) was equal to zero to within experimental uncertainty and $\Delta \rho$ was given by $\Delta \rho=\rho_{i}-\rho_{\text {ref }}=\left(S_{\text {ref }}-S_{i}\right)$

The specific worth ( $\mathrm{Ih} / \mathrm{kg}$ ) is also listed in Table LXXXII for comparison with the measured small sample central worth in the assembly. The uncertainties shown on the experimental data include the standard deviation for the repeated rod drop subcriticality measurements, propagated in the usual manner, and the configuration reproducibility uncertainty of $\pm 2.0 \mathrm{Ih}$, added in quadrature.

\section{Analysis}

The initial cross-section base library generated for the analysis of the GCFR Phase I experiments did not include hydrogen. Processing of hydrogen ross-sections was not possible with the earlier versions of $M^{2}-2 / S D X$ codes cause of algorithm errors. With the improved algorithms, hydrogen crosssections were generated by introducing trace amounts $\left(10^{-10}\right.$ atoms/cm-barn) of hydrogen in the core composition mix within an $M C^{2}-2$ (inconsistent $\mathrm{P}_{1}$ ) 
spectrum calculation and thus generating a 226 intermediate group base library which included hydrogen.

The calcualtional $\mathrm{R}-\mathrm{Z}$ model used for the analysis is shown in Fig. 94. In addition to the explicit representation of the steam zone, an adjacent core zone was defined to account for effects introduced in the adjoining core by the presence of $\mathrm{CH}_{2}$ in the experimental zone. For a zone of the size chosen, the reactivity worth calculation could only be done by eigenvalue difference and exact perturbation theory methods. Introduction of the $\mathrm{CH}_{2}$ would be expected to soften the spectrum and hence alter the cross-sections of al1 isotopes within the zone and the adjacent regions. In addition, the Benoist bi-directional diffusion coefficient modifiers would be altered relative to the normal core configuration. A11 these effects were explicitly taken into account for each steam entry simulation step. The calculational steps are summarized below.

\section{Broad Group Cross Section Generation}

The 29-broad group cross-section set used in the analys is of the experiment were generated from the intermediate group 1ibrary using the SDX code. The one dimensional SDX model included the central steam zone, the adjacent core, the normal core and normal blanket. A space-dependent spectrum calculation was performed to collapse the cross-sections. Figure 95 shows the normalized 226-group spectra at the core center for the normal $\left(\mathrm{no}-\mathrm{CH}_{2}\right)$ core and three densities of $\mathrm{CH}_{2}$ in the experimental zone (full, nominal half and quarter densities) identified as steam densities 1,2 , and 3 , respectively. An individual cross-section set was not generated for the Step 2B (nominal half density $\mathrm{CH}_{2}$ using perforated polyethylene foam strips) because the spectral effects in this case were clearly very close to the effects calculated for Step 2. The cross-sections calculated for Step 2 were used for this case also. Figures 96-98 show the differences in the intermediate group spectra with and without steam for the three densities separately to focus on individual differences. In all cases appreciable softening of the spectrum is observed with the addition of $\mathrm{CH}_{2}$.

\section{Neutron Streaming Effects}

As in earlier calculations the Benoist bi-directional diffusion coefficients were used to include the anisotropic effects of neutron streaming. The Benoist coefficients were generated in directions parallel and perpendicular to the steam filled void slots for the steam zone composition and in directions parallel and perpendicular to the void slots in the region adjacent to the steam zone. For the other regions the coefficients generated earlier were used in the calculations. In the R-Z calculations, $D_{z}$ was taken to be $D_{/}$and $D_{r}$ was set equal to $1 / 2\left(D_{\mid} \mid+D_{1}\right)$. Table LXXXIII gives the Benoist coefficients for the core region.

\section{Eigenvalue Calculations}

The R-Z model used for the analysis of the steam zone is shown Fig. 94. The dimensions of the different regions and the mesh spacings us are shown. The radial dimensions were evaluated such that the circular region contained the same area as the matrix locations actually comprising these 
regions. The homogeneous atom concentrations of all isotopes in the assembly are the same as in Table XXII except for the $\mathrm{C}$ and $\mathrm{H}$ atom concentrations which are listed in Table LXXX.

Two-dimensional diffusion calculations in 29-energy groups were performed for the reference and from steam simulaton cases. The results of the eigenvalue difference calculations are shown in Table LXXXIV.

\section{Exact Perturbation Theory Ca1culations}

Since the experimental worth of adding $\mathrm{CH}_{2}$ in the zone was small, two-dimensional 29-group exact perturbation theory calculations were performed for each $\mathrm{CH}_{2}$ addition case to get the most accurate reactivity values. In the exact perturbation theory model, the real flux was taken from the steam-filled zone cases, while the adjoint $f l u x$ was taken from the normal core (no steam) calculation. This was done to avoid introducing errors in the results due to possible errors in the adjoint flux calculation for the "with-steam" calculations. In the present cross section generation codes, the last (thermal) group cross-sections are not correctly computed. This presents no problem in a real flux calculation, since there is no coupling up in energy (no upscatter) and in all fast reactor situations encountered so far, the neutron flux in the last energy group $(0.414 \mathrm{eV}$ to $0.0 \mathrm{eV})$ is negligibly small. However, in the adjoint calculation, because of the transposed matrix, the lowest group is coupled to all other groups by the "downscatter" term. This is seen clearly from the following expression for the group adjoint fluxes.

$$
\phi *=\frac{1}{\left(D_{j} B^{2}+R_{j}\right)}\left(v \Sigma_{j j} \sum_{k} x_{k} \phi_{k}^{*}+\sum \Sigma_{j k} \phi_{k}^{*}\right)
$$

Since the thermal group cross-sections are known to be inaccurate, the adjoint flux in the thermal group is inaccurate. If the coupling terms $\Sigma_{j k}$ (transfer cross-sections) are large, then this inaccuracy in the thermal group adjoint propagates to the other groups.

A sensitivity calculation showed that there is no such propagation for the adjoint calculation for the no-steam case - the transfer cross-sections to the last group are small. However, for the case where hydrogen was in the system in appreciable quantities, the transfer cross-sections to the last group are large and the adjoint flux might be in error. The choice of the real flux from the steam $z$ one case and the adjoint flux from the normal case for the exact perturbation theory calculation results in no errors. The shape of the adjoint spectrum in the center of axial core is shown in Fig. 99. An interesting side-light to this discussion is that the calculation of $\mathrm{CH}_{2}$ foam central worths (Chapter V) was unaffected by the problem because it was done by First Order Perturbation theory methods.

In the calculations the perturbation consisted of introducing the "steam zone" and core adjacent to the steam zone compositions at the appropriate locations. The perturbations in cross-sections for the steam zone and jacent zone are given by the following expressions:

$$
\Delta \Sigma^{S}=\Sigma_{\mathrm{CH}_{2}}+\left(\Sigma_{\text {core }}^{S}-\Sigma_{\text {core }}\right)
$$




$$
\Delta \Sigma^{\mathrm{A}}=\left(\Sigma_{\text {core }}^{\mathrm{A}}-\Sigma_{\text {core }}\right)
$$

whese $\quad \Sigma_{\text {core }}^{S}=\begin{aligned} & \text { macroscopic cross-section of core material in the steam } \\ & \text { filled environment }\end{aligned}$ and

$$
\begin{aligned}
\Sigma_{\text {core }}^{A}= & \text { macroscopic cross-section of core material in the region } \\
& \text { adjacent to steam zone, the spectral effects of the zone } \\
& \text { having been explicitly taken into account. }
\end{aligned}
$$

This formulation gave the most rigorous treatment of the problem.

Figures 100 - 103 show the comparative 29 group real fluxes (at core center) within a11 the individual steam entry conditions relative to the normal core environment. Once again the spectral softening effects of $\mathrm{CH}_{2}$ are clearly seen in al1 cases. The large spectral softening effect is seen to become important just below the energy range of the iron resonance. The results of the $\mathrm{R}-\mathrm{Z}$ exact perturbation theory calculation are shown in Table LXXXIV.

\section{X-Y Exact Perturbation Theory Mode1}

In the $\mathrm{R}-\mathrm{Z}$ calculation an exact representation of the cross-section of the zone was not possible. An X-Y exact perturbation calculation was done for the highest density steam entry case to study the effect of the radial representation of the model.

In the $X-Y$ calculation the zone was represented exactly in the $X-Y$ plane; however, the axial dimension of the zone was not included in the spectrum calculations. A group and region independent buckling was used and the real flux was calculated for the "steam-in" case and the adjoint flux was calculated for the normal core case. The exact perturbation calculation was performed in the manner described for the $\mathrm{R}-\mathrm{Z}$ calculation with the exception that the actual axial height of the zone was not input for the transverse integration. The results of these calculations are shown in Table LXXXIV. The results are in good agreement with the results of the $R-Z$ exact perturbation calculation.

\section{E. Discussion}

Figure 104 shows all the experimental and calculated results in a composite plot which also includes the experimental central worth of a small polyethylene foam sample and the corresponding calculated value. The reactivity worth for theaddition of steam is seen to be positive for all densities investigated (in contradiction to earlier predictions) and the calculations correctly predictedthe sign of the reactivity change. The response of an actual GCFR to steam entrycannot be obtained by direct extrapolation of these results within the central zone of a critical assembly mockup because the response in an operating GCFR will depend on the presence of poisons (fissi products, control rods), core Doppler effect and true heterogeneity effect the core. However, the experiment does provide a good means of testing calculational methods used for the design analysis of such effects. 
Figure 104 shows that the trends observed in the experiments were reproduced by the calculations (in particular, the divergence of the worth from the central worth line with increased steam density because of spectral effects). However, the agreement between the magnitudes of the calculated and experimental worths is poor at the lowest and highest densities of steam investigated. It will be noted that the central worth of the small steam sample determines the slope at the origin of the steam mass vs. reactivity plot. The reactivity worth per unit mass was always mispredicted by calculation, going from the wrong sign for "zero" density (small sample) to too positive for high densities. This error results in the discrepancies seen in the plot.

Table LXXXV shows the results of neutron balance calculations for the dry reference Phase I assembly and the assembly with the steam zone in place. It is seen that the addition of steam causes a decrease in the $k_{\infty}$ of the zone (and the assembly) and a decrease in the leakage faction L. Both these effects result from the softening of the spectrum on steam entry. For the cases studied, the positive reactivity effect of decreased leakage counteracted the negative effect of decreased $k_{\infty}$ resulting in a net positive reactivity change. Tables LXXXVI and LXXXVII lists the detailed reaction rates in the steam filled zone and the dry reference central zone respecitvely for a closer look at the change in $k_{\infty}$.

The components of the steam reactivity worth are listed in Table LXXXVIII. All the components are seen to be small relative to the scattering terms, and the net reactivity is given by the difference between the scattering gain and the scattering loss terms. Since these are of comparable size and opposite sign, the magnitude and sign of the overall reactivity effects for steam is most sensitive to the calculation of these terms (both terms are very sensitive to the calculated shapes of the real and adjoint spectra). As discussed earlier, the calculational methods used were as rigorous as possible within the confines of the $\mathrm{MC}^{2}-2 / \mathrm{SDX}$ cross-section processing codes and two-dimensional diffusion theory methods. The disparity between the experimental and calculated results, particularly the inability of the calculations to predict the sign of the central worth points to the need for refinement of analytical methods to enable an accurate treatment of the problem.

\section{SUMMARY AND CONCLUSIONS}

The GCFR Phase I critical experiments program was started in early March 1975, and was completed in June 1975. A large volume of experimental data was accumulated during the program. These data formed the first set of information ever produced for GCFR systems and provided valuable input for validation of design methods. The general conclusions of the GCFR Phase I program are summarized below.

1. As in earlier fast critical assemblies, the approach-to-critical measurements gave good indications of the critical mass from the early stages of fuel loading especially from centrally located in-core detectors. 
2. The spectrum in the GCFR assembly was appreciably harder than comparable LMFBR assemblies (e.g., ZPR-6 Assembly-7). This was seen from comparisons of actual experimental spectra, from the various spectral indices and from the reactivity worths of several isotopes.

3. The Benoist formulation for the treatment of the anisotropy caused by neutron streaming within the framework of diffusion theory was used in the analysis of experiments in the assembly. The present experimental analyses were the first direct test of the methods. The predicted eigenvalue from standard two-dimensional diffusion calculations was lower than the eigenvalues calculated for earlier LMFBR assemblies. It was shown ${ }^{6}$ that errors of about $2 \%$ can be anticipated in the calculation of the Benoist modifiers by present methods and that could cause the eigenvalue discrepancy of about $1 \%$. The low experimental $\Delta \mathrm{k}$ compared to the calculated $\Delta \mathrm{k}$ for the checkerboard streaming experiment supported this conclusion. More recent calculations using three-dimensional diffusion theory methods revealed a large reflection effect from the empty matrix tubes beyond the axial blankets which was neglected in the two-dimensional analysis. Additionally, the azimuthal asymmetry introduced by the massive bed located at the base of the reactor caused a two-dimensional $\mathrm{R}-\mathrm{Z}$ modeling of it to be inaccurate. Since the $\mathrm{R}-\mathrm{Z}$ calculation was the basic one used for the two-dimensional analysis (the $X-Y$ model derived $i t s$ transverse buckling from a keff match with the R-Z calculation) all the two-dimensional eigenvalue results are in error. The eigenvalue produced by a threedimensional model of the reactor (which included an accurate representation of the bed and knees) was quite typical of earlier LMFBR mockup assembly results.

It is of interest to note that the calculation of central integral parameters is not affected by the above mentioned difficulties in the calculation of the eigenvalue. The $\mathrm{C} / \mathrm{E}$ values for the parameters are comparable to those obtained in earlier LMFBR assemblies. This suggests that the discrepancy in eigenvalue calculation can be attributed to the leakage component.

4. Because of the large size of the GCFR Phase I Assembly and the large leakage component, the effect of neutron-reflections from the reactor knees and bed turned out to be significant - particularly in the measurements of the reaction rate profiles. The reflection effects masked the effects of neutron streaming in the radial reaction rate traverses. In addition the reaction rate traverses in the "checkerboard" octant gave inconclusive results because the foils were located at the boundary between the checkerboard and normal environments.

5. The $\mathrm{C} / \mathrm{E}$ values for the central reactivity worths of most materials followed the usual trends seen in the past. The chronic problems associated with predicting the worths of light scatterers were observed again. For the $\mathrm{CH}_{2}$ samples the sign of the central worth was mispredicted. The worth of light scatterers in general and hydrogeneous materials in particular are extremely sensitive to the real and adjoint energy spectrum. The worth of polyethylene has been measured to be positive in all assemblies. In sodium-filled LMFBR assemblies, a positive worth of polyethylene has been calculated (e.g., in ZPPR- 2 and ZEBRA assemblies). However, in sodium-voided LMFBR and GCFR assemblies the calculations have given negative results. It appears that First Order Perturbation theory methods are inadequate to analyze the worth of even a small hydrogeneous sample. More analysis is needed to resolve this problem. 
6. The experimental reactivity worth of steam entry in the simulated steamentry experiment was positive for all densities of "steam" studied. This was in contradiction to earlier analytical results. The present analysis properly predicted the sign of the reactivity worths, although the $\mathrm{C} / \mathrm{E}$ values were not good for the highest and lowest densities of "steam". The calculations took into account the effect of spectral softening caused by the steam on the cross-sections of materials (in the experimental zone and surrounding core region). A full core simulated steam entry experiment is needed to predict the actual response of a GCFR system to steam entry. Such an experiment will be performed in the GCFR Phase II As sembly.

7. An unambiguous experimental validation of the Benoist method for computing streaming effects was not possible during the Phase I program because the asymetry caused by neutron streaming were masked by the anisotropy caused by reflection. The experimental reaction rate traverses were thus unable to provide definitive information about neutron streaming effects. However, the criticality prediction (which is very sensitive to the leakage component) was consistent with earlier results for an adequate assembly model. The prediction included a sizeable $(\sim 2 \% \Delta \mathrm{k})$ streaming connection and the indications are that the Benoist directional diffusion coefficients as computed by present methods cannot be in error by more than $\pm 5 \%$.

A calculational validation of the Benoist method is presently being undertaken by Wade. $5 \%$ He has compared the non-leakage probabilities calculated by the VIM code (continuous energy Monte Carlo) with the results obtained from the diffusion theory methods including the Benoist prescription for neutron streaming. His preliminary conclusions are that for the same unit cell model, the leakage probability parallel and perpendicular to the void channels are preserved by the diffusion theory methods relative to the Monte Carlo method to within the $\mathrm{VIM}^{58}$ statistics. While it still remains to be proved that the three-dimensional unit cell can be modeled in one-dimension wi thout introducing an appreciable error in leakage probability, it appears to be safe to say that the Benoist method of computing anisotropic streaming effects is accurate enough for most design analysis. 


\section{APPENDIX A \\ CORRECTION FOR DECAY OF CAPTURE PRODUCT IN CENTRAL REACTION RATE MEASUREMENT}

The determination of absolute reaction rates from irradiated foil data requires that the power $h$ istory of the reactor $r$ un be used to determine the number of reactions that have occurred in the foils. After the irradiation, the transitions per unit time that occur in the foils follow the usual one or two step radiative decay equations. The power history determines the initial number of nuclei that decay.

The method chosen for use is an exact formulation, rather than the approximation of one short half-life and one much longer in a two-step decay (which is the case for both ${ }^{232} \mathrm{Th}$ and ${ }^{238} \mathrm{U}$ capture). In the following, subscript 1 denotes parent activities (e.g., ${ }^{23}{ }^{3} \mathrm{U}$ or ${ }^{23}{ }^{3} \mathrm{Th}$ for capture, or any number of fission products) and subscript 2 denotes daughter activities $\left(\mathrm{e} . \mathrm{g} \cdot{ }^{239} \mathrm{~Np}\right.$ or $\left.233^{3 \mathrm{~Pa}}\right)$.

Define $t=0$ as the start of the irradiation and $t=t_{0}$ as the end of the irradiation. Assume that the power history can be divided into $n$ equal, short time intervals of time length $t=t_{0} / n$. The equation describing the formation and decay of a given reaction product is

$$
\frac{d N_{1}}{d t}=\sum \phi(t)-\lambda_{1} N_{1}
$$

where $\sum$ is the macroscopic cross section for either fission or capture, $N_{1}$ is the number of fission or capture products with decay constant $\lambda_{1}$ and $\phi(t)$ is the flux level in the reactor. Integrating from $t=0$ to $t$.

$$
e^{\lambda_{1} t} N_{I}(t)=\int_{0}^{t} \sum \phi(t) e^{\lambda_{1} t} d t .
$$

Approximate the integral by a sum to get

$$
\int_{0}^{t} \sum \phi(t) e^{\lambda_{1} t} d t=\sum_{i=1}^{n} \sum \phi_{i} e^{\lambda_{1} i \Delta t} \Delta t
$$

Solving (2) for $\mathrm{N}_{1}(t)$.

$$
\begin{aligned}
N_{l}\left(t_{j}\right) & =\sum_{i=1}^{j} \sum_{i} \Delta t e^{\lambda_{1} i \Delta t} e^{-\lambda_{1} j \Delta t} \\
& =\sum_{i=1}^{j} \sum \phi_{i} \Delta t e^{-\lambda_{1}(j=i) \Delta t}
\end{aligned}
$$


Thus,

$N_{l}\left(t_{0}\right)=\sum_{i=1}^{n} \sum \phi_{i} \Delta t e^{-\lambda_{1}(n-i) \Delta t}$

The number of daughter products formed during the irradiation is given by $\mathrm{dN}_{2}$

$\overline{\mathrm{dt}}=\lambda_{1} \mathrm{~N}_{1}-\lambda_{2} \mathrm{~N}_{2}$

where $N_{1}(t)$ is given by (3) above. Rearranging (5) and integrating from $t=0$ to $t=t_{0}$.

$$
e^{\lambda_{2} t_{0}} N_{2}\left(t_{0}\right)=\int_{0}^{t_{0}} \lambda_{1} N_{1}(t) e^{\lambda_{2} t} d t
$$

Again approximating the integral by a sum,

$$
e^{\lambda_{2} t_{0}} N_{2}\left(t_{0}\right)=\sum_{i=1}^{n} \lambda_{1}\left(\sum_{j=1}^{i} \sum \phi_{j} \Delta t e^{-\lambda_{1}(i-j) \Delta t} e^{\lambda_{2} i \Delta t} \Delta t\right.
$$

or

$$
N_{2}\left(t_{0}\right)=\sum_{i=1}^{n} \lambda_{1}\left(\sum_{j=1}^{i} \sum \phi_{j} \Delta t e^{-\lambda_{1}(i-j) \Delta t}\right) e^{-\lambda_{2}(n-i) \Delta t} \Delta t
$$

Equation (4) gives the number of capture or fission products left at the end of the irradiation, and equation (6) gives the number of daughter nuclei present at that time.

Now let $t$ ime $t=0$ be the end of the irradiation. For $t>0$ the following equations describe $\mathrm{N}_{1}$ and $\mathrm{N}_{2}$ :

$$
\begin{aligned}
& \frac{\mathrm{dN}_{1}}{\mathrm{dt}}=-\lambda_{1} \mathrm{~N}_{1} \\
& \frac{\mathrm{dN}_{2}}{\mathrm{dt}}=\lambda_{1} \mathrm{~N}_{1}-\lambda_{2} \mathrm{~N}_{2} .
\end{aligned}
$$


Solving,

$$
\begin{aligned}
& N_{1}(t)=N_{1}\left(t_{0}\right) e^{-\lambda_{1} t} \\
& N_{2}(t)=N_{2}\left(t_{0}\right) e^{-\lambda_{2} t}+\frac{\lambda_{1}}{\lambda_{2}-\lambda_{1}} e^{-\lambda_{2} t} N_{1}\left(t_{0}\right)\left(e^{-\left(\lambda_{1}-\lambda_{2}\right) t}-1\right) .
\end{aligned}
$$

The common factor in $\mathrm{N}_{1}\left(\mathrm{t}_{0}\right)$ and $\mathrm{N}_{2}\left(\mathrm{t}_{0}\right)$ is $\sum$. If the experimental data can be normalized and decay corrected to yield $\sum$, then the total number of captures or fissions $c$ an be calculated according to

$$
\sum_{i=1}^{n} \sum_{i} \Delta t
$$

Let the counting interval begin at time $t_{1}$ and end at $t_{2}$. Both times are recorded by the computer and written on magnetic tape with the spectrum. Define the following: $A=$ area of the photopeak in the decay spectrum; $E$ = efficiency of the detector system; $Y=$ net gamma-ray yield per nuclear decay (for fission, this factor would include the fission yield for a given isotope); $\mathrm{L}$ = fractional live time of the counting system, also recorded and out put by the computer. For the case of parent activities, $i . e .$, some fission products, the number decaying between times $t_{1}$ and $t_{2}$ is

$$
\Delta N_{1}=N_{1}\left(t_{0}\right)\left(e^{-\lambda_{1} t_{1}}-e^{-\lambda_{1} t_{2}}\right)
$$

The area of the photopeak in the spectrum is

$$
A=\Delta N_{1} * E * Y * L=N_{1}\left(t_{0}\right)\left(e^{-\lambda_{1} t_{1}}-e^{-\lambda_{1} t_{2}}\right) * E * Y * L
$$

When calculating $\mathrm{N}_{1}\left(t_{0}\right)$ and $\mathrm{N}_{2}\left(t_{0}\right)$, the cross section is set equal to 1 , so the result is really $N_{1}\left(t_{0}\right) / \sum$ or $N_{2}\left(t_{0}\right) / \sum$. Using this fact Eq. (10) can be solved for $\sum$.

$$
\sum=\frac{A}{N_{1}\left(t_{0}\right)\left(e^{-\lambda_{1} t_{1}}-e^{-\lambda_{1} t_{2}}\right) * E * Y * L}
$$

Each count taken on each foil gives a value of $\sum$. These values are averaged to give a value of $\sum$ for each foil. The total number of fissions during the irradiation in the foil is then calculated to be

$$
\sum_{i=1}^{n} \phi_{i} \Delta t \bar{\sum}
$$

For capture- or fission-product daughters, the same reasoning applies, but with $\mathrm{N}_{2}(t)$. From time $\mathrm{t}_{1}$ to $\mathrm{t}_{2}$, 


$$
\begin{aligned}
\Delta N_{2} & =N_{2}\left(t_{0}\right)\left(e^{-\lambda_{2} t_{1}}-e^{-\lambda_{2} t_{2}}\right) \\
& +\frac{\lambda_{1}}{\lambda_{2}-\lambda_{1}} N_{1}\left(t_{0}\right)\left(\left(e^{-\lambda_{1} t_{1}}-e^{-\lambda_{2} t_{1}}\right)-\left(e^{-\lambda_{1} t_{2}}-e^{-\lambda_{2} t_{2}}\right)\right) .
\end{aligned}
$$

This gives

$$
\begin{aligned}
\Sigma & =A\left\{\left\{N_{2}\left(t_{0}\right)\left(e^{-\lambda_{2} t_{1}}-e^{-\lambda_{2} t_{2}}\right)+\frac{\lambda_{1}}{\lambda_{2}-\lambda_{1}} N_{1}\left(t_{0}\right)\right.\right. \\
& \left.\left.*\left[\left(e^{-\lambda_{1} t_{1}}-e^{-\lambda_{2} t_{1}}\right)-\left(e^{-\lambda_{1} t_{2}}-e^{-\lambda_{2} t_{2}}\right)\right]\right\} * E * Y * L\right\}^{-1},
\end{aligned}
$$

and the total number of captures can be calculated with Eq. (11). 
APPENDIX B

DETERMINATION OF ISOTOPIC FISSION RATES FROM FOIL DATA

\section{Uranium Foils}

In order to determine the fission rates in ${ }^{235} \mathrm{U}$ and ${ }^{238} \mathrm{U}$ from the relative reaction rates obtained using COMBO, it is necessary to know the isotopic fission rate ratio at each foil location since the relative fission rates in these two isotopes depend on the neutron spectrum. Calculated values of the isotopic fission rate ratio, ${ }^{238} \mathrm{U} / 235 \mathrm{U}$ (F8/F5) at each foil location were used to convert the depleted and enriched uranium fission rates to ${ }^{238} \mathrm{U}$ and ${ }^{235} \mathrm{U}$ fission rates. This method of isotopic correction is not as exact as the method using measured fission rates in fission chambers at the center of the core for normalization of the foil data but the difference between the two methods was found to be negligible except for ${ }^{238_{U}}(n, f)$ at the extreme edge of the blanket where a $10 \%$ difference was noted.

Let ENR and DEP be the observed relative fission rates (per gram) in the enriched and depleted uranium foils respectively, obtained from COMBO.

Let the weight-fractions of ${ }^{234} \mathrm{U},{ }^{236} \mathrm{U}$ and ${ }^{238} \mathrm{U}$ in the enriched uranium foils be denoted by I4E, I5E, I6E and I8E, respectively, and the weight-fractions in the depleted uranium foils by I5D, I6D and I8D.

Let F4, F5, F6 and F8 be the calculated fission rates per gram of $2{ }^{34} \mathrm{U}, 235 \mathrm{U}, 236 \mathrm{U}$ and $238 \mathrm{U}$ respectively. Values for the ratios $\mathrm{F} 4 / \mathrm{F} 8$ and F6/F8 at every point of measurement were not needed, since the required corrections are smal1. In this report, the central calculated values of 7.63 and 2.40 will be used for $\mathrm{F} 4 / \mathrm{F} 8$, and $\mathrm{F} 6 / \mathrm{F} 8$ respectively.

The isotopic correction equations can then be written. The measured $235 \mathrm{U}$ fission rate (F2 35) derived from the enriched uranium foil data is given by:

$$
F 235=\frac{E N R}{I 5 E+I 8 E+I 6 E\left(\frac{F 6}{F 8}\right)+I 4 E\left(\frac{F 4}{F 8}\right)\left(A \frac{F 8}{F 5}\right)}
$$

and the measured ${ }^{23} 8_{U}$ fission rate of (F238) derived from the depleted uranium foil is given by:

$$
F 238=\frac{D E P}{I 8 D+I 6 D\left(\frac{F 6}{F 8}\right)+I 5 D\left(A \frac{F 8}{F 5}\right)^{-1}}
$$

where the factor A takes into account the different decay rates of the fission products from $235 \mathrm{U}$ and $238 \mathrm{U}$ fission. An experimental value of the factor $A$ has been determined ${ }^{59}$ in an auxiliary measurement to be 0.95 . Although $A$ is a function of the time after the irradiation, most of the fission-product c is done within a 20-hour period commencing 4-hours after the irradiation the value of $A$ is reasonably constant from experiment to experiment. 
The maximum value of F8/F5 for fast reactor spectra is approximately 0.03 and it can get considerably smaller when the neutron spectrum softens. In the GCFR Phase I assemb1y, F8/F5 has a range from 0.029 at the core center to $\sim 0.004$ at the outer edge of the radial blanket. Table B-I 1ists the ca1culated ratio of F8/F5 for each matrix location used in the foil traverses of the normal and checkerboard core configurations.

F235, the isotopic fission rate calculated from the enriched enriched uranium foil data is insensitive to the correction term:

$$
I 8 E+I 6 E\left(\frac{F 6}{F 8}\right)+I 4 E\left(\frac{F 4}{F 8}\right)\left(A \frac{F 8}{F 5}\right)
$$

A $10 \%$ change in this term will result in a change in $\mathrm{F} 235$ of approximately $0.04 \%$.

The situation for the depleted uranium foils is different. Here the minimum value of the correction term 15D/A(F8/F5) corresponding to the maximum value of $\mathrm{F} 8 / \mathrm{F} 5$, is approximately 0.12 (for $0.21 \%$ depleted uranium) so that a $10 \%$ change in this term will cause a change of approximately $1 \%$ in $\mathrm{F} 238$.

\section{Plutonium Foils}

Similar isotopic corrections were applied to the plutonium foil results as those applied to the depleted and enriched uranium foils results.

Let $\mathrm{Pu}$ be the observed relative fission rate (per gram) in the plutonium foils, obtained from COMBO. Let the weight-fraction of $239 \mathrm{Pu}, 240 \mathrm{Pu}$ and ${ }^{241} \mathrm{Pu}$ in the plutonium foils be denoted by I9P, IOP and IIP, respectively. Let $\mathrm{F} 9$, F0 and $\mathrm{F} 1$ be the calculated fission rates per gram of ${ }^{239} \mathrm{Pu}, 240 \mathrm{Pu}$ and $241 \mathrm{Pu}$ respective1y.

The measured ${ }^{2}{ }^{39} \mathrm{Pu}$ fission rate (F239) is given by:

$$
F 239=\frac{P u}{I 9 P+I O P\left(\frac{F 0}{F 9}\right)+I I P\left(\frac{F 1}{F 9}\right)}
$$




\section{REFERENCES}

1. P. Benoist, Streaming Effects and Collision Probabilities in Lattices, Nucl. Sci. Eng. 34, 285-307 (1968).

2. E. M. Bohn et a1., Private Communieation (Sept 1971).

3. A. P. Olson and R. G. Palmer, "Approach to Critical Study by Moda1 Analysis for Plutonium-Fueled Fast Criticals, Reactor Physics Division Annual Report, JuIy 1, 1968, to June 30, 1969, ANL-7610, pp. 235-239.

4. C. E. Till et al., "ZPR-6 Assemblies 6A and 7: Benchmark Specifications for the Two Large Single-Core-Zone Critical Assemblies -- $235 \mathrm{U}$-Fueled Assembly 6A and P1utonium-Fueled Assembly 77 -- LMFBR Demonstration Reactor Benchmark Program," Applied Physics Division Annual Report, July 1, 1970, to June 30, 1971, ANL-7910, pp. 86-101.

5. H. D. Lemmel, The Third IAEA Evaluation of the $2200 \mathrm{~m} / \mathrm{s}$ and $20^{\circ} \mathrm{C}$ MaxweZZian Neutron Data for U-233, U-235, Pu-239 and Pu-241, Paper presented at the Conference on Nuclear Cross Sections and Technology, Washington, D. C., March 3-7, 1975, CONF-750303.

6. D. C. Wade and E. M. Gelbard, Neutron Strecoming in Plate Criticals, Paper presented at the ANS Topical Meeting on Advanced Reactors: Physics, Design and Economics, Atlanta, Georgia, September 1974, CONF-740903.

7. H. Henryson II, B. J. Toppel, and C. G. Stenberg, ETOE-2/MC 2 -2/SDX MuZtigroup Neutron Cross Section Processing, Seminar on Nuclear Data Processing Codes, Ispra, Italy, December 5-7, 1973, EACRP-U-52.

8. C. G. Stenberg, "ETOE-2, A Program for Conversion of ENDF/B to $\mathrm{MC}^{2}-2$," Applied Physics Division Annual Report, July 1, 1970, to June 30, 1971, ANL-7910, pp. 442-445.

9. H. Henryson II, B. J. Toppel, and C. G. Stenberg, MC ${ }^{2}-2$ : A Code to CaZcuLate Fast Neutron Spectra and Multigroup Cross Sections, ANL-8144, Argonne Nationa1 Laboratory (1976).

10. R. B. Pond, Reactor Physics Studies in the Engineering Mockup Critical of the Fast Test Reactor, ANL-76-42. Argonne National Laboratory (1976).

11. W. M. Stacey et al., A New Space-Dependent Fast Neutron Multigroup CrossSection Preparation Capability, Trans. Am. Nucl. Soc. 15, 292 (1972).

12. P. H. Kier, Private Communication (1974).

13. S. K. Bhattacharyya, Neutron Streaming Effects in Simulated GCFR Core, Trans. Am. Nuc1. Soc. 21, 497 (1975).

14. R. D. McKnight, Private Communication, October 1975.

15. P. J. Collins, Private Communication, 1976.

16. D. C. Wade and K. R. Krack, Private Communication (Nov 1975).

17. C. H. Adams, SYN3D: A Single-channel, Spatial Elux Synthesis Code for Diffusion Theory Calculations, ANL-76-21, Argonne National Laboratory (1976).

18. D. R. Ferguson et a1., Private Communication (Jan 1976). 
19. S. Seth et a1., GCFR Benchmarks: Experiments and Analysis, Nuclear CrossSections and Technology, Vol. I, NBS Special Publication 425, pp. 464-468 (1975).

20. R. Richmond et al., Measurements of Neutron Spectrum and Reaction Rates in a Gas-Cooled Fast Reactor Lattice, EIR Report 239 (1973).

21. T. J. Yule and E. F. Bennett, Measured Neutron Spectra in a Number of Uranium- and Plutonium-Fueled Reactor Assemblies, Nucl. Sci. Eng. 46, 236 (1971).

22. E. F. Bennett and T. J. Yule, Techniques and Analyses of Fast-reactor Neutron Spectroscopy with Proton-recoil Proportional Counters, ANL-7763 (1971).

23. E. F. Bennett, T. J. Yule, and D. C. Wade, Spatialiy Dependent Neutron Spectra in an Engineering Mockup of the FTR, Trans. Am. Nucl. Soc. 16, 236 (1971).

24. J. A. Morman and C. E. Cohn, Private Communication.

25. W. C. Schick, Jr., SKEWGAUS: A Fortran Progrom for Fitting Peaks in Semiconductor Detector Spectra, USAEC Report IS-3460 (October 1974).

26. P. H. Kier and M. Salvatores, The Effect of Local Flux Distortions on the Doppler Effect of Small Fissile Samples, Nuc1. Sci. Eng. 53, 479-482 (1974).

27. D. W. Maddison and J. M. Gasid1o, Foil Activation Analysis in Critical Facilities Assemblies, Trans. Am. Nuc1. Soc. 15, 930 (1972).

28. R. Böhme and H. Seufert, Uranium Reaction Rate Measurements in the SteamCooled Fast Reactor SNEAK, Assembly 3A-2, KFK-811, EUR3970e, Kernforschung Kernforschungszentrum Karlsruhe, Germany (1968).

29. E. F. Bennett and R. L. Long, Precision Limitations in the Measurement of Small Reactivity Changes, Nuc1. Sci. Eng. 17(3), 425-432 (Nov 1963).

30. C. D. Swanson, A. B. Long, and J. F. Meyer, "On-Line Acquisition and Analysis of Data From Reactivity Measurements Using an Autorod," Applied Physics Division Annual Report, July 1, 1971, to June 30, 1972, ANL-8010, pp. 410-413.

31. C. E. Cohn, R. A. Karam, J. E. Marsha11, and J. M. VanDoorninch, ComputerAided Calibration of a Fine Autorod, Nucl. Appl. Technol. 7, 342-346 (Oct 1969).

32. R. A. Karam, Measurements of the Normalization Integral and the Spatial Distribution of the Importance of Fission Neutrons, Nuc1. Sci. Eng. 37, 192 (1969).

33. M. M. Bretscher and W. C. Redman, Low Flux Measurements of ${ }^{239} P u$ and $235 U$ Capture-to-Fission Ratios in a Fast Reactor Spectrum, Nuc1. Sci. Eng. 39,368 (1970).

34. W. C. Redman and M. M. Bretscher, Experimental Determination of the Perturbation Denominator in Fast Critical Assemblies, Nuc1. Sci. Eng. 44, 450 (1971).

L. G. LeSage, "Comment on the Experimental Normalization of Reactivity Measurements," Applied Physics Division Annual Report, July 1, 1972, to June 30, 1973, ANL-8010, pp. 318-321. 
36. M. M. Bretscher, Private Communication (1975).

37. M. M. Bretscher, "Perturbation Denominator Measurements in 2PR-6 Assemb1y 7," Applied Physics Division Annual Report, July 1, 1970, to June 30, 1971, ANL-7910, pp. 154-156.

38. E. M. Bohn, The Central Worth Discrepancy in Three Fast Reactor Benchmark Critical Assemblies, ANL-75-14 (June 1975).

39. L. G. LeSage, E. M. Bohn, and J. E. Marsha11, "Sodium-Void and Sma11Sample Reactivity Worth Measurements in ZPR-6 Assembly 7," Applied Physics Division Annual Report, July 1, 1970, to June 30, 1971, ANL-7910, pp. 141-154.

40. D. C. Wade, M. M. Bretscher, and R. G. Bucher, Private Communication (1976).

41. R. D. McKnight, Benchmark Testing Using ENDF/B-III and IV, Trans. Am. Nucl. Soc. 22, 723 (1975).

42. E. F. Bennett, Methods and Errors in Subcriticality Measurements by RodDrop-Elux-Profile Analysis, ANL-7966 (1976).

43. W. W. Graham III, D. S. Harmer, and C. E. Cohn, Accurate DeZayed-Neutron Parameter Measurements in a Heavy Water Reactor, Nucl. Sci. Eng. 38, 33 (1969).

44. W. C. Davidon, Variable Metric Method for Minimization, ANL/5990 (1959).

45. J. J. Mihalczo, Neutron Importance and Fission Density in Uranium-235Enriched Uraniom and Plutonium Metal Spheres, Nuc1. Sci. Eng. 56, 271 (1975).

46. D. C. Wade and K. R. Krack, Private Communication (1976).

47. K. E. Plumlee and M. T. Wiggins, Automatic Foil Activity Counting Facility and Data-reduction Program, ANL-6628 (1962).

48. G. S. Stanford, The Codes NURF, COMBO, and TWOSORCE for Processing Foir Counting Data, ANL-7356 (1967).

49. G. S. Stanford, Private Communication (Dec 1970).

50. R. Sher, Gomma-Gomma Coincidence Method for Measuring Resonance Escape Probability in ${ }^{238}$ U Lattices, Nucl. Sci. Eng. 7, 479 (1960).

51. D. C. Wade, J. A. Morman, K. R. Krack, W. R. Robinson, S. K. Bhattacharyya, and R. D. McKnight, Measurements and Analyses of Neutron Leakage Effects in the GCFR Phase I Assembly, Trans. Am. Nuc1. Soc. 23, 564 (1976).

52. J. H. Broido and M. P. Rothstein, The Effect of Steam Entry on the GasCooled Fast Breeder Reactor Demonstration Plant, Gulf-GA-A12041, Gulf General Atomic (1972).

53. E. Eisemann, Considerations on the Accident: Water Ingress in the Primary Loop of a Helium Cooled Fast Breeder Reactor with Secondary Steam Cycle, Institut für Neutronenphysik and Reactortechnik, Karlsruhe, Report KFK 1487 (1971).

54. B. Pellaud, The Physics Design of the Gas-Cooled Fast Reactor, USAEC Report GA-10509, Gulf General Atomic (1971). 
55. W. Heer, M. Jermann, R. Richmond, and S. Seth, Simulation of Steam Entry in the PROTEUS GCFR Lattice, Trans. Am. Nuc1. Soc. 22, 700 (1975).

56. G. L. Grasseschi, Private Communication (1976).

57. D. C. Wade, Private Communication (1976).

58. L. B. Levitt and R. C. Lewis, VIM-1, Atomics International Report AI-AEC-12951 (1970).

59. G. S. Stanford, Private Communication (1970). 


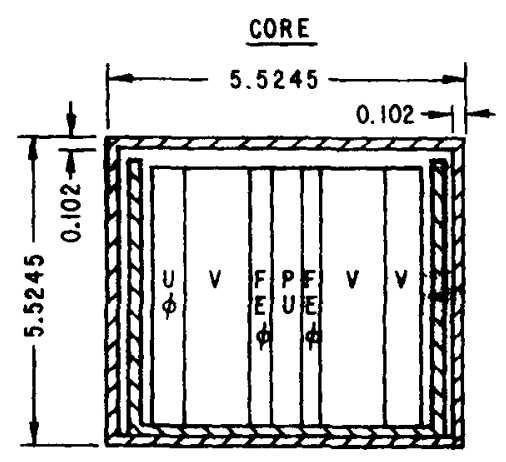

UNIT CELL XS

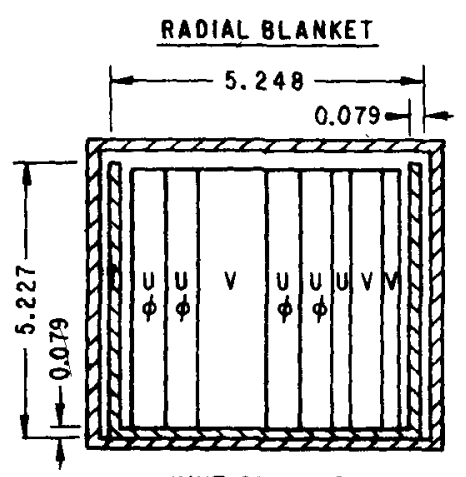

UNIT CELL XS

AXIAL BLAKKET

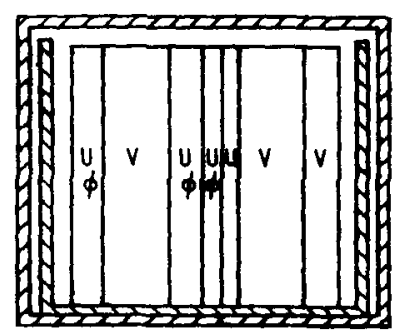

UNIT CELL XS

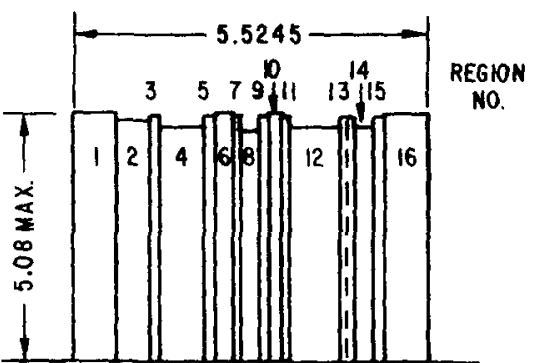

HETE ROGENEOUS MODE L

Uo $=\mathrm{U}_{3} \mathrm{O}_{B}$ Plate $\quad \mathrm{FEO}=\mathrm{Fe}_{2} \mathrm{O}_{3}$ Plate

$\mathrm{U}=$ DepI. U Plate $\mathrm{V}$ = Vold Can

$\mathrm{Pu}=\mathrm{Pu}-\mathrm{U}-\mathrm{Mo} \mathrm{Can}$

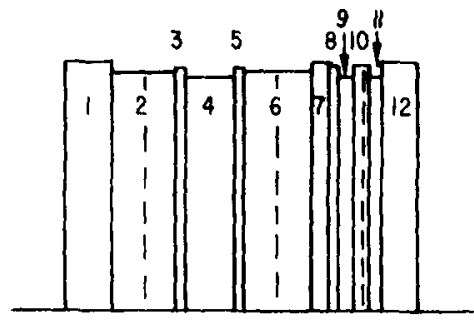

HETEROGENEOUS MODEL

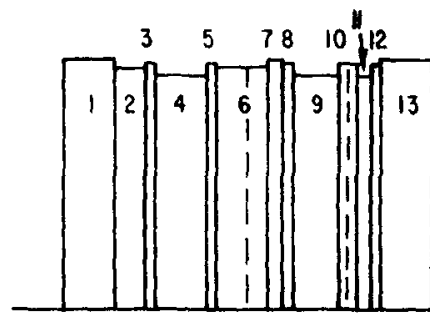

HETEROGENEOUS MOOEL

Fig. 1. Unit Cell Structures (actual and model) for the Core, Radial Blanket and Axial Blanket in the ZPR-GCFR Phase I Critical Assembly. All dimensions in centimeters. (ANL Neg. No. 116-76312).

DRAWER MASTER NO. 2801-F S.1

GCFR PHASE 1

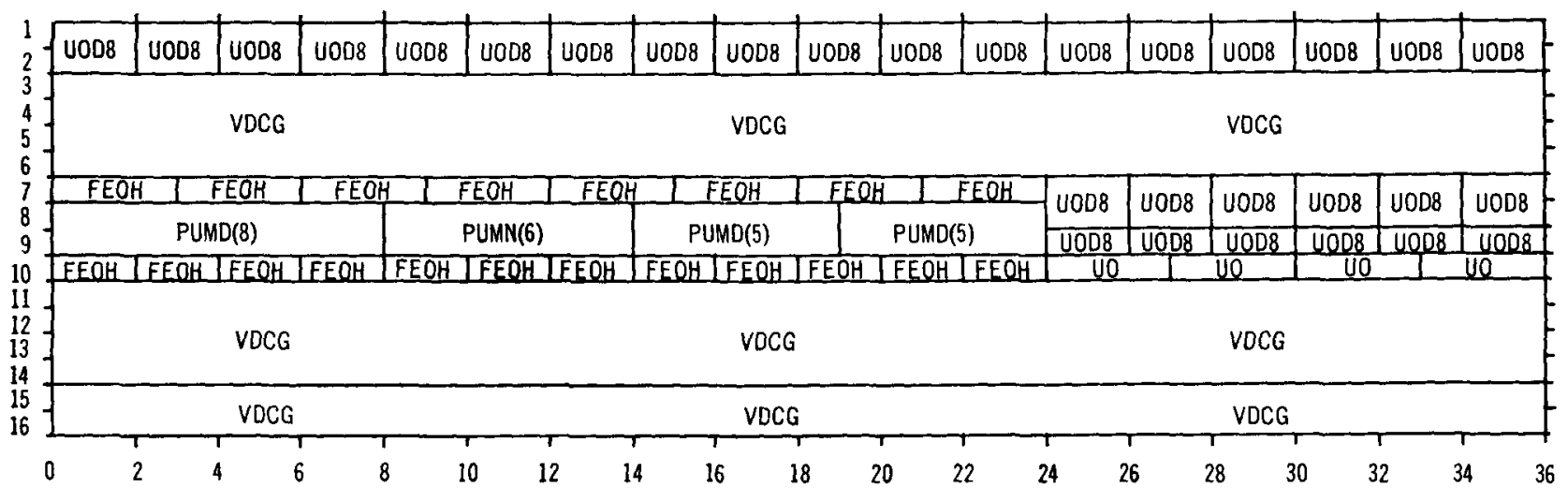

TOTAL U-235 IN CORE $\quad 0.006590 \mathrm{KG}$

TOTAL. PU-239 IN PU FUEL TOTAL PU-241 IN PU FUEL
$0.658530 \mathrm{KG}$ $0.008780 \mathrm{KG}$

Fig. 2. Drawer Master for Drawers in Core Region 1 (See Fig. 4). The actual layout of the various plates are depicted. (ANL Neg. No. 116-76-322). 
DRAWER MASTER NO. 2810-F S-1

GCFR PHASE 1

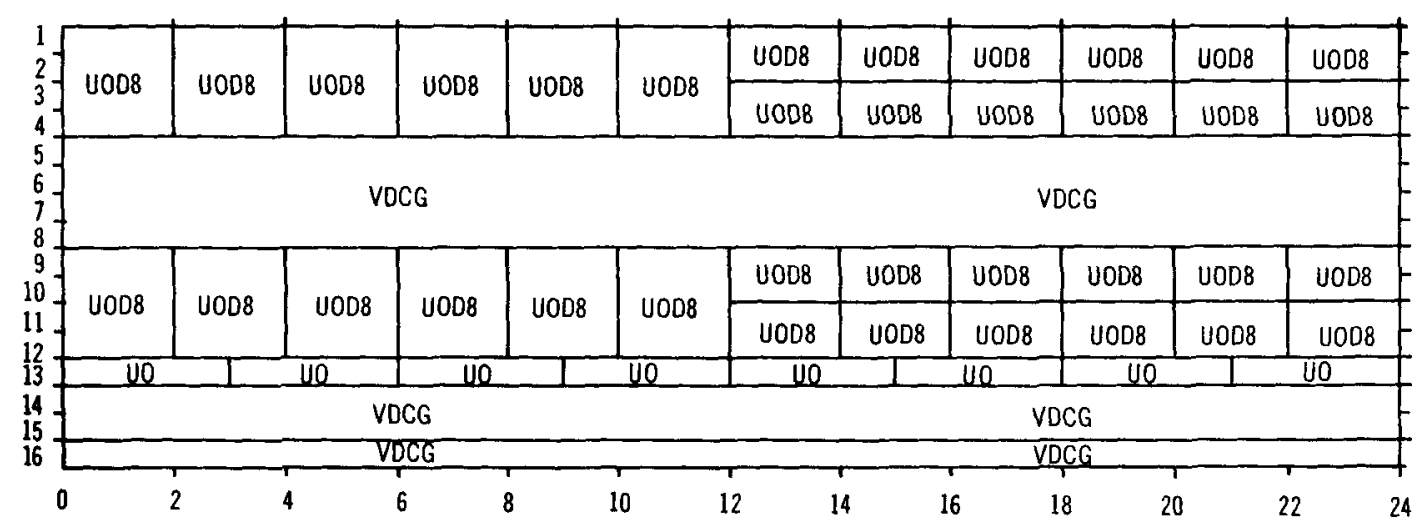

DRAWER MASTER NO. 2810-B S-1

RADIAL REFLECTOR-LAST 12 in.

\begin{tabular}{|c|c|c|c|c|c|}
\hline U0D8 & UOD8 & U0D8 & U0D8 & U008 & UOD8 \\
\hline U0D8 & U0D8 & UOD8 & UOD8 & UOD8 & U0DB \\
\hline \multicolumn{3}{|c|}{ VDCG } & \multicolumn{3}{|c|}{ VDCG } \\
\hline U008 & UOD8 & UODB & U008 & U0D8 & U0D8 \\
\hline UOD8 & UOD8 & U0D8 & UOD8 & UOD8 & U0D8 \\
\hline \multicolumn{2}{|l|}{$\mathrm{UO}$} & UO & \multicolumn{3}{|c|}{40} \\
\hline \multicolumn{3}{|c|}{ VDCG } & \multicolumn{3}{|c|}{ VDCG } \\
\hline \multicolumn{3}{|c|}{ VDCG } & \multicolumn{3}{|c|}{ YOCG } \\
\hline & & & & & \\
\hline
\end{tabular}

Fig. 3. Drawer Master for Drawers in the Radial Blanket Region. The layout of plates is shown in the $24 \mathrm{in.} \mathrm{front} \mathrm{drawer}$ and the 12 in. back drawer. VDCG stands for Void Cans, UODS for $\mathrm{U}_{3} \mathrm{O}_{8}$ and UD for depleted uranium. (ANL Neg. No. 116-76-323). 


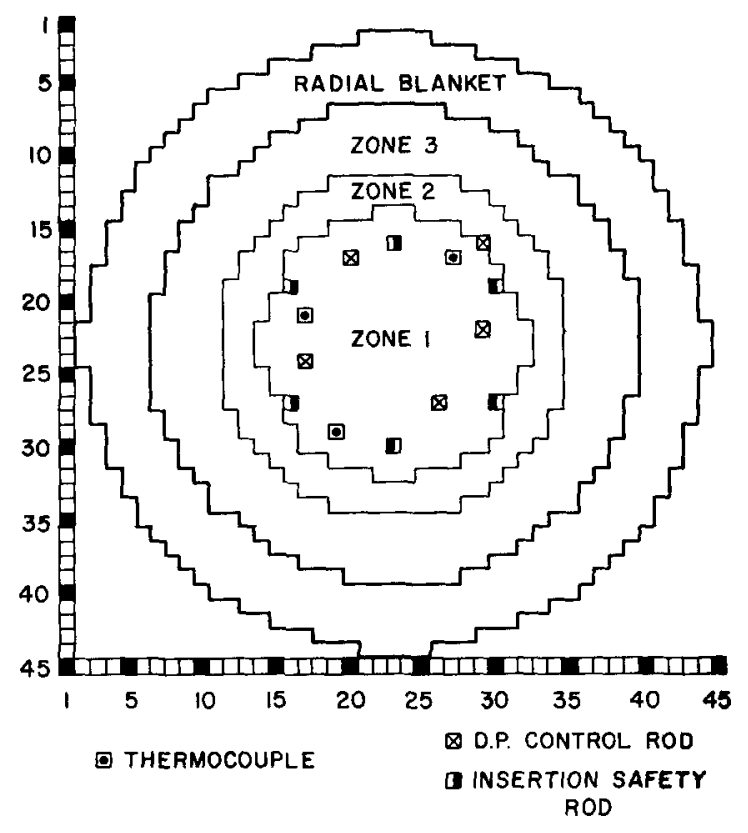

STATIONARY HALF

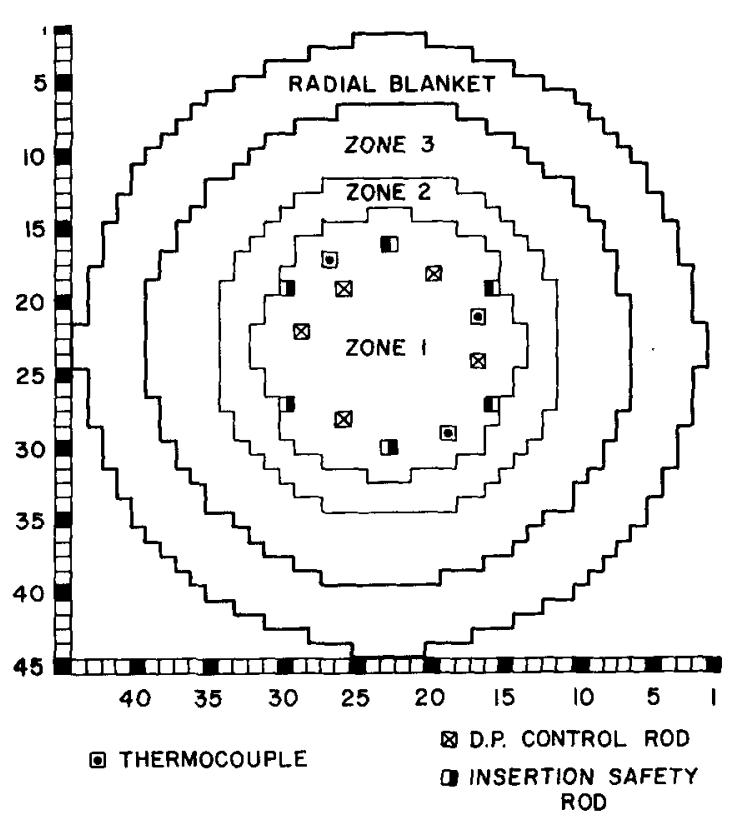

MOVABLE HALF
Fig. 4. Sketch of the Stationary Half of the Assembly Showing the As-Built Configuration. The locations of the ${ }^{10} \mathrm{~B}$ and dual purpose fuel bearing control rods are shown along with thermocouple locations in the core. (ANL Neg. No. 116-76267).

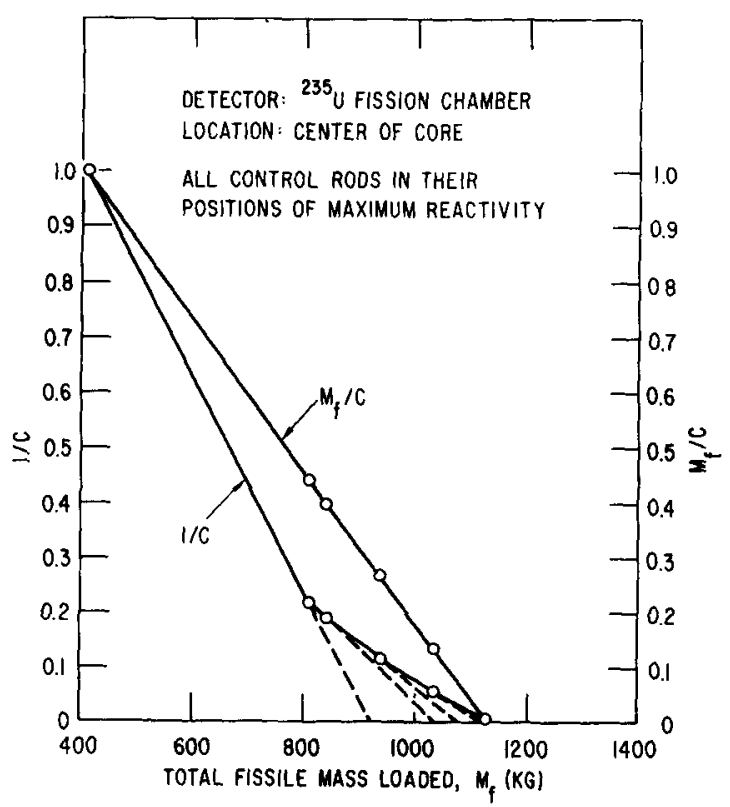

Fig. 5. Sketch of the Movable Half of the Assembly Showing the AsBuilt Configuration. (ANL Neg. No. 116-76-268).

Fig. 6. Approach-to-Critical Plots from ${ }^{235} \mathrm{U}$ Fission Chamber Located at the Core Center. (ANL Neg. No. 116-76-310). 


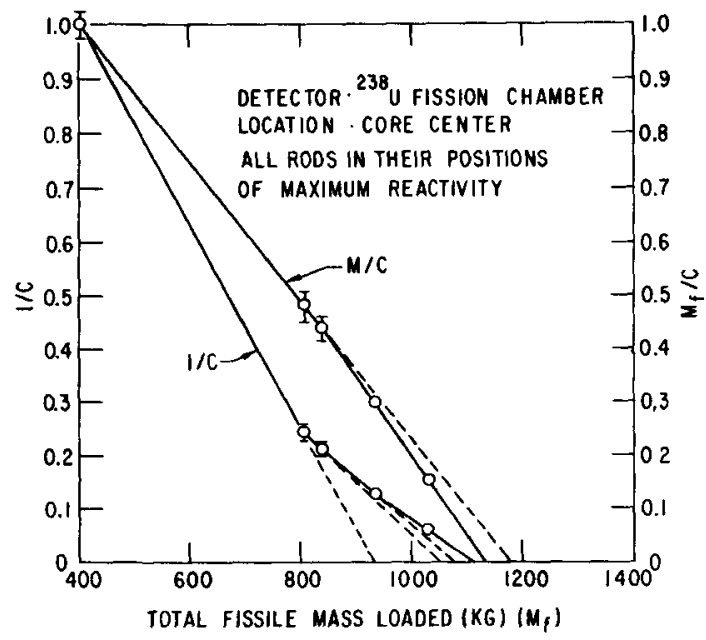

Fig. 7. Approach-to-Critical Plots from ${ }^{2}{ }^{8} \mathrm{U}$ Fission Chamber Located at Core Center. (ANL Neg. No. 116-76-305).

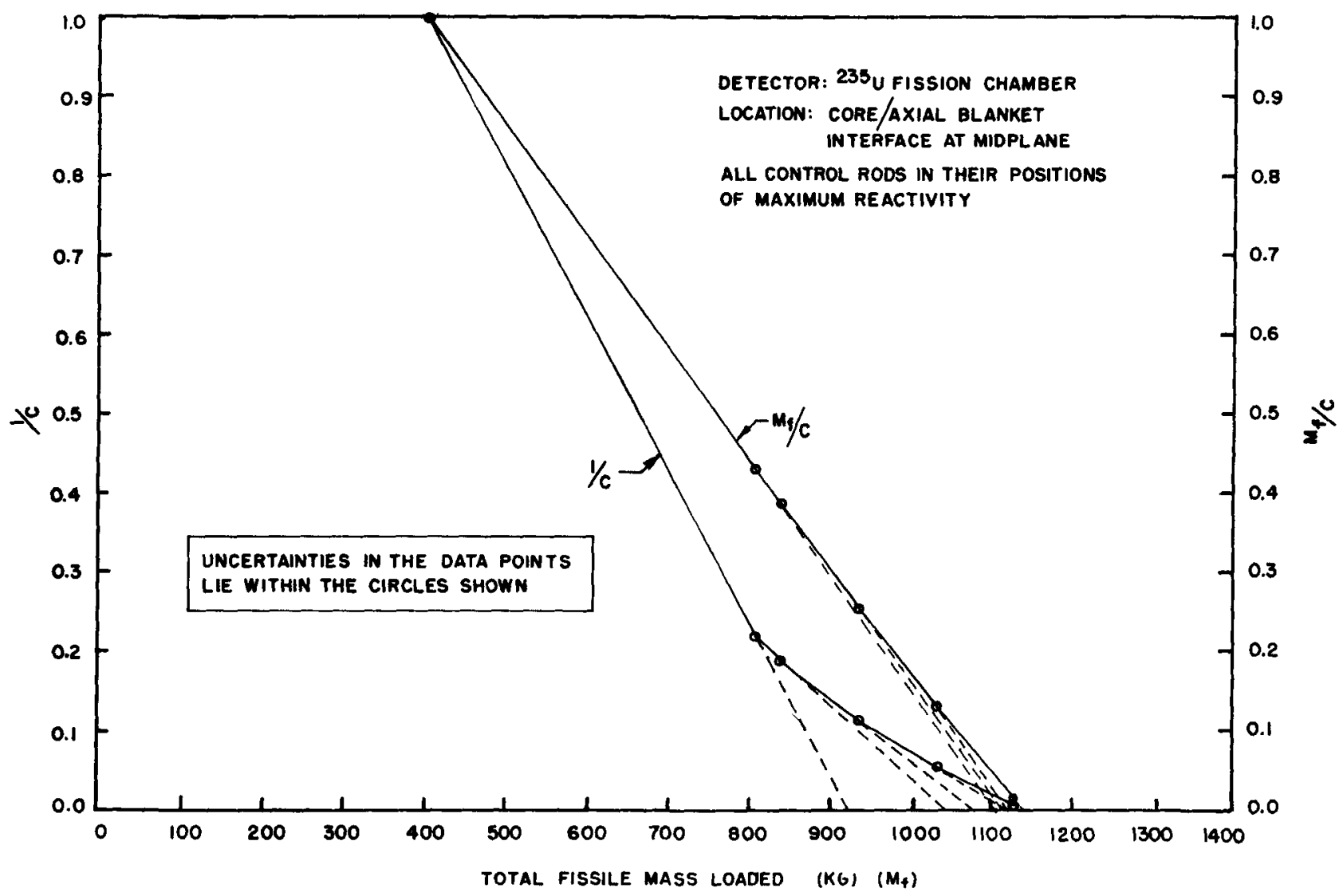

Fig. 8. Approach-to-Critical Plots from ${ }^{235} \mathrm{U}$ Fission Chamber Located at Core/Axial Blanket Interface. (ANL Neg. No. 116-76-274). 


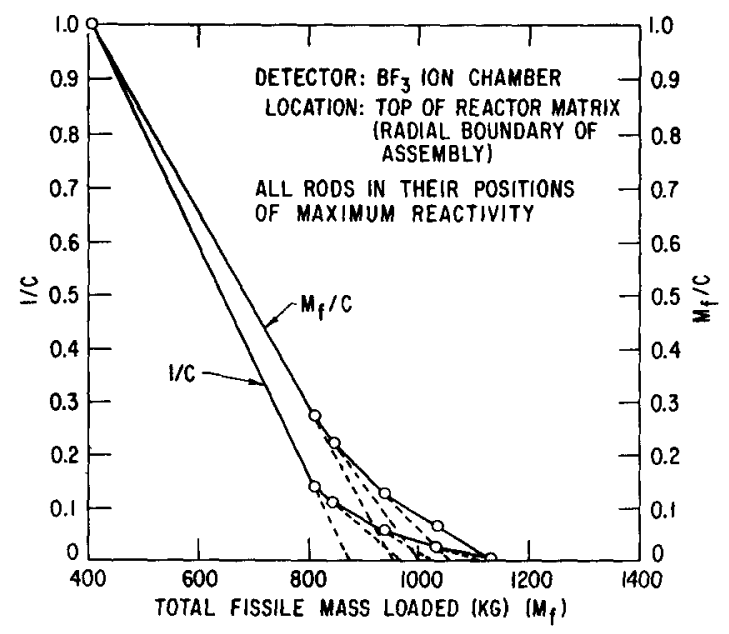

Fig. 9. Approach-to-Critical Plots from $\mathrm{BF}_{3}$ Ionization Chamber Located at Outside Boundary of Radial Blanket. (ANL Neg. No. 116-76-270).

\section{CRITICAL RADIUS PREDICTION USING OLSON-PALMER METHOD}

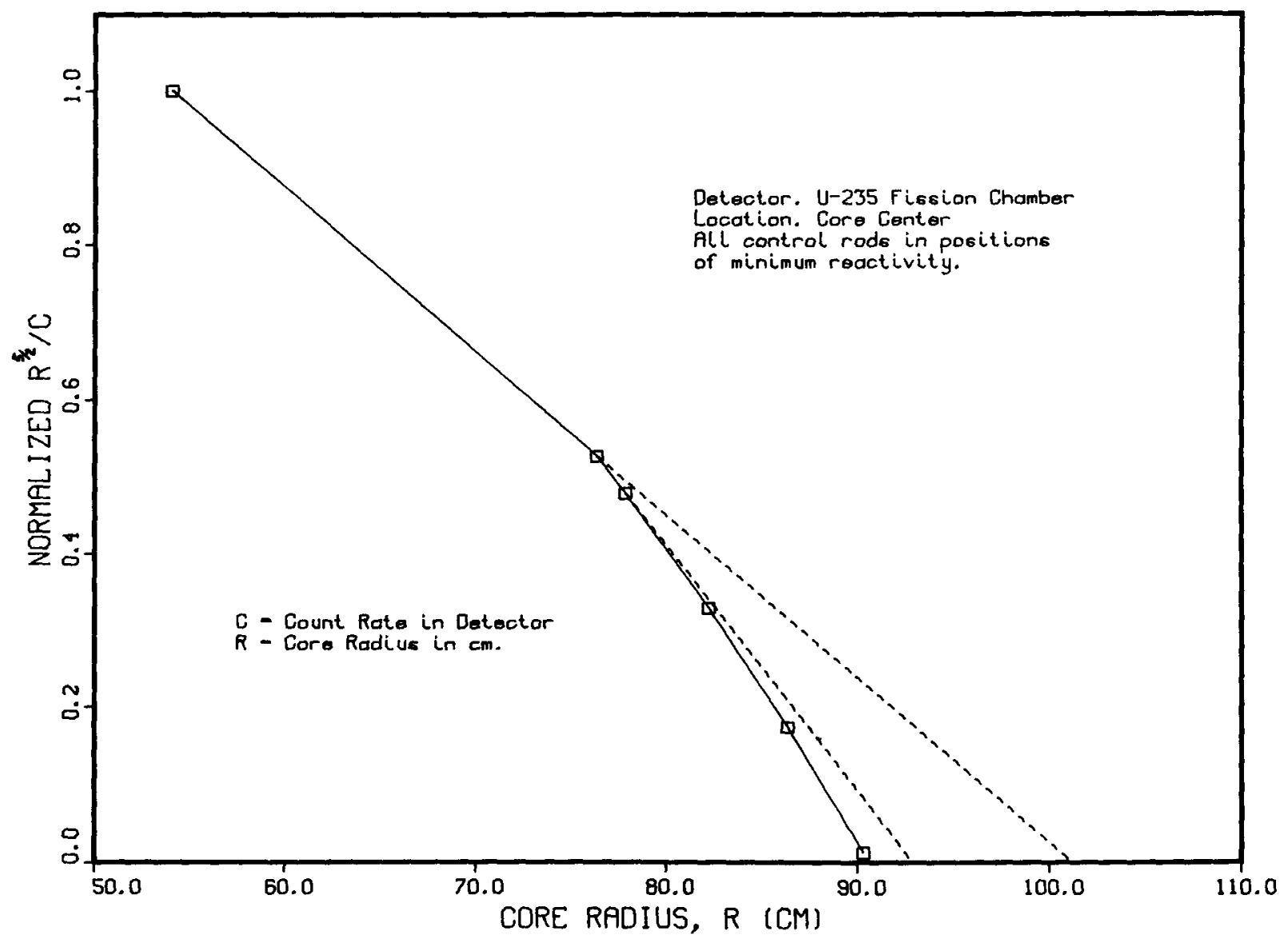

Fig. 10. Approach-to-Critical Plots from ${ }^{235} \mathrm{U}$ Fission Chamber Located at Core Center, using the 0lson-Palmer Method. (ANL Neg. No. 116-7 297). 


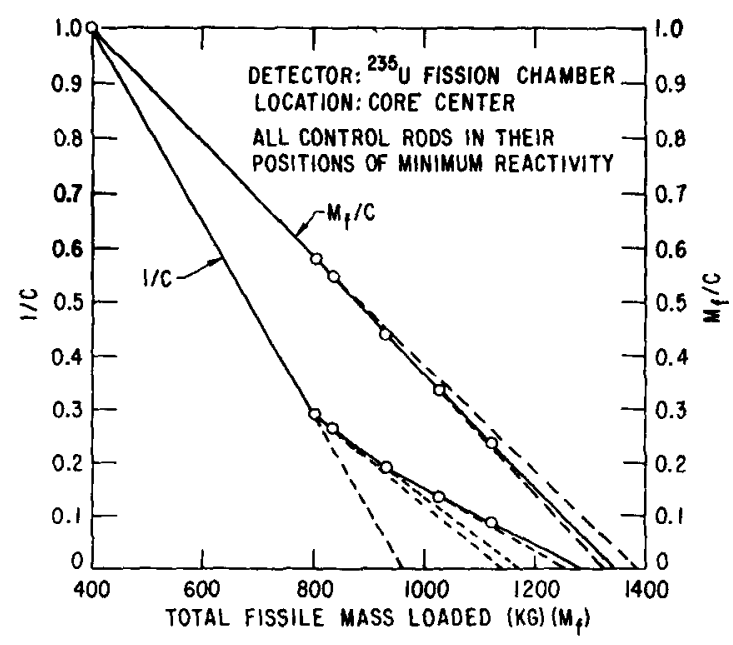

Fig. 11. Approach-to-Critical Plots from ${ }^{235} \mathrm{U}$ Fission Chamber Located at Core Center, with All Control Rods in their Positions of Least Reactivity. (ANL Neg. No.116-76-273).

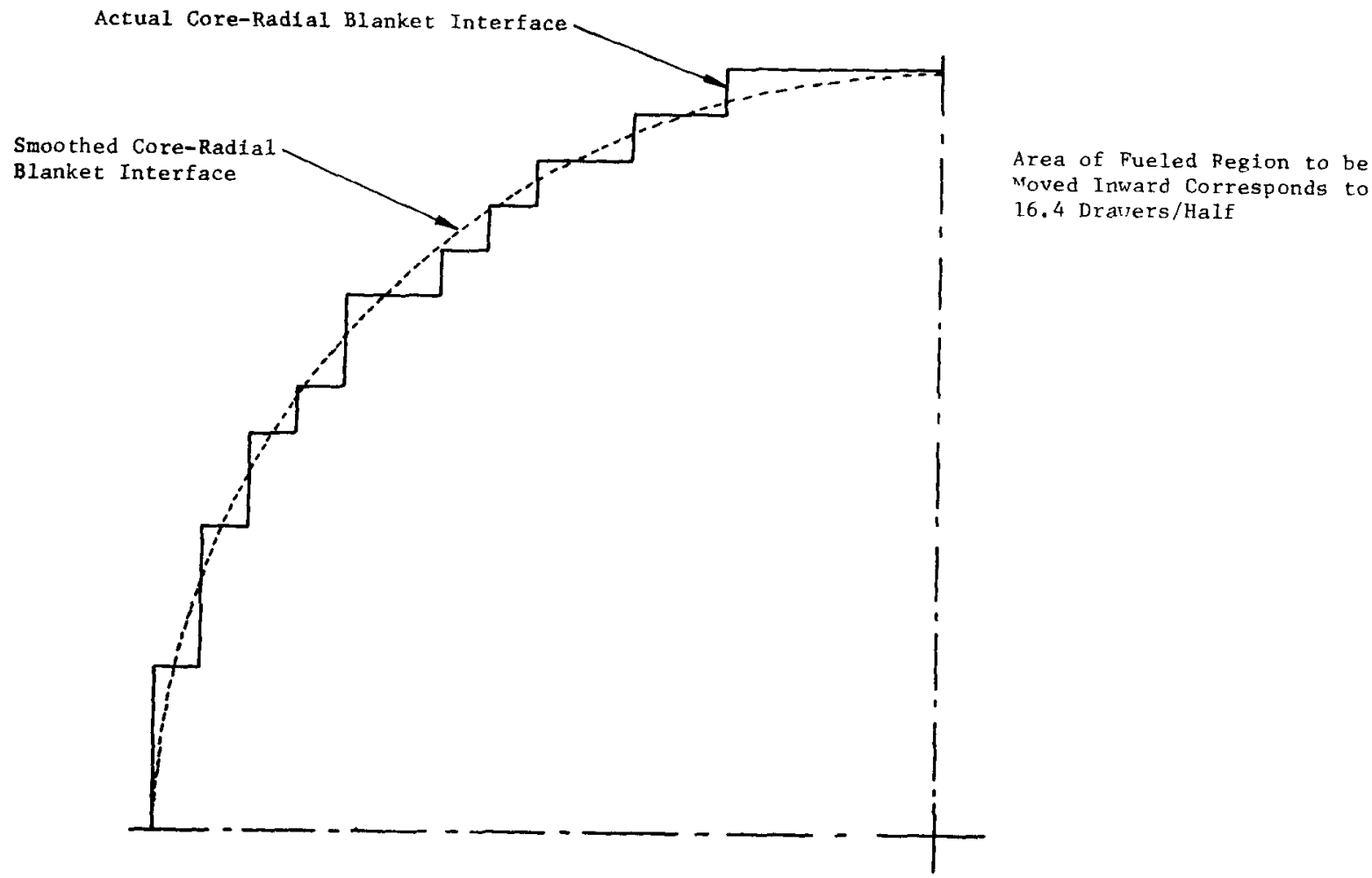

Fig. 12. Outline of Quadrant of the Core of the Assembly showing the Smoothed Core-Blanket Interface. (ANL Neg. No. 116-76-290). 


\section{DETERMINATION OF TEMPERATURE COEFFICIENT}

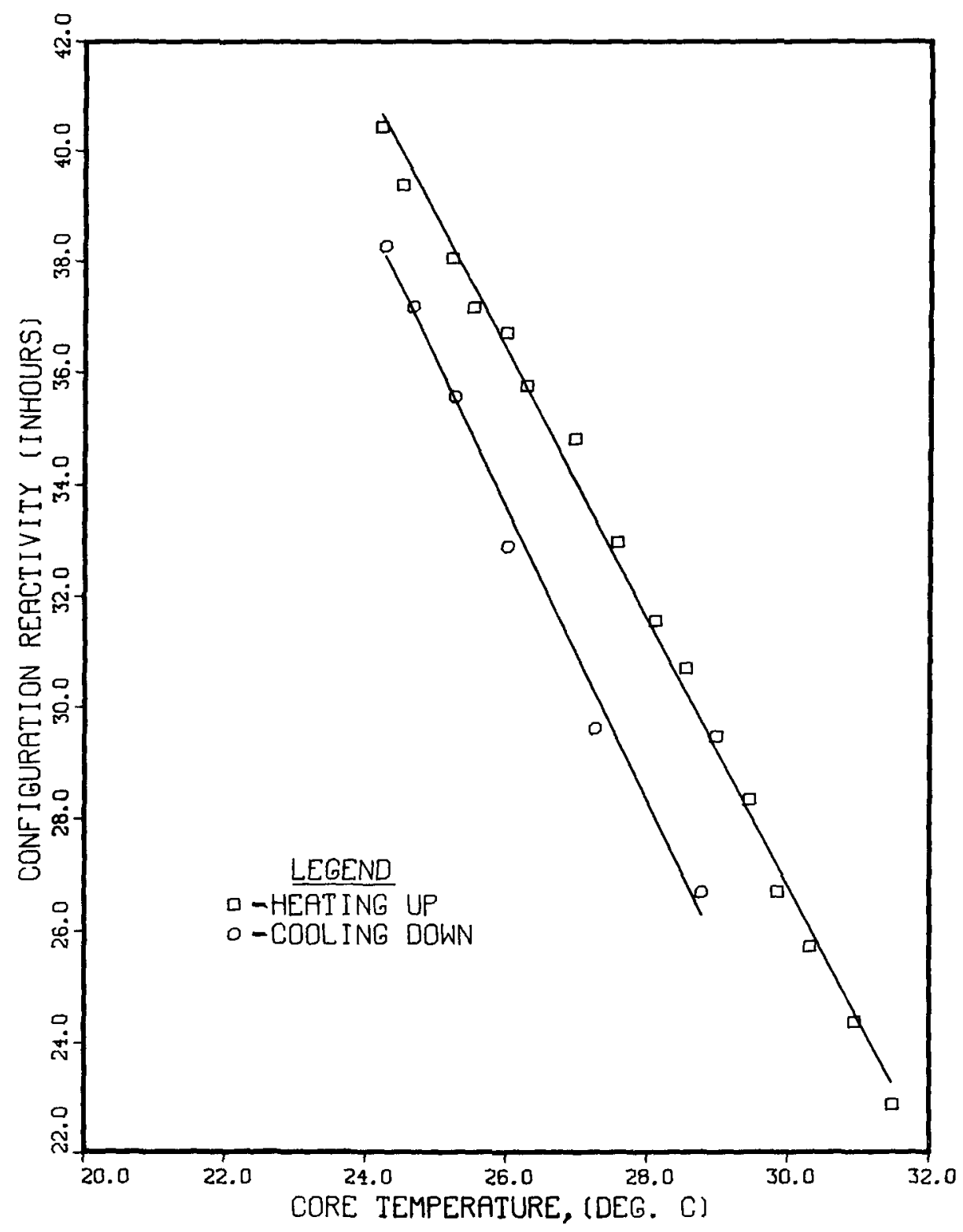

Fig. 13. Experimental Data and Least Squares Fitted Straight Lines used in the Determination of the Temperature "Coefficient" of the Assembly. (ANL Neg. No. 116-76336). 
REACTIVITY EFFECT OF PU-241 DECAY

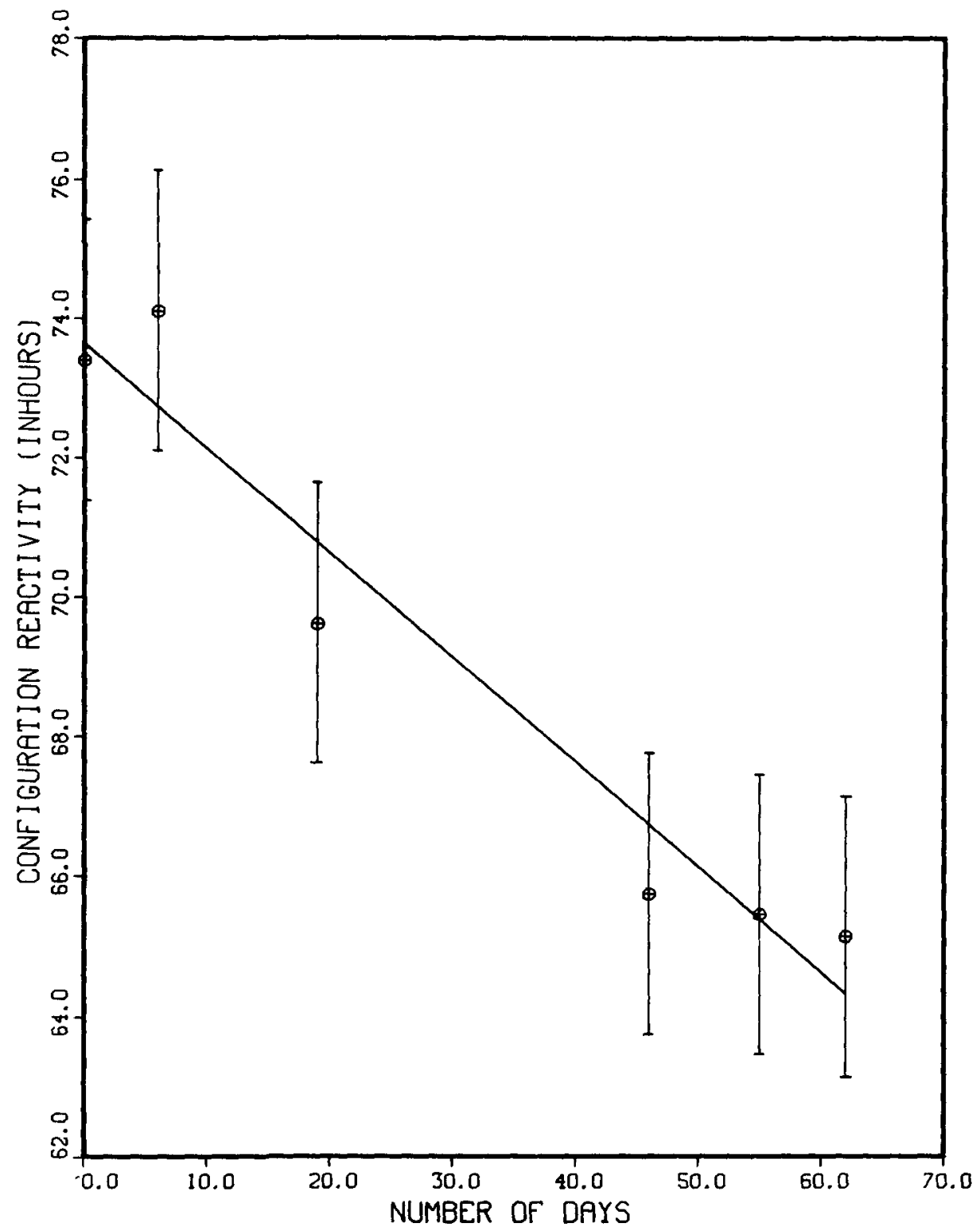

Fig. 14. Experimental Data and Least Squares Fitted Line used in the Determination of the Reactivity Worth of $24 \mathrm{P} \mathrm{Pu}$ Decay. The uncertainties are comprised primarily of the configuration reproducibility uncertainty $( \pm 2.0 \mathrm{Ih})$. (ANL Neg. No. 116-76-272). 
FLOWCHART OF CALCLLATION METHOD

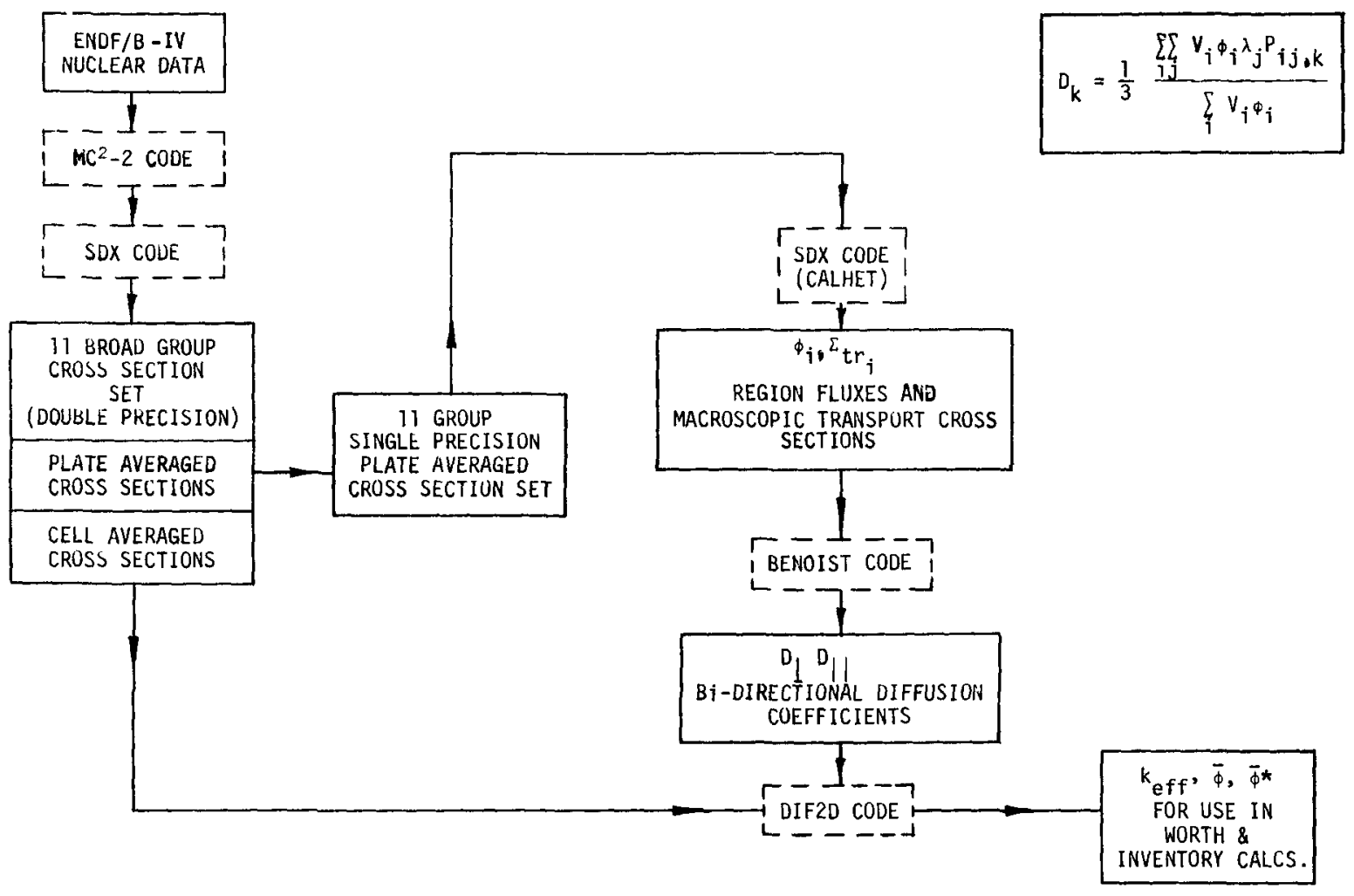

Fig. 15. Flowchart of the Calculation Method. (ANL Neg. No. 116-76-313). 
77

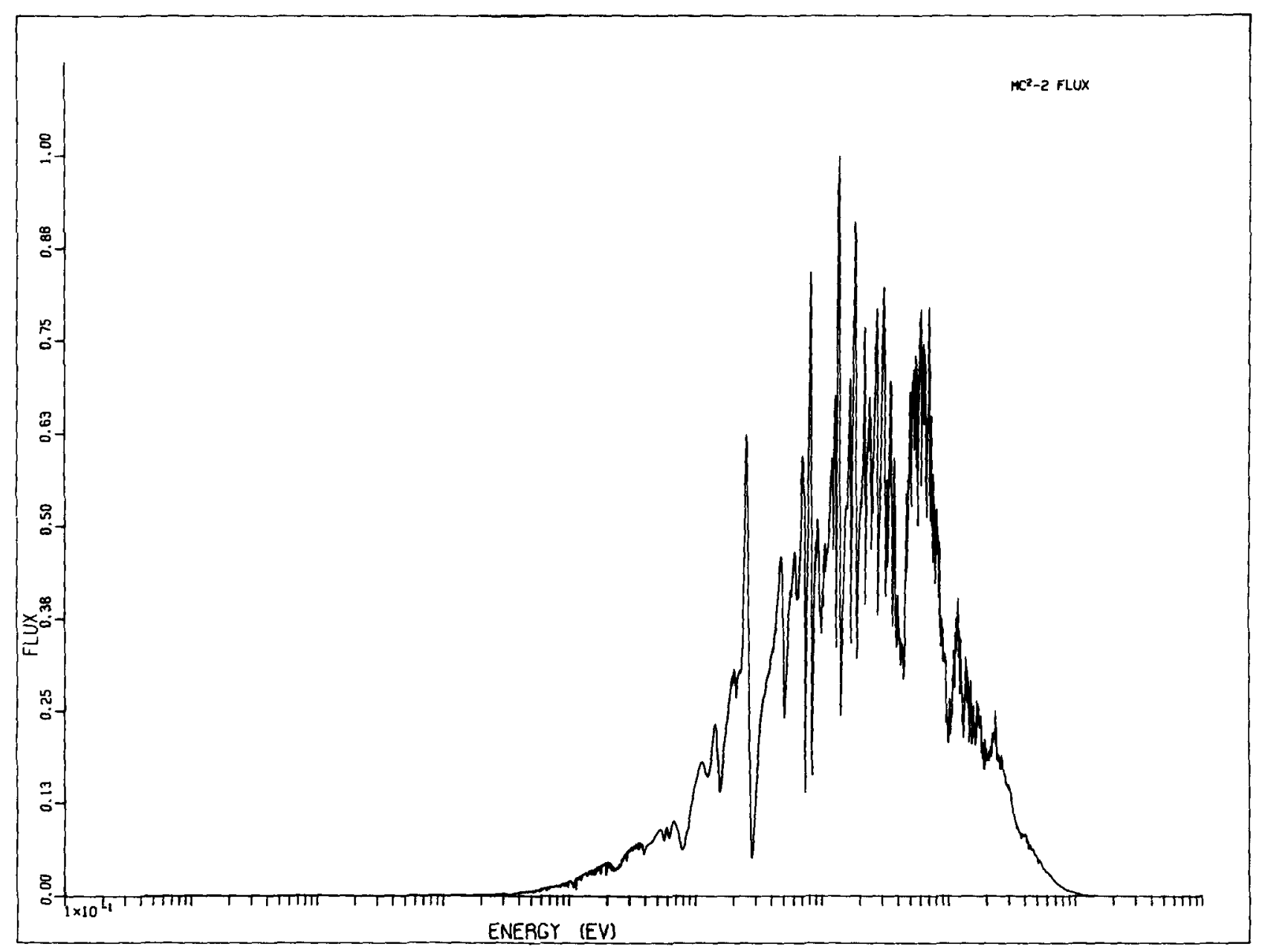

Fig. 16. MC ${ }^{2}$ Ultrafine Group Spectrum at the Core Center. (ANL Neg. No. 116-76-307). 
$\mathbf{Z}$

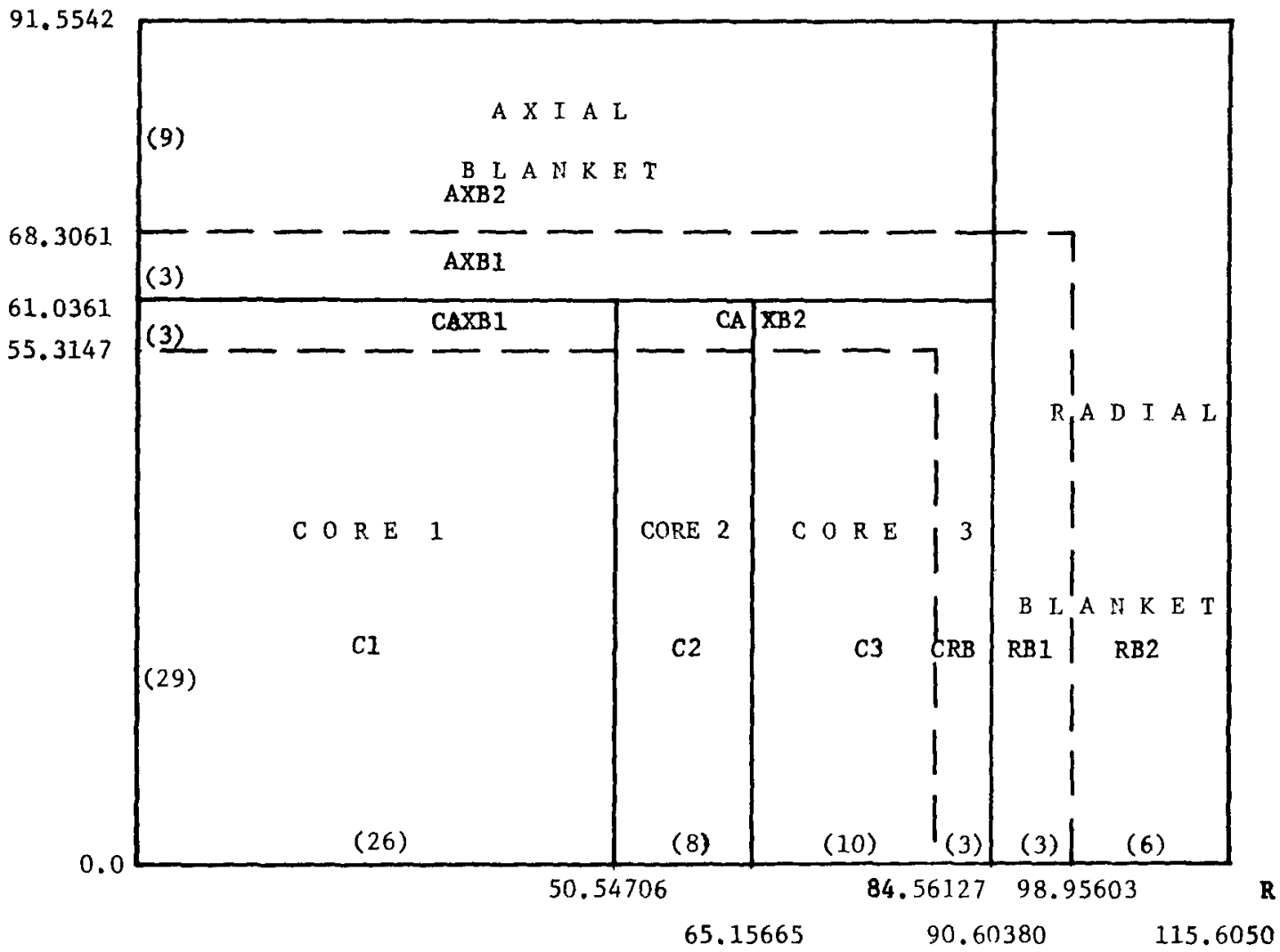

Regions and compositions are indicated by solid lines; cross section types are indicated by dashed lines. The number of mesh intervals is shown in parentheses. A11 dimensions are in $\mathrm{cm}$.

Fig. 17. The R-Z Model of the GCFR Phase-I Assembly. (ANL Neg. No. 116-76-325). 


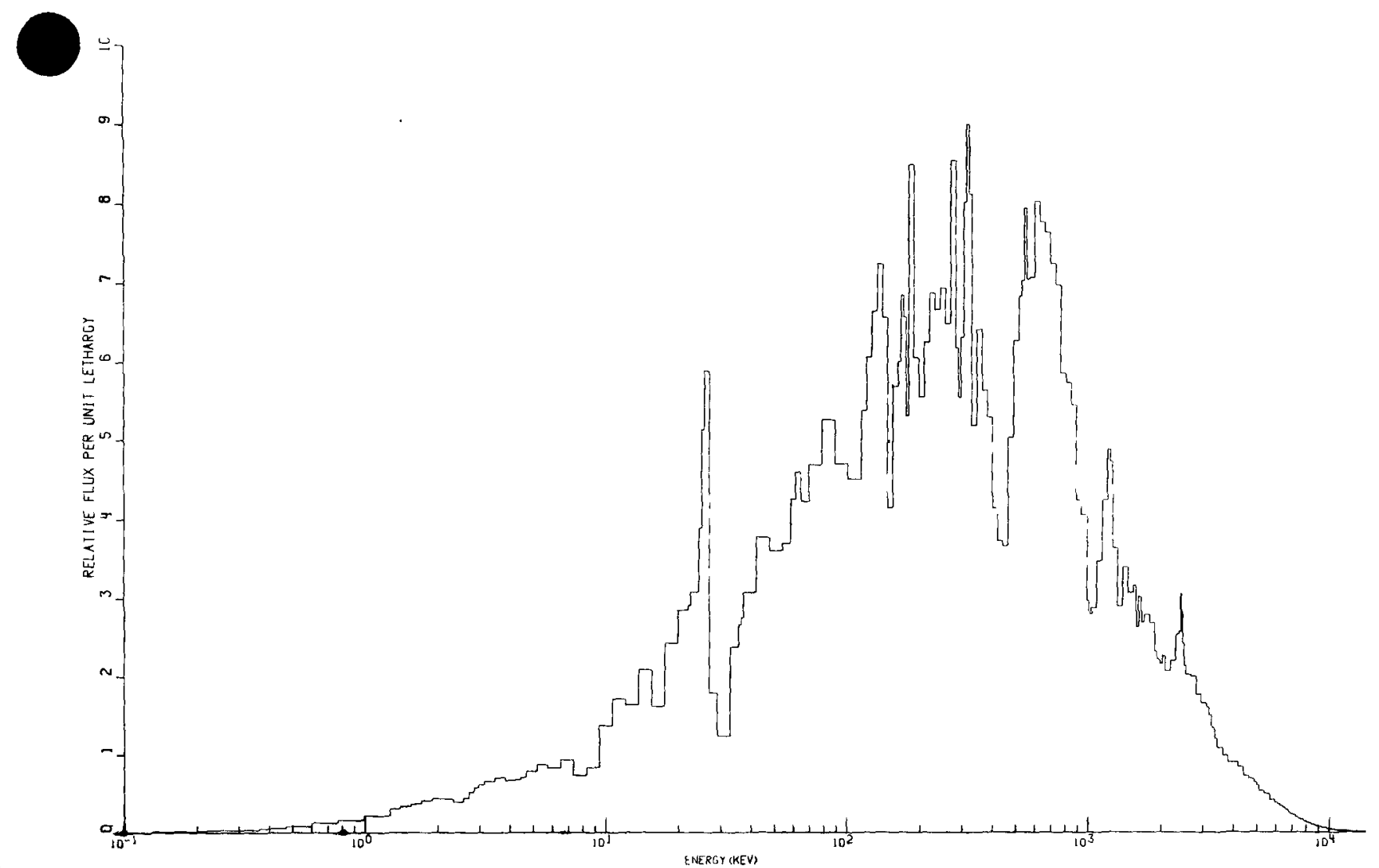

Fig. 18. SDX Fine-Group (USS226) Spectra for the Core Center of the GCFR Phase-I Assembly. (ANL Neg. No. 116-76-324).

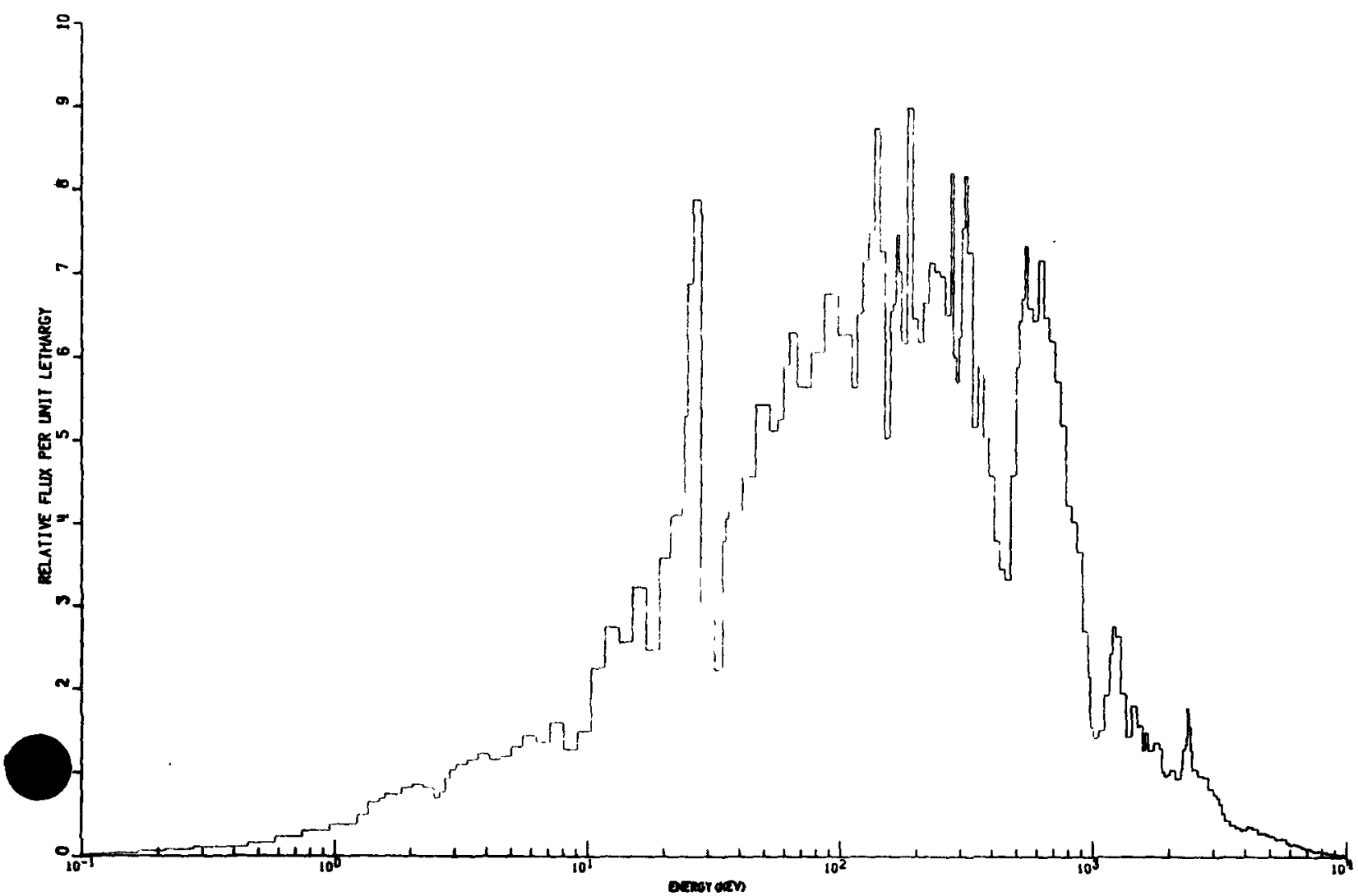

Fig. 19. SDX Fine-Group (USS226) Spectra for the Axial Blanket of the GCFR Phase-I Assembly. (ANL Neg. No. 116-76-296). 
GCFR PHASE 1

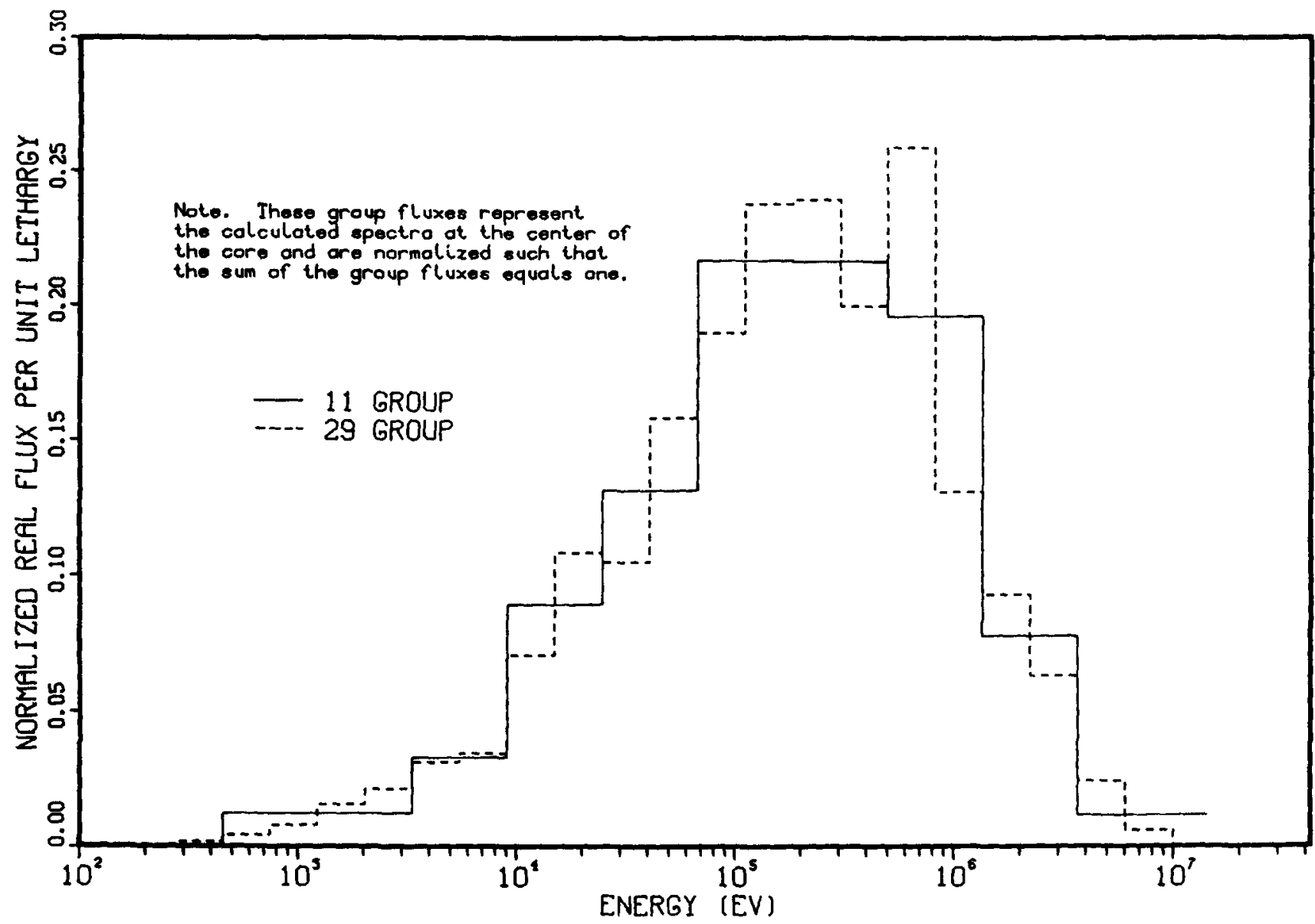

Fig. 20. Comparison of 11- and 29-Broad Group Real Flux for the GCFR Phase-I Assembly. (ANL Neg. No. 116-76-280). 


\section{GCFR PHASE 1}

$\simeq$

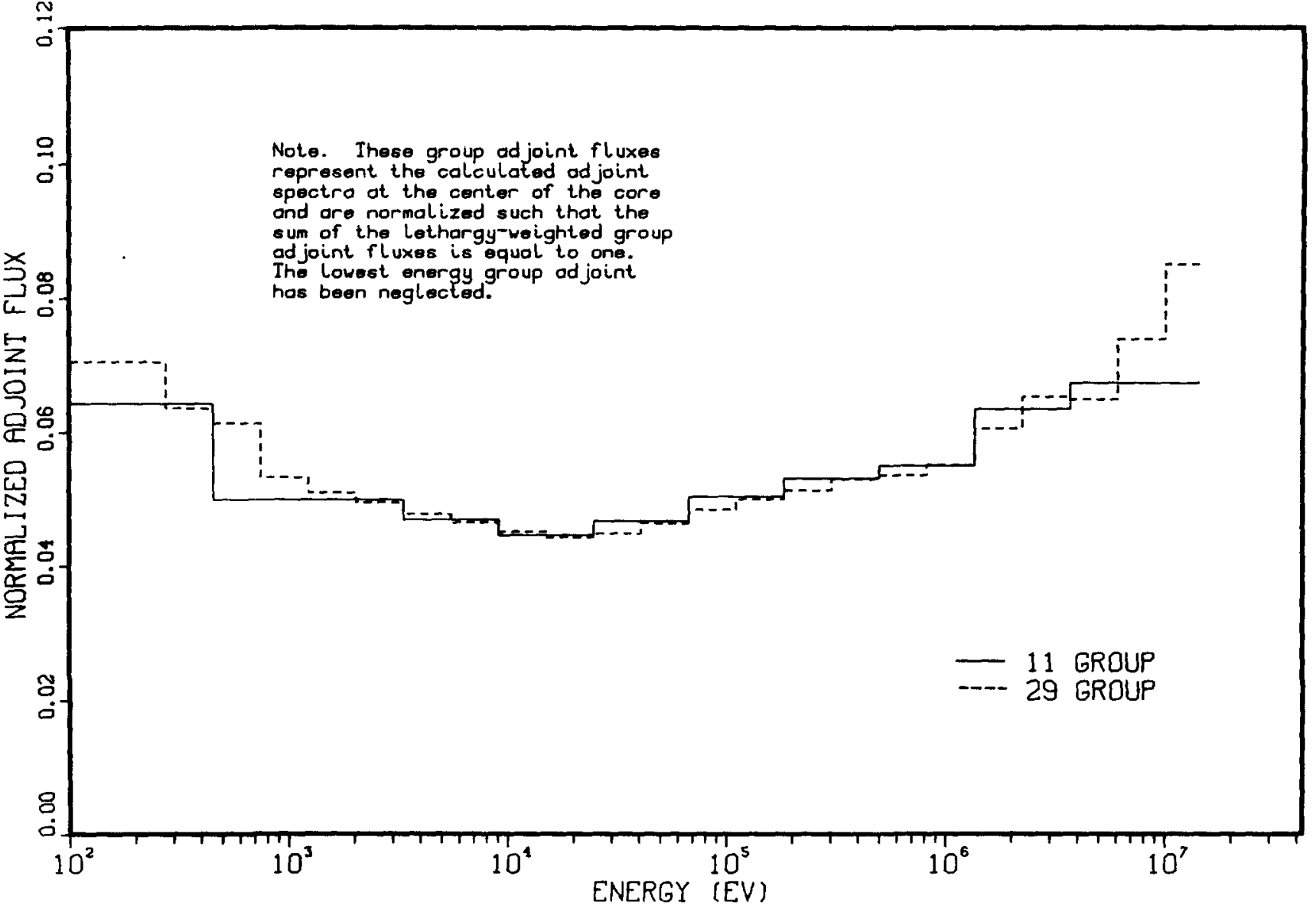

Fig. 21. Comparison of 11- and 29-Broad Group Adjoint Flux for the GCFR Phase-I Assembly. (ANL Neg. No. 116-76-292). 
GCFR PHASE 1

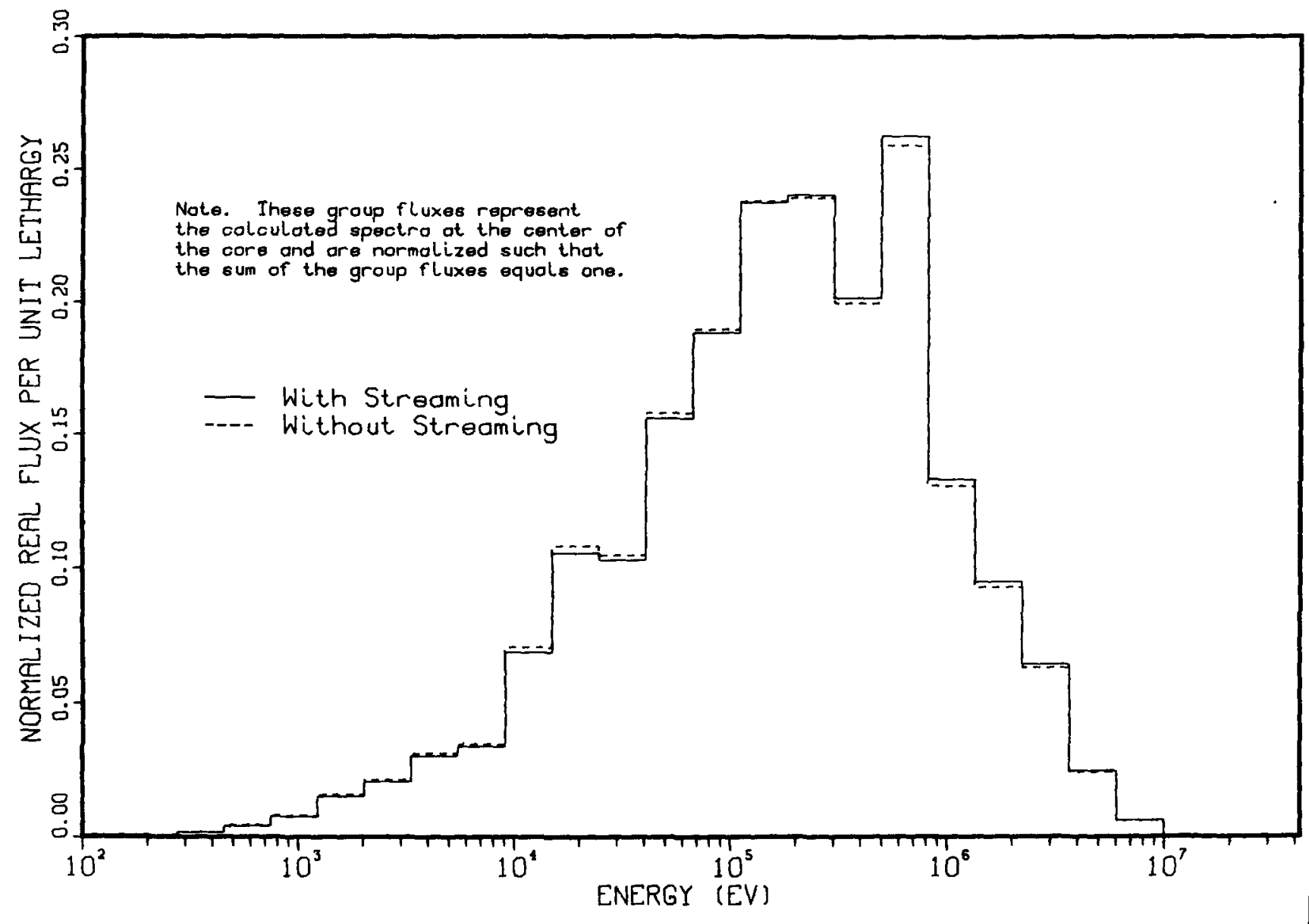

Fig. 22. Comparison of Real Group Flux for Streaming and Nonstreaming Calculations. (ANL Neg. No. 116-76-358). 


\section{GCFR PHASE 1}

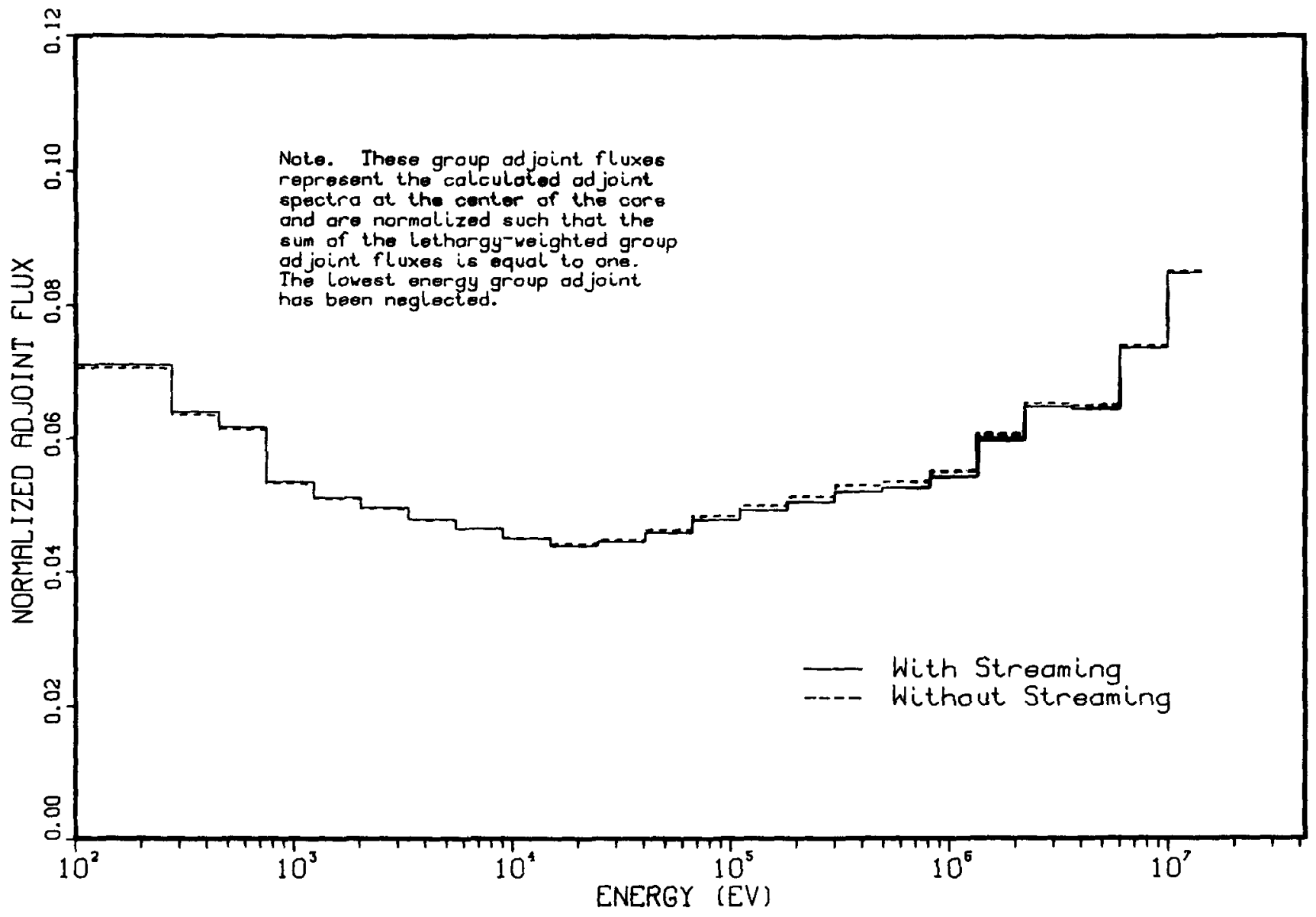

Fig. 23. Comparison of Adjoint Group Flux for Streaming and Nonstreaming Calculations. (ANL Neg. No. 116-76-276).

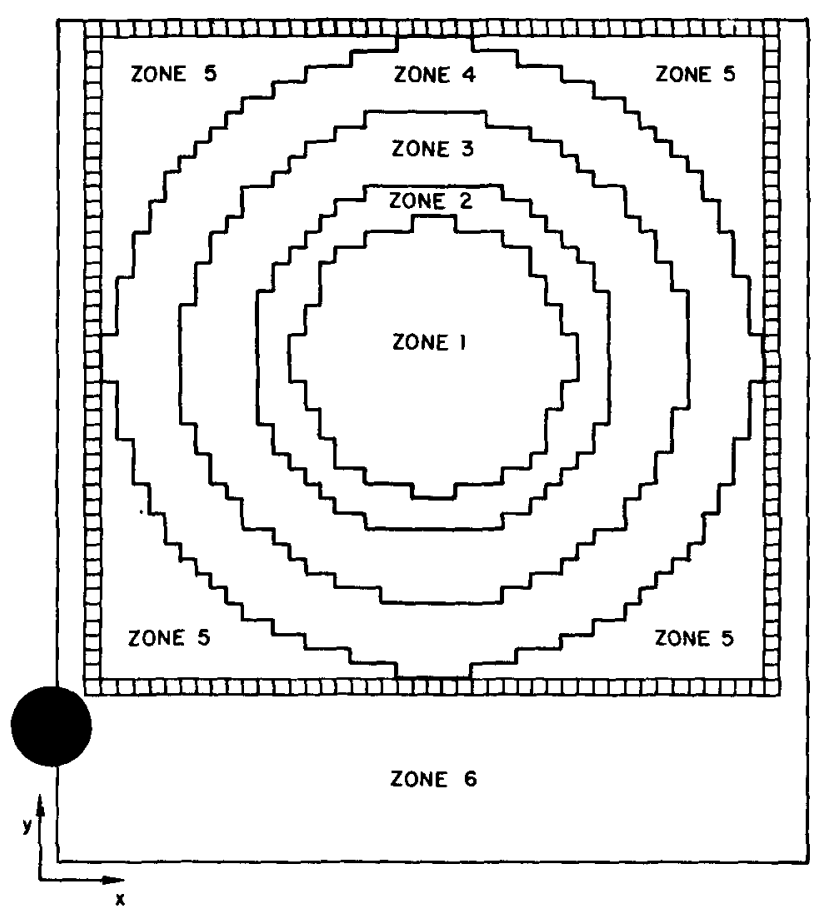

Fig. 24. The XY Full Plane Model Used in the 3D Calculation.

(ANL Neg. No. 116-76-317). 


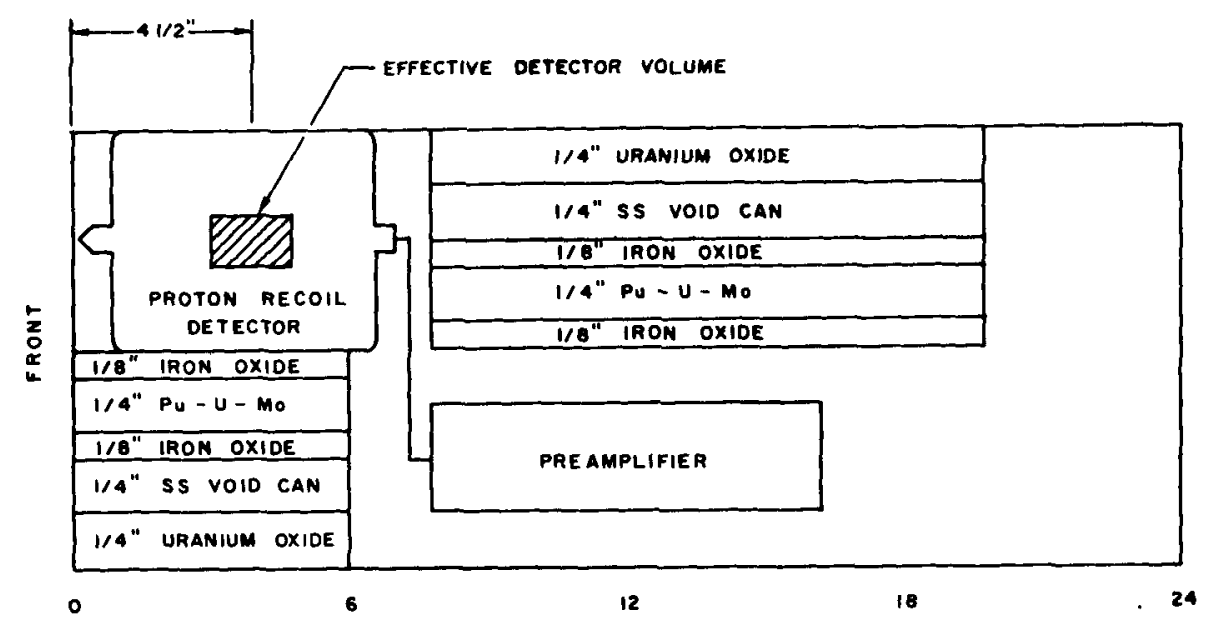

Fig. 25. Schematic Showing In-Core Detector, Preamplifier, and Reactor Material Placement. (ANL Neg. No. 116-76-269).

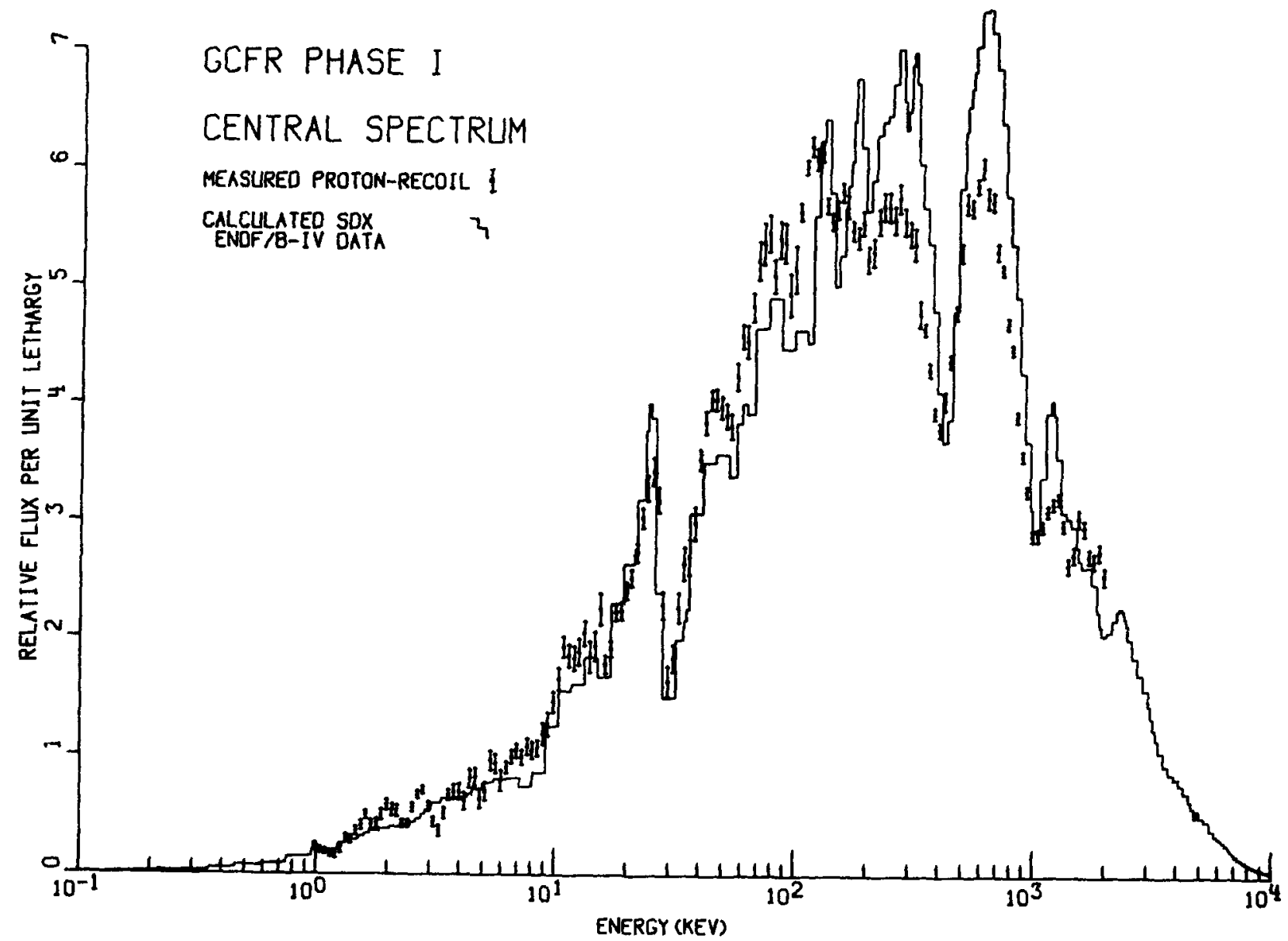

Fig. 26. Measured and Calculated Spectra at the Center of the GCFR Phase-I Reference Core. (ANL Neg. No. 116-76-319). 

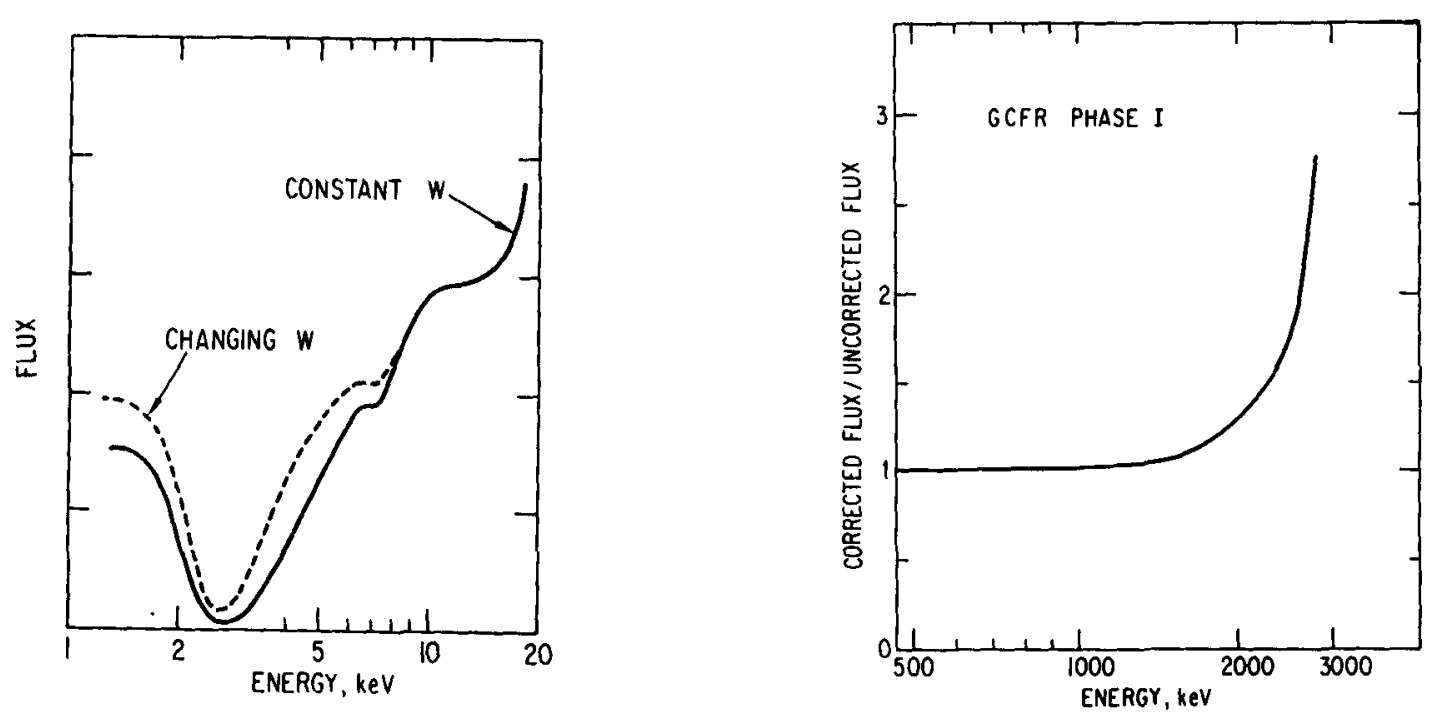

Fig. 27. Effect of an Uncertainty in Fig. 28. Correction to Account for W on a Typical LMFBR Spectrum. (ANL Neg. No. Wall and End Effects. (ANL Neg. No. 116-76-278). 116-76-316).

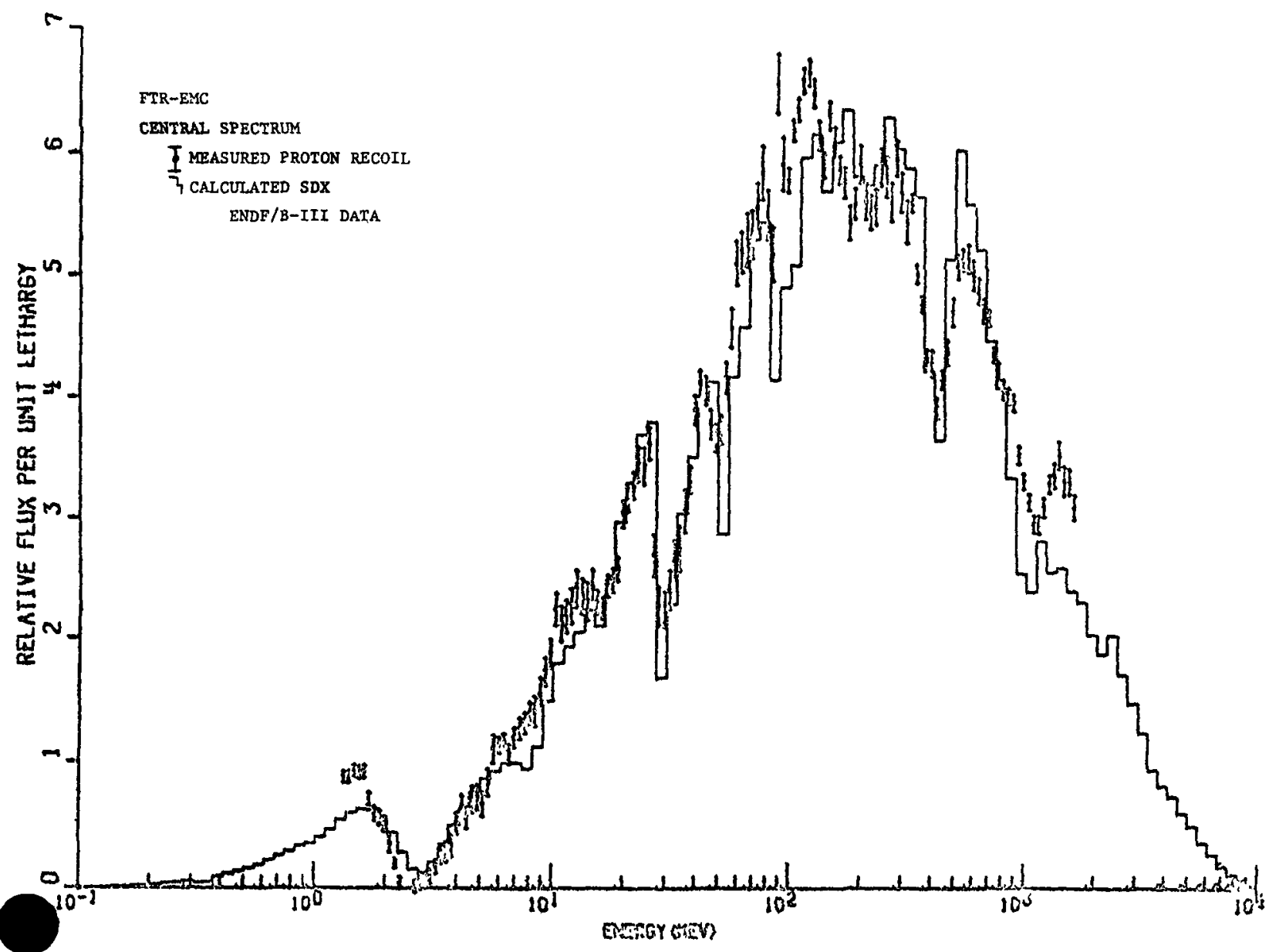

Fig. 29. Measured and Calculated Spectra at the Center of the FTP-EMC Core. (ANL Neg. No. 116-76-100). 

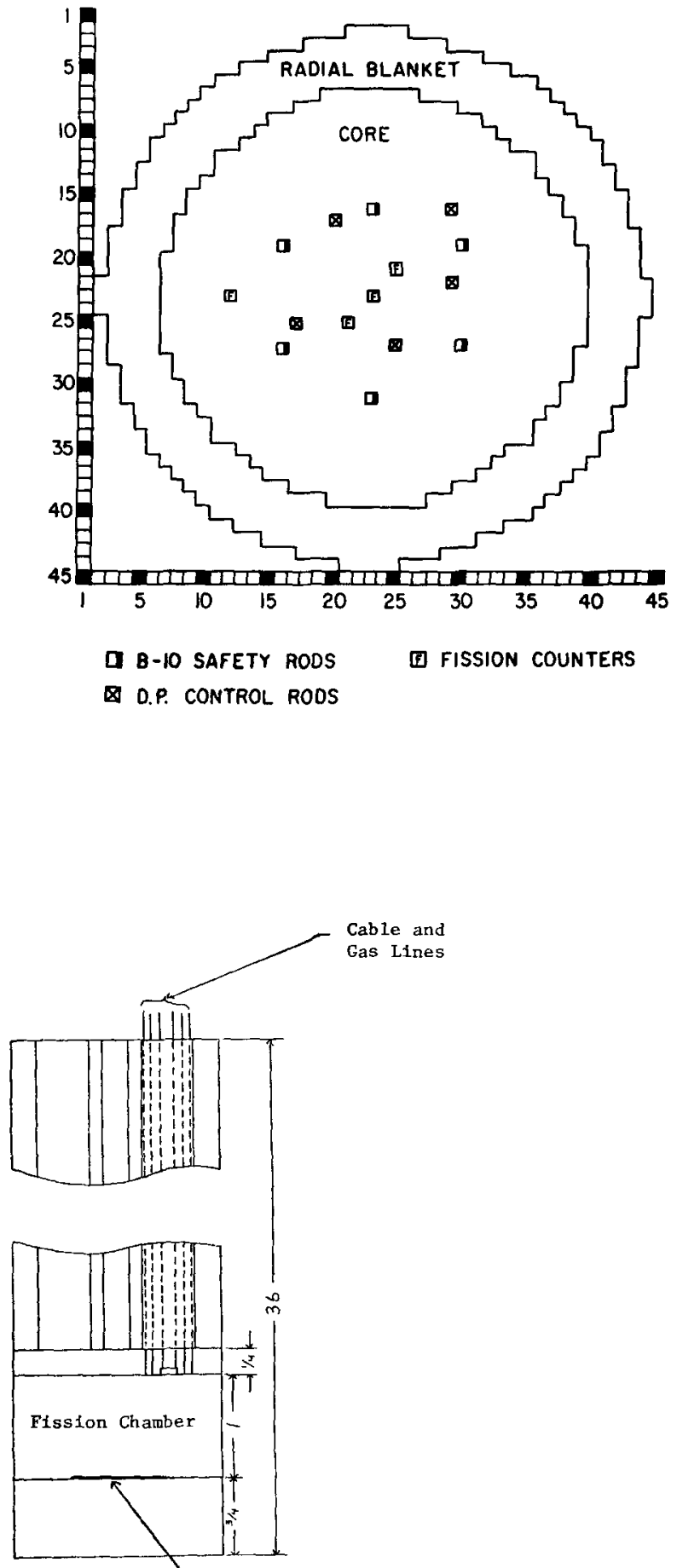

Source, $7 / 8$ diameter
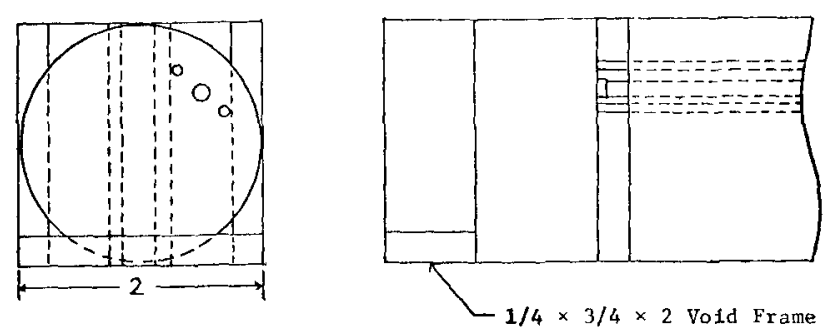

Fig. 30. ZPR-9 Core Configuration for the GCFR Phase-I Assembly. (ANL Neg. No. 116-76-279).
Fig. 31. Position of Fission Chamber Within Stationary Half Drawers. (A1I dimensions in inches.) (ANL Neg. No. 116-76-354). 


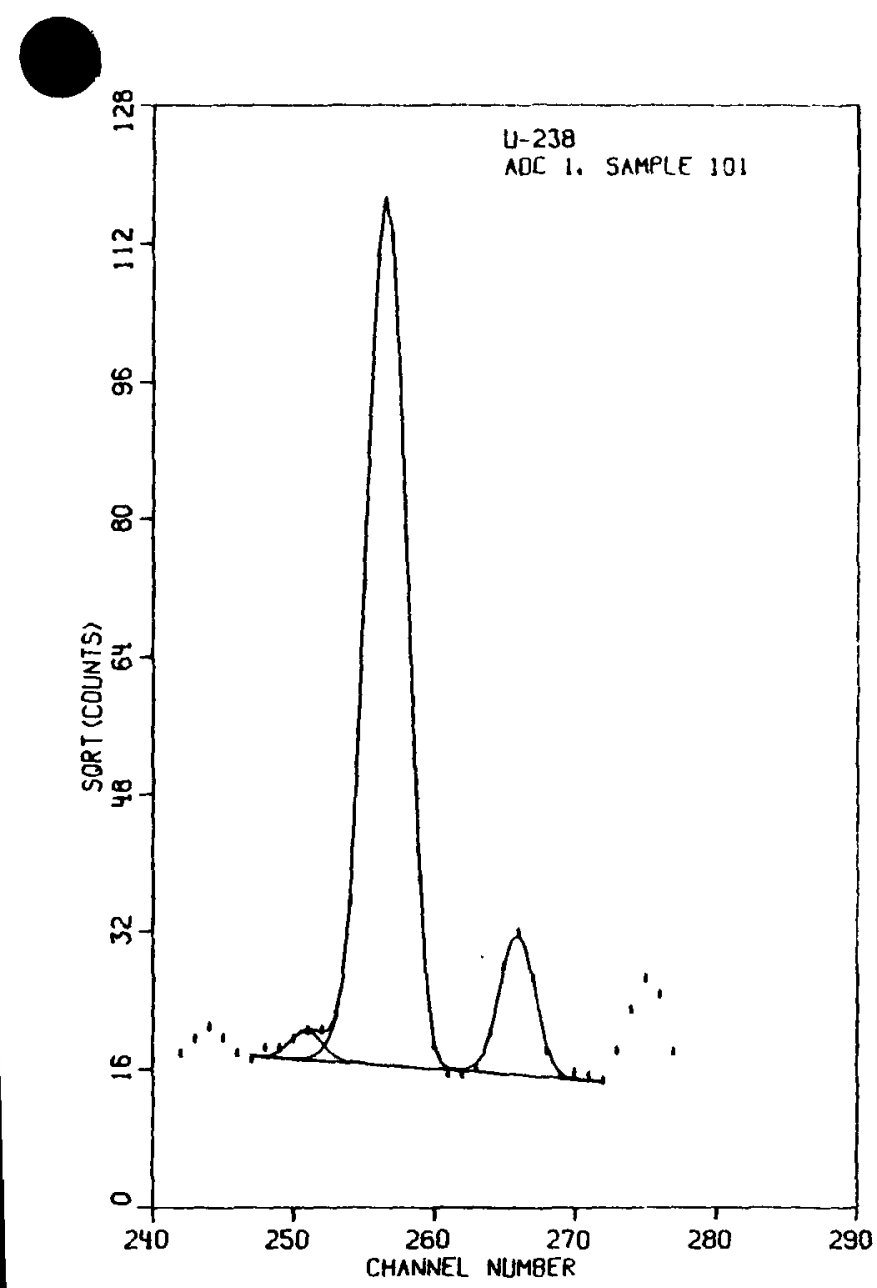

Fig. 32. Example of Computer Fit to the $227.6-\mathrm{keV}$ Photopeak in the Decay Spectrum of ${ }^{239} \mathrm{~Np}$. (ANL Neg. No. 116-76-306).

Fig. 33. Exapmple of Computer Fit to the 311.9-keV Photopeak in the Decay Spectrum of $233 \mathrm{~Pa}$. (ANL Neg. No. 116-76-327).

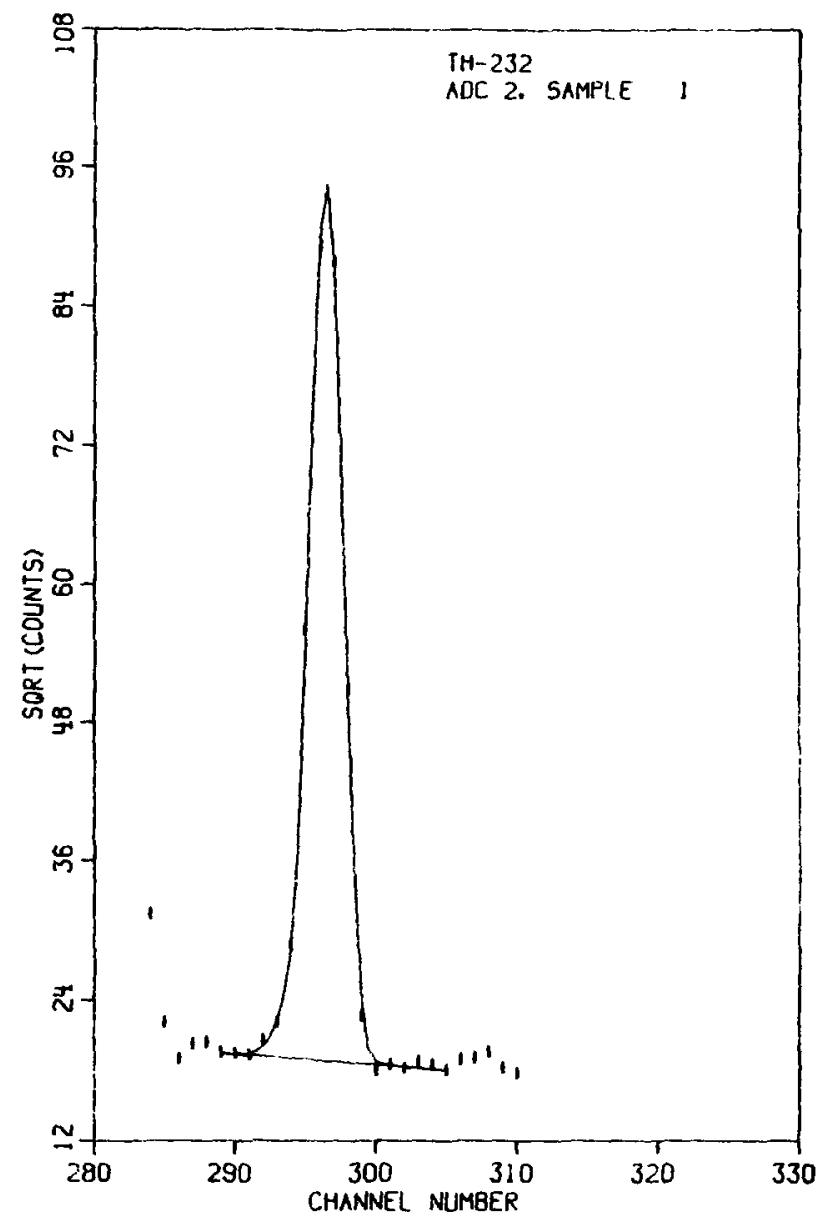




\section{GCFR PHASE 1}

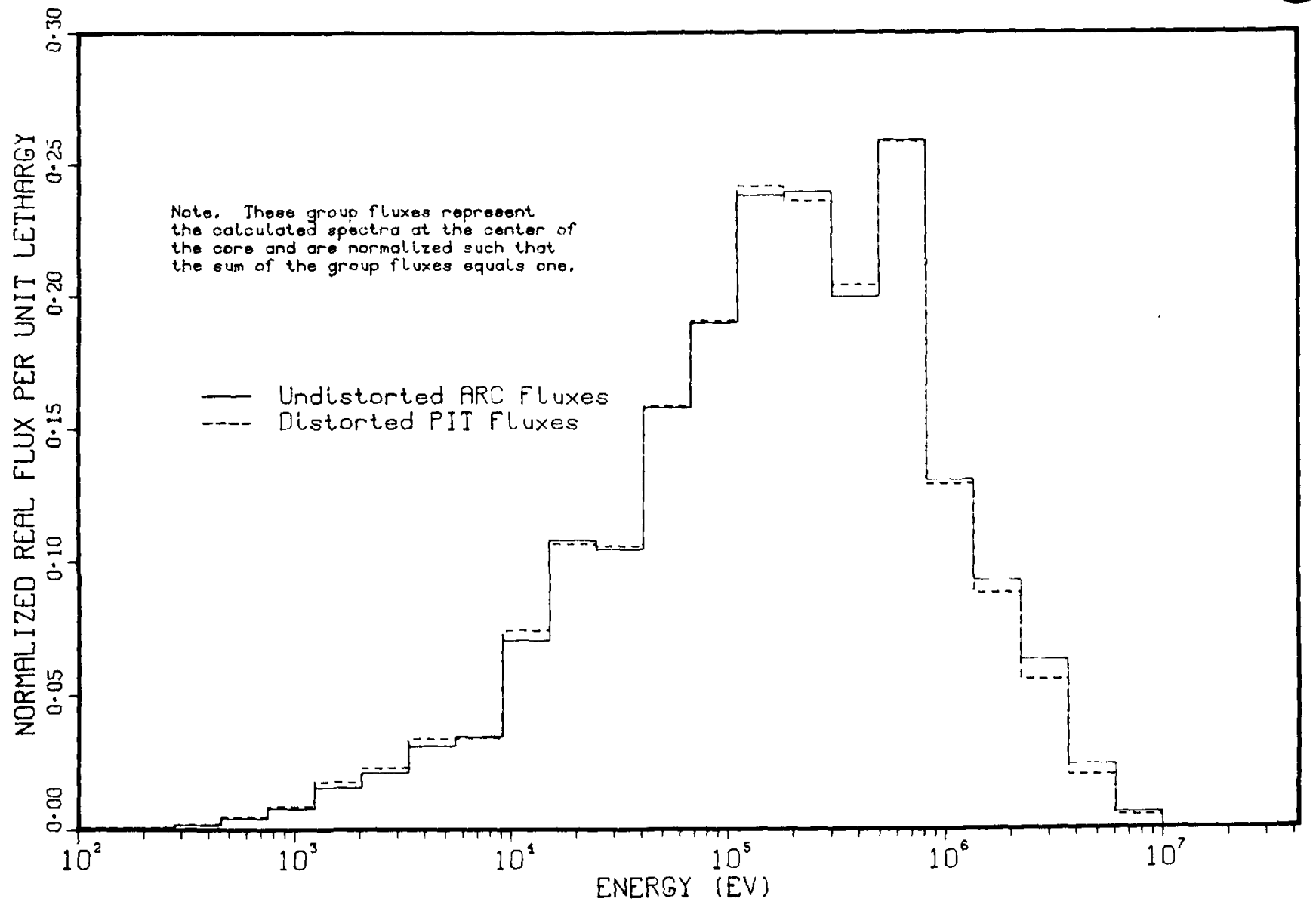

Fig. 34. Comparison of Undistorted ARC and Distorted PIT Broad Group Flux Spectra. (ANL Neg. No. 116-76-300).

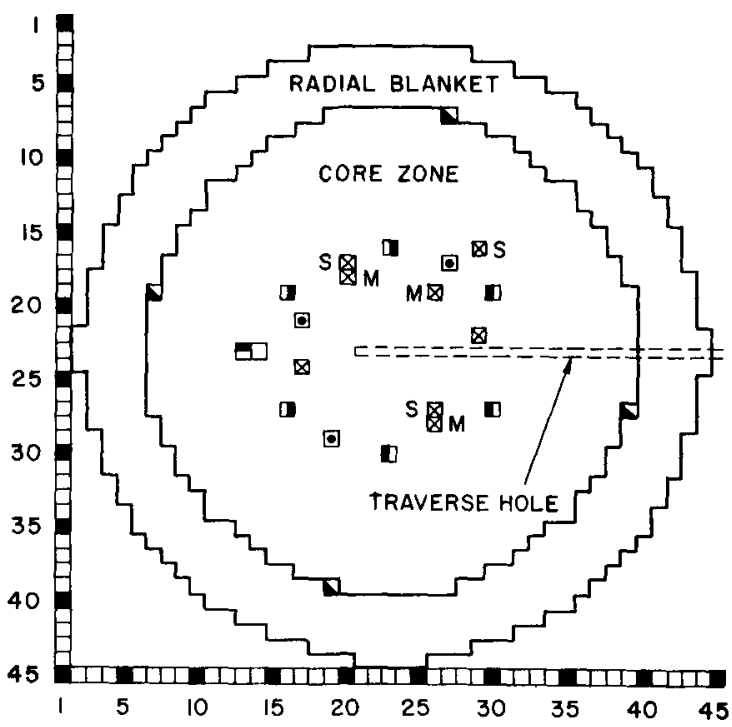

Fig. 35. Reactor Configuration for the Small Sample Central Reactivity Worth Measurements in the GCFR Phase-I Assembly. (ANL Neg. No. 116-76-360).

$\otimes$ DUAL PURPOSE CONTROL ROD

D $10_{B}$ SAFETy BLADES

$S \otimes$ STATIONARY HALF ONLY

$M \otimes$ MOVABLE HALF ONLY
- DOUBLE FUEL LOADING

๑ THERMOCOUPLE

$\square$ AUTOROD

D REACTIVITY ADJUSTMENT TUBE 


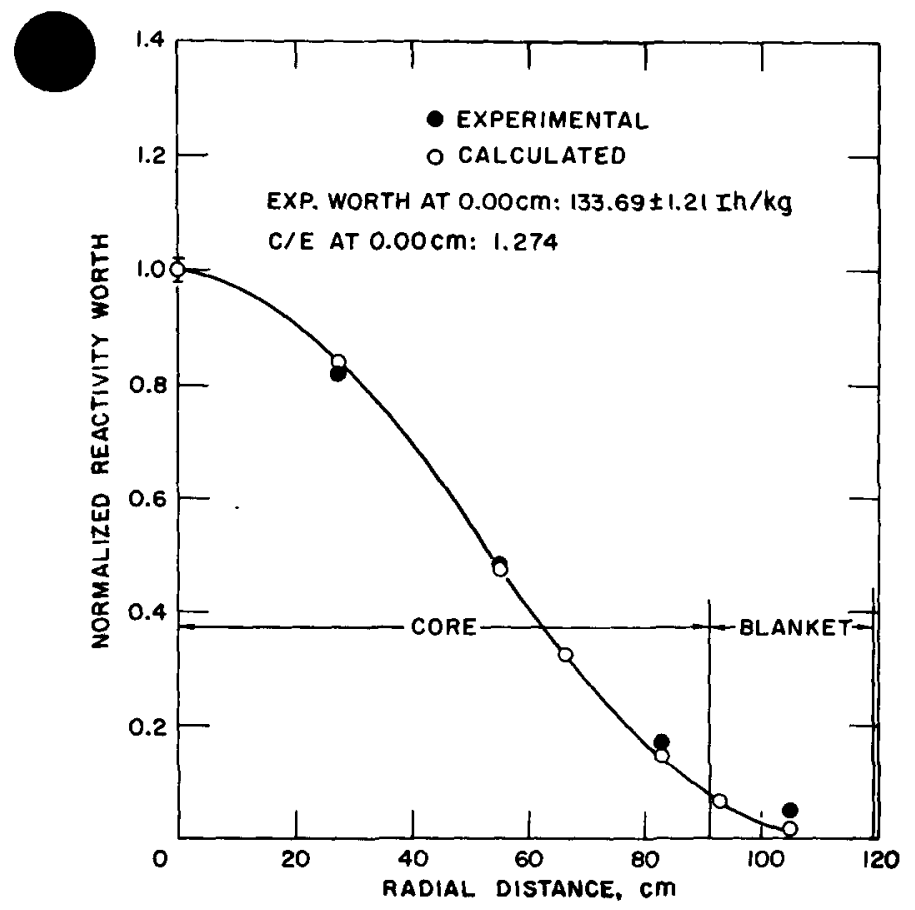

Fig. 36. Radial Traverse of $M B-10$ $\left({ }^{239} \mathrm{Pu}\right.$ ). (ANL Neg. No. 116-76-328).

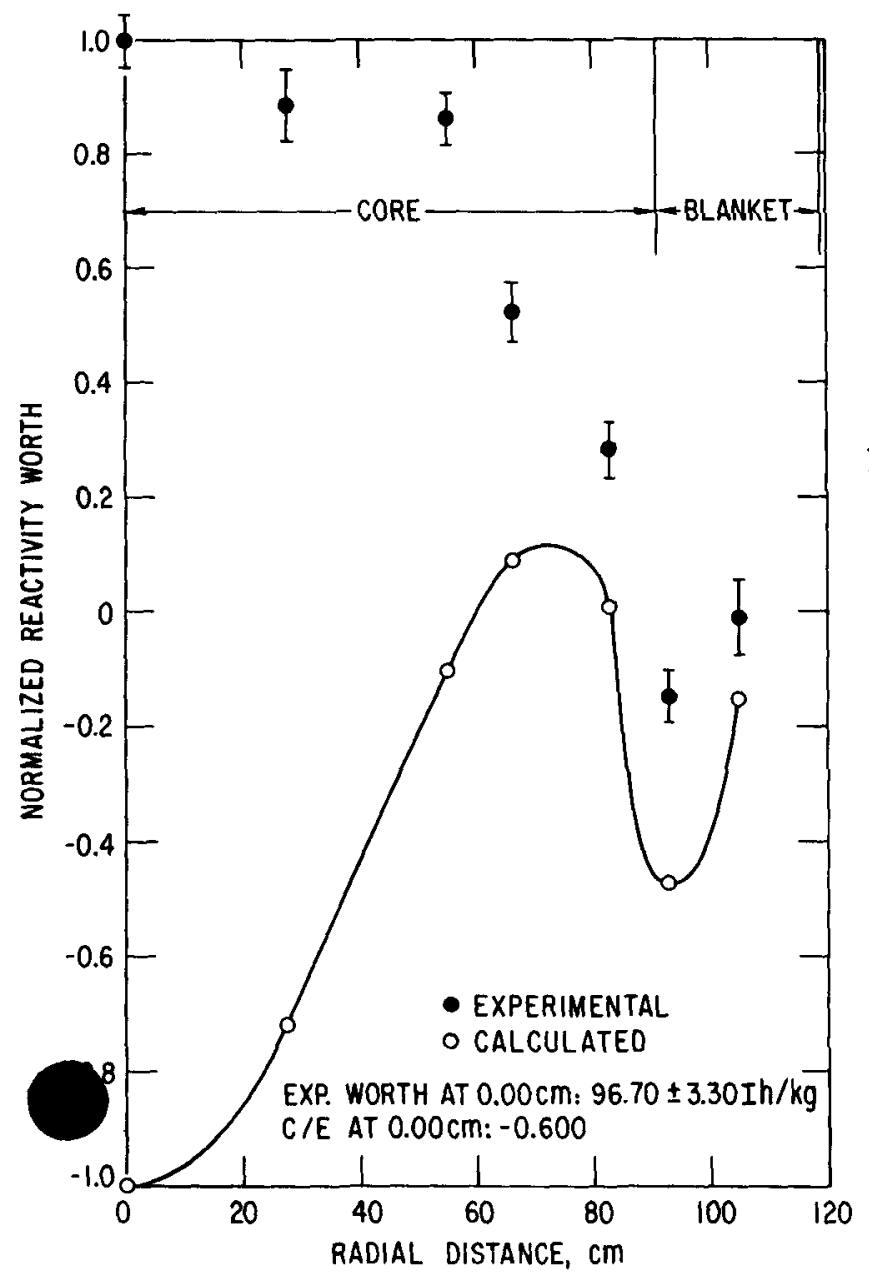

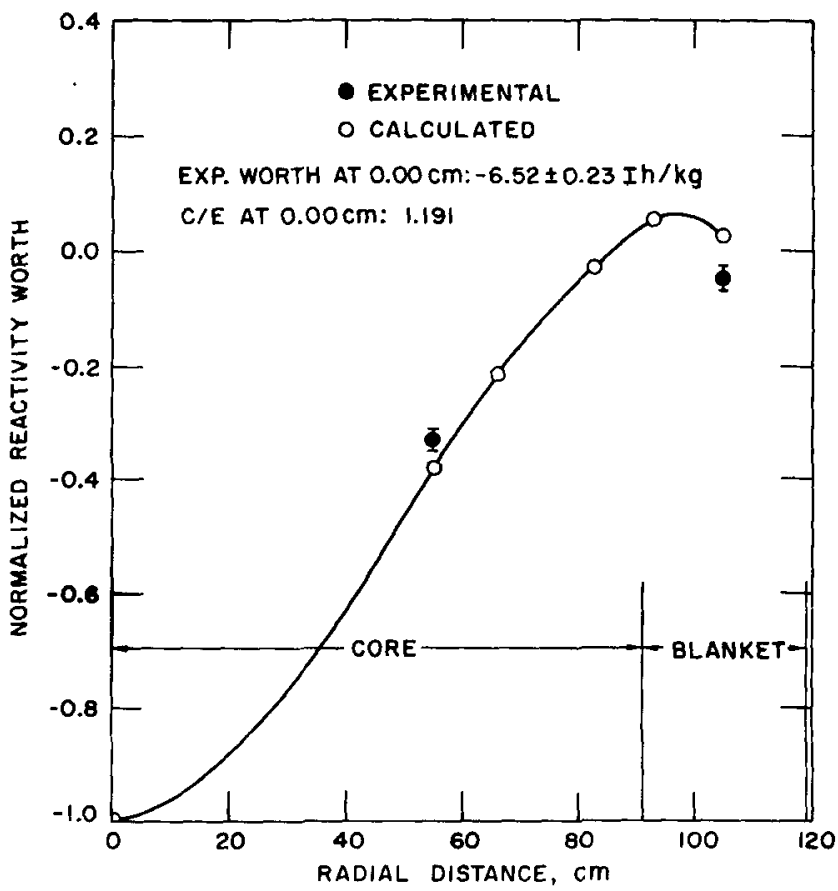

Fig. 37. Radial Traverse of MB-35 $\left({ }^{238} \mathrm{U}\right)$. (ANL Neg. No. 116-76-304).

Fig. 38. Radial Traverse of $\mathrm{CH}_{2}$ (F)-4 (Polyethylene Foam). ANL Neg. No. 116-76-288). 


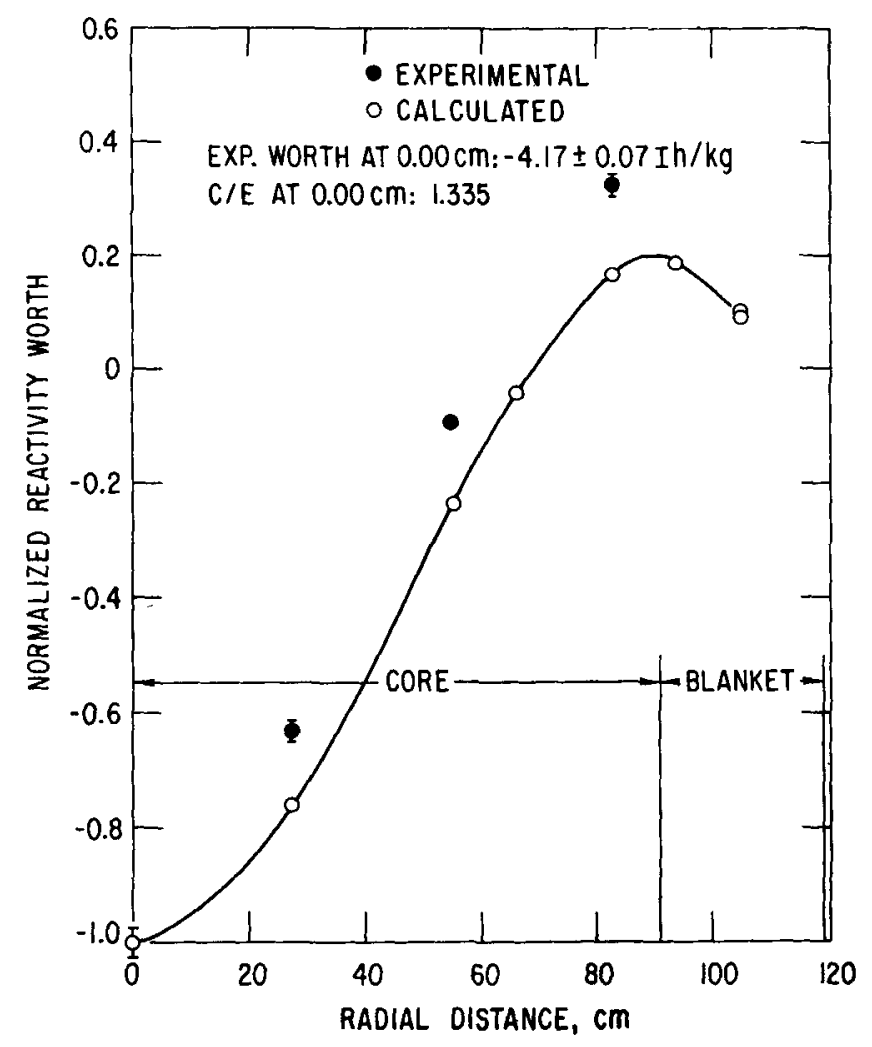

Fig. 39. Radial Traverse of 304 SST. (ANL Neg. No. 116-76-347). 


\section{REACTIVITY WORTH OF CALIFORNIUM}

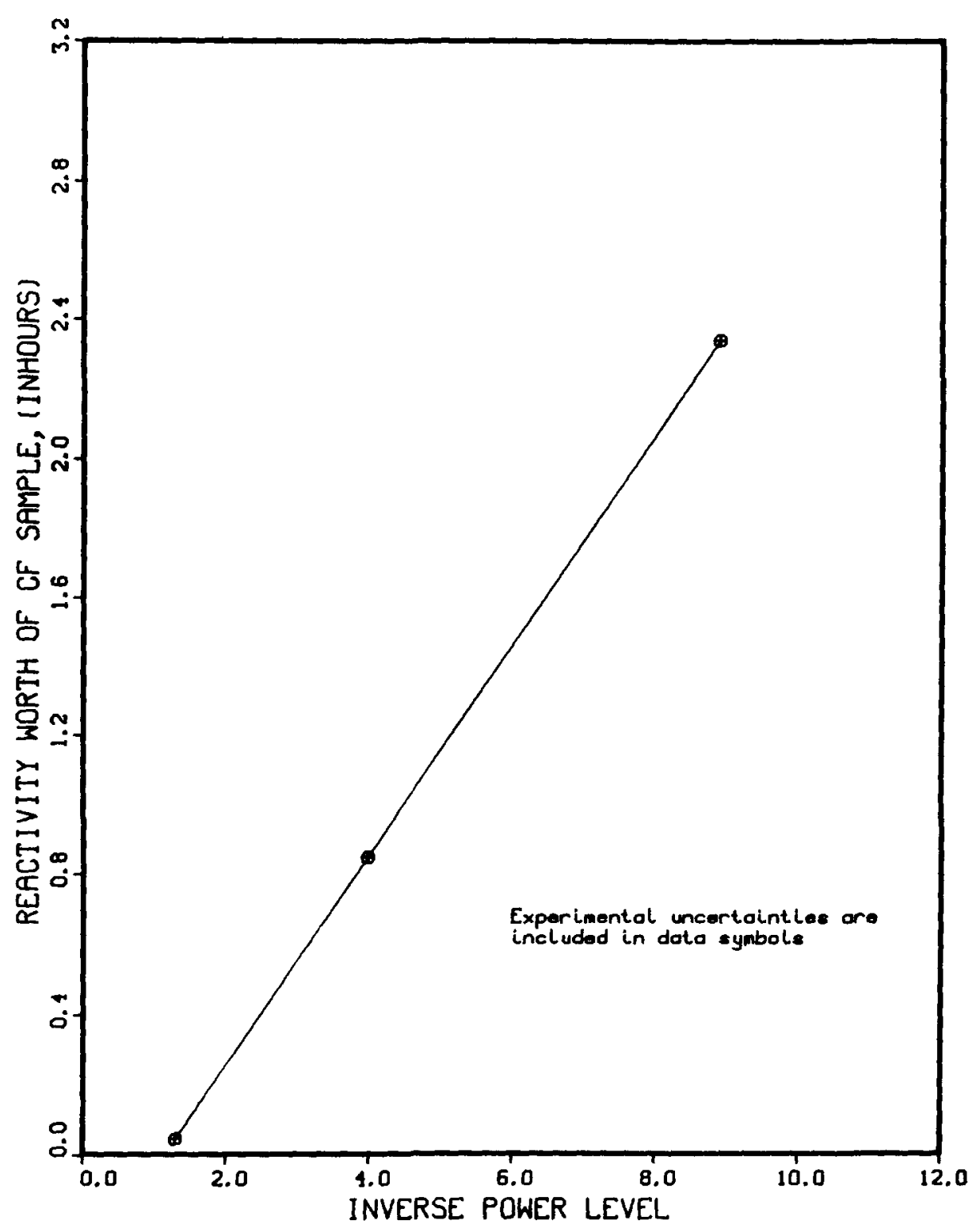

Fig. 40. Variation of the Reactivity Worth of ${ }^{252}$ Cf With Inverse Power Leve1. (ANL Neg. No. 116-76-294) 


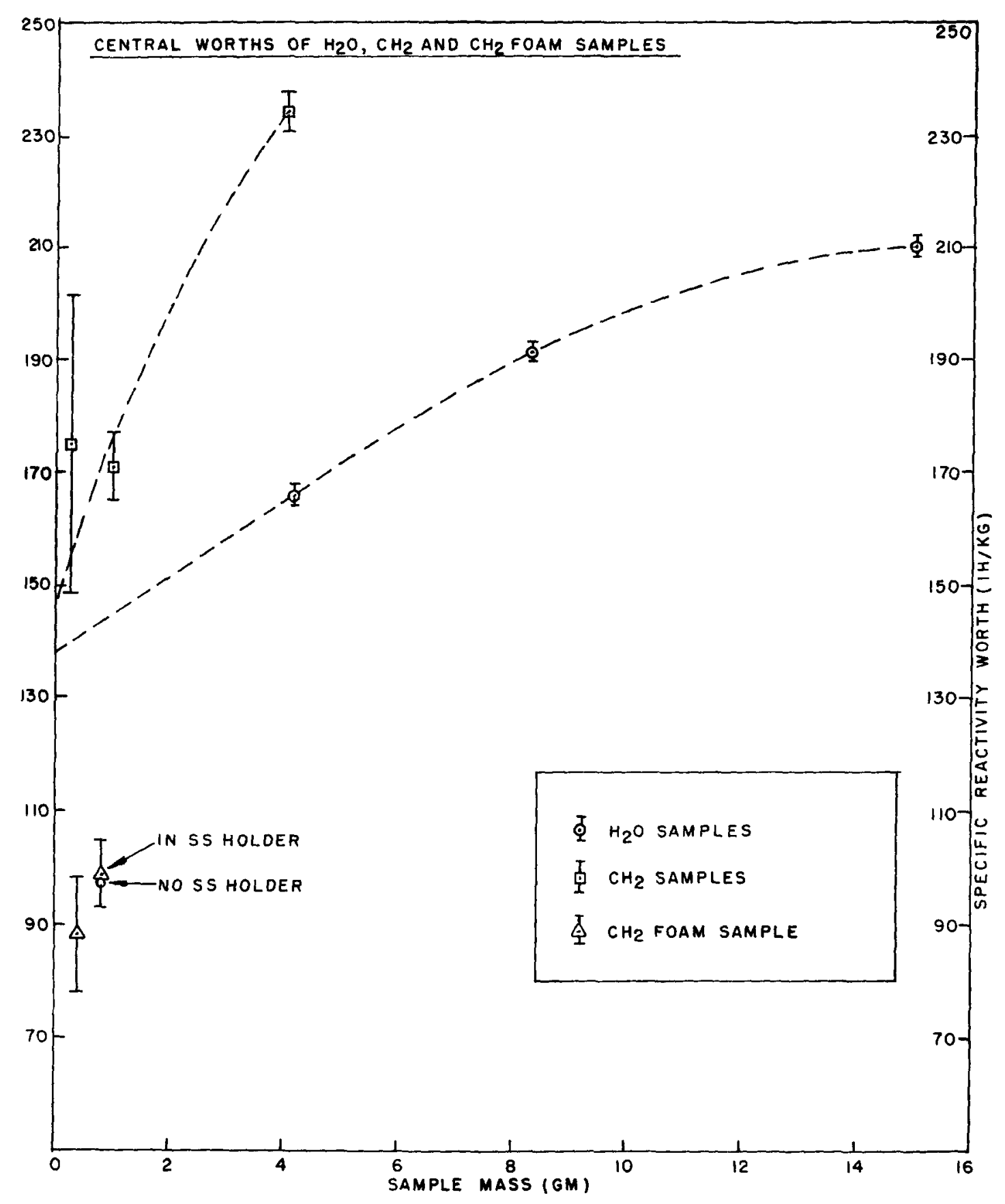

Fig. 41. Central Reactivity Worth vs Sample Mass of $\mathrm{H}_{2} \mathrm{O}, \mathrm{CH}_{2}$, and $\mathrm{CH}_{2}$ Foam Samples. (ANL Neg. No. 116-76-311). 


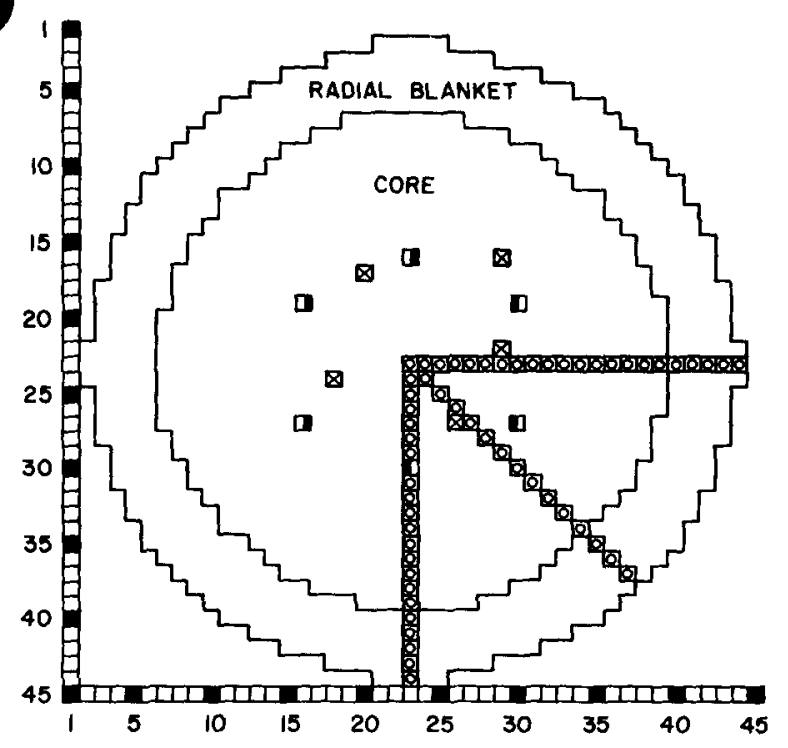

Fig. 42. ZPR-9 Core Configuration for the GCFR Phase-I Assemb1y. (ANL Neg. No. 116-76-379).

D. B-10 SAFETY RODS

GOIL LOCATIONS

$\triangle$ D.P. CONTROL RODS

Fig. 43. Illustration of the Checkerboard Loading Concept. The actual unit cell compositions in the drawers are shown in Fig. 2. (ANL Neg. No.
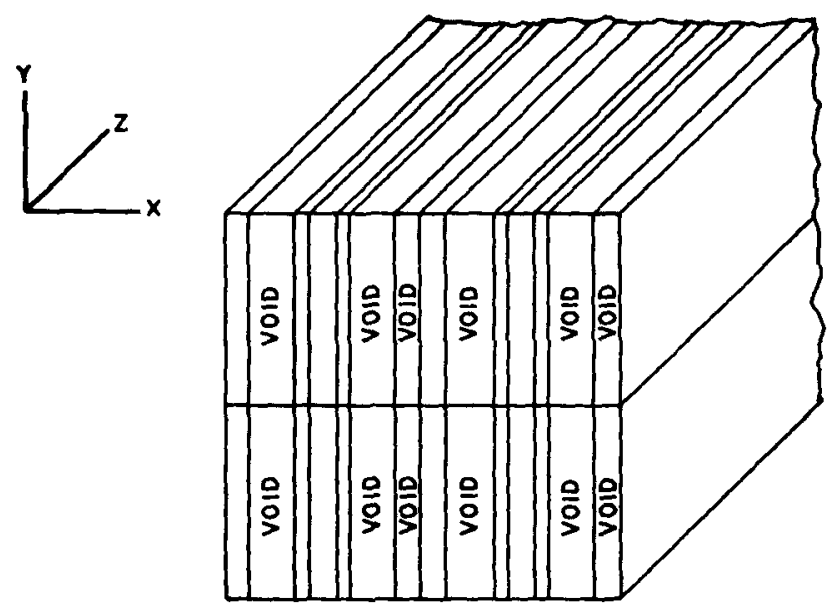
116-76-318).

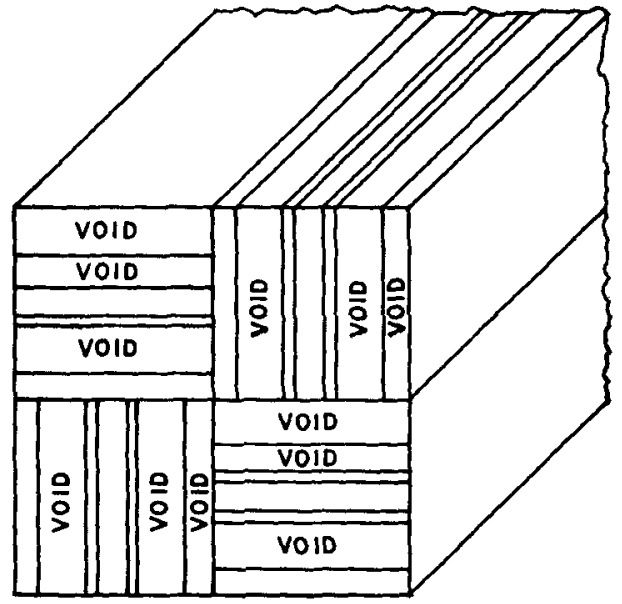




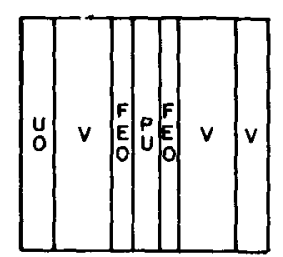

CORE REGION:

NORMAL, VERTICAL PLATE STRUCTURE

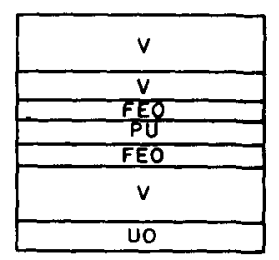

CORE REGION:

hORIZONTAL PLATE STRUCTURE

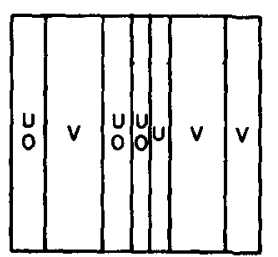

AXIAL BLANKET

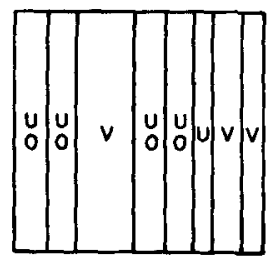

RADIAL BLANKET

KEY:

UO $=\mathrm{U}_{3} \mathrm{O}_{3}$ PLATE

$U=$ DUPL. U PLATE

$\mathrm{PU}=\mathrm{PU}-\mathrm{U}-\mathrm{MO}$ CAN

$F E O=\mathrm{F}_{2} \mathrm{O}_{3}$ PLATE

$V=V O I D$ CAN
Fig. 45. Foil and Horizontally Loaded Drawer Locations for the Checkerboard Streaming Experiment in the GCFR Phase-I Assembly. (ANL Neg. No. 116-76-309).
Fig. 44. Unit Cell Structures for the Checkerboard Streaming Experiment in the GCFR Phase-I Assembly as Viewed From BeTween the Assembly Halves, Facing the Stationary Half. (ANL Neg. No. 116-76-351).

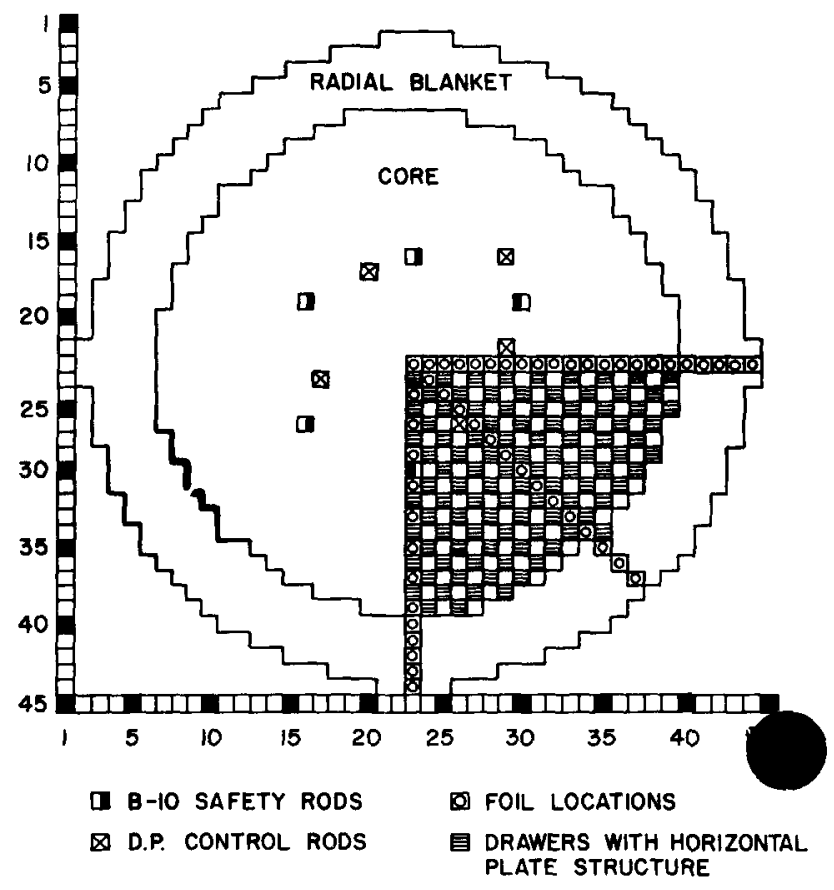



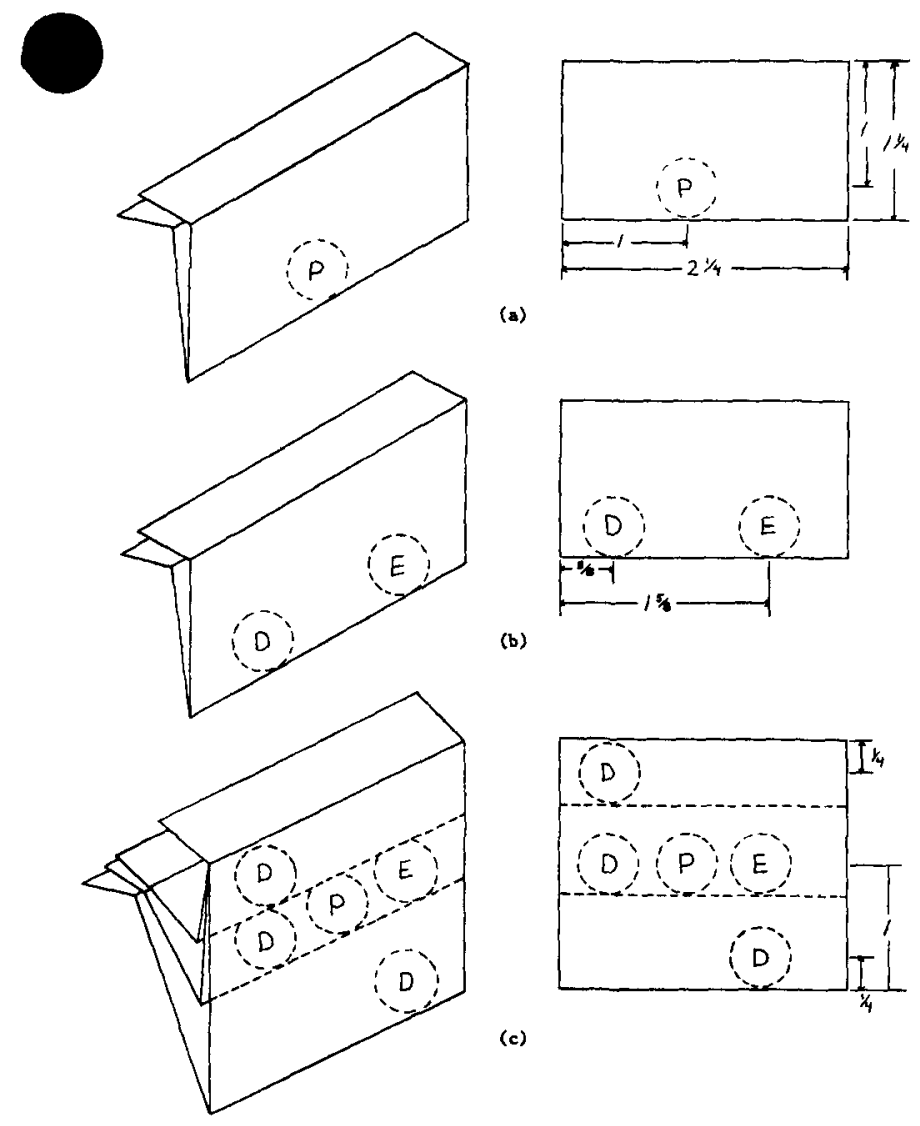

(a)

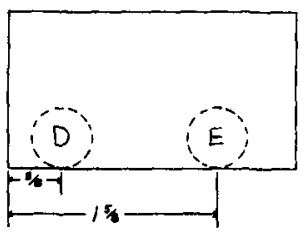

Fig. 46. Aluminum (0.001 in. thick)

Foil Holders for Foil

Irradiations Showing Positions of Plutonium (P) Foils and

(b)

Enriched (E) and Depleted

(D) Uranium Foils. (ANL

Neg. No. 116-76-303).

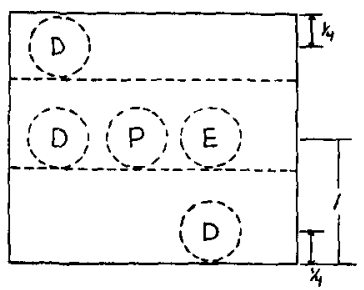

(c)

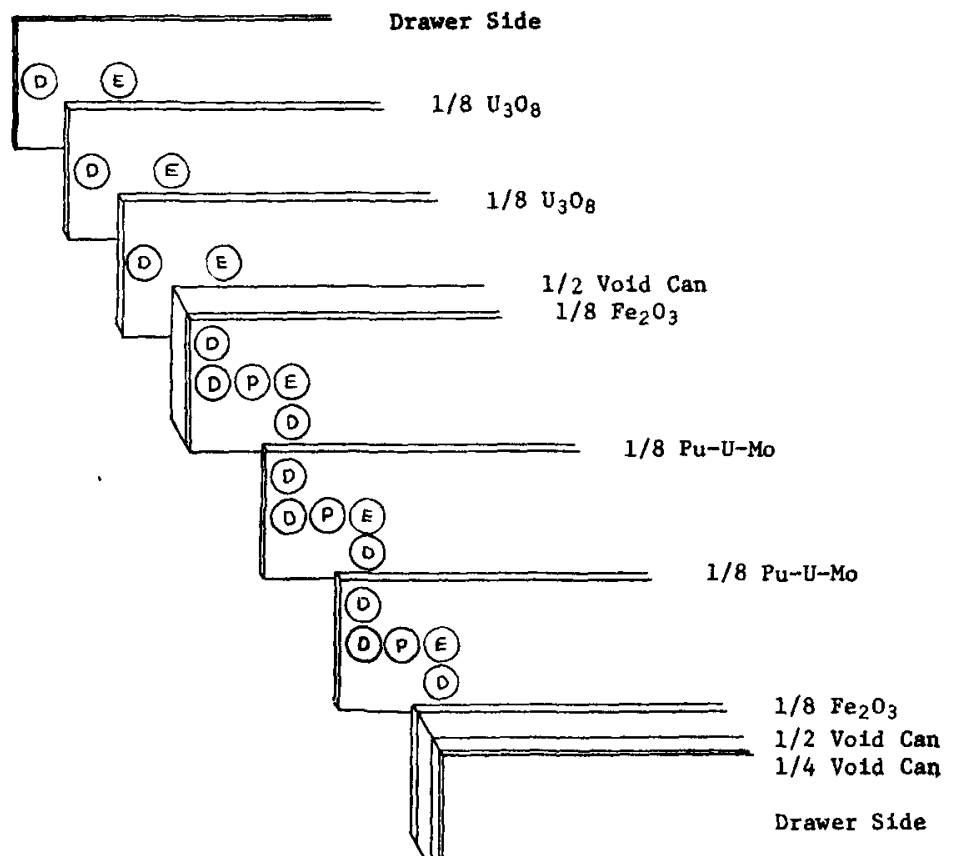

Fig. 47. Depleted (D) and Enriched (E) Uranium and Plutonium (P) Foil Locations for Unit Ce11 Measurements in the Core Region. (ANL Neg. No. 116-76-321). 


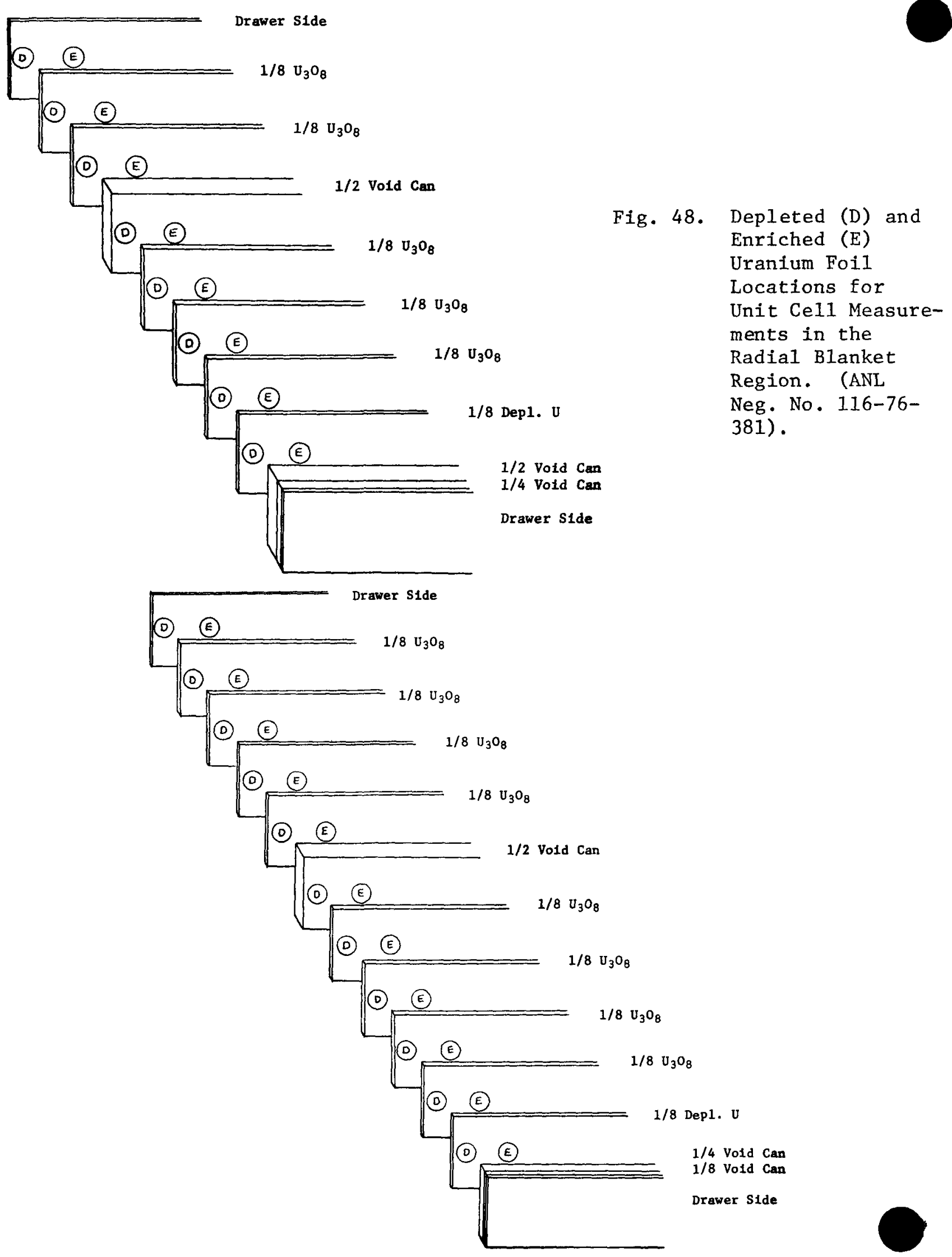

Fig. 49. Depleted (D) and Enriched (E) Uranium Foil Locations for Unit Cell Measurements in the Axial Blanket Region. (ANL Neg. No. 116-76-289). 


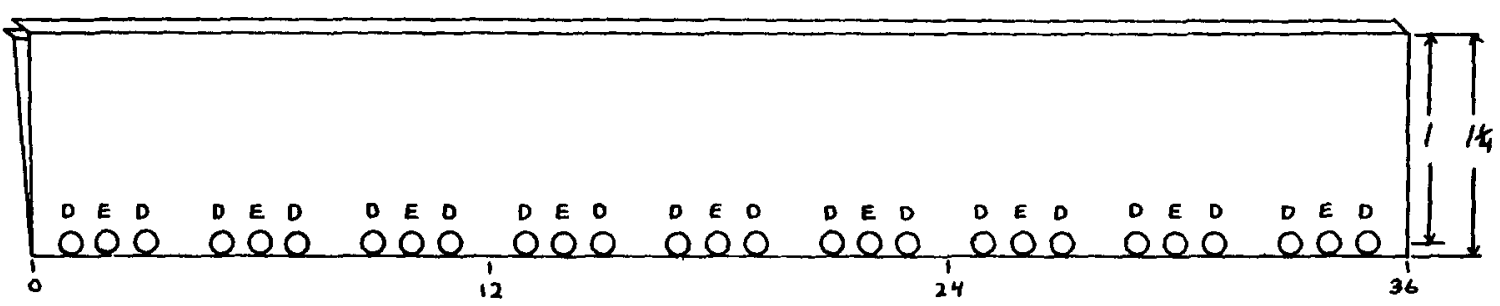

(b)

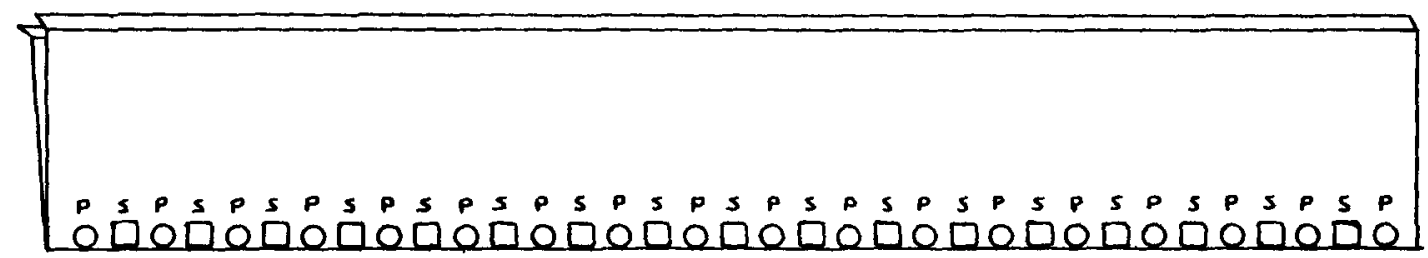

(a)

Fig. 50. Axial Traverse Foil Holders (0.001 in. aluminum) Showing Positions of Enriched (E) and Depleted (D) Uranium Foils, and Plutonium (F) Foils and Spacers (S). (ANL Neg. No. 11676-345).

Fig. 51. Pin Zone Location in the Movable Half of ZPR-9. (ANL Neg. No. 116-76-356).

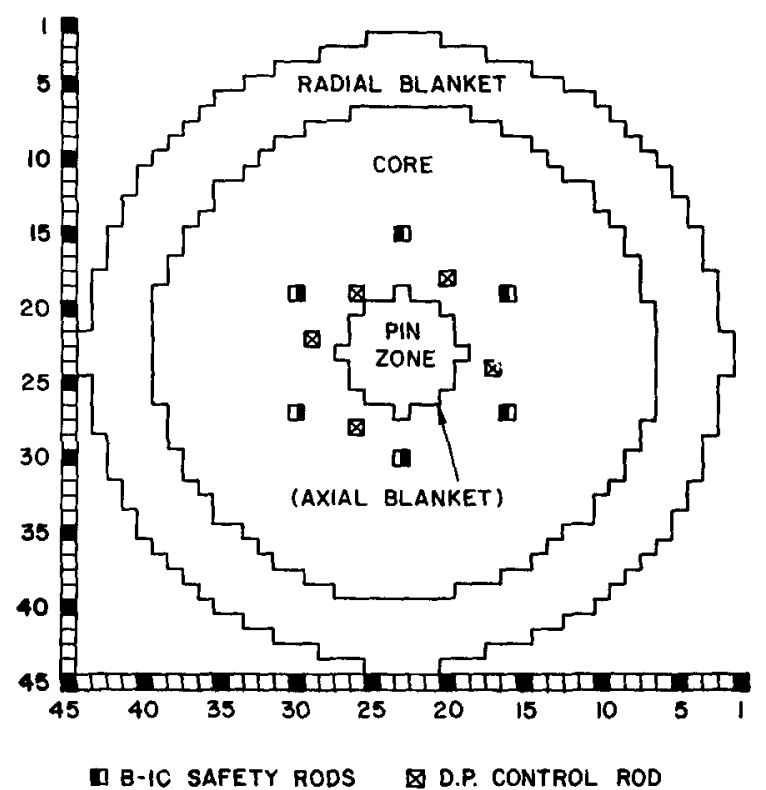



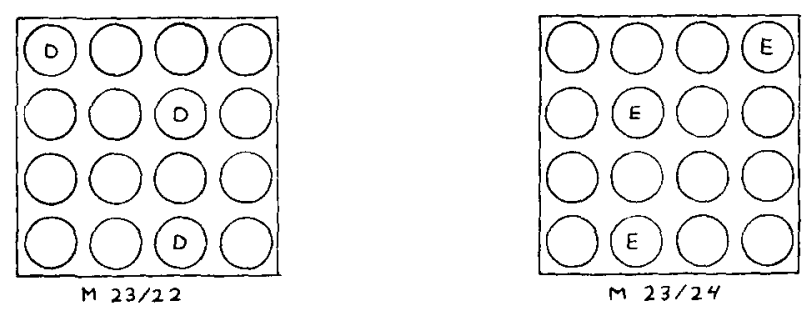

Fig. 52. Location of Pins Containing Enriched (E) and Depleted (D) Uranium Foils in the Pin Zone, and Position of Foils on top of Pins (Depleted on top of M23/22, Enriched on top of M23/24). (ANL Neg. No. 116-76-314).

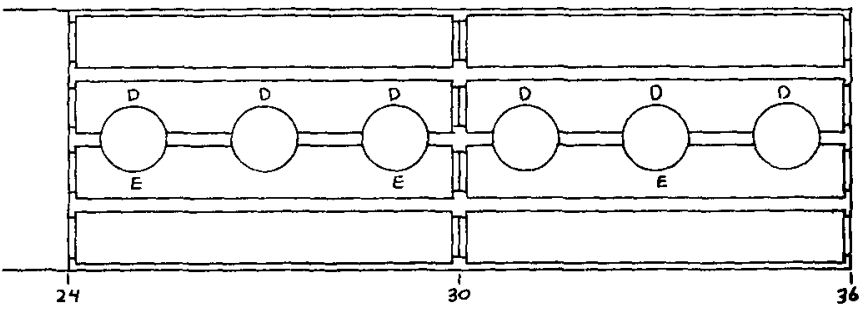

12 in. Calandria Can

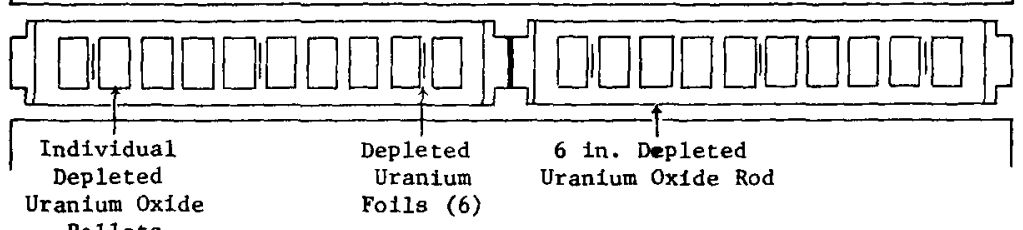

Pellets

(a) Foll positions for three rods in drawer $M 23 / 22$.

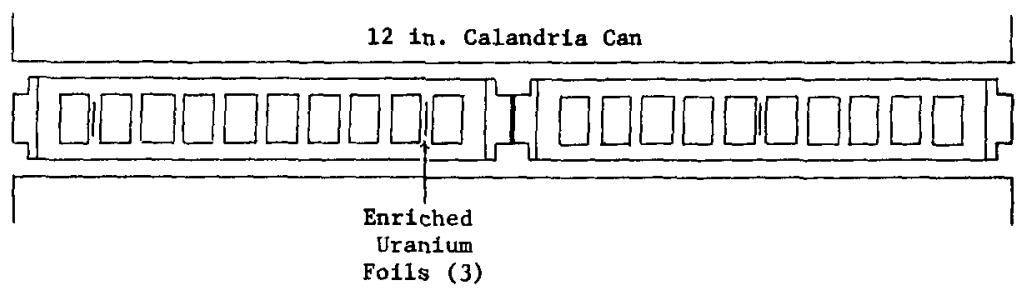

(b) Pol1 posttions for three rods in drawer M23/24.

Fig. 53. Approximate Location of Enriched and Depleted Uranium Foils Within Selected Rods of the Pin Zone. (ANL Neg. No. 116-76-284). 
Core Region Unit Cell

s22/22 $0-2$ in.

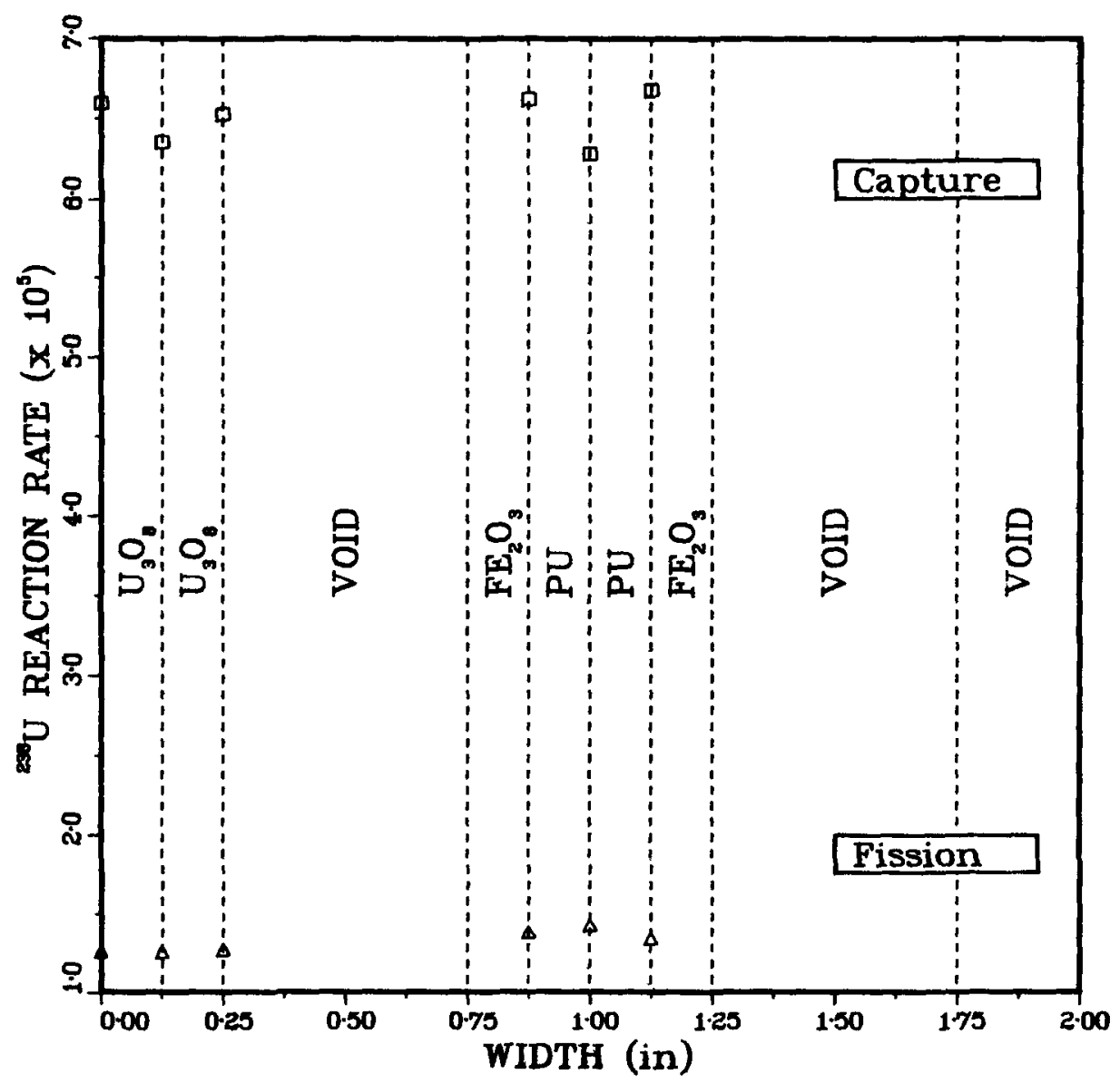

Fig. 54. Typical ${ }^{238} \mathrm{U}$ Reaction Rate Distributions in a Core Region Unit Cell. (ANL Neg. No. 116-76-348). 
Radial Blanket Unit Cell

\$40/22 $0-2$ in.

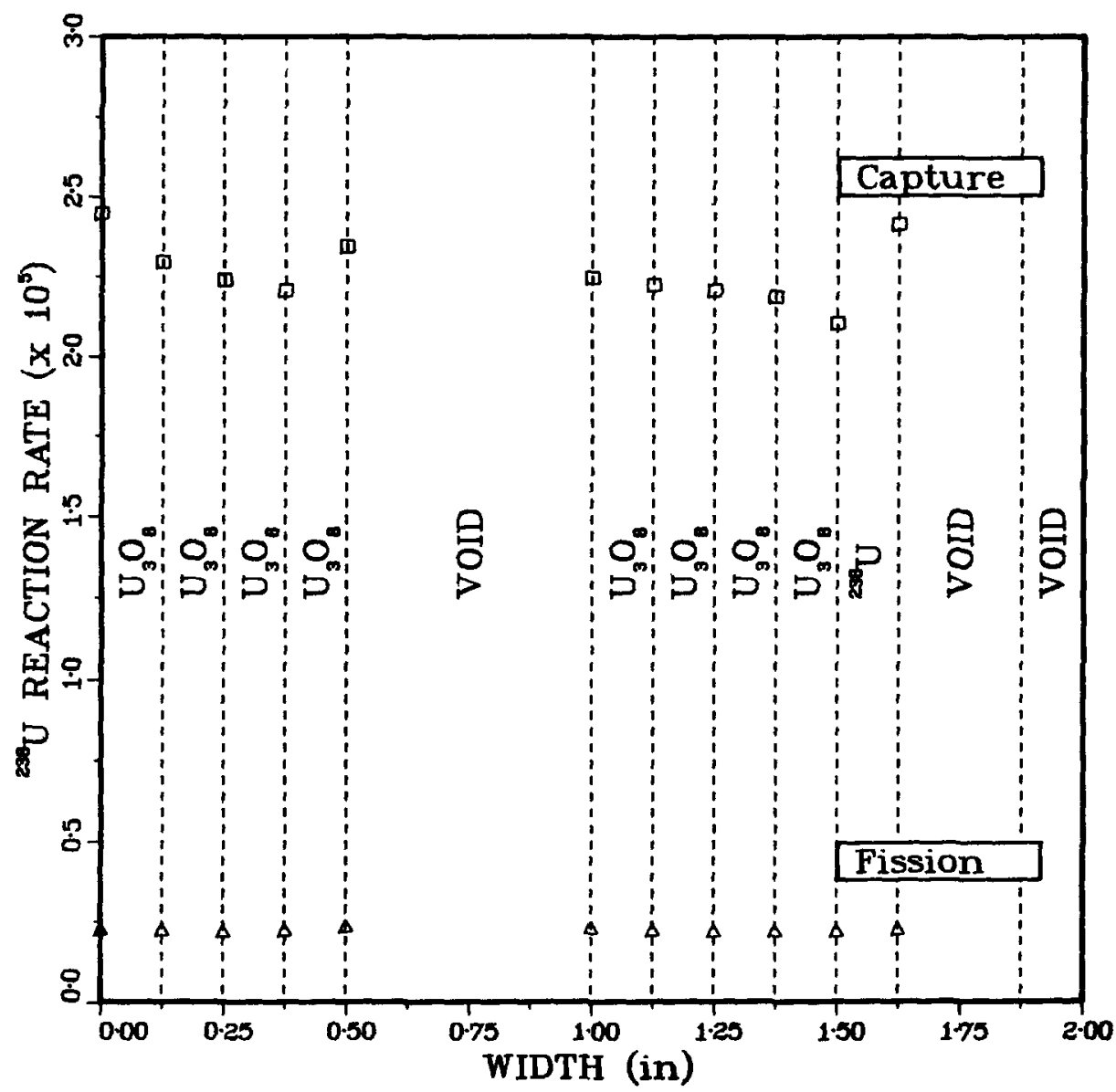

Fig. 55. Typical ${ }^{238} \mathrm{U}$ Reaction Rate Distributions in a Radial Blanket Region Unit Cell. (ANL Neg. No. 116-76-355). 


\section{Axial Blanket Unit Cell}

s22/22 24-26 in.

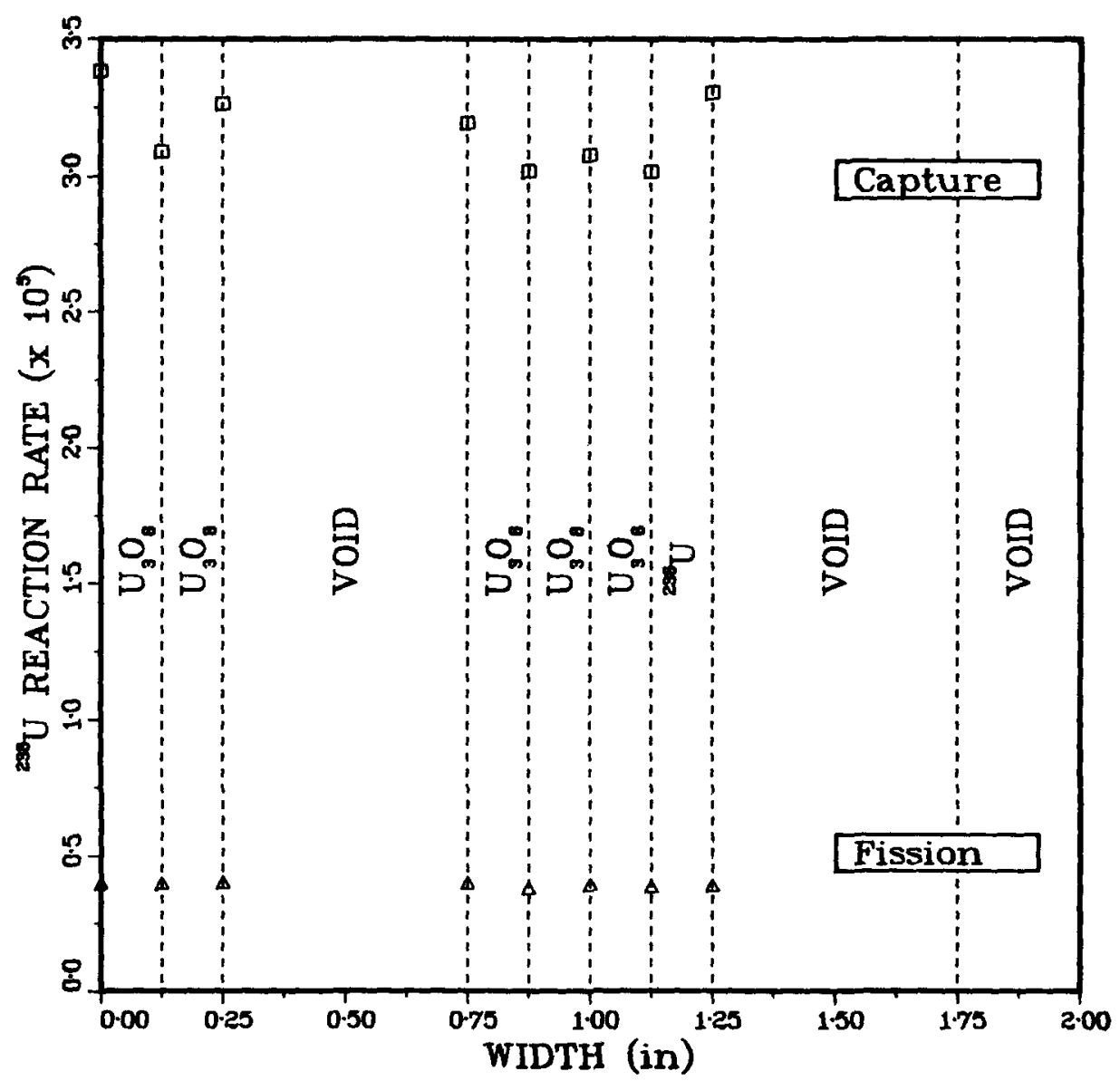

Fig. 56. Typical ${ }^{238} \mathrm{U}$ Reaction Rate Distributions in an Axial Blanket Region Unit Cell. (ANL Neg. No. 116-76-295). 
${ }^{230} \mathrm{U}$ Fission Rate, $0^{\circ}$ Traverse

Normal Loading

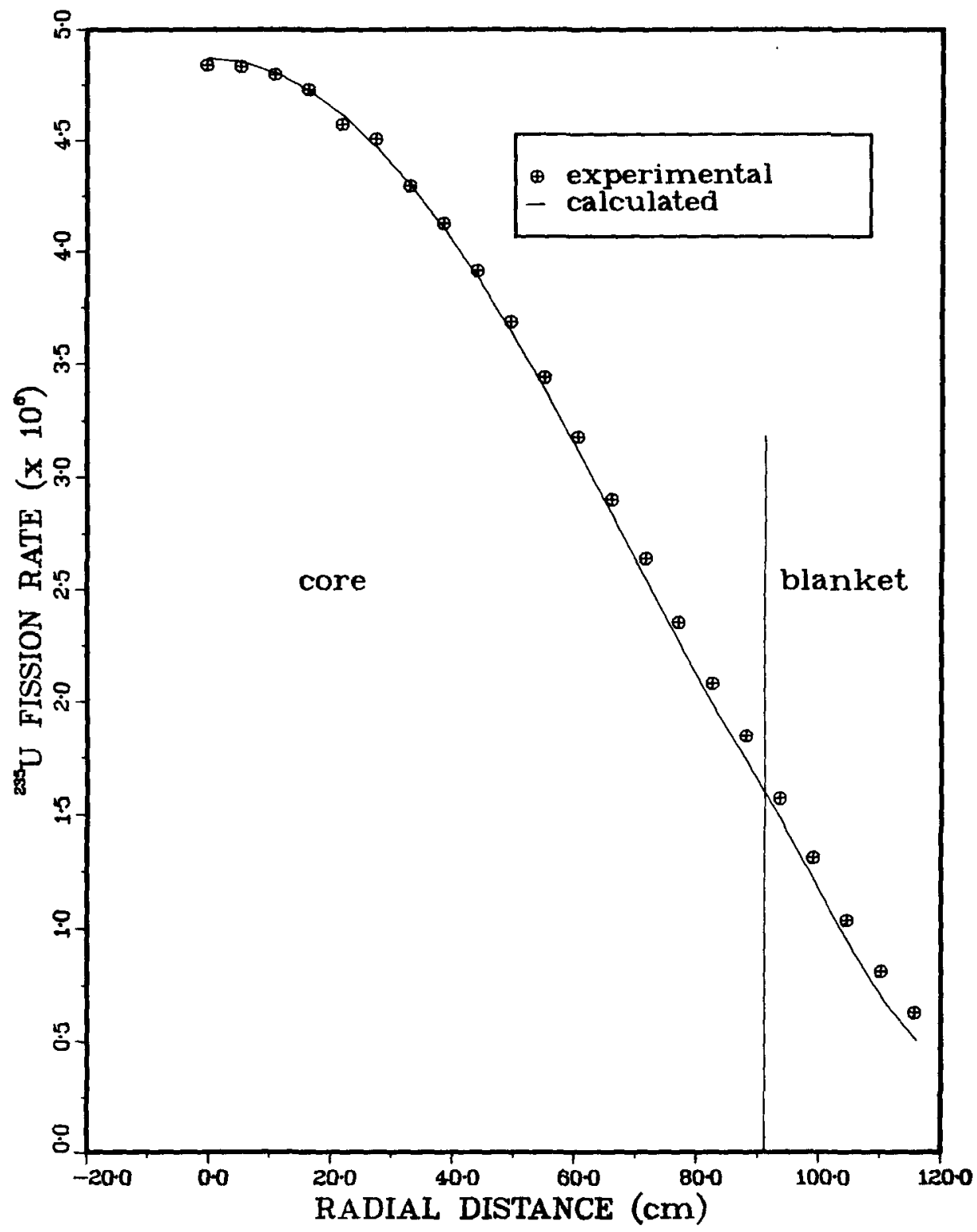

Fig. 57. Radial ${ }^{235} \mathrm{U}$ Fission Rate Distribution, Diagonal. (ANL Neg. No. 116-76-343). 
${ }^{230} \mathrm{U}$ Fission Rate, $45^{\circ}$ Traverse

Normal Loading

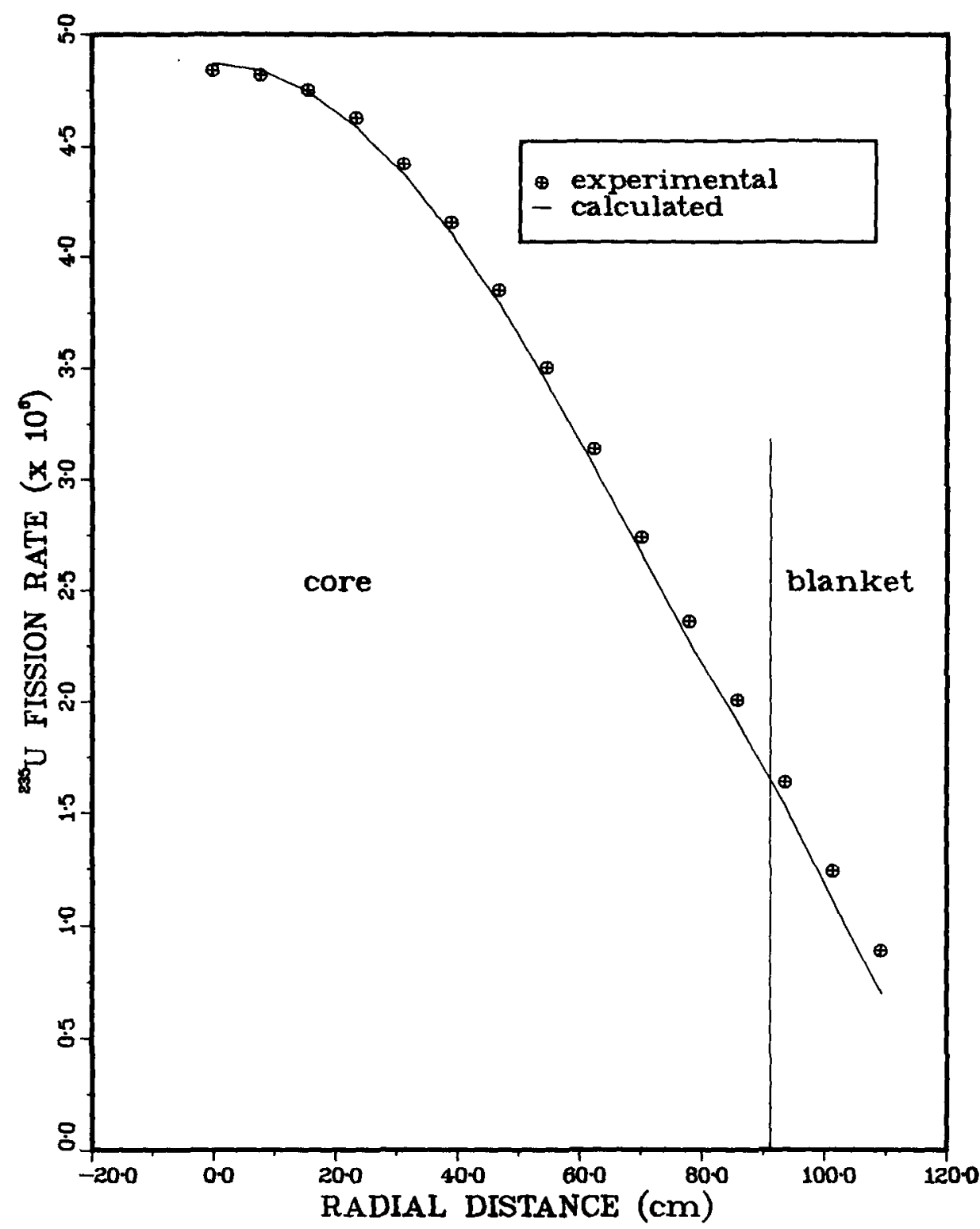

Fig. 58. Radial ${ }^{235} \mathrm{U}$ Fission Rate Distribution, Diagonal. (ANL Neg. No. 116-76-341). 
${ }^{230} \mathrm{U}$ Fission Rate, $90^{\circ}$ Traverse

Normal Loading

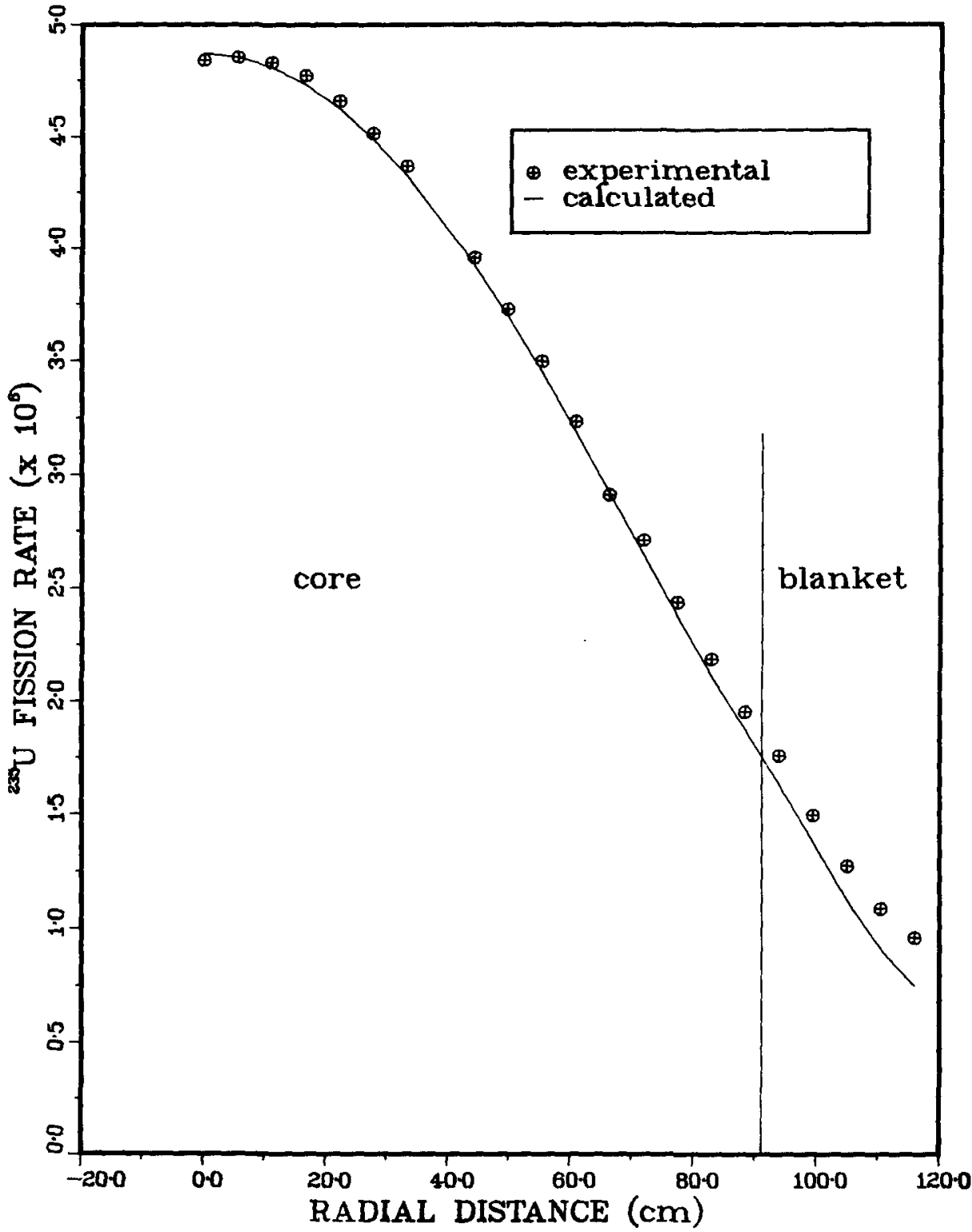

Fig. 59. Radial ${ }^{235} \mathrm{U}$ Fission Rate Distribution, Column 23. (ANL Neg. No. 116-76-344). 
${ }^{230} \mathrm{U}$ Fission Rate, $0^{\circ}$ Traverse

Normal Loading

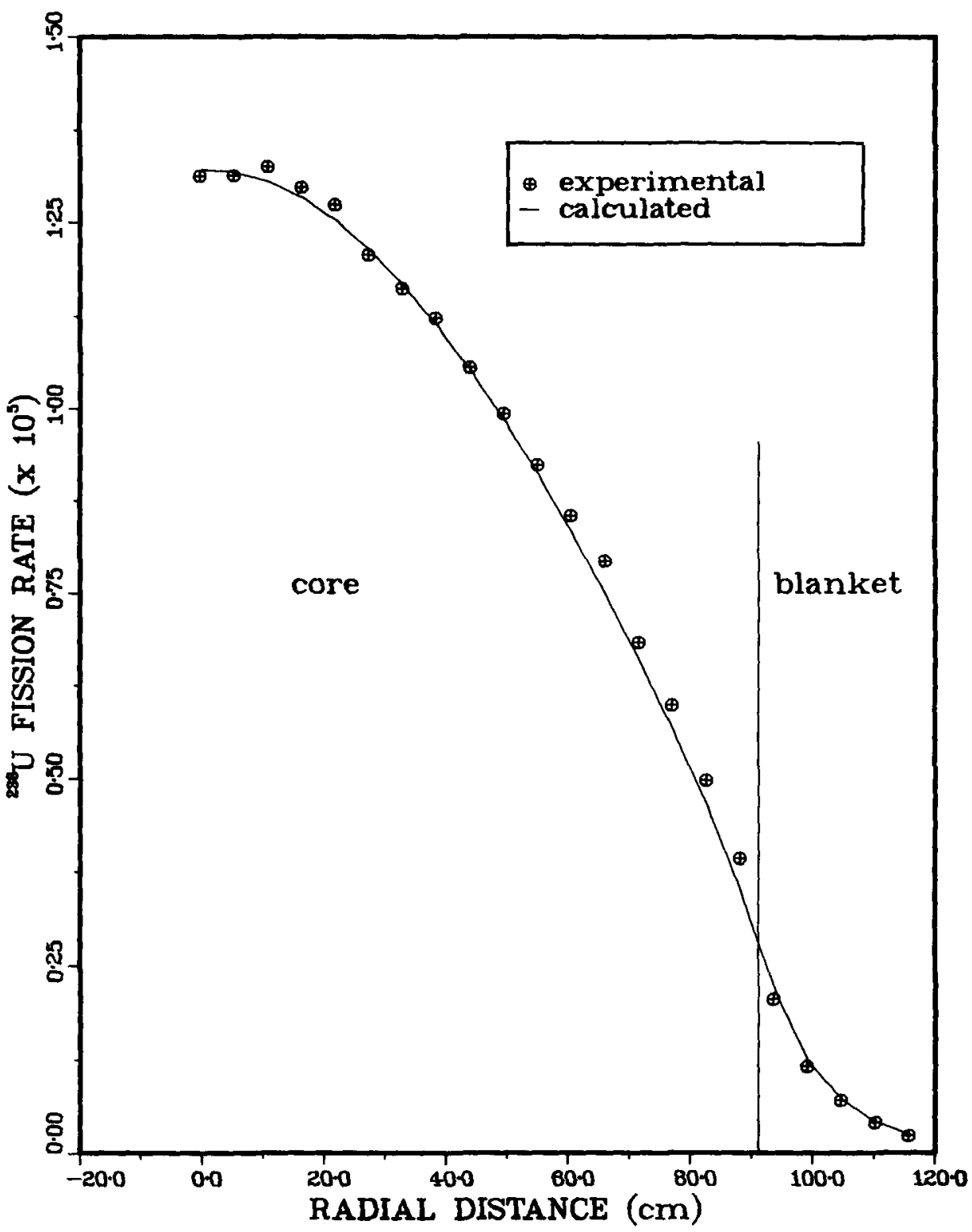

Fig. 60. Radial ${ }^{238}$ U Fission Rate Distribution, Row 23. (ANL Neg. No. 116-76-357). 
200 Fission Rate, $45^{\circ}$ Traverse

Normal Loading

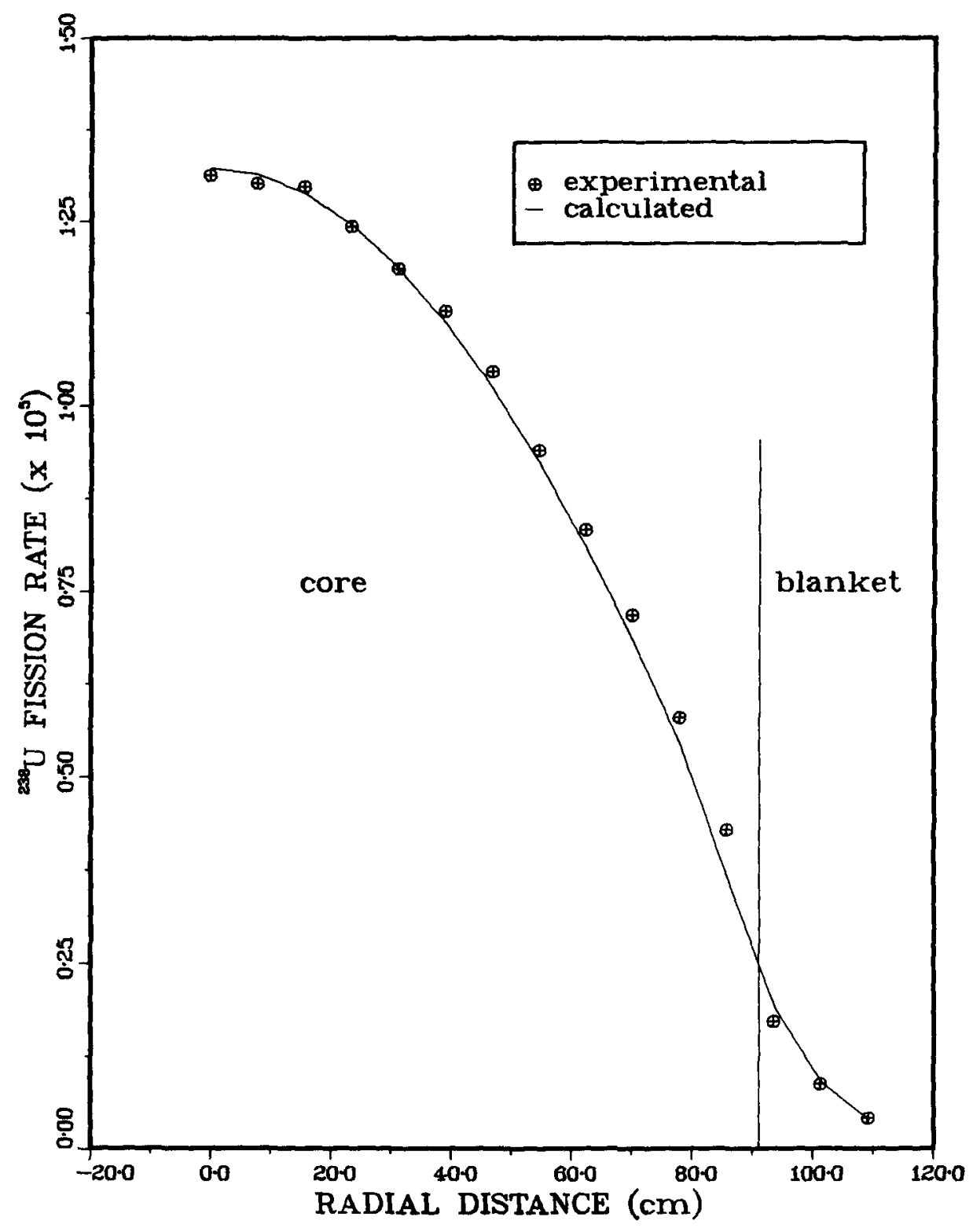

Fig. 61. Radial ${ }^{238} \mathrm{U}$ Fission Rate Distribution, Diagona1. (ANL Neg. No. 116-76-359). 
${ }^{230} \mathrm{U}$ Fission Rate, $90^{\circ}$ Traverse

Normal Loading

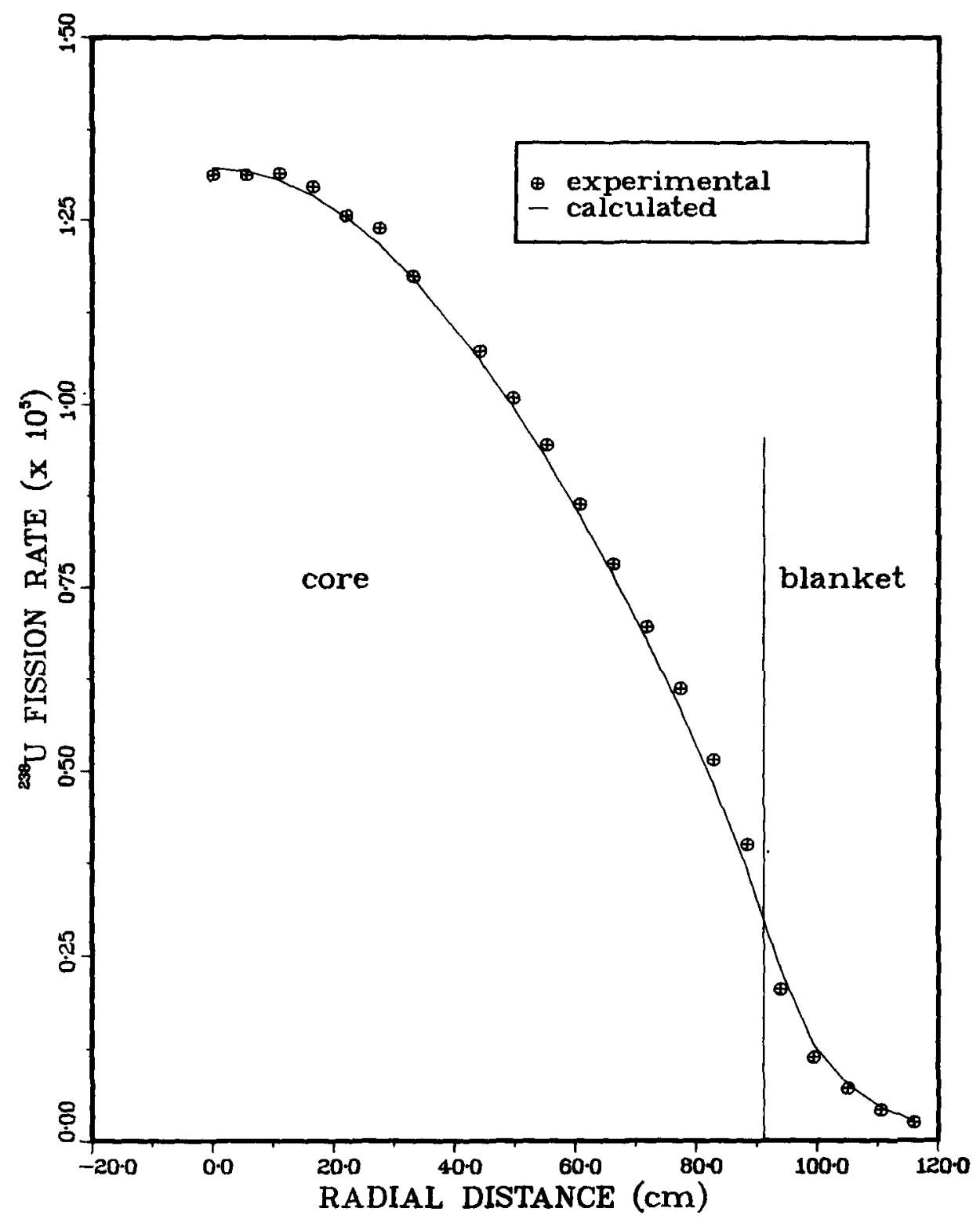

Fig. 62. Radial ${ }^{238}$ U Fission Rate Distribution, Column 23. (ANL Neg. No. 116-76-338). 
Pu Foil Fission Rate, $0^{\circ}$ Traverse

Normal Loading

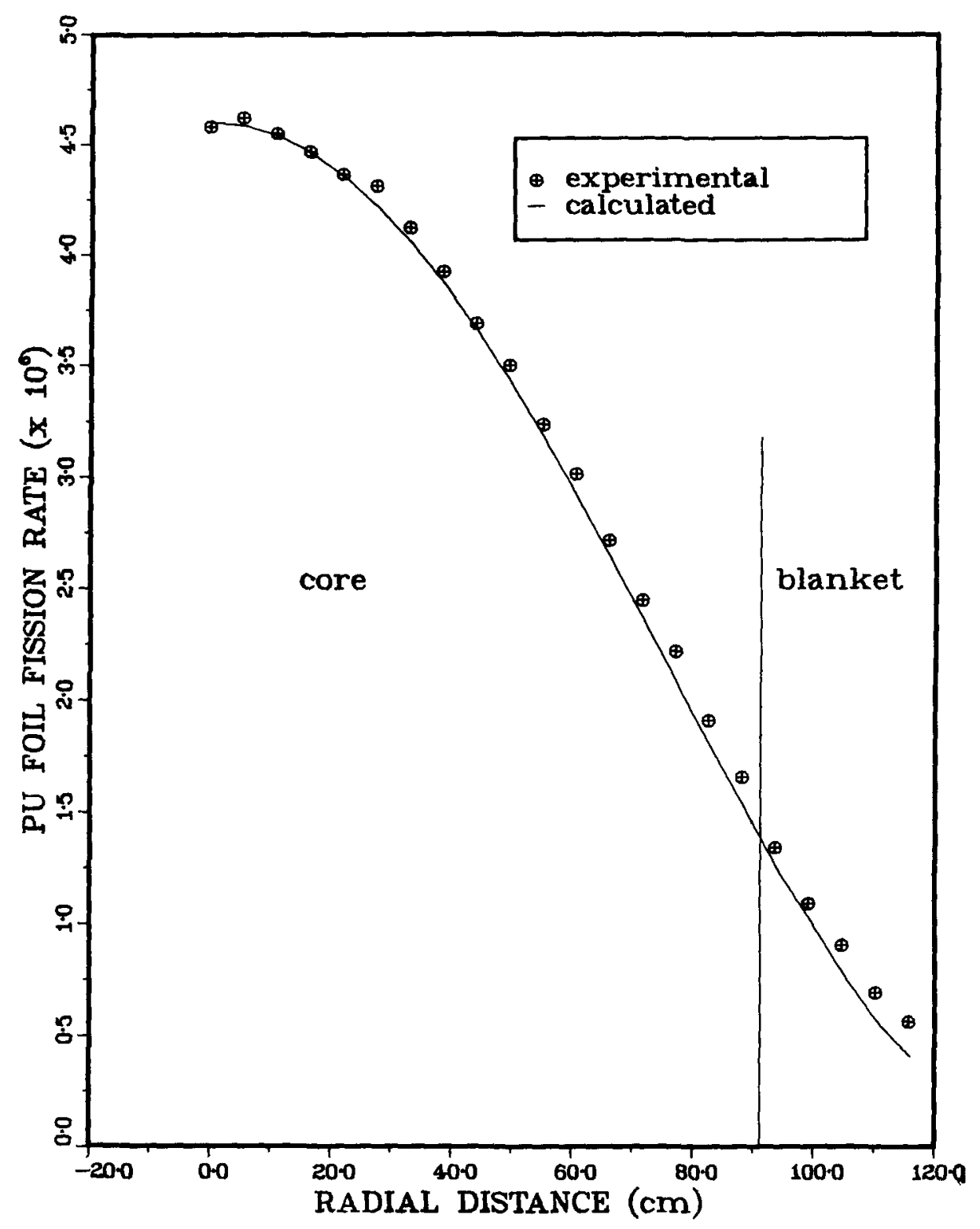

Fig. 63. Radial Pu Foil Fission Rate Distribution, Row 23. (ANL Neg. No. 116-76-339). 
Pu Foil Fission Rate, $45^{\circ}$ Traverse

Normal Loading

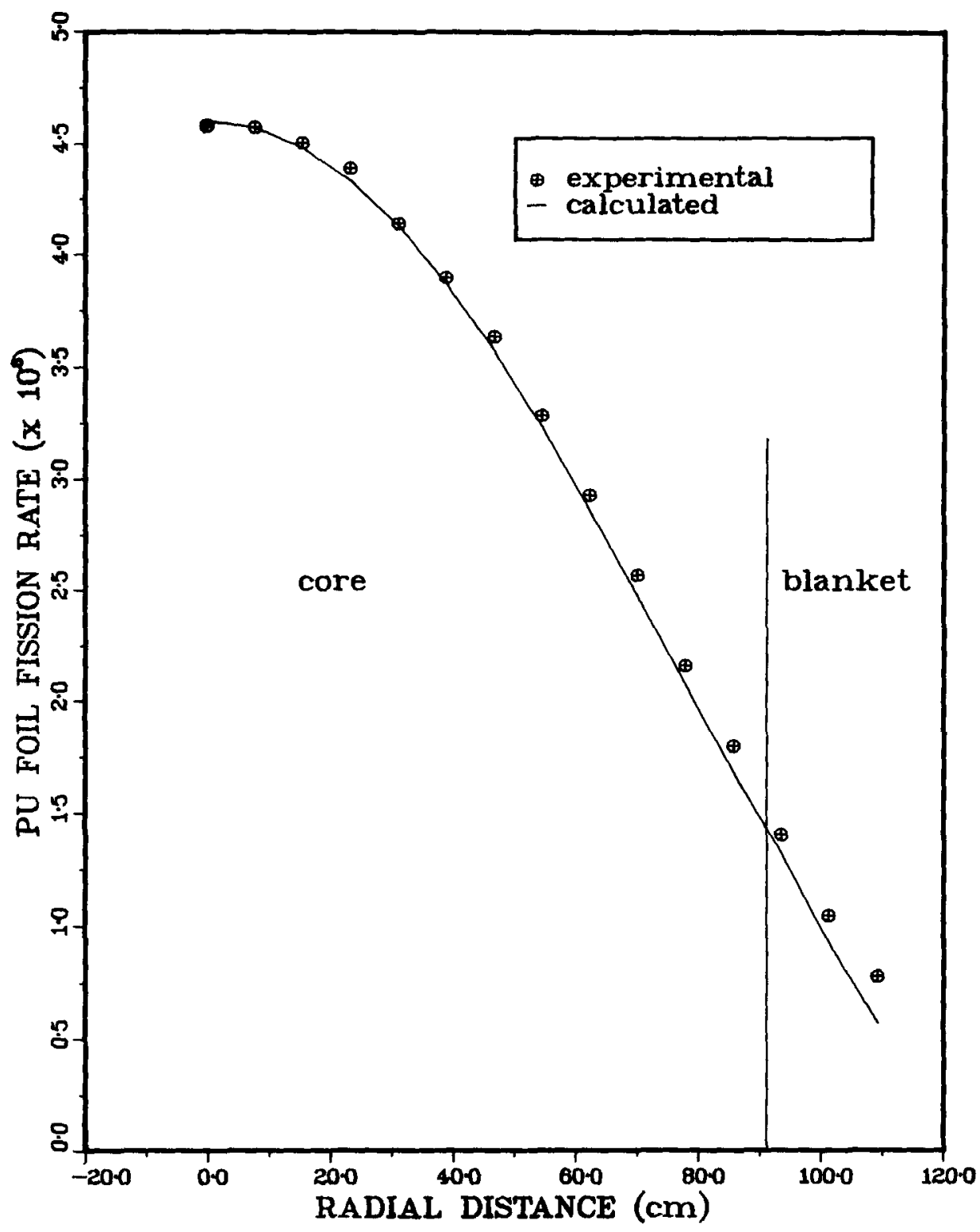

Fig. 64. Radial Pu Foil Fission Rate Distribution, Diagonal. (ANL Neg. No, 116-76-301). 
Pu Foil Fission Rate, $90^{\circ}$ Traverse

Normal Loading

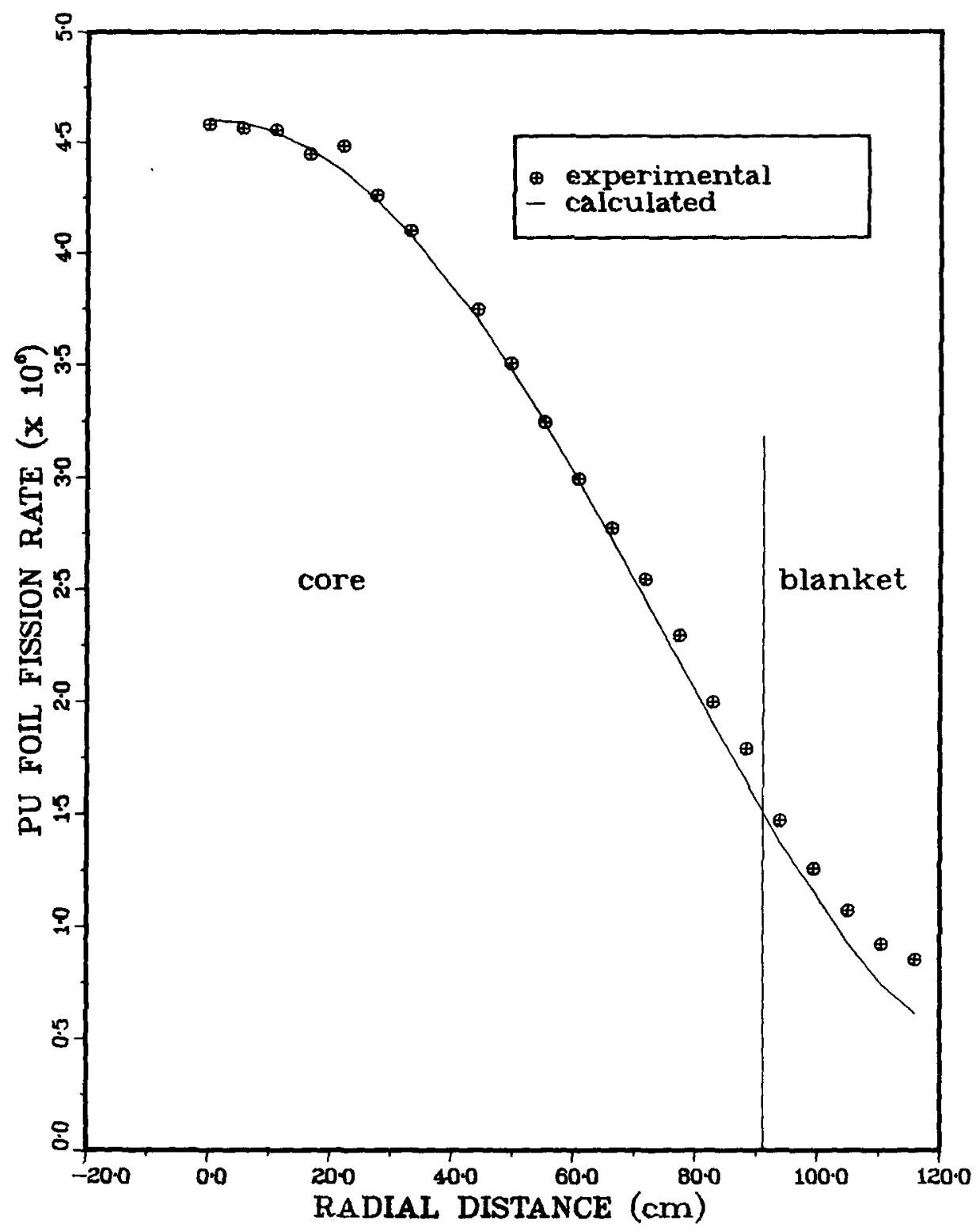

Fig. 65. Radial Pu Foil Fission Rate Distribution, Column 23. (ANL Neg. No. 116-76-353). 
${ }^{230} \mathrm{U}$ Capture Rate, $0^{\circ}$ Traverse

Normal Loading

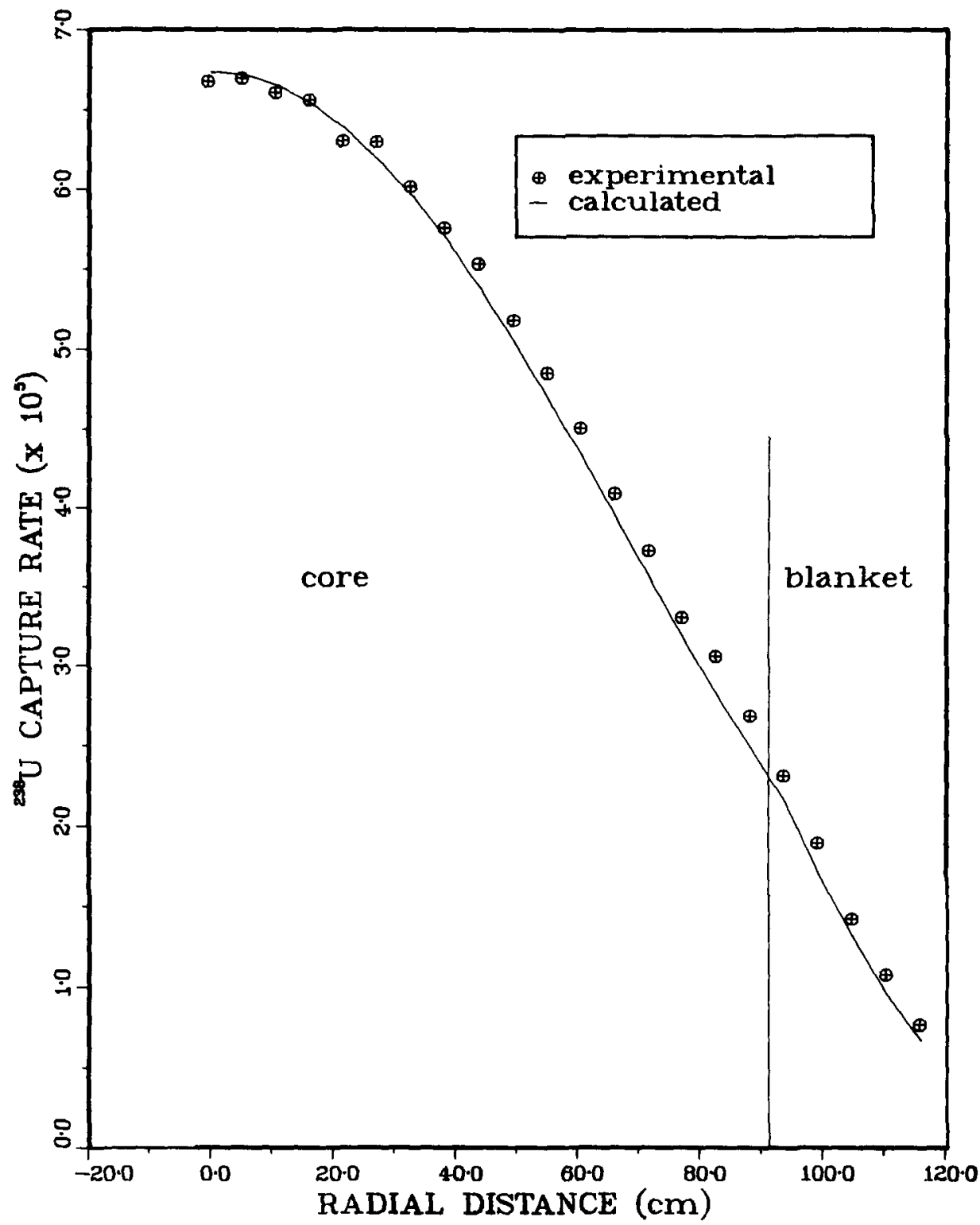

Fig. 66. Radial ${ }^{238}$ U Capture Rate Distribution, Row 23. (ANL Neg. No. 116-76-340). 
200 Capture Rate, $45^{\circ}$ Traverse

Normal Loading

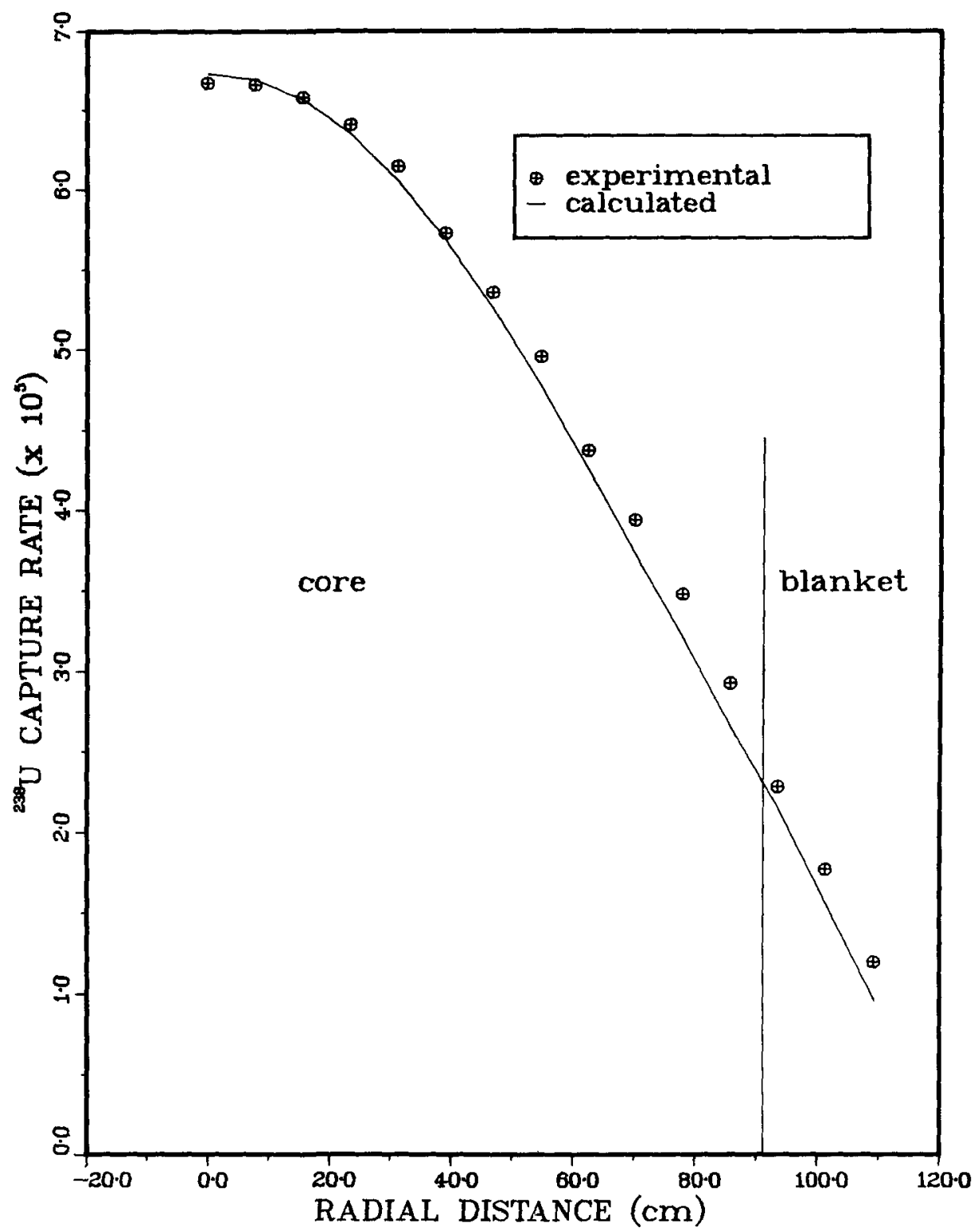

Fig. 67. Radial ${ }^{238} \mathrm{U}$ Capture Rate Distribution, Diagonal. (ANL Neg. No. 116-76-302). 
${ }^{230} \mathrm{U}$ Capture Rate, $90^{\circ}$ Traverse

Normal Loading

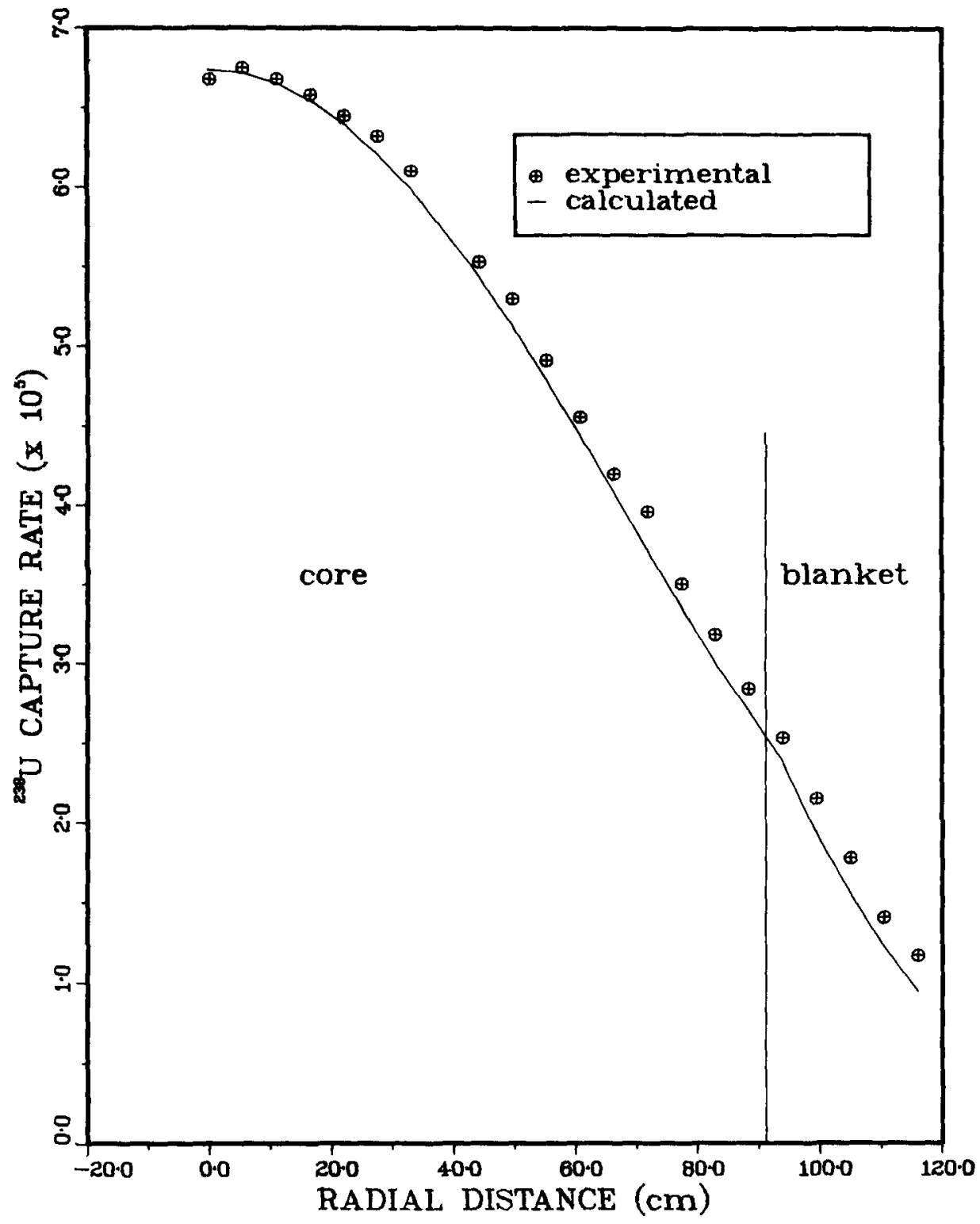

Fig. 68. Radial ${ }^{238} \mathrm{U}$ Capture Rate Distribution, Column 23. (ANL Neg. No. 116-76-286). 
${ }^{230} \mathrm{U}$ Fission Rate, Axial Traverse

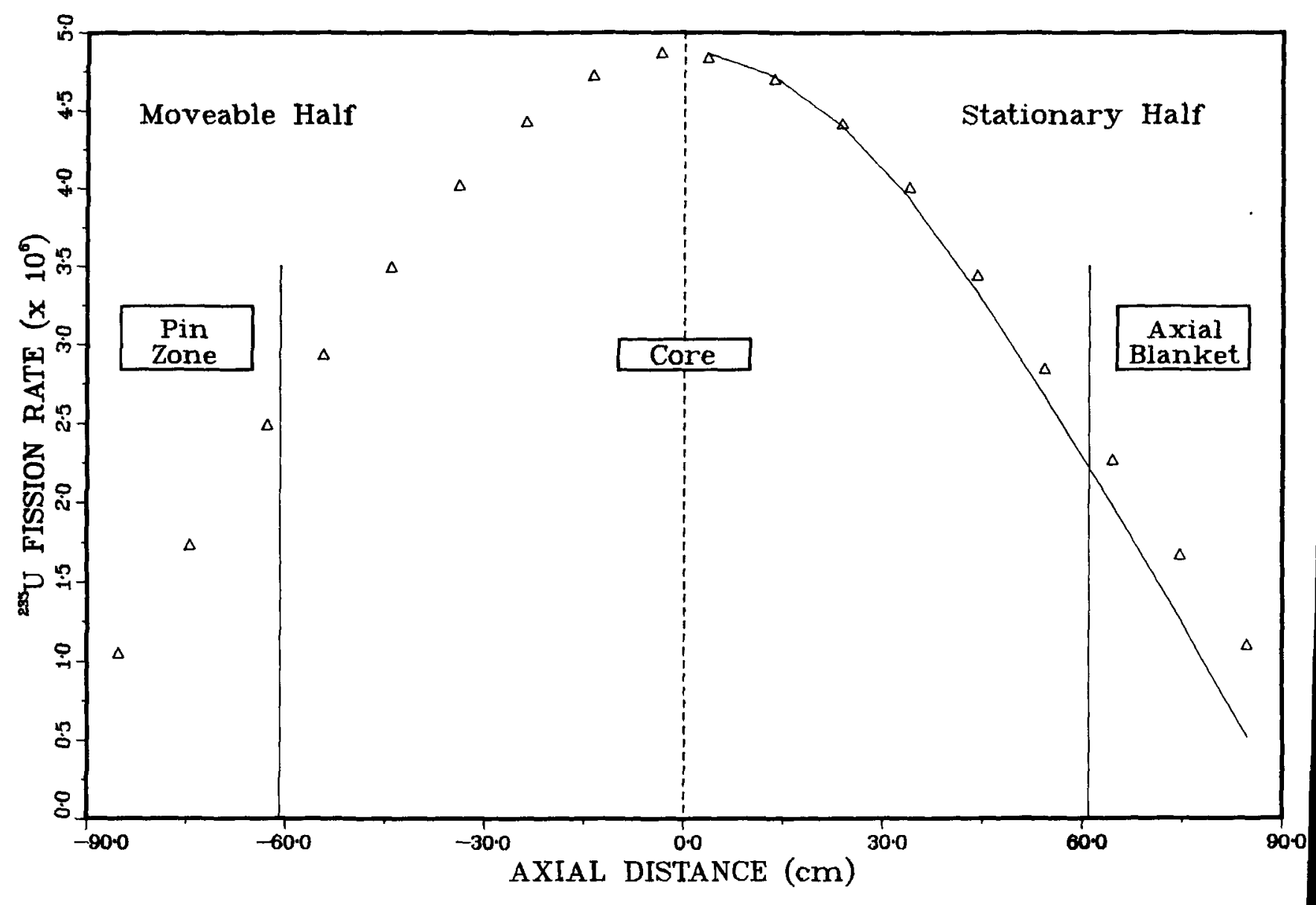

Fig. 69. Axial ${ }^{235} \mathrm{U}$ Fission Rate Distribution, Experimental (Symbols) and Calculated (Line). (ANL Neg. No. 116-76-333). 
${ }^{20}$ Fission Rate, Axial Traverse

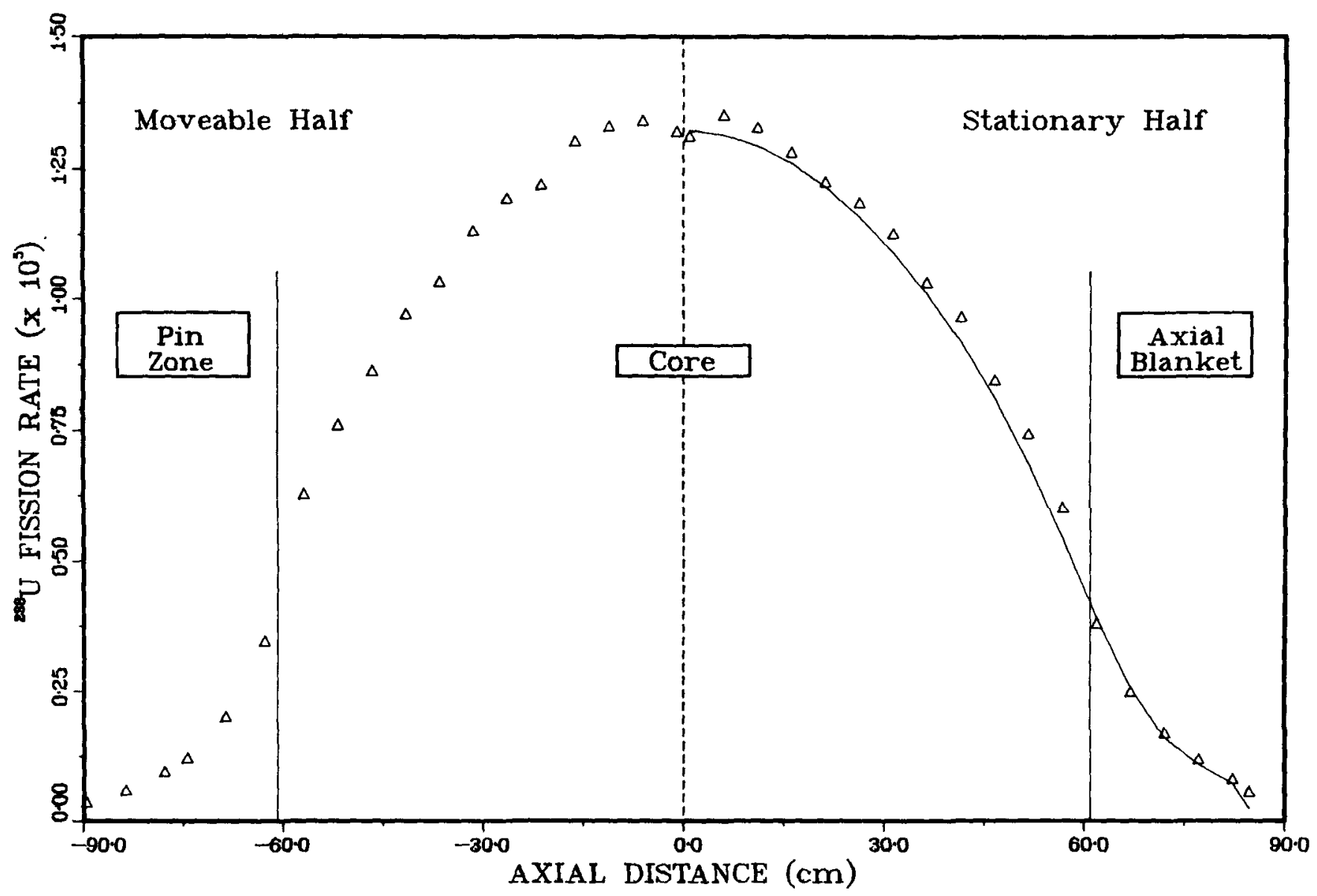

Fig. 70. Axia1 ${ }^{238}$ U Fission Rate Distribution, Experimental (Symbols) and Calculated (Line). (ANL Neg. No. 116-76-262). 
${ }^{230}$ Capture Rate, Axial Traverse

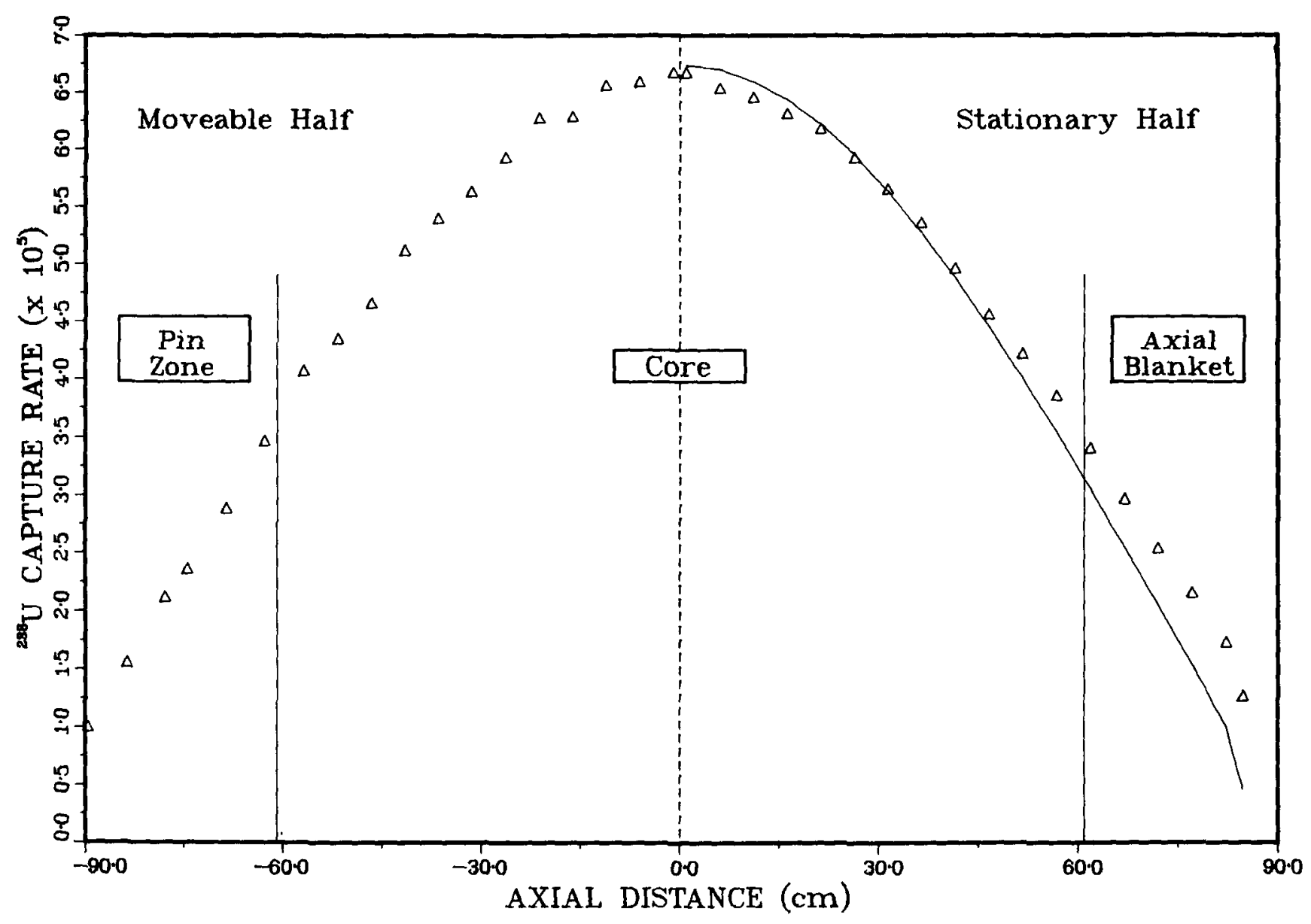

Fig. 71. Axial ${ }^{238} \mathrm{U}$ Capture Rate Distribution, Experimental (Symbols) and Calculated (Line). (ANL Neg. No. 116-76-261). 
Pu Foil Fission Rate

Axial Traverse

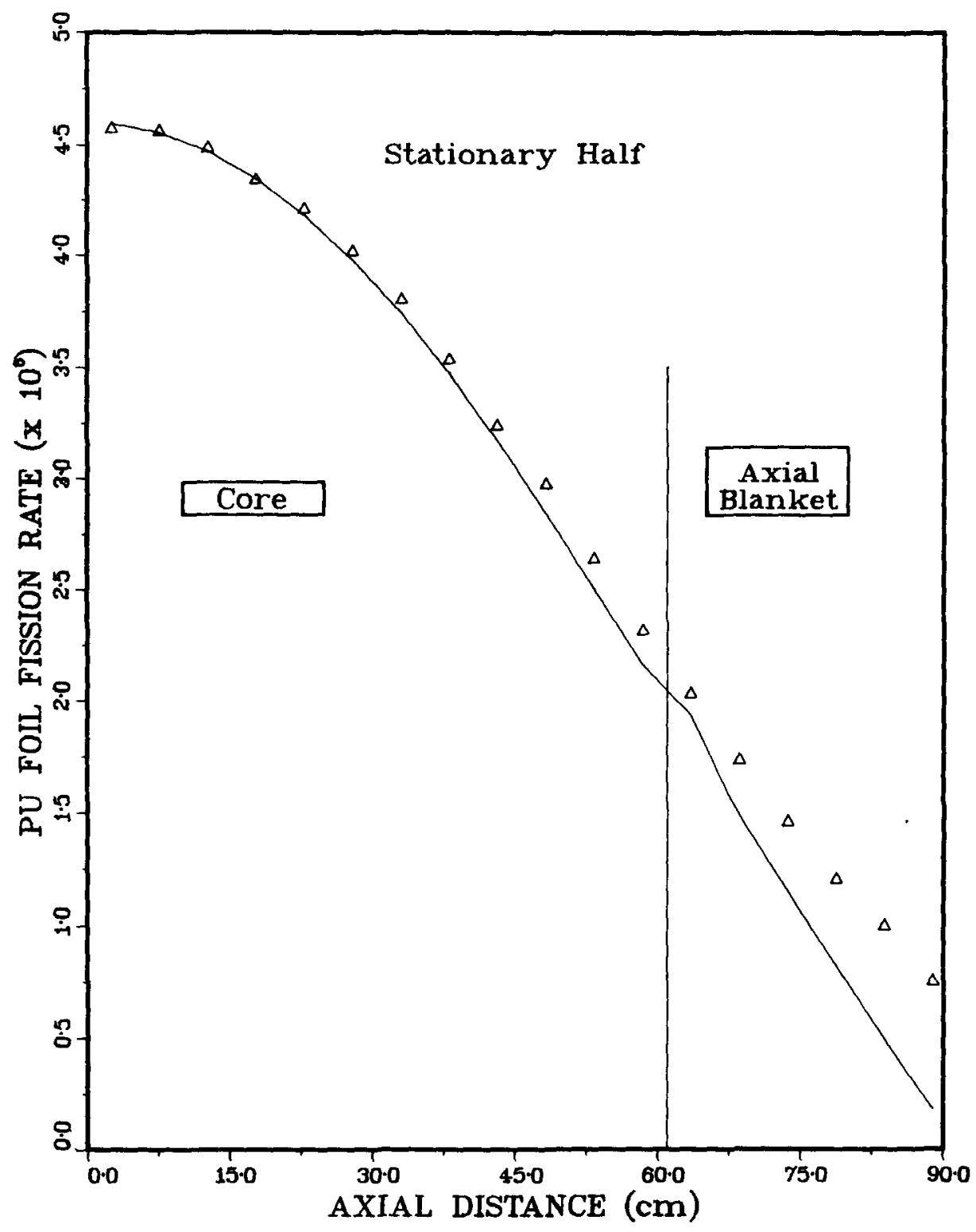

Fig. 72. Axial Pu Foil Fission Rate Distribution, Stationary Half of ZPR-9, Experimental (Symbols) and Calculated (Line). (ANL Neg. No. 11676-349). 


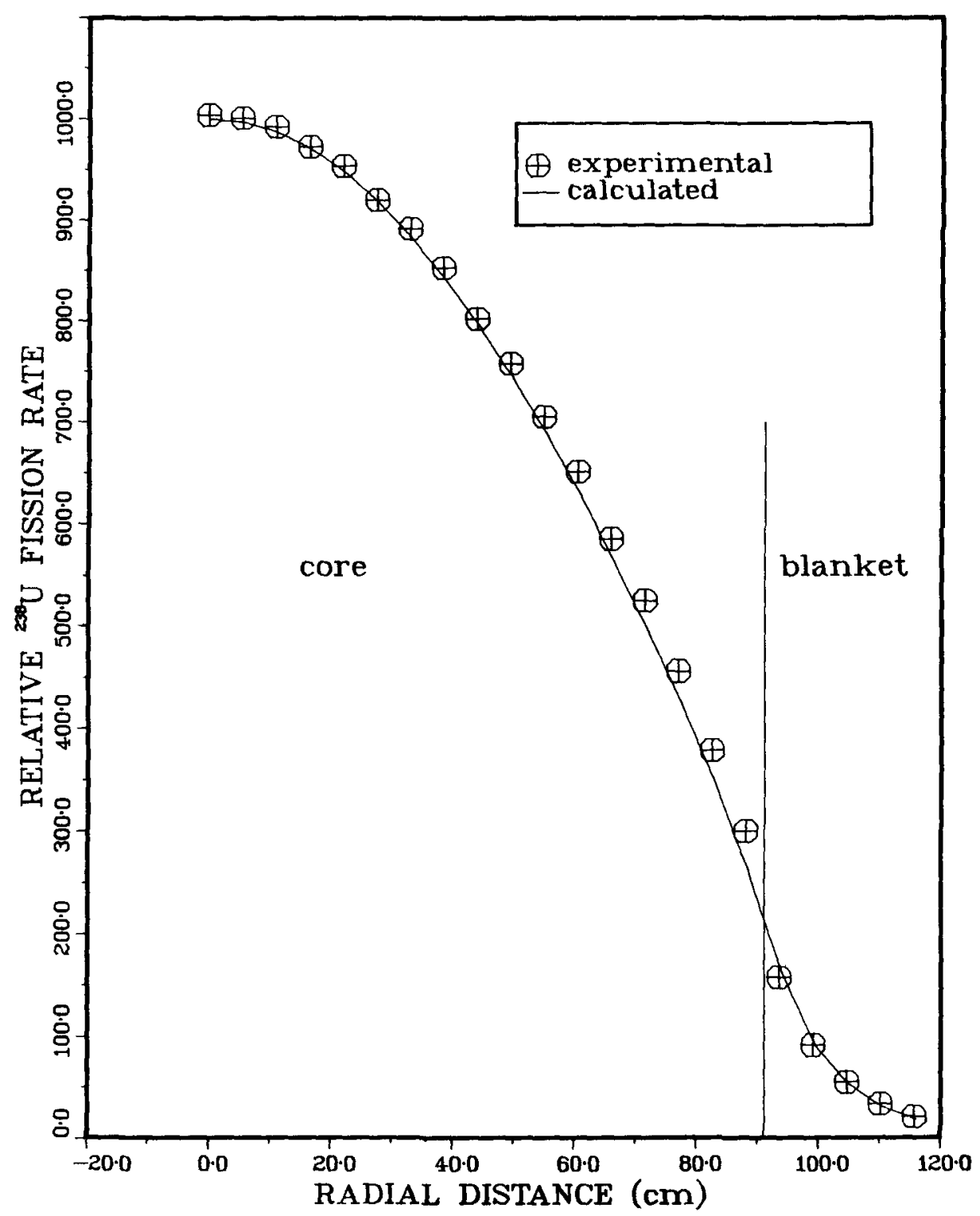

Fig. 73. Radial ${ }^{238} \mathrm{U}$ Fission Rate Distribution (Row 23) in the Checkerboard Octant. (ANL Neg. No. 116-76-332). 


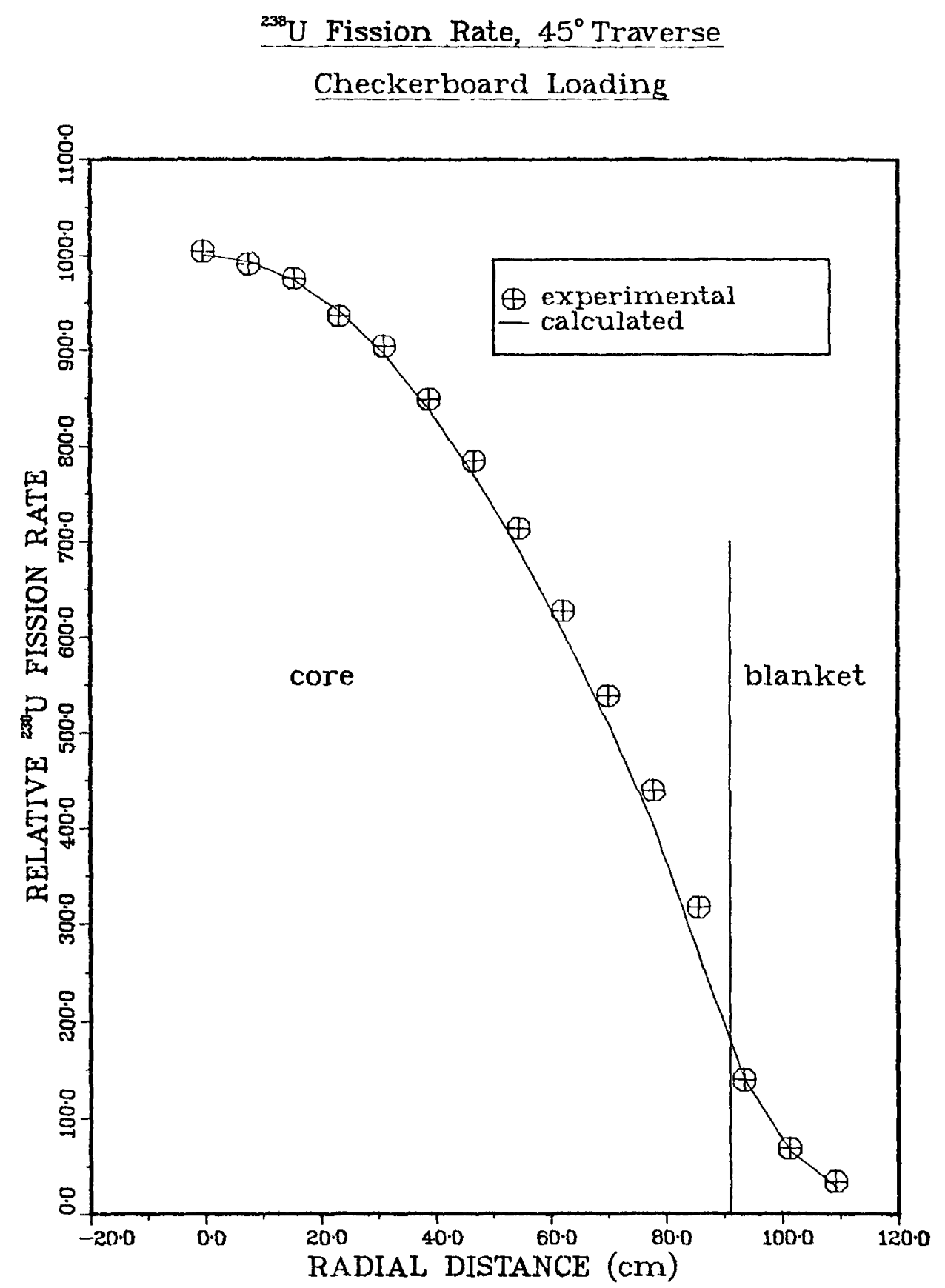

Fig. 74. Radial ${ }^{238} \mathrm{U}$ Fission Rate Distribution (Diagona1) in the Checkerboard Octant. (ANL Neg. No. 116-76-350). 


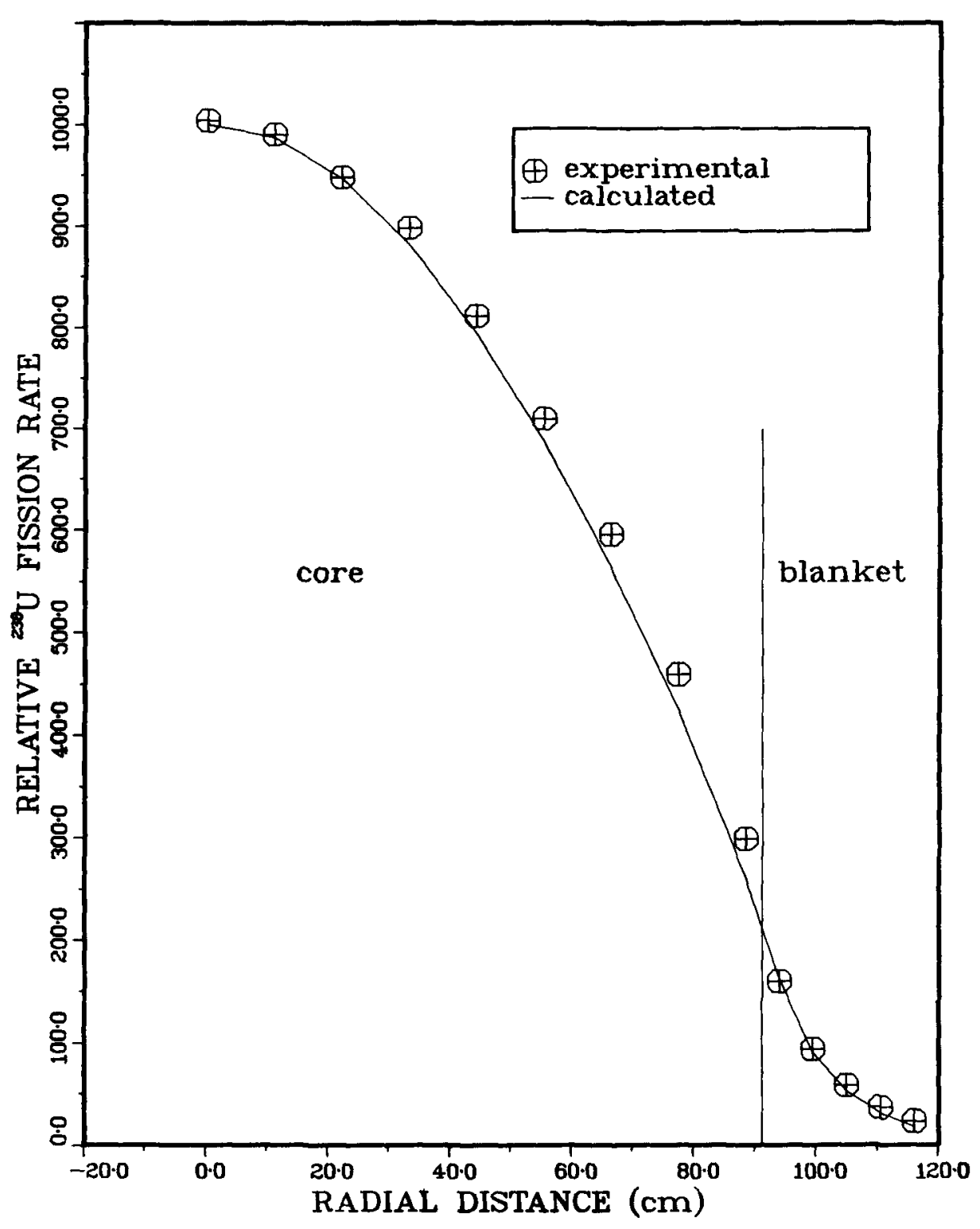

Fig. 75. Radial ${ }^{238} \mathrm{U}$ Fission Rate Distribution (Column 23) in the Checkerboard Octant. (ANL Neg. No. 116-76-335). 
${ }^{200} \mathrm{Pu}$ Fission Rate, $0^{\circ}$ Traverse

Checkerboard Loading

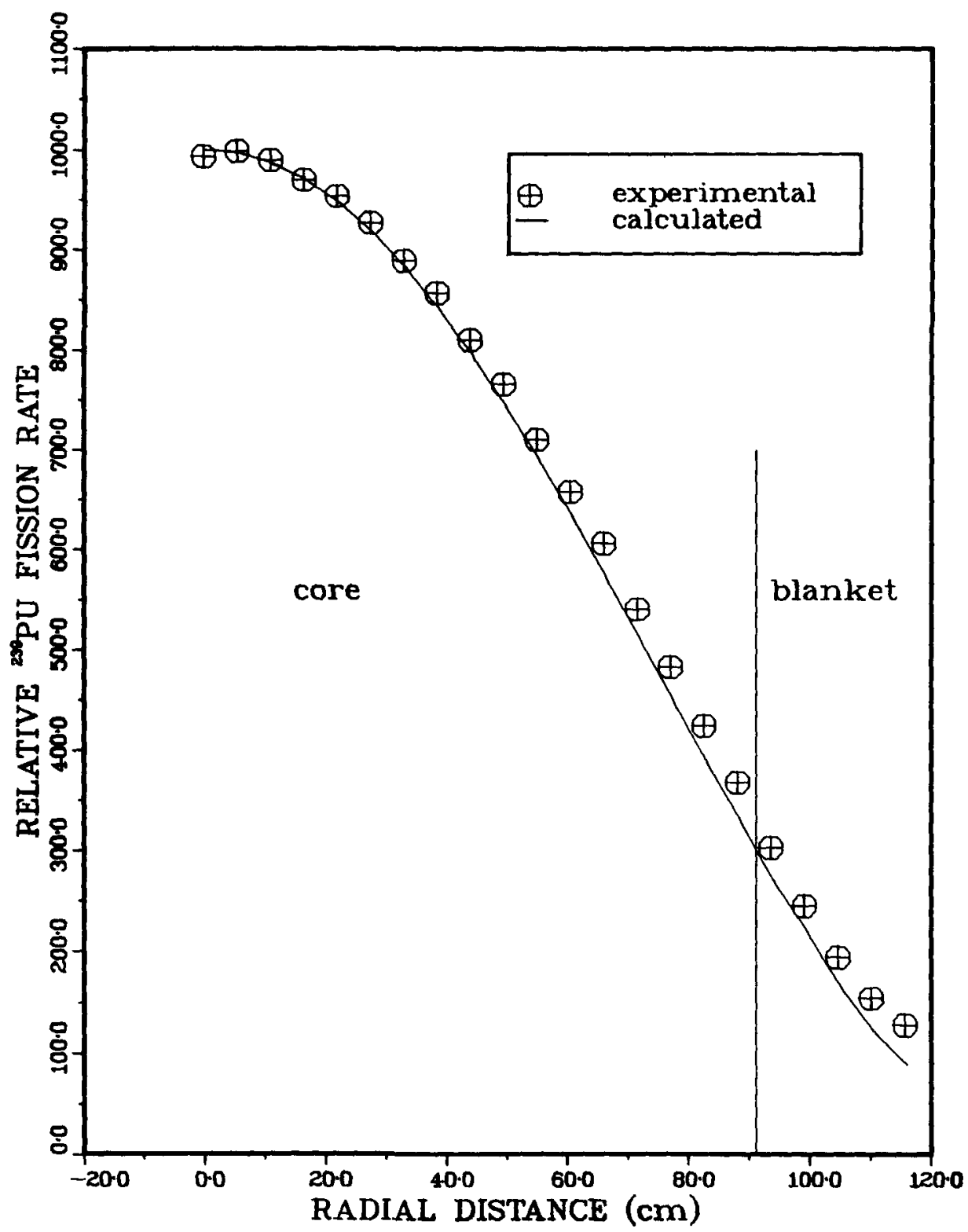

Fig. 76. Radial ${ }^{239} \mathrm{Pu}$ Fission Rate Distribution (Row 23) in the Checkerboard Octant. (ANL Neg. No. 11676-330). 
${ }^{200} \mathrm{Pu}$ Fission Rate, $45^{\circ}$ Traverse

Checkerboard Loading

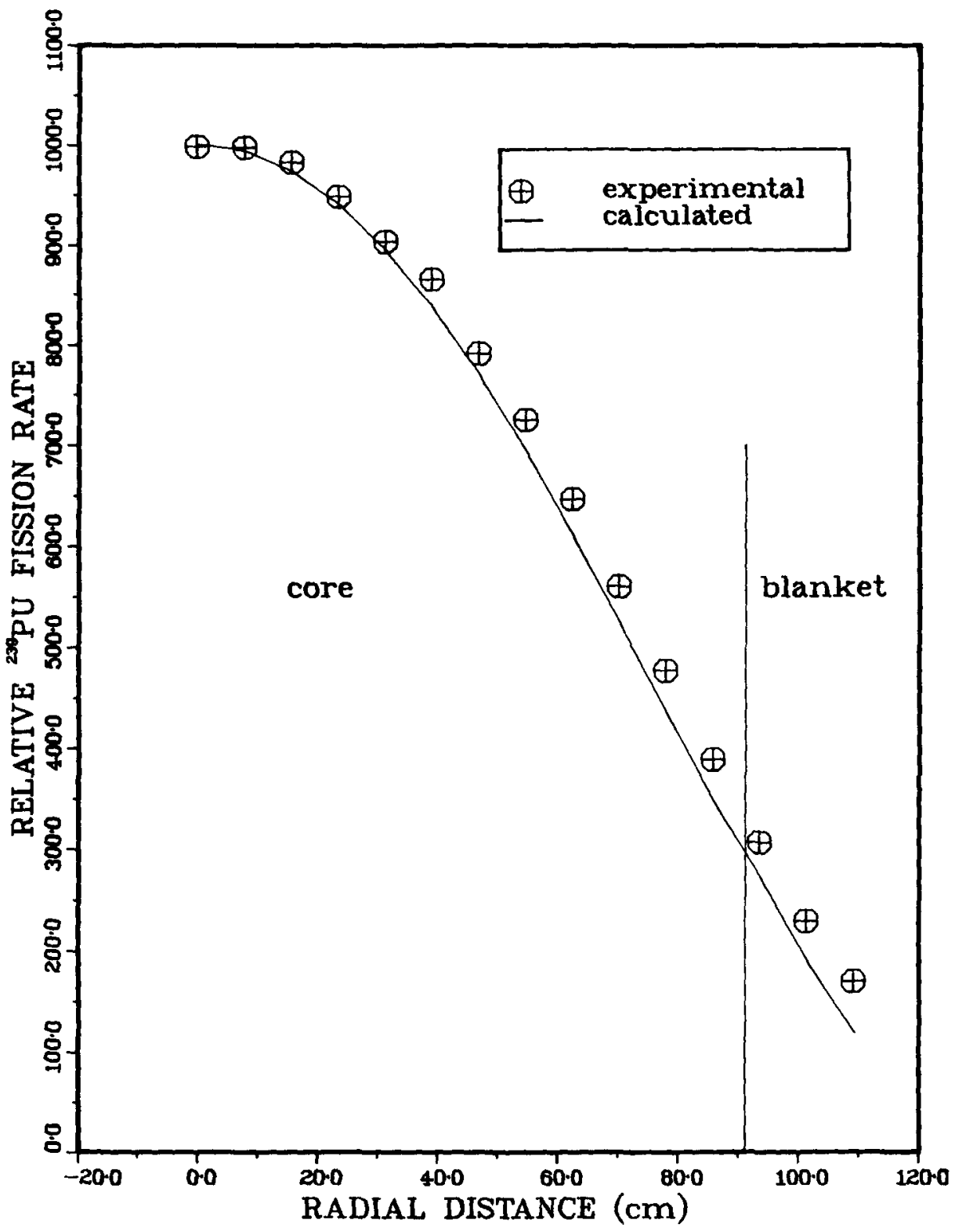

Fig. 77. Radial ${ }^{239} \mathrm{Pu}$ Fission Rate Distribution (Diagonal) in the Checkerboard Octant. (ANL Neg. No. 116-76342). 
${ }^{200}$ Pu Fission Rate, $90^{\circ}$ Traverse

Checkerboard Loading

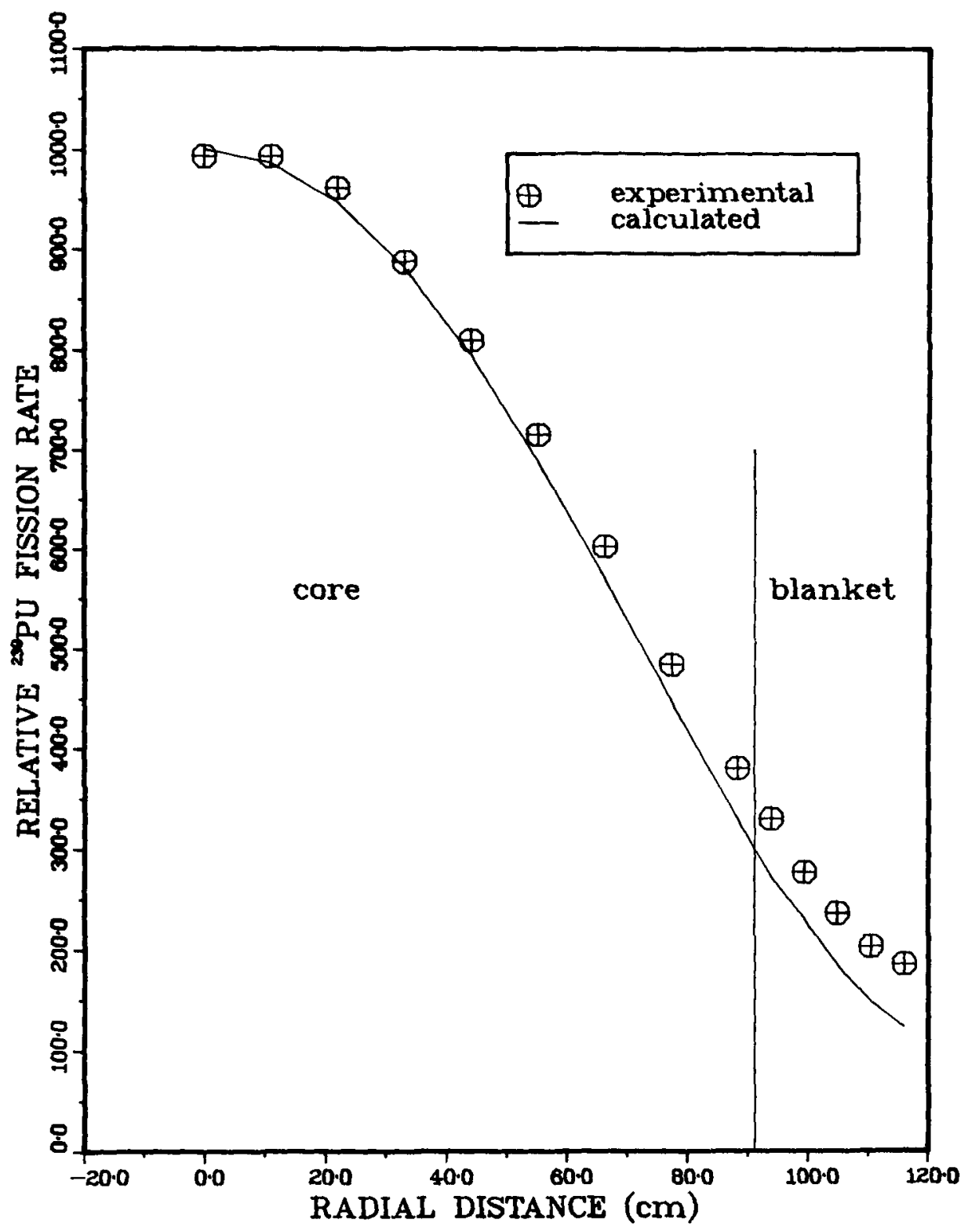

Fig. 78. Radial ${ }^{239} \mathrm{Pu}$ Fission Rate Distribution (Column 23) in the Checkerboard Octant. (ANL Neg. No. 116-76331). 
${ }^{236} \mathrm{U}$ Capture Rate, $0^{\circ}$ Traverse

Checkerboard Loading

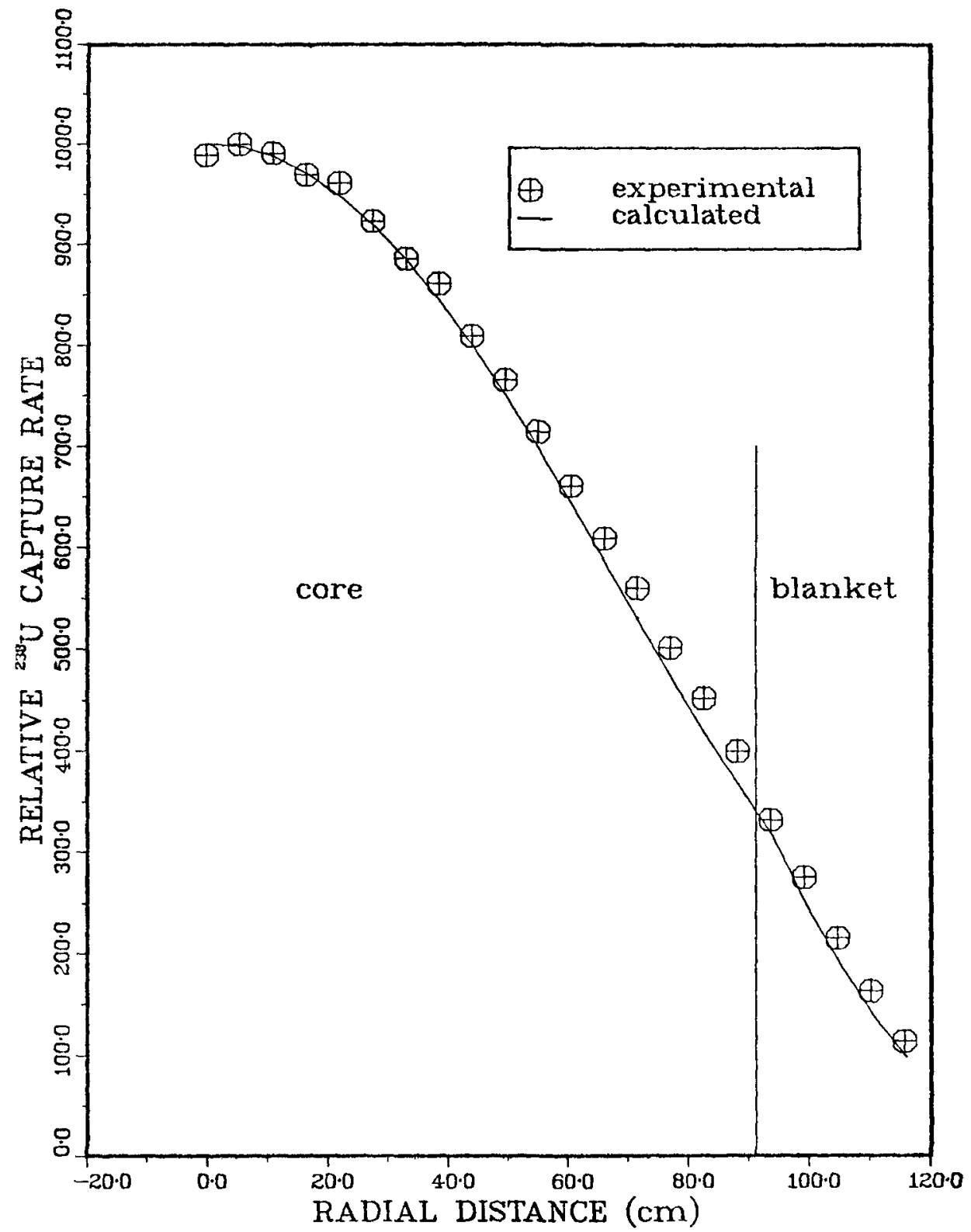

Fig. 79. Radial ${ }^{238} \mathrm{U}$ Capture Rate Distribution (Row 23) in the Checkerboard Octant. (ANL Neg. No. 116-76-334). 
${ }^{230} \mathrm{U}$ Capture Rate, $45^{\circ}$ Traverse

Checkerboard Loading

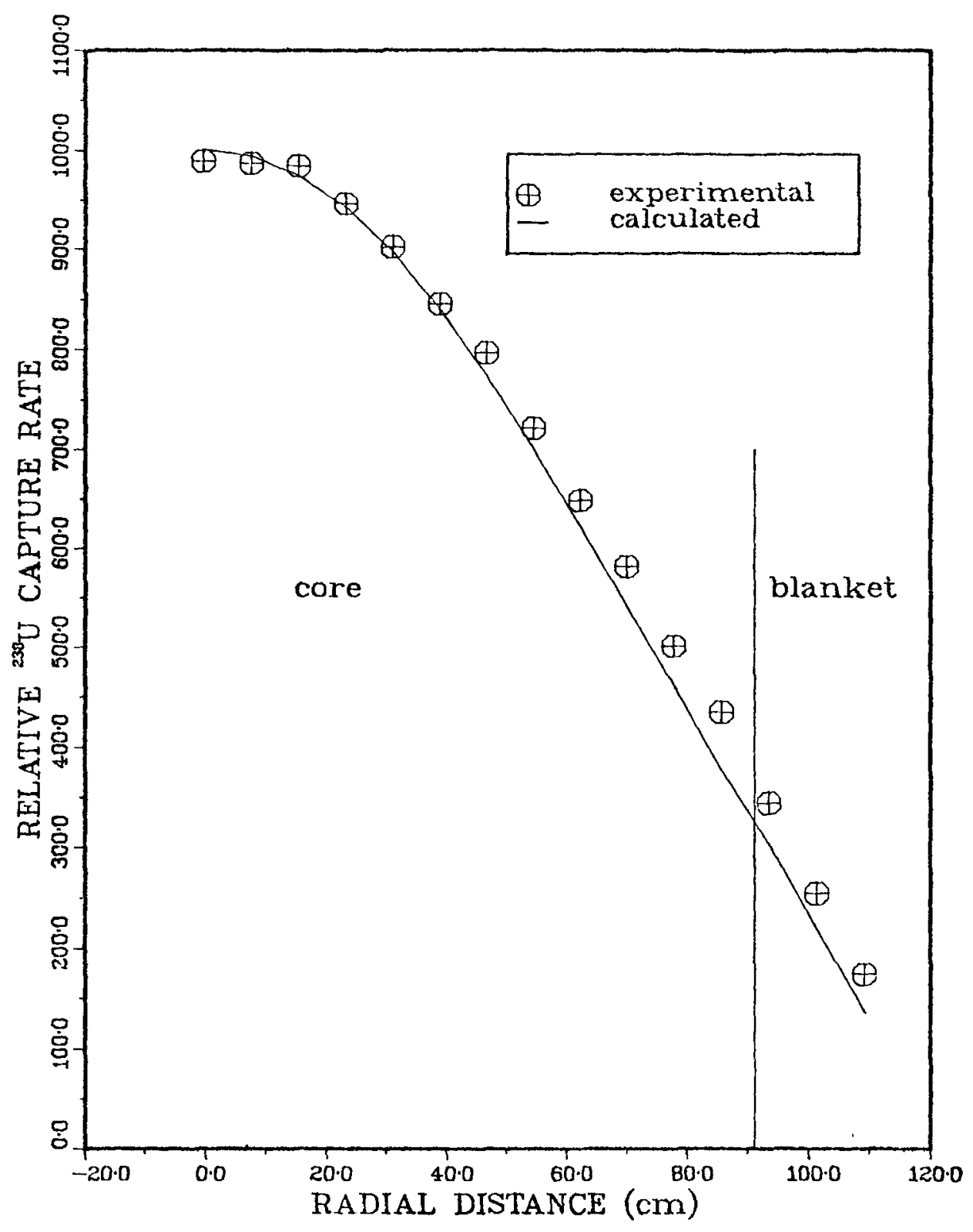

Fig. 80. Radial ${ }^{238} \mathrm{U}$ Capture Rate Distribution (Diagonal) in the Checkerboard Octant. (ANL Neg. No. 116-76-291). 
${ }^{238} \mathrm{U}$ Capture Rate, $90^{\circ}$ Traverse

Checkerboard Loading

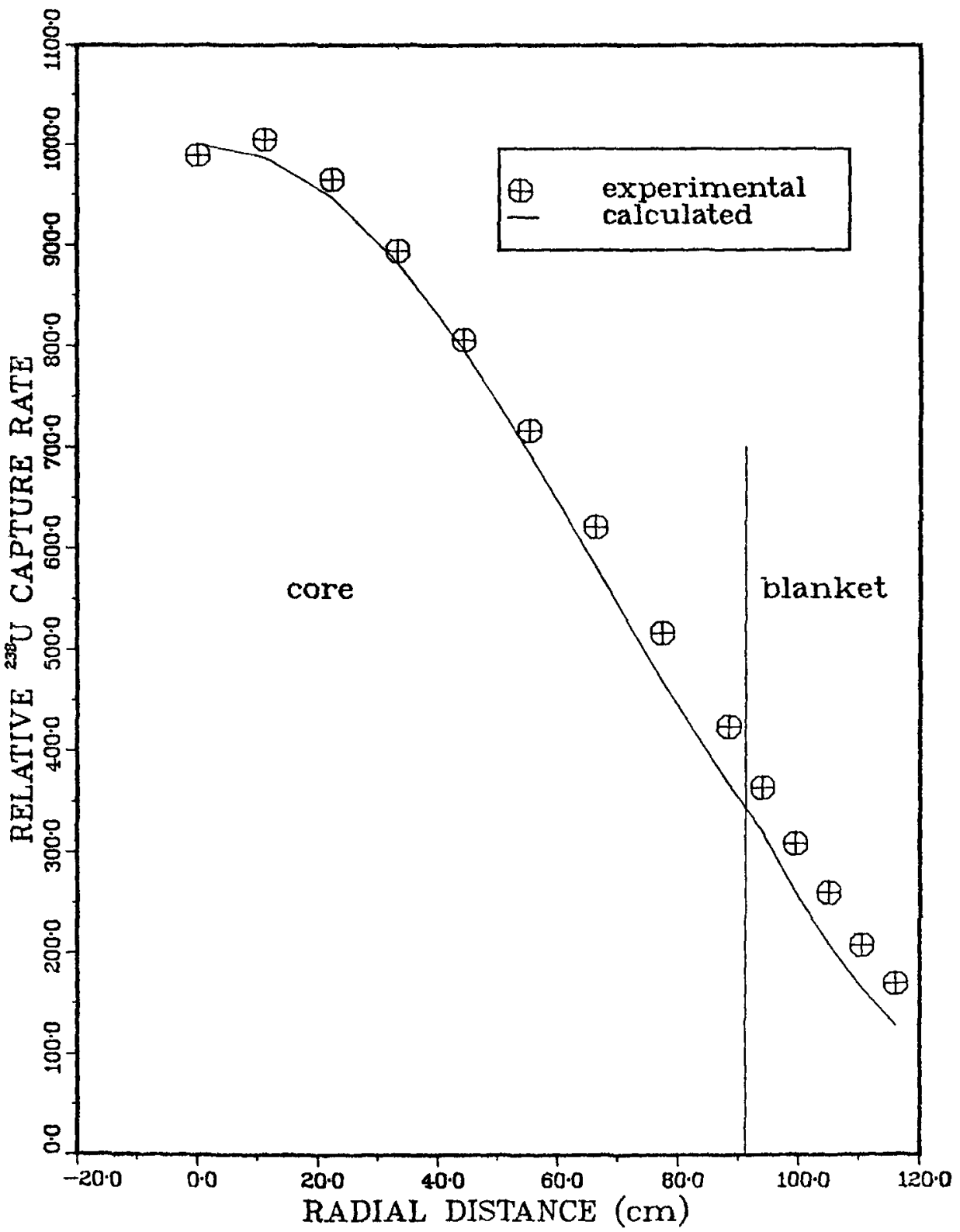

Fig. 81. Radial ${ }^{238}$ U Capture Rate Distribution (Column 23) in the Checkerboard Octant. (ANL Neg. No. 116-76277). 


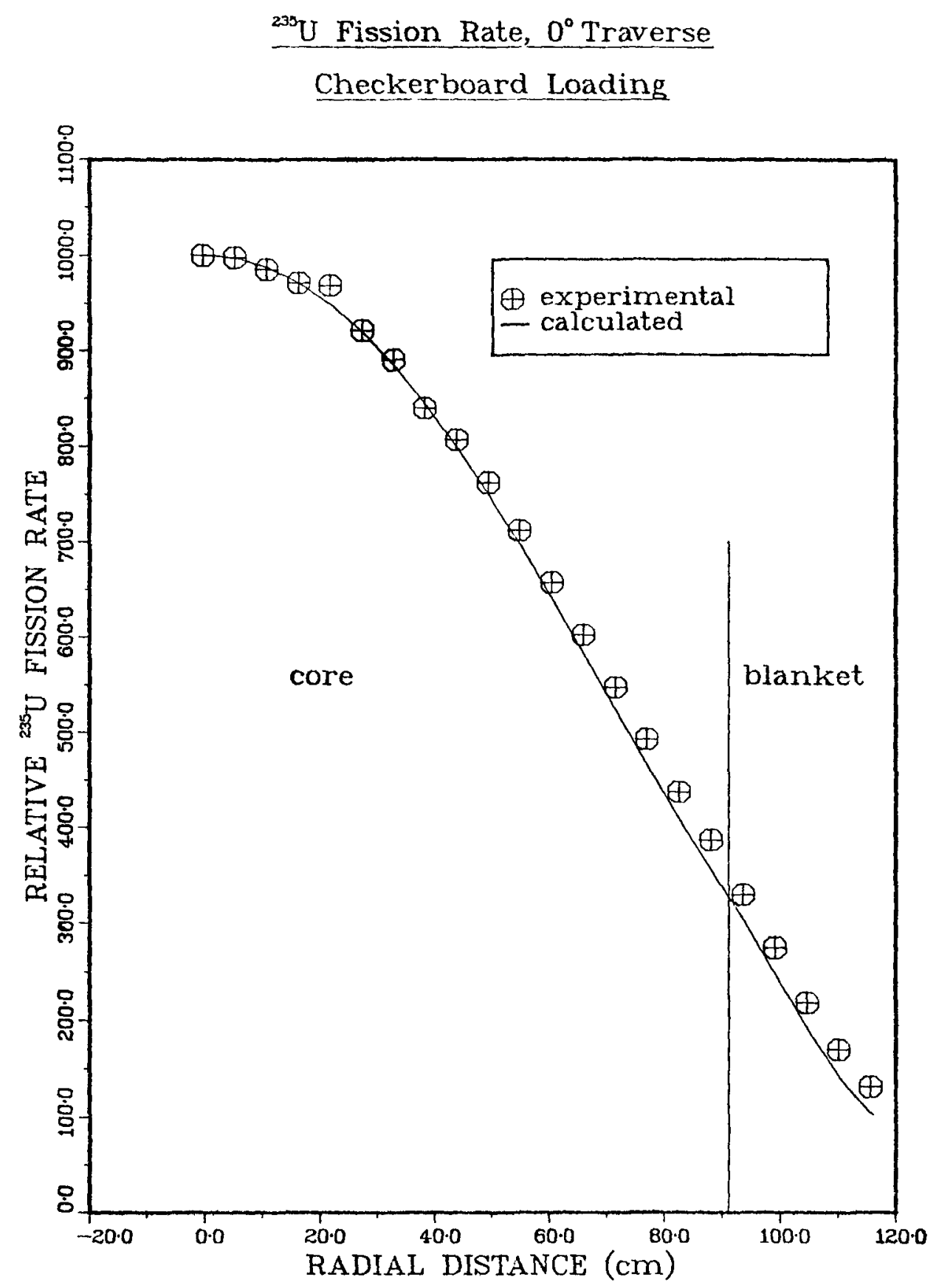

Fig. 82. Radial $235 \mathrm{U}$ Fission Rate Distribution (Row 23) in the Checkerboard Octant. (ANL Neg. No. 116-76-285). 
${ }^{230} \mathrm{U}$ Fission Rate, $45^{\circ}$ Traverse

Checkerboard Loading

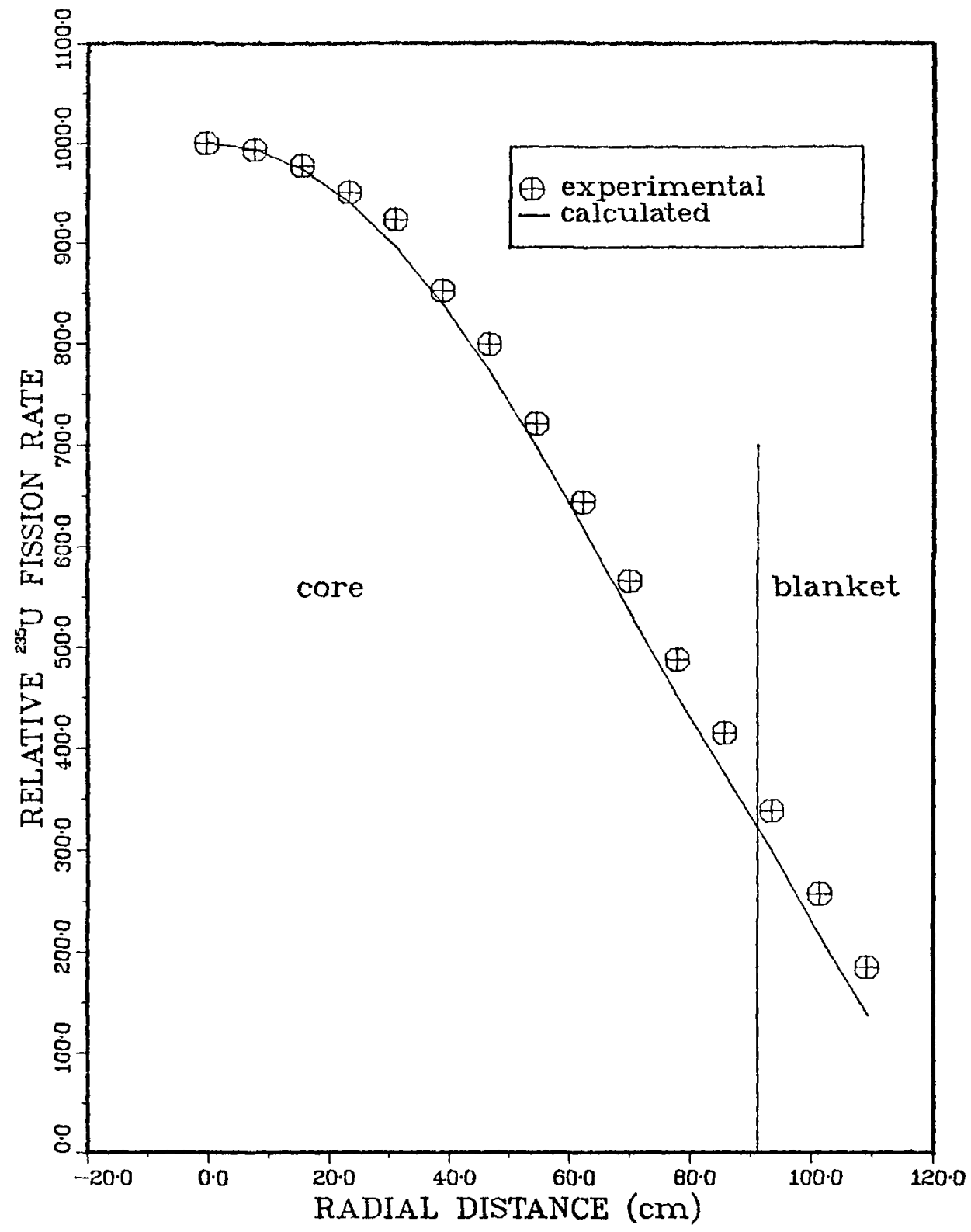

Fig. 83. Radial $235_{\mathrm{U}}$ Fission Rate Distribution (Diagonal) in the Checkerboard Octant. (ANL Neg. No. 116-76287). 
${ }^{230} \mathrm{U}$ Fission Rate, $90^{\circ}$ Traverse

Checkerboard Loading

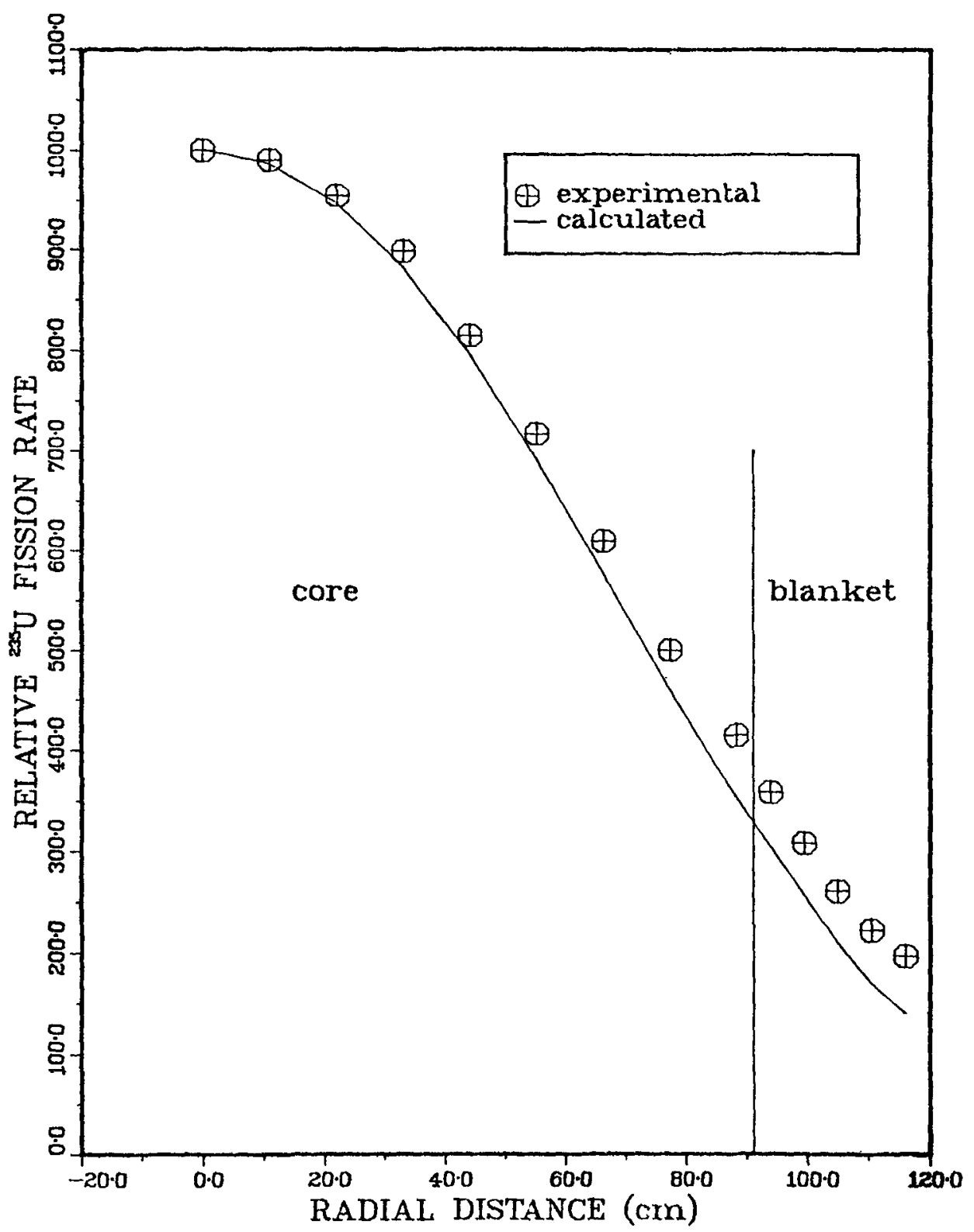

Fig. 84. Radial 235 U Fission Rate Distribution (Column 23) in the checkerboard Octant. (ANL Neg. No. 116-76282). 
${ }^{239} \mathrm{Pu}$ Fission Rate

Normal Loading

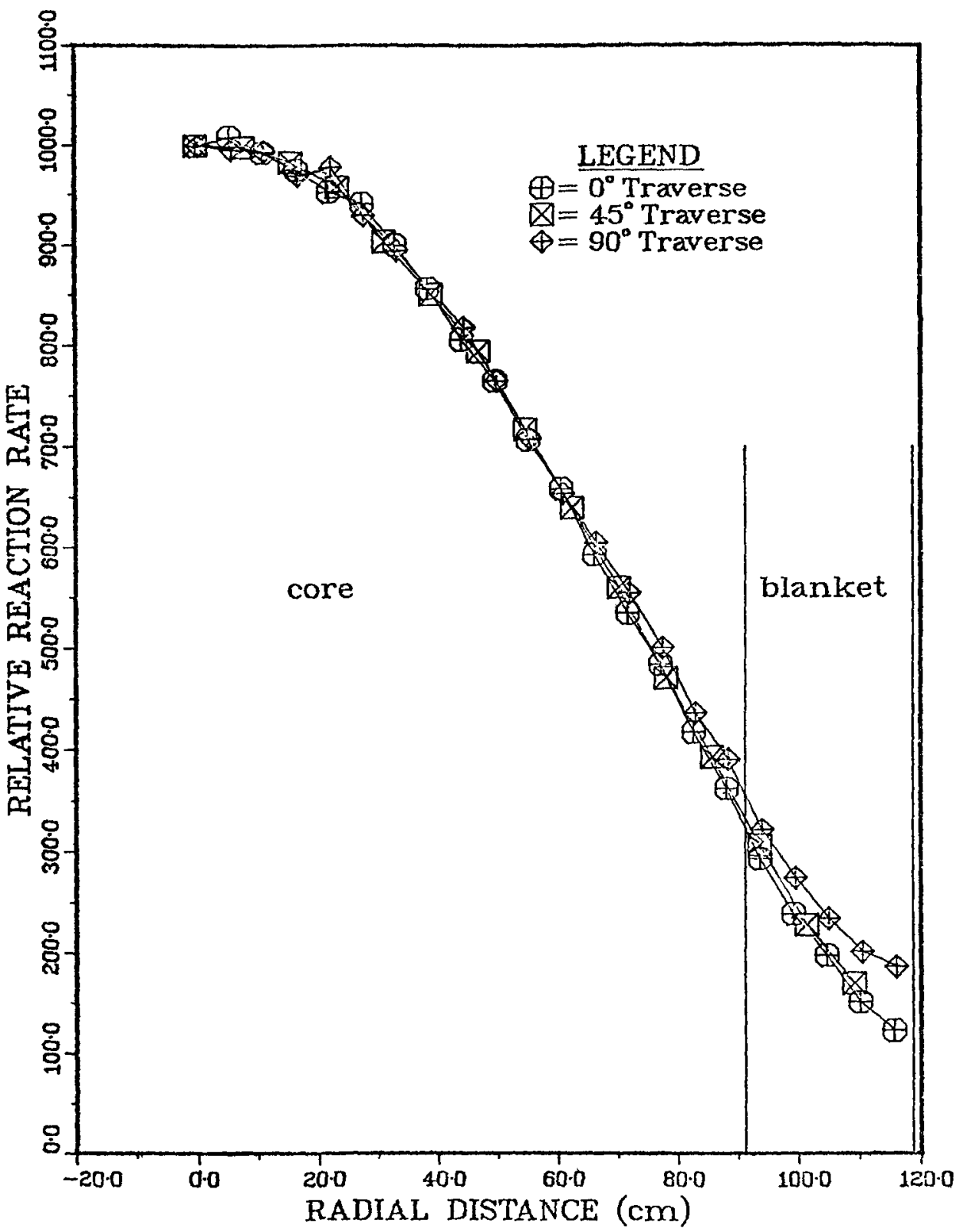

Fig. 85. Comparison of the ${ }^{239} \mathrm{Pu}$ Fission Rate Distribution in the Three Radial Directions. (ANL Neg. No. 116-76281). 
${ }^{230} \mathrm{U}$ Fission Rate

Normal Loading

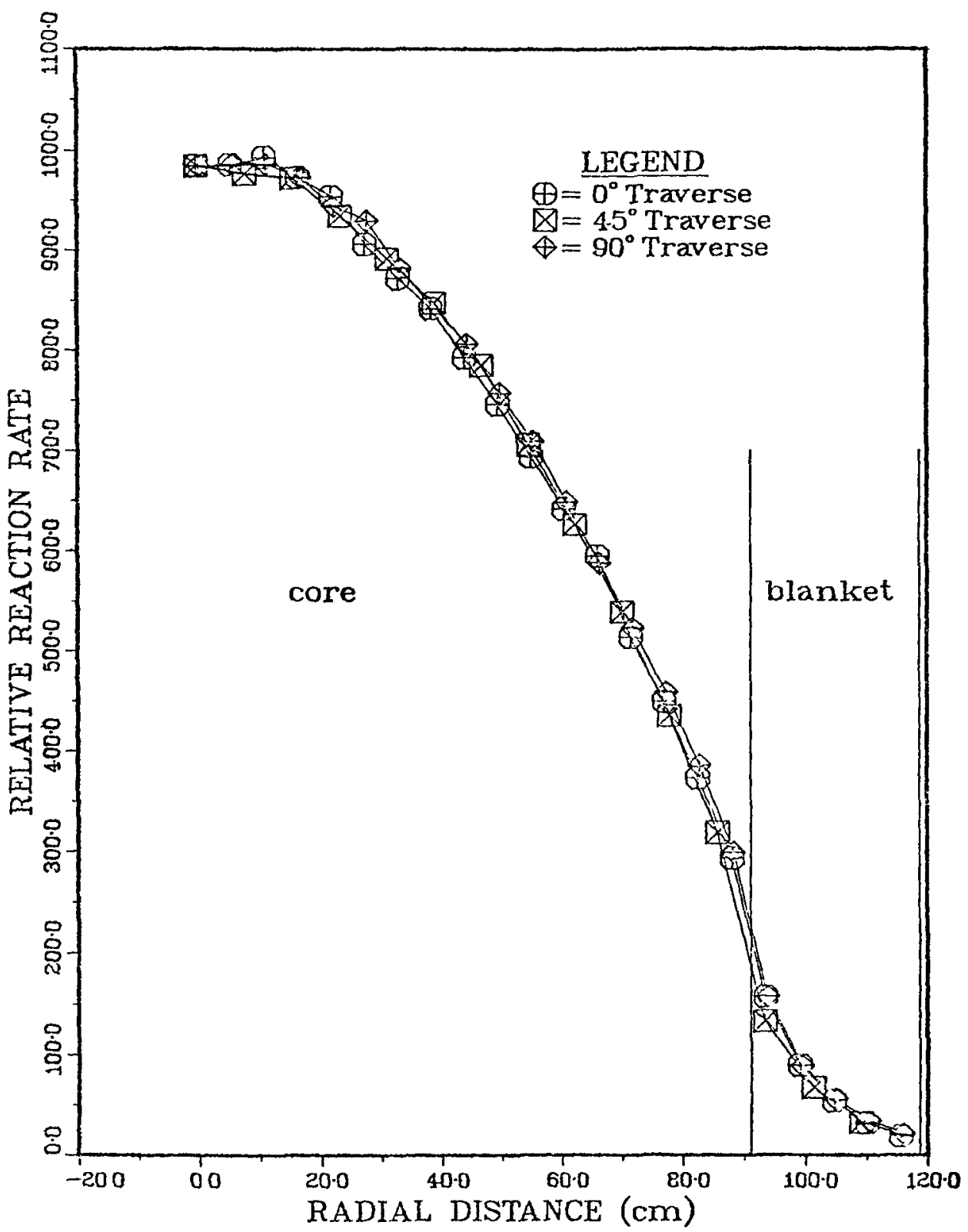

Fig. 86. Comparison of the ${ }^{238} \mathrm{U}$ Fission Rate in the Three Radial Directions. (ANL Neg. No. 116-76-329). 
${ }^{239} \mathrm{Pu}$ Fission Rate

$90^{\circ}$ Traverse

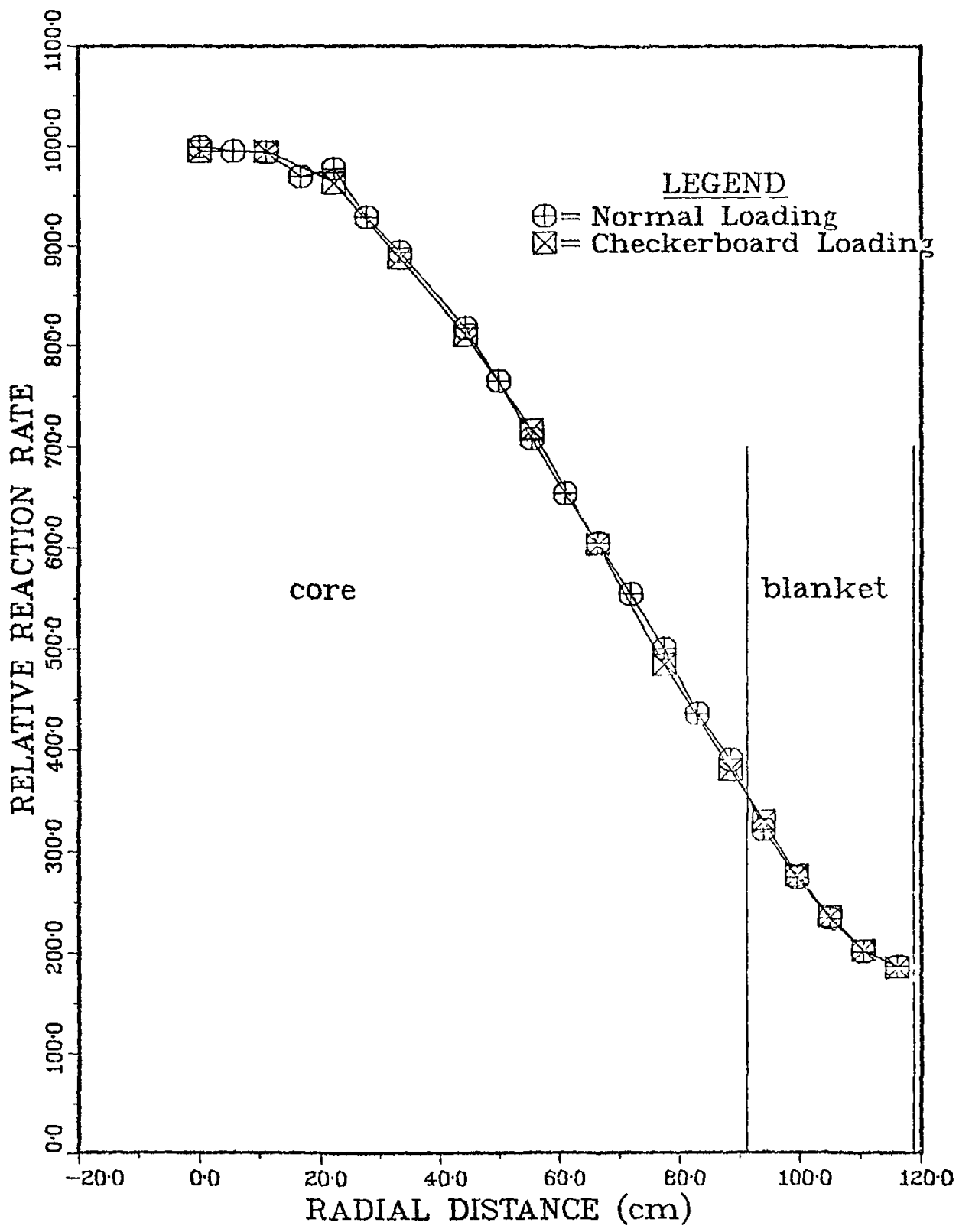

Fig. 87. Comparison of the Experimental ${ }^{239} \mathrm{Pu}$ Fission Rate in the $90^{\circ}$ Direction (Column 23) for the Normal and Checkerboard Cases. (ANL Neg. No. 116-76-346). 


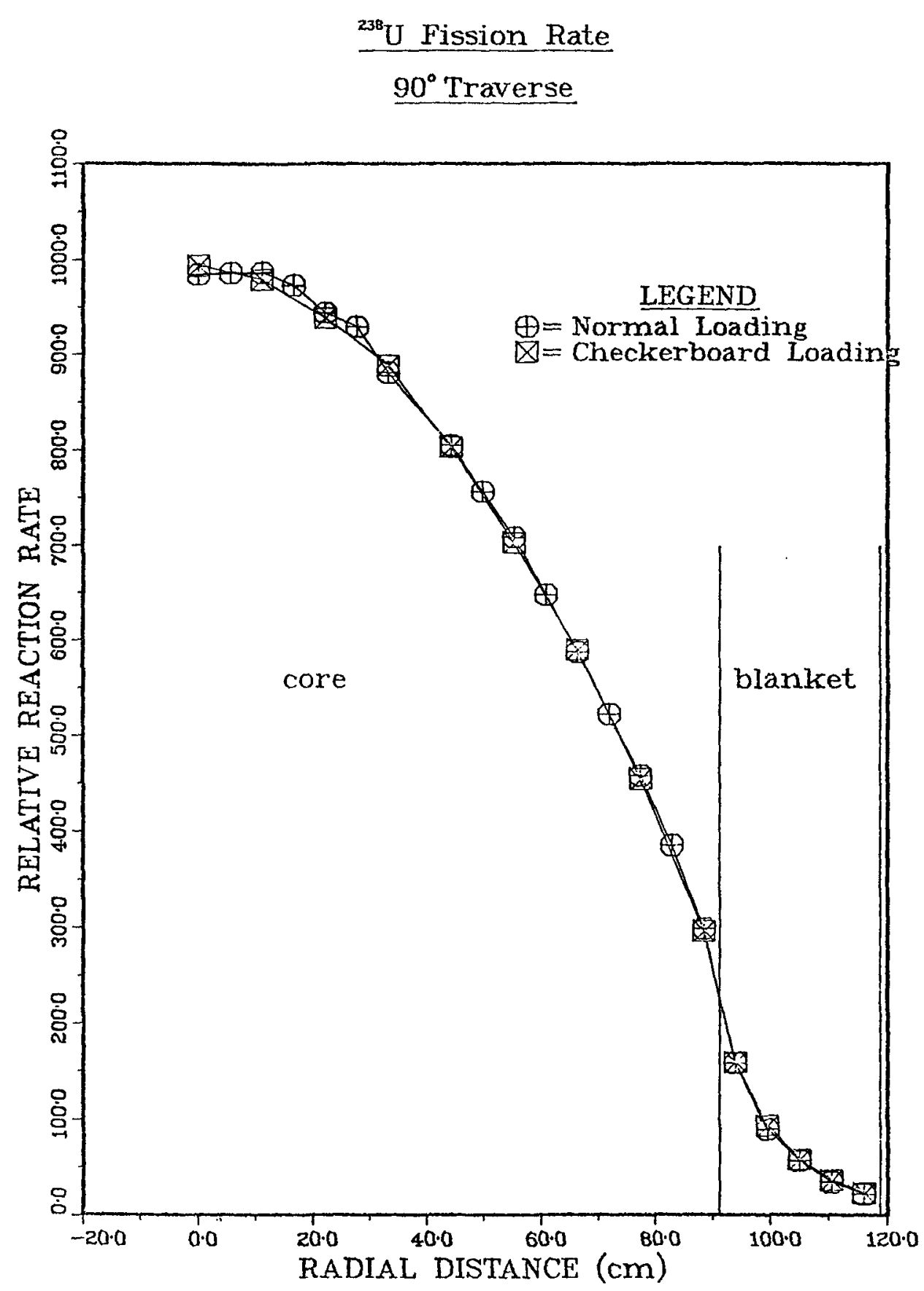

Fig. 88. Comparison of the Experimental ${ }^{238} \mathrm{U}$ Fission Rate in the $90^{\circ}$ Direction (Column 23) for the Normal and Checkerboard Cases. (ANL Neg. No. 116-76-298). 
${ }^{238} \mathrm{U}$ Fission Rate

$0^{\circ}$ Traverse

Calculated Data

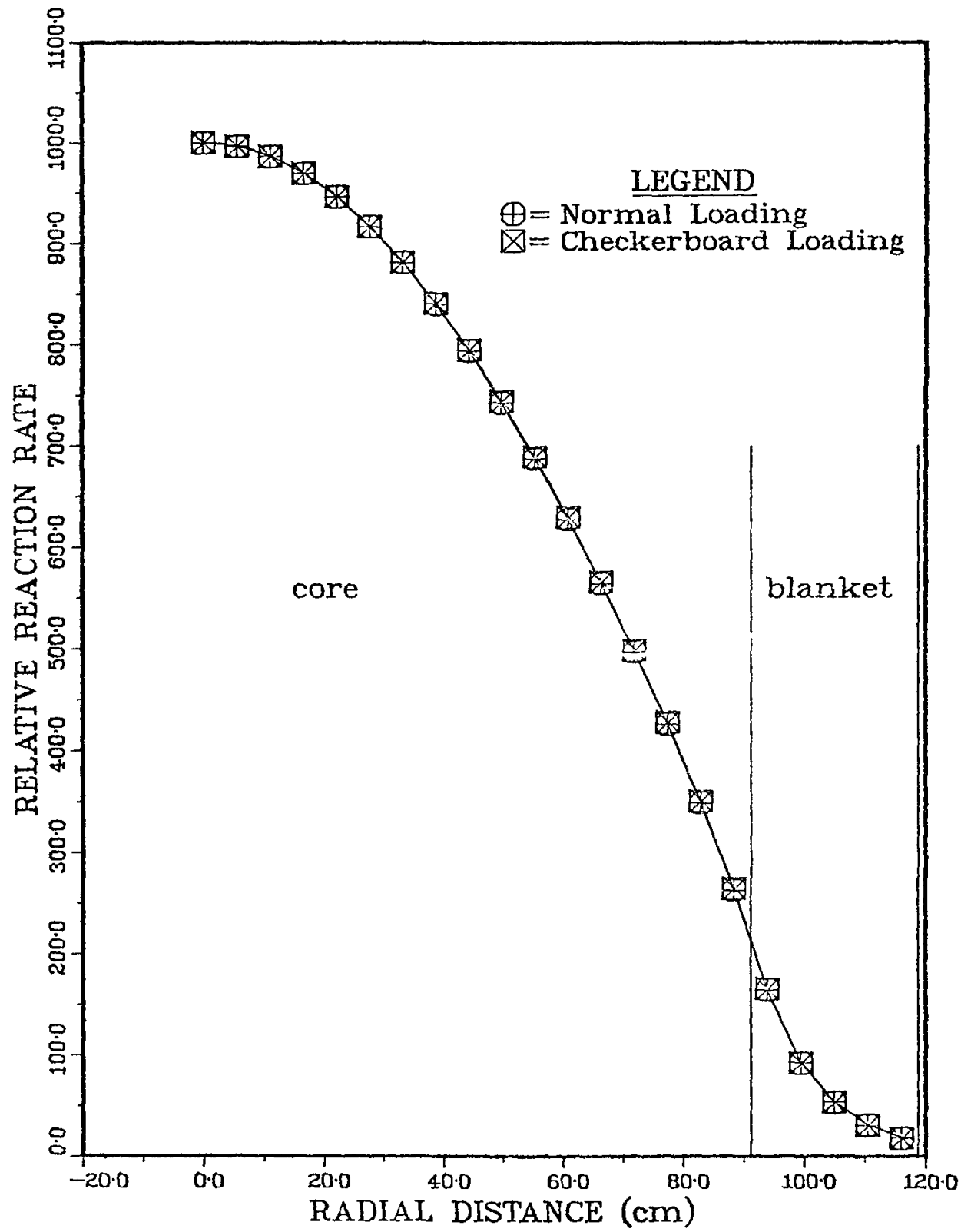

Fig. 89. Comparison of the Calculated $238 \mathrm{U}$ Fission Rate Distribution in the $0^{\circ}$ Direction (Row 23) for the Normal and Checkerboard Cases. (ANL Neg. No. 11676-337). 


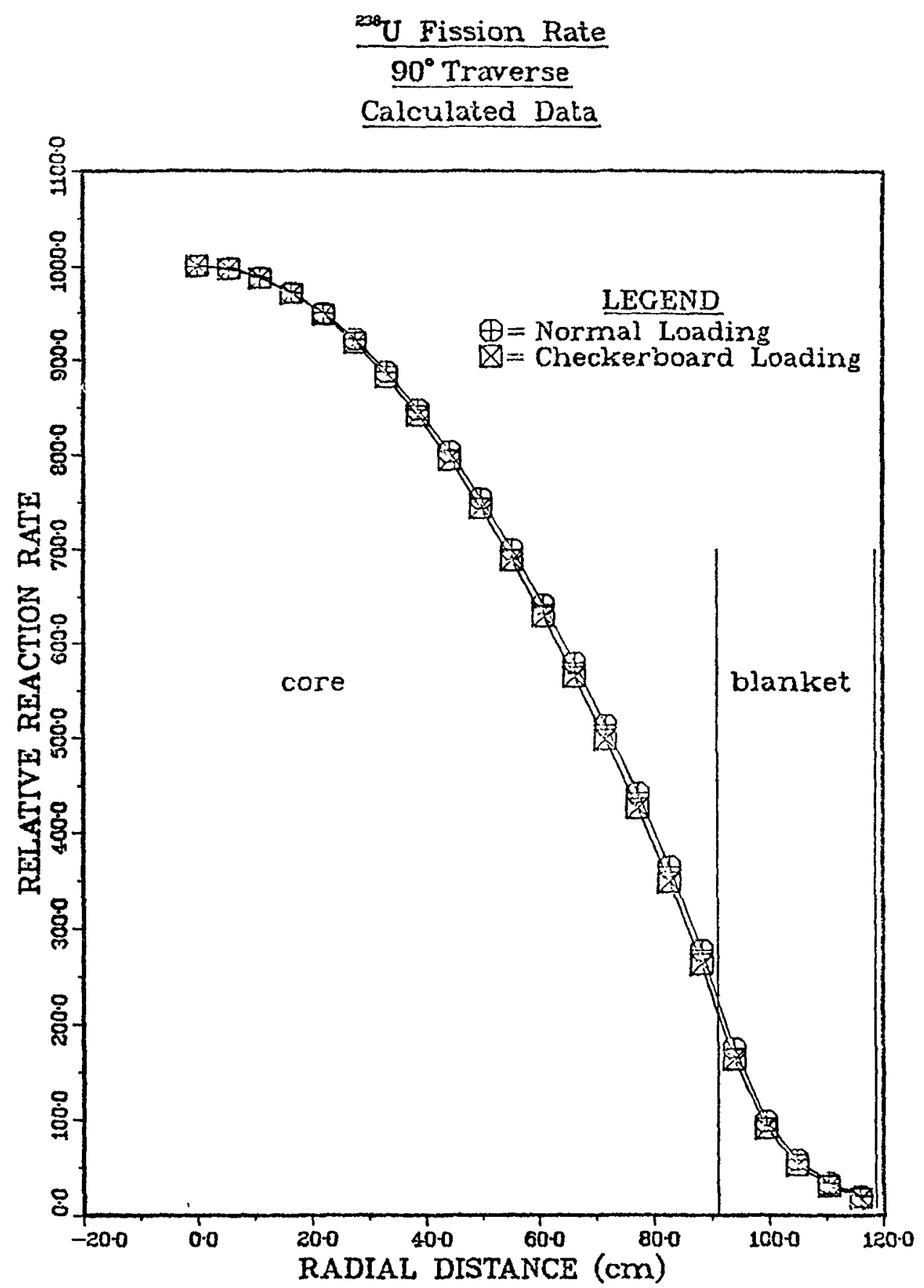

Fig. 90. Comparison of the Calculated ${ }^{238} \mathrm{U}$ Fission Rate Distribution in the $90^{\circ}$ Direction (Column 23) for the Normal and Checkerboard Cases. (ANL Neg. No. 116-76-271). 
${ }^{230} \mathrm{Pu}$ Fission Rate

$0^{\circ}$ Traverse

Calculated Data

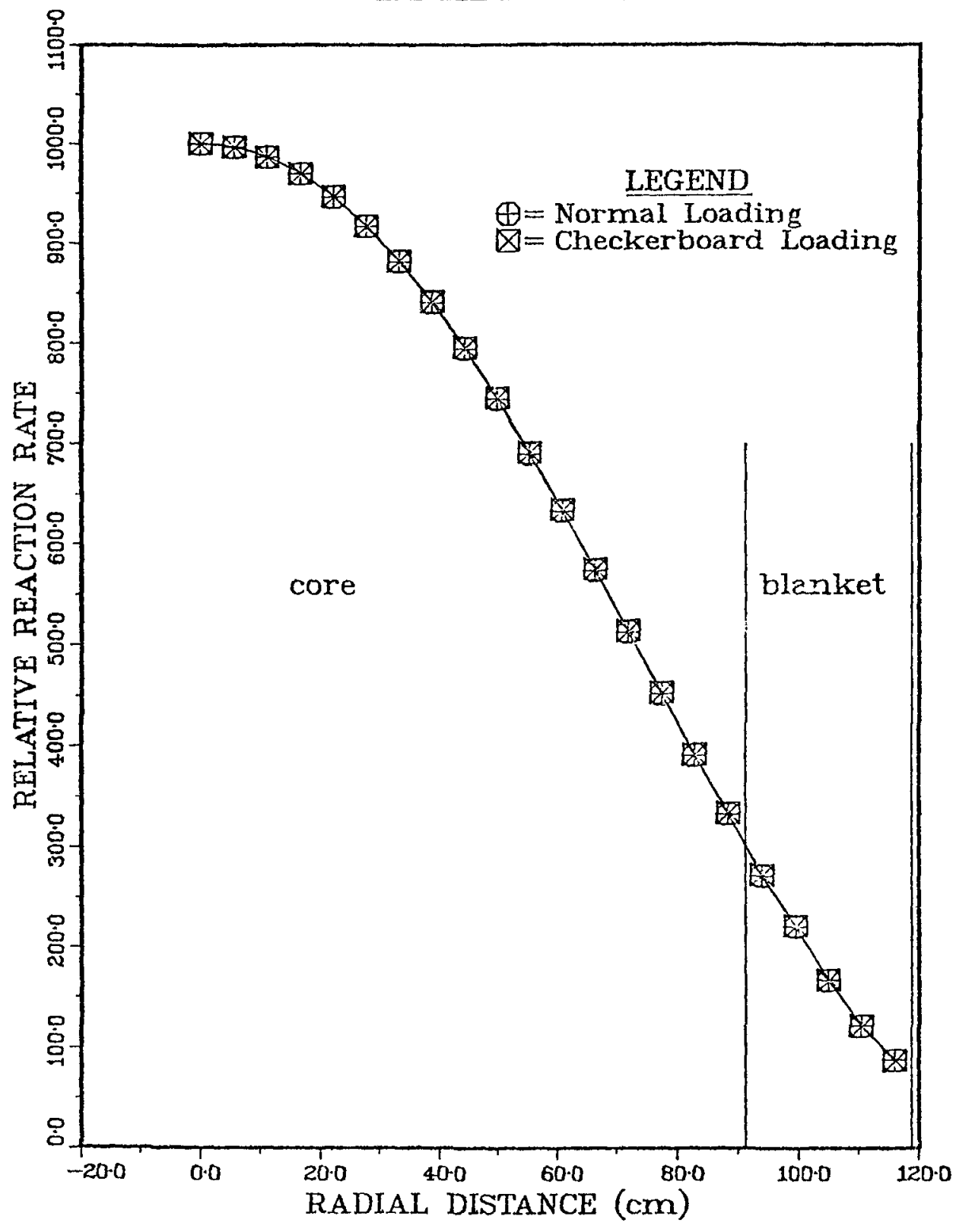

Fig. 91. Comparison of the Calculated $239 \mathrm{Pu}$ Fission Rate Distribution in the $0^{\circ}$ Direction (Row 23) for the Normal and Checkerboard Cases. (ANL Neg. No. 11676-380). 


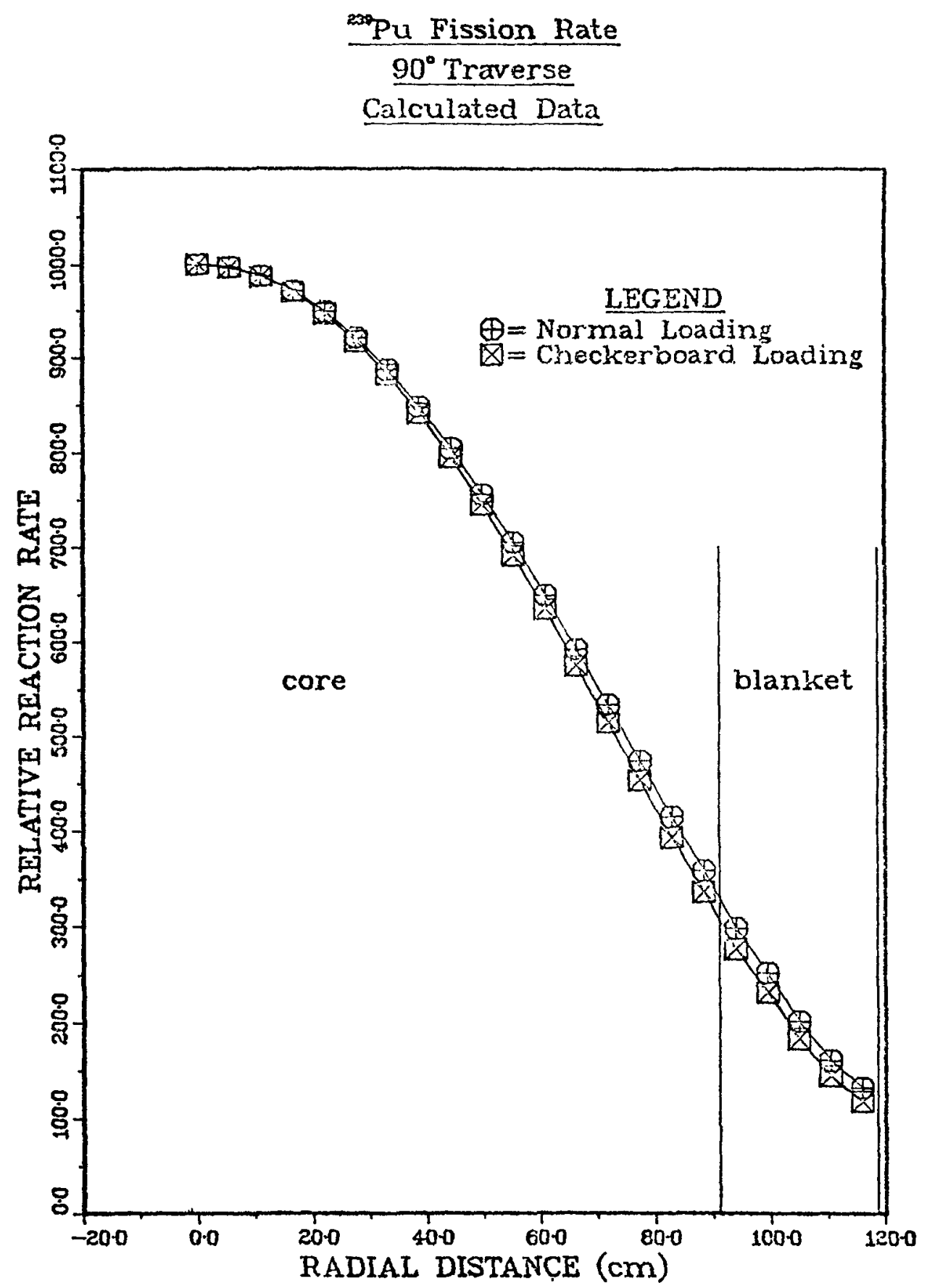

Fig. 92. Comparison of the Calculated ${ }^{239} \mathrm{Pu}$ Fission Rate Distribution in the $90^{\circ}$ (Column 23) for the Normal and Checkerboard Cases. (ANL Neg. No. 116-76308). 


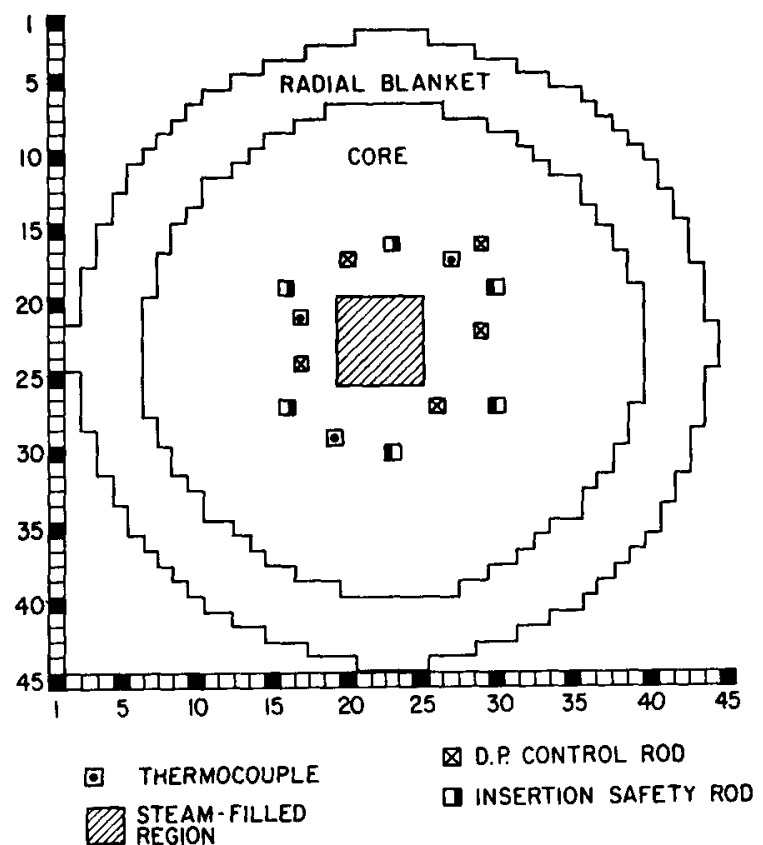

STATIONARY MALF
Fig. 93. Location of the Experimenta1 Zone for the Steam Entry Measurements. (ANL Neg. No. 116-76-352).

Fig. 94. RZ Model Used in the Analysis

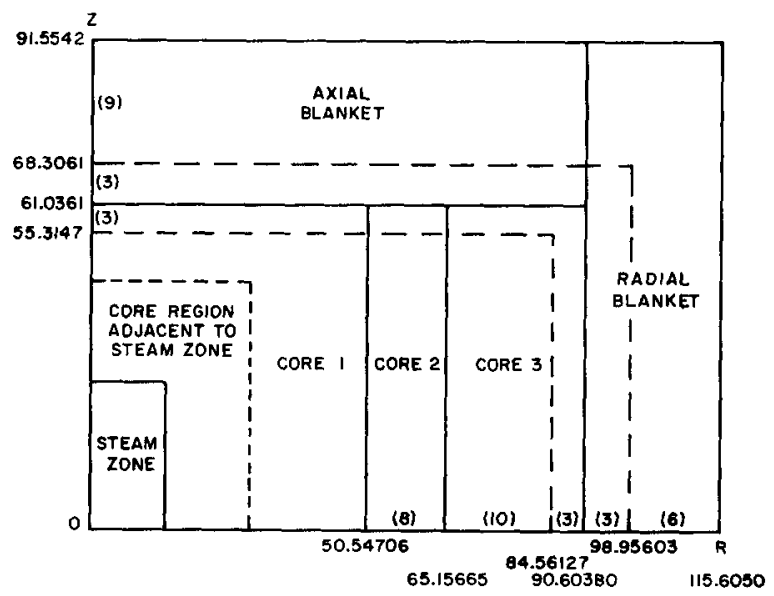
ments (ANL Neg. No. 116 76-283). 


\section{GCFR PHASE 1 STEAM ZONE}

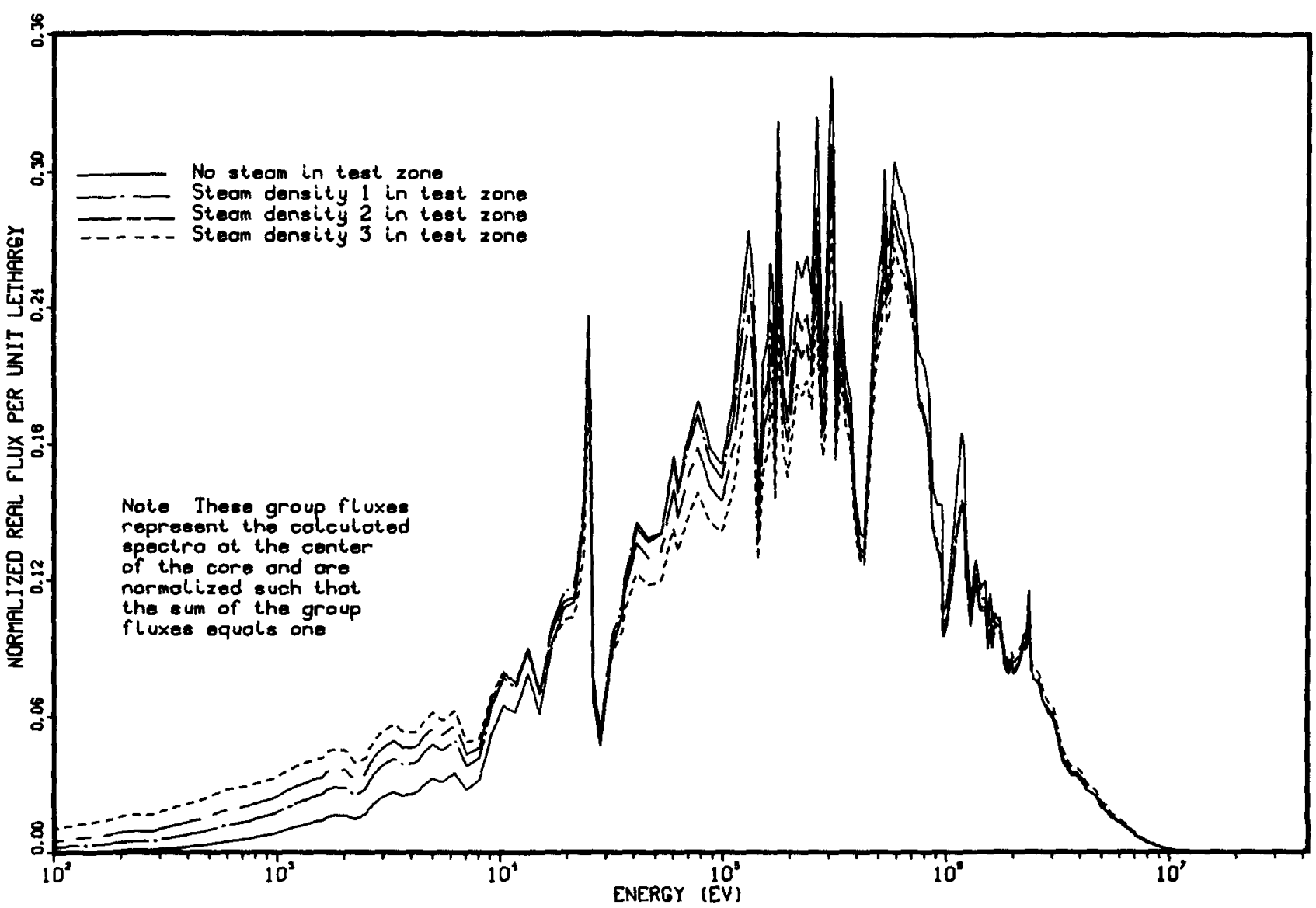

F1g. 95. Intermediate Group Spectra at Core Center for "Normal" and "SteamFilled" Cases. (ANL Neg. No. 116-76-275). 


\section{GCFR PHASE I STEAM ZONE}

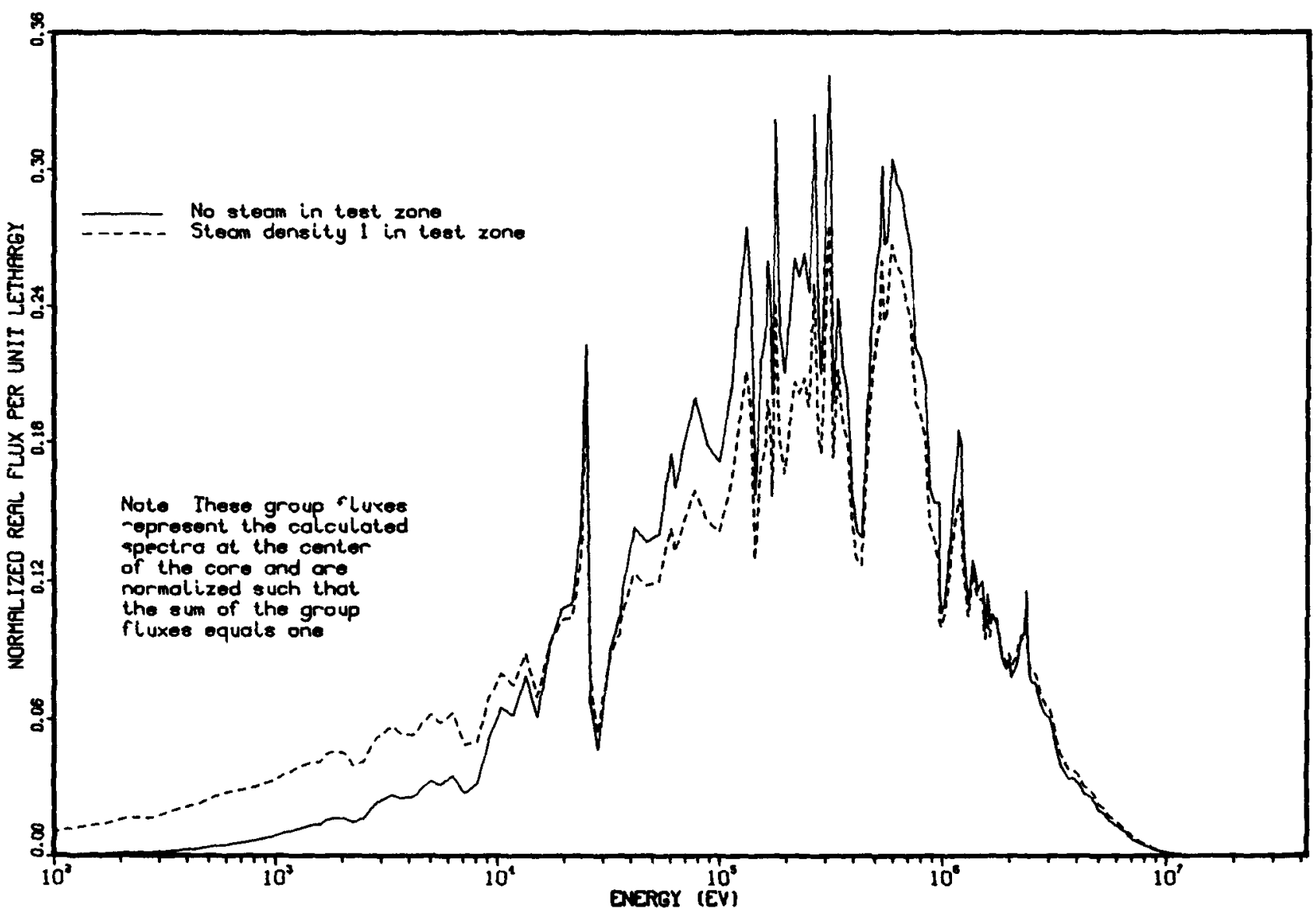

Fig. 96. Intermediate Group Spectra at Center of Test Zone for Normal and Steam-Filled Cases (Steam Density No. 1). (ANL Neg. No. 116-76264). 


\section{GCFR PHASE 1 STEAM ZONE}

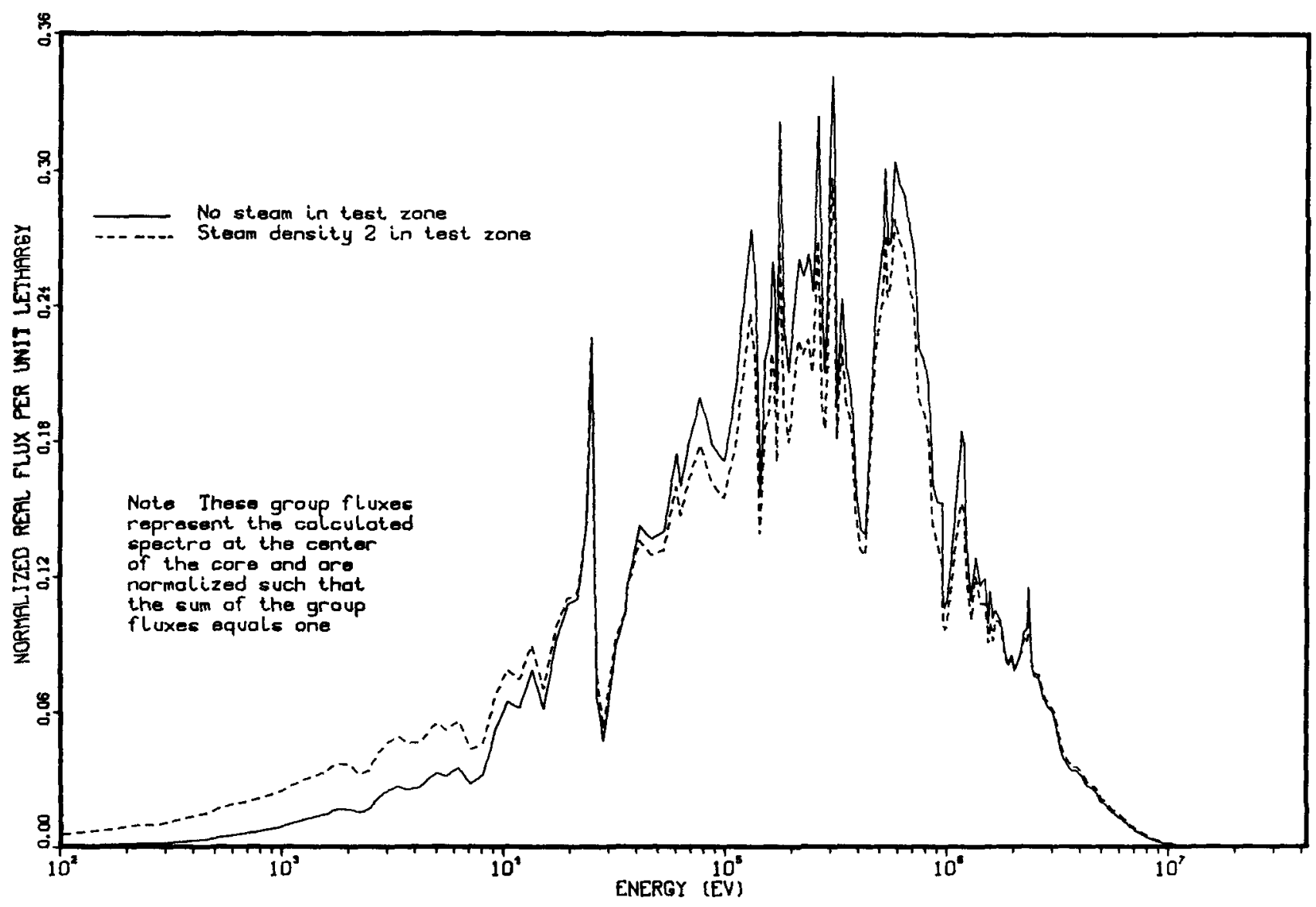

Fig. 97. Intermediate Group Spectra at Center of Test Zone for Normal and Steam-Filled Cases (Steam Density No. 2). (ANL Neg. No. 116-76263). 


\section{GCFR PHASE 1 STEAM ZONE}

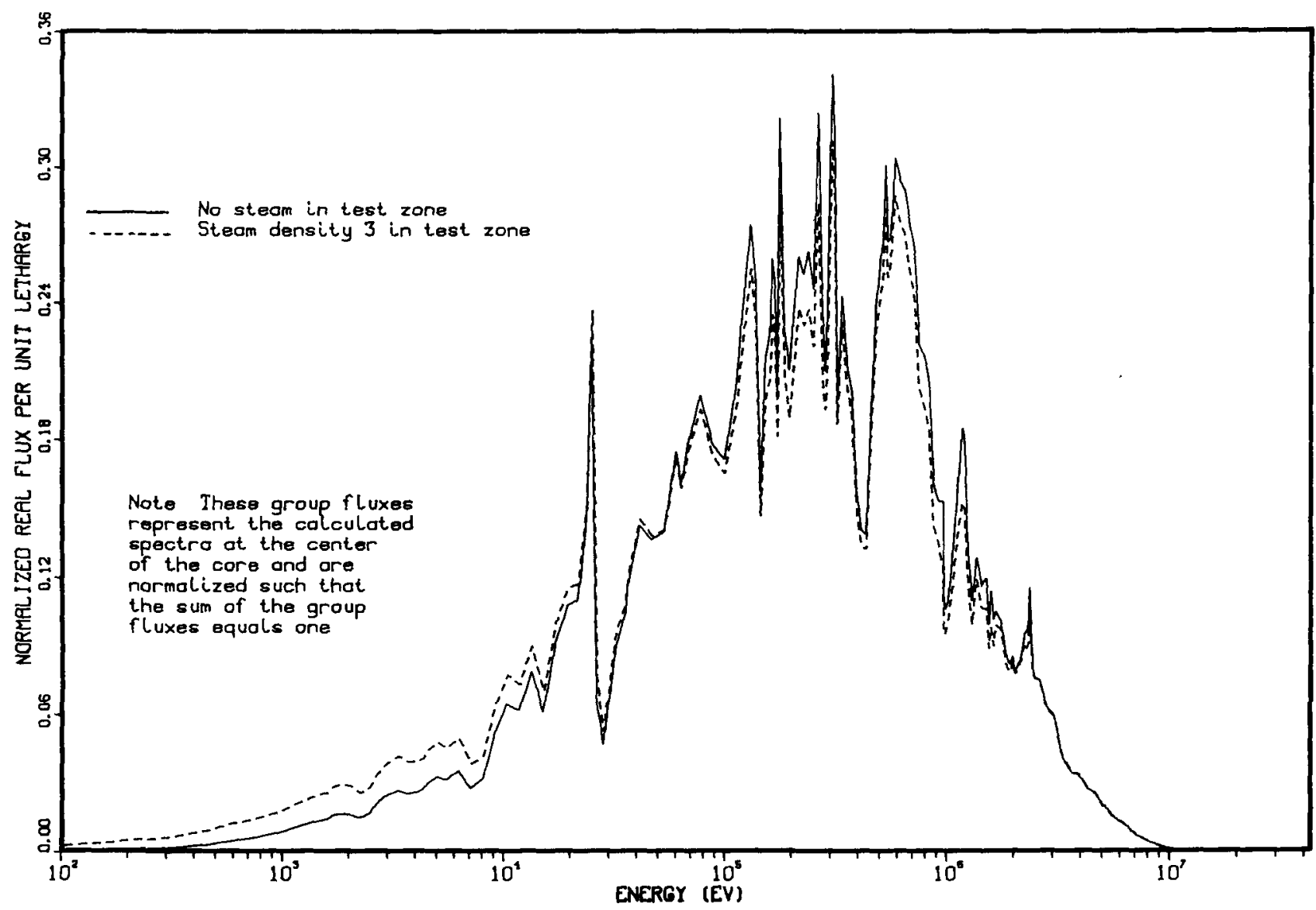

Fig. 98. Intermediate Group Spectra at Center of Test Zone for Normal and Steam-Filled Case (Steam Density No. 3). (ANL Neg. No. 116-76265). 


\section{GCFR PHASE I (UNPERTURBED ASSEMBLY)}

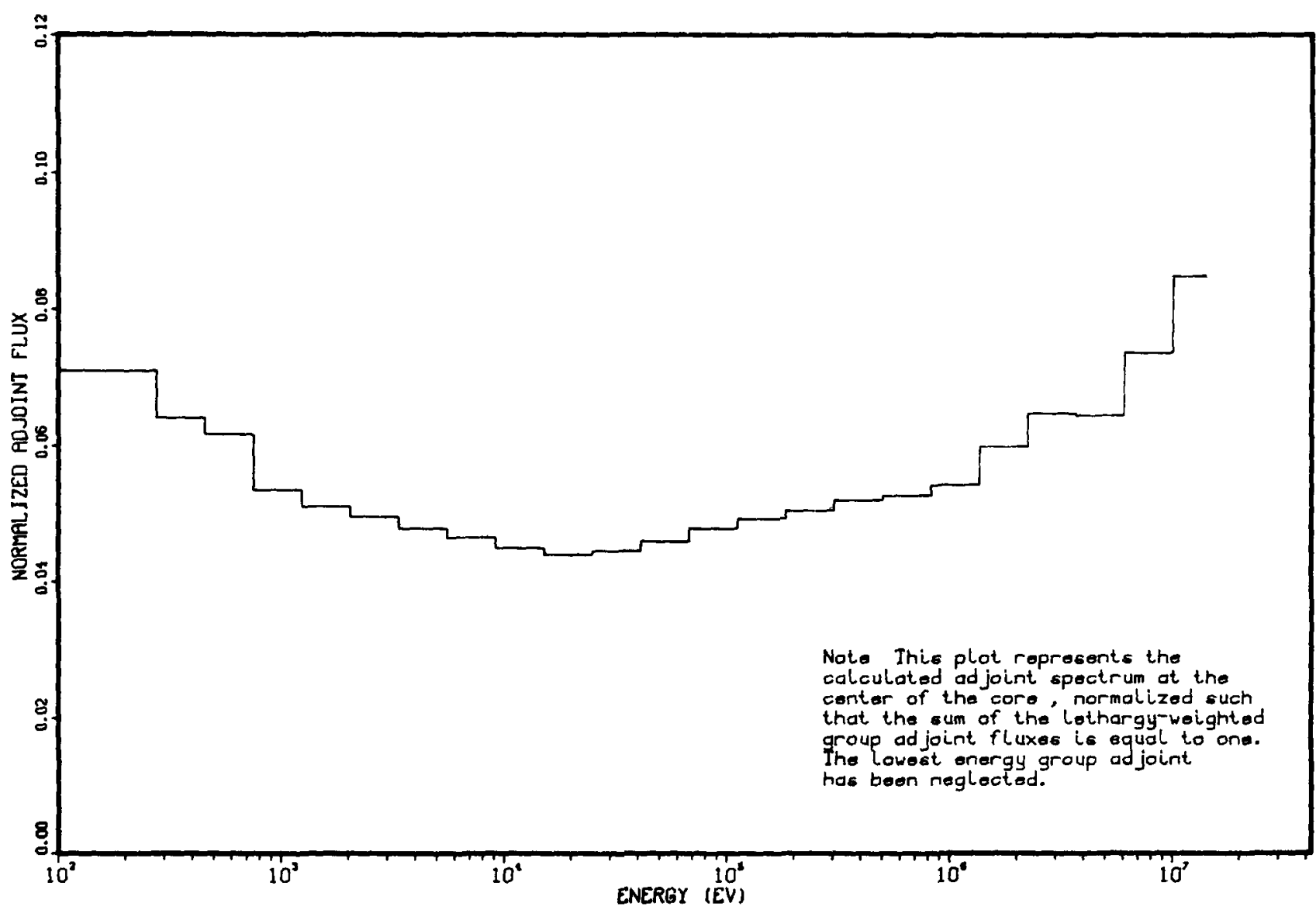

Fig. 99. Broad Group Adjoint Spectrum at Core Center for Reference Configuration. (ANL Neg. No. 116-76-293). 


\section{GCFR PHASE 1 STEAM ZONE FLUXES}

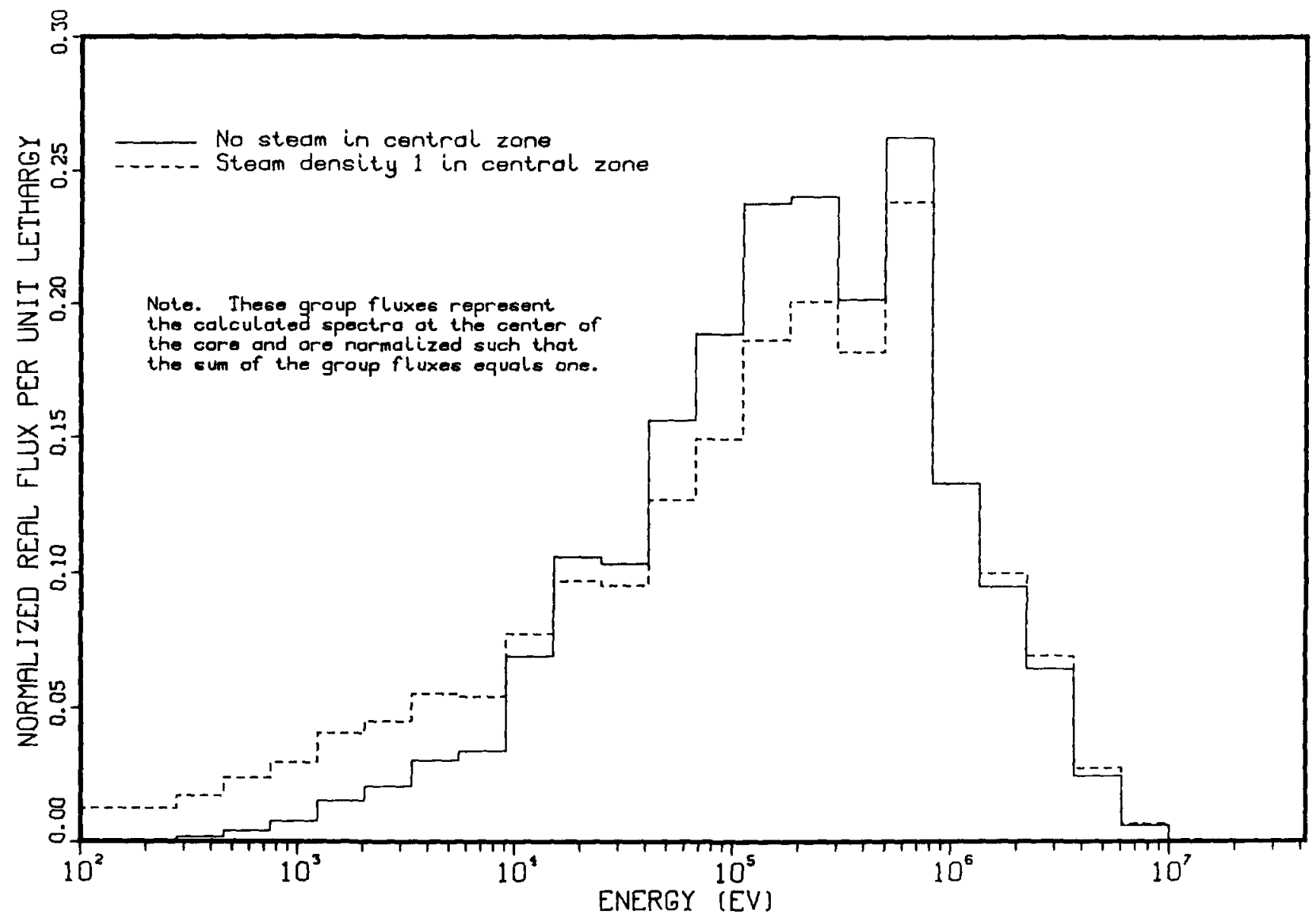

Fig. 100. Comparative Broad (29) Group Spectra at Center of Test Zone for the Reference and Steam Density No. 1 Filled Zone Cases. (ANL Neg. No. 116-76-266). 


\section{GCFR PHASE 1 STEAM ZONE FLUXES}

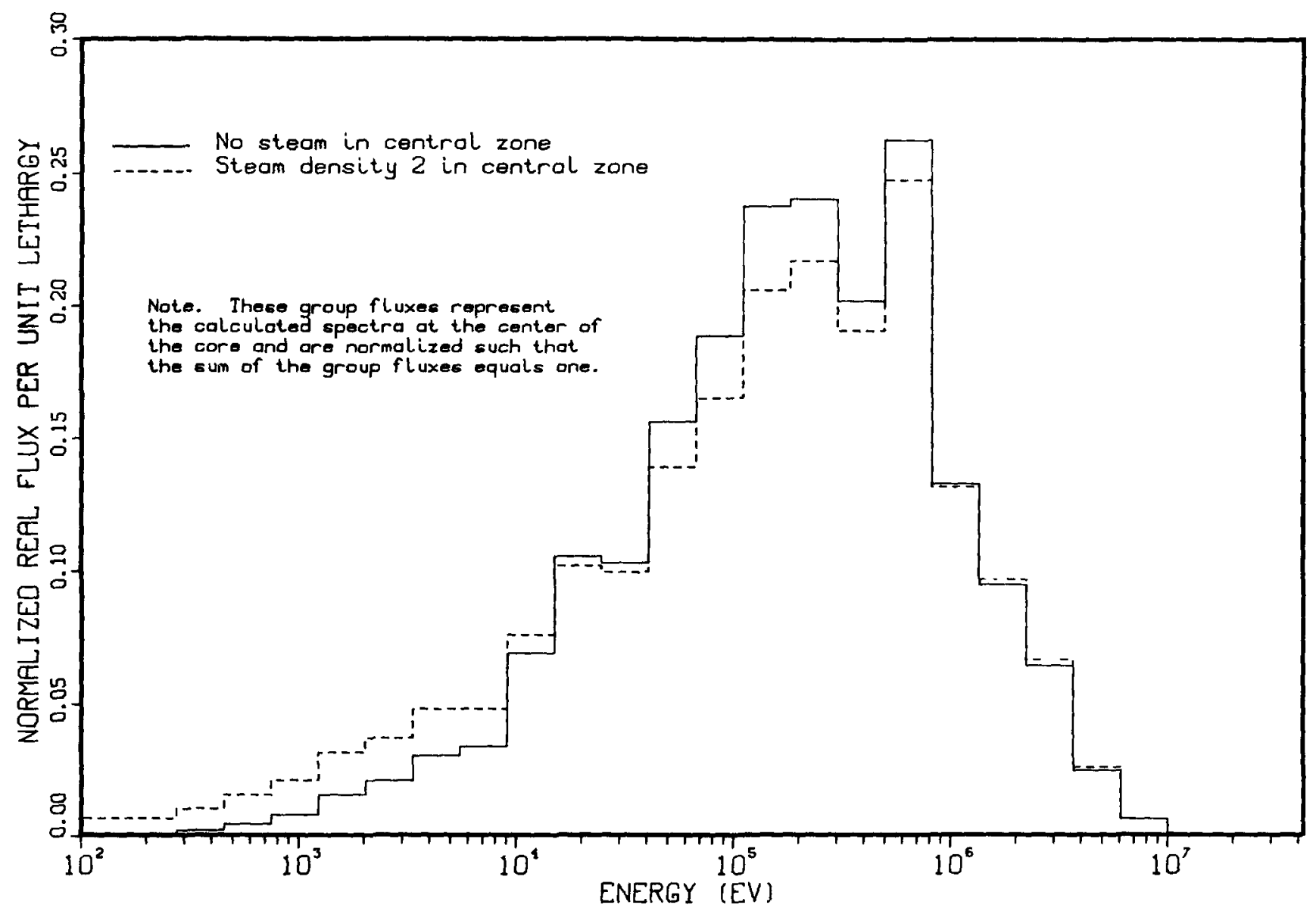

Fig. 101. Comparative Broad (29) Group Spectra at Center of Test Zone for the Reference and Steam Density No. 2 Filled Zone Cases. (ANL Neg. No. 116-76-320). 


\section{CCFR PHASE 1 STEAM ZONE FLUXES}

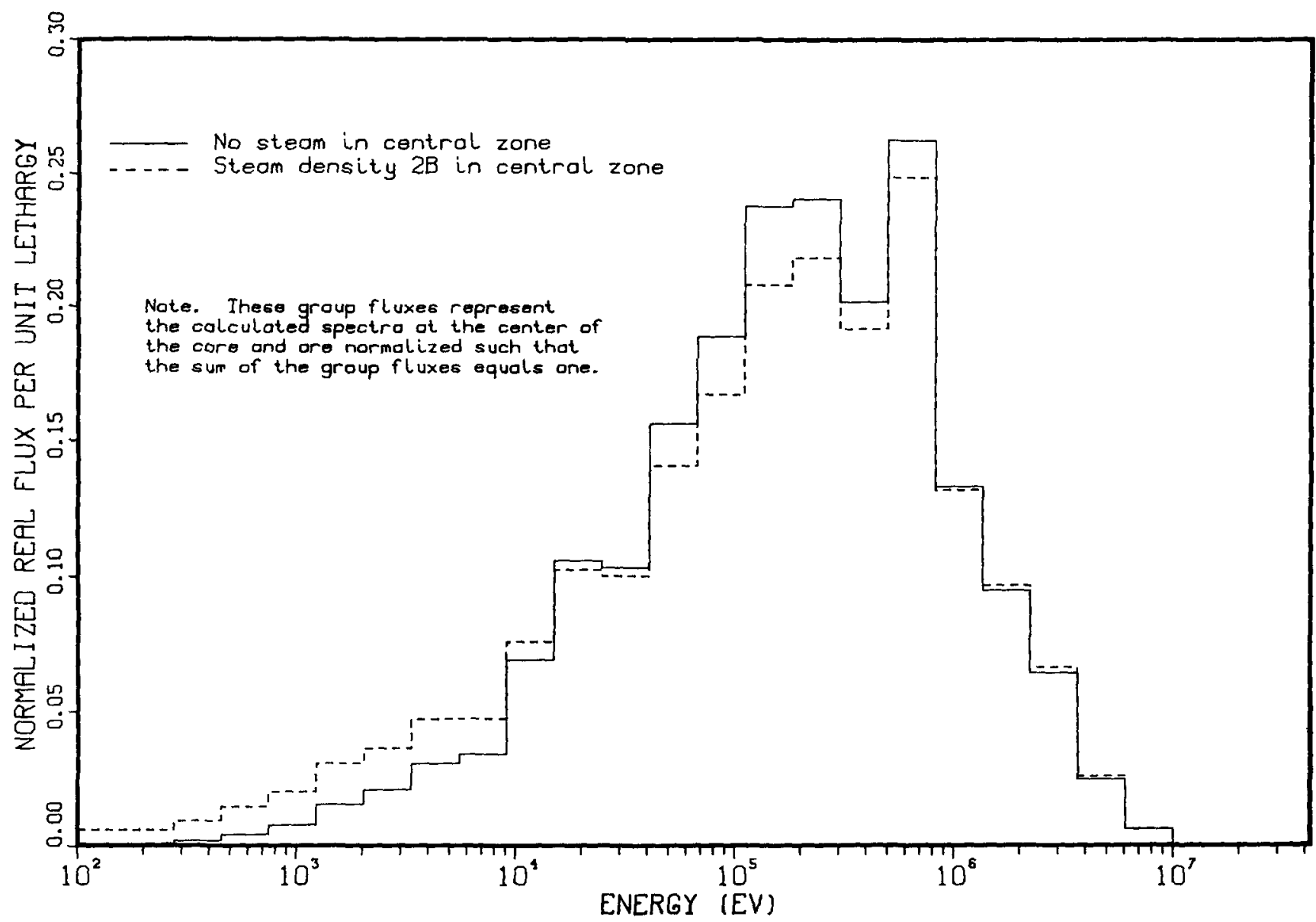

Fig. 102. Comparative Broad (29) Group Spectra at Center of Test Zone for the Reference and Steam Density No. 2b Filled Zone Cases. (ANL Neg. No. 116-76-299). 


\section{GCFR PHASE 1 STEAM ZONE FLUXES}

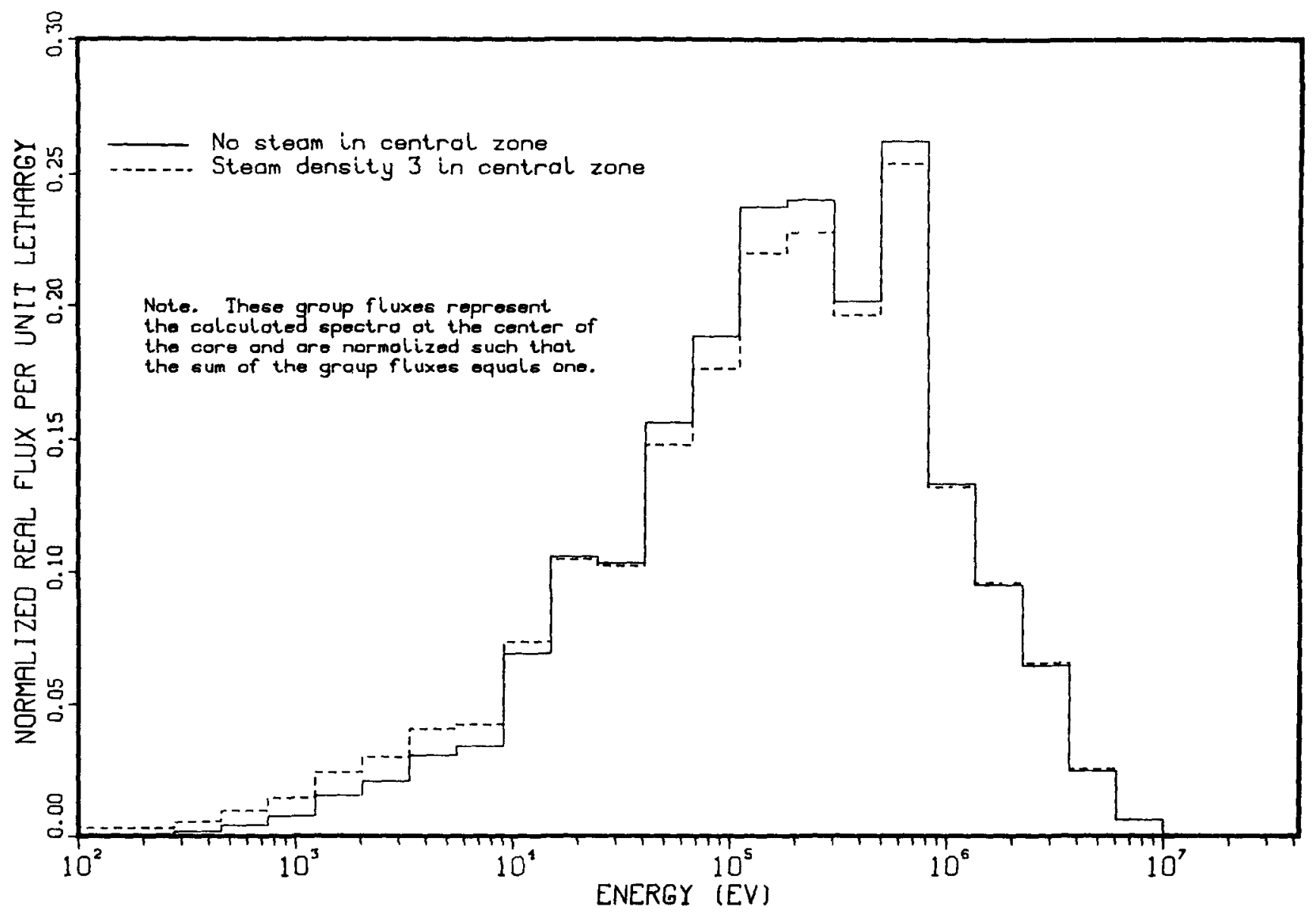

Fig. 103. Comparative Broad (29) Group Spectra at Center of Test Zone for the Reference and Steam Density No. 3 Filled Zone Cases. (ANL Neg. No. 116-76-315). 
ANALYSIS OF GCFR PHASE I STEAM ENTRY EXPERIMEN'.

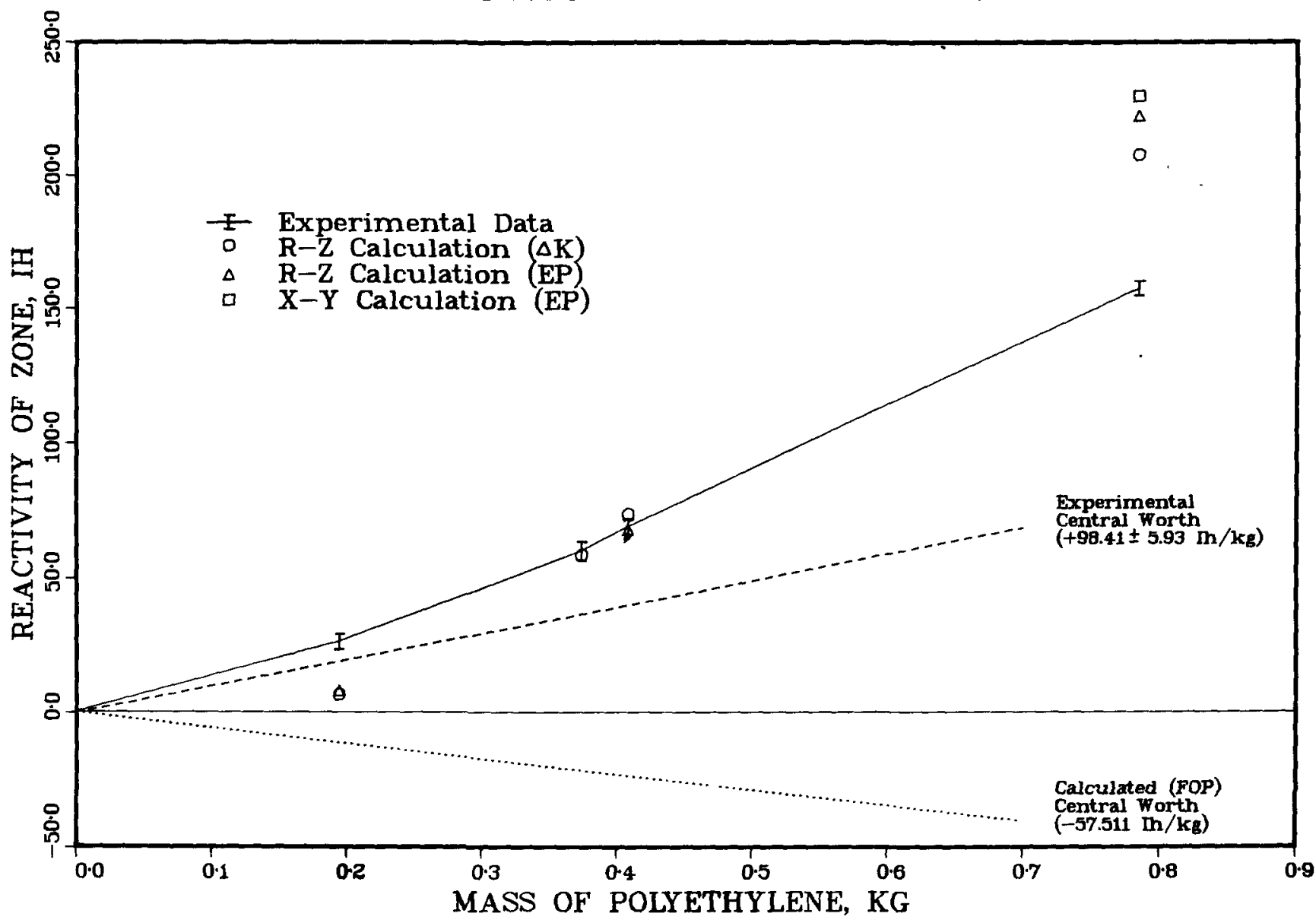

Fig. 104. Results of Small Zone Steam-Entry Experiments. (ANL Neg. No. $116-76-326)$. 
I. Geometric Specifications of Core Unit Cell

\begin{tabular}{|c|c|c|c|c|}
\hline \multicolumn{2}{|r|}{ Intra-Cell Region } & \multirow{2}{*}{$\underset{\mathrm{cm}}{\text { Width }}, a$} & \multirow{2}{*}{$\underset{\mathrm{cm}}{\operatorname{He} \mathrm{ght}, \mathrm{b}}$} & \multirow{2}{*}{$\begin{array}{l}\text { Unit } \\
\text { Volume, } \\
\mathrm{cm}^{3}\end{array}$} \\
\hline No. & Description & & & \\
\hline $\begin{array}{l}1 \\
2 \\
3 \\
4 \\
5 \\
6 \\
7 \\
8 \\
9 \\
10 \\
11 \\
12 \\
13 \\
14 \\
15 \\
16\end{array}$ & 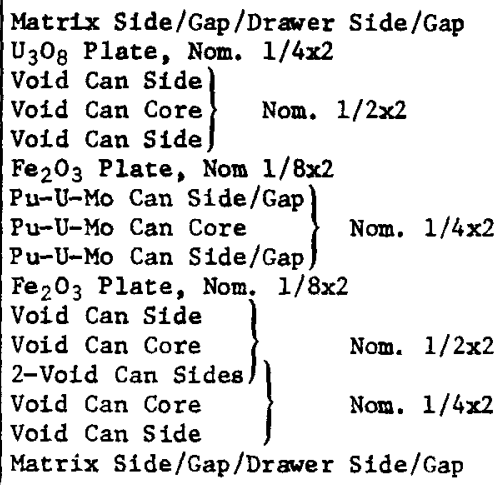 & $\begin{array}{l}0.28215 \\
0.599 \\
0.038 \\
1.173 \\
0.038 \\
0.3175 \\
0.0521 \\
0.510 \\
0.0521 \\
0.3175 \\
0.038 \\
1.173 \\
0.076 \\
0.538 \\
0.038 \\
0.28215\end{array}$ & $\begin{array}{l}5.08 \\
5.048 \\
5.067 \\
4.991 \\
5.067 \\
5.08 \\
5.07 \\
4.920 \\
5.07 \\
5.08 \\
5.067 \\
4.991 \\
5.067 \\
4.991 \\
5.067 \\
5.08\end{array}$ & $\begin{array}{l}1.433 \\
3.024 \\
0.1925 \\
5.854 \\
0.1925 \\
1.613 \\
0.2641 \\
2.509 \\
0.2641 \\
1.613 \\
0.1925 \\
5.854 \\
0.3851 \\
2.685 \\
0.1925 \\
1.433\end{array}$ \\
\hline$\sum$ & Sum - All Regions & 5.5245 & $\mathrm{~N} / \mathrm{A}$ & $27.7013^{c}$ \\
\hline $\mathbb{N} / \mathrm{A}$ & Homogeneous Unit-Cell & 5.5245 & 5.5245 & $1862.8^{d}$ \\
\hline
\end{tabular}

a"Direction of calculation" dimension in a 1-D neutronics input module. bsed for computation of densities (See Table III). Unit length $=1 \mathrm{~cm}$. $c^{c}$ That portion of the $(5.5245)^{2}$, or $30.5201 \mathrm{~cm}^{2}$, cell cross section

assigned to the slab regions.

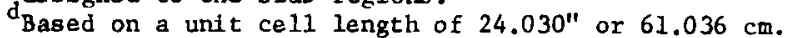

TABLE II. Geometric Specifications of Blanket Unit Cell

\begin{tabular}{|c|c|c|c|c|}
\hline \multicolumn{2}{|r|}{ Intra-Cell Region } & \multirow{2}{*}{ Widtha } & \multirow{2}{*}{$\underset{\mathrm{cm}}{H e},,^{b}$} & \multirow{2}{*}{$\begin{array}{c}\text { Unit } \\
\text { Volume } \\
\mathrm{cm}^{3}\end{array}$} \\
\hline No. & Description & & & \\
\hline \multicolumn{5}{|c|}{ RADIAL BLANKET } \\
\hline $\begin{array}{l}1 \\
2 \\
3 \\
4 \\
5 \\
6 \\
7 \\
8 \\
9 \\
10 \\
11 \\
12\end{array}$ & 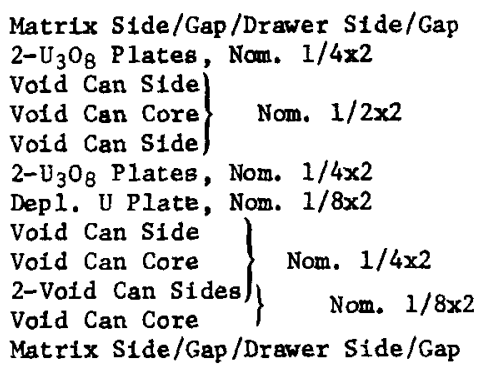 & $\begin{array}{l}0.3205 \\
1.198 \\
0.038 \\
1.173 \\
0.038 \\
1.198 \\
0.3175 \\
0.038 \\
0.538 \\
0.076 \\
0.269 \\
0.3205\end{array}$ & $\begin{array}{l}5.08 \\
5.048 \\
5.067 \\
4.991 \\
5.067 \\
5.048 \\
5.08 \\
5.067 \\
4.991 \\
5.067 \\
4.991 \\
5.08\end{array}$ & $\begin{array}{l}1.628 \\
6.048 \\
0.1925 \\
5.854 \\
0.1925 \\
6.048 \\
1.613 \\
0.1925 \\
2.685 \\
0.3851 \\
1.343 \\
1.628\end{array}$ \\
\hline$\sum$ & Sum - All Regions & 5.5245 & $\mathrm{~N} / \mathrm{A}$ & $27.8096^{c}$ \\
\hline $\mathrm{N} / \mathrm{A}$ & Homogeneous Unt $t$-Cell & 5.5245 & 5.5245 & $2793.0^{d}$ \\
\hline \multicolumn{5}{|c|}{ AXIAL BLANKET } \\
\hline $\begin{array}{r}1 \\
2 \\
3 \\
4 \\
5 \\
6 \\
7 \\
8 \\
9 \\
10 \\
11 \\
12 \\
13\end{array}$ & 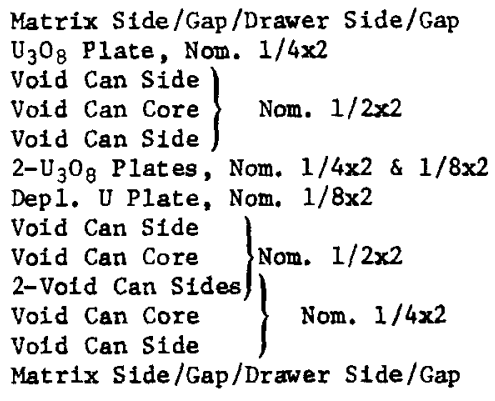 & $\begin{array}{l}0.2950 \\
0.599 \\
0.038 \\
1.173 \\
0.038 \\
0.906 \\
0.3175 \\
0.038 \\
1.173 \\
0.076 \\
0.538 \\
0.038 \\
0.2950\end{array}$ & $\begin{array}{l}5.08 \\
5.048 \\
5.067 \\
4.991 \\
5.067 \\
5.052 \\
5.08 \\
5.067 \\
4.991 \\
5.067 \\
4.991 \\
5.067 \\
5.08\end{array}$ & $\begin{array}{l}1.499 \\
3.024 \\
0.1925 \\
5.854 \\
0.1925 \\
4.577 \\
1.613 \\
0.1925 \\
5.854 \\
0.3851 \\
2.685 \\
0.1925 \\
1.499\end{array}$ \\
\hline$\sum$ & Sum - All Regions & 5.5245 & $\mathrm{~N} / \mathrm{A}$ & $27.7601^{c}$ \\
\hline $\mathrm{N} / \mathrm{A}$ & Homogeneous Un $1 \mathrm{t}-\mathrm{Ce} 11$ & 5.5245 & 5.5245 & $930.25^{e}$ \\
\hline
\end{tabular}

a "Direction of calculation" dimension in a l-D neutrontcs 1nput module. b Used for computation of densities (See Table III). Unit length - $1 \mathrm{~cm}$ c That portion of the $(5.5245)^{2}$, or $30.5201 \mathrm{~cm}^{2}$, ceil cross section assigned to the slab regions.

assigned to the slab regions.

e Based on a unit-cell length of 12.000 " or $30.480 \mathrm{~cm}$. 
TABLE III. GCFR-Phase I Thermocouple Locations

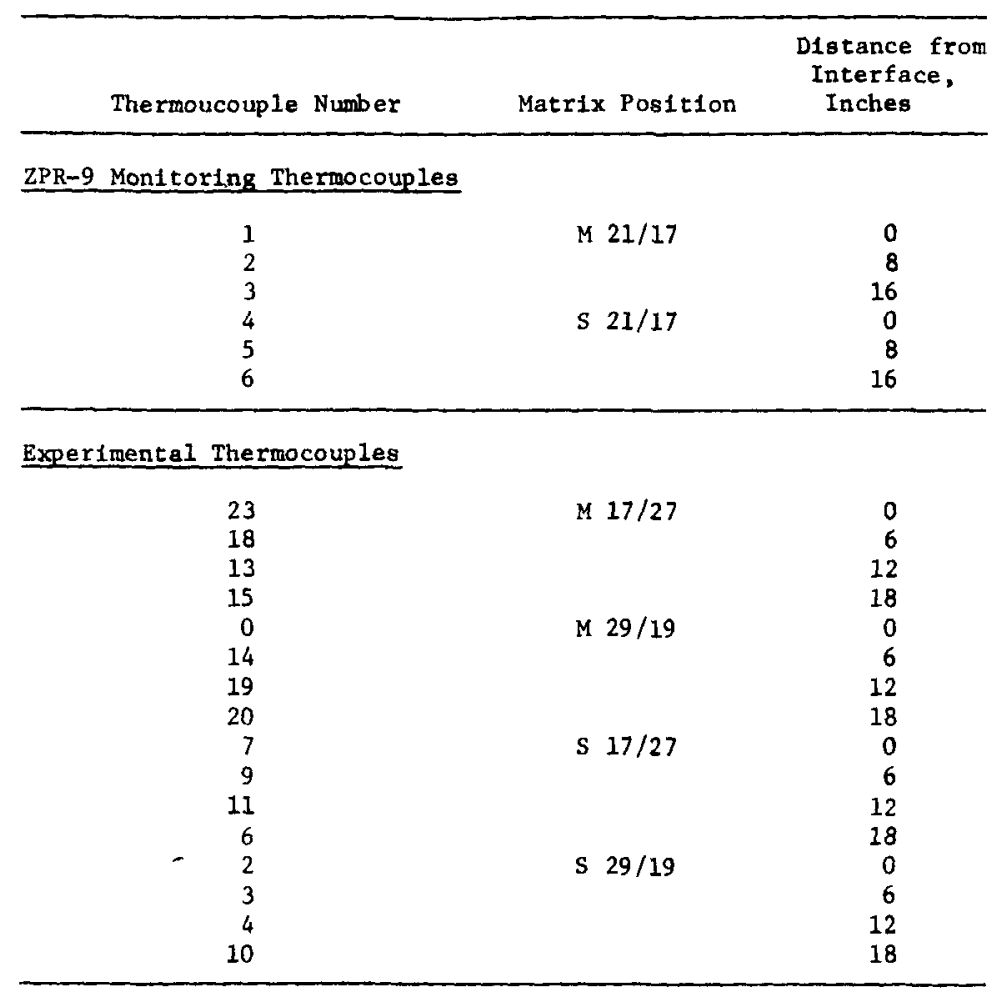

TABLE IV. Location of Detectors for Use in Approach-to-Critical Measurement

\begin{tabular}{|c|c|c|c|c|c|}
\hline $\begin{array}{l}\text { Detector } \\
\text { No. }\end{array}$ & Detector Type & Detector Location & & Comment & \\
\hline 1 & $2{ }^{38}$ U Fission Chamber & Matrix location $523 / 23$ in axtal midplane & Incore det & tector & \\
\hline 2 & ${ }^{235} U$ Fission Chamber & $\begin{array}{l}\text { Matrix location } 523 / 23,24 \text { in. from axial } \\
\text { midplane }\end{array}$ & $"$ & $"$ & \\
\hline 3 & ${ }^{235} \mathrm{U}$ F1ssion Chamber & Matrix location $\mathrm{M} 23 / 23$ in axtal midplane & $"$ & 11 & \\
\hline 4 & 235 U Fission Chamber & $\begin{array}{l}\text { Matrix location } \mathrm{M} 23 / 23,24 \text { in. from axial } \\
\text { mfdplane }\end{array}$ & $"$ & $"$ & \\
\hline 5 & $\mathrm{BF}_{3}$ Proportional Counter & Top of stationary half at axial midplane & Reactor & Operattonal & Instrument \\
\hline 6 & $\mathrm{BF}_{3}$ Proportional Counter & Top of movable half at axial midplane & $"$ & $"$ & $"$ \\
\hline 7 & $\mathrm{BF}_{3}$ Ionization Chamber & Top of stationary half at axial midplane & $"$ & $"$ & $" 1$ \\
\hline 9 & $\mathrm{BF}_{3}$ Ionization Chamber & Top of stationary half at axial midplane & $"$ & $"$ & $"$ \\
\hline 10 & $\mathrm{BF}_{3}$ Ion Ization Chamber & Top of movable half at axial midplane & $"$ & $"$ & $"$ \\
\hline
\end{tabular}


BLE V. Fuel Loading Steps in the Approach to Critical

\begin{tabular}{ccccc}
$\begin{array}{c}\text { Loading } \\
\text { Step }\end{array}$ & Date & $\begin{array}{c}\text { Loading } \\
\text { No. }\end{array}$ & $\begin{array}{c}\text { Fissile Mass } \\
\text { Loaded }(\mathrm{kg}) \\
2{ }^{39} \mathrm{Pu}^{24} \mathrm{Pu}{ }^{235} \mathrm{U}\end{array}$ & $\begin{array}{c}\text { Core Radius } \\
(\mathrm{cm})\end{array}$ \\
\hline 1 & $3 / 12 / 75$ & 25 & 407.40 & 54.03 \\
2 & $3 / 19 / 75$ & 28 & 809.00 & 76.34 \\
3 & $3 / 20 / 75$ & 29 & 841.06 & 77.85 \\
4 & $3 / 25 / 75$ & 30 & 937.23 & 82.21 \\
5 & $3 / 27 / 75$ & 31 & 1033.40 & 86.35 \\
6 & $3 / 31 / 75$ & 32 & 1129.59 & 90.31 \\
\hline
\end{tabular}

TABLE VI. Predicted Critical Masses from the Responses of A11 Detectors during the Approach to Critical Measurements Using a $1 / \mathrm{C}$ vs. $M_{f}$

\begin{tabular}{|c|c|c|c|c|c|c|}
\hline & Estimated & Critical & Mass (kg) & in Loading & Step & \\
\hline Number & Loading Step: & 2 & 3 & 4 & 5 & 6 \\
\hline 1 & & 940 & 1060 & 1085 & 1119 & 1135 \\
\hline 2 & & 913 & 1017 & 1082 & 1113 & 1135 \\
\hline 3 & & 920 & 1035 & 1080 & 1122 & 1136 \\
\hline 4 & & 922 & 1044 & 1075 & 1118 & 1136 \\
\hline 5 & & 877 & 1027 & 1073 & $1132^{a}$ & a \\
\hline 6 & & 877 & 1054 & 1061 & $1129^{a}$ & $-{ }^{a}$ \\
\hline 7 & & 860 & $-b$ & 1035 & 1100 & 1130 \\
\hline 8 & & 875 & 970 & 1030 & 1110 & 1137 \\
\hline 9 & & 870 & 998 & 1060 & 1109 & 1136 \\
\hline 10 & & 872 & 999 & 1035 & 1111 & 1136 \\
\hline
\end{tabular}

High instrument dead time. Results unreliable.

${ }^{b}$ Error in data point.
TABLE VII. Predicted Critical Masses from the Responses of A11

Detectors during the Approach to Critical Measurements Using a $M_{f} / C$ vs. $M_{f}$ Formulation

\begin{tabular}{|c|c|c|c|c|c|c|}
\hline \multirow{2}{*}{$\begin{array}{l}\text { Detector } \\
\text { Number }\end{array}$} & \multicolumn{6}{|c|}{ Estimated Critical Mass (kg) in Loading Step } \\
\hline & Loading Step: & 2 & 3 & 4 & 5 & 6 \\
\hline 1 & & 1180 & 1175 & 1140 & 1135 & 1136 \\
\hline 2 & & 1090 & 1090 & 1130 & 1138 & 1136 \\
\hline 3 & & 1130 & 1130 & 1130 & 1130 & 1136 \\
\hline 4 & & 1115 & 1114 & 1130 & 1156 & 1136 \\
\hline 5 & & 971 & 1084 & 1116 & $1156^{\mathrm{a}}$ & $-{ }^{a}$ \\
\hline 6 & & 977 & 1100 & 1108 & $1151^{\mathrm{a}}$ & $-a^{a}$ \\
\hline 7 & & 910 & $-b$ & 1050 & 1137 & 1140 \\
\hline 8 & & 955 & 995 & 1055 & 1122 & 1137 \\
\hline 9 & & 945 & 1031 & 1094 & 1122 & 1137 \\
\hline 10 & & 957 & 1045 & 1059 & 1130 & 1136 \\
\hline
\end{tabular}

High instrument dead time. Results unreliable.

brror in data point. 
TABLE VIII. Summary of Reactivity and Mass Correction for the GCFR Phase I Assembly

\begin{tabular}{lccc}
\hline \multicolumn{1}{c}{ Correction } & $\begin{array}{c}\Delta \rho \text { (Ih) } \\
\left.\text { (Corrected to } 25^{\circ} \mathrm{C}\right)\end{array}$ & $\begin{array}{c}\text { Equivalent } \Delta M \\
(\mathrm{~kg})^{\mathrm{a}}\end{array}$ \\
\hline $\begin{array}{l}\text { 1. Excess Reactivity Cor- } \\
\text { rected to } 25^{\circ} \mathrm{C}\end{array}$ & $+75.3 \pm 2.0$ & $-7.61 \pm 0.35$ \\
$\begin{array}{l}\text { 2. Edge Smooth1ng between } \\
\text { core and radia1 blanket }\end{array}$ & $+1.350 \pm 0.675$ & $-0.136 \pm 0.068$ \\
3. Interface Gap Worth & $0 \quad \pm 2.0$ & 0 & \pm 0.20 \\
4. Source Subcriticality & $-1.957 \pm 0.004$ & $0.198 \pm 0.008$ \\
\hline $\begin{array}{l}\text { at operating power } \\
\text { leve1 }\end{array}$ & & & \\
\hline
\end{tabular}

Loaded Fissile Mass (kg) Loading 37)

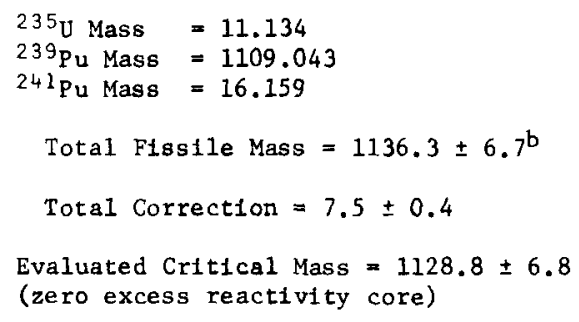

$a_{\Delta \rho} / \Delta M$ is the edge drawer worth $(6.67 \pm 0.25 \mathrm{Ih} /$ drawer $)$ divided by the fissile mass in the drawer $(0.674 \pm 0.003 \mathrm{~kg})=$ $9.90 \pm 0.38 \mathrm{Ih} / \mathrm{kg}$.

${ }^{b}$ An uncertainty of $0.5 \%$ has been assumed for the fissile mass. 
TABLE IX. Heterogeneous Atom Concentrations in Core Unit Cell (atom $/ \mathrm{cc} \times 10^{-22}$ )

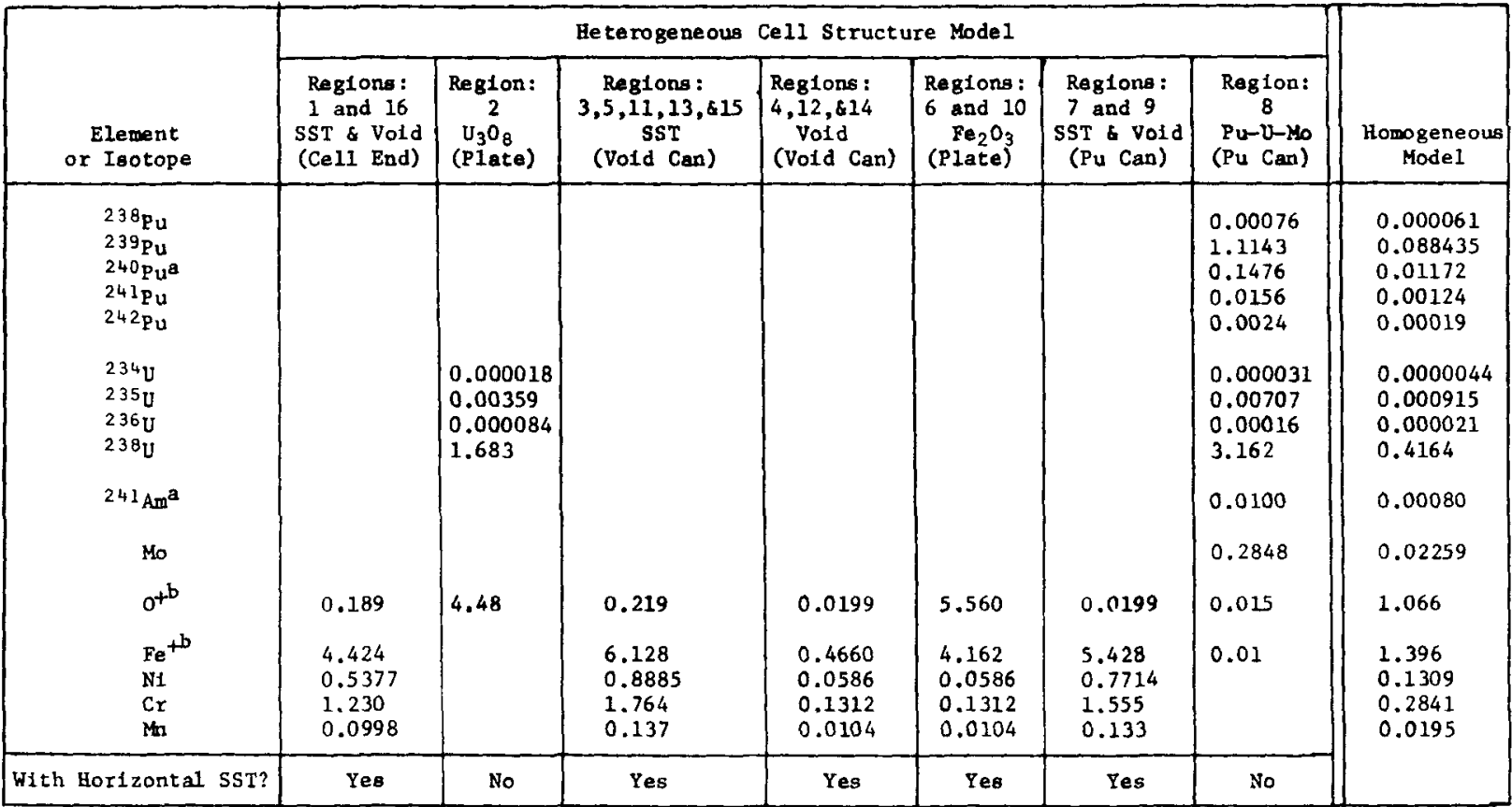

a. $241 \mathrm{Pu} \rightarrow 241$ Am decay corrected to 6-30-74.

b. $0^{+}=0+$ (Total Impurities with Atomic Weights $\leq \mathrm{S} 1$ ).

$\mathrm{Fe}^{+}=\mathrm{Fe}+($ Total Impurities with Atomlc Weights $>\mathrm{Si})$.

TABLE X. Heterogeneous Atom Concentrations in Radial Blanket Unit Cell

(atoms/cc $\times 10^{-22}$ )

-RADIAL BLANKET-

\begin{tabular}{|c|c|c|c|c|c|c|}
\hline \multirow[b]{2}{*}{$\begin{array}{l}\text { Element } \\
\text { or Ieotope }\end{array}$} & \multicolumn{5}{|c|}{ Heterogeneous Cell Structure Model } & \multirow[b]{2}{*}{$\begin{array}{l}\text { Homogeneous } \\
\text { Model }\end{array}$} \\
\hline & $\begin{array}{l}\text { Regions: } \\
1 \text { and } 12 \\
\text { SST \& Vold } \\
\text { (Ce11 End) }\end{array}$ & $\begin{array}{l}\text { Reglons: } \\
2 \text { and } 6 \\
\mathrm{U}_{3} \mathrm{O}_{8} \\
\text { (Plate) }\end{array}$ & $\begin{array}{l}\text { Reglons: } \\
3,5,8, \& 10 \\
\text { SST } \\
\text { (Vofd Can) }\end{array}$ & $\begin{array}{l}\text { Regions: } \\
4,9, \text { \& } 11 \\
\text { Void } \\
\text { (Void Can) }\end{array}$ & $\begin{array}{l}\text { Region: } \\
7 \\
\text { Dep I. U } \\
\text { (Plate) }\end{array}$ & \\
\hline $\begin{array}{l}{ }^{234} \mathrm{U} \\
235 \mathrm{U} \\
236 \mathrm{U} \\
238 \mathrm{U} \\
\mathrm{O}^{+8} \\
\mathrm{Fe}^{+a} \\
\mathrm{N1} \\
\mathrm{Cr} \\
\mathrm{Mr}\end{array}$ & $\begin{array}{l}0.181 \\
4.138 \\
0.5065 \\
1.159 \\
0.0938\end{array}$ & $\begin{array}{l}0.000018 \\
0.00359 \\
0.000084 \\
1.683 \\
4.48\end{array}$ & $\begin{array}{l}0.230 \\
6.344 \\
0.9147 \\
1.826 \\
0.141\end{array}$ & $\begin{array}{l}0.0304 \\
0.6819 \\
0.08476 \\
0.1918 \\
0.0152\end{array}$ & $\begin{array}{l}0.000046 \\
0.00984 \\
0.00024 \\
4.5815\end{array}$ & $\begin{array}{l}0.0000095 \\
0.00192 \\
0.000046 \\
0.89733 \\
1.789 \\
0.8564 \\
0.1095 \\
0.2413 \\
0.0162\end{array}$ \\
\hline W1th Horizontal SST? & Yes & No & Yes & Yes & No & \\
\hline
\end{tabular}


TABLE XI. Heterogeneous Atom Concentrations in Axial Blanket (atoms/cc $\times 10^{-22}$ )

-AXIAL BLANKET-

\begin{tabular}{|c|c|c|c|c|c|c|}
\hline \multirow[b]{2}{*}{$\begin{array}{l}\text { Element } \\
\text { or Inotope }\end{array}$} & \multicolumn{5}{|c|}{ Heterogeneous Cell Structure Model } & \multirow[b]{2}{*}{$\begin{array}{c}\text { Homogeneous } \\
\text { Model }\end{array}$} \\
\hline & $\begin{array}{l}\text { Regions: } \\
1 \text { and } 13 \\
\text { SST \& Vold } \\
\text { (Cel1 End) }\end{array}$ & $\begin{array}{l}\text { Regions: } \\
2 \text { and } 6 \\
\mathrm{U}_{3} \mathrm{O}_{8} \\
\text { (P1ate) }\end{array}$ & $\begin{array}{c}\text { Reglons: } \\
3,5,8,10,812 \\
\text { SST } \\
\text { (Vold Can) }\end{array}$ & $\begin{array}{l}\text { Reglons: } \\
4,9, \text { il } \\
\text { Void } \\
\text { (Vold Can) }\end{array}$ & $\begin{array}{l}\text { Region: } \\
7 \\
\text { Dep1. U } \\
\text { (P1ate) }\end{array}$ & \\
\hline $\begin{array}{l}234 \mathrm{U} \\
235 \mathrm{U} \\
236 \mathrm{U} \\
238 \mathrm{U}\end{array}$ & & $\begin{array}{l}0.000018 \\
0.00359 \\
0.000084 \\
1.683\end{array}$ & & & $\begin{array}{l}0.000046 \\
0.00984 \\
0.00024 \\
4.5815\end{array}$ & \multirow{4}{*}{$\begin{array}{l}0.0000069 \\
0.00140 \\
0.000033 \\
0.65203 \\
1.132 \\
0.9097 \\
0.1173 \\
0.2567 \\
0.0204\end{array}$} \\
\hline$\sigma^{+8}$ & 0.186 & 4.48 & 0.223 & 0.0238 & & \\
\hline $\begin{array}{l}\mathrm{Fe}^{+^{a}} \\
\mathrm{~N} 1 \\
\mathrm{Cr} \\
\mathrm{Mn}\end{array}$ & $\left\{\begin{array}{l}4.294 \\
0.5253 \\
1.202 \\
0.0975\end{array}\right.$ & & $\begin{array}{l}6.201 \\
0.8973 \\
1.785 \\
0.138\end{array}$ & $\begin{array}{l}0.5394 \\
0.06732 \\
0.1519 \\
0.0121\end{array}$ & & \\
\hline With Horizontal SST? & Yes & No & Yas & Yes & No & \\
\hline
\end{tabular}

a. $0^{+}=0+$ (Total Impurities w1th Atomic Welghts $\leq S 1$ ).

$\mathrm{Fe}^{+}=\mathrm{Fe}+$ (Total Impurities with Atomic WeIghts $>\mathrm{Si}$ ).

TABLE XII. As-Built ZPR-GCFR Phase I Composition Specification Homogeneous UnIt Ce11 Atom Densities (atoms/cc $\times 10^{-21}$ )

\begin{tabular}{|c|c|c|c|c|c|c|c|}
\hline & 1 & 2 & 3 & 4 & 5 & $\begin{array}{c}\text { Axial } \\
\text { Blanket }\end{array}$ & $\begin{array}{l}\text { Radial } \\
\text { Blanket }\end{array}$ \\
\hline $2{ }^{38} \mathrm{Pu}$ & 0.0004 & 0.0004 & 0.0006 & 0,0005 & 0,0004 & - & - \\
\hline $2{ }^{39} \mathrm{Pu}$ & 0.8905 & 0.8903 & 0.8842 & 0.8845 & 0.8897 & - & - \\
\hline $240^{\circ} \mathrm{Pu}$ & 0.1182 & 0.1182 & 0,1171 & 0.1173 & 0.1180 & - & - \\
\hline $241^{1} \mathrm{Pu}$ & 0.0118 & 0.0117 & 0.0134 & 0.0132 & 0.0121 & - & - \\
\hline${ }^{242} \mathrm{Pu}$ & 0.0016 & 0.0016 & 0,0019 & 0.0019 & 0.0017 & - & - \\
\hline $\begin{array}{l}2{ }^{35} \mathrm{U} \\
2{ }^{38} \mathrm{U}\end{array}$ & $\begin{array}{l}0.0091 \\
4.1529\end{array}$ & $\begin{array}{l}0.0091 \\
4.1485\end{array}$ & $\begin{array}{l}0.0091 \\
4.1612\end{array}$ & $\begin{array}{l}0.0091 \\
4.1573\end{array}$ & $\begin{array}{l}0.0091 \\
4.1550\end{array}$ & $\begin{array}{l}0.0139 \\
6.5191\end{array}$ & $\begin{array}{l}0.0191 \\
8.9616\end{array}$ \\
\hline $\mathrm{Fe}$ & 14.1256 & 14.1286 & 14.1216 & 14.1238 & 14.1346 & 9.2617 & 9.1206 \\
\hline Nf & 1.3270 & 1.3274 & 1.3264 & 1.3267 & 1.3283 & 1.1947 & 1.1627 \\
\hline $\mathrm{Cr}$ & 2.8706 & 2.8715 & 2.8694 & 2.8701 & 2.8732 & 2.6098 & 2.5655 \\
\hline $\operatorname{Mn}$ & 0.2284 & 0.2285 & 0.2283 & 0.2283 & 0.2286 & 0.2060 & 0.2038 \\
\hline 0 & 10.4582 & 10.4583 & 10.458 & 10.4581 & 10.4585 & 11.2722 & 17.8871 \\
\hline Am & 0.0086 & 0.0090 & 0.0048 & 0.0056 & 0.0076 & - & - \\
\hline Mo & 0.2275 & 0.2271 & 0.2257 & 0.2263 & 0.2287 & - & - \\
\hline
\end{tabular}


TABLE XIII. Atom Concentrat tons of Isotopes in the Core Unft Cell Computed us ing the Wade-Gelbard Prescription (atoms/cc $\times 10^{-2 I}$ )

\begin{tabular}{|c|c|c|c|c|c|c|c|c|}
\hline & $\begin{array}{l}\text { Regions } \\
(1 \& 16) \\
\text { SST\& VoId } \\
\text { (Cel1 End) }\end{array}$ & $\begin{array}{c}\text { Region } 2 \\
\mathrm{U}_{3} \mathrm{O}_{8} \\
\text { Plate }\end{array}$ & $\begin{array}{c}\text { Reglons } \\
3,5,11,13 \& 15 \\
\text { SST Void Can } \\
(1 / 2)\end{array}$ & $\begin{array}{l}\text { Reglon } 6 \\
\mathrm{Fe}_{2} \mathrm{O}_{3} \\
\text { Plate } \\
2 \text { In. Size }\end{array}$ & $\begin{array}{l}\text { Region } 10 \\
\mathrm{Fe}_{2} \mathrm{O}_{3} \\
\text { Plate } \\
3 \text { In. Size }\end{array}$ & $\begin{array}{c}\text { Regton } 4,14 \\
\text { Vold } \\
\text { Void Can }(1 / 2)\end{array}$ & $\begin{array}{l}\text { Region } 7,9 \\
\text { SST, Void } \\
\text { Pu Can }\end{array}$ & $\begin{array}{l}\text { Region } 8 \\
\text { Pu-U-Mo } \\
\text { (Pu Can) }\end{array}$ \\
\hline $23^{8} \mathrm{Pu}$ & - & - & - & - & - & - & - & 0.004 \\
\hline $239 \mathrm{Pu}$ & - & - & - & - & - & - & - & 9.647 \\
\hline $240 \mathrm{Pu}$ & - & - & - & - & - & - & - & 1.280 \\
\hline $241 \mathrm{Pu}$ & - & - & - & - & - & - & - & 0.128 \\
\hline $242 \mathrm{Pu}$ & - & - & - & - & - & - & - & 0.017 \\
\hline $235 \mathrm{U}$ & - & 0.032 & - & - & - & - & - & 0.061 \\
\hline $238_{U}$ & - & 15.212 & - & - & - & - & - & 27.120 \\
\hline $\mathrm{Fe}$ & 39.500 & - & 58.306 & 38.822 & 39.037 & 4.425 & 49.236 & - \\
\hline $\mathrm{Ni}$ & 4.829 & - & 8.482 & 0.556 & 0.556 & 0.556 & 7.053 & - \\
\hline $\mathrm{Cr}$ & 11.045 & - & 16.713 & 1.244 & 1.244 & 1.244 & 14.221 & - \\
\hline Mn & 0.897 & - & 1.253 & 0.099 & 0.099 & 0.099 & 1.206 & - \\
\hline 0 & 1.505 & 40.591 & 2.131 & - & - & 0.163 & 1.643 & - \\
\hline Am & - & - & - & 49.443 & 49.750 & - & - & 0.093 \\
\hline Mo & - & - & - & - & - & - & - & 2.465 \\
\hline
\end{tabular}

TABLE XIV. Atom Concentrations of Isotopes in the Axial Blanket Unit Cell Computed Using the Wade-Gelbard Prescription (atoms/cc $\times 10^{-21}$ )

\begin{tabular}{|c|c|c|c|c|c|c|c|c|c|}
\hline & $\begin{array}{rrl}1 & \& & 12 \\
\text { SST } \& \text { Void } \\
(C e I 1 & \text { End })\end{array}$ & $\begin{array}{c}2 \\
\mathrm{U}_{3} \mathrm{O}_{\mathrm{B}} \\
\text { Plate }\end{array}$ & $\begin{array}{c}3,5,8 \\
\text { SST } \\
\text { Void Can } \\
(1 / 2)\end{array}$ & $\begin{array}{c}6 \\
\mathrm{U}_{3} \mathrm{O}_{8} \\
\text { Plate }\end{array}$ & $\begin{array}{c}4,9 \\
\text { Void } \\
\text { Void Can } \\
(1 / 2)\end{array}$ & $\begin{array}{c}7 \\
\text { Dep1 U } \\
\text { Plate }\end{array}$ & $\begin{array}{c}10 \\
\text { SST } \\
\text { Vofd Can }\end{array}$ & $\begin{array}{c}11 \\
\text { Void } \\
\text { Void Can } \\
(1 / 4)\end{array}$ & $\begin{array}{c}12 \\
\text { SST } \\
\text { void Can } \\
(1 / 4)\end{array}$ \\
\hline $238_{\mathrm{Pu}}$ & - & - & - & - & - & - & - & - & - \\
\hline $239 \mathrm{Pu}$ & - & - & - & - & - & - & - & - & - \\
\hline $240 \mathrm{Pu}$ & - & - & - & - & - & - & - & - & - \\
\hline $241 \mathrm{Pu}$ & - & - & - & - & - & - & - & - & - \\
\hline $242 \mathrm{Pu}$ & - & - & - & - & - & - & - & - & - \\
\hline $\begin{array}{l}235 \mathrm{U} \\
238_{\mathrm{U}}\end{array}$ & - & $\begin{array}{r}0.032 \\
15.213\end{array}$ & - & $\begin{array}{r}0.032 \\
14.941\end{array}$ & - & $\begin{array}{r}0.090 \\
42.097\end{array}$ & - & - & - \\
\hline $\begin{array}{l}\mathrm{Fe} \\
\mathrm{Ni} \\
\mathrm{Cr} \\
\mathrm{Mn}\end{array}$ & $\begin{array}{r}38.876 \\
4.752 \\
10.870 \\
0.883\end{array}$ & $\begin{array}{l}- \\
- \\
-\end{array}$ & $\begin{array}{r}59.215 \\
8.591 \\
16.966 \\
1.273\end{array}$ & $\begin{array}{l}- \\
- \\
-\end{array}$ & $\begin{array}{l}5.332 \\
0.665 \\
1.497 \\
0.119\end{array}$ & $\begin{array}{l}- \\
- \\
-\end{array}$ & $\begin{array}{r}59.264 \\
8.599 \\
16.980 \\
1.274\end{array}$ & $\begin{array}{l}5.332 \\
0.665 \\
1.497 \\
0.119\end{array}$ & $\begin{array}{r}59.322 \\
8.607 \\
16.997 \\
1.276\end{array}$ \\
\hline 0 & 1.481 & 40.594 & 2.166 & 39.788 & 0.198 & - & 2.168 & 0.198 & 2.170 \\
\hline An & - & - & - & - & - & - & - & - & - \\
\hline Mo & - & - & - & - & - & - & - & - & - \\
\hline
\end{tabular}

TABLE XV. ZPR-GCFR Phase I - Radial Blanket Composition Specifications Atom Densities; Atoms/cc $\times 10^{-21}$

Stretched Heterogeneous Model

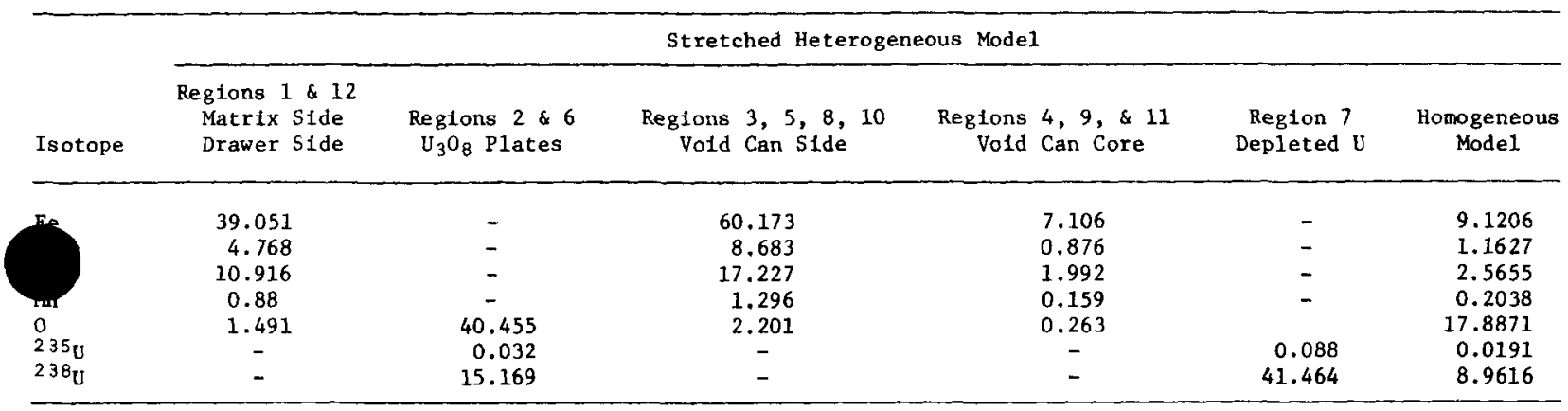


TABLE XVI. Results of Operational Measurements

\begin{tabular}{lc}
\hline \multicolumn{1}{c}{ Measurement } & \multicolumn{1}{c}{ Result } \\
\hline Temperature "Coefficient" of Assembly & $-2.42 \pm 0.04 \mathrm{Ih} /{ }^{\circ} \mathrm{C}$ \\
Edge Core Drawer Worth & $6.68 \pm 0.25 \mathrm{In} / \mathrm{drawer}$ \\
Central Core Drawer Worth & $62.99 \pm 1.09 \mathrm{Ih} / \mathrm{drawer}$ \\
Control Rod Worths & Shown on Table XIV \\
$24{ }^{\text {Pu Decay Reactivity Coefficient }}$ & $-0.150 \pm 0.019 \mathrm{Ih} /$ day \\
Configuration Reproductbility & $\pm 1.42 \mathrm{Ih}^{\mathrm{a}}$ \\
As-Built Critical Mass (Fissile Pu and $235 \mathrm{U})$ & $1136 \pm 6.0 \mathrm{~kg}$ \\
\hline
\end{tabular}

The actual value used for the evaluation of experimental worths is $\pm 2.0 \mathrm{Ih}$.

TABLE XVII. Experimentally Determined Control Rod Worths in GCFR Phase I Assembly

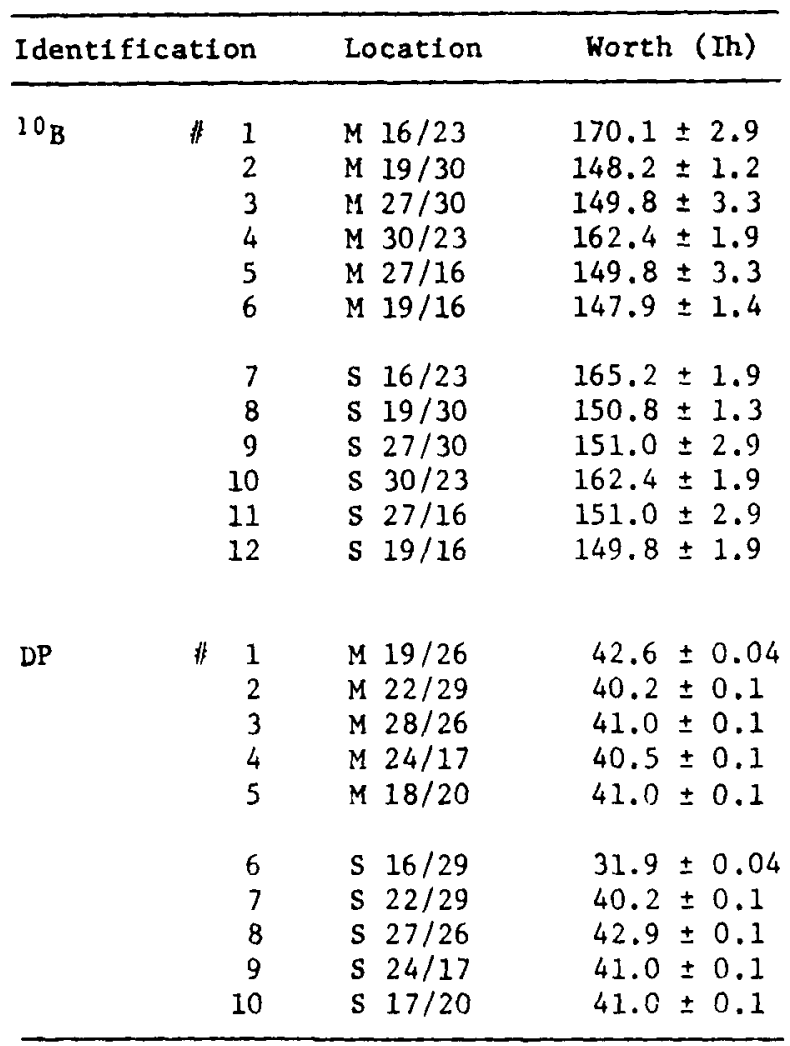

TABLE XVIII. Energy Group Structure for the 11-Group Cross Section Set

\begin{tabular}{ccc}
\hline $\begin{array}{c}\text { Group } \\
\text { No. }\end{array}$ & Upper Energy (eV) & Lethargy \\
\hline 1 & $1.4191 \times 10^{7}$ & 0.0 \\
2 & $3.6788 \times 10^{6}$ & 1.35 \\
3 & $1.3534 \times 10^{6}$ & 2.35 \\
4 & $4.9787 \times 10^{5}$ & 3.35 \\
5 & $1.8316 \times 10^{5}$ & 4.35 \\
6 & $6.7379 \times 10^{4}$ & 5.35 \\
7 & $2.4788 \times 10^{4}$ & 6.35 \\
8 & $9.1188 \times 10^{3}$ & 7.35 \\
9 & $3.3546 \times 10^{3}$ & 8.35 \\
10 & $4.5400 \times 10^{2}$ & 10.35 \\
11 & $4.1399 \times 10^{-1}$ & 17.35 \\
\hline
\end{tabular}


TABLE XIX. Energy Group Structure

for the 29-Group Cross-Section Set

\begin{tabular}{|c|c|c|c|}
\hline $\begin{array}{c}\text { Group } \\
\text { No. }\end{array}$ & Upper Ene & rgy $(\mathrm{eV})$ & I.ethargy \\
\hline 1 & 1.4191 & $\times 10^{7}$ & 0.0 \\
\hline 2 & 1.0000 & $\times 10^{7}$ & 0.3500 \\
\hline 3 & 6.0653 & $\times 10^{6}$ & 0.8500 \\
\hline 4 & 3.6788 & $\times 10^{6}$ & 1.3500 \\
\hline 5 & 2.2313 & $\times 10^{6}$ & 1.8500 \\
\hline 6 & 1.3534 & $\times 10^{6}$ & 2.3500 \\
\hline 7 & 8.2085 & $\times 10^{5}$ & 2.8500 \\
\hline 8 & 4.9787 & $\times 10^{5}$ & 3.3500 \\
\hline 9 & 3.0197 & $\times 10^{5}$ & 3.8500 \\
\hline 10 & 1.8316 & $\times 10^{5}$ & 4.3500 \\
\hline 11 & 1.1109 & $\times 10^{5}$ & 4.8500 \\
\hline 12 & 6.7379 & $\times 10^{4}$ & 5.3500 \\
\hline 13 & 4.0868 & $\times 10^{4}$ & 5.8500 \\
\hline 14 & 2.4788 & $\times 10^{4}$ & 6.3500 \\
\hline 15 & 1.5034 & $\times 10^{4}$ & 6.8500 \\
\hline 16 & 9.1188 & $\times 10^{3}$ & 7.3500 \\
\hline 17 & 5.5308 & $\times 10^{3}$ & 7.8500 \\
\hline 18 & 3,3546 & $\times 10^{3}$ & 8.3500 \\
\hline 19 & 2.0347 & $\times 10^{3}$ & 8.8500 \\
\hline 20 & 1.2341 & $\times 10^{3}$ & 9.3500 \\
\hline 21 & 7.4852 & $\times 10^{2}$ & 9.8500 \\
\hline 22 & 4.5400 & $\times 10^{2}$ & 10.3500 \\
\hline 23 & 2.7536 & $\times 10^{2}$ & 10.8500 \\
\hline 24 & 1.0130 & $\times 10^{2}$ & 11.8500 \\
\hline 25 & 3.7267 & $\times 10^{1}$ & 12.8500 \\
\hline 26 & 1.3740 & $\times 10^{1}$ & 13.8500 \\
\hline 27 & 5.0435 & $\times 10^{0}$ & 14.8500 \\
\hline 28 & 1.8554 & $\times 10^{0}$ & 15.8500 \\
\hline 29 & 4.1399 & $\times 10^{-1}$ & 17.3500 \\
\hline
\end{tabular}

TABLE XX. Homogeneous Atom Densities $\left(\times 10^{-21}\right)$ used in $R-Z$ Mode 1 of GCFR Phase I Assemb1y

\begin{tabular}{lrrrrr}
\hline & Core 1 & Core 2 & Core $3^{\mathrm{a}}$ & Ax1al Blanket & Radial Blanket \\
\hline $2{ }^{38} \mathrm{Pu}$ & 0.0004 & 0.0004 & 0.0006 & - & - \\
$2{ }^{39} \mathrm{Pu}$ & 0.8905 & 0.8903 & 0.8843 & - & - \\
$240 \mathrm{Pu}$ & 0.1182 & 0.1182 & 0.1171 & - & - \\
$241_{\mathrm{Pu}}$ & 0.0118 & 0.0117 & 0.01315 & - & - \\
$242 \mathrm{Pu}$ & 0.0016 & 0.0016 & 0.0019 & - & 0.0191 \\
& & & & & \\
$2{ }^{35} \mathrm{U}$ & 0.0091 & 0.0091 & 0.0091 & 0.0139 & 8.9616 \\
$2{ }^{38} \mathrm{U}$ & 4.1529 & 4.1485 & 4.1607 & 6.5191 & 9.1206 \\
$\mathrm{Fe}$ & 14.1256 & 14.1286 & 14.1219 & 9.2617 & 1.1627 \\
$\mathrm{Ni}$ & 1.3270 & 1.3274 & 1.3264 & 1.1947 & 2.5655 \\
$\mathrm{Cr}$ & 2.8706 & 2.8715 & 2.8696 & 2.6098 & 0.2038 \\
$\mathrm{Mn}$ & 0.2284 & 0.2285 & 0.2283 & 0.2060 & 17.8871 \\
0 & 10.4582 & 10.4583 & 10.4580 & 11.2722 & - \\
$\mathrm{Am}$ & 0.0086 & 0.0090 & 0.0049 & - & - \\
$\mathrm{Mo}$ & 0.2275 & 0.2271 & 0.2258 & - & - \\
\hline
\end{tabular}

The atom densitles indicated for core region 3 represent a volume-weighted average of the actual number densities listed in Table IX for core reglons 3 , 4 , and 5 . 
TABLE XXI. B1-directional Diffusion Coefficients for Core Composttion in GCFR Phase I Assembly

\begin{tabular}{cccccc}
\hline $\begin{array}{c}\text { Group } \\
\text { Ho. }\end{array}$ & $D_{\text {Homogeneous }}$ & $D_{\text {Average }}$ & $D_{1}$ & $D_{\|}\left(=D_{z}\right)$ & $D_{R}=1 / 2\left(D_{1}+D_{\|}\right)$ \\
\hline 1 & 4.5848 & 4.7567 & 4.5926 & 4.8387 & 4.7157 \\
2 & 3.9236 & 4.1079 & 3.9336 & 4.1951 & 4.0644 \\
3 & 3.3558 & 3.5871 & 3.3703 & 3.6954 & 3.5329 \\
4 & 2.3220 & 2.5606 & 2.3427 & 2.6696 & 2.5062 \\
5 & 2.0753 & 2.2991 & 2.0979 & 2.3997 & 2.2488 \\
6 & 1.6292 & 1.8231 & 1.6535 & 1.9079 & 1.7807 \\
7 & 1.7525 & 1.9528 & 1.7760 & 2.0413 & 1.9087 \\
8 & 0.9812 & 1.1080 & 1.0041 & 1.1599 & 1.0820 \\
9 & 1.1007 & 1.2506 & 1.1265 & 1.3127 & 1.2196 \\
10 & 0.92684 & 1.07448 & 0.95745 & 1.1335 & 1.04548 \\
11 & 0.49627 & 0.98370 & 0.74036 & 1.1054 & 0.92288 \\
\hline
\end{tabular}

TABLE XXII. Bi-directional Diffusion Coefficients for Axial Blanket Composition in GCFR Phase I Assembly

\begin{tabular}{cccccc}
\hline $\begin{array}{c}\text { Group } \\
\text { No. }\end{array}$ & $\mathrm{D}_{\text {Homogeneous }}$ & $\mathrm{D}_{\text {Average }}$ & $\mathrm{D}_{1}$ & $\mathrm{D}_{\|}\left(\mathrm{D}_{2}\right)$ & $\mathrm{D}_{\mathrm{R}}=1 / 2\left(\mathrm{D}_{\perp}+\mathrm{D}_{\|}\right)$ \\
\hline 1 & 4.9620 & 5.0905 & 4.9671 & 5.1522 & 5.0597 \\
2 & 4.1937 & 4.3336 & 4.2003 & 4.4003 & 4.3003 \\
3 & 3.4040 & 3.6008 & 3.4161 & 3.6931 & 3.5486 \\
4 & 2.3089 & 2.5158 & 2.3274 & 2.6100 & 2.4687 \\
5 & 2.0531 & 2.2366 & 2.0711 & 2.3194 & 2.1863 \\
6 & 1.6463 & 1.7857 & 1.6620 & 1.8476 & 1.7548 \\
7 & 1.4857 & 1.6743 & 1.5135 & 1.7546 & 1.6341 \\
8 & 1.0724 & 1.1652 & 1.0900 & 1.2028 & 1.1464 \\
9 & 1.2306 & 1.3338 & 1.2462 & 1.3776 & 1.3119 \\
10 & 1.1298 & 1.2242 & 1.1467 & 1.2629 & 1.2048 \\
11 & 1.3211 & 1.4142 & 1.3369 & 1.4528 & 1.3949 \\
\hline
\end{tabular}


TABLE XXIII. Bi-directional Diffusion Coefficients for Radial Blanket Composition in GCFR Phase I Assembly

\begin{tabular}{cccccc}
\hline $\begin{array}{c}\text { Group } \\
\text { No. }\end{array}$ & $D_{\text {Homogeneous }}$ & $D_{\text {Average }}$ & $D_{1}$ & $D_{\mid}\left(=D_{z}\right)$ & $D_{R}=1 / 2\left(D_{1}+D_{f}\right)$ \\
\hline 1 & 3.9852 & 4.0550 & 3.9889 & 4.0880 & 4.0385 \\
2 & 3.3284 & 3.4036 & 3.3328 & 3.4390 & 3.3859 \\
3 & 2.5728 & 2.6809 & 2.5800 & 2.7313 & 2.6557 \\
4 & 1.7274 & 1.8424 & 1.7386 & 1.8944 & 1.8165 \\
5 & 1.5776 & 1.6773 & 1.5885 & 1.7218 & 1.6552 \\
6 & 1.3086 & 1.3829 & 1.3189 & 1.4150 & 1.3670 \\
7 & 1.3203 & 1.4043 & 1.3315 & 1.4407 & 1.3861 \\
8 & 0.91359 & 0.96618 & 0.92824 & 0.98514 & 0.95669 \\
9 & 1.0234 & 1.0789 & 1.0354 & 1.1007 & 1.06805 \\
10 & 0.95784 & 1.0106 & 0.9716 & 1.0301 & 1.00089 \\
11 & 1.1261 & 1.1786 & 1.1389 & 1.1984 & 1.16865 \\
\hline
\end{tabular}

TABLE XXIV. Bi-directional Diffusion Coefficient Modifiers for GCFR Phase I Assembly

\begin{tabular}{|c|c|c|c|c|c|c|}
\hline \multirow[b]{2}{*}{$\begin{array}{l}\text { Group } \\
\text { No. }\end{array}$} & \multicolumn{2}{|c|}{ Core Composition } & \multicolumn{2}{|c|}{$\begin{array}{l}\text { Axial Blanket } \\
\text { Composition }\end{array}$} & \multicolumn{2}{|c|}{$\begin{array}{l}\text { Radial Blanket } \\
\text { Composition }\end{array}$} \\
\hline & $\mathrm{D}_{z} / \mathrm{D}_{\text {Homo }}$ & $\mathrm{D}_{\mathrm{R}} / \mathrm{D}_{\text {Homo }}$ & $\mathrm{D}_{\mathrm{z}} / \mathrm{D}_{\text {Homo }}$ & $\mathrm{D}_{\mathrm{R}} / \mathrm{D}_{\text {Homo }}$ & $\mathrm{D}_{\mathrm{z}} / \mathrm{D}_{\text {Homo }}$ & $\mathrm{D}_{\mathrm{R}} / \mathrm{D}_{\text {Homo }}$ \\
\hline 1 & 1.0554 & 1.0286 & 1.0383 & 1.0197 & 1.0258 & 1.0134 \\
\hline 2 & 1.0692 & 1.0359 & 1.0493 & 1.0254 & 1.0332 & 1.0173 \\
\hline 3 & 1.1012 & 1.0528 & 1.0849 & 1.0425 & 1.0967 & 1.0516 \\
\hline 4 & 1.1497 & 1.0793 & 1.1304 & 1.0692 & 1.0967 & 1.0516 \\
\hline 5 & 1.1563 & 1.0836 & 1.1297 & 1.0649 & 1.0914 & 1.0492 \\
\hline 6 & 1.1711 & 1.0930 & 1.1223 & 1.0659 & 1.0813 & 1.0446 \\
\hline 7 & 1.1648 & 1.0891 & 1.1810 & 1.0999 & 1.0912 & 1.0498 \\
\hline 8 & 1.1821 & 1.1027 & 1.1216 & 1.0690 & 1.0783 & 1.0472 \\
\hline 9 & 1.1926 & 1.1080 & 1.1195 & 1.0661 & 1.0755 & 1.0436 \\
\hline 10 & 1.2230 & 1.1280 & 1.1178 & 1.0664 & 1.0754 & 1.0449 \\
\hline 11 & 2.2274 & 1.8596 & 1.0997 & 1.0558 & 1.0642 & 1.0378 \\
\hline
\end{tabular}


TABLE XXV. Results of Critlcality Calculations of As-Built Phase I Assembly

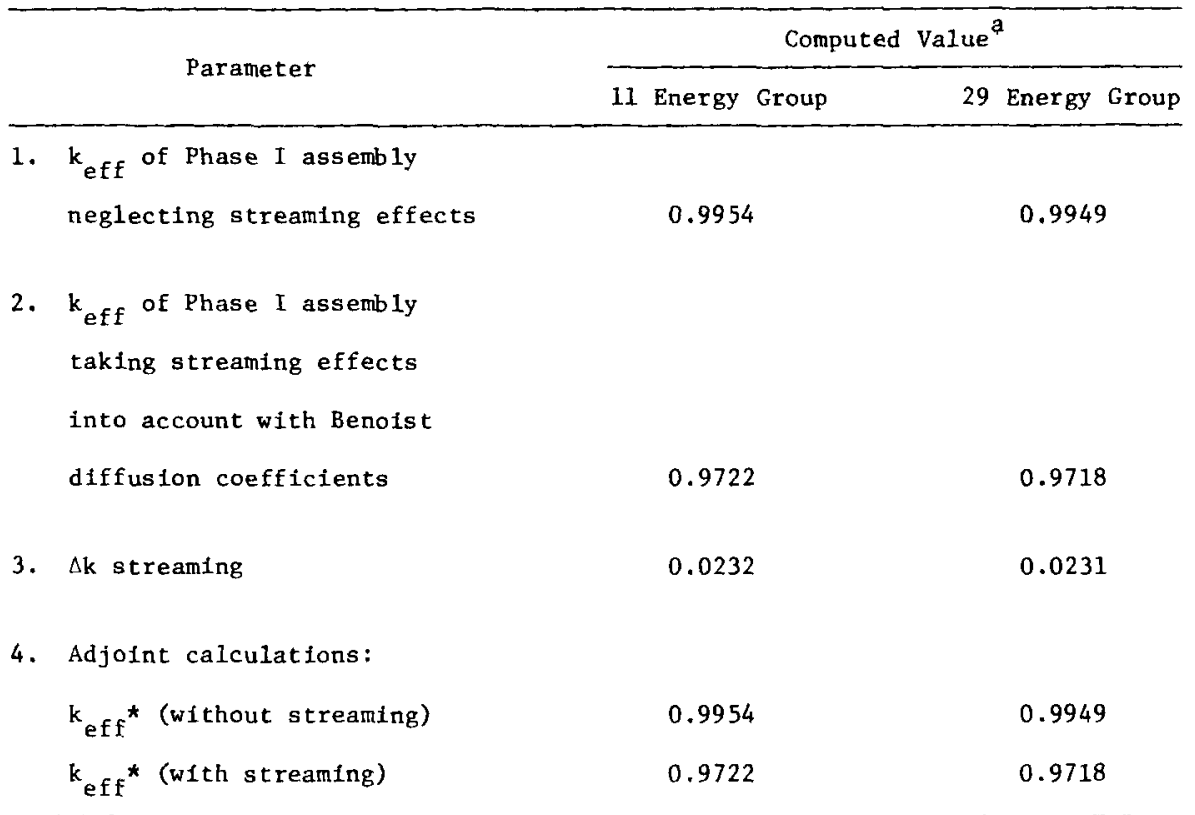

These calculations utilized two-dimensional ( $Z-R)$ diffusion theory and ENDF/B Version 4 data.

TABLE XXVI. Sensitivity of Eigenvalue to Calculated Parameters

\begin{tabular}{|c|c|}
\hline Parameter & Change in $k, \Delta k$ \\
\hline 1. Change in Benoist Coefflctents & \\
\hline Uniform $5 \%$ Change (Decrease/Increase) in $D^{\prime} s$ & \pm 0.0146 \\
\hline $\begin{array}{l}\text { 2. Change in Energy Group Structure (From } 29 \text { Groups } \\
\text { to } 11 \text { Groups) }\end{array}$ & +0.0004 \\
\hline $\begin{array}{l}\text { 3. Neglecting the Reflection Effects of the Knees } \\
\text { and Bed of ZPR-9 Reactor }\end{array}$ & -0.0031 \\
\hline $\begin{array}{l}\text { 4. Ef fect of Using a Zero F1ux Condition at Outer } \\
\text { Boundary as Opposed to Using the Zero Flux } \\
\text { at Extrapolated Boundary Condition }\end{array}$ & -0.0014 \\
\hline
\end{tabular}

TABLE XXVII. Final Results of Criticality Calculations Performed in Two-Dimensions
1. $k_{\text {eff }}$ of Assembly Neglecting Neutron Streaming 0.9992 effects
2. $k_{\text {eff }}$ of Assemb1y Taking Streaming Effects 1nto Account with Benolst Diffusion Coefficlents
3. $\Delta \mathrm{k}$ st reaming


TABLE XXVIII. Comparison of Rea1 and Adfoint Spectra for 11- and 29-Group Nonstreaming Calculations

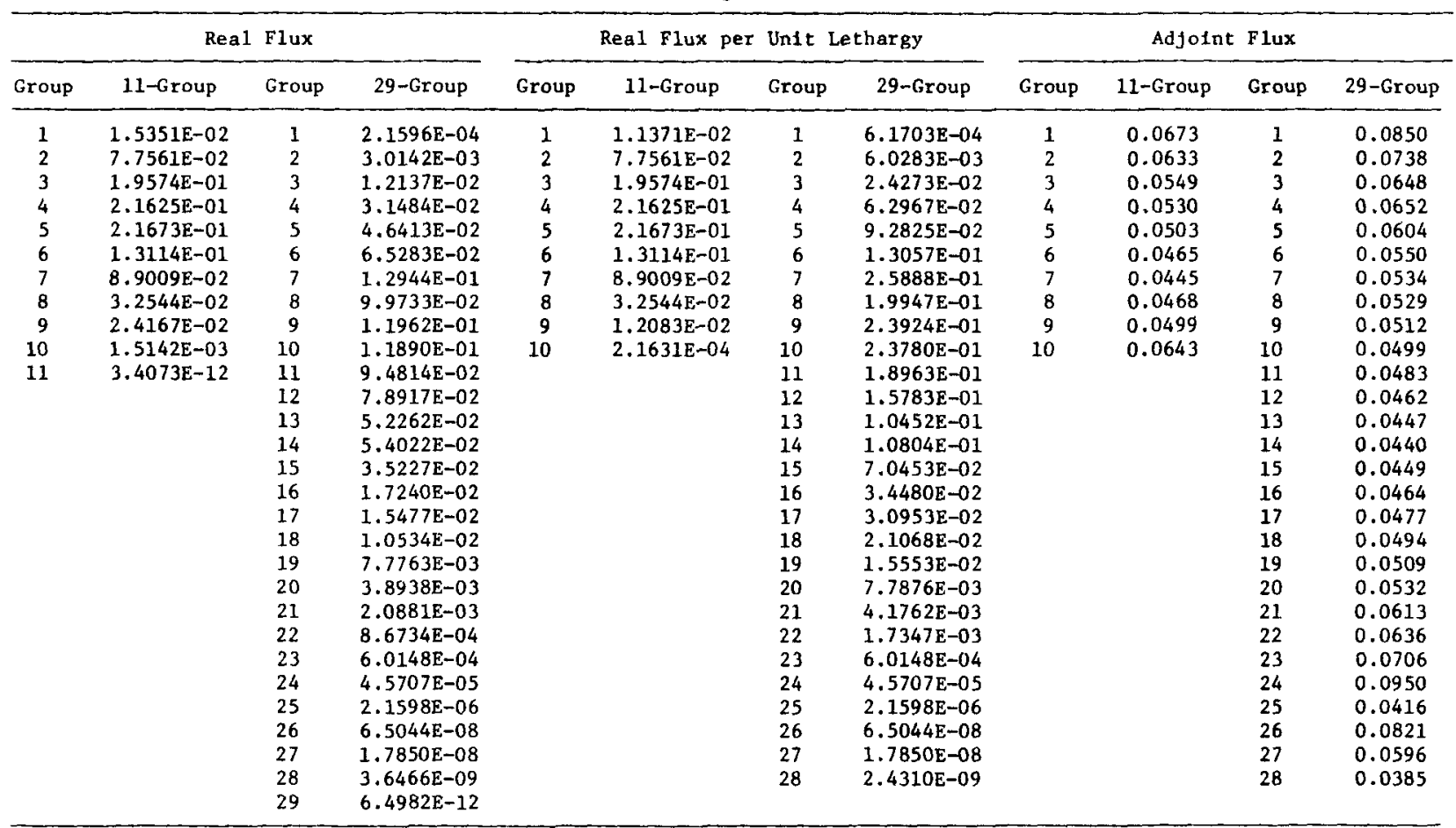

Real Group Fluxes, $\phi_{\mathrm{g}}$, are normalized such that $\sum_{\mathrm{a} I 1 \mathrm{~g}} \phi_{\mathrm{g}}=1.0$.

Adjoint Group Fluxes, $\phi_{g}{ }^{\star}$, are normalized such that $\sum_{a 11}{ }_{g} \neq 1$ 
TABLE XXIX. Comparison of 29-Group Rea1 and Adjoint Spectra for the With Streaming and Without Streaming Calculations

\begin{tabular}{|c|c|c|c|c|c|c|c|c|c|}
\hline \multirow[b]{2}{*}{ Group } & \multicolumn{2}{|c|}{ Real Group Flux } & \multicolumn{2}{|c|}{$\begin{array}{l}\text { Real Group F1ux } \\
\text { per Un1t Lethargy }\end{array}$} & \multirow{2}{*}{$\begin{array}{l}\text { Ratio of } \\
\text { Real Flux } \\
\text { With } \\
\text { Streaming/ } \\
\text { W1thout } \\
\text { Streaming }\end{array}$} & \multirow[b]{2}{*}{ Group } & \multicolumn{2}{|c|}{ Adjoint Group Flux } & \multirow{2}{*}{$\begin{array}{l}\text { Ratio of } \\
\text { Adjoint } \\
\text { Flux With } \\
\text { Streaming/ } \\
\text { Without } \\
\text { Streaming }\end{array}$} \\
\hline & $\begin{array}{c}\text { With } \\
\text { Streaming }\end{array}$ & $\begin{array}{l}\text { Without } \\
\text { Streaming }\end{array}$ & $\begin{array}{c}\text { With } \\
\text { Strramtnb }\end{array}$ & $\begin{array}{l}\text { Without } \\
\text { streaming }\end{array}$ & & & $\begin{array}{c}\text { With } \\
\text { Streaming }\end{array}$ & $\begin{array}{l}\text { W1 thout } \\
\text { Streaming }\end{array}$ & \\
\hline 1 & $2.2287 E-04$ & $2.1596 \mathrm{E}-04$ & $6.3677 \mathrm{E}-04$ & $6.1703 \mathrm{E}-04$ & $1.0320 \mathrm{E}-00$ & 1 & $8.4746 \mathrm{E}-02$ & $2.4980 \mathrm{E}-02$ & $9.9724 \mathrm{E}-01$ \\
\hline 2 & $3.0910 \mathrm{E}-03$ & $3.0142 \mathrm{E}-03$ & $6.1819 \mathrm{E}-03$ & $6.0283 \mathrm{E}-03$ & $1.0255 \mathrm{E}-00$ & 2 & $7.3459 E-02$ & $7.3790 \mathrm{E}-02$ & $9.9552 \mathrm{E}-01$ \\
\hline 3 & 1. $2438 \mathrm{E}-02$ & $1.2137 \mathrm{E}-02$ & $2.4876 \mathrm{E}-02$ & $2.4273 \mathrm{E}-02$ & $1.0248 E-00$ & 3 & $6.4297 E-02$ & $6.4771 E-02$ & $9.9268 \mathrm{E}-01$ \\
\hline 4 & $3.2237 \mathrm{E}-02$ & $3.1484 \mathrm{E}-02$ & $6.4475 \mathrm{E}-02$ & $6.2967 \mathrm{E}-02$ & $1.0239 \mathrm{E}-00$ & 4 & $6.4739 \mathrm{E}-02$ & $6.5242 \mathrm{E}-02$ & $9.9228 \mathrm{E}-01$ \\
\hline 5 & $4.7464 \mathrm{E}-02$ & $4.6413 \mathrm{E}-02$ & $9,4929 \mathrm{E}-02$ & $9.2825 \mathrm{E}-02$ & $1.0227 \mathrm{E}-00$ & 5 & $5.9748 \mathrm{E}-02$ & $6.0421 \mathrm{E}-02$ & $9.8887 E-01$ \\
\hline 6 & $6.6543 \mathrm{E}-02$ & $6.5283 \mathrm{E}-02$ & $1.3309 \mathrm{E}-01$ & $1.3057 \mathrm{E}-01$ & $1.0193 \mathrm{E}-00$ & 6 & $6.4117 \mathrm{E}-02$ & $5.4965 E-02$ & $9.8456 \mathrm{E}-01$ \\
\hline 7 & 1. $3123 \mathrm{E}-01$ & $1.2933 \mathrm{E}-01$ & $2,6246 \mathrm{E}-01$ & $2.5888 \mathrm{E}-01$ & $1.0138 \mathrm{E}-00$ & 7 & $5.2546 \mathrm{E}-02$ & $5.3531 \mathrm{E}-02$ & $9.8343 \mathrm{E}-01$ \\
\hline 8 & $1.0067 \mathrm{E}-01$ & $9.9733 \mathrm{E}-02$ & $2.0135 E-01$ & $1.9947 \mathrm{E}-01$ & $1.0094 \mathrm{E}-00$ & 8 & $5.1942 \mathrm{E}-02$ & $5.2873 E-02$ & $9.8240 \mathrm{E}-01$ \\
\hline 9 & $1.2016 \mathrm{E}-01$ & $1.1962 \mathrm{E}-01$ & $2.4032 E-01$ & $2.3924 E-01$ & $1.0045 \mathrm{E}-00$ & 9 & $5.0384 \mathrm{E}-02$ & $5.1192 \mathrm{E}-02$ & $9.8422 \mathrm{E}-01$ \\
\hline 10 & $1.1866 \mathrm{E}-01$ & $1.1890 \mathrm{E}-01$ & $2.3733 \mathrm{E}-01$ & $2.3780 \mathrm{E}-01$ & $9.9804 \mathrm{E}-0 \mathrm{I}$ & 10 & $4.9176 \mathrm{E}-02$ & $4.9880 \mathrm{E}-02$ & $9.8590 \mathrm{E}-01$ \\
\hline 11 & $9.4135 \mathrm{E}-02$ & $9.4814 \mathrm{E}-02$ & $1.8827 \mathrm{E}-01$ & $1.8963 \mathrm{E}-01$ & $9.9284 \mathrm{E}-01$ & 11 & $4.7718 \mathrm{E}-02$ & $4.8258 E-02$ & $9.8881 \mathrm{E}-01$ \\
\hline 12 & $7.7925 \mathrm{E}-02$ & $7.8917 \mathrm{E}-02$ & $1.5585 \mathrm{E}-01$ & $1.5783 \mathrm{E}-01$ & $9.8743 \mathrm{E}-01$ & 12 & $4.5772 E-02$ & $4.6198 \mathrm{E}-02$ & $9.9078 \mathrm{E}-01$ \\
\hline 13 & $5.1412 \mathrm{E}-02$ & $5.2262 \mathrm{E}-02$ & $1.0282 \mathrm{E}-01$ & $1.0452 \mathrm{E}-01$ & $9.8373 E-01$ & 13 & $4.4369 \mathrm{E}-02$ & $4.4680 \mathrm{E}-02$ & $9.9303 \mathrm{E}-01$ \\
\hline 14 & $5.2813 E-02$ & $5.4022 E-02$ & $1.0563 \mathrm{E}-01$ & $2.0804 E-01$ & $9.7762 \mathrm{E}-01$ & 14 & $4.3805 \mathrm{E}-02$ & $4.4045 E-02$ & $9.9454 \mathrm{E}-01$ \\
\hline 15 & $3.4316 \mathrm{E}-02$ & $3.5227 E-02$ & $6.8632 \mathrm{E}-02$ & $7.0453 \mathrm{E}-02$ & $9.7414 \mathrm{E}-01$ & 15 & $4.4903 \mathrm{E}-02$ & $4.4950 \mathrm{E}-02$ & $9.9895 \mathrm{E}-01$ \\
\hline 16 & $1.6763 \mathrm{E}-02$ & $1.7240 \mathrm{E}-02$ & $3.3526 \mathrm{E}-02$ & $3.4480 \mathrm{E}-02$ & $9.7232 \mathrm{E}-01$ & 16 & $4.6452 \mathrm{E}-02$ & $4.6385 \mathrm{E}-02$ & $1.0014 \mathrm{E}-00$ \\
\hline 17 & $1.5015 \mathrm{E}-02$ & $1.5477 \mathrm{E}-02$ & $3.0029 \mathrm{E}-02$ & $3.0953 \mathrm{E}-02$ & $9.7014 \mathrm{E}-01$ & 17 & $4.7802 E-02$ & $4.7698 E-02$ & $1.0022 \mathrm{E}-00$ \\
\hline 18 & $1.0197 \mathrm{E}-02$ & $1.0534 \mathrm{E}-02$ & $2.0394 \mathrm{E}-02$ & $2.1068 \mathrm{E}-02$ & $9.6800 E-01$ & 18 & $4.9561 \mathrm{E}-02$ & $4.9415 E-02$ & $1.0029 \mathrm{E}-00$ \\
\hline 19 & $7.5034 \mathrm{E}-03$ & $7.7763 \mathrm{E}-03$ & $1.5007 E-02$ & $1.5553 E-02$ & $9.6490 \mathrm{E}-01$ & 19 & $5.1093 \mathrm{E}-02$ & $5.0923 \mathrm{E}-02$ & $1.0033 \mathrm{E}-00$ \\
\hline 20 & $3.7473 \mathrm{E}-03$ & $3.8938 \mathrm{E}-03$ & $7.4947 \mathrm{E}-03$ & $7.7876 \mathrm{E}-03$ & $9.6238 \mathrm{E}-01$ & 20 & $5.3480 \mathrm{E}-02$ & $5.3220 \mathrm{E}-02$ & $1.0049 \mathrm{E}-00$ \\
\hline 21 & $2.0041 \mathrm{E}-03$ & $2.0881 E-03$ & $4.0082 \mathrm{E}-03$ & $4.1762 \mathrm{E}-03$ & $9.5979 \mathrm{E}-01$ & 21 & $6.1693 \mathrm{E}-02$ & $6.1350 E-02$ & $1.0056 E-00$ \\
\hline 22 & $8.3103 E-04$ & $8.6734 \mathrm{E}-04$ & $1.6621 \mathrm{E}-03$ & $1.7347 \mathrm{E}-03$ & $9.5814 \mathrm{E}-01$ & 22 & $6.4025 E-02$ & 6. $3595 E-02$ & $1.0068 \mathrm{E}-00$ \\
\hline 23 & $3.7434 \mathrm{E}-04$ & $6.0148 \mathrm{E}-04$ & $5.7434 \mathrm{E}-04$ & $6.0148 \mathrm{E}-04$ & $9.5488 \mathrm{E}-01$ & 23 & $7.1055 \mathrm{E}-02$ & $7.0562 \mathrm{E}-02$ & $1.0070 \mathrm{E}-00$ \\
\hline 24 & $4.3523 E-05$ & $4.5707 \mathrm{E}-05$ & $4.3523 \mathrm{E}-05$ & $4.5707 E-05$ & $9.5221 \mathrm{E}-01$ & 24 & $9.5804 \mathrm{E}-02$ & $9.5010 \mathrm{E}-02$ & $1.0084 \mathrm{E}-00$ \\
\hline 25 & $2.0720 E-06$ & $2.1598 E-06$ & $2.0720 \mathrm{E}-06$ & $2.1598 \mathrm{E}-06$ & $9.5935 \mathrm{E}-01$ & 25 & $4.1873 \mathrm{E}-02$ & $4.1554 \mathrm{E}-02$ & $1.0077 \mathrm{E}-00$ \\
\hline 26 & $6.4134 \mathrm{E}-08$ & $6.5044 \mathrm{E}-08$ & $6.4134 \mathrm{E}-08$ & $6.5044 E-08$ & $9.8601 \mathrm{E}-01$ & 26 & $8.2793 \mathrm{E}-02$ & 8. $2100 \mathrm{E}-02$ & $1.0084 \mathrm{E}-00$ \\
\hline 27 & $1.7784 \mathrm{E}-08$ & $1.7850 \mathrm{E}-08$ & $1.7784 \mathrm{E}-08$ & $1.7850 \mathrm{E}-08$ & $9.9627 E-01$ & 27 & $6.0014 E-02$ & $5.9581 E-02$ & $1.0073 \mathrm{E}-00$ \\
\hline 28 & $3.6779 E-09$ & $3.6466 \mathrm{E}-09$ & $2.4519 E-09$ & $2.4310 E-09$ & $1.0086 E-00$ & 28 & $3.8841 E-02$ & $3.8540 E-02$ & $1.0078 E-00$ \\
\hline 29 & $6.4757 \mathrm{E}-12$ & $6.4982 \mathrm{E}-12$ & & & & & & & \\
\hline
\end{tabular}

Real Group Fluxes, $\phi_{g}$, are normalized such that $\sum_{\text {all }} \phi_{g}=1.0$.

Adjoint Group Fluxes, $\phi_{g}{ }^{*}$, are normalized such that $\sum_{\text {al1 }} \neq 1 \neq \phi_{g}^{* \Delta u_{g}}=1.0$. 
TABLE XXX. Basic Physics Parameters of the ZPR-GCFR Phase I Core

\begin{tabular}{|c|c|c|}
\hline Physics Parameter & $\begin{array}{l}\text { Without } \\
\text { Streaming }\end{array}$ & $\begin{array}{l}\text { With } \\
\text { Streaming }\end{array}$ \\
\hline $\begin{array}{l}\text { 1. Neutron generation time } \\
\left(\phi / V, \phi^{*}\right) /\left(F \phi, \phi^{*}\right)\end{array}$ & $4,496108 \times 10^{-7}$ & $4.475272 \times 10^{-7}$ \\
\hline 2. Prompt neutron lifetime & $4.473623 \times 10^{-7}$ & $4.357195 \times 10^{-7}$ \\
\hline 3. Normalized perturbation denominator & $4,227584 \times 10^{5}$ & $4.339371 \times 10^{5}$ \\
\hline 4. Effective delayed neutron fraction ( $\left.\beta_{\text {eff }}\right)$ & $3.225338 \times 10^{-3}$ & $3.238769 \times 10^{-3}$ \\
\hline 5. Inhours per percent reactivity & $9.772558 \times 10^{2}$ & $9.766388 \times 10^{2}$ \\
\hline 6. $o(3600 \mathrm{sec})$ & $1.023273 \times 10^{-5}$ & $1.023920 \times 10^{-5}$ \\
\hline 7. $k_{\text {eff }}$ (from real flux) & 0.9954 & 0.9722 \\
\hline 8. $k_{\text {eff }}$ (from adjoint flux) & 0.9954 & 0.9722 \\
\hline
\end{tabular}

a11-Group calculations using ENDF/B Version 4 data for a cylindrical reactor model.

botained using homogeneous diffusion coefficients,

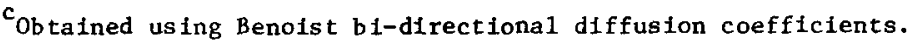

TABLE XXXI. Kinetics Parameters of the ZPR-GCFR Phase I Core

\begin{tabular}{|c|c|c|c|c|c|}
\hline \multirow[b]{2}{*}{ Isotope } & \multirow[b]{2}{*}{ Index } & \multicolumn{2}{|c|}{$B_{1}$} & \multirow{2}{*}{\multicolumn{2}{|c|}{$\lambda_{1}$}} \\
\hline & & w/o Streaming ${ }^{a, b}$ & With Streaming ${ }^{a, c}$ & & \\
\hline $235 \mathrm{U}$ & $\begin{array}{l}1 \\
2 \\
3 \\
4 \\
5 \\
6\end{array}$ & $\begin{array}{l}1.784554 \times 10^{-6} \\
1.060477 \times 10^{-5} \\
8.933034 \times 10^{-6} \\
1.944694 \times 10^{-5} \\
6.115992 \times 10^{-6} \\
1.242311 \times 10^{-6}\end{array}$ & $\begin{array}{l}1.779738 \times 10^{-6} \\
1.056445 \times 10^{-5} \\
8.906866 \times 10^{-6} \\
1.938586 \times 10^{-5} \\
6.096780 \times 10^{-6} \\
1.238408 \times 10^{-6}\end{array}$ & $\begin{array}{l}1.272 \\
3.174 \\
1.160 \\
3.11 \\
1.400 \\
3.87\end{array}$ & $\begin{array}{l}\times 10^{-2} \\
\times 10^{-2} \\
\times 10^{-1} \\
\times 10^{-1}\end{array}$ \\
\hline${ }^{238} \mathrm{U}$ & $\begin{array}{l}1 \\
2 \\
3 \\
4 \\
5 \\
6\end{array}$ & $\begin{array}{l}1.855877 \times 10^{-5} \\
2.069849 \times 10^{-4} \\
2.341152 \times 10^{-4} \\
5.616857 \times 10^{-4} \\
3.243282 \times 10^{-4} \\
1.081094 \times 10^{-4}\end{array}$ & $\begin{array}{l}1.888407 \times 10^{-5} \\
2.104182 \times 10^{-4} \\
2.381710 \times 10^{-4} \\
5.713120 \times 10^{-4} \\
3.299351 \times 10^{-4} \\
1.099784 \times 10^{-4}\end{array}$ & $\begin{array}{l}1.323 \\
3.212 \\
1.39 \\
3.59 \\
1.41 \\
4.03\end{array}$ & $\begin{array}{ll}\times & 10^{-2} \\
\times & 10^{-2} \\
\times & 10^{-1} \\
\times & 10^{-1}\end{array}$ \\
\hline $239_{\mathrm{Pu}}$ & $\begin{array}{l}1 \\
2 \\
3 \\
4 \\
5 \\
6\end{array}$ & $\begin{array}{l}5.884044 \times 10^{-5} \\
4.559963 \times 10^{-4} \\
3.354436 \times 10^{-4} \\
5.136262 \times 10^{-4} \\
1.612912 \times 10^{-4} \\
5.480768 \times 10^{-5}\end{array}$ & $\begin{array}{l}5.845605 \times 10^{-5} \\
4.527117 \times 10^{-4} \\
3.332017 \times 10^{-4} \\
5.100306 \times 10^{-4} \\
1.601621 \times 10^{-4} \\
5.442400 \times 10^{-5}\end{array}$ & $\begin{array}{l}1.29 \\
3.11 \\
1.34 \\
3.32 \\
1.26 \\
3.21\end{array}$ & $\begin{array}{ll}\times & 10^{-2} \\
\times & 10^{-2} \\
\times & 10^{-1} \\
\times & 10^{-1}\end{array}$ \\
\hline $240 \mathrm{Pu}$ & $\begin{array}{l}1 \\
2 \\
3 \\
4 \\
5 \\
6\end{array}$ & $\begin{array}{l}2.014815 \times 10^{-6} \\
2.065517 \times 10^{-5} \\
1.385624 \times 10^{-5} \\
2.546833 \times 10^{-5} \\
9.314133 \times 10^{-6} \\
2.110233 \times 10^{-6}\end{array}$ & $\begin{array}{l}2.026214 \times 10^{-6} \\
2.075844 \times 10^{-5} \\
1.393250 \times 10^{-5} \\
2.560028 \times 10^{-5} \\
9.362390 \times 10^{-6} \\
2.121166 \times 10^{-6}\end{array}$ & $\begin{array}{l}1.294 \\
3.131 \\
1.35 \\
3.33 \\
1.36 \\
4.03\end{array}$ & $\begin{array}{ll}\times & 10^{-2} \\
\times & 10^{-2} \\
\times & 10^{-1} \\
\times & 10^{-1}\end{array}$ \\
\hline $\begin{array}{c}{ }^{241} \mathrm{Pu} \\
+ \\
242 \mathrm{Pu}\end{array}$ & $\begin{array}{l}1 \\
2 \\
3 \\
4 \\
5 \\
6\end{array}$ & $\begin{array}{l}6.869020 \times 10^{-7} \\
1.654944 \times 10^{-5} \\
1.191850 \times 10^{-5} \\
2.709378 \times 10^{-5} \\
1.264376 \times 10^{-5} \\
1.111540 \times 10^{-6}\end{array}$ & $\begin{array}{l}6.804222 \times 10^{-7} \\
1.638182 \times 10^{-5} \\
1.180429 \times 10^{-5} \\
2.682555 \times 10^{-5} \\
1.251859 \times 10^{-5} \\
1.100535 \times 10^{-6}\end{array}$ & $\begin{array}{l}1.28 \\
2.99 \\
1.24 \\
3.52 \\
1.61 \\
3.47\end{array}$ & $\begin{array}{ll}\times 10^{-2} \\
\times 10^{-2} \\
\times 10^{-1} \\
\times 10^{-1}\end{array}$ \\
\hline
\end{tabular}

${ }^{a} 11$-group calculations using ENDF/B Version 4 data for a cylindrical reactor model.

botalned using homogeneous diffusion coefficlents.

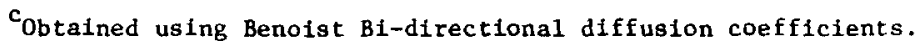


TABLE XXXIT. Delayed Neutron Kinetics Parameters for the GCFR Phase I Assembly (29 Energy Group R-Z Diffusion Theory Calculation, With Streaming)

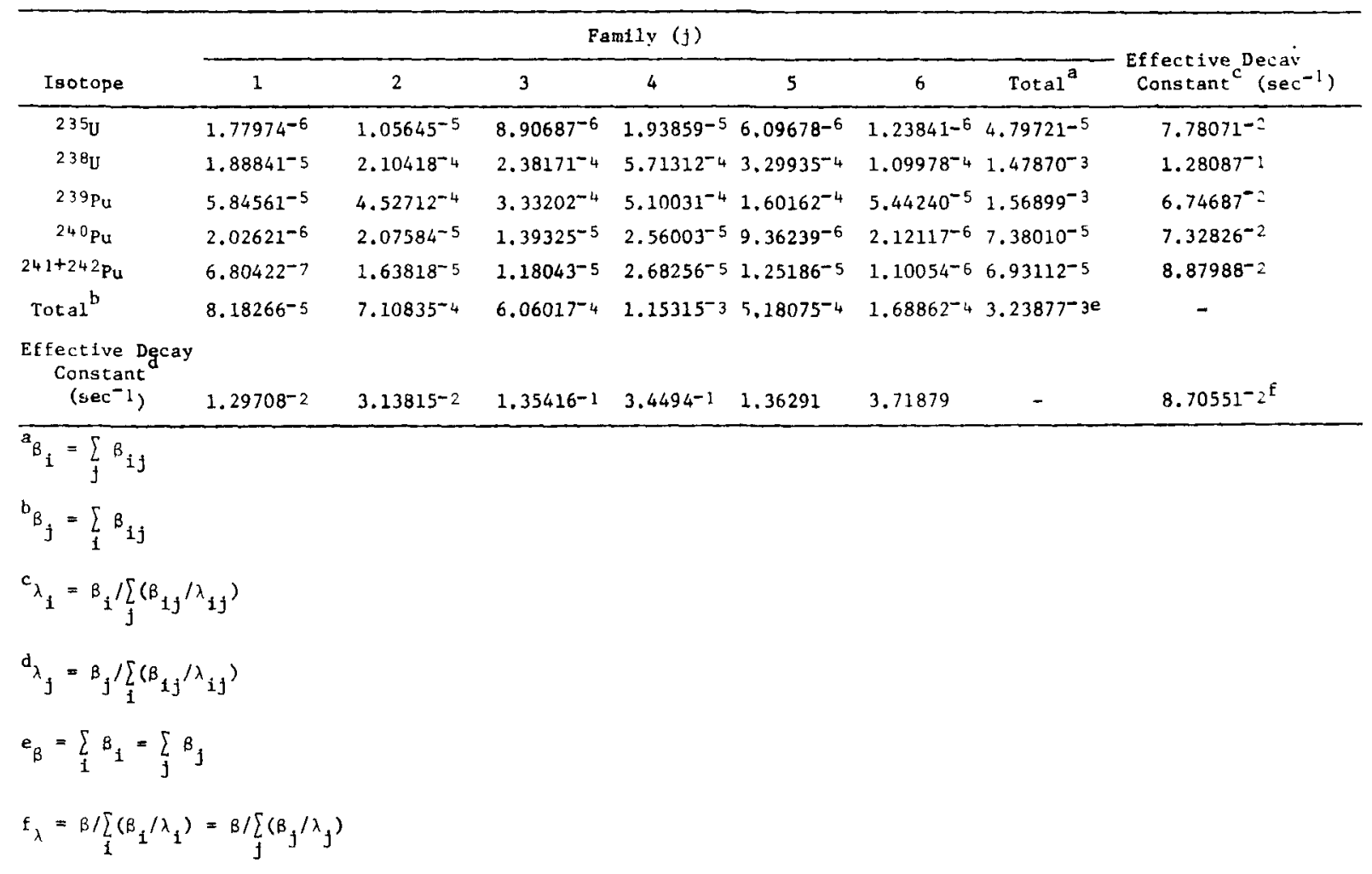

TABLE XXXIII. Condensed Kinetics Parameters Used

in the Analyses of the Phase I Experiments

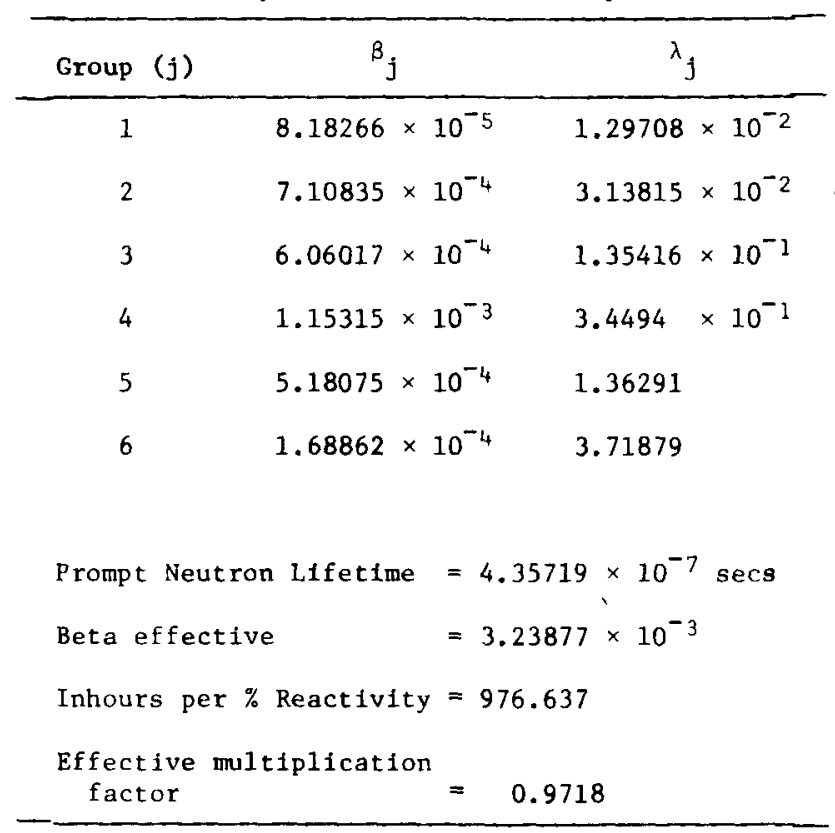


TABLE XXXIV. Breakdown of Reaction Rates by Regton ${ }^{a}$ in the GCFR Phase I Assembly

\begin{tabular}{lllllll}
\hline Region & Total Source & Fission Source & Absorption & Fission & Capture & Leakage \\
\hline C1 & $4.0444^{-1}$ & $4.0278^{-1}$ & $2.5793^{-1}$ & $1.3744^{-1}$ & $1.2048^{-1}$ & $1.4651^{-1}$ \\
C2 & $2.0332^{-1}$ & $2.0249^{-1}$ & $1.3032^{-1}$ & $6.9108^{-2}$ & $6.1216^{-2}$ & $7.2997^{-2}$ \\
C3 & $2.4608^{-1}$ & $2.4511^{-1}$ & $1.6081^{-1}$ & $8.3727^{-2}$ & $7.7079^{-2}$ & $8.5272^{-2}$ \\
CRB & $7.9359^{-2}$ & $7.9093^{-2}$ & $5.4256^{-2}$ & $2.7081^{-2}$ & $2.7175^{-2}$ & $2.5102^{-2}$ \\
CAXB1 & $2.3139^{-2}$ & $2.3059^{-2}$ & $1.5502^{-2}$ & $7.8889^{-3}$ & $7.6136^{-3}$ & $7.6366^{-3}$ \\
CAXB2 & $1.1649^{-2}$ & $1.1609^{-2}$ & $7.8449^{-3}$ & $3.9721^{-3}$ & $3.8723^{-3}$ & $3.8036^{-3}$ \\
RB1 & $1.1312^{-2}$ & $1.0987^{-2}$ & $4.1247^{-2}$ & $4.0395^{-3}$ & $3.7208^{-2}$ & $2.9935^{-2}$ \\
RB2 & $7.9832^{-3}$ & $7.7772^{-3}$ & $4.1741^{-2}$ & $2.8870^{-3}$ & $3.8854^{-2}$ & $3.3758^{-2}$ \\
AXB1 & $6.3294^{-3}$ & $6.1438^{-3}$ & $2.1152^{-2}$ & $2.2546^{-3}$ & $1.8898^{-2}$ & $1.4823^{-2}$ \\
AXB2 & $6.3959^{-3}$ & $6.2251^{-3}$ & $2.9072^{-2}$ & $2.3036^{-3}$ & $2.6769^{-2}$ & $2.2677^{-2}$ \\
Tota1s & $1.0000^{\mathrm{d}}$ & 0.99528 & 0.75987 & 0.34071 & $0.41917^{\mathrm{e}}$ & 0.2401 \\
\hline
\end{tabular}

${ }^{a}$ See Figure 17 for location of regions. Regions $\mathrm{Cl}$ - BAXB2 contain core composition RB, and RB2 contain radial blanket composition and AXB1 and AXB2 contain axla1 blanket composition.

botal source is defined as fission source plus $2 \times(n-2 n)$ source.

cAbsorption includes the $(n-2 n)$ term.

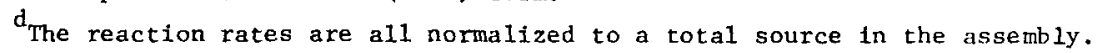

$e_{K_{\infty}}$ of the central core region 151.5680 and for the whole assembly is 1.3160 .

TABLE XXXV. Isotopic Breakdown of Reaction Rate In Central Core Region

\begin{tabular}{llllll}
\hline Isotope $^{\mathrm{a}}$ & Total Source & F1ssion Source & F1ss1on & Absorption & Capture \\
\hline $238 \mathrm{Pu}$ & $2.9005^{-4}$ & $2.9121^{-4}$ & $2.9907^{-4}$ & $2.0000^{-4}$ & $8.8876^{-5}$ \\
$239 \mathrm{Pu}$ & $8.3658^{-1}$ & $8.3980^{-1}$ & $8.3449^{-1}$ & $5.3845^{-1}$ & $2.0636^{-1}$ \\
$240 \mathrm{Pu}$ & $2.9669^{-2}$ & $2.9779^{-2}$ & $2.8301^{-2}$ & $2.8749^{-2}$ & $2.9252^{-2}$ \\
$24 \mathrm{Pu}^{\mathrm{P}}$ & $1.5254^{-2}$ & $1.5302^{-2}$ & $1.5003^{-2}$ & $9.3034^{-3}$ & $2.9100^{-3}$ \\
$242 \mathrm{Pu}$ & $3.3099^{-4}$ & $3.3172^{-4}$ & $3.1857^{-4}$ & $3.0392^{-4}$ & $2.8748^{-4}$ \\
$235 \mathrm{U}$ & $7.2156^{-3}$ & $7.2412^{-3}$ & $8.6349^{-3}$ & $5.8075^{-3}$ & $2.6360^{-3}$ \\
$238 \mathrm{U}$ & $1.0859^{-1}$ & $1.0530^{-1}$ & $1.1123^{-1}$ & $3.4404^{-1}$ & $6.0519^{-1}$ \\
$\mathrm{Fe}$ & $4.3803^{-5}$ & $0.0000^{-1}$ & $0.0000^{-1}$ & $3.3423^{-2}$ & $7.0914^{-2}$ \\
$\mathrm{~N} 1$ & $2.8352^{-6}$ & $0.0000^{-1}$ & $0.0000^{-1}$ & $1.1032^{-2}$ & $2.3406^{-2}$ \\
$\mathrm{Cr}$ & $3.1296^{-5}$ & $0.0000^{-1}$ & $0.0000^{-1}$ & $1.2113^{-2}$ & $2.5701^{-2}$ \\
$\mathrm{Mn}$ & $3.3504^{-6}$ & $0.0000^{-1}$ & $0.0000^{-1}$ & $2.7157^{-3}$ & $5.7620^{-3}$ \\
$16 \mathrm{O}$ & $0.0000^{-1}$ & $0.0000^{-1}$ & $0.0000^{-1}$ & $3.0852^{-3}$ & $6.5460^{-3}$ \\
$\mathrm{Am}$ & $1.9443^{-3}$ & $1.9522^{-3}$ & $1.7196^{-3}$ & $2.9005^{-3}$ & $4.2252^{-3}$ \\
$\mathrm{Mo}$ & $4.6521^{-5}$ & $0.0000^{-1}$ & $0.0000^{-1}$ & $7.8815^{-3}$ & $1.6722^{-2}$ \\
\hline & & & & &
\end{tabular}

a Each column is normalized to untty.

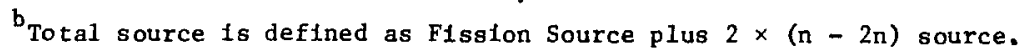

${ }^{c}$ Absorption includes the $(n-2 n)$ term. 
TABLE XXXVI. Summary of Calculated E1genvalues ${ }^{a}$ for Several Fast Critical Assembiles

\begin{tabular}{|c|c|c|}
\hline & $\begin{array}{l}\text { Calculated } \\
\text { Value }\end{array}$ & Bias, $\delta \mathrm{k}$ \\
\hline ZPR-3 As semb 1y 48 & 0.9911 & -0.0089 \\
\hline ZPR-6 Assemb 1y 6A & 0.9893 & -0.0107 \\
\hline ZPR-6 Assemb 1 y 7 & 0.9844 & -0.0156 \\
\hline GCFR Phase I Without Streaming & 0.9992 & -0.0008 \\
\hline With Streaming & 0.9763 & -0.0237 \\
\hline
\end{tabular}

TABLE XXXVII. Summary of Eigenvalue Calculations
of GCFR Phase I Assembly

\begin{tabular}{|c|c|}
\hline $\begin{array}{l}\text { Two dimensional calculation } \\
\text { (with streaming, logarithmic boundary } \\
\text { conditions, knees and bed included in } \\
x-y \text { model) }\end{array}$ & 0.9763 \\
\hline$\Delta k$ streaming from $2 D$ calculations & $2.29 \%$ \\
\hline $\begin{array}{l}\text { Three dimenstonal calculation } \\
\text { (SYN3D, full plane half helght } \\
\text { logarithmic boundary conditions, axial } \\
\text { reflector } 30 \mathrm{~cm} \text {, knees and bed } \\
\text { included) }\end{array}$ & 0.9909 \\
\hline$\Delta k$ streaming from $3 \mathrm{D}$ calculations & $1.91 \%$ \\
\hline
\end{tabular}

TABLE XXXVIII, Fission Rates Measured in Three Central Drawers ${ }^{a}$

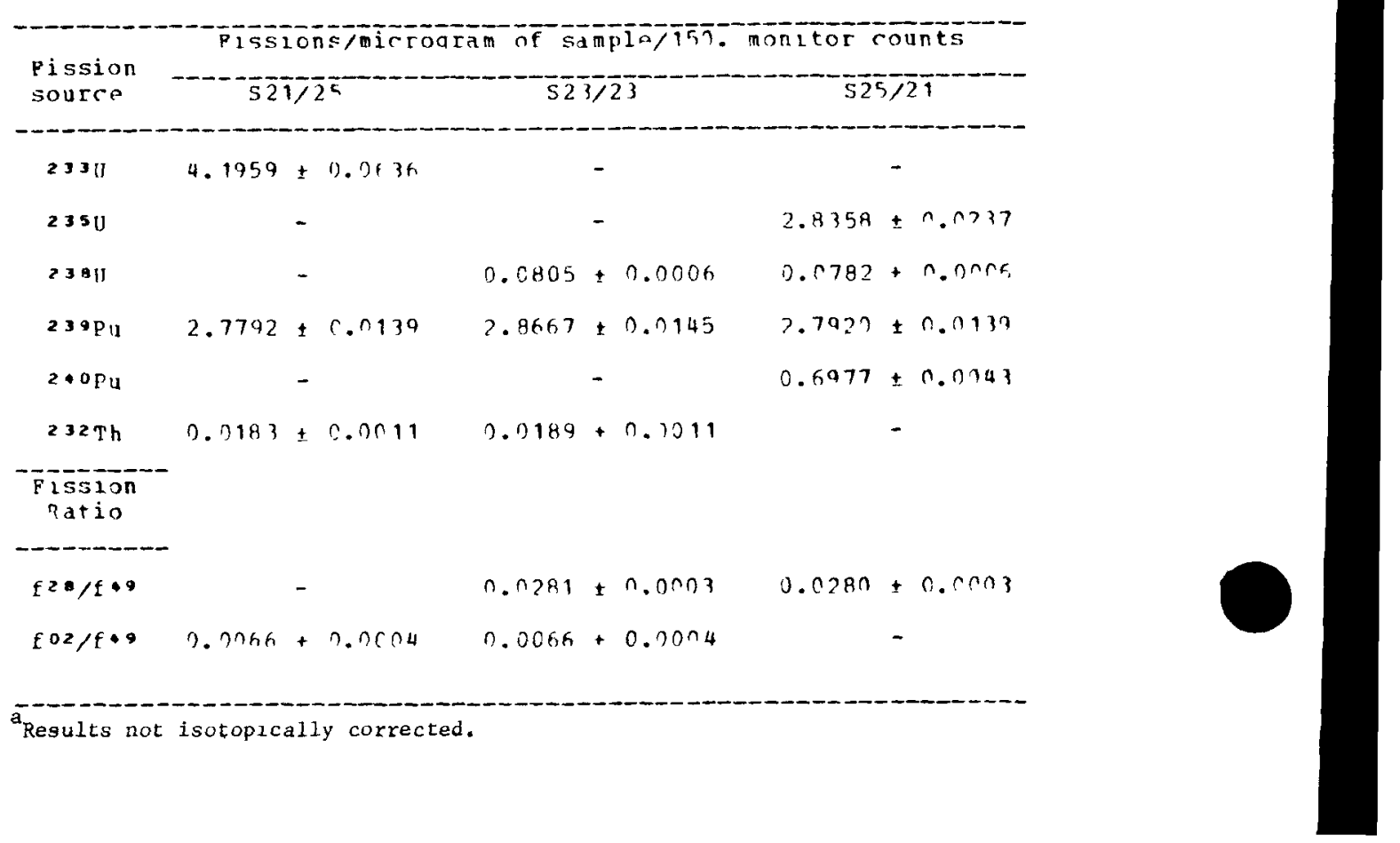


TABLE XXXIX. Mass and Composition of Fission Counter Sources

\begin{tabular}{|c|c|c|c|c|c|c|c|}
\hline \multirow{2}{*}{ 1 sotnpe } & \multicolumn{2}{|c|}{ (m1crograms) } & \multicolumn{5}{|c|}{$w+a$} \\
\hline & -- & $\cdots$ & 2730 & $-\frac{-5}{3} \cdot \bar{U}$ & $-\overline{2} 35 \overline{4}$ & $-\overline{3} 367$ & गुणा \\
\hline 2330 & 44.455 & \pm 0.657 & 99.538 & 0.184 & 3.762 & $n . n 13$ & $0.2^{n}$ \\
\hline $235 \mathrm{U}$ & 78.167 & $\pm 0.6,33$ & - & 1.074 & 93.39 & 0.446 & 0.13 \\
\hline \multirow[t]{2}{*}{2384} & 416.87 & \pm 3.33 & - & - & $n .014$ & - & 99.78 \\
\hline & & & $--\frac{1}{2} \overline{9} \overline{\mathrm{Fu}}$ & 540511 & $29 \overline{\mathrm{Fu}}$ & 242011 & \\
\hline $239 \mathrm{Pu}^{\mathrm{a}}$ & 81.47 & \pm 0.41 & 99.940 & 1.012 & 1.049 & - & \\
\hline $239 \mathrm{pu}$ & 27.054 & \pm 0.135 & 98.947 & 1.112 & 10.549 & - & \\
\hline \multirow[t]{2}{*}{$z+0 \mathrm{Pu}$} & 76.593 & \pm 0.475 & $0.77 n$ & 98.578 & 0.541 & 0.111 & \\
\hline & & & $-\overline{3} 2 \mathrm{Th}$ & & 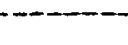 & & \\
\hline $232 \mathrm{Th}$ & 535. & \pm 32. & 100.0 & & & & \\
\hline
\end{tabular}

Used as the monitor counter.

TABLE XL, Reaction Rate Ratios at the Center of the GCFR Phase I Assembly

\begin{tabular}{llcl}
\hline Ratio & Experimental & Calculated $^{\mathrm{a}}$ & $\mathrm{C} / \mathrm{E}$ \\
\hline $\mathrm{f}^{23} / \mathrm{f}^{49}$ & $1.467 \pm 0.026$ & 1.490 & 1.016 \\
$\mathrm{f}^{25} / \mathrm{f}^{49}$ & $1.004 \pm 0.012$ & 1.018 & 1.014 \\
$\mathrm{f}^{28} / \mathrm{f}^{49}$ & $0.0276 \pm 0.0003$ & 0.0246 & 0.891 \\
$\mathrm{c}^{28} / \mathrm{f}^{49}$ & $0.1419 \pm 0.0016$ & $0.1427^{\mathrm{b}}$ & 1.002 \\
$\mathrm{f}^{40} / \mathrm{f}^{49}$ & $0.2374 \pm 0.0025$ & 0.2360 & 0.994 \\
$\mathrm{f}^{02} / \mathrm{f}^{49}$ & $0.0064 \pm 0.0004$ & 0.0055 & 0.859 \\
$\mathrm{c}^{02} / \mathrm{f}^{49}$ & $0.1974 \pm 0.0023$ & $0.1989^{\mathrm{b}}$ & 1.008 \\
\hline
\end{tabular}

a Obtained using homogeneous cross sections with

29 broad group distorted fluxes from PIT calculations of the effects of the stainless steel surroundings.

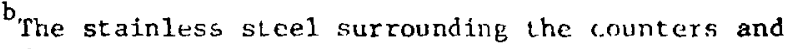
foils may have an effect on the resonance capture rates $c^{28}$ and $c^{02}$, and this is not accounted for in the calculations.

TABLE XLI. Calculated Reaction Rates at the Center of the GCFR Phase I Assembly

\begin{tabular}{|c|c|c|c|c|c|c|c|}
\hline \multirow{2}{*}{$\begin{array}{l}\text { Reaction } \\
\text { Rate }\end{array}$} & \multicolumn{2}{|c|}{$\begin{array}{l}\text { Homogeneous } \\
\text { Cross Sections }\end{array}$} & \multicolumn{2}{|c|}{$\begin{array}{l}\text { Heterogeneous } \\
\text { Cross Sections }\end{array}$} & \multicolumn{2}{|c|}{ Heter./Homog. } & \multirow{2}{*}{$\frac{\text { Homogeneous }}{\text { Perturbed/ARC }}$} \\
\hline & ARC & Perturbed & ARC & Perturbed & ARC & Perturbed & \\
\hline$f^{49}$ & 1.7276 & 1.7385 & 1.7322 & 1.7380 & 1.0027 & 0.9997 & 1.0063 \\
\hline $\mathbf{f}^{23}$ & 2.5533 & 2.5905 & 2.5531 & 2.5903 & 0.9999 & 0.9999 & 1.0146 \\
\hline $\mathrm{f}^{25}$ & 1.7406 & 1.7704 & 1.7362 & 1.7637 & 0.9974 & 0.9962 & 1.0171 \\
\hline $\mathrm{f}^{28}$ & 0.0473 & 0.0427 & 0.0499 & 0.0451 & 1.0566 & 1.0553 & 0.9037 \\
\hline$c^{28}$ & 0.2415 & 0.2473 & 0.2341 & 0.2391 & 0.9692 & 0.9669 & 1.0240 \\
\hline $\mathrm{f}^{40}$ & 0.4238 & 0.4102 & 0.4486 & 0.4330 & 1.0585 & 1.0555 & 0.9680 \\
\hline $\mathrm{f}^{02}$ & 0.0107 & 0.0096 & 0.0107 & 0.0096 & 0.9990 & 0.9990 & 0.8985 \\
\hline$c^{02}$ & 0.3335 & 0.3457 & 0.3333 & 0.3456 & 0.9997 & 0.9997 & 1.0369 \\
\hline
\end{tabular}


TABLE XLII. Calculated Reaction Rate Ratios (Relative to ${ }^{239} \mathrm{Pu}$ ) at the Center of GCFR Phase I Assembly

\begin{tabular}{|c|c|c|c|c|c|c|c|}
\hline \multirow{2}{*}{$\begin{array}{l}\text { Reaction } \\
\text { Rate } \\
\text { Ratios }\end{array}$} & \multicolumn{2}{|c|}{$\begin{array}{l}\text { Homogeneous } \\
\text { Cross Sections }\end{array}$} & $\begin{array}{l}\text { Heter } \\
\text { Cross }\end{array}$ & $\begin{array}{l}\text { ogeneous } \\
\text { Sections }\end{array}$ & \multicolumn{2}{|c|}{ Heter. /Homog. } & \multirow{2}{*}{$\frac{\text { Homogeneous }}{\text { Perturbed/ARC }}$} \\
\hline & ARC & Perturbed & ARC & Perturbed & ARC & Perturbed & \\
\hline $\mathrm{f}^{23} / \mathrm{f}^{49}$ & 1.4778 & 1.4901 & 1.4739 & 1.4904 & 0.9973 & 1.0002 & 1.0082 \\
\hline$f^{25} / f^{49}$ & 1.0076 & 1.0183 & 1.0023 & 1.0148 & 0.9948 & 0.9965 & 1.0107 \\
\hline$f^{28} / f^{49}$ & 0.0274 & 0.0246 & 0.0288 & 0.0259 & 1.0538 & 1.0556 & 0.8980 \\
\hline$c^{28} / f^{49}$ & 0.1398 & 0.1423 & 0.1351 & 0.1376 & 0.9666 & 0.9671 & 1.0176 \\
\hline$f^{40} / f^{49}$ & 0.2453 & 0.2360 & 0.2590 & 0.2492 & 1.0556 & 1.0558 & 0.9619 \\
\hline$f^{02} / f^{49}$ & 0.0062 & 0.0055 & 0.0062 & 0.0055 & 0.9964 & 0.9992 & 0.8928 \\
\hline$c^{02} / f^{49}$ & 0.1930 & 0.1989 & 0.1924 & 0.1989 & 0.9970 & 0.9999 & 1.0304 \\
\hline
\end{tabular}

TABLE XLIII. Comparison of ARC and PIT Group Fluxes and ${ }^{238} \mathrm{U}$ Fission Rate by Group

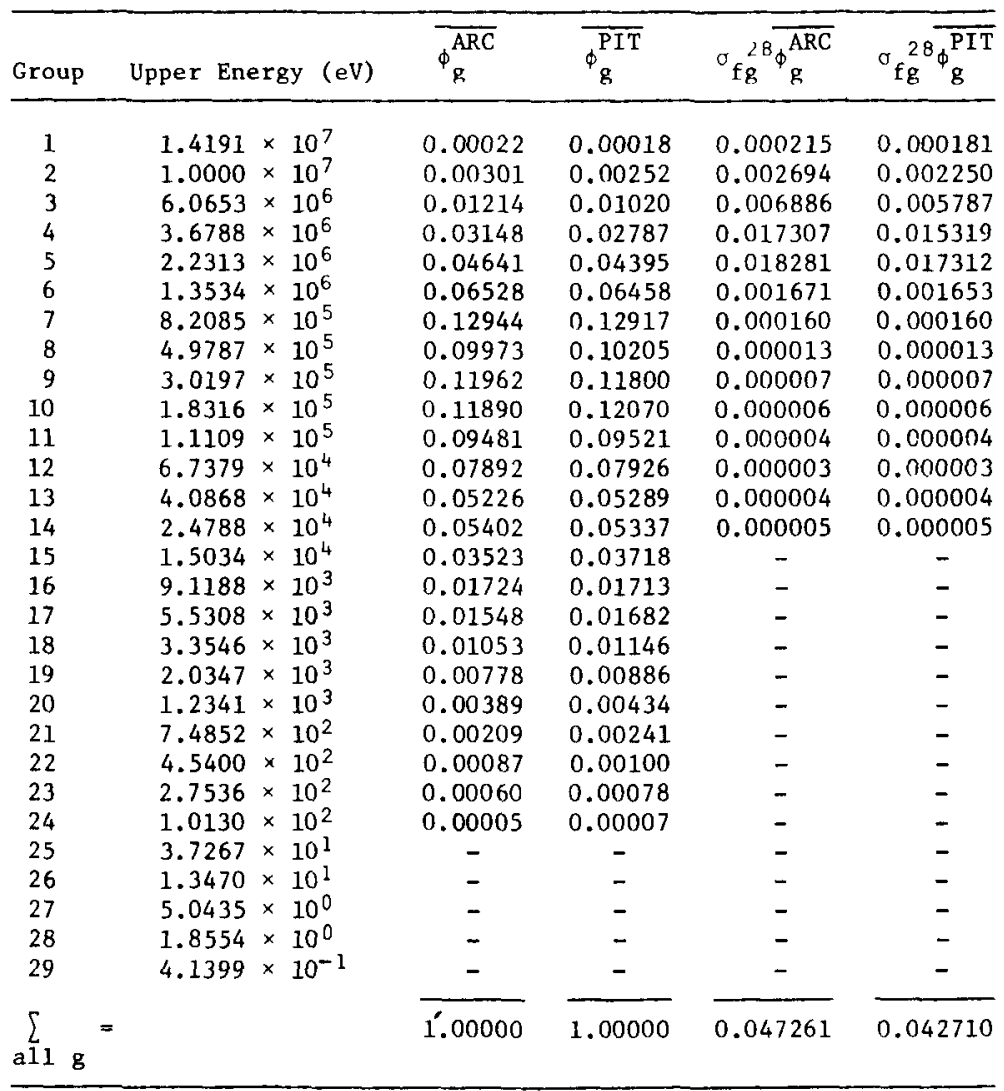


TABLE XLIV. Comparison of Reaction Rate Ratios in GCFR Phase I As sembly and ZPR-6 Assemb1y 7

\begin{tabular}{ccccccc}
\hline & \multicolumn{2}{c}{ ZPR-6 Assemb1y 7} & & \multicolumn{2}{c}{ GCFR Phase I Assemb 1y } \\
Ratio & $\begin{array}{c}\text { Experimental } \\
\text { Value }\end{array}$ & $\mathrm{C} / \mathrm{E}$ & & $\begin{array}{c}\text { Experimental } \\
\text { Value }\end{array}$ & $\mathrm{C} / \mathrm{E}$ \\
\hline $25_{\mathrm{f}} / 49_{\mathrm{f}}$ & $1.0474 \pm 0.0227$ & 1.040 & $1.0210 \pm 0.012$ & 0.987 \\
$28_{\mathrm{f}} / 49_{\mathrm{f}}$ & $0.0215 \pm 0.0002$ & 1.079 & $0.0779 \pm 0.0003$ & 0.988 \\
$40_{\mathrm{f} / 49_{\mathrm{f}}}$ & $0.1850 \pm 0.0027$ & 1.089 & $0.2364 \pm 0.0025$ & 1.038 \\
\hline
\end{tabular}

Note: The calculated results shown in this table do not include the effects of flux perturbation by the counter.

TABLE XLV. Sample Description

\begin{tabular}{|c|c|c|c|c|c|c|c|c|c|c|}
\hline \multirow[b]{2}{*}{$\begin{array}{c}\text { Sample } \\
\text { Identification }\end{array}$} & \multirow{2}{*}{$\begin{array}{c}\text { Major } \\
\text { Sample } \\
\text { Constituent }\end{array}$} & \multirow[b]{2}{*}{ State } & \multirow[b]{2}{*}{ Geometry } & \multicolumn{3}{|c|}{ Dimensions, In. } & \multirow{2}{*}{$\begin{array}{c}\text { Sample } \\
\text { Mass, } \\
\mathrm{g}\end{array}$} & \multirow[b]{2}{*}{$\begin{array}{c}\text { Sample } \\
\text { Composition }\end{array}$} & \multirow{2}{*}{$\begin{array}{c}\text { Capsule } \\
\text { Mass, } \\
\text { g }\end{array}$} & \multirow{2}{*}{$\begin{array}{c}\text { Capsule } \\
\text { Composition }\end{array}$} \\
\hline & & & & O.D. & Length & $\begin{array}{l}\text { Wall } \\
\text { Thickness }\end{array}$ & & & & \\
\hline MB-06 & $239 \mathrm{Pu}$ & Solid & Annulus & 0.835 & 1.250 & 0.005 & 3.524 & $C-1$ & 13.218 & 304 SST $^{b}$ \\
\hline$M B-10$ & 11 & 11 & "1 & 11 & 1.156 & 0.015 & 9.956 & "1 & 13.485 & 11201 \\
\hline$M B-11$ & $"$ & $"$ & $" 1$ & $"$ & 1.250 & 0.030 & 21.409 & " & 13.577 & $"$ \\
\hline Pu-240-1D & $240 \mathrm{PuO}_{2}$ & Powder & $"$ & 0.526 & 1.800 & 0.166 & 30.735 & $C-2$ & 24.045 & Inconel $600^{\mathrm{C}}$ \\
\hline $\mathrm{Pu}-240-2 \mathrm{D}$ & & " & $"$ & 0.604 & $\begin{array}{l}2.000 \\
1.800\end{array}$ & 0.205 & 45.135 & "1" & 28.821 & " \\
\hline $\mathrm{Pu}-50$ & $241 \mathrm{PuO}_{2}$ & $"$ & Cylinder & 0.210 & 0.157 & + & 0.5817 & $c-3$ & 17.722 & $304 \mathrm{SST}$ \\
\hline$P u-242-4-1$ & $242 \mathrm{Pu}$ & Solid & "1 & 0.366 & 0.813 & - & 27.295 & $C-4$ & 20.885 & 304 L SST \& A \\
\hline $\mathrm{U}-233-1$ & $233_{\mathrm{U}}$ & " & Rectangular & $0.415^{d}$ & 1.805 & 0.005 & 1.035 & $C-5$ & 16.136 & 304 SST \\
\hline $\mathrm{U}-233-2$ & $"$ & $"$ & " & $0.830^{\mathrm{d}}$ & 11 & " & 2.046 & $"$ & 15.651 & " \\
\hline $\mathrm{U}-233-3$ & " & $"$ & $"$ & " & $"$ & 0.010 & 4.158 & $"$ & 16.513 & 11 \\
\hline$M B-20$ & $235 \mathrm{U}$ & $"$ & Annulus & 0.835 & 1.688 & 0.005 & 5.231 & $c-6$ & 13.907 & $"$ \\
\hline $\mathrm{MB}-21$ & " & $" 1$ & " & $"$ & " & 0.015 & 15.778 & $"$ & 13.729 & $"$ \\
\hline $\mathrm{MB}-22$ & " & $"$ & $" \prime$ & $"$ & $" 1$ & 0.030 & 30.838 & $"$ & 13.580 & $"$ \\
\hline $\mathrm{MB}-23$ & $23 \mathrm{~B}_{\mathrm{U}}$ & $n$ & $"$ & $"$ & $"$ & 0.006 & 6.315 & $C-7$ & 13.867 & $"$ \\
\hline$M B-24$ & $"$ & $"$ & $"$ & $"$ & $"$ & 0.018 & 19.074 & " & 13.859 & $"$ \\
\hline MB-25 & $"$ & $"$ & $"$ & $"$ & $"$ & 0.036 & 38.163 & $"$ & 13.691 & $"$ \\
\hline Th-2 & Th & $"$ & $"$ & $"$ & 1.773 & 0.024 & 19.802 & $\mathrm{C}-8$ & 12.811 & $"$ \\
\hline $\begin{array}{l}\text { Th-3 } \\
\text { The }\end{array}$ & $"$ & $"$ & $"$ & $"$ & 1.750 & 0.047 & 37.977 & $"$ & 13.898 & $"$ \\
\hline Th-4 & $"$ & " & $"$ & $"$ & 1.805 & 0.094 & 71.910 & $"$ & 13.216 & $"$ \\
\hline B-7 & ${ }^{10} \mathrm{~B}$ & Powder & $"$ & 0.400 & 2.173 & 0.0094 & 0.4968 & C-9 & 20.776 & $"$ \\
\hline $\mathrm{B}(\mathrm{L})$ & $\mathrm{II}_{\mathrm{B}}$ & " & Cylinder & 0.125 & 1.969 & - & 0.5553 & $C-10$ & 8.489 & $"$ \\
\hline $\mathrm{Eu}_{2} \mathrm{O}_{3}-3$ & $\mathrm{Eu}_{2} \mathrm{O}_{3}$ & Solid & " & 0.402 & 2.163 & - & 9.963 & $99.9 \%$ Pure & 10.578 & $"$ \\
\hline $\mathrm{BeO}-1$ & $\mathrm{BeO}$ & Powder & $" r$ & 0.390 & 2.173 & - & 12.342 & $99 \%$ Pure & 10.348 & $"$ \\
\hline $\mathrm{H}_{2} \mathrm{O}-1 \mathrm{~A}$ & $\mathrm{H}_{2} \mathrm{O}$ & Liquid & $"$ & 0.440 & 1.710 & - & 4.177 & $<0.1$ PPM Impurtties & 6.648 & $"$ \\
\hline $\mathrm{H}_{2} \mathrm{O}-2 \mathrm{~A}$ & 11 & " & $"$ & 0.620 & 11 & - & 8.274 & " " & 9.299 & $"$ \\
\hline $\mathrm{H}_{2} \mathrm{O}-3 \mathrm{~A}$ & $"$ & $"$ & $"$ & 0.835 & $"$ & - & 15.047 & " " & 13.623 & $"$ \\
\hline Poly-3 & Polyethylene & Solid & $"$ & 0.102 & 2.172 & - & 0.269 & {$\left[\mathrm{CH}_{2} \cdot \mathrm{CH}_{2}\right]$} & 10.959 & $"$ \\
\hline Poly -2 & " & $" 1$ & $"$ & 0.201 & 11 & - & 1.062 & 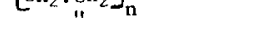 & 11.303 & $"$ \\
\hline Poly-1 & $"$ & 11 & $"$ & 0.390 & 2.173 & - & 4.024 & " & 10.567 & $"$ \\
\hline $\mathrm{CH}_{2}(\mathrm{~F})-\mathrm{I}$ & $"$ & Foan & $"$ & 0.875 & 2.656 & - & 0.843 & $C-11$ & - & - \\
\hline $\mathrm{CH}_{2}(F)-2 \mathrm{~A}$ & $"$ & 11 & $" 1$ & 0.975 & 2.00 & - & 0.910 & $"$ & - & - \\
\hline $\mathrm{CH}_{2}(\mathrm{~F})-3 \mathrm{~A}$ & Polyethylene & Foame & Cylinder & 0.975 & 2.00 & - & 0.448 & $\mathrm{C}-11$ & - & - \\
\hline$C(S)$ & C & Solid & " & 0.829 & 1.806 & - & 27.336 & $99.9 \%$ Pure & 13.421 & 304 SST \\
\hline $\mathrm{Fe}-1$ & $\mathrm{Fe}$ & & $"$ & 0.389 & 2.172 & - & 33.277 & $99.99 \%$ Pure & 10.611 & $"$ \\
\hline $\mathrm{Fe}_{2} \mathrm{O}_{3}-2$ & $\mathrm{Fe}_{2} \mathrm{O}_{3}$ & Powder & $"$ & 0.401 & 2.169 & - & 5.265 & $99.999 \%$ Pure & 10.624 & $"$ \\
\hline 304 SST & $304 \mathrm{SST}$ & Soldd & $"$ & 0.870 & 2.00 & - & 154.205 & b & - & - \\
\hline D-1 & Dummy & - & - & 0.420 & 2.625 & 0.010 & - & - & 10.667 & 304 SST \\
\hline$M B-19$ & $"$ & - & - & 0.865 & 2.00 & 0.015 & - & - & 13.807 & 11 \\
\hline DUM-2D & $"$ & - & - & 0.670 & 2.00 & 0.033 & - & - & 24.473 & Inconel 600 \\
\hline$D-242-4-1$ & $"$ & - & - & 0.580 & 2.19 & 0.031 & - & - & 15.743 & $304 \mathrm{~L}$ SST \\
\hline & & & & 0.468 & 1.66 & 0.031 & - & - & 5.030 & Al \\
\hline Cap. Hold. No. 3 & $" 1$ & - & - & 0.938 & 2.72 & 0.031 & - & - & 57.573 & 304 SST \\
\hline Cap. Hold. No. & $"$ & - & - & & $"$ & 11 & - & - & 57.579 & \\
\hline
\end{tabular}

Table II.

pical analysis: $\mathrm{Fe}-70.39 \mathrm{w} / \mathrm{o}, \mathrm{Cr}-18.45 \mathrm{w} / \mathrm{o}, \mathrm{N} i-9.71 \mathrm{w} / \mathrm{o}, \mathrm{Mn}-1.46 \mathrm{w} / \mathrm{o}$

Typical analysis: $\mathrm{Ni}-75.8 \mathrm{w} / \mathrm{o}, \mathrm{Cr}-15.74 \mathrm{w} / \mathrm{o}, \mathrm{Fe}-7.72 \mathrm{w} / \mathrm{o}, \mathrm{Si}-0.22 \mathrm{w} / \mathrm{o}, \mathrm{Mn}-0.20 \mathrm{w} / 0, \mathrm{Cu}-0.11 \mathrm{w} / \mathrm{o}$

dimension 1 s of sample width.

Foam has been perforated with resultant density one-half that of normal foam. 
TABLE XLVI. Sample Composition

\begin{tabular}{|c|c|c|c|c|c|c|c|}
\hline \multirow{2}{*}{$\begin{array}{l}\text { Sample } \\
\text { Identification } \\
M B-06 \\
M B-10 \\
M B-11\end{array}$} & \multirow{2}{*}{$\begin{array}{c}\begin{array}{c}\text { Composition } \\
\text { Identification }\end{array} \\
C-1\end{array}$} & \multirow{2}{*}{ Material } & \multirow{2}{*}{$\begin{array}{c}\begin{array}{c}\text { Material } \\
\text { Mass, } \\
\mathrm{g}\end{array} \\
3,524 \\
9.956 \\
21.409\end{array}$} & \multicolumn{2}{|c|}{$\begin{array}{c}\text { Material } \\
\text { Composition, } \\
\text { w/o }\end{array}$} & \multicolumn{2}{|c|}{$\begin{array}{c}\text { Isotopic } \\
\text { Composition, } \\
\text { w/o }\end{array}$} \\
\hline & & & & $\begin{array}{l}\mathrm{Pu} \\
\mathrm{Al}\end{array}$ & $\begin{array}{l}-97.985 \\
-\quad 1.2075\end{array}$ & $\begin{array}{l}239 \mathrm{Pu} \\
240 \mathrm{Pu} \\
241 \mathrm{Pu} \\
242 \mathrm{Pu}\end{array}$ & $\begin{array}{r}-98.9315 \\
-\quad 1.0123 \\
-\quad 0.0528 \\
-\quad 0.0034\end{array}$ \\
\hline $\begin{array}{l}P u-240-1 D \\
P u-240-1 D\end{array}$ & $C-2$ & $\mathrm{PuO}_{2}$ & $\begin{array}{l}30.735 \\
45.135\end{array}$ & $\begin{array}{c}\mathrm{Pu} \\
0 \\
\mathrm{H} \\
\mathrm{N}\end{array}$ & $\begin{array}{l}-86.1 \\
-11.97 \\
-\quad 0.035 \\
-\quad 0.02\end{array}$ & $\begin{array}{l}239 \mathrm{Pu} \\
240 \mathrm{Pu} \\
241 \mathrm{Pu} \\
242 \mathrm{Pu}\end{array}$ & $\begin{array}{l}-\quad 1.05 \\
-\quad 93.73 \\
-\quad 0.57 \\
-\quad 4.65\end{array}$ \\
\hline Pu -50 & $c-3$ & $\mathrm{PuO}_{2}$ & 0.5817 & $\begin{array}{r}\mathrm{Pu} \\
\mathrm{Am} \\
\mathrm{O} \\
\mathrm{N} \\
\mathrm{H} \\
\mathrm{O}\end{array}$ & $\begin{array}{l}-66.64^{a} \\
-20.21^{a} \\
-12.05 \\
-<0.1 \\
-<0.1 \\
-<0.1\end{array}$ & $\begin{array}{l}239 \mathrm{Pu} \\
240 \mathrm{Pu} \\
241 \mathrm{Pu} \\
242 \mathrm{Pu}\end{array}$ & $\begin{array}{l}-1.508^{a} \\
-\quad 4.034^{a} \\
-92.436^{a} \\
-\quad 2.023^{a}\end{array}$ \\
\hline$P u-242-4-1$ & $C-4$ & $\mathrm{Pu}$ & 27.295 & $\mathrm{Pu}$ & -99.8 & $\begin{array}{l}239 \mathrm{Pu} \\
240 \mathrm{Pu} \\
241 \mathrm{Pu} \\
242 \mathrm{Pu}\end{array}$ & $\begin{array}{r}1.4800 \\
-\quad 0.0927 \\
-\quad 0.0069 \\
-\quad 98.4204\end{array}$ \\
\hline $\begin{array}{l}U-233-1 \\
U-233-2 \\
U-233-3\end{array}$ & $c-5$ & $\mathbf{U}$ & $\begin{array}{l}1.0351 \\
2.0457 \\
4.1576\end{array}$ & $\begin{array}{l}\mathrm{U} \\
\mathrm{Fe} \\
\mathrm{Cr} \\
\mathrm{Na}\end{array}$ & $\begin{array}{l}-99.82 \\
-\quad 0.15 \\
-\quad 0.024^{b} \\
-<0.001\end{array}$ & $\begin{array}{l}233 U \\
234 U \\
235 U \\
236 U \\
23{ }^{8} U\end{array}$ & $\begin{array}{c}-99.47 \\
-\quad 0.183 \\
-\quad 0.071 \\
-\quad 0.014 \\
-\quad 0.266\end{array}$ \\
\hline $\begin{array}{l}M B-20 \\
M B-21 \\
M B-22\end{array}$ & $C-6$ & $\mathrm{U}$ & $\begin{array}{r}5.231 \\
15.778 \\
30.838\end{array}$ & $\mathrm{U}$ & -100.0 & $\begin{array}{l}234 \mathrm{U} \\
235 \mathrm{U} \\
236 \mathrm{U} \\
238 \mathrm{U}\end{array}$ & $\begin{array}{r}0.6651 \\
93.2438 \\
0.3162 \\
5.7750\end{array}$ \\
\hline $\begin{array}{l}M B-23 \\
M B-24 \\
M B-25\end{array}$ & $\mathrm{C}-7$ & $\mathrm{U}$ & $\begin{array}{r}6.315 \\
19.074 \\
38.163\end{array}$ & $\mathrm{v}$ & -100.0 & $\begin{array}{l}235 \mathrm{U} \\
238 \mathrm{U}\end{array}$ & $\begin{array}{r}0.2328 \\
99.7672\end{array}$ \\
\hline $\begin{array}{l}\text { Th-2 } \\
\text { Th-3 } \\
\text { Th-4 }\end{array}$ & $C-8$ & Th & $\begin{array}{l}19.802 \\
37.977 \\
71.910\end{array}$ & $\begin{array}{r}\mathrm{Th} \\
\mathrm{Fe} \\
\mathrm{Zn} \\
\mathrm{Ni} \\
\mathrm{P} \\
\mathrm{U}\end{array}$ & $\begin{array}{l}-99.93 \mathrm{~b} \\
-\quad 0.025^{\mathrm{b}} \\
-\quad 0.020^{\mathrm{b}} \mathrm{b} \\
-\quad 0.010 \mathrm{p}^{\mathrm{b}} \\
-<0.020^{\mathrm{b}} \\
-4 \mathrm{PPM}\end{array}$ & $232 \mathrm{Th}$ & -100.0 \\
\hline B-7 & $C-9$ & Boron & 0.4968 & $\begin{array}{r}\mathrm{B} \\
0 \\
\mathrm{C} \\
\mathrm{Si} \\
\mathrm{H} \\
\mathrm{A} 1\end{array}$ & $\begin{array}{l}-94.50 \\
-\quad 1.43 \\
-\quad 0.96 \\
-\quad 0.26 \\
-\quad 0.09 \\
-\quad 0.05\end{array}$ & ${ }^{11} n_{B}$ & $\begin{array}{r}-92.19 \\
-\quad 7.81\end{array}$ \\
\hline$B(L)$ & $C-10$ & Boron & 0.5553 & B & -100.0 & $\begin{array}{l}10_{B} \\
111_{B}\end{array}$ & $\begin{array}{l}-19.88 \\
-80.12\end{array}$ \\
\hline $\begin{array}{l}\mathrm{CH}_{2}(\mathrm{~F})-1 \\
\mathrm{CH}_{2}(\mathrm{~F})-2 \mathrm{~A} \\
\mathrm{CH}_{2}(\mathrm{~F})-3 \mathrm{~A}\end{array}$ & $C-11$ & $\begin{array}{l}\text { Polyethyl ene } \\
\text { Foam }\end{array}$ & $\begin{array}{l}0.843 \\
0.910 \\
0.448\end{array}$ & $\begin{array}{r}{\left[\mathrm{CH}_{2}\right.} \\
\mathrm{Zr} \\
\mathrm{C} \\
\mathrm{F}\end{array}$ & $\begin{array}{ll}\left.2 . \mathrm{CH}_{2}\right]_{\mathrm{n}}-99.76_{\mathrm{b}} & -0.12^{\mathrm{b}} \\
\mathrm{n} & -0.11_{\mathrm{b}} \\
\mathrm{a} & -0.01\end{array}$ & $\begin{array}{l}\mathrm{C} \\
\mathrm{H}\end{array}$ & $\begin{array}{l}-85.50^{c} \\
-15.50^{c}\end{array}$ \\
\hline
\end{tabular}

${ }^{\text {a }}$ Corrected to $5 / 2 / 75$ from original analysis date of 5/1/72. A 3.003 year decay correction was made assuming a 14.5 year half-11 fe for $241 \mathrm{Pu}+241 \mathrm{Am}$ decay.

${ }^{b}$ Factor of 2 accuracy.

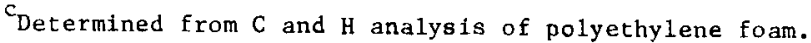


TABLE XLVII. Experimental Small Sample Central Reactivity Worths in the GCFR Phase I Assembly

\begin{tabular}{|c|c|c|c|c|c|c|c|c|c|c|c|c|}
\hline $\begin{array}{l}\text { Sample } \\
\text { I.D. }\end{array}$ & $\begin{array}{l}\text { Principal } \\
\text { Mater1al } \\
\text { or Isotope }\end{array}$ & $\begin{array}{l}\text { Sample, Sample } \\
\text { Capsule and } \\
\text { SST Worth, } \\
\text { Ih }\end{array}$ & $\begin{array}{c}\text { Sample } \\
\text { Capsule and } \\
\text { SST Mass. } \\
\text { M }\end{array}$ & $\begin{array}{l}\text { Durmy } \\
\text { Capsule } \\
\text { I.D. }\end{array}$ & $\begin{array}{l}\text { Dunaty } \\
\text { and } 5 \\
\text { Worth } \\
\text { Ih }\end{array}$ & $\begin{array}{l}\text { Capsule } \\
\text { ssta } \\
\text { h, }\end{array}$ & $\begin{array}{l}\text { Duminy } \\
\text { Capsule and } \\
\text { SST }^{2} \text { Mass, } \\
\mathrm{g}\end{array}$ & $\begin{array}{l}\text { Ratio of Sample } \\
\text { Capsule Plus SST } \\
\text { Mass to Durmy } \\
\text { Capsule Plus } \\
\text { SST }^{\mathrm{a}} \text { Mass }\end{array}$ & $\begin{array}{l}\text { Ad justed worth } \\
\text { of Dumnyy. } \\
\text { Ih }\end{array}$ & $\begin{array}{l}\text { Net Sample } \\
\text { Worth, } \\
\text { Ih }\end{array}$ & $\begin{array}{l}\text { Sample } \\
\text { Mass, } \\
\text { g }\end{array}$ & $\begin{array}{l}\text { Specific Sample } \\
\text { Worth, } \\
\mathrm{Ih} / \mathrm{kg}\end{array}$ \\
\hline$M B-06$ & ${ }^{239} \mathrm{Pu}$ & $0.184 \pm 0.005^{c}$ & 70.791 & MB-19 & $-0.28 ?$ & \pm 0.005 & 71.386 & 0.9917 & $-0.385 \pm 0.005$ & $0.469 \pm 0.007$ & 3.524 & $133.09 \pm 2.01$ \\
\hline MB-10 & ${ }^{239} \mathrm{Pu}$ & $1.048 \pm 0.012$ & 71.128 & MB-19 & & $"$ & $"$ & 0.9964 & $-0.286 \pm 0.005$ & $1.334 \pm 0.013$ & 9.956 & $133.99 \pm 1.31$ \\
\hline MB-11 & ${ }^{239} \mathrm{Pu}$ & $2.596 \pm 0.026$ & 71.215 & MB-19 & & $"$ & $"$ & 0.9976 & $-0.286 \pm 0.005$ & $2.882 \pm 0.026$ & 21.409 & $134.62 \pm 1.23$ \\
\hline Pu-140-1D & ${ }^{24} \mathrm{P}_{\mathrm{PLO}}$ & $0.186 \pm 0.004$ & 92.368 & DIM-2D & -0.420 & \pm 0.007 & 95.794 & 0.9642 & $-0.405 \pm 0.007$ & $0.591 \pm 0.010$ & 30.735 & $19.23 \pm 0.32$ \\
\hline Pu-240-2D & ${ }^{24} \mathrm{PuO}_{2}$ & $0.444 \pm 0.005$ & 96.168 & $D U M-2 D$ & & $"$ & $"$ & 1.0039 & $-0.422 \pm 0.007$ & $0.866 \pm 0.009$ & 45.135 & $19.19 \pm 0.19$ \\
\hline$P u-50$ & ${ }^{24} \mathrm{i}_{\mathrm{PuO}}$ & $-0.249 \pm 0.003$ & 77.661 & $D-1$ & -0.292 & \pm 0.005 & 70.606 & 1.0999 & $-0.321 \pm 0.005$ & $0.072 \pm 0.006$ & 0.5817 & $123.78 \pm 10.02$ \\
\hline Pu-242-4-1 & ${ }^{242} \mathrm{Pu}$ & $0.155 \pm 0.003$ & 83.995 & $D-242-4-1$ & -0.349 & \pm 0.005 & 83.873 & 1.0015 & $-0.350 \pm 0.005$ & $0.505 \pm 0.006$ & 27.295 & $18.50 \pm 0.21$ \\
\hline $4-233-1$ & ${ }^{233} \mathrm{u}$ & $-0.112 \pm 0.005$ & 73.774 & MB-19 & $-0.28 i$ & \pm 0.005 & 71.386 & 1.0335 & $-0.297 \pm 0.005$ & $0.185 \pm 0.007$ & 1.0351 & $178.73 \pm 6.83$ \\
\hline v- $933-2$ & ${ }^{23}{ }^{3} \mathrm{U}$ & $0.056 \pm 0.004$ & 73.231 & MB-19 & & $"$ & $"$ & 1.0258 & $-0.294 \pm 0.005$ & $0.350 \pm 0.006$ & 2.0457 & $171.09 \pm 3.13$ \\
\hline$U-233-3$ & ${ }^{233} \mathrm{U}$ & $0.415 \pm 0.006$ & 74.092 & MB-19 & & $"$ & " & 1.0379 & $-0.298 \pm 0.005$ & $0.713 \pm 0.008$ & 4.1576 & $171.49 \pm 1.88$ \\
\hline MB-20 & $235 \mathrm{U}$ & $0.197 \pm 0.006$ & 73.372 & MB-19 & & $"$ & " & 0.9998 & $-0.287 \pm 0.005$ & $0.484 \div 0.008$ & 5.231 & $92.53 \pm 1.49$ \\
\hline$M B-21$ & ${ }^{235} \mathrm{U}$ & $1.163 \pm 0.013$ & 71.273 & MB-19 & & $"$ & $"$ & 0.9984 & $-0.287 \pm 0.005$ & $1.450 \pm 0.014$ & 15.778 & $91.90 \pm 0.88$ \\
\hline MB-22 & $235_{\mathrm{J}}$ & $2.529=0.027$ & 71.250 & MB-19 & & $"$ & $"$ & 0.9981 & $-0.286 \pm 0.005$ & $2.815 \div 0.027$ & 30.838 & $91.28 \pm 0.89$ \\
\hline MB-23 & ${ }^{238} \mathrm{U}$ & $-0.328 \pm 0.006$ & 71.537 & MB-19 & & $"$ & $"$ & 1.0021 & $-0.288 \pm 0.005$ & $-0.040 \pm 0.008$ & 6.315 & $6.33 \pm 1.24$ \\
\hline MB-24 & ${ }^{238} \mathrm{U}$ & $-0.421 \pm 0.005$ & 71.497 & MB- 19 & & $"$ & $"$ & 1.0016 & $-0.287 \pm 0.005$ & $-0.134 \pm 0.007$ & 19.074 & $7.03 \pm 0.37$ \\
\hline MB-25 & ${ }^{238} \mathrm{U}$ & $-0.550 \pm 0.007$ & 71.156 & MB-19 & & $"$ & $"$ & 0.9968 & $-0.286 \pm 0.005$ & $-0.264 \pm 0.009$ & 38.163 & $6.92 \pm 0.23$ \\
\hline Th-2 & ${ }^{232} \mathrm{Th}$ & $-0.540 \pm 0.007$ & 70.481 & MB-19 & -0.287 & $\pm 0,005$ & 71.386 & 0.9873 & $-0.283 \pm 0005$ & $-0.257 \pm 0.009$ & 19.802 & $-12.98 \pm 0.43$ \\
\hline Th-3 & ${ }^{232} \mathrm{Th}$ & $-0.768 \pm 0.010$ & 71.536 & MB-19 & & $"$ & $"$ & 1.0021 & $-0.288 \pm 0.005$ & $-0.480 \pm 0.011$ & 37.977 & $-12.64 \pm 0.29$ \\
\hline Th-4 & ${ }^{232} \mathrm{Th}$ & $-1.176 \pm 0.012$ & 70.796 & $M B-19$ & & " & $"$ & 0.9917 & $-0.285 \pm 0.005$ & $-0.891 \pm 0.013$ & 71.910 & $-12.39 \pm 0.18$ \\
\hline B-7 & ${ }^{10} \mathrm{~B}$ & $-1.218 \div 0.014$ & 80.619 & $\mathrm{D}-1$ & -0.292 & \pm 0.005 & 70.606 & 1.1418 & $-0.333 \pm 0.006$ & $-0.885 \pm 0.015$ & 0.4968 & $-1781.40 \pm 30.66$ \\
\hline $\mathrm{B}(\mathrm{L})$ & $11_{\mathrm{B}}$ & $-0.494 \pm 0.008$ & 66.068 & $\mathrm{D}-1$ & & $"$ & $"$ & 0.9357 & $-0.273 \pm 0.005$ & $-0.221 \pm 0.009$ & 0.5553 & $-397.98 \pm 16.99$ \\
\hline $\mathrm{Eu}_{2} \mathrm{O}_{3}-3$ & $\mathrm{Eu}_{2} \mathrm{O}_{3}$ & $-1.516 \pm 0.015$ & 70.421 & $\mathrm{D}-1$ & & $"$ & $"$ & 0.9974 & $-0.291 \pm 0.005$ & $-1.225 \pm 0.016$ & 9.963 & $-122.95 \pm 1.59$ \\
\hline
\end{tabular}


TABLE XIVII. (Contd.)

\begin{tabular}{|c|c|c|c|c|c|c|c|c|c|c|c|}
\hline $\begin{array}{c}\text { Sample } \\
\text { I.D. }\end{array}$ & $\begin{array}{l}\text { Principal } \\
\text { Material } \\
\text { or Isatope }\end{array}$ & $\begin{array}{l}\text { Sample, Sample } \\
\text { Capsule and } \\
\text { SST Th }^{2} \text { Worth, } \\
\text { Ih }\end{array}$ & $\begin{array}{c}\text { Sample } \\
\text { Capsule and } \\
\text { SST }^{\mathrm{a}} \text { Mass, } \\
\text { g }\end{array}$ & $\begin{array}{l}\text { Dummy } \\
\text { Capsule } \\
\text { I.D. }\end{array}$ & $\begin{array}{l}\text { Dummy Capsule } \\
\text { and SST } \\
\text { Worth, } \\
\text { Ih }\end{array}$ & $\begin{array}{l}\text { Dunmy } \\
\text { Capsule and } \\
\text { SST T Mass, }^{2} \\
\mathbf{8}\end{array}$ & $\begin{array}{l}\text { Ratio of Sample } \\
\text { Capsule Plus SST } \\
\text { Mass to Dumny } \\
\text { Capsule Plus } \\
\text { SST }^{\text {a }} \text { Mass }\end{array}$ & $\begin{array}{l}\text { Adjusted Worth } \\
\text { of Durnmy, } \\
\mathrm{Ih}\end{array}$ & $\begin{array}{l}\text { Net Sample } \\
\text { Worth, } \\
\text { Ih }\end{array}$ & $\begin{array}{l}\text { Sample } \\
\text { Mass, } \\
\text { 8 }\end{array}$ & $\begin{array}{l}\text { Specific Sample } \\
\text { Worth, } \\
\mathrm{Ih} / \mathrm{kg}\end{array}$ \\
\hline $\mathrm{Be} 0-1$ & $\mathrm{BeO}$ & $-0.430 \pm 0.005$ & 70.177 & D-1 & $-0.292 \pm 0.005$ & 70.606 & 0.9939 & $-0.290 \pm 0.005$ & $-0.140 \div 0.007$ & 12.342 & $-11.34 \pm 0.57$ \\
\hline $\mathrm{H}_{2} \mathrm{O}-1 \mathrm{~A}$ & $\mathrm{H}_{2} \mathrm{O}$ & $0.409 \pm 0.006$ & 70.959 & MB-19 & $-0.287 \pm 0.005$ & 71.386 & 0.9940 & $-0.285 \pm 0.005$ & $0.694 \div 0.008$ & 4.177 & $166.15 \pm 1.87$ \\
\hline $\mathrm{H}_{2} \mathrm{O}-2 \mathrm{~A}$ & $\mathrm{H}_{2} \mathrm{O}$ & $1.287 \pm 0.013$ & 74.968 & MB-19 & $"$ & $"$ & 1.0502 & $-0.301 \pm 0.005$ & $1.588 \pm 0.014$ & 8.274 & $191.93 \pm 1.68$ \\
\hline $\mathrm{H}_{2} \mathrm{O}-3 \mathrm{~A}$ & $\mathrm{H}_{2} \mathrm{O}$ & $2.877 \pm 0.030$ & 71.153 & MB-19 & $"$ & $"$ & 0.9967 & $-0.286 \pm 0.005$ & $3.163=0.030$ & 15.047 & $210.21 \pm 2.02$ \\
\hline Poly-3 & $\mathrm{CH}_{2}$ (Sol1d) & $-0.246 \pm 0.005$ & 70.857 & $D-1$ & $-0.292 \pm 0.005$ & 70.606 & 1.0036 & $-0.293 \pm 0.005$ & $0.047 \pm 0.007$ & 0.269 & $174.72 \pm 26.29$ \\
\hline Poly-2 & $\mathrm{CH}_{2}$ (SolHd) & $-0.213 \pm 0.003$ & 71.307 & $0-1$ & " & " & 1.0099 & $-0.295 \pm 0.005$ & $0.182 \pm 0.006$ & 1.062 & $171.37 \pm 5.49$ \\
\hline Poly-1 & $\mathrm{CH}_{2}$ (Solid) & $0.653 \pm 0.009$ & 70.452 & $D-1$ & $"$ & " & 0.9978 & $-0.291 \pm 0.005$ & $0.944 \pm 0.010$ & 4.024 & $234.59=2.56$ \\
\hline $\mathrm{CH}_{2}(\mathrm{~F})-1$ & $\mathrm{CH}_{2}$ (FoAm) & $-0.158 \pm 0.004$ & 57.573 & C.H. No. 3 & $-0.241 \pm 0.004$ & 57.573 & 1.0000 & $-0.241 \pm 0.004$ & $0.083 \pm 0.006$ & 0.8434 & $98.41 \pm 6.71$ \\
\hline $\mathrm{c}(\mathrm{S})$ & c & $-0.619 \pm 0.007$ & 71.000 & MB-19 & $-0.287 \pm 0.005$ & 71.386 & 0.9946 & $-0.285 \pm 0.005$ & $-0.334 \pm 0.009$ & 27.336 & $-12.22 \pm 0.31$ \\
\hline $\mathrm{Fe}-1$ & $\mathrm{Fe}$ & $-0.403 \pm 0.005$ & 70.373 & $\mathrm{D}-1$ & $-0.292 \pm 0.005$ & 70.606 & 3.9967 & $-0.291 \pm 0.005$ & $-0.112 \pm 0.007$ & 33.277 & $-3.37 \pm 0.21$ \\
\hline $\mathrm{Fe}_{2} \mathrm{O}_{3}-2$ & $\mathrm{Fe}_{2} \mathrm{O}_{3}$ & $-0.305 \pm 0.005$ & 70.462 & $D-1$ & " & $"$ & 0.9980 & $-0.291 \pm 0.005$ & $-0.014 \pm 0.007$ & 5.265 & $-2.66 \pm 1.34$ \\
\hline $304 \mathrm{SST}$ & SST & $-0.803 \pm 0.011$ & 57.573 & C.H. No. 3 & $-0.241 \pm 0.004$ & 57.573 & 1.0000 & $-0.241 \pm 0.004$ & $-0.562 \pm 0.012$ & 154.205 & $-3.64 \pm 0.08$ \\
\hline$D-1$ & Dusumy & $-0.292 \pm 0.004$ & 59.939 & C.H. No. 3 & " & $"$ & 1.0411 & $-0.251 \pm 0.003$ & $-0.041 \pm 0.005$ & 10.667 & $-3.84 \pm 0.47$ \\
\hline$M B-19$ & Dumny & $-0.287 \pm 0.004$ & 57.579 & C.H. No. 3 & $"$ & " & 1.0001 & $-0.241 \pm 0.003$ & $-0.046 \pm 0.005$ & 13.807 & $-3.33 \pm 0.36$ \\
\hline DUM 2D & Dumby & $-0.420 \pm 0.005$ & 67.321 & С.н. No. 3 & " & $"$ & 1.1693 & $-0.282 \pm 0.004$ & $-0.138 \pm 0.006$ & 28.473 & $-4.85+0.22$ \\
\hline$D 0242-4-1$ & numomy & $-0.349 \pm 0.005$ & 63.101 & C.H. No. 3 & " & $"$ & 1.0960 & $-0.264 \pm 0.003$ & $-0.085 \pm 0.004$ & 20.773 & $-4.09 \pm 0.20$ \\
\hline $\begin{array}{l}\text { Capsule } \\
\text { Holder } \\
\text { No. } 3\end{array}$ & Dummy & $-0.241 \pm 0.003$ & - & None & - & - & - & - & $-0.241 \pm 0.003$ & 57.573 & $-4.19 \pm 0.05$ \\
\hline
\end{tabular}

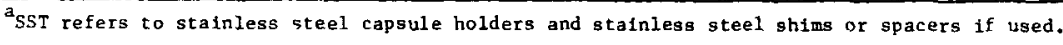


TABLE XIVIII. Calculated Small Sample Central Reactivity Worths in the GCFR Phase I Assembly with Comparison to Experimental Worths

\begin{tabular}{|c|c|c|c|c|}
\hline Sample & $\begin{array}{l}\text { Principle } \\
\text { Material } \\
\quad \text { or } \\
\text { Isotope }\end{array}$ & $\begin{array}{c}\text { Calculated } \\
\text { Sample Worth, } \\
\text { Ih/kg }\end{array}$ & $\begin{array}{c}\text { Experimental } \\
\text { Sample Worth } \\
\text { Ih } / \mathrm{kg}\end{array}$ & $C / E$ \\
\hline$M B-06$ & ${ }^{239} \mathrm{Pu}$ & 170.31 & $133.09 \pm 2.01^{b}$ & 1.28 \\
\hline$P u-240-1 D$ & $240 \mathrm{PuO}_{2}$ & 26.12 & $19.23 \pm 0.32^{b}$ & 1.36 \\
\hline $\mathrm{Pu}-50$ & $241 \mathrm{PuO}_{2}$ & 149.55 & $123.78 \pm 10.02$ & 1.21 \\
\hline $\mathrm{Pu}-242-4-1$ & ${ }^{24}+2 \mathrm{Pu}$ & 26.19 & $18.50 \pm 0.21$ & 1.42 \\
\hline$U-233-1$ & $233 \mathrm{U}$ & 217.75 & $178.73 \pm 6.83^{b}$ & 1.22 \\
\hline $\mathrm{MB}-20$ & $235 U$ & 121.55 & $92.53 \pm 1.49^{b}$ & 1.31 \\
\hline$M B-23$ & $23^{8} \mathrm{U}$ & -7.76 & $6.33 \pm 1.24^{b}$ & 1.23 \\
\hline Th-2 & ${ }^{232} \mathrm{Th}$ & -16.46 & $-12.98 \pm 0.43^{b}$ & 1.27 \\
\hline$B-7$ & $10 \mathrm{~B}$ & -1989.99 & $-1781.40 \pm 30.66^{c}$ & 1.12 \\
\hline$B(L)$ & ${ }^{11} \mathrm{~B}$ & -473.90 & $-397.98 \pm 16.99$ & 1.19 \\
\hline $\mathrm{Eu}_{2} \mathrm{O}_{3}-3$ & $\mathrm{Eu}_{2} \mathrm{O}_{3}$ & -138.09 & $-122.95 \pm 1.59$ & 1.12 \\
\hline $\mathrm{BeO}-1$ & $\mathrm{BeO}$ & -22.32 & $-11.34 \pm 0.57$ & 1.97 \\
\hline $\mathrm{H}_{2} \mathrm{O}-1 \mathrm{~A}$ & $\mathrm{H}_{2} \mathrm{O}$ & -42.53 & $166.15 \pm 1.87^{\mathrm{d}}$ & -0.26 \\
\hline Poly-3 & $\mathrm{CH}_{2}$ & $-\quad 57.36$ & $174.72 \pm 26.29^{d}$ & -0.33 \\
\hline $\mathrm{CH}_{2}(\mathrm{~F})-1$ & $\mathrm{CH}_{2}$ (Foam) & $-\quad 57.51$ & $98.41 \pm 6.71$ & -0.58 \\
\hline$c(S)$ & C & -22.48 & $12.22 \pm 0.31$ & 1.84 \\
\hline $\mathrm{Fe}-1$ & $\mathrm{Fe}$ & 4.86 & $3.37 \pm 0.21$ & 1.44 \\
\hline $\mathrm{Fe}_{2} \mathrm{O}_{3}-2$ & $\mathrm{Fe}_{2} \mathrm{O}_{3}$ & 7.75 & $2.66 \pm 1.34$ & 2.92 \\
\hline 304 SST & SST & 5.52 & $3.84 \pm 0.47^{\mathrm{e}}$ & 1.44 \\
\hline
\end{tabular}

For cases where more than one sample size was measured, the sample of smallest mass is reported here. No sample size effect corrections were made.

${ }^{b}$ The data in Table $V$ indicates that the fissile and fertile material sample worths are not a strong function of sample size. At the most, a factor of only 3 to 4 percent need be applied to the heaviest samples.

${ }^{c}$ Expected magnitude of sample size correction for $10_{B}$ sample is about $3 \%$ from previous evaluation for ZPR-6 Assembly $7^{16}$

$\mathrm{d}_{\text {Table }} \mathrm{V}$ and Figure 8 show that the sample worths of the hydrogeneous materials vary rapidly with changing sample size probably because of multiple scattering effects. It should be noted that the calculations mispredicted the sign of the central worth for these materials.

e The sample for this measurement was the $10.667 \mathrm{~g} \mathrm{D}-1$ dumy capsule. 
TABLE XLIX. Isotop1c or Elemental Central Reactivity Worths In the GCFR Phase I Assembly;

Experimental and Calculated

\begin{tabular}{|c|c|c|c|c|}
\hline $\begin{array}{l}\text { Isotope } \\
\text { or Element }\end{array}$ & $\begin{array}{r}\text { Experime } \\
\text { Central } \\
\text { Ih } /\end{array}$ & $\begin{array}{l}\text { nental } \\
\text { Worth, } \\
\text { kg }\end{array}$ & $\begin{array}{l}\text { Calculated } \\
\text { Central Worth, } \\
\text { Ih } / \mathrm{kg}\end{array}$ & $C / E$ \\
\hline $2{ }^{39} \mathrm{Pu}$ & 137.01 & $\pm 2.07^{\mathrm{a}}$ & 175.25 & 1.28 \\
\hline $240 \mathrm{Pu}$ & 22.41 & $\pm 0.44^{\mathrm{a}}$ & 29.91 & 1.33 \\
\hline${ }^{241} \mathrm{Pu}$ & 197.62 & $\pm 16.30^{a}$ & 238.35 & 1.21 \\
\hline $242 \mathrm{Pu}$ & 16.74 & $\pm 0.22^{\mathrm{a}}$ & 23.98 & 1.43 \\
\hline${ }^{2}{ }^{3} 3 U$ & 178.73 & $\pm 6.83^{b}$ & 219.22 & 1.22 \\
\hline $234 \mathrm{U}$ & & - & 8.35 & - \\
\hline $235 \mathrm{U}$ & 99.64 & $\pm 1.60^{\mathrm{a}}$ & 130.85 & 1.31 \\
\hline $236 \mathrm{U}$ & & - & -13.06 & - \\
\hline $238_{U}$ & $-6.58=$ & $\pm 1.24^{\mathrm{a}}$ & $-\quad 8.09$ & 1.23 \\
\hline $2{ }^{32} \mathrm{Th}$ & $-12.98=$ & $\pm 0.43^{\mathrm{b}}$ & $-\quad 16.47$ & 1.27 \\
\hline $241 \mathrm{Am}$ & & - & 7.88 & - \\
\hline $10_{B}$ & -2045.70 & $\pm 35.99^{\mathrm{a}}$ & -2281.57 & 1.12 \\
\hline $11_{B}$ & & - & $-\quad 25.37$ & - \\
\hline${ }^{151} \mathrm{Eu}$ & & - & -194.55 & - \\
\hline $153 \mathrm{Eu}$ & & - & -123.75 & - \\
\hline${ }^{9} \mathrm{Be}$ & & - & $-\quad 36.21$ & - \\
\hline $\mathrm{H}$ & & - & -265.04 & - \\
\hline $\mathrm{C}$ & $-\quad 12.22$ & \pm 0.31 & $-\quad 22.49$ & 1.84 \\
\hline 0 & & - & -14.50 & - \\
\hline $\mathrm{Fe}$ & $3.37 \pm$ & \pm 0.21 & $-\quad 4.86$ & 1.44 \\
\hline $\mathrm{Cr}$ & & - & -6.06 & - \\
\hline Ni & & - & $-\quad 8.35$ & - \\
\hline $\operatorname{Mn}$ & & - & -11.62 & - \\
\hline
\end{tabular}

${ }^{a}$ The isotopic worths of the isotope of $235 \mathrm{U}$ and $238 \mathrm{U},{ }^{10} \mathrm{~B}$, and the $\mathrm{Pu}$ 1sotopes were determined from the experimental worths of enriched and depleted $U$, enriched and natural $\mathrm{B}$, and $\mathrm{Pu}$ samples respectively by solving simultaneous equations relating isotopic worths to sample worths. The sample worths of samples of smallest mass were used for cases where more than one sample size was meesured.

${ }^{b}$ For the Th and ${ }^{233} \mathrm{U}$ samples, the sample of smallest mass was reported. No sample size effect corrections were made. 
TABLE L. Experimencal Sma11 Sample Radial Reactivity Worth Traverses
in the GCFR Fhase I Assembly

\begin{tabular}{|c|c|c|c|c|c|c|c|c|c|c|c|c|c|}
\hline $\begin{array}{l}\text { Sample } \\
\text { I.D. }\end{array}$ & $\begin{array}{l}\text { Principle } \\
\text { Materaial } \\
\text { or Isotope }\end{array}$ & $\begin{array}{l}\text { Radial } \\
\text { Postion, } \\
\text { cem } \\
\end{array}$ & $\begin{array}{c}\text { Sample, Sample } \\
\text { Capsule and } \\
\text { SST Worth, } \\
\text { Ih } \\
\end{array}$ & $\begin{array}{l}\text { Sample } \\
\text { Capssule and } \\
\text { SST TS }^{\text {Mass, }} \\
\text { B } \\
\end{array}$ & $\begin{array}{l}\text { Dumy y } \\
\text { Capsule } \\
\text { I.D. }\end{array}$ & $\begin{array}{l}\text { Dumny Capsule } \\
\text { and } \mathrm{SST}^{\mathrm{a}} \\
\text { Worth, } \\
\mathrm{Ih}\end{array}$ & $\begin{array}{l}\text { Dunmy } \\
\text { Capssule and } \\
\text { SsT }^{2} \text { Mass, } \\
\mathbf{g} \\
\end{array}$ & $\begin{array}{l}\text { Ratio of Sample } \\
\text { Capsule Plus SST } \\
\text { Mass to Dummy } \\
\text { Capsule Plus } \\
\text { SST Mass }\end{array}$ & $\begin{array}{l}\text { Adjusted worth } \\
\text { of Dumay, } \\
\text { Ih }\end{array}$ & $\begin{array}{l}\text { Net Sample } \\
\text { Worth, b } \\
\text { Ih }\end{array}$ & $\begin{array}{l}\text { Sample } \\
\text { Mass, } \\
8\end{array}$ & $\begin{array}{c}\text { Spectfic } \\
\text { Worth, } \\
\text { Ih } / \mathrm{kg}\end{array}$ & Sample \\
\hline \multirow[t]{5}{*}{ MB -10} & ${ }^{239} \mathrm{Pu}$ & 0.0 & $1.035 \pm 0.011$ & 71.058 & MB-19 & $-0.298 \pm 0.005$ & 71.477 & 0.9941 & $-0.296=0.005$ & $1.331 \pm 0.012$ & 9.956 & $133.69 \pm$ & \pm 1.21 \\
\hline & & 27.62 & $0.900=0.010$ & & & $-0.188 \pm 0.003$ & & & $-0.187 \pm 0.003$ & $1.087 \pm 0.010$ & & $109.18 \pm$ & \pm 1.00 \\
\hline & & 55.25 & $0.609 \pm 0.006$ & & & $-0.028 \pm 0.002$ & & & $-0.028 \pm 0.002$ & $0.637 \pm 0.006$ & & $63.98=$ & $=0.60$ \\
\hline & & 82.87 & $0.320 \pm 0.007$ & & & $0.098 \pm 0.004$ & & & $0.097 \pm 0.004$ & $0.223 \div 0.008$ & & $22.40+$ & +0.81 \\
\hline & & 104.97 & $0.092+0.006$ & & & $0.031 \div 0.003$ & & & $0.031 \pm 0.003$ & $0.061 \pm 0.007$ & & $6.13 \neq$ & $=0.67$ \\
\hline \multirow[t]{3}{*}{ MB- 25} & ${ }^{238} 8_{U}$ & 0.0 & $-0.546 \pm 0.007$ & 71.156 & MB-19 & $-0.298 \pm 0.005$ & 71.477 & 0.9955 & $-0.297 \pm 0.005$ & $-0.249=0.009$ & 38.163 & $-6.52=$ & $=0.23$ \\
\hline & & .55 .25 & $-0.111=0.003$ & & & $-0.028 \pm 0.002$ & & & $-0.028 \pm 0.002$ & $-0.083 \pm 0.004$ & & $-2.17 \pm$ & 0.09 \\
\hline & & 104.97 & $0.042=0.004$ & & & $0.031 \pm 0.003$ & & & $0.031 \pm 0.003$ & $0.011 \pm 0.005$ & & $0.288 \pm$ & 0.131 \\
\hline $\mathrm{CH}_{2}(F)-1$ & $\mathrm{CH}_{2}\langle$ (Foan $)$ & 0.0 & $-0.156 \pm 0.008$ & 57.580 & Hold. No. 9 & $-0.241 \pm 0.005$ & 57.579 & 1.0000 & $-0.241=0.005$ & $0.085 \pm 0.009$ & 0.843 & $100.83 \pm$ & $=11.19$ \\
\hline \multirow[t]{7}{*}{$\mathrm{CH}_{2}(\mathrm{~F})-2 \mathrm{~A}$} & $\mathrm{CH}_{2}$ (Foarn) & 0.0 & $0.088=0.003$ & - & - & - & - & - & - & $0.088 \div 0.003$ & 0.910 & $96.70 \pm$ & $=3.30$ \\
\hline & & 27.62 & $0.078 \pm 0.005$ & - & - & - & - & - & - & $0.078 \pm 0.005$ & & $85.71 \pm$ & 5.49 \\
\hline & & 55.25 & $0.076 \pm 0.003$ & - & - & - & - & - & - & $0.076 \pm 0.003$ & & $83.52 \div$ & 3.30 \\
\hline & & 66.29 & $0.046=0.004$ & - & - & - & - & - & - & $0.046 \pm 0.004$ & & $50.55 \pm$ & 4.40 \\
\hline & & 82.87 & $0.025 \pm 0.004$ & - & - & - & - & - & - & $0.025 \pm 0.004$ & & $27.47 \pm$ & 4.40 \\
\hline & & 93.92 & $-0.013 \neq 0.004$ & - & - & - & - & - & - & $-0.013 \pm 0.004$ & & $-14.29 \pm$ & 4.40 \\
\hline & & 104.97 & $-0.001=0.006$ & - & - & - & - & - & - & $-0.001 \pm 0.006$ & & $-1.10 \pm$ & $\quad 6.59$ \\
\hline $\mathrm{CH}_{2}(\mathrm{~F})-3 \mathrm{~A}$ & $\mathrm{CH}_{2}$ (Fo am) & 0.0 & $0.036 \pm 0.004$ & - & - & - & - & - & - & $-0.036 \pm 0.004$ & 0.448 & $80.36 \pm$ & +8.93 \\
\hline MB-19 and & 304 SST & 0.0 & $-0.298 \pm 0.005$ & - & - & - & - & - & - & $-0.298 \pm 0.005$ & 71.477 & $-4.17=$ & 0.07 \\
\hline \multirow{4}{*}{$\begin{array}{l}\text { Capsule Holder } \\
\text { No. } 4\end{array}$} & & 27.62 & $-0.188 \pm 0.003$ & - & - & - & - & - & - & $-0.188 \div 0.003$ & & $-2.63 \pm$ & 0.04 \\
\hline & & 55.25 & $-0.028=0.002$ & - & - & - & - & - & - & $-0.028 \mp 0.002$ & & $-0.392=$ & $=0.028$ \\
\hline & & 82.87 & $0.098 \pm 0.004$ & - & - & - & - & - & - & $0.098 \pm 0.004$ & & $1.37 \pm$ & 0.06 \\
\hline & & 104.97 & $0.031=0.003$ & - & - & - & - & - & - & $0.031 \pm 0.003$ & & $0.434=$ & $=0.042$ \\
\hline $\begin{array}{l}\text { Capsule Holcer } \\
\text { No. } 9\end{array}$ & $304 \mathrm{SST}$ & 0.0 & $-0.241 \pm 0.005$ & - & - & - & - & - & & $-0.241 \pm 0.005$ & 57.579 & $-4.19=$ & 0.09 \\
\hline
\end{tabular}

SST refers to statnless steel capsule holders and stainless steel shims or spacers if used.

$b_{\mathbb{N} \text { o }}$ sample aize effect corrections have been usde. 
TABLE LI. Comparison of Experimental and Calculated Small Sample Radial Reactivity Worth Traverses in the GCFR Phase I Assembly

\begin{tabular}{|c|c|c|c|c|c|c|c|}
\hline \multirow{2}{*}{$\frac{\text { Sample }}{\mathrm{MB}-10}$} & \multirow{2}{*}{$\frac{\begin{array}{c}\text { Radial } \\
\text { Position } \\
\mathrm{cm}\end{array}}{0.0}$} & \multicolumn{2}{|c|}{$\begin{array}{c}\text { Experimental } \\
\text { Worth } \\
\text { Ih } / \mathrm{kg}\end{array}$} & $\begin{array}{l}\text { Normalized } \\
\text { Experimental } \\
\text { Worth }\end{array}$ & \multirow{2}{*}{$\frac{\begin{array}{c}\text { Calculated } \\
\text { worth } \\
\text { Ih/kg }\end{array}}{170.28}$} & \multirow{2}{*}{$\begin{array}{c}\text { Normallzed } \\
\begin{array}{c}\text { Calculated } \\
\text { Worth }\end{array} \\
1.000\end{array}$} & \multirow{2}{*}{$\frac{C / E}{1.274}$} \\
\hline & & 133.69 & \pm 1.21 & $1.000 \pm 0.013$ & & & \\
\hline \multirow[t]{6}{*}{$\left({ }^{239} \mathrm{Pu}\right)$} & 27.62 & 109.18 & \pm 1.00 & $0.817 \pm 0.011$ & 142.73 & 0.838 & 1.307 \\
\hline & 55.25 & 63.98 & \pm 0.60 & $0.479 \pm 0.006$ & 80.13 & 0.471 & 1.252 \\
\hline & 66.29 & & - & - & 54.83 & 0.322 & - \\
\hline & 82.87 & 22.40 & \pm 0.81 & $0.168 \pm 0.006$ & 24.65 & 0.145 & 1.100 \\
\hline & 93.92 & & - & - & 11.23 & 0.066 & - \\
\hline & 104.97 & 6.13 & \pm 0.67 & $0.046 \pm 0.005$ & 2.85 & 0.017 & 0.465 \\
\hline M8- 25 & 0.0 & -6.52 & \pm 0.23 & $-1.000 \pm 0.050$ & -7.763 & -1.000 & 1.191 \\
\hline \multirow[t]{6}{*}{$\left({ }^{238} U\right)$} & 27.62 & & - & - & - & - & - \\
\hline & 55.25 & -2.17 & \pm 0.09 & $-0.333 \pm 0.018$ & -2.952 & -0.380 & 1.360 \\
\hline & 66.29 & & - & - & -1.659 & -0.214 & - \\
\hline & 82.87 & & - & - & -0.206 & -0.027 & - \\
\hline & 93.92 & & - & - & 0.448 & 0.058 & - \\
\hline & 104.97 & 0.288 & \pm 0.131 & $0.044 \pm 0.021$ & 0.208 & 0.027 & 0.722 \\
\hline $\mathrm{CH}_{2}(\mathrm{~F})-2 \mathrm{~A}$ & 0.0 & 96.70 & \pm 3.30 & $1.000 \pm 0.048$ & -58.035 & -1.000 & -0.600 \\
\hline (Poly & 27.62 & 85.71 & \pm 5.49 & $0.886 \pm 0.064$ & -41.712 & -0.719 & -0.487 \\
\hline \multirow[t]{5}{*}{ Foam) } & 55.25 & 83.52 & \pm 3.30 & $0.862 \pm 0.045$ & -5.994 & -0.103 & -0.072 \\
\hline & 66.29 & 50.55 & \pm 4.40 & $0.523 \pm 0.049$ & 5.159 & 0.089 & 0.102 \\
\hline & 82.87 & 27.47 & \pm 4.40 & $0.284 \pm 0.047$ & 0.472 & 0.008 & 0.017 \\
\hline & 93.92 & -14.29 & \pm 4.40 & $-0.148 \pm 0.046$ & -27.428 & -0.473 & 1.919 \\
\hline & 104.97 & -1.10 & \pm 6.59 & $-0.011 \pm 0.066$ & -8.878 & -0.153 & 8.071 \\
\hline$M B-19$ and & 0.0 & -4.17 & \pm 0.07 & $-1.000 \pm 0.024$ & -5.568 & -1.000 & 1.335 \\
\hline Capsule Holder No. 4 & 27.62 & -2.63 & \pm 0.04 & $-0.631 \pm 0.014$ & -4.235 & -0.761 & 1.610 \\
\hline \multirow[t]{5}{*}{$(304 \mathrm{SST})$} & 55.25 & -0.392 & \pm 0.028 & $-0.094 \pm 0.007$ & -1.327 & -0.238 & 3.385 \\
\hline & 66.29 & & - & - & -0.229 & -0.041 & - \\
\hline & 82.87 & 1.37 & \pm 0.06 & $0.329 \pm 0.015$ & 0.928 & 0.167 & 0.677 \\
\hline & 93.92 & & - & - & 1.047 & 0.188 & - \\
\hline & 104.97 & 0.434 & \pm 0.042 & $0.104 \pm 0.010$ & 0.510 & 0.092 & 1.175 \\
\hline
\end{tabular}

No sample size effect corrections have been made for the experimental worths.

TABLE LII. Isotopic Fission Rates at the Center of the GCFR Phase I Assembly

\begin{tabular}{|c|c|c|c|c|c|}
\hline Isotope & $\begin{array}{c}\overline{\mathrm{R}}_{\mathrm{f} f} \\
\text { Experimental } \\
\text { Isotopic Fission } \\
\text { Rate at Power } \\
\text { Level of } \\
\text { Measurement } \\
\text { (fissions/ } / \mathrm{gm-sec} \text { ) }\end{array}$ & $\begin{array}{l}\text { Experimental Isotopic } \\
\text { Fission Rate at Power } \\
\text { Level of Interest } \\
\text { (fissions/gm-gec) }\end{array}$ & $\begin{array}{l}\quad \mathrm{N}_{\mathrm{f}} \\
\text { Isotopic Atom } \\
\text { Concentration } \\
\text { at Core } \\
\text { Center } \\
\text { (atom/cm- } \\
\text { barn) }\end{array}$ & $\frac{\mathrm{N}_{f} \cdot \mathrm{A}_{1}}{(\mathrm{gm} / \mathrm{cc})}$ & $\begin{array}{c}R_{\mathrm{fj}} \\
(\text { fission } / \mathrm{sec}-\mathrm{cc}) \\
\left(\times 10^{6}\right)\end{array}$ \\
\hline $235 \mathrm{U}$ & $2.9498 \pm 0.0324$ & $1.2545 \pm 0.0137 \times 10^{7}$ & 0.0000091 & 0.0035526 & \pm 0.00049 \\
\hline $238 \mathrm{U}$ & $0.08012 \pm 0.00064$ & $0.34074 \pm 0.00272 \times 10^{6}$ & 0.0041529 & 1.642025 & $0.55956 \pm 0.00441$ \\
\hline $239 \mathrm{Pu}$ & $2.8886 \pm 0.0146$ & $1.22848 \pm 0.00622 \times 10^{7}$ & 0.0008905 & 0.353578 & \pm 0.0220 \\
\hline $240 \mathrm{Pu}$ & $0.6830 \pm 0.0065$ & $0.29047 \pm 0.00274 \times 10^{7}$ & 0.0001182 & 0.047129 & $0.13689 \pm 0.00129$ \\
\hline $241 \mathrm{Pu}^{\mathrm{a}}$ & - & - & 0.0000118 & - & $0.075204 \pm 1 \%^{\mathrm{a}}$ \\
\hline${ }^{242} \mathrm{Pu}^{\mathrm{a}}$ & - & - & 0.0000016 & - & $0.015219 \pm 1 \%^{a}$ \\
\hline
\end{tabular}

${ }^{a}$ Calculated values. The uncertalnites were assigned based upon earlier estimations. 
TABLE LIII. Parameters Used in the Determination of the Perturbation Denominator

\begin{tabular}{|c|c|c|c|c|c|}
\hline \multicolumn{2}{|r|}{ Parametér } & \multirow{2}{*}{ Symbol } & \multirow{2}{*}{$\begin{array}{l}\text { Un1ts } \\
\text { n/sec }\end{array}$} & \multicolumn{2}{|c|}{$\begin{array}{l}\text { Numerical } \\
\text { Value }\end{array}$} \\
\hline 1. & $\begin{array}{l}{ }^{252} \mathrm{Cf} \text { source strength at } \\
\text { the time of the experiment }\end{array}$ & & & 1.473 & $\pm 0.010 \times 10^{7}$ \\
\hline 2. & $\begin{array}{l}\text { Reactivity worth of }{ }^{252} \mathrm{Cf} \\
\text { at power level of interest } \\
\text { at core center }\end{array}$ & $\rho^{\prime}(\mathrm{Cf})$ & Ih & 0.0821 & \pm 0.0006 \\
\hline 3. & $\begin{array}{l}\text { The average number of } \\
\text { neut rons emitted per } \\
\text { fission (average over } \\
\text { lsotopes) }\end{array}$ & $\vec{v}$ & n/fission & 2.931 & \pm 0.0029 \\
\hline 4. & $\begin{array}{l}\text { The total fission rate at } \\
\text { core center summed over } \\
\text { all fissionable isotopes }\end{array}$ & $R_{f}$ & ftssion/sec-cc & 5.175 & $\times 10^{6} \pm 2 \%^{a}$ \\
\hline 5. & $\begin{array}{l}\text { Reactivity conversion } \\
\text { factor (calculated) }\end{array}$ & Ih $/ \Delta \mathrm{k} / \mathrm{k}$ & - & 0.9694 & $\times 10^{5}$ \\
\hline 6. & Normalized Pert. Denominator & & & & \\
\hline & a) Experimental & - & - & 21.834 & \pm 0.173 \\
\hline & b) Calculated & - & - & 8.919 & \\
\hline & c) $\mathrm{C} / \mathrm{E}$ & - & - & 0.75 & \\
\hline
\end{tabular}

$a_{\text {Estimated uncertainty. }}$

TABLE LIV. A Comaprison of the C/E Values for Isotopic Central Worths

\begin{tabular}{llcc}
\hline Isotope & $\begin{array}{c}\text { C/E } \\
\text { (from FoP } \\
\text { calculation) }\end{array}$ & $\begin{array}{c}\text { C/E Adjusted } \\
\text { by the C/E of } \\
\text { the Perturbation } \\
\text { Denominator }\end{array}$ & $\begin{array}{c}\text { C/E Adjusted } \\
\text { by.the C/E of } \\
\text { 239Pu worth }\end{array}$ \\
\hline${ }^{239} \mathrm{Pu}$ & 1.28 & 0.96 & - \\
$240 \mathrm{Pu}$ & 1.33 & 1.00 & 1.04 \\
$241 \mathrm{Pu}$ & 1.21 & 0.91 & 0.95 \\
$242 \mathrm{Pu}$ & 1.43 & 1.07 & 1.12 \\
$23{ }^{3} \mathrm{U}$ & 1.22 & 0.92 & 0.95 \\
$235 \mathrm{U}$ & 1.31 & 0.98 & 1.02 \\
$23{ }^{2} \mathrm{U}$ & 1.23 & 0.92 & 0.96 \\
$232 \mathrm{Th}$ & 1.27 & 0.95 & 0.99 \\
$10 \mathrm{~B}$ & 1.12 & 0.84 & 0.88 \\
$\mathrm{C}$ & 1.84 & 1.38 & 1.44 \\
$\mathrm{Fe}$ & 1.44 & 1.08 & 1.13 \\
\hline
\end{tabular}


TABLE LV. A Comparison of Reactivity Worth Results For GCFR Phase I Assembly

and ZPR- 6 Assembly 7

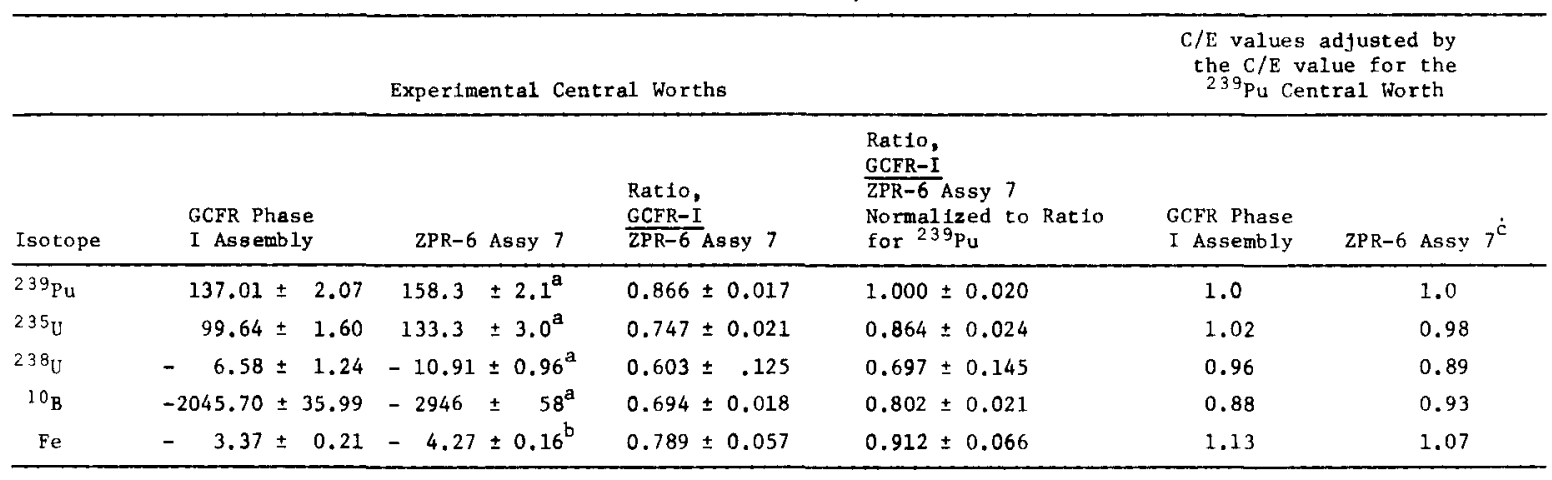

Note: The experimental values for 2 PR- 6 Assembly 7 used sample size correction factors which were experimentally determined. No such corrections were made for the GCFR Phase I Assembly results.

a values obtained from Ref. 16.

balue obtained from Ref. 14 .

${ }^{c}$ Calculated results obtained from Ref. 19.

TABLE LVI. VMM-Fitted Amplitudes and Decay Constants $\left(a_{1}, \lambda_{i}\right)$

\begin{tabular}{|c|c|c|c|c|}
\hline Group & $\begin{array}{c}\text { Fitted } \\
\text { Relative Yields } \\
a_{i}\end{array}$ & $\begin{array}{c}\text { F1tted } \\
\text { Decay Constants } \\
\lambda_{1}\left(\sec ^{-1}\right)\end{array}$ & $\begin{array}{c}\text { Final } \\
\text { Subcriticality } \\
(\$)\end{array}$ & $\sum \frac{a_{1}}{\lambda_{1}}$ \\
\hline 1 & 0.02304 & 0.01499 & - & - \\
\hline 2 & 0.16102 & 0.02657 & - & - \\
\hline 3 & 0.19521 & 0.08484 & 7.18922 & 11.209 \\
\hline 4 & 0.39371 & 0.33671 & - & - \\
\hline 5 & 0.17133 & 1.35210 & - & - \\
\hline 6 & 0.05569 & 3.72012 & - & - \\
\hline 1 & 0.02242 & 0.01300 & - & - \\
\hline 2 & 0.20238 & 0.03109 & - & - \\
\hline 3 & 0.17152 & 0.12378 & 6.38129 & 10.831 \\
\hline 4 & 0.36921 & 0.34492 & - & - \\
\hline 5 & 0.17621 & 1.41050 & - & - \\
\hline 6 & 0.05827 & 3.72494 & - & - \\
\hline 1 & 0.02231 & 0.01369 & - & - \\
\hline 2 & 0.18869 & 0.02916 & - & - \\
\hline 3 & 0.17977 & 0.10553 & 6.11117 & 11.048 \\
\hline 4 & 0.38088 & 0.34477 & - & - \\
\hline 5 & 0.17132 & 1.37197 & - & - \\
\hline 6 & 0.05703 & 3.73796 & - & - \\
\hline 1 & 0.02024 & 0.01179 & - & - \\
\hline 2 & 0.21465 & 0.03047 & - & - \\
\hline 3 & 0.17243 & 0.12460 & 6.95199 & 11.348 \\
\hline 4 & 0.36747 & 0.34541 & - & - \\
\hline 5 & 0.16916 & 1.37242 & - & - \\
\hline 6 & 0.05605 & 3.73289 & - & - \\
\hline 1 & 0.01655 & 0.01143 & - & - \\
\hline 2 & 0.20629 & 0.02964 & - & - \\
\hline 3 & 0.17477 & 0.11992 & 6.001713 & 11.046 \\
\hline 4 & 0.36233 & 0.34988 & - & - \\
\hline 5 & 0.18154 & 1.41368 & - & - \\
\hline 6 & 0.05851 & 3.70236 & - & - \\
\hline
\end{tabular}


TABLE LVII. Compartson of Calculated and Collapsed Set of Measured Values for Relative Yields and Decay Constants

\begin{tabular}{|c|c|c|c|c|c|c|}
\hline Group & $\begin{array}{c}\text { Keepin } \\
\text { Calculated } \\
\text { Relative Yield }\end{array}$ & $\begin{array}{c}\text { Calculated } \\
\text { Decay Constant } \\
\left(\sec ^{-1}\right)\end{array}$ & $\begin{array}{c}\text { ENDF/B-4 } \\
\text { Calculated } \\
\text { Relative Yield }\end{array}$ & $\begin{array}{c}\text { Calculated } \\
\text { Decay Constant } \\
\left(\sec ^{-1}\right)\end{array}$ & $\begin{array}{c}\text { Derived } \\
\text { Relative Yield }\end{array}$ & $\begin{array}{c}\text { Derived } \\
\text { Decay Constant } \\
\left(\sec ^{-1}\right)\end{array}$ \\
\hline 1 & 0.02608 & 0.0130 & 0.02528 & 0.0130 & 0.02091 & 0.01298 \\
\hline 2 & 0.21111 & 0.0314 & 0.21973 & 0.0314 & 0.19461 & 0.02945 \\
\hline 3 & 0.18796 & 0.1360 & 0.18728 & 0.1350 & 0.17874 & 0.10857 \\
\hline 4 & 0.35947 & 0.3440 & 0.35540 & 0.3450 & 0.37472 & 0.34417 \\
\hline 5 & 0.16207 & 1.3600 & 0.16008 & 1.3600 & 0.17391 & 1.38423 \\
\hline 6 & 0.05331 & 3.7200 & 0.05223 & 3.7200 & 0.05711 & 3.72343 \\
\hline
\end{tabular}

TABLE LVIII. Variation of $\tau \sigma^{2}$ Versus $\tau$ as Multiples of the Basic Sampling Interval, DT $=0.1 \mathrm{Sec}$

\begin{tabular}{lccc}
$\tau(\mathrm{sec})$ & $\begin{array}{c}\text { Delayed-Neutron } \\
\text { Correction Factor }\end{array}$ & $\begin{array}{c}\tau \sigma^{2} \times 10^{-5} \\
\text { (Corrected) }\end{array}$ & $\begin{array}{c}\% \text { Statistical } \\
\text { Error in } \tau \sigma^{2}\end{array}$ \\
\hline 0.1 & 1.000 & 0.1869 & 0.72 \\
0.2 & 1.006 & 0.1940 & 0.63 \\
0.4 & 1.021 & 0.2048 & 1.00 \\
0.8 & 1.057 & 0.2070 & 1.40 \\
1.6 & 1.129 & 0.2106 & 2.10 \\
\hline
\end{tabular}

TABLE LIX. Description of Activation Foils

\begin{tabular}{|c|c|c|c|c|}
\hline $\begin{array}{l}\text { Target } \\
\text { Isotope }\end{array}$ & Material and Form & $\begin{array}{c}\text { Dlameter, } \\
\text { in. }\end{array}$ & $\begin{array}{l}\text { Nominal } \\
\text { Thickness } \\
\text { m1ls }\end{array}$ & $\begin{array}{l}\text { Approximate } \\
\text { Welght of Target } \\
\text { Element, } g\end{array}$ \\
\hline U & Enriched Urantum ${ }^{a}$ & 0.500 & 4.4 & 0.25 \\
\hline $\mathbf{U}$ & Depleted Uranium & 0.500 & 5.5 & 0.31 \\
\hline $\mathrm{Pu}$ & $\begin{array}{l}\text { Pu-Al alloy, clad } \\
\text { in 5-mil aluminum }\end{array}$ & 0.425 & 0.4 & 0.02 \\
\hline${ }^{a}$ Isoto & composttion of en & d uranium & $\begin{array}{l}234 \mathrm{U}: \\
235 \mathrm{U}: \\
236 \mathrm{U}: \\
238_{\mathrm{U}}:\end{array}$ & $\begin{array}{l}1.03 \mathrm{wt} \% \\
93.07 \\
0.27 \\
5.63\end{array}$ \\
\hline${ }^{b}$ Isotopi & composition of depl & urantum fo: & $\begin{array}{l}235 \mathrm{U}: \\
236 \mathrm{U}: \\
238_{\mathrm{U}}:\end{array}$ & $\begin{array}{c}0.215 \\
0.005 \\
99.78\end{array}$ \\
\hline $\begin{array}{l}{ }^{c} \text { Isotopi } \\
\text { alloy w }\end{array}$ & $\begin{array}{l}\text { composition of the } \\
\text { ch } 1 \mathrm{~s} 98.0 \text { wt } \% \mathrm{Pu} \text { at }\end{array}$ & $\begin{array}{l}\text { nium foll } \\
3 \text { wt } \% \text { Al: }\end{array}$ & $\begin{array}{l}239 \mathrm{Pu}: \\
240 \mathrm{Pu}: \\
241_{\mathrm{Pu}} \\
242 \mathrm{Pu}:\end{array}$ & $\begin{array}{l}94.98 \\
4.7 \\
0.303 \\
0.019\end{array}$ \\
\hline
\end{tabular}

dAluminum cladding is 0.5 in.-diameter and 5 mils thick. 
TABLE LX. Unit Cell Measurement Locations

\begin{tabular}{llc}
\hline $\begin{array}{c}\text { Matrix } \\
\text { Position }\end{array}$ & Region & $\begin{array}{c}\text { Distance from } \\
\text { Axial Midplane } \\
\text { (in.) }\end{array}$ \\
\hline S22/22 & Core & $0-2$ \\
S22/22 & Core & $22-24$ \\
S22/22 & Ax. Blanket & $24-26$ \\
$522 / 22$ & Ax. Blanket & $34-36$ \\
S40/22 & Core & $0-2$ \\
S4I/22 & Rad. Blanket & $0-2$ \\
\hline
\end{tabular}

TABLE LXI. Cel1-Averaged Reaction Rate to Mapping Foll Reaction Rate Ratios

\begin{tabular}{|c|c|c|c|c|c|c|}
\hline \multirow[b]{2}{*}{$\begin{array}{l}\text { Matrix } \\
\text { Position }\end{array}$} & \multirow{2}{*}{$\begin{array}{l}\text { Axial Distance } \\
\text { From Midplane } \\
\text { (in.) }\end{array}$} & \multirow[b]{2}{*}{ Region } & \multicolumn{4}{|c|}{$\frac{\text { (Reaction Rate) Cell Avg. }}{\text { (Reaction Rate) }}$} \\
\hline & & & ${ }^{239} \mathrm{Pu}(\mathrm{n}, \mathrm{f})$ & ${ }^{235} U(n, f)$ & $2{ }^{38} U(n, f)$ & $238_{U}(n, \gamma)$ \\
\hline $\mathrm{S} 22 / 22$ & $0-2$ & Core & 1.0018 & 1.0014 & 0.9687 & 0.9852 \\
\hline $\mathrm{S} 22 / 22$ & $22-24$ & Core & 1.0041 & 1.0041 & 0.9638 & 0.9805 \\
\hline $\mathrm{s} 22 / 22$ & $24-26$ & $A x, B 1$ & 1.0 & 1.0100 & 1.0279 & 1.0322 \\
\hline $\mathrm{S} 22 / 22$ & $34-36$ & $A x . B 1$ & 1.0 & 1.0110 & 1.0557 & 1.0527 \\
\hline $\mathrm{S} 39 / 22$ & $0-2$ & Core & 0.9995 & 0.9987 & 0.9815 & 0.9559 \\
\hline $540 / 22$ & $0-2$ & Rad. 81. & 1.0 & 1.0014 & 1.0137 & 1.0177 \\
\hline
\end{tabular}

TABLE LXII. Average Unit Cell Correction Factors

\begin{tabular}{lcccc}
\hline & \multicolumn{2}{c}{ Radial } & Traverses & \multicolumn{2}{c}{ Axial } & Traverses \\
\cline { 2 - 5 } $\begin{array}{l}\text { Reaction } \\
\text { Rate }\end{array}$ & Core & B1anket & Core & Blanket \\
\hline $\mathrm{f}^{25}$ & 1.0000 & 1.0014 & 1.0028 & 1.0105 \\
$\mathrm{f}^{28}$ & 0.9751 & 1.0137 & 0.9662 & 1.0418 \\
$\mathrm{c}^{28}$ & 0.9706 & 1.0177 & 0.9828 & 1.0424 \\
$\mathrm{f}^{49}$ & 1.0006 & 1.0000 & 1.0030 & 1.0000 \\
\hline
\end{tabular}

TARLE LXIII. Reactivity Worths of Steps Toward the Establishment of the Stationary Half of Checkerboard Quadrant

\begin{tabular}{|c|c|c|c|c|c|}
\hline Watr:/Day & $\begin{array}{l}\text { Loading } \\
\text { No. }\end{array}$ & $\begin{array}{l}\text { Measurement: } \\
\text { Numb } \leqslant \text { of } \\
\text { Drawers Changed } \\
\text { to the } \\
\text { Horizontul } \\
\text { plate Structure }\end{array}$ & $\begin{array}{l}\text { Excess Ruactivity } \\
\left.\text { (Corrected to } 25^{\circ} \mathrm{C}\right) \\
\text { Ih }\end{array}$ & $\begin{array}{l}\text { Measured } \\
\text { Reactivity } \\
\text { Change, b } \\
\text { Ih }\end{array}$ & $\begin{array}{c}\text { Calculated } \\
\text { Reactivity } \\
\text { Change, } \\
\text { Ih }\end{array}$ \\
\hline $6-5-75 / 156$ & 63 & Reference & $-238.8 \pm 2.1$ & - & - \\
\hline $6-10-75 / 161$ & 64 & 48 & $-228.3 \pm 1.1$ & $11.3 \pm 3.4$ & - \\
\hline $6-12-75 / 163$ & 65 & 54 & $-216.6+2.7$ & $23.3 \pm 4.0$ & 111 \\
\hline
\end{tabular}


TABLE LXIV. Comparison of Experimental and Calculated Uranium Fission Rates, $0^{\circ}$ Radial Traverse

\begin{tabular}{|c|c|c|c|c|c|c|c|c|}
\hline \multirow[b]{2}{*}{ Row/Col } & \multicolumn{2}{|c|}{$\begin{array}{l}\text { Experimental Uranium } \\
\text { Fission Rate }\end{array}$} & \multicolumn{2}{|c|}{$2{ }^{35}$ U Fission Rate } & \multicolumn{4}{|c|}{${ }^{238}$ U F1ssion Rate ${ }^{b}$} \\
\hline & $\begin{array}{l}\text { Enriched } \\
\left(\times 10^{6}\right)\end{array}$ & $\begin{array}{l}\text { Deplated } \\
\left(\times 10^{5}\right)\end{array}$ & $\begin{array}{l}\text { Exper1mental } \\
\qquad\left(\times 10^{6}\right)\end{array}$ & $\begin{array}{c}\text { Calculated } \\
\left(\times 10^{6}\right)\end{array}$ & $C / E$ & $\begin{array}{l}\text { Exper 1mental } \\
\left(\times 10^{5}\right)\end{array}$ & $\begin{array}{l}\text { Calculated } \\
\left(\times 10^{5}\right)\end{array}$ & $\mathrm{C} / \mathrm{E}$ \\
\hline $23 / 23$ & 4.521 & 1.414 & 4.838 & 4.868 & 1.006 & 1.313 & 1.322 & 1.007 \\
\hline $23 / 24$ & 4.516 & 1.415 & 4.832 & 4.852 & 1.004 & 1.314 & 1.318 & 1.003 \\
\hline $23 / 25$ & 4.482 & 1.426 & 4.796 & 4.802 & 1.001 & 1.326 & 1.304 & 0.983 \\
\hline $23 / 26$ & 4.420 & 1.396 & 4.729 & 4.720 & 0.998 & 1.297 & 1.282 & 0.988 \\
\hline $23 / 27$ & 4.273 & 1.369 & 4.572 & 4.607 & 1.008 & 1.273 & 1.251 & 0.983 \\
\hline $23 / 28$ & 4.211 & 1.300 & 4.506 & 4.464 & 0.991 & 1.206 & 1.212 & 1.005 \\
\hline $23 / 29$ & 4.014 & 1.251 & 4.295 & 4.293 & 1.000 & 1.161 & 1.165 & 1.003 \\
\hline $23 / 30$ & 3.856 & 1.207 & 4.126 & 4.096 & 0.993 & 1.121 & 1.111 & 0.991 \\
\hline $23 / 31$ & 3.659 & 1.137 & 3.915 & 3.875 & 0.990 & 1.055 & 1.049 & 0.994 \\
\hline $23 / 32$ & 3.445 & 1.070 & 3.686 & 3.634 & 0.986 & 0.9928 & 0.9819 & 0.989 \\
\hline $23 / 33$ & 3.213 & 0.9947 & 3.438 & 3.375 & 0.982 & 0.9227 & 0.9084 & 0.985 \\
\hline $23 / 34$ & 2.970 & 0.9213 & 3.178 & 3.103 & 0.976 & 0.8547 & 0.8297 & 0.971 \\
\hline $23 / 35$ & 2.709 & 0.8538 & 2.899 & 2.822 & 0.973 & 0.7931 & 0.7458 & 0.940 \\
\hline $23 / 36$ & 2.466 & 0.7384 & 2.639 & 2.537 & 0.961 & 0.6830 & 0.6571 & 0.962 \\
\hline $23 / 37$ & 2.197 & 0.6479 & 2.352 & 2.253 & 0.958 & 0.5986 & 0.5628 & 0.940 \\
\hline $23 / 38$ & 1.944 & 0.5411 & 2.081 & 1.978 & 0.951 & 0.4973 & 0.4607 & 0.926 \\
\hline $23 / 39$ & 1.722 & 0.4311 & 1.844 & 1.725 & 0.935 & 0.3922 & 0.3470 & 0.884 \\
\hline $23 / 40$ & 1.464 & 0.2391 & 1.570 & 1.471 & 0.937 & 0.2057 & 0.2166 & 1.053 \\
\hline $23 / 41$ & 1.218 & 0.1437 & 1.307 & 1.200 & 0.918 & 0.1158 & 0.1207 & 1.042 \\
\hline $23 / 42$ & 0.9635 & 0.09215 & 1.034 & 0.9276 & 0.897 & 0.0701 & 0.0699 & 0.997 \\
\hline $23 / 43$ & 0.7515 & 0.05793 & 0.8068 & 0.6871 & 0.852 & 0.0406 & 0.0402 & 0.990 \\
\hline $23 / 44$ & 0.5828 & 0.03684 & 0.6258 & 0.5011 & 0.801 & 0.0233 & 0.0237 & 1.017 \\
\hline
\end{tabular}

Tission/sec/g of foil material at a power level of approximately 100 watts.

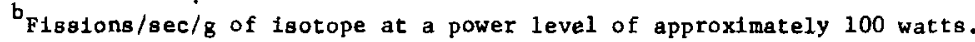

TABLE LXV. Comparison of Experimental and Calculated Uranium F1salon Rates, $45^{\circ}$ Radial Traverse

\begin{tabular}{|c|c|c|c|c|c|c|c|c|}
\hline \multirow[b]{2}{*}{ Row/Col } & \multicolumn{2}{|c|}{$\begin{array}{l}\text { Experimental Uranium } \\
\text { Fission Rate }\end{array}$} & \multicolumn{2}{|c|}{235 U Fission Rate } & \multicolumn{4}{|c|}{${ }^{238} \mathrm{U}$ Fission Rate ${ }^{b}$} \\
\hline & $\begin{array}{c}\text { Enriched } \\
\left(\times 10^{6}\right)\end{array}$ & $\begin{array}{l}\text { Depleted } \\
\left(\times 10^{5}\right)\end{array}$ & $\begin{array}{l}\text { Experimental } \\
\left(\times 10^{6}\right)\end{array}$ & $\begin{array}{l}\text { Calculated } \\
\left(\times 10^{6}\right)\end{array}$ & $C / E$ & $\begin{array}{l}\text { Experimental } \\
\qquad\left(\times 10^{5}\right)\end{array}$ & $\begin{array}{l}\text { Calculated } \\
\left(\times 10^{5}\right)\end{array}$ & $\mathrm{C} / \mathrm{E}$ \\
\hline $23 / 23$ & 4.521 & 1.414 & 4.838 & 4.868 & 1.006 & 1.313 & 1.322 & 1.007 \\
\hline $24 / 24$ & 4.503 & 1.402 & 4.819 & 4.836 & 1.004 & 1.301 & 1.313 & 1.009 \\
\hline $25 / 25$ & 4.440 & 1.396 & 4.751 & 4.740 & 0.998 & 1.297 & 1.287 & 0.992 \\
\hline $26 / 26$ & 4.325 & 1.340 & 4.628 & 4.583 & 0.990 & 1.243 & 1.244 & 1.001 \\
\hline $27 / 27$ & 4.131 & 1.279 & 4.421 & 4.367 & 0.988 & 1.186 & 1.185 & 0.999 \\
\hline $28 / 28$ & 3.882 & 1.215 & 4.154 & 4.099 & 0.988 & 1.128 & 1.111 & 0.985 \\
\hline $29 / 29$ & 3.602 & 1.126 & 3.854 & 3.784 & 0.982 & 1.045 & 1.022 & 0.978 \\
\hline $30 / 30$ & 3.276 & 1.013 & 3.506 & 3.431 & 0.979 & 0.9396 & 0.9215 & 0.981 \\
\hline $31 / 31$ & 2.932 & 0.8995 & 3.138 & 3.050 & 0.972 & 0.8337 & 0.8082 & 0.969 \\
\hline $32 / 32$ & 2.560 & 0.7760 & 2.740 & 2.654 & 0.969 & 0.7186 & 0.6826 & 0.950 \\
\hline $33 / 33$ & 2.206 & 0.6296 & 2.361 & 2.259 & 0.957 & 0.5800 & 0.5422 & 0.935 \\
\hline $34 / 34$ & 1.873 & 0.4700 & 2.006 & 1.899 & 0.947 & 0.4277 & 0.3629 & 0.848 \\
\hline $35 / 35$ & 1.525 & 0.2066 & 1.636 & 1.520 & 0.929 & 0.1717 & 0.1912 & 1.114 \\
\hline $36 / 36$ & 1.154 & 0.1132 & 1.239 & 1.094 & 0.883 & 0.0867 & 0.0879 & 1.014 \\
\hline $37 / 37$ & 0.8276 & 0.0604 & 0.8886 & 0.6951 & 0.782 & 0.0413 & 0.0382 & 0.925 \\
\hline
\end{tabular}

$a_{\mathrm{Fission}} / \mathrm{sec} / \mathrm{g}$ of foll matertal at a power level of approximately 100 watts.

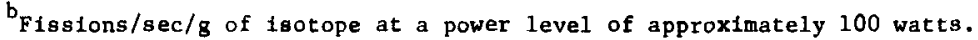


TABLE LXVI. Comparison of Experimental and Calculated Uraniun Fission Rates, $90^{\circ}$ Radial Traverse

\begin{tabular}{|c|c|c|c|c|c|c|c|c|}
\hline \multirow[b]{2}{*}{ Row/Co 1} & \multicolumn{2}{|c|}{$\begin{array}{l}\text { Experimental Uranium } \\
\text { Fission Rate }\end{array}$} & \multicolumn{3}{|c|}{${ }^{235}$ U Fission Rate ${ }^{b}$} & \multicolumn{3}{|c|}{238 U Fission Rate ${ }^{b}$} \\
\hline & $\begin{array}{l}\text { Enriched } \\
\left(\times 10^{6}\right)\end{array}$ & $\begin{array}{l}\text { Depleted } \\
\left(\times 10^{5}\right)\end{array}$ & $\begin{array}{l}\text { Experfmental } \\
\qquad\left(\times 10^{6}\right)\end{array}$ & $\begin{array}{l}\text { Calculated } \\
\quad\left(\times 10^{6}\right)\end{array}$ & $C / E$ & $\begin{array}{l}\text { Exper imental } \\
\quad\left(\times 10^{5}\right)\end{array}$ & $\begin{array}{l}\text { Calculated } \\
\left(\times 10^{5}\right)\end{array}$ & $C / E$ \\
\hline $23 / 23$ & 4.521 & 1.414 & 4.838 & 4.868 & 1.006 & 1.313 & 1.322 & 1.007 \\
\hline $24 / 23$ & 4.535 & 1.415 & 4.853 & 4.852 & 1.000 & 1.313 & 1.318 & 1.004 \\
\hline $25 / 23$ & 4.513 & 1.416 & 4.829 & 4.806 & 0.995 & 1.315 & 1.305 & 0.992 \\
\hline $26 / 23$ & 4.458 & 1.397 & 4.770 & 4.728 & 0.991 & 1.297 & 1.284 & 0.990 \\
\hline $27 / 23$ & 4.353 & 1.354 & 4.658 & 4.621 & 0.992 & 1.256 & 1.254 & 0.998 \\
\hline $28 / 23$ & 4.217 & 1.333 & 4.512 & 4.485 & 0.994 & 1.239 & 1.217 & 0.982 \\
\hline $29 / 23$ & 4.081 & 1.265 & 4.367 & 4.322 & 0.990 & 1.174 & 1.172 & 0.998 \\
\hline $31 / 23$ & 3.698 & 1.156 & 3.957 & 3.925 & 0.992 & 1.073 & 1.061 & 0.989 \\
\hline $32 / 23$ & 3.482 & 1.087 & 3.726 & 3.695 & 0.992 & 1.009 & 0.9962 & 0.987 \\
\hline $33 / 23$ & 3.266 & 1.018 & 3.495 & 3.448 & 0.987 & 0.9448 & 0.9250 & 0.979 \\
\hline $34 / 23$ & 3.020 & 0.9318 & 3.232 & 3.188 & 0.986 & 0.8641 & 0.8483 & 0.982 \\
\hline $35 / 23$ & 2.774 & 0.8448 & 2.969 & 2.919 & 0.983 & 0.7826 & 0.7659 & 0.979 \\
\hline $36 / 23$ & 2.531 & 0.7531 & 2.709 & 2.646 & 0.977 & 0.6963 & 0.6780 & 0.974 \\
\hline $37 / 23$ & 2.276 & 0.6625 & 2.436 & 2.374 & 0.975 & 0.6114 & 0.5835 & 0.954 \\
\hline $38 / 23$ & 2.042 & 0.5604 & 2.186 & 2.113 & 0.967 & 0.5144 & 0.4808 & 0.935 \\
\hline $39 / 23$ & 1.821 & 0.4403 & 1.951 & 1.874 & 0.961 & 0.3992 & 0.3654 & 0.915 \\
\hline $40 / 23$ & 1.639 & 0.2413 & 1.758 & 1.631 & 0.928 & 0.2039 & 0.2303 & 1.129 \\
\hline $41 / 23$ & 1.390 & 0.1447 & 1.492 & 1.374 & 0.921 & 0,1128 & 0.1292 & 1.145 \\
\hline $42 / 23$ & 1.183 & .0978 & 1.270 & 1.120 & 0.882 & 0.0706 & 0.0757 & 1.072 \\
\hline $43 / 23$ & 1.008 & .0647 & 1.082 & 0.9040 & 0.835 & 0.0415 & 0.0445 & 1.072 \\
\hline $44 / 23$ & 0.8892 & .0457 & 0.9550 & 0.7441 & 0.779 & 0.0252 & 0.0261 & 1.036 \\
\hline
\end{tabular}

Fission/sec/g of foll material at a power level of approximately 100 watts.

$\mathrm{b}_{\mathrm{Fiss}}$ ions/sec/g of lootope at a power level of approximately 100 watts.

TABLE LXVII. Comparison of Experimental and Calculated Plutonium Fission and $238 \mathrm{U}$ Capture Rates, $0^{\circ}$ Radial Traverse

\begin{tabular}{|c|c|c|c|c|c|c|}
\hline \multirow[b]{2}{*}{ Row/Co1 } & \multicolumn{2}{|c|}{ Plutonfum Fission Rate ${ }^{a}$} & \multicolumn{4}{|c|}{238 U Capture Rate } \\
\hline & $\begin{array}{l}\text { Exper imental } \\
\left(\times 10^{6}\right)\end{array}$ & $\begin{array}{l}\text { Calculated } \\
\left(\times 10^{6}\right)\end{array}$ & $C / E$ & $\begin{array}{l}\text { Experimental } \\
\qquad\left(\times 10^{5}\right)\end{array}$ & $\begin{array}{l}\text { Calculated } \\
\left(\times 10^{5}\right)\end{array}$ & $C / E$ \\
\hline $23 / 23$ & 4.581 & 4.600 & 1.004 & 6.675 & 6.733 & 1.009 \\
\hline $23 / 24$ & 4.621 & 4.584 & 0.992 & 6.695 & 6.714 & 1.003 \\
\hline $23 / 25$ & 4.549 & 4.537 & 0.997 & 6.606 & 6.647 & 1.006 \\
\hline $23 / 26$ & 4.469 & 4.460 & 0.998 & 6.559 & 6.532 & 0.996 \\
\hline $23 / 27$ & 4.365 & 4.352 & 0.997 & 6.303 & 6.376 & 1.012 \\
\hline $23 / 28$ & 4.310 & 4.216 & 0.978 & 6.298 & 6.178 & 0.981 \\
\hline $23 / 29$ & 4.123 & 4.053 & 0.983 & 6.010 & 5.943 & 0.989 \\
\hline $23 / 30$ & 3.924 & 3.865 & 0.985 & 5.749 & 5.671 & 0.986 \\
\hline $23 / 31$ & 3.690 & 3.654 & 0.990 & 5.528 & 5.368 & 0.971 \\
\hline $23 / 32$ & 3.502 & 3.423 & 0.977 & 5.176 & 5.038 & 0.973 \\
\hline $23 / 33$ & 3.237 & 3.174 & 0.981 & 4.849 & 4.685 & 0.966 \\
\hline $23 / 34$ & 3.015 & 2.911 & 0.966 & 4.501 & 4.314 & 0.958 \\
\hline $23 / 35$ & 2.716 & 2.638 & 0.971 & 4.088 & 3.934 & 0.962 \\
\hline $23 / 36$ & 2.448 & 2.357 & 0.963 & 3.727 & 3.549 & 0.952 \\
\hline $23 / 37$ & 2.217 & 2.074 & 0.935 & 3.304 & 3.170 & 0.959 \\
\hline $23 / 38$ & 1.910 & 1.795 & 0.940 & 3.060 & 2.808 & 0.918 \\
\hline $23 / 39$ & 1.656 & 1.527 & 0.922 & 2.687 & 2.477 & 0.922 \\
\hline $23 / 40$ & 1.340 & 1.246 & 0.930 & 2.313 & 2.145 & 0.927 \\
\hline $23 / 41$ & 1.091 & 1.015 & 0.930 & 1.889 & 1.688 & 0.894 \\
\hline $23 / 42$ & 0.9028 & 0.7680 & 0.851 & 1.421 & 1.298 & 0.913 \\
\hline $23 / 43$ & 0.6908 & 0.5610 & 0.812 & 1.073 & 0.9439 & 0.880 \\
\hline $23 / 44$ & 0.5606 & 0.4065 & 0.725 & 0.7645 & 0.6626 & 0.867 \\
\hline
\end{tabular}

$\mathrm{a}_{\mathrm{Fissions}} / \mathrm{sec} / \mathrm{g}$ of foil material at a power level of approximately 100 watts.

${ }^{b}$ Captures/sec/g of isotope at a power level of approximately 100 watts. 
TABLE LXVIII. Comparison of Experimental and Calculated Plutonium Fission and $238 \mathrm{U}$ Capture Rates, $45^{\circ}$ Radial Traverse

\begin{tabular}{|c|c|c|c|c|c|c|}
\hline \multirow[b]{2}{*}{ Row/Col } & \multicolumn{2}{|c|}{ Plutonium Fission Rate } & \multicolumn{4}{|c|}{$238_{\text {U Capture Rate }}{ }^{b}$} \\
\hline & $\begin{array}{c}\text { Experimental } \\
\left(\times 10^{6}\right)\end{array}$ & $\begin{array}{l}\text { Calculated } \\
\left(\times 10^{6}\right)\end{array}$ & $C / E$ & $\begin{array}{l}\text { Experimental } \\
\left(\times 10^{5}\right)\end{array}$ & $\begin{array}{l}\text { Calculated } \\
\left(\times 10^{5}\right)\end{array}$ & $C / E$ \\
\hline $23 / 23$ & 4.581 & 4.600 & 1.004 & 6.675 & 6.733 & 1.009 \\
\hline $24 / 24$ & 4.573 & 4.569 & 0.999 & 6.663 & 6.692 & 1.004 \\
\hline $25 / 25$ & 4.505 & 4.478 & 0.994 & 6.582 & 6.561 & 0.997 \\
\hline $28 / 28$ & 3.898 & 3.867 & 0.992 & 5.727 & 5.677 & 0.991 \\
\hline $29 / 29$ & 3.636 & 3.565 & 0.980 & 5.357 & 5.246 & 0.979 \\
\hline $30 / 30$ & 3.284 & 3.224 & 0.982 & 4.957 & 4.765 & 0.961 \\
\hline $31 / 31$ & 2.928 & 2.853 & 0.974 & 4.374 & 4.249 & 0.971 \\
\hline $32 / 32$ & 2.565 & 2.462 & 0.960 & 3.940 & 3.718 & 0.944 \\
\hline $37 / 37$ & 0.7767 & 0.5650 & 0.727 & 1.194 & 0.9536 & 0.799 \\
\hline
\end{tabular}

${ }^{a}$ Fissions/sec/g of foil material at a power level of approximately 100 watts.

${ }^{\mathrm{b}}$ Captures/sec/g of isotope at a power level of approximately 100 watts.

TABLE IXIX. Comparison of Experimental and Calculated Plutonium Fission and ${ }^{238} \mathrm{U}$ Capture Rates, $90^{\circ}$ Radial Traverse

\begin{tabular}{|c|c|c|c|c|c|c|}
\hline \multirow[b]{2}{*}{ Row/Col } & \multicolumn{2}{|c|}{ Plutonium Flssion Rate } & \multicolumn{4}{|c|}{${ }^{238} \mathrm{U}$ Capture Rate } \\
\hline & $\begin{array}{l}\text { Experimental } \\
\left(\times 10^{6}\right)\end{array}$ & $\begin{array}{c}\text { Calculated } \\
\left(\times 10^{6}\right)\end{array}$ & $C / E$ & $\begin{array}{l}\text { Experimental } \\
\left(\times 10^{5}\right)\end{array}$ & $\begin{array}{l}\text { Calculated } \\
\left(\times 10^{5}\right)\end{array}$ & $C / E$ \\
\hline $23 / 23$ & 4.581 & 4.600 & 1.004 & 6.675 & 6.733 & 1.009 \\
\hline $24 / 23$ & 4.562 & 4.585 & 1.005 & 6.748 & 6.714 & 0.995 \\
\hline $25 / 23$ & 4.555 & 4.540 & 0.997 & 6.677 & 6.650 & 0.996 \\
\hline $26 / 23$ & 4.443 & 4.467 & 1.005 & 6.578 & 6.542 & 0.995 \\
\hline $27 / 23$ & 4.481 & 4.364 & 0.974 & 6.449 & 6.396 & 0.992 \\
\hline $28 / 23$ & 4.257 & 4.235 & 0.995 & 6.324 & 6.208 & 0.982 \\
\hline $29 / 23$ & 4.100 & 4.080 & 0.995 & 6.102 & 5.984 & 0.981 \\
\hline $31 / 23$ & 3.745 & 3.699 & 0.988 & 5.529 & 5.439 & 0.984 \\
\hline $32 / 23$ & 3.504 & 3.477 & 0.992 & 5.300 & 5.124 & 0.967 \\
\hline $33 / 23$ & 3.243 & 3.238 & 0.998 & 4.911 & 4.789 & 0.975 \\
\hline $34 / 23$ & 2.994 & 2.985 & 0.997 & 4.551 & 4.436 & 0.975 \\
\hline $35 / 23$ & 2.771 & 2.721 & 0.982 & 4.190 & 4.074 & 0.972 \\
\hline $36 / 23$ & 2.543 & 2.450 & 0.963 & 3.955 & 3.709 & 0.938 \\
\hline $37 / 23$ & 2.292 & 2.177 & 0.950 & 3.499 & 3.349 & 0.957 \\
\hline $38 / 23$ & 1.996 & 1.906 & 0.955 & 3.179 & 3.006 & 0.946 \\
\hline $39 / 23$ & 1.787 & 1.647 & 0.922 & 2.836 & 2.696 & 0.951 \\
\hline $40 / 23$ & 1.472 & 1.370 & 0.931 & 2.529 & 2.379 & 0.941 \\
\hline $41 / 23$ & 1.254 & 1.156 & 0.922 & 2.143 & 1.923 & 0.897 \\
\hline $42 / 23$ & 1.071 & 0.9247 & 0.863 & 1.772 & 1.550 & 0.875 \\
\hline $43 / 23$ & 0.9194 & 0.7386 & 0.803 & 1.401 & 1.215 & 0.867 \\
\hline $44 / 23$ & 0.8515 & 0.6083 & 0.714 & 1.165 & 0.9404 & 0.807 \\
\hline
\end{tabular}

${ }^{a}$ Fissions/sec/g of foll material at a power level of approximately 100 watts.

${ }^{b}$ Captures $/ \mathrm{sec} / \mathrm{g}$ of 1 sotope at a power level of approximately 100 watts. 
TABLE LXX. Comparison of Experimental and Calculated Uranium Fission Rates, Stationary Half Axial Traverse

\begin{tabular}{|c|c|c|c|c|c|c|c|c|}
\hline \multirow{2}{*}{$\begin{array}{l}\text { Axial } \\
\text { Distance } \\
\text { from } \\
\text { Midplane } \\
\text { (cm) }\end{array}$} & \multicolumn{2}{|c|}{$\begin{array}{l}\text { Experimental Urantum } \\
\text { Fission Rate }\end{array}$} & \multicolumn{2}{|c|}{235 U Fission Rate ${ }^{b}$} & \multicolumn{4}{|c|}{$23{ }^{8}$ U Fission Rate } \\
\hline & $\begin{array}{l}\text { Enriched } \\
\left(\times 10^{6}\right)\end{array}$ & $\begin{array}{l}\text { Depleted } \\
\left(\times 10^{5}\right)\end{array}$ & $\begin{array}{c}\text { Experimental } \\
\left(\times 10^{6}\right)\end{array}$ & $\begin{array}{l}\text { Calculated } \\
\left(\times 10^{6}\right)\end{array}$ & $C / E$ & $\begin{array}{l}\text { Experimental } \\
\left(\times 10^{5}\right)\end{array}$ & $\begin{array}{l}\text { Calculated } \\
\left(\times 10^{5}\right)\end{array}$ & $C / E$ \\
\hline 0.95 & & 1.414 & & & & 1.312 & 1.322 & 1.008 \\
\hline 3.49 & 4.521 & & 4.837 & 4.858 & 1.004 & & & \\
\hline 6.03 & & 1.454 & & & & 1.353 & 1.313 & 0.970 \\
\hline 11.11 & & 1.429 & & & & 1.329 & 1.292 & 0.972 \\
\hline 13.65 & 4.395 & & 4.702 & 4.711 & 1.002 & & & \\
\hline 16.19 & & 1.379 & & & & 1.282 & 1.260 & 0.983 \\
\hline 21.27 & & 1.320 & & & & 1.226 & 1.215 & 0.991 \\
\hline 23.81 & 4.129 & & 4.418 & 4.395 & 0.995 & & & \\
\hline 26.35 & & 1.277 & & & & 1.186 & 1.157 & 0.976 \\
\hline 31.43 & & 1.212 & & & & 1.126 & 1.087 & 0.965 \\
\hline 33.97 & 3.745 & & 4.007 & 3.928 & 0.980 & & & \\
\hline 36.51 & & 1.112 & & & & 1.031 & 1.007 & 0.977 \\
\hline 41.59 & & 1.043 & & & & 0.9667 & 0.9169 & 0.948 \\
\hline 44.13 & 3.218 & & 3.444 & 3.331 & 0.967 & & בטגד & 0.240 \\
\hline 46.67 & & 0.9148 & & & & 0.8451 & 0.8113 & 0.960 \\
\hline 51.75 & & 0.8073 & & & & 0.7444 & 0.6855 & 0.921 \\
\hline 54.29 & 2.658 & & 2.846 & 2.666 & 0.937 & & & \\
\hline 56.83 & & 0.6590 & & & & 0.6021 & 0.5408 & 0.898 \\
\hline 61.91 & & 0.4299 & & & & 0.3793 & 0.3897 & 1.027 \\
\hline 64.45 & 2.117 & & 2.270 & 1.980 & 0.872 & & & \\
\hline 66.99 & & 0.2929 & & & & 0.2486 & 0.2549 & 1.025 \\
\hline 72.07 & & 0.2079 & & & & 0.1696 & 0.1593 & 0.939 \\
\hline 74.61 & 1.557 & & 1.671 & 1.260 & 0.754 & & & \\
\hline 77.15 & & 0.1502 & & & & 0.1182 & 0.1067 & 0.903 \\
\hline 82.23 & & 0.1071 & & & & 0.0808 & 0.0702 & 0.869 \\
\hline 84.77 & 1.024 & & 1.099 & 0.5102 & 0.464 & & & \\
\hline 87.31 & & 0.0773 & & & & 0.0567 & 0.0229 & 0.404 \\
\hline
\end{tabular}

Q Fissions/sec/g of foll material at a power level of approximately 100 watts.

${ }^{b}$ Fissions/sec/g of isotope at a power level of approximately 100 watts. 
TABLE LXXI. Comparison of Experimental and Calculated Plutonium Fission and ${ }^{238} \mathrm{U}$ Capture Rates, Stationary Half Axial Traverse

\begin{tabular}{|c|c|c|c|c|c|c|}
\hline \multirow{2}{*}{$\begin{array}{l}\text { Axtal } \\
\text { Distance } \\
\text { from } \\
\text { Midplane } \\
\text { (cm) }\end{array}$} & \multicolumn{3}{|c|}{ Plutonium Flssion Rate } & \multicolumn{3}{|c|}{$238_{U}$ Capture Rate ${ }^{b}$} \\
\hline & $\begin{array}{l}\text { Experimental } \\
\qquad\left(\times 10^{6}\right)\end{array}$ & $\begin{array}{c}\text { Calculated } \\
\left(\times 10^{6}\right)\end{array}$ & $\mathrm{c} / \mathrm{E}$ & $\begin{array}{c}\text { Experimental } \\
\left(\times 10^{5}\right)\end{array}$ & $\begin{array}{c}\text { Calculated } \\
\left(\times 10^{5}\right)\end{array}$ & $\mathrm{C} / \mathrm{E}$ \\
\hline $\begin{array}{l}0.95 \\
2.54\end{array}$ & 4.581 & 4503 & 1003 & 6.675 & 6.731 & 1.008 \\
\hline $\begin{array}{l}2.34 \\
6.03\end{array}$ & 4.501 & נ. & 1.000 & 6.539 & 6.693 & 1.024 \\
\hline 7.62 & 4.569 & 4.553 & 0.996 & & & \\
\hline 11.11 & & & & 6.461 & 6.589 & 1.020 \\
\hline 12.70 & 4.493 & 4.471 & 0.995 & & & \\
\hline 16.19 & & & & 6.315 & 6.432 & 1.019 \\
\hline 17.78 & 4.348 & 4.343 & 0.999 & & & \\
\hline 21.27 & & & & 6.186 & 6.219 & 1.005 \\
\hline 22.86 & 4.214 & 4.177 & 0.994 & & & \\
\hline 26.35 & & & & 5.929 & 5.950 & 1.004 \\
\hline $\begin{array}{l}27.94 \\
31.43\end{array}$ & 4.023 & 3.976 & 0.991 & 5.654 & 5.632 & 0.996 \\
\hline 33.02 & 3.811 & 3.741 & 0.982 & & & \\
\hline 36.51 & & & & 5.364 & 5.270 & 0.982 \\
\hline 38.10 & 3.542 & 3.472 & 0.980 & & & \\
\hline 41.59 & & & & 4.964 & 4.875 & 0.982 \\
\hline 43.18 & 3.246 & 3.170 & 0.977 & & & \\
\hline 46.67 & & & & 4.563 & 4.451 & 0.975 \\
\hline 48.26 & 2.982 & 2.843 & 0.953 & & & \\
\hline 51.75 & & & & 4.221 & 4.004 & 0.949 \\
\hline 53.34 & 2.643 & 2.501 & 0.946 & & & \\
\hline 56.83 & & & & 3.861 & 3.536 & 0.916 \\
\hline 58.42 & 2.322 & 2.157 & 0.929 & & & \\
\hline 61.91 & & & & 3.403 & 3.050 & 0.896 \\
\hline 63.50 & 2.037 & 1.933 & 0.949 & & & \\
\hline 66.99 & & & & 2.974 & 2.551 & 0.858 \\
\hline 68.58 & 1.740 & 1.481 & 0.851 & & & \\
\hline 72.07 & & & & 2.541 & 2.042 & 0.804 \\
\hline 73.66 & 1.464 & 1.150 & 0.786 & & & \\
\hline 77.15 & & & & 2.156 & 1.526 & 0.708 \\
\hline 78.74 & 1.211 & 0.8197 & 0.677 & & & \\
\hline 82.23 & & & & 1.730 & 0.9972 & 0.576 \\
\hline 83.82 & 1.009 & 0.4949 & 0.490 & & & \\
\hline 87.31 & & & & 1.267 & 0.4579 & 0.361 \\
\hline 88.90 & 0.7583 & 0.1769 & 0.233 & & & \\
\hline
\end{tabular}

a,b (See Table VIII) 
TABLE LXXII. Experimental Urantum Fission Rates, and $238_{\mathrm{U}}$ Capture Rate, Moveable Half Axlal Traverse

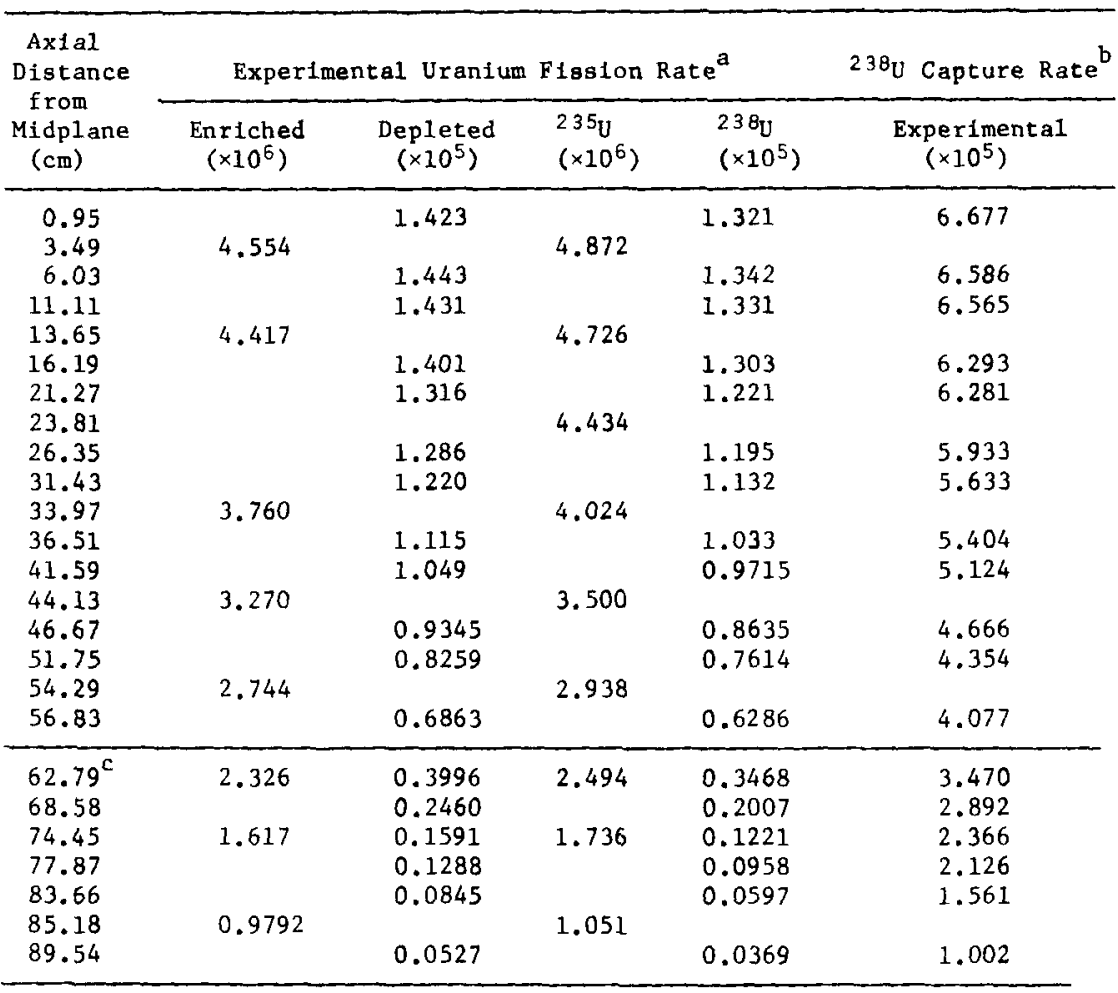

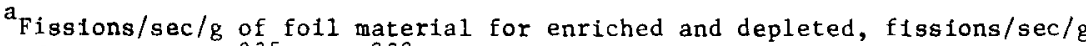
of isotope for ${ }^{235} \mathrm{U}$ and ${ }^{238} \mathrm{U}$ at a power level of approximately 100 watts. ${ }^{b}$ Captures/sec/g of isotope at a power level of approximately 100 watts.

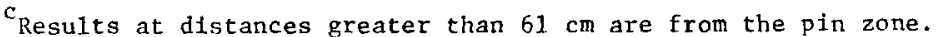

TABLE IXXIII. Normalized Relattve Calrilated and Measured Uranium Fisaion Rates for the Row 23, $0^{\circ}$ Traverse, in the GCFR Phase I Normal and Checkerboard Core Configurations

\begin{tabular}{|c|c|c|c|c|c|c|c|c|c|c|c|c|}
\hline $\begin{array}{c}\text { Matrix } \\
\text { Location }\end{array}$ & $\begin{array}{l}\text { Meas i } \\
\text { Dop }\end{array}$ & $\begin{array}{l}\text { Meas }_{2} \\
\text { Depf }_{f}\end{array}$ & $\begin{array}{c}\text { Meas } \\
238\end{array}$ & $\begin{array}{c}\text { Meas } 2 \\
239\end{array}$ & $\begin{array}{c}\mathrm{Calcl} \\
238\end{array}$ & $\begin{array}{c}\mathrm{Calc}_{2} \\
238\end{array}$ & $\begin{array}{l}\text { Meas } 1 \\
\text { Ett: }_{f}\end{array}$ & $\begin{array}{l}\text { Meas } 2_{2} \\
\text { Ent }_{E}\end{array}$ & $\begin{array}{l}\text { Meas } 1 \\
235\end{array}$ & ${ }_{\mathrm{f}}^{\mathrm{Mcas}_{2}}$ & $\begin{array}{c}\mathrm{C}_{\Delta L} \mathrm{C}_{\mathrm{l}} \\
235\end{array}$ & $\begin{array}{c}\mathrm{CaLC}_{\mathrm{f}} \\
235\end{array}$ \\
\hline $523 / 23$ & 1068.3 & 1079.0 & 993.9 & 1003.5 & 1000 & 1000 & 931.4 & 934.2 & 996.5 & 999.6 & 1000 & 1000 \\
\hline $523 / 24$ & 1069.2 & 1076.9 & 994.7 & $100 \mathrm{I} .1$ & 997.0 & 996.6 & 930.3 & 932.4 & 995.3 & 997.6 & 996.7 & 996.6 \\
\hline$\$ 23 / 25$ & 1077.7 & 1067.3 & 1002.6 & 992.2 & 986.4 & 986.3 & 923.4 & 920.7 & 988.0 & 985.1 & 986.4 & 986.4 \\
\hline $523 / 26$ & 1055.4 & 1045.9 & 981.9 & 972.3 & 969.7 & 969.6 & 910.5 & 907.6 & 974.2 & 971.1 & 969.6 & 969.6 \\
\hline $523 / 27$ & 1034.4 & 1025.1 & 952.3 & 953.0 & 946.3 & 946.2 & 880.3 & 935.0 & 941.9 & 968.3 & 946.4 & 946.5 \\
\hline $523 / 28$ & 282.5 & 988.9 & 914.0 & 919.3 & 916.8 & 916.9 & 887.6 & 860.4 & 928.3 & 920.6 & 017.0 & 917.1 \\
\hline $523 / 29$ & 945.5 & 958.7 & 879.6 & 891.2 & 881.2 & 881.1 & 827.0 & 831.5 & 884.3 & 889.7 & 881.9 & 882.0 \\
\hline $523 / 30$ & 922,4 & 917.2 & 848.8 & 852.7 & 840.4 & 840.1 & 794.5 & 784.3 & 850.0 & 839.7 & 841.4 & 841.5 \\
\hline $523 / 31$ & 859.3 & 862.8 & 799.2 & 801.9 & 793.5 & 793.8 & 753.8 & 753.7 & 806.5 & 806.4 & 796.0 & 796.1 \\
\hline $523 / 32$ & 808.8 & 815.1 & 752.3 & 757.4 & 742.7 & 742.8 & 709.7 & 711.4 & 759.3 & 761.2 & 746.5 & 746.5 \\
\hline $523 / 33$ & 751.9 & 759.4 & 699.0 & 705.4 & 687.1 & 687.3 & 662.0 & 664.9 & 708.3 & 711.4 & 693.3 & 693.3 \\
\hline$\$ 23 / 34$ & 695.9 & 701.3 & 647.1 & 651.3 & 627.6 & 627.5 & 611.9 & 613.7 & 654.7 & 658.7 & 637.4 & 637.2 \\
\hline$\$ 23 / 35$ & 645.3 & 631.0 & 599.1 & 585.4 & 564.1 & 564.1 & 558.2 & 552.6 & 597.3 & 602.0 & 579.1 & 579.2 \\
\hline $523 / 36$ & 558.1 & 566.9 & 517.5 & 525.2 & 497.0 & 496.8 & 508.0 & 510.9 & 543.6 & 546.8 & 521.2 & 520.2 \\
\hline$\$ 23 / 37$ & 489.7 & 493.6 & 452.8 & 456.0 & 425.7 & 425.2 & 452.5 & 459.7 & 494.3 & 492.0 & 462.8 & 461.5 \\
\hline $523 / 38$ & 408.9 & 412.1 & 375.9 & 378.6 & 348.5 & 348.1 & 400.4 & 407.3 & 428.7 & 436.0 & 406.3 & 404.7 \\
\hline $523 / 39$ & 325.8 & 330.8 & 295.7 & 300.0 & 262.5 & 261.9 & 354.8 & 350.3 & 330.0 & 385.9 & 354.4 & 352.3 \\
\hline $523 / 40$ & 180,8 & 179.5 & 158.7 & 157.3 & 163.8 & 163.3 & 301.5 & 306.4 & 323.2 & 328.5 & 302.2 & 299.7 \\
\hline $523 / 41$ & 108.7 & 110.6 & 90.13 & 91.57 & 91.30 & 90.93 & 250.9 & 255.3 & 269.2 & 273.9 & 246.5 & 244.1 \\
\hline $523 / 42$ & 69.64 & 70.63 & 54.68 & 55.25 & 52.87 & 52.55 & 198.5 & 202.7 & 213.0 & 217.5 & 190.6 & 188.4 \\
\hline $523 / 43$ & 43.77 & 45.81 & 32.36 & 33.74 & 30.41 & 30.24 & 154.7 & 157.7 & 166.1 & 169.3 & 141.1 & \\
\hline $523 / 44$ & 27.84 & 29.96 & 19.40 & 20.78 & 17.93 & 17.82 & 120.1 & 222.1 & 128.9 & 131.1 & 102.9 & 10 \\
\hline
\end{tabular}

Note: Subscript 1 refers to normal assembly loading. Subuvdpt 2 refers to checkerboard assembly 1oading. 
TABLE LXXIV. Normalized Relative Calculated and Measured Plutonium Fission Rates and $238 \mathrm{U}$ Capture Rates for the Row 23, $0^{\circ}$ Traverse in the GCFR Phase I Normal and Checkerboard Core Configurations

\begin{tabular}{|c|c|c|c|c|c|c|c|c|}
\hline $\begin{array}{l}\text { Matrix } \\
\text { Location }\end{array}$ & $\begin{array}{c}\text { Meas }_{1} \\
239_{\mathrm{f}}\end{array}$ & $\begin{array}{c}\mathrm{Meas}_{2} \\
239 \\
\mathrm{f}\end{array}$ & $\begin{array}{c}\mathrm{Calc}_{1} \\
2.39 \\
\mathrm{f}\end{array}$ & $\begin{array}{c}\mathrm{Calc}_{2} \\
239 \\
\mathrm{f}\end{array}$ & $\begin{array}{c}\text { Meas } 1 \\
238 \\
c\end{array}$ & $\begin{array}{c}\text { Meas }_{2} \\
238 \\
c\end{array}$ & $\begin{array}{c}\mathrm{CaIc}_{1} \\
238 \\
\mathrm{c}\end{array}$ & $\begin{array}{c}\mathrm{CaIc}_{2} \\
238 \\
\mathrm{c}\end{array}$ \\
\hline $\begin{array}{l}\mathrm{s} 23 / 23 \\
\mathrm{~s} 23 / 24 \\
\mathrm{~s} 23 / 25 \\
\mathrm{~s} 23 / 26 \\
\mathrm{~s} 23 / 27 \\
\mathrm{~s} 23 / 28 \\
\mathrm{~s} 23 / 29 \\
\mathrm{~s} 23 / 30 \\
\mathrm{~s} 23 / 31 \\
\mathrm{~s} 23 / 32 \\
\mathrm{~s} 23 / 33 \\
\mathrm{~s} 23 / 34 \\
\mathrm{~s} 23 / 35 \\
\mathrm{~s} 23 / 36 \\
\mathrm{~s} 23 / 37 \\
\mathrm{~s} 23 / 38 \\
\mathrm{~s} 23 / 39 \\
\mathrm{~s} 23 / 40 \\
\mathrm{~s} 23 / 41 \\
\mathrm{~s} 23 / 42 \\
\mathrm{~s} 23 / 43 \\
\mathrm{~s} 23 / 44\end{array}$ & $\begin{array}{r}995.9 \\
1004.6 \\
988.9 \\
971.6 \\
948.9 \\
937.0 \\
896.3 \\
853.1 \\
802.2 \\
761.4 \\
703.8 \\
655.5 \\
590.6 \\
532.4 \\
482.3 \\
415.7 \\
360.7 \\
292.3 \\
238.5 \\
197.5 \\
151.2 \\
122.8\end{array}$ & $\begin{array}{l}993.3 \\
998.6 \\
989.5 \\
969.8 \\
953.2 \\
925.9 \\
888.2 \\
856.2 \\
809.0 \\
765.3 \\
709.9 \\
657.1 \\
605.6 \\
539.9 \\
482.2 \\
423.8 \\
366.7 \\
302.1 \\
244.1 \\
193.8 \\
153.5 \\
127.0\end{array}$ & $\begin{array}{c}1000 . \\
996.6 \\
986.4 \\
969.6 \\
946.2 \\
916.6 \\
881.2 \\
840.3 \\
794.5 \\
744.3 \\
690.2 \\
633.0 \\
573.6 \\
512.6 \\
451.2 \\
390.7 \\
332.6 \\
271.8 \\
221.8 \\
168.0 \\
122.8 \\
89.03\end{array}$ & $\begin{array}{c}1000 . \\
996.6 \\
986.4 \\
969.6 \\
946.3 \\
916.7 \\
881.4 \\
840.5 \\
794.7 \\
744.4 \\
690.3 \\
633.0 \\
573.4 \\
512.2 \\
450.5 \\
389.5 \\
331.1 \\
270.0 \\
220.0 \\
166.4 \\
121.4 \\
87.79\end{array}$ & $\begin{array}{l}991.1 \\
993.9 \\
980.8 \\
973.7 \\
935.9 \\
935.0 \\
892.4 \\
853.6 \\
820.7 \\
768.3 . \\
791.9 \\
668.2 \\
606.8 \\
553.3 \\
490.5 \\
454.3 \\
399.0 \\
343.3 \\
280.5 \\
210.9 \\
159.4 \\
113.5\end{array}$ & $\begin{array}{r}989.9 \\
1000.0 \\
991.1 \\
969.6 \\
961.5 \\
923.2 \\
885.9 \\
861.5 \\
809.3 \\
765.4 \\
714.5 \\
660.9 \\
608.8 \\
559.4 \\
500.8 \\
451.4 \\
399.0 \\
331.6 \\
274.7 \\
214.7 \\
162.1 \\
113.0\end{array}$ & $\begin{array}{c}1000 . \\
997.2 \\
987.2 \\
970.1 \\
947.0 \\
917.6 \\
882.7 \\
842.3 \\
797.3 \\
748.3 \\
695.8 \\
640.7 \\
584.3 \\
527.1 \\
470.8 \\
417.1 \\
367.9 \\
318.6 \\
250.7 \\
192.8 \\
140.2 \\
98.41\end{array}$ & $\begin{array}{c}1000 . \\
997.2 \\
987.0 \\
969.8 \\
947.0 \\
917.8 \\
882.8 \\
842.4 \\
794.4 \\
748.1 \\
695.6 \\
640.4 \\
583.6 \\
526.1 \\
469.3 \\
415.0 \\
365.3 \\
315.6 \\
247.9 \\
190.3 \\
138.2 \\
96.78\end{array}$ \\
\hline
\end{tabular}

Note: Subscript 1 refers to normal assembly loading. Subscript 2 refers to checkerboard assembly loading.

TABLF LXXV. Normalized Relative Calculated and Measured Uranium Fission Rates for the Diagonal, $45^{\circ}$ Traverse, In the GCFR Phase I Normal and Checkerboard Core Conf Iguration

\begin{tabular}{|c|c|c|c|c|c|c|c|c|c|c|c|c|}
\hline $\begin{array}{l}\text { Natrix } \\
\text { Location }\end{array}$ & $\begin{array}{l}\text { Meas } \\
\text { Depf }\end{array}$ & $\begin{array}{l}\mathrm{Keas}_{2} \\
\text { Depf }\end{array}$ & $\begin{array}{l}\text { Meass } \\
23 b_{f}\end{array}$ & $\begin{array}{l}\text { Meas } 2 \\
238_{f}^{2}\end{array}$ & 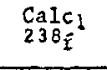 & $\begin{array}{l}\mathrm{CaIc}_{2} \\
23 \mathrm{~B}_{\mathrm{f}}\end{array}$ & $\begin{array}{l}\text { Meas } \\
\text { Enr }_{\mathrm{f}}\end{array}$ & $\begin{array}{l}\mathrm{Keas}_{2} \\
\mathrm{Enr}_{\mathrm{E}}\end{array}$ & $\begin{array}{l}\text { Meas } 1 \\
235_{1}\end{array}$ & $\begin{array}{l}\text { Meas } 2 \\
235 \mathrm{f}\end{array}$ & $\begin{array}{l}\operatorname{Cal} c_{1} \\
235 \mathrm{f}\end{array}$ & $\begin{array}{l}\operatorname{Calc} c_{2} \\
235_{f}\end{array}$ \\
\hline $523 / 23$ & 1068.3 & 1079.9 & 993.9 & 1003.9 & 1000 & 1000 & 931.4 & 934.2 & 996.5 & 999.6 & 1000 & 1000 \\
\hline $24 / 24$ & 1059.4 & 1066.3 & 985.6 & 991.4 & 993.2 & 993.1 & 927.8 & 928.3 & 992.7 & 993.2 & 993.4 & 993.1 \\
\hline $25 / 25$ & 1055.0 & 1049.6 & 981.5 & 975.7 & 973.5 & 972.8 & 914.6 & 913.4 & 978.6 & 977.3 & 973.7 & 972.8 \\
\hline $26 / 26$ & 1012.9 & 1006.1 & 942.3 & 935.3 & 941.0 & 939.2 & 891.0 & 888.2 & 953.3 & 950.3 & 241.5 & 939.5 \\
\hline $27 / 27$ & 966.4 & 972.0 & 899.1 & 903.6 & 896.4 & 893.3 & 851.1 & 862.5 & 910.6 & 922.8 & 897.1 & 894.0 \\
\hline $28 / 28$ & 918.5 & 912.8 & 854.3 & 848.4 & 840.4 & 835.6 & 799.8 & 796.5 & 855.7 & 952.2 & 842.0 & 837.2 \\
\hline $29 / 29$ & 850.8 & 843.1 & 791.3 & 783.6 & 773.1 & 767.3 & 742.0 & 746.6 & 793.9 & 798.8 & 777.3 & 770.6 \\
\hline $30 / 30$ & 765.8 & 767.8 & 711.9 & 713.4 & 697.0 & 689.2 & 674.9 & 673.5 & 722.1 & 720.7 & 704.8 & 696.0 \\
\hline $31 / 31$ & 679.9 & 675.8 & 631.4 & 627.1 & 611.3 & 602.0 & 604.1 & 601.5 & 646.4 & 643.6 & 626.5 & 615.5 \\
\hline $32 / 32$ & 586.6 & 581.1 & 543.5 & 538.2 & 516.3 & 505.9 & 527.4 & 528.3 & 564.4 & 565.4 & 545.2 & 531.8 \\
\hline $33 / 33$ & 475.8 & 475.8 & 438.5 & 438.4 & 410.1 & 399.3 & 454.5 & 455.3 & 486.5 & 487.4 & 464.1 & 448.2 \\
\hline $34 / 34$ & 355.3 & 350.4 & 320.9 & 316.3 & 274.5 & 264.3 & 385.9 & 386.5 & 413.4 & 414.0 & 390.1 & 372.1 \\
\hline $35 / 35$ & 156.1 & 262.4 & 134.1 & 139,3 & 144.6 & 137.9 & 314.2 & 314.7 & 336.9 & 337.5 & 312.2 & 295.8 \\
\hline $36 / 36$ & 85.58 & 85.61 & 68.04 & 67.89 & 66.49 & 63.47 & 237.8 & 239.0 & 255.2 & 256.5 & 224.7 & 213.0 \\
\hline $37 / 37$ & 45.68 & 46.73 & 33,17 & 33.84 & 28.90 & 27.60 & 170.6 & 171.1 & 183.1 & .183 .7 & 142,8 & 135.2 \\
\hline
\end{tabular}

Note: Subscript 1 refers to normal assembly loading. Subassembly 2 refers to checkerboard assembly loading. 
TABLE LXXVI. Normalized Relative Calculated and Measured Plutonium Fission Rates and $238 \mathrm{U}$ Capture Rates for the Diagonal, $45^{\circ}$ Traverse, in the GCFR Phase I Normal and Checkerboard Core Conf 1 gurations

\begin{tabular}{|c|c|c|c|c|c|c|c|c|}
\hline $\begin{array}{l}\text { Matrix } \\
\text { Location }\end{array}$ & $\begin{array}{c}\text { Meas } 1 \\
239 \\
f\end{array}$ & $\begin{array}{c}\text { Meas }_{2} \\
239\end{array}$ & $\begin{array}{l}\mathrm{Calc}_{1} \\
239\end{array}$ & $\begin{array}{c}\mathrm{Calc}_{2} \\
239 \\
\mathrm{f}\end{array}$ & $\begin{array}{c}\text { Meas } \\
238 \\
\mathrm{C}\end{array}$ & $\begin{array}{c}\mathrm{Meas}_{2} \\
238 \\
\mathrm{c}\end{array}$ & $\begin{array}{c}\mathrm{Calc}_{1} \\
2.38 \\
\mathrm{c}\end{array}$ & $\begin{array}{c}\mathrm{CaIc}_{2} \\
238 \\
\mathrm{c}\end{array}$ \\
\hline $523 / 23$ & 995.9 & 998.3 & 1000 & 1000 & 991.1 & 988.9 & 1000 & 1000 \\
\hline S24/24 & 994.2 & 997.5 & 993.4 & 993.2 & 989.1 & 986.7 & 993.9 & 993.5 \\
\hline $\mathrm{S} 25 / 25$ & 979.4 & 982.7 & 973.6 & 972.8 & 977.2 & 984.2 & 974.5 & 973.3 \\
\hline$S 26 / 26$ & 953.9 & 948.4 & 941.1 & 939.3 & 951.3 & 945.3 & 941.9 & 940.1 \\
\hline $527 / 27$ & 899.8 & 903.6 & 896.4 & 893.4 & 912.5 & 901.1 & 897.8 & 794.7 \\
\hline $\mathrm{S} 28 / 28$ & 847.4 & 865.4 & 840.7 & 836.1 & 850.2 & 845.2 & 843.2 & 838.2 \\
\hline $529 / 29$ & 790.5 & 791.2 & 775.1 & 768.8 & 795.3 & 796.3 & 779.1 & 772.2 \\
\hline $\mathrm{S} 30 / 30$ & 714.0 & 724.8 & 701.0 & 692.8 & 735.9 & 721.0 & 707.7 & 698.5 \\
\hline $\mathrm{S} 31 / 31$ & 636.7 & 646.5 & 620.4 & 610.2 & 649.5 & 647.4 & 631.1 & 619.5 \\
\hline $\mathrm{S} 32 / 32$ & 557.9 & 560.2 & 535.5 & 523.1 & 585.0 & 580.6 & 552.2 & 538.0 \\
\hline $533 / 33$ & 469.2 & 476.7 & 448.7 & 434.4 & 516.2 & 500.2 & 474.5 & 457.8 \\
\hline $534 / 34$ & 391.7 & 388.8 & 363.8 & 347.6 & 434.6 & 433.6 & 392.2 & 373.4 \\
\hline $535 / 35$ & 306.1 & 306.5 & 285.6 & 270.9 & 339.0 & 342.5 & 317.5 & 300.1 \\
\hline $\mathrm{S} 36 / 36$ & 228.2 & 228.9 & 198.8 & 188.7 & 262.8 & 253.3 & 228.4 & 216.0 \\
\hline $537 / 37$ & 170.1 & 169.8 & 123.7 & 117.3 & 117.3 & 173.1 & 141.6 & 133.9 \\
\hline
\end{tabular}

Note: Subscript 1 refers to normal assembly loading.

Subscript 2 refers to checkerboard assembly loading.

TABLE LXXVII. Normalized Relative Calculated and Measured Uraniun Fission Rates for the Column 23 , $90^{\circ}$ Traverse, in the GCFR Phase I Normal and Checkerboard Core Conf1gurations

\begin{tabular}{|c|c|c|c|c|c|c|c|c|c|c|c|c|}
\hline $\begin{array}{l}\text { Matrix } \\
\text { Locat1on }\end{array}$ & $\begin{array}{l}\text { Neas } 1 \\
\text { Dopi }\end{array}$ & $\begin{array}{l}\text { Meas }_{2} \\
\text { Lept }\end{array}$ & $\begin{array}{l}\text { Meas } 1 \\
238_{f}\end{array}$ & $\begin{array}{l}\operatorname{Meas}_{2} \\
23 y \leq\end{array}$ & $\begin{array}{l}\mathrm{Calc}_{1} \\
23 \mathrm{f}_{\mathrm{f}}\end{array}$ & $\begin{array}{l}\mathrm{Calc}_{2} \\
238_{4}\end{array}$ & $\begin{array}{l}\text { Meas } 1 \\
\text { Enrf }\end{array}$ & $\begin{array}{l}\text { Meas }_{2} \\
\text { Enr }_{5}\end{array}$ & $\begin{array}{l}\text { Meas } 1 \\
235_{\mathrm{f}}\end{array}$ & $\begin{array}{l}\operatorname{Meas}_{2} \\
235_{f}\end{array}$ & $\begin{array}{l}\text { Calc } \\
235 f\end{array}$ & $\begin{array}{l}\operatorname{call}_{2} \\
235 \mathrm{f}\end{array}$ \\
\hline$\$ 23 / 23$ & 1068.3 & 1079.9 & 393.9 & 1003.9 & 1000 & 1000 & 931.4 & 934.2 & 996.5 & 999.6 & 1000 & 1000 \\
\hline $24 / 23$ & 1069.7 & - & 995.2 & - & 997.0 & 996.6 & 934.3 & - & 999.6 & - & 996.7 & 996.5 \\
\hline $25 / 23$ & 1070.3 & 1064.7 & 995.7 & 989.8 & 987.1 & 986.3 & 929.9 & 925.6 & 994.9 & 990.4 & 987.3 & 986.3 \\
\hline $26 / 23$ & 1056.1 & - & 982.5 & - & 971.3 & 969.1 & 918.5 & - & 982.7 & - & 971.2 & 969.3 \\
\hline $27 / 23$ & 1023.6 & 1019.6 & 952.3 & 947.9 & 948.6 & 945.8 & 896.8 & 392.1 & 959.5 & 954.5 & 949.3 & 945.9 \\
\hline $28 / 23$ & 1007.6 & - & 937.4 & - & 920.6 & 916.0 & 868.7 & - & 929.4 & - & 921.3 & 916.3 \\
\hline $29 / 23$ & 956.1 & 966.0 & 889.5 & 898.0 & 886.5 & 880.2 & 840.7 & 839.0 & 899.5 & 897.7 & 837.8 & 880.8 \\
\hline $30 / 23$ & - & - & - & - & - & 838.6 & - & - & - & - & - & 839.9 \\
\hline $31 / 23$ & 873.9 & 873.5 & 812.8 & 811.8 & 802.6 & 791.9 & 761.8 & 760.6 & 815.1 & 813.8 & 806.3 & 794.3 \\
\hline $32 / 23$ & 821.3 & - & 763.7 & - & 753.6 & 740.0 & 717.3 & - & 767.5 & - & 759.0 & 744.2 \\
\hline $33 / 23$ & 769.5 & 764.7 & 715.4 & 710.4 & 699.7 & 684.1 & 672.8 & 669.8 & 719.9 & 716.7 & 708.3 & 690.6 \\
\hline $34 / 23$ & 704.1 & - & 654.1 & - & 641.7 & 623.9 & 622.1 & - & 655.7 & - & 654.9 & 634.3 \\
\hline $35 / 23$ & 638.5 & 642.2 & 592.5 & 595.8 & 579.3 & 559.6 & 571.5 & 569.0 & 611.5 & 608.9 & 599.6 & 576.1 \\
\hline $36 / 23$ & 569.2 & - & 527.2 & - & 512.9 & 491.9 & 521.5 & - & 558.1 & - & 543.5 & 517.2 \\
\hline $37 / 27$ & 500.7 & 497.6 & 462.3 & 459.5 & 441.4 & 419.9 & 468.9 & 467.0 & 501.9 & 499.9 & 487.7 & 458.8 \\
\hline $38 / 27$ & 423.5 & - & 388.6 & - & 363.7 & 342.3 & 421.0 & - & 450.8 & - & 434.1 & 402.7 \\
\hline $39 / 2$ & 332.8 & 330.2 & 301.1 & 298.9 & 276.4 & 256.2 & 375.3 & 387.0 & 402.0 & 414.6 & 385.0 & 351.7 \\
\hline $40 / 23$ & 182.3 & 183.7 & 159.1 & 160.2 & 174.2 & 159.5 & 337.6 & 333.7 & 351.9 & 357.8 & 335.0 & 303.8 \\
\hline $41 / 23$ & 109.4 & 115.0 & 89.60 & 94.07 & 97.73 & 89.46 & 286.4 & 280.6 & 307.3 & 307.5 & 282.3 & 256.2 \\
\hline $42 / 23$ & 73.94 & 76.48 & 56.59 & 58.42 & 57.26 & 52.44 & 243.6 & 242.8 & 261.5 & 260.6 & 230.1 & 209.0 \\
\hline $43 / 23$ & 48.91 & 52.39 & 34.42 & 36.79 & 33.66 & 30.83 & 207.6 & 206.8 & 222.9 & 222.0 & 185.7 & 168.9 \\
\hline $44 / 23$ & 34.54 & 37.21 & 21.72 & 23.33 & 19.74 & 18.11 & 183.2 & 182.9 & 196.7 & 196.4 & 152.9 & 139.4 \\
\hline
\end{tabular}

NOTE: Subscript 1 refers to normal assembly loading. Subscript 2 refers to checkerboard assembly loading. 
TABLE LXXVIII. Normalized Relative Calculated and Measured Plutonium Fission Rates and $238 \mathrm{U}$ Capture Rates for the Colunn 23,

$90^{\circ}$ Traverse, in the GCFR Phase I Normal and Checkerboard Core Configurations

\begin{tabular}{|c|c|c|c|c|c|c|c|c|}
\hline $\begin{array}{c}\text { Matrix } \\
\text { Location }\end{array}$ & $\begin{array}{l}\text { Meas }_{1} \\
239 \\
f\end{array}$ & $\begin{array}{c}\mathrm{Meas}_{2} \\
238 \\
\mathrm{~F}\end{array}$ & $\begin{array}{c}\mathrm{Calc}_{1} \\
239\end{array}$ & $\begin{array}{c}\mathrm{CaIc}_{2} \\
239\end{array}$ & $\begin{array}{c}\text { Meas } \\
238 \\
\mathrm{c}\end{array}$ & $\begin{array}{c}\text { Meas }_{2} \\
238 \\
c\end{array}$ & $\begin{array}{c}\mathrm{Calc}_{1} \\
238 \\
\mathrm{c}\end{array}$ & $\begin{array}{c}\mathrm{Calc}_{2} \\
238 \\
\mathrm{c}\end{array}$ \\
\hline $\mathrm{S} 23 / 23$ & 995.9 & 993.3 & 1000 & 1000 & 991.1 & 988.9 & 1000 & 1000 \\
\hline $\mathrm{S} 24 / 23$ & 991.8 & - & 996.8 & 996.6 & 1000.9 & - & 997.2 & 997.2 \\
\hline$S 25 / 23$ & 990.3 & 993.1 & 987.1 & 986.3 & 991.2 & 1005.2 & 987.7 & 968.5 \\
\hline $\mathrm{S} 26 / 23$ & 965.9 & - & 971.1 & 969.3 & 976.6 & - & 971.6 & 969.8 \\
\hline $\mathrm{S} 27 / 23$ & 974.2 & 961.4 & 948.9 & 945.7 & 957.4 & 964.9 & 949.9 & 946.6 \\
\hline$S 28 / 23$ & 925.5 & - & 920.8 & 915.9 & 938.9 & - & 922.0 & 917.0 \\
\hline $\mathrm{S} 29.23$ & 891.3 & 886.7 & 887.0 & 880.2 & 906.0 & 894.1 & 888.8 & 881.6 \\
\hline $\mathrm{S} 30 / 23$ & - & - & - & 839.0 & - & - & - & 840.9 \\
\hline $\mathrm{s} 31 / 23$ & 814.2 & 808.7 & 804.1 & 792.7 & 820.8 & 805.5 & 807.8 & 795.5 \\
\hline$\$ 32 / 23$ & 761.8 & - & 756.0 & 742.0 & 786.8 & - & 761.0 & 746.0 \\
\hline $\mathrm{S} 33 / 23$ & 705.1 & 715.2 & 704.1 & 687.4 & 729.1 & 715.8 & 711.3 & 693.0 \\
\hline $534 / 23$ & 651.0 & - & 649.1 & 629.8 & 675.6 & - & 658.8 & 637.6 \\
\hline$S 35 / 23$ & 602.6 & 602.1 & 591.8 & 569.8 & 622.1 & 620.2 & 605.1 & 580.7 \\
\hline $536 / 23$ & 553.1 & - & 532.9 & 508.4 & 587.1 & - & 550.9 & 523.3 \\
\hline $537 / 23$ & 498.6 & 483.8 & 473.5 & 446.7 & 519.4 & 515.3 & 497.4 & 466.9 \\
\hline$S 38 / 23$ & 434.5 & - & 414.9 & 386.0 & 472.0 & - & 446.5 & 413.3 \\
\hline $539 / 23$ & 389.3 & 380.1 & 358.7 & 328.4 & 421.1 & 422.9 & 400.4 & 365.1 \\
\hline $540 / 23$ & 321.2 & 329.8 & 298.8 & 271.1 & 375.4 & 362.7 & 353.3 & 319.7 \\
\hline $541 / 23$ & 274.2 & 276.7 & 252.8 & 229.8 & 318.1 & 307.5 & 285.6 & 258.4 \\
\hline$\$ 42 / 23$ & 234.4 & 236.6 & 202.4 & 184.0 & 263.1 & 259.0 & 230.2 & 208.4 \\
\hline $543 / 23$ & 201.4 & 203.0 & 161.8 & 147.4 & 208.2 & 207.3 & 180.5 & 163.6 \\
\hline$\$ 44 / 23$ & 186.7 & 185.8 & 133.3 & 212.8 & 172.9 & 169.2 & 139.7 & 126.8 \\
\hline
\end{tabular}

Note: Subscript 1 refers to normal assembly loading.

Subscript 2 refers to checkerboard assembly loading.

TABLE LXXIX. Ratio of Reaction Rates at Monftor Foil Location (S16/12) and at Diagonally Opposite Location in Checkerboard Octant.

\begin{tabular}{lccc}
\hline Reaction Rate & Normal Core & Checkerbourd Core & Ratio Checkerboard/Norma] \\
\hline 235 U Fission & 0.9639 & 0.9630 & 0.9991 \\
$238_{\mathrm{U}}$ Fission & 0.9641 & 0.9592 & 0.9949 \\
$238_{\mathrm{U}}$ Capture & 0.9542 & 0.9539 & 0.9997 \\
\hline
\end{tabular}

TABLE LXXX. Sunmary of the $\mathrm{CH}_{2}$ Mass and Atom Concentrations Used in the Simulated Steam Entry Experiment

\begin{tabular}{|c|c|c|c|c|c|c|c|}
\hline \multirow[b]{2}{*}{ No. } & \multirow{2}{*}{$\begin{array}{l}\text { Weight of } \mathrm{CH}_{2} \text { in } \\
\text { 2one } \\
(\mathrm{gm})\end{array}$} & \multirow{2}{*}{$\begin{array}{c}\text { Effective Density } \\
\text { of } \mathrm{CH}_{2} \text { in Zone } \\
\text { (Homogeneous) } \\
\mathrm{g} / \mathrm{cm}^{3}\end{array}$} & \multirow{2}{*}{$\begin{array}{l}\text { Equivalent Steam } \\
\left(\mathrm{H}_{2} \mathrm{O}\right) \text { Density in } \\
\text { Zone } \\
\text { (Homogeneous) } \\
\mathrm{g} / \mathrm{cm}^{3}\end{array}$} & \multicolumn{2}{|c|}{$\begin{array}{c}\text { Concentration of Hydrogen } \\
\text { (atoms/cm-barn) }\end{array}$} & \multicolumn{2}{|c|}{$\begin{array}{l}\text { Concentration of } \mathrm{C} \\
\text { (atoms/cm-barn) }\end{array}$} \\
\hline & & & & $\begin{array}{l}\text { In Unit } \\
\text { Cel1 }\end{array}$ & $\begin{array}{l}\text { In Vold } \\
\text { Channel }\end{array}$ & $\begin{array}{c}\text { In Unit } \\
\text { Cell }\end{array}$ & $\begin{array}{l}\text { In Void } \\
\text { Channel }\end{array}$ \\
\hline 1 & 784.0 & 0.0169 & 0.0217 & 0.001450 & 0.003064 & 0.000725 & 0.001532 \\
\hline 2 & 408.2 & 0.00878 & 0.0113 & 0.000755 & 0.001595 & 0.000378 & 0.000798 \\
\hline 3 & 373.8 & 0.00804 & 0.0103 & 0.000691 & 0.001461 & 0.000346 & 0.000730 \\
\hline 4 & 194.4 & 0.00418 & 0.00537 & 0.000360 & 0.000760 & 0.000180 & 0.000380 \\
\hline
\end{tabular}

a The vold fraction (in the unit cell) was taken to be $53 \%$.

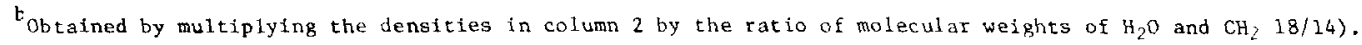


TABLE LXXXI. Inverse Kinetics Data

\begin{tabular}{|c|c|c|c|c|c|}
\hline \multirow[t]{2}{*}{ Date } & \multirow[t]{2}{*}{ Configuration } & \multirow[t]{2}{*}{$\begin{array}{l}\text { Core Temp } \\
{ }^{\circ} \mathrm{C}\end{array}$} & \multirow[t]{2}{*}{$\begin{array}{l}\text { ZPR-9 }{ }^{10} \mathrm{~B} \\
\text { Rods } \\
\text { Dropped }\end{array}$} & \multicolumn{2}{|c|}{$\begin{array}{l}\text { Subcriticality of configuration } \\
\text { corrected to } 25^{\circ} \mathrm{C}, \mathrm{Ih}\end{array}$} \\
\hline & & & & $S P-10$ & $\mathrm{SP}-11$ \\
\hline \multirow[t]{5}{*}{$5 / 21 / 75$} & Full density steam & 26.77 & 9 & -81.013 & -81.248 \\
\hline & & 26.03 & 8 & -78.870 & -79.060 \\
\hline & & 26.00 & 6 & -78.398 & -78.206 \\
\hline & & 25.98 & 5 & -76.594 & -76.576 \\
\hline & & Average & & $-78.687 \pm 0.906$ & $-78.737 \pm 0.968$ \\
\hline \multirow[t]{5}{*}{$5 / 22 / 75$} & Half density steam, & 25.96 & 9 & -167.686 & -168.247 \\
\hline & checkerboard loading & 25.50 & 8 & -170.343 & -170.236 \\
\hline & & 25.23 & 6 & -166.402 & -165.420 \\
\hline & & 25.15 & 5 & -164.805 & -164.777 \\
\hline & & Average & & $-167.289 \pm 1.166$ & $-167.141 \pm 1.266$ \\
\hline \multirow[t]{5}{*}{$5 / 23 / 75$} & Half density steam, & 26.32 & 9 & -178.065 & -178.083 \\
\hline & perforated foam loading & 25.74 & 8 & -180.868 & -181.775 \\
\hline & & 25.47 & 6 & -178.744 & -171.396 \\
\hline & & 25.36 & 5 & -176.012 & -174.860 \\
\hline & & Average & & $-178.405 \pm 1.001$ & $-176.445 \pm 2.217$ \\
\hline \multirow[t]{5}{*}{$5 / 27 / 75$} & Quarter density steam, & 25.76 & 9 & -210.437 & -211.265 \\
\hline & checkerboard loading & 25.40 & 8 & -211.383 & -210.793 \\
\hline & of perforated foam & 25.04 & 6 & -213.327 & -213.512 \\
\hline & & 24.94 & 5 & -206.722 & -206.139 \\
\hline & & Average & & $-210.440 \pm 1.394$ & $-210.393 \pm 1.560$ \\
\hline
\end{tabular}

TABLE LXXXII. Analysis of Simulated Steam Entry Experiment

$p_{1}=$ Worth of $10_{\mathrm{B}}$ Rods Inserted - (Subcriticality)

$P_{i}-\rho_{R E F}=$ (Subcriticality) REF - (Subcriticality) ${ }_{1}$

\begin{tabular}{|c|c|c|c|c|c|c|c|}
\hline \multirow{2}{*}{$\frac{\text { Date/Day }}{5-21-75 / 141}$} & \multirow{2}{*}{$\begin{array}{l}\text { Configuration } \\
\text { Full density polyethylene } \\
\text { in zone }\end{array}$} & \multirow{2}{*}{$\frac{\begin{array}{c}\text { Mass of } \\
\text { Polyethylene } \\
(\mathrm{kg})\end{array}}{0.7840}$} & \multirow{2}{*}{$\begin{array}{c}\begin{array}{c}\text { Excess }\left(25^{\circ} \mathrm{C}\right) \\
\text { Ih } \\
(- \text { Subcritfcalify })\end{array} \\
-78.737 \pm 0.968\end{array}$} & \multicolumn{2}{|c|}{$\begin{array}{l}\text { Worth } \\
\left.\operatorname{lin}_{i}{ }^{a, b}\right) \\
\text { IhEF }\end{array}$} & \multicolumn{2}{|c|}{$\begin{array}{c}\text { Specific } \\
\text { Worth } \\
\mathrm{Ih} / \mathrm{kg}\end{array}$} \\
\hline & & & & 157.21 & \pm 2.60 & $200.52 \pm$ & 3.32 \\
\hline $5-22-75 / 142$ & $\begin{array}{l}\text { llalf density (checkerboard } \\
\text { polyethylene in zone }\end{array}$ & 0.4082 & $-167.141 \pm 1.266$ & 68.99 & \pm 2.72 & $169.01 \pm$ & 6.66 \\
\hline $5-23-75 / 143$ & $\begin{array}{l}\text { Half density (perforated) } \\
\text { polyethylene in zone }\end{array}$ & 0.3738 & $-176.445 \pm 2.217$ & 59.87 & \pm 3.28 & $160.17 \pm$ & 8.77 \\
\hline $5-27-75 / 144$ & $\begin{array}{l}\text { Quarter density (perforated) } \\
+ \text { checkerboard) polyethylene } \\
\text { in zone }\end{array}$ & 0.1944 & $-210.393 \pm 1.560$ & 26.107 & \pm 2.87 & $134.30 \pm$ & 14.76 \\
\hline $5-29-75 / 148$ & Reference & 0.0 & $-237.232 \pm 1.349$ & 0.0 & & - & \\
\hline
\end{tabular}

Wcrth of reference configuration $=61.97 \pm 3.96 \mathrm{Ih}$

${ }^{b}$ Decay worth of $241 \mathrm{Pu} \quad=-0.183 \pm 0.003 \mathrm{Ih} / \mathrm{day}$

${ }^{C}$ On 1y the experimentally determined subcritfcalty values were used in this evaluation. 
TABLE LXXXIII. Comparis on of the Benoist Coefficients for the Normal and Steam-filled Cores

\begin{tabular}{|c|c|c|c|c|}
\hline \multirow[b]{2}{*}{ Group No. } & \multicolumn{2}{|c|}{${ }^{\mathrm{D}} \perp$} & \multicolumn{2}{|c|}{${ }^{D} \|$} \\
\hline & Normal & With steam ${ }^{\mathrm{a}}$ & Normal & With steam \\
\hline 1 & 1.0012 & 1.0015 & 1.0477 & 1.0500 \\
\hline 2 & 1.0014 & 1.0017 & 1.0502 & 1.0517 \\
\hline 3 & 1.0018 & 1.0022 & 1.0572 & 1.0576 \\
\hline 4 & 1.0021 & 1.0026 & 1.0609 & 1.0598 \\
\hline 5 & 1.0029 & 1.0035 & 1.0759 & 1.0737 \\
\hline 6 & 1.0047 & 1.0055 & 1.1099 & 1.1020 \\
\hline 7 & 1.0042 & 1.0049 & 1.0973 & 1.0867 \\
\hline 8 & 1.0093 & 1.0109 & 1.1595 & 1.1434 \\
\hline 9 & 1.0087 & 1.0100 & 1.1427 & 1.1248 \\
\hline 10 & 1.0100 & 1.0116 & 1.1543 & 1.1317 \\
\hline 11 & 1.0123 & 1.0141 & 1.1607 & 1.1392 \\
\hline 12 & 1.0140 & 1.0162 & 1.1695 & 1.1468 \\
\hline 13 & 1.0166 & 1.0197 & 1.1752 & 1.1569 \\
\hline 14 & 1.0126 & 1.0144 & 1.1675 & 1.1378 \\
\hline 15 & 1.0154 & 1.0178 & 1.1653 & 1.1464 \\
\hline 16 & 1.0259 & 1.0301 & 1.1901 & 1.1843 \\
\hline 17 & 1.0212 & 1.0244 & 1.1755 & 1.1651 \\
\hline 18 & 1.0224 & 1.0264 & 1.1825 & 1.1733 \\
\hline 19. & 1.0235 & 1.0274 & 1.1973 & 1.1822 \\
\hline 20 & 1.0260 & 1.0310 & 1.2060 & 1.1949 \\
\hline 21 & 1.0275 & 1.0334 & 1.2116 & 1.2025 \\
\hline 22 & 1.0296 & 1.0364 & 1.2037 & 1.2052 \\
\hline 23 & 1.0381 & 1.0477 & 1.2505 & 1.2500 \\
\hline 24 & 1.0591 & 1.0789 & 1.3187 & 1.3405 \\
\hline 25 & 1.0536 & 1.0758 & 1.3174 & 1.3469 \\
\hline 26 & 1.0935 & 1.1229 & 1.4202 & 1.4707 \\
\hline 27 & 1.0294 & 1.0377 & 1. 2160 & 1.2157 \\
\hline 28 & 1.1359 & 1.2936 & 1.5200 & 1.8073 \\
\hline 29 & 1.4960 & 1.6262 & 2.2336 & 2.4027 \\
\hline
\end{tabular}

${ }^{a_{F u} 11}$ density steam loading.

TABLE LXXXIV. Summary of Experimental and Calculated Results for Steam Zone Experiments

\begin{tabular}{|c|c|c|c|c|c|c|}
\hline \multirow[b]{2}{*}{ No. } & \multirow[b]{2}{*}{$\begin{array}{l}\text { Weight of } \\
\mathrm{CH}_{2} \text { in Zone } \\
\text { (kg) }\end{array}$} & \multirow{2}{*}{\multicolumn{2}{|c|}{ Experimental }} & \multicolumn{3}{|c|}{ Calculated Reactivity Worth (Ih) } \\
\hline & & & & $\begin{array}{l}\text { Rz Model, } \\
\Delta \mathrm{k} \text { Result }\end{array}$ & $\begin{array}{l}\text { RZ Model, } \\
\text { Exact } \\
\text { Perturbation } \\
\text { Results }\end{array}$ & $\begin{array}{l}\text { XY Model, } \\
\text { Exact } \\
\text { Perturbation } \\
\text { Results }\end{array}$ \\
\hline 1 & 0.7840 & 157.21 & \pm 2.60 & 207.82 & 222.39 & 229.84 \\
\hline 2 & 0.4082 & 68.99 & \pm 2.72 & 73.75 & 67.77 & - \\
\hline 3 & 0.3738 & 59.87 & \pm 3.28 & 58.28 & - & - \\
\hline 4 & 0.194 & 26.107 & \pm 2.87 & 6.57 & 7.95 & - \\
\hline
\end{tabular}


TABLE LXXXV. Comparison of the Integral Parameters of the Dry Reference Assemb1y and the Experimental Assembly with $\mathrm{CH}_{2}$ in Test Zone

\begin{tabular}{|c|c|c|}
\hline Parameter & Dry As sembly & $\begin{array}{l}\text { Agsembly with } \\
\mathrm{CH}_{2} \text { in Test Zone }\end{array}$ \\
\hline 1. $k_{\text {eff }}$ & 0.9718 & 0.9724 \\
\hline 2. $k_{\infty}$ (Zone) & 1.5629 & 1.4966 \\
\hline 3. $L^{b}$ (zone) & $37.94 \%$ & $35.16 \%$ \\
\hline 4. $k_{\infty}($ Co $r e)$ & 1.5439 & 1.5397 \\
\hline 5. L (Core) & $37.18 \%$ & $36.96 \%$ \\
\hline 6. $k_{\infty}$ (As semb1y) & 1.3169 & 1.3152 \\
\hline 7. L (As semb $1 y)$ & $26.38 \%$ & $26.24 \%$ \\
\hline
\end{tabular}

TABLE LXXXVI. Isotopic Breakdown of Reaction Rates in the Central Test Zone - With Steam in Test Zone

\begin{tabular}{|c|c|c|c|c|c|}
\hline Isotope & Total Source & Fission Source & Fission & Absorption & Capture \\
\hline${ }^{23}{ }^{8} \mathrm{Pu}$ & $2.4810^{-4}$ & $2.4907^{-4}$ & $2.5566^{-4}$ & $1.8125^{-4}$ & $1.0386^{-4}$ \\
\hline $239{ }^{9} \mathrm{Pu}$ & $8.3873^{-1}$ & $8.4191^{-1}$ & $8.3681^{-1}$ & $5.4110^{-1}$ & $2.3354^{-1}$ \\
\hline $240 \mathrm{Pu}$ & $2.8516^{-2}$ & $2.8619^{-2}$ & $2.7148^{-2}$ & $3.0518^{-2}$ & $3.4022^{-2}$ \\
\hline $241 \mathrm{Pu}$ & $1.5845^{-2}$ & I. $5894^{-2}$ & $1.5601^{-2}$ & $9.4689^{-3}$ & $3.0907^{-3}$ \\
\hline${ }^{242} \mathrm{Pu}$ & $3.0016^{-4}$ & $3.0078^{-4}$ & $2.8808^{-4}$ & $3.5564^{-4}$ & $4.2591^{-4}$ \\
\hline $235 \mathrm{U}$ & $7.6717^{3}$ & $7.6987^{-3}$ & $9.1926^{-3}$ & $6.0944^{-3}$ & $2.8722^{-3}$ \\
\hline${ }^{2}{ }^{38} \mathrm{U}$ & $1.0633^{-1}$ & $1.0308^{-1}$ & $1.0873^{-1}$ & $3.3483^{-1}$ & $5.6999-1$ \\
\hline $\mathrm{Fe}$ & $4.3091^{-5}$ & $0.0000^{-1}$ & $0.0000^{-1}$ & $3.4064^{-2}$ & $6.9493^{-2}$ \\
\hline $\mathrm{Ni}$ & $2.7856^{-6}$ & $0.0000^{-1}$ & $0.0000^{-1}$ & $1.0212^{-2}$ & $2.0832^{-2}$ \\
\hline $\mathrm{Cr}$ & $3.0719^{-5}$ & $0.0000^{-1}$ & $0.0000^{-1}$ & $1.2179^{-2}$ & $2.4845^{-2}$ \\
\hline Mn & $3.2942^{-6}$ & $0.0000^{-1}$ & $0.0000^{-1}$ & $4.7321^{-3}$ & $9.6537^{-3}$ \\
\hline${ }^{16} 0$ & $0.0000^{-1}$ & $0.0000^{-1}$ & $0.0000^{-1}$ & $2.9102^{-3}$ & $5.9368^{-3}$ \\
\hline$A m$ & $2.2300^{3}$ & $2.2389^{-3}$ & $1.9767^{-3}$ & $4.0217-^{3}$ & $6.1485^{-3}$ \\
\hline Mo & $4.6041^{-5}$ & $0.0000^{-1}$ & $0.0000^{-1}$ & $9.2816^{-3}$ & $1.8935^{-2}$ \\
\hline C & $0.0000^{-1}$ & $0.0000^{-1}$ & $0.0000^{-1}$ & $7.9161^{-6}$ & $1.6149^{-5}$ \\
\hline Hyd & $0.0000^{-1}$ & $0.0000^{-1}$ & $0.0000^{-1}$ & $4.3327^{-5}$ & $8.8389^{-5}$ \\
\hline
\end{tabular}


TABLE LXXXVII. Isotopic Breakdown of Reaction Rates in the Centra1 Test Zone - Dry Reference Case

\begin{tabular}{llllll}
\hline Isotope & Total Source & Fission Source & Fission & Absorption & Capture \\
\hline${ }^{2{ }^{8} \mathrm{Pu}}$ & $5.0196^{-5}$ & $5.1319^{-5}$ & $5.0202^{-5}$ & $1.0067^{-4}$ & $1.514^{-4}$ \\
$2{ }^{39} \mathrm{Pu}$ & $8.939 \mathrm{I}^{-1}$ & $9.1570^{-1}$ & $9.2290^{-1}$ & $6.8980^{-1}$ & $4.5508^{-1}$ \\
$2{ }^{40} \mathrm{Pu}$ & $5.5056^{-3}$ & $5.5547^{-3}$ & $4.5357^{-3}$ & $6.1538^{-2}$ & $1.1894^{-1}$ \\
$2{ }^{4} \mathrm{I}_{\mathrm{Pu}}$ & $1.7756^{-2}$ & $1.8168^{-2}$ & $1.7995^{-2}$ & $1.1637^{-2}$ & $5.2354^{-3}$ \\
$2{ }^{242} \mathrm{Pu}$ & $6.7306^{-5}$ & $6.5143^{-5}$ & $5.2899^{-5}$ & $1.4600^{-3}$ & $2.8769^{-3}$ \\
$235_{\mathrm{U}}$ & $7.5257^{-3}$ & $7.7002^{-3}$ & $9.2705^{-3}$ & $6.6732^{-3}$ & $4.0579^{-3}$ \\
$238 \mathrm{U}$ & $7.1634^{-2}$ & $5.1993^{-2}$ & $4.4575^{-2}$ & $1.4091^{-1}$ & $2.3792^{-1}$ \\
$\mathrm{Fe}$ & $1.2464^{-3}$ & $0.0000^{-1}$ & $0.0000^{-1}$ & $4.2482^{-2}$ & $8.5258^{-2}$ \\
$\mathrm{Ni}$ & $6.7889^{-5}$ & $0.0000^{-1}$ & $0.0000^{-1}$ & $8.9026^{-3}$ & $1.7867^{-2}$ \\
$\mathrm{Cr}$ & $5.6132^{-4}$ & $0.0000^{-1}$ & $0.0000^{-1}$ & $1.0337^{-2}$ & $2.0745^{-2}$ \\
$\mathrm{Mn}$ & $9.5334^{-5}$ & $0.0000^{-1}$ & $0.0000^{-1}$ & $6.4813^{-3}$ & $1.3008^{-2}$ \\
$16 \mathrm{O}$ & $0.0000^{-1}$ & $0.0000^{-1}$ & $0.0000^{-1}$ & $5.5242^{-3}$ & $1.1087^{-2}$ \\
$\mathrm{Am}$ & $7.4728^{4}$ & $7.6620^{-4}$ & $6.2064^{-4}$ & $9.0536^{-3}$ & $1.7545^{-2}$ \\
$\mathrm{Mo}$ & $8.3546^{-4}$ & $0.0000^{-1}$ & $0.0000^{-1}$ & $5.1008^{-3}$ & $1.0237^{-2}$ \\
\hline
\end{tabular}

TABLE LXXXVIII. Analysis of Steam Worth Components

\begin{tabular}{|c|c|c|}
\hline & $\begin{array}{l}\text { Central } \delta k / k^{2} \\
\text { Steam Zone (with } \\
\text { Full density steam) }\end{array}$ & $\begin{array}{c}\text { Central } \\
\text { Smal1 Sample }\end{array}$ \\
\hline Capture & $-3.078679 \times 10^{-5}$ & $-2.58711 \times 10^{-5}$ \\
\hline Scattering Loss & $-1.082172 \times 10^{-1}$ & $-1.2277353 \times 10^{-1}$ \\
\hline Scattering Gain & $1.111151 \times 10^{-1}$ & $1.201843 \times 10^{-1}$ \\
\hline Fission Loss & $3.701872 \times 10^{-4}$ & - \\
\hline Fission Gain & $-2.484402 \times 10^{-4}$ & - \\
\hline Net Fission & $-6.484402 \times 10^{-4}$ & - \\
\hline Net Scattering & $+2.8979 \times 10^{-3}$ & -2.58923 \\
\hline Leakage & $5.9293 \times 10^{-5}$ & 2.950132 \\
\hline Total $\Delta \mathrm{k} / \mathrm{k}^{2}$ & $2.369328 \times 10^{-3}$ & $-2.585683 \times 10^{-3}$ \\
\hline
\end{tabular}

NOTE: The calculated (FOP) Small $\mathrm{CH}_{2}$ Sample Central Worth was negative while the measured worth was positive. From this Table it is clear that the magnitude and sign of the calculated worth depends sensitively on the Scattering Loss and Scattering Gain terms. Small changes in the shapes of the real and adjoint spectra can alter the sign of the calculated worth. 
TABLE B-I. Calculated $238 \mathrm{U} / 235 \mathrm{U}$ (F8/F5) Fission Rate Ratios

\begin{tabular}{|c|c|c|c|c|c|c|c|c|}
\hline \multicolumn{3}{|c|}{$0^{\circ}$ Traverse } & \multicolumn{3}{|c|}{$45^{\circ}$ Traverse } & \multicolumn{3}{|c|}{$90^{\circ}$ Traverse } \\
\hline \multirow{2}{*}{$\begin{array}{l}\text { Matrix } \\
\text { Location }\end{array}$} & \multicolumn{2}{|c|}{$(F 8 / F 5) \times 10^{-2}$} & \multirow{2}{*}{$\begin{array}{l}\text { Matrix } \\
\text { Location }\end{array}$} & \multicolumn{2}{|c|}{$(F 8 / F 5) \times 10^{-2}$} & \multirow{2}{*}{$\begin{array}{l}\text { Matrix } \\
\text { Location }\end{array}$} & \multicolumn{2}{|c|}{$(\mathrm{F} 8 / \mathrm{F} 5) \times 10^{-2}$} \\
\hline & Normal & Checkerboard & & Normal & Checkerboard & & Norma1 & Checkerboard \\
\hline S23/23 & 2.94 & 2.91 & S23/23 & 2.94 & 2.91 & $523 / 23$ & 2.94 & 2.91 \\
\hline S23/24 & 2.94 & 2.91 & S24/24 & 2.94 & 2.91 & S24/23 & 2.94 & \\
\hline $\mathrm{S} 23 / 25$ & 2.94 & 2.91 & $S 25 / 25$ & 2.94 & 2.91 & $S 25 / 23$ & 2.94 & 2.91 \\
\hline$S 23 / 26$ & 2.94 & 2.91 & $S 26 / 26$ & 2.94 & 2.91 & $S 26 / 23$ & 2.94 & \\
\hline S23/27 & 2.94 & 2.91 & $527 / 27$ & 2.94 & 2.91 & $527 / 23$ & 2.94 & 2.91 \\
\hline S23/28 & 2.94 & 2.91 & $528 / 28$ & 2.93 & 2.90 & $S 28 / 23$ & 2.94 & \\
\hline S2 3/29 & 2.94 & 2.91 & S29/29 & 2.93 & 2.90 & $S 29 / 23$ & 2.94 & 2.91 \\
\hline$S 23 / 30$ & 2.94 & 2.91 & $\mathrm{~s} 30 / 30$ & 2.91 & 2.88 & $531 / 23$ & 2.93 & 2.90 \\
\hline$S 23 / 31$ & 2.93 & 2.90 & $531 / 31$ & 2.87 & 2.84 & $S 32 / 23$ & 2.92 & \\
\hline$S 23 / 32$ & 2.93 & 2.89 & $532 / 32$ & 2.78 & 2.77 & S33/23 & 2.91 & 2.88 \\
\hline$S 23 / 33$ & 2.91 & 2.88 & $533 / 33$ & 2.60 & 2.59 & $534 / 23$ & 2.88 & \\
\hline$S 23 / 34$ & 2.90 & 2.87 & $534 / 34$ & 2.07 & 2.06 & $535 / 23$ & 2.84 & 2.83 \\
\hline$S 23 / 35$ & 2.86 & 2.83 & S35/35 & 1.36 & 1.35 & $536 / 23$ & 2.77 & \\
\hline$S 23 / 36$ & 2.81 & 2.78 & $536 / 36$ & 0.871 & 0.86 & $537 / 23$ & 2.66 & 2.66 \\
\hline $523 / 37$ & 2.71 & 2.68 & S37/37 & 0.597 & 0.591 & $538 / 23$ & 2.46 & \\
\hline$S 23 / 38$ & 2.52 & 2.50 & & & & S39/23 & 2.11 & 2.12 \\
\hline S23/39 & 2.18 & 2.16 & & & & $540 / 23$ & 1.53 & 1.52 \\
\hline$S 23 / 40$ & 1.60 & 1.58 & & & & $S 41 / 23$ & 1.02 & 1.01 \\
\hline S23/41 & 1.09 & 1.08 & & & & $S 42 / 23$ & 0.733 & 0.727 \\
\hline S23/42 & 0.821 & 0.807 & & & & $543 / 23$ & 0.535 & 0.531 \\
\hline$S 23 / 43$ & 0.638 & 0.629 & & & & $544 / 23$ & 0.382 & 0.379 \\
\hline$S 23 / 44$ & 0.518 & 0.510 & & & & & & \\
\hline
\end{tabular}


Internal:
J. A. Kyger
A. Amorosi
R. Avery
L. Burris
S. A. Davis
B. R. T. Frost
D. C. Rardin
R. G. Staker
R. J. Teunis
C. E. Ti 11
R. S. Zeno
C. E. Dickerman
H. K. Fauske
S. Fistedis
B. D. LaMar
J. F. Marchaterre
H. O. Monson
R. Sevy
L. G. LeSage (5)
W. M. Stacey
W. J. Sturm
H. Bigelow

T. J. Yule

N. M. O'Fallon

D. C. Wade

R. D. McKnight

R. W. Schaefer

G. K. Rusch

A. B. Smith

E. M. Gelbard

R. E. Grajek

R. J. Armani

J. D. Williams

W. L. Woodruff

F. W. Thalgott

P. I. Amund son

S. G. Carpenter

M. Lineberry

G. G. Simons

R. J. Forrester

A. B. Krisciunas

ANL Contract File

ANL Libraries (5)

TIS Files

\section{Externa1:}

ERDA-TIC, for distribution per UC-77 (176)
Manager, Chicago Operations Office
Chief, Chicago Patent Group
Director, ERDA-NRA (2)
Director, Reactor Programs Div., ERDA-CH
Director, CH-INEL
President, Argonne Universities Association
Applied Physics Division Review Committee:
R. M. Brugger, Univ. of Missouri, Columbia
Paul Greebler, General Electric Co., Sunnyvale
R. L. Hellens, Combustion Engineering, Inc.
J. M. Hendrie, Brookhaven National Laboratory
J. S. King, Univ. of Michigan
W. B. Loewenstein, Electric Power Research Institute 